\title{
Rhodium-Catalyzed Denitrogenative Diazole-Triazole Coupling Toward aza-Bridged Structures and Imidazole-Based Chelating Ligands
}

Julia O. Strelnikova, Alexander N. Koronatov, Nikolai V. Rostovskii, Alexander F. Khlebnikov, Olesya V. Khoroshilova, Mariya A. Kryukova, and Mikhail S. Novikov*

Institute of Chemistry, St. Petersburg State University, 7/9 Universitetskaya nab., St. Petersburg 199034, Russia

* E-mail: m.novikov@spbu.ru

\section{Table of Contents}

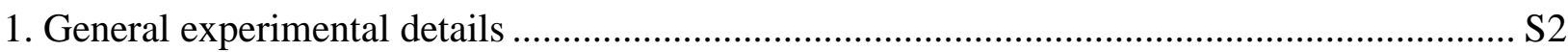

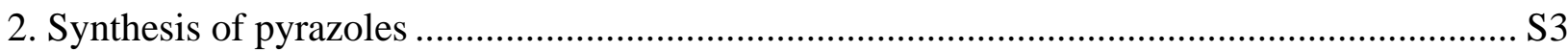

3. Synthesis of 1-((4-methyl-3-nitrophenyl)sulfonyl)-4-phenyl-1H-1,2,3-triazole (2e) ............. S7

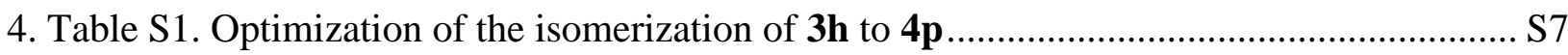

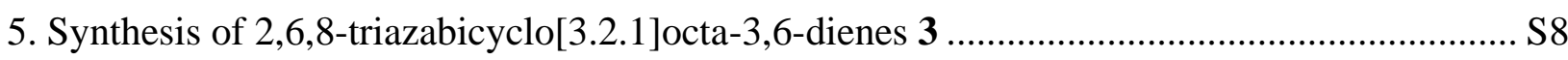

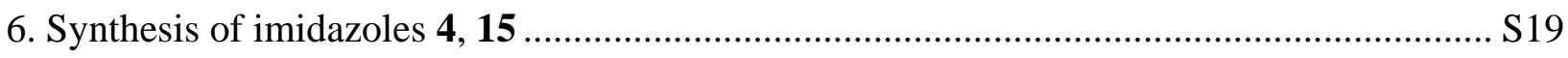

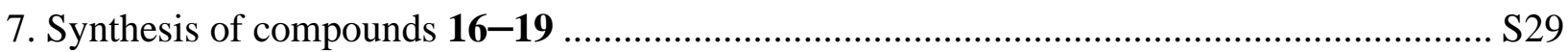

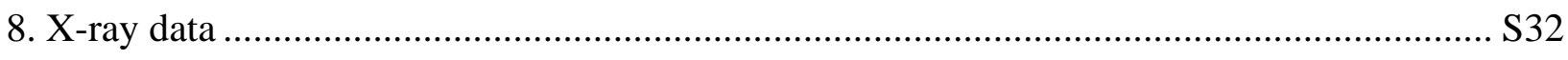

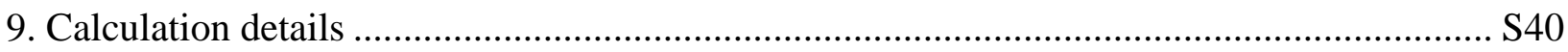

10. Figure S6. Energy profile for the transformation of compound 6 to bicycle 3zb. .............. S60

11. Figure S7. Energy profile for the transformation of compound 3zb to imidazole $4 \mathbf{r} . . . . . . . . . S 61$

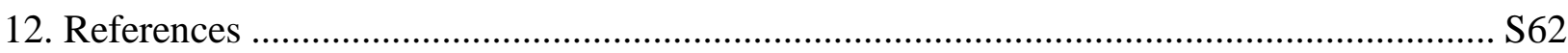

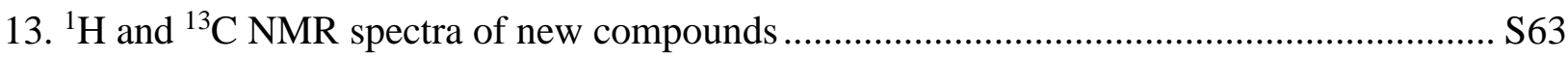




\section{General experimental details}

Melting points were determined on a Stuart Melting Point Apparatus SMP30 and are uncorrected. ${ }^{1} \mathrm{H}(400 \mathrm{MHz}),{ }^{13} \mathrm{C}(100 \mathrm{MHz})$ and ${ }^{19} \mathrm{~F}(376.5 \mathrm{MHz})$ NMR spectra were recorded on a Bruker AVANCE 400 spectrometer in solvents indicated below. Chemical shifts $(\delta)$ are reported in parts per million downfield from tetramethylsilane. IR spectra were recorded on a TENSOR 27 (Bruker) FT-IR spectrometer with $\mathrm{KBr}$ pellets in the range $4000-400 \mathrm{~cm}^{-1}$. High-resolution mass spectra were recorded on a Bruker MaXis mass spectrometer, electrospray ionization, positive mode. Thin-layer chromatography (TLC) was conducted on aluminum sheets precoated with $\mathrm{SiO}_{2}$ ALUGRAM SIL G/UV254. Column chromatography was performed on silica gel $60 \mathrm{M}$ $(0.04-0.063 \mathrm{~mm})$. All solvents were distilled and dried prior to use. Acetonitrile was distilled from $\mathrm{P}_{2} \mathrm{O}_{5}$ and redistilled from $\mathrm{K}_{2} \mathrm{CO}_{3}$. Toluene and tetrahydrofuran were distilled and stored over sodium metal. The catalyst $\mathrm{Rh}_{2}(\mathrm{Piv})_{4}$ was prepared by the reported procedure. ${ }^{1}$

Pyrazoles $1 \mathbf{a}, \mathbf{b},{ }^{2} \mathbf{1 c}-\mathbf{f},{ }^{3} \mathbf{1 h},{ }^{4} \mathbf{1 p}, \mathbf{1 z b}, \mathbf{1 z k},{ }^{5} \mathbf{1 r},{ }^{6} 1 \mathbf{s},{ }^{7} \mathbf{1 t}-\mathbf{y}, \mathbf{1 z a}, \mathbf{1 z c}, 1 \mathrm{ze},{ }^{8} \mathbf{1 z},{ }^{9} \mathbf{1 z d},{ }^{10} \mathbf{1 z f},{ }^{11} \mathbf{1 z g},{ }^{12}$ $\mathbf{1 z h},{ }^{13} \mathbf{1 z j},{ }^{14}$ and triazoles $\mathbf{2 a}-\mathbf{i}^{15}$ are known compounds and have full characterization data. All these compounds were prepared according to the reported procedures.

\section{Structures of starting pyrazoles 1}<smiles>c1ccc(-c2cnn(-c3ccccc3)c2)cc1</smiles>

1a<smiles>[R]c1cnn(-c2ccc([N+](=O)[O-])cc2[N+](=O)[O-])c1</smiles><smiles>Cc1ccccc1-n1cc(-c2ccccc2)cn1</smiles>

1b<smiles>[R]c1cnn(-c2ccc([N+](=O)[O-])cc2)c1</smiles>

1c, $R=P h$

1d, $\mathrm{R}=4-\mathrm{MeC}_{6} \mathrm{H}_{4}$

1e, $\mathrm{R}=4-\mathrm{MeOC}_{6} \mathrm{H}_{4}$

1f, $\mathrm{R}=4-\mathrm{ClC}_{6} \mathrm{H}_{4}$

1g, $\mathrm{R}=3-\mathrm{BrC}_{6} \mathrm{H}_{4}$

1h, $\mathrm{R}=4-\mathrm{NO}_{2} \mathrm{C}_{6} \mathrm{H}_{4}$

$1 \mathrm{i}, \mathrm{R}=$ naphthalen-2-yl

1j, $R=B r$

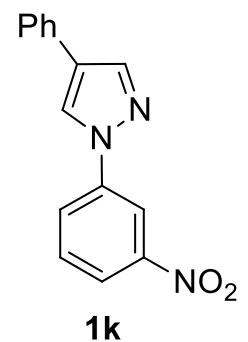

$1 \mathrm{k}$<smiles>c1ccc(-c2cnn(-c3cc(-c4ccccc4)no3)c2)cc1</smiles><smiles>[R]n1ncc2ccccc21</smiles><smiles>CCOCCO</smiles><smiles>CCCc1cnn(-c2ccccc2)c1C</smiles>

1q, $\mathrm{R}=4-\mathrm{NO}_{2} \mathrm{C}_{6} \mathrm{H}_{4}$ $1 \mathrm{r}, \mathrm{R}=\mathrm{Me}$

1s<smiles>[R]c1cnn(C)c1</smiles>

$$
\begin{aligned}
& \text { 1t, } \mathrm{R}=\mathrm{Ph} \\
& \text { 1u, } \mathrm{R}=4-\mathrm{MeC}_{6} \mathrm{H}_{4} \\
& \text { 1v, } \mathrm{R}=4-\mathrm{MeOC}_{6} \mathrm{H}_{4} \\
& \text { 1w, } \mathrm{R}=4-\mathrm{ClC}_{6} \mathrm{H}_{4} \\
& \text { 1x, } \mathrm{R}=4-\mathrm{BrC}_{6} \mathrm{H}_{4} \\
& \text { 1y, } \mathrm{R}=4-\mathrm{NO}_{2} \mathrm{C}_{6} \mathrm{H}_{4} \\
& \text { 1z, } \mathrm{R}=\text { naphthalen-2-yl } \\
& \text { 1za, } \mathrm{R}=\text { COPh }
\end{aligned}
$$

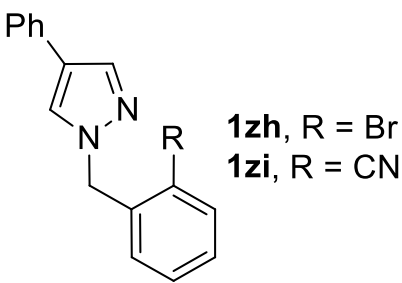

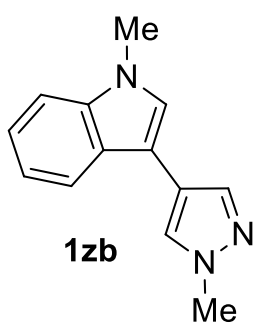<smiles>[R]n1cc(-c2ccccc2)cn1</smiles>
1zc, $R=E t$ 1zd, $\mathrm{R}=i-\mathrm{Pr}$ 1ze, $R=B n$ 1zf, $\mathrm{R}=\mathrm{CH}_{2} \mathrm{CH}_{2} \mathrm{Br}$ 1zg, $\mathrm{R}=\mathrm{CH}_{2} \mathrm{CO}_{2} \mathrm{Me}$<smiles>[X]C(=O)c1c(-c2ccccc2)cnn1C</smiles>

1zj, $X=O E t$ 1zk, $\mathrm{X}=\mathrm{NMe}_{2}$<smiles>COc1c(-c2ccccc2)cnn1C</smiles>

$1 z \mid$ 


\section{Structures of starting triazoles 2}

$\mathrm{Ph}$<smiles>[R]S(=O)(=O)n1cc(I)nn1</smiles><smiles>[R]c1cn([AsH2-])nn1</smiles>
2a, $\mathrm{R}=p$-Tol
2b, $\mathrm{R}=\mathrm{Me}$
2c, $\mathrm{R}=2,4,6-\mathrm{Me}_{3} \mathrm{C}_{6} \mathrm{H}_{2}$
2d, $\mathrm{R}=4-\mathrm{MeOC}_{6} \mathrm{H}_{4}$

2f, $\mathrm{R}=4-\mathrm{MeOC}_{6} \mathrm{H}_{4}$

2g, $\mathrm{R}=4-\mathrm{ClC}_{6} \mathrm{H}_{4}$

2h, $\mathrm{R}=4-\mathrm{CF}_{3} \mathrm{C}_{6} \mathrm{H}_{4}$

2i, $\mathrm{R}=4-\mathrm{NO}_{2} \mathrm{C}_{6} \mathrm{H}_{4}$

\section{Synthesis of pyrazoles}

\section{Synthesis of pyrazoles $1 \mathrm{~g}, \mathbf{1 i}, \mathbf{1 j}, \mathbf{1 1 - 0 , 1 q ^ { 3 }}$}

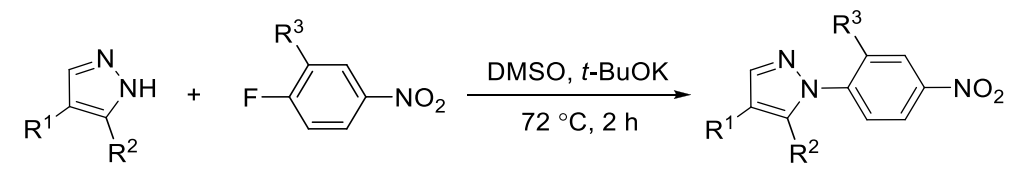

General procedure: To a solution of the substituted pyrazole $(1.25 \mathrm{mmol})$ in DMSO $(1.5 \mathrm{~mL})$ solid potassium tert-butoxide $(1.38 \mathrm{mmol})$ was added followed by the addition of 1-fluoro-4nitrobenzene or 1-fluoro-2,4-dinitrobenzene $(1.31 \mathrm{mmol})$ in DMSO $(0.5 \mathrm{~mL})$. The resulting mixture was heated in an oil bath at $72{ }^{\circ} \mathrm{C}$ under stirring for $2 \mathrm{~h}$. Then the mixture was cooled to room temperature and diluted with water $(20 \mathrm{~mL})$. The resulting precipitate of $N$-arylpyrazole 1 was filtered off, washed with water $(20 \mathrm{~mL})$ and dried in air at $50{ }^{\circ} \mathrm{C}$.

\section{4-(3-Bromophenyl)-1-(4-nitrophenyl)-1H-pyrazole (1g)}

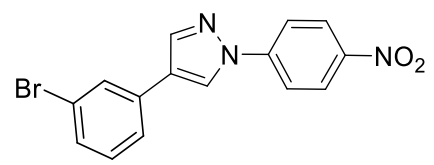

Obtained from 4-(3-bromophenyl)pyrazole and 1-fluoro-4-nitrobenzene according to the general procedure as a yellow solid (370 mg, yield 86\%); mp $191-193{ }^{\circ} \mathrm{C} ;{ }^{1} \mathrm{H}$ NMR (DMSO- $d_{6}$ ) $\delta 9.32$ $(\mathrm{s}, 1 \mathrm{H}), 8.44(\mathrm{~s}, 1 \mathrm{H}), 8.41(\mathrm{~d}, J=9.2 \mathrm{~Hz}, 2 \mathrm{H}), 8.15(\mathrm{~d}, J=9.2 \mathrm{~Hz}, 2 \mathrm{H}), 8.00(\mathrm{~s}, 1 \mathrm{H}), 7.76(\mathrm{~d}, J=$ $7.7 \mathrm{~Hz}, 1 \mathrm{H}), 7.47(\mathrm{~d}, J=8.6 \mathrm{~Hz}, 1 \mathrm{H}), 7.39(\mathrm{t}, J=7.8 \mathrm{~Hz}, 1 \mathrm{H}) ;{ }^{13} \mathrm{C}\left\{{ }^{1} \mathrm{H}\right\}$ NMR (DMSO-d $) \delta 145.4$, 144.3, 141.1, 134.2, 131.5, 130.1, 128.4, 126.6, 126.0, 124.8, 124.4, 122.9, 118.8; HRMS-ESI [M - $\mathrm{H}^{-}$calcd for $\mathrm{C}_{15} \mathrm{H}_{9}{ }^{79} \mathrm{BrN}_{3} \mathrm{O}_{2}{ }^{-}$341.9873; found 341.9873 .

\section{4-(Naphthalen-2-yl)-1-(4-nitrophenyl)-1H-pyrazole (1i)}

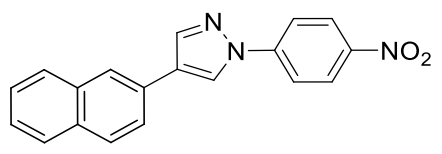

Obtained from 4-(naphthalen-2-yl)pyrazole and 1-fluoro-4-nitrobenzene according to the general procedure as a yellow solid (335 mg, yield 85\%); mp $203-205{ }^{\circ} \mathrm{C} ;{ }^{1} \mathrm{H}$ NMR (DMSO- $\left.d_{6}\right) \delta 9.37$ $(\mathrm{s}, 1 \mathrm{H}), 8.53(\mathrm{~s}, 1 \mathrm{H}), 8.42(\mathrm{~d}, J=9.1 \mathrm{~Hz}, 2 \mathrm{H}), 8.29(\mathrm{~s}, 1 \mathrm{H}), 8.20(\mathrm{~d}, J=9.1 \mathrm{~Hz}, 2 \mathrm{H}), 8.01-7.96$ $(\mathrm{m}, 1 \mathrm{H}), 7.95-7.88(\mathrm{~m}, 3 \mathrm{H}), 7.57-7.48(\mathrm{~m}, 2 \mathrm{H}) ;{ }^{13} \mathrm{C}\left\{{ }^{1} \mathrm{H}\right\} \mathrm{NMR}\left(\mathrm{DMSO}-d_{6}\right) \delta 145.3,144.4,141.3$, 
133.8, 132.6, 129.2, 129.0, 128.2, 128.1, 127.1, 126.3, 126.2, 126.0, 125.9, 124.8, 123.9, 118.8; HRMS-ESI [M - H] $]^{-}$calcd for $\mathrm{C}_{19} \mathrm{H}_{12} \mathrm{~N}_{3} \mathrm{O}_{2}{ }^{-}$314.0924; found 314.0912.

\section{4-Bromo-1-(4-nitrophenyl)-1H-pyrazole (1j)}

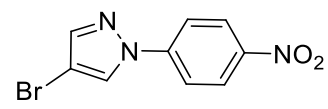

Obtained from 4-bromopyrazole and 1-fluoro-4-nitrobenzene according to the general procedure as a white solid (308 mg, yield 92\%); mp 168-170 ${ }^{\circ} \mathrm{C} ;{ }^{1} \mathrm{H}$ NMR (DMSO- $\left.d_{6}\right) \delta 9.01(\mathrm{~s}, 1 \mathrm{H}$ ), 8.39-8.34 (m, 2H), 8.12-8.07 (m, 2H), $8.02(\mathrm{~s}, 1 \mathrm{H}) ;{ }^{13} \mathrm{C}\left\{{ }^{1} \mathrm{H}\right\}$ NMR (DMSO- $\left.d_{6}\right) \delta 145.7,143.8$, 143.4, 129.6, 125.9, 119.0, 97.2; HRMS-ESI [M - H] calcd for $\mathrm{C}_{9} \mathrm{H}_{5}{ }^{79} \mathrm{BrN}_{3} \mathrm{O}_{2}{ }^{-}$265.9560; found 265.9561 .

\section{1-(2,4-Dinitrophenyl)-4-phenyl-1H-pyrazole (11)}

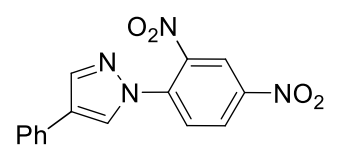

Obtained from 4-phenylpyrazole and 1-fluoro-2,4-dinitrobenzene according to the general procedure as a yellow solid (250 mg, yield 64\%); mp 203-205 ${ }^{\circ} \mathrm{C} ;{ }^{1} \mathrm{H} \mathrm{NMR}\left(\mathrm{CDCl}_{3}\right) \delta 8.73(\mathrm{~d}, J$ $=2.5 \mathrm{~Hz}, 1 \mathrm{H}), 8.56(\mathrm{dd}, J=8.9,2.5 \mathrm{~Hz}, 1 \mathrm{H}), 8.11(\mathrm{~s}, 1 \mathrm{H}), 8.03(\mathrm{~s}, 1 \mathrm{H}), 7.92(\mathrm{~d}, J=8.9 \mathrm{~Hz}, 1 \mathrm{H})$, $7.59-7.54(\mathrm{~m}, 2 \mathrm{H}), 7.45(\mathrm{t}, J=7.6 \mathrm{~Hz}, 2 \mathrm{H}), 7.36(\mathrm{t}, J=7.4 \mathrm{~Hz}, 1 \mathrm{H}) ;{ }^{13} \mathrm{C}\left\{{ }^{1} \mathrm{H}\right\} \mathrm{NMR}\left(\mathrm{CDCl}_{3}\right) \delta$ $145.5,143.0,142.0,137.2,130.5,129.1,127.9,127.4,127.3,126.0,125.5,125.4,121.2$; HRMS-ESI [M + H] $]^{+}$calcd for $\mathrm{C}_{15} \mathrm{H}_{11} \mathrm{~N}_{4} \mathrm{O}_{4}{ }^{+} 311.0775$; found 311.0783 .

\section{1-(2,4-Dinitrophenyl)-4-(4-fluorophenyl)-1H-pyrazole (1m)}

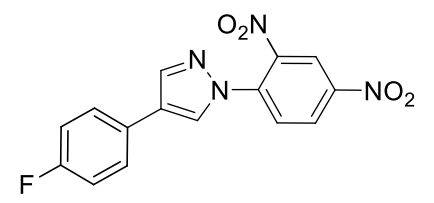

Obtained from 4-(4-fluorophenyl)pyrazole and 1-fluoro-2,4-dinitrobenzene according to the general procedure as a yellow solid (291 mg, yield 71\%); mp 242-244 ${ }^{\circ} \mathrm{C} ;{ }^{1} \mathrm{H}$ NMR (DMSO- $d_{6}$ ) $\delta 9.05(\mathrm{~s}, 1 \mathrm{H}), 8.88(\mathrm{~d}, J=2.4 \mathrm{~Hz}, 1 \mathrm{H}), 8.68(\mathrm{dd}, J=8.9,2.5 \mathrm{~Hz}, 1 \mathrm{H}), 8.38(\mathrm{~s}, 1 \mathrm{H}), 8.20(\mathrm{~d}, J=$ $9.0 \mathrm{~Hz}, 1 \mathrm{H}), 7.79(\mathrm{dd}, J=8.6,5.4 \mathrm{~Hz}, 2 \mathrm{H}), 7.30(\mathrm{t}, J=8.8 \mathrm{~Hz}, 2 \mathrm{H}) ;{ }^{13} \mathrm{C}\left\{{ }^{1} \mathrm{H}\right\}$ NMR (DMSO- $d_{6}$, $\left.60{ }^{\circ} \mathrm{C}\right) \delta 161.2(\mathrm{~d}, J=244.3 \mathrm{~Hz}), 145.1,142.2,141.1,135.6,127.6,127.4(\mathrm{~d}, J=7.7 \mathrm{~Hz}), 127.2$, 127.1, 124.9, 124.4, 120.8, $115.5(\mathrm{~d}, J=21.6 \mathrm{~Hz})$; HRMS-ESI $[\mathrm{M}+\mathrm{H}]^{+}$calcd for $\mathrm{C}_{15} \mathrm{H}_{10} \mathrm{FN}_{4} \mathrm{O}_{4}{ }^{+}$ 329.0681; found 329.0675 .

\section{4-(2-Chlorophenyl)-1-(2,4-dinitrophenyl)-1H-pyrazole (1n)}

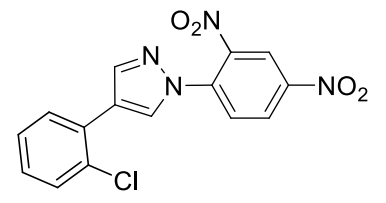

Obtained from 4-(2-chlorophenyl)pyrazole and 1-fluoro-2,4-dinitrobenzene according to the general procedure as a yellow solid $(267 \mathrm{mg}$, yield $62 \%)$; mp $168-170{ }^{\circ} \mathrm{C} ;{ }^{1} \mathrm{H} \mathrm{NMR}\left(\mathrm{CDCl}_{3}\right) \delta$ 
$8.74(\mathrm{~d}, J=2.5 \mathrm{~Hz}, 1 \mathrm{H}), 8.57(\mathrm{dd}, J=8.9,2.5 \mathrm{~Hz}, 1 \mathrm{H}), 8.23(\mathrm{~s}, 1 \mathrm{H}), 8.13(\mathrm{~s}, 1 \mathrm{H}), 7.94(\mathrm{~d}, J=8.9$ $\mathrm{Hz}, 1 \mathrm{H}), 7.56-7.50(\mathrm{~m}, 2 \mathrm{H}), 7.39-7.30(\mathrm{~m}, 2 \mathrm{H}) ;{ }^{13} \mathrm{C}\left\{{ }^{1} \mathrm{H}\right\} \mathrm{NMR}\left(\mathrm{CDCl}_{3}\right) \delta$ 145.6, 143.7, 143.1, 137.1, 132.2, 130.6, 130.0, 129.4, 128.9, 128.5, 127.5, 127.3, 125.7, 123.7, 121.2; HRMS-ESI [M $+\mathrm{H}]^{+}$calcd for $\mathrm{C}_{15} \mathrm{H}_{10}{ }^{35} \mathrm{ClN}_{4} \mathrm{O}_{4}{ }^{+}$345.0385; found 345.0388.

\section{4-(4-Bromophenyl)-1-(2,4-dinitrophenyl)-1H-pyrazole (10)}

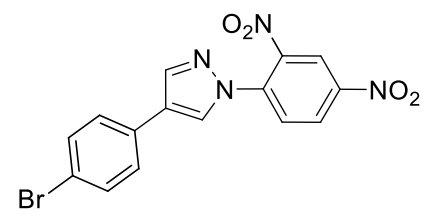

Obtained from 4-(4-bromophenyl)pyrazole and 1-fluoro-2,4-dinitrobenzene according to the general procedure as a yellow solid (350 mg, yield 72\%); mp 244-246 ${ }^{\circ} \mathrm{C} ;{ }^{1} \mathrm{H}$ NMR (DMSO- $d_{6}$ ) $\delta 9.09(\mathrm{~s}, 1 \mathrm{H}), 8.88(\mathrm{~d}, J=2.5 \mathrm{~Hz}, 1 \mathrm{H}), 8.67(\mathrm{dd}, J=9.0,2.5 \mathrm{~Hz}, 1 \mathrm{H}), 8.40(\mathrm{~s}, 1 \mathrm{H}), 8.19$ (d, $J=$ $9.0 \mathrm{~Hz}, 1 \mathrm{H}), 7.73-7.68(\mathrm{~m}, 2 \mathrm{H}), 7.67-7.62(\mathrm{~m}, 2 \mathrm{H}) ;{ }^{13} \mathrm{C}\left\{{ }^{1} \mathrm{H}\right\}$ NMR (DMSO- $\left.d_{6}\right) \delta 145.8,142.9$, 142.0, 136.1 132.4, 130.6, 128.5, 128.4, 128.1, 125.5, 124.8, 121.7, 120.7; HRMS-ESI [M + H] ${ }^{+}$ calcd for $\mathrm{C}_{15} \mathrm{H}_{10}{ }^{79} \mathrm{BrN}_{4} \mathrm{O}_{4}{ }^{+} 388.9880$; found 388.9871 .

\section{1-(4-Nitrophenyl)-1H-indazole (1q)}

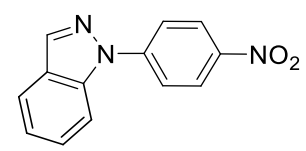

Obtained from indazole and 1-fluoro-4-nitrobenzene according to the general procedure (column chromatography on silica gel (eluent $\left.\mathrm{CHCl}_{3}\right)$ ) as a yellow solid (144 mg, yield 48\%); mp 121-123 ${ }^{\circ} \mathrm{C} ;{ }^{1} \mathrm{H}$ NMR $\left(\mathrm{CDCl}_{3}\right) \delta 8.46-8.41(\mathrm{~m}, 2 \mathrm{H}), 8.30(\mathrm{~s}, 1 \mathrm{H}), 8.03-7.98(\mathrm{~m}, 2 \mathrm{H}), 7.90-7.85(\mathrm{~m}, 2 \mathrm{H})$, 7.59-7.53 (m, 1H), 7.37-7.32 (m, 1H); ${ }^{13} \mathrm{C}\left\{{ }^{1} \mathrm{H}\right\}$ NMR $\left(\mathrm{CDCl}_{3}\right) \delta 145.4,145.1,138.6,137.6$, 128.4, 126.3, 125.3, 122.7, 121.9, 121.4, 110.5; HRMS-ESI $[\mathrm{M}+\mathrm{Na}]^{+}$calcd for $\mathrm{C}_{13} \mathrm{H}_{9} \mathrm{NaN}_{3} \mathrm{O}_{2}{ }^{+}$ 262.0587; found 262.0591.

\section{Synthesis of 1-(3-nitrophenyl)-4-phenyl-1H-pyrazole (1k)}

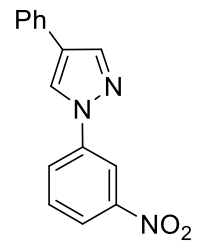

$N$-(3-(Dimethylamino)-2-phenylallylidene)- $N$-methylmethanaminium perchlorate $(6.9 \mathrm{~g}, 22.8$ mmol) and $\mathrm{NaOH}(3.31 \mathrm{~g}, 82.8 \mathrm{mmol})$ were dissolved in water $(20 \mathrm{~mL})$, then 3 $\mathrm{NO}_{2} \mathrm{C}_{6} \mathrm{H}_{4} \mathrm{NHNH}_{2} \cdot \mathrm{HCl}(6.54 \mathrm{~g}, 34.5 \mathrm{mmol})$ was added. The reaction mixture was stirred at room temperature for $5 \mathrm{~min}$. Then the reaction mixture was acidify with $\mathrm{HCl}$ to $\mathrm{pH}=5$, diluted with $\mathrm{MeOH}(30 \mathrm{~mL})$ and heated in an oil bath at $55^{\circ} \mathrm{C}$ under stirring for 2 days. The resulting orange precipitate was filtered off, washed with water and dried in air. The precipitate was purified by column chromatography on silica gel (eluent petroleum ether-EtOAc 3:1). Orange solid (4.58 g, yield $76 \%) ; \mathrm{mp} 159-160{ }^{\circ} \mathrm{C} ;{ }^{1} \mathrm{H} \mathrm{NMR}\left(\mathrm{CDCl}_{3}\right) \delta 8.61(\mathrm{t}, J=2.1 \mathrm{~Hz}, 1 \mathrm{H}), 8.26(\mathrm{~s}, 1 \mathrm{H}), 8.18-8.13$ $(\mathrm{m}, 2 \mathrm{H}), 8.06(\mathrm{~s}, 1 \mathrm{H}), 7.67(\mathrm{t}, J=8.2 \mathrm{~Hz}, 1 \mathrm{H}), 7.61-7.57(\mathrm{~m}, 2 \mathrm{H}), 7.48-7.42(\mathrm{~m}, 2 \mathrm{H}), 7.36-7.30$ $(\mathrm{m}, 1 \mathrm{H}) ;{ }^{13} \mathrm{C}\left\{{ }^{1} \mathrm{H}\right\} \mathrm{NMR}\left(\mathrm{CDCl}_{3}\right) \delta 149.0,140.8,139.9,131.3,130.5,129.0,127.3,126.1,125.8$, 
124.2, 123.1, 120.8, 113.5; HRMS-ESI $[\mathrm{M}+\mathrm{Na}]^{+}$calcd for $\mathrm{C}_{15} \mathrm{H}_{11} \mathrm{NaN}_{3} \mathrm{O}_{2}{ }^{+}$288.0743; found 288.0738 .

\section{Synthesis of 2-((4-phenyl-1H-pyrazol-1-yl)methyl)benzonitrile (1zi)}

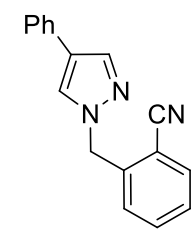

To a suspension of $\mathrm{NaH}$ (244 $\mathrm{mg}, 6.1 \mathrm{mmol})$ in THF $(22 \mathrm{~mL})$ was added 4-phenylpyrazole (763 $\mathrm{mg}, 5.3 \mathrm{mmol}$ ) and the mixture was stirred at $0{ }^{\circ} \mathrm{C}$ for $30 \mathrm{~min}$. Then 2-(bromomethyl)benzonitrile (1143 mg, $5.8 \mathrm{mmol}$ ) was added and the reaction mixture was stirred at room temperature for 2 days. The reaction mixture was quenched with water, extracted with EtOAc and the organic layer was washed with $1 \mathrm{M} \mathrm{HCl}(3 * 20 \mathrm{~mL}), 5 \% \mathrm{NaHCO}_{3}(3 * 20 \mathrm{~mL})$, water and brine. The combined organic layers were dried over $\mathrm{Na}_{2} \mathrm{SO}_{4}$, filtered, and concentrated under reduced pressure. Purification by column chromatography on silica gel (eluent hexane-EtOAc, 4:1) afforded crude product which was recrystallized (hexane- $\mathrm{Et}_{2} \mathrm{O}, 5: 1$ ). White solid (990 $\mathrm{mg}$, yield 66\%); mp 99-101 ${ }^{\circ} \mathrm{C} ;{ }^{1} \mathrm{H}$ NMR $\left(\mathrm{CDCl}_{3}\right) \delta 7.87(\mathrm{~s}, 1 \mathrm{H}), 7.82(\mathrm{~s}, 1 \mathrm{H}), 7.71(\mathrm{dd}, J=7.7,0.9 \mathrm{~Hz}, 1 \mathrm{H}), 7.59(\mathrm{td}$, $J=7.7,1.2 \mathrm{~Hz}, 1 \mathrm{H}), 7.53-7.49(\mathrm{~m}, 2 \mathrm{H}), 7.46-7.35(\mathrm{~m}, 3 \mathrm{H}), 7.31(\mathrm{~d}, J=7.9 \mathrm{~Hz}, 1 \mathrm{H}), 7.28-7.23$ $(\mathrm{m}, 1 \mathrm{H}), 5.57(\mathrm{~s}, 2 \mathrm{H}) ;{ }^{13} \mathrm{C}\left\{{ }^{1} \mathrm{H}\right\} \mathrm{NMR}\left(\mathrm{CDCl}_{3}\right) \delta 140.1,137.8,133.4,132.9,132.1,129.0,128.9$, 128.6, 126.9, 126.6, 125.6, 123.9, 117.2, 111.4, 53.9; HRMS-ESI $[\mathrm{M}+\mathrm{H}]^{+}$calcd for $\mathrm{C}_{17} \mathrm{H}_{14} \mathrm{~N}_{3}{ }^{+}$ 260.1182 ; found 260.1172 .

\section{Synthesis of 5-methoxy-1,4-diphenyl-1H-pyrazole (1zl)}

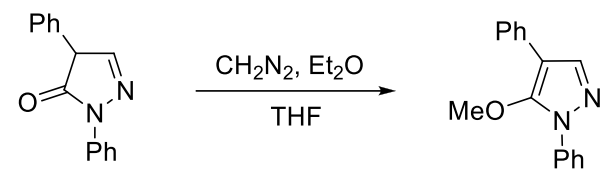

To a stirred solution of 2,4-diphenyl-2,4-dihydro-3H-pyrazol-3-one ${ }^{16}(1.34 \mathrm{~g}, 5.7 \mathrm{mmol})$ in dry THF $(50 \mathrm{~mL})$ a solution of diazomethane $(11.4 \mathrm{mmol})$ in $\mathrm{Et}_{2} \mathrm{O}(50 \mathrm{~mL})$, prepared from $N$-nitroso$\mathrm{N}$-methylurea and $\mathrm{KOH}$, was added dropwise at $0{ }^{\circ} \mathrm{C}$. The resulting mixturewas stirred at $\mathrm{rt}$ for 2 h. Then acetic acid was added $(342 \mathrm{mg}, 5.7 \mathrm{mmol})$ and mixture was stirred at $\mathrm{rt}$ for additional 30 min. The reaction mixture was concentrated in vacuo, and the residue was purified by column chromatography on silica gel (eluent petroleum ether-EtOAc 9:1). White solid (1.22 mg, yield 85\%); mp 58-60 ${ }^{\circ} \mathrm{C} ;{ }^{1} \mathrm{H}$ NMR $\left(\mathrm{CDCl}_{3}\right) \delta 7.83(\mathrm{~s}, 1 \mathrm{H}), 7.82-7.77(\mathrm{~m}, 2 \mathrm{H}), 7.68-7.63(\mathrm{~m}, 2 \mathrm{H})$, 7.54-7.48 (m, 2H), $7.44(\mathrm{t}, J=7.7 \mathrm{~Hz}, 2 \mathrm{H}), 7.40-7.34(\mathrm{~m}, 1 \mathrm{H}), 7.33-7.27(\mathrm{~m}, 1 \mathrm{H}), 3.75(\mathrm{~s}, 1 \mathrm{H})$; ${ }^{13} \mathrm{C}\left\{{ }^{1} \mathrm{H}\right\} \mathrm{NMR}\left(\mathrm{CDCl}_{3}\right) \delta 150.9,138.7,138.5,131.6,129.0,128.7,126.9,126.34,126.26,122.3$, 108.5, 61.2; HRMS-ESI [M + H $]^{+}$calcd for $\mathrm{C}_{16} \mathrm{H}_{15} \mathrm{~N}_{2} \mathrm{O}^{+}$251.1179; found 251.1176. 


\section{Synthesis of 1-((4-methyl-3-nitrophenyl)sulfonyl)-4-phenyl-1H-1,2,3-triazole}

(2e)

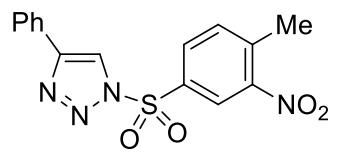

Triazole 2e was prepared by the reported procedure ${ }^{15}$ as a white solid (yield 55\%). Mp 138-139 ${ }^{\circ} \mathrm{C}(\mathrm{dec}.) ;{ }^{1} \mathrm{H} \mathrm{NMR}\left(\mathrm{CDCl}_{3}\right) \delta 8.72(\mathrm{~d}, J=2.0 \mathrm{~Hz}, 1 \mathrm{H}), 8.37(\mathrm{~s}, 1 \mathrm{H}), 8.31(\mathrm{dd}, J=8.2,2.0 \mathrm{~Hz}$, $1 \mathrm{H}), 7.88-7.83(\mathrm{~m}, 2 \mathrm{H}), 7.66(\mathrm{~d}, J=8.2 \mathrm{~Hz}, 1 \mathrm{H}), 7.50-7.39(\mathrm{~m}, 3 \mathrm{H}), 2.74(\mathrm{~s}, 3 \mathrm{H}) ;{ }^{13} \mathrm{C}\left\{{ }^{1} \mathrm{H}\right\} \mathrm{NMR}$ $\left(\mathrm{CDCl}_{3}\right) \delta$ 149.3, 147.8, 142.1, 135.3, 134.8, 132.1, 129.4, 129.1, 128.4, 126.1, 125.0, 119.0, 20.9; HRMS-ESI [M + H] calcd for $\mathrm{C}_{15} \mathrm{H}_{13} \mathrm{~N}_{4} \mathrm{O}_{4} \mathrm{~S}^{+} 345.0652$; found 356.0648 .

\section{Table S1. Optimization of the isomerization of $3 \mathrm{~h}$ to $4 \mathrm{p}$}
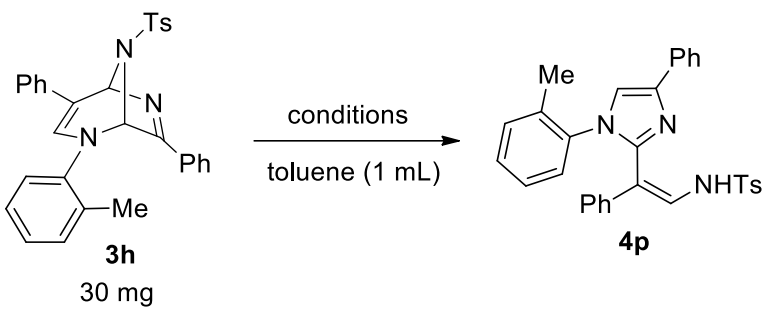

\begin{tabular}{|c|c|c|c|}
\hline Entry & Catalyst & Conditions & NMR yield of $\mathbf{4 p}, \%$ \\
\hline 1 & none & $160^{\circ} \mathrm{C}, 6 \mathrm{~h}$ & $0^{a}$ \\
\hline 2 & TsOH (5 mol\%) & $120^{\circ} \mathrm{C}, 1 \mathrm{~h}$ & $0^{b}$ \\
\hline 3 & $\mathrm{TsNH}_{2}(5 \mathrm{~mol} \%)$ & $120^{\circ} \mathrm{C}, 1 \mathrm{~h}$ & $0^{a}$ \\
\hline 4 & $\mathrm{DABCO}(5 \mathrm{~mol} \%)$ & $120^{\circ} \mathrm{C}, 1 \mathrm{~h}$ & $0^{a}$ \\
\hline 5 & $\mathrm{Rh}_{2}(\mathrm{Piv})_{4}(5 \mathrm{~mol} \%)$ & $120^{\circ} \mathrm{C}, 1 \mathrm{~h}$ & $0^{a}$ \\
\hline 6 & $\mathrm{Rh}_{2}(\mathrm{OAc})_{4}(5 \mathrm{~mol} \%)$ & $120^{\circ} \mathrm{C}, 1 \mathrm{~h}$ & $0^{a}$ \\
\hline 7 & $\mathrm{RhCl}_{3} \cdot 4 \mathrm{H}_{2} \mathrm{O}(5 \mathrm{~mol} \%)$ & $120^{\circ} \mathrm{C}, 1 \mathrm{~h}$ & $100 \%$ \\
\hline \multirow[t]{2}{*}{8} & a few drops of the reaction mixture: & $120^{\circ} \mathrm{C}, 4.5 \mathrm{~h}$ & $100 \%$ \\
\hline & $\stackrel{\mathrm{Rh}_{2}(\mathrm{Piv})_{4}}{\longrightarrow}$ & & \\
\hline
\end{tabular}

$9 \quad$ Silica gel $(500 \mathrm{wt} \%)$

$120^{\circ} \mathrm{C}, 2 \mathrm{~h} \quad 100 \%^{\circ}$

${ }^{c}$ Isolated yield is $80 \%$. 


\section{Synthesis of 2,6,8-triazabicyclo[3.2.1]octa-3,6-dienes 3}

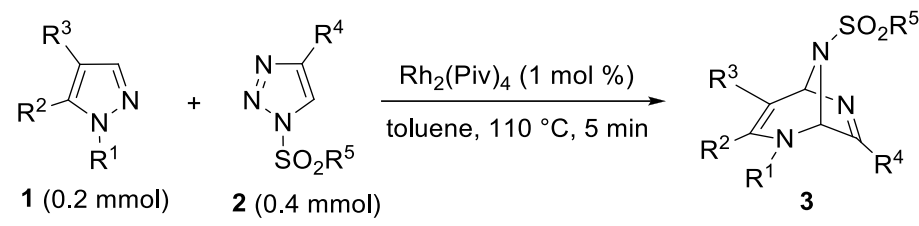

General procedure. Pyrazole $1(0.2 \mathrm{mmol})$, triazole $2(0.4 \mathrm{mmol})$, and toluene $(1 \mathrm{~mL})$ were placed into a screw cap glass tube. After the addition of $\mathrm{Rh}_{2}(\mathrm{Piv})_{4}(1.2 \mathrm{mg}, 0.01$ equiv), cap was rapidly screwed and the tube was placed into an oil bath preheated to $110^{\circ} \mathrm{C}$. The reaction mixture was stirred at this temperature under stirring until nitrogen evolution had ceased (ca. 2-5 min). The solvent was removed in vacuo, and the residue was purified by column chromatography on silica gel (eluent hexane-EtOAc, 2:1) to give the desired product 3.

rac-(1R,5R)-8-(4-Methylphenylsulfonyl)-2,4,7-triphenyl-2,6,8-triazabicyclo[3.2.1]octa-3,6diene (3a)

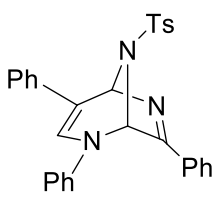

Compound 3a was obtained as a yellow oil (95 mg, yield 97\%) according to the general procedure (eluent hexane-EtOAc, 2:1) from pyrazole 1a and triazole $2 \mathbf{a} ;{ }^{1} \mathrm{H} \mathrm{NMR}\left(\mathrm{CDCl}_{3}\right) \delta 7.64(\mathrm{~d}, J=8.3$ $\mathrm{Hz}, 2 \mathrm{H}), 7.50-7.45(\mathrm{~m}, 2 \mathrm{H}), 7.39-7.30(\mathrm{~m}, 5 \mathrm{H}), 7.26-7.18(\mathrm{~m}, 5 \mathrm{H}), 7.15(\mathrm{t}, J=7.4 \mathrm{~Hz}, 1 \mathrm{H})$, $7.09-7.04(\mathrm{~m}, 4 \mathrm{H}), 6.63(\mathrm{~s}, 1 \mathrm{H}), 6.17(\mathrm{~s}, 1 \mathrm{H}), 6.12(\mathrm{~s}, 1 \mathrm{H}), 2.34(\mathrm{~s}, 3 \mathrm{H}) ;{ }^{13} \mathrm{C}\left\{{ }^{1} \mathrm{H}\right\} \mathrm{NMR}\left(\mathrm{CDCl}_{3}\right)$ $\delta$ 157.6, 144.0, 143.4, 136.4, 134.9, 131.0, 130.3, 129.9, 129.1, 128.6, 128.5, 128.0, 127.9, 125.9, 124.5, 123.8, 123.7, 120.7, 111.8, 80.7, 74.6, 21.4; HRMS-ESI $[\mathrm{M}+\mathrm{H}]^{+}$calcd for $\mathrm{C}_{30} \mathrm{H}_{26} \mathrm{~N}_{3} \mathrm{O}_{2} \mathrm{~S}^{+}$ 492.1740; found 492.1745.

rac-(1R,5R)-8-(Methylsulfonyl)-2,4,7-triphenyl-2,6,8-triazabicyclo[3.2.1]octa-3,6-diene (3b)

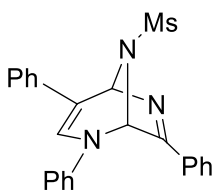

Compound $\mathbf{3 b}$ was obtained as a pale yellow solid (82 $\mathrm{mg}$, yield 99\%) according to the general procedure (eluent hexane-EtOAc, 2:1) from pyrazole 1a and triazole $\mathbf{2 b}$; mp $154-156{ }^{\circ} \mathrm{C} ;{ }^{1} \mathrm{H}$ $\operatorname{NMR}\left(\mathrm{CDCl}_{3}\right) \delta 7.58(\mathrm{~d}, J=7.3 \mathrm{~Hz}, 2 \mathrm{H}), 7.51-7.46(\mathrm{~m}, 2 \mathrm{H}), 7.44-7.36(\mathrm{~m}, 5 \mathrm{H}), 7.32-7.28(\mathrm{~m}$, 2H), 7.28-7.16 (m, 4H), $6.68(\mathrm{~s}, 1 \mathrm{H}), 6.62(\mathrm{~s}, 1 \mathrm{H}), 6.18(\mathrm{~s}, 1 \mathrm{H}), 2.99(\mathrm{~s}, 3 \mathrm{H}) ;{ }^{13} \mathrm{C}\left\{{ }^{1} \mathrm{H}\right\} \mathrm{NMR}$ $\left(\mathrm{CDCl}_{3}\right) \delta 157.5,143.5,136.2,131.2,130.1$ (2C), 129.0, 128.6, 128.0, 126.3, 125.0, 124.7, 123.9, 121.0, 112.5, 80.6, 74.4, 39.4; IR (KBr): 3061, 3030, 3007, 2927, 1587, 1490, 1343, 1158, 759, $692,560,509 \mathrm{~cm}^{-1}$; HRMS-ESI $[\mathrm{M}+\mathrm{H}]^{+}$calcd for $\mathrm{C}_{24} \mathrm{H}_{22} \mathrm{~N}_{3} \mathrm{O}_{2} \mathrm{~S}^{+} 416.1427$; found 416.1429 . 


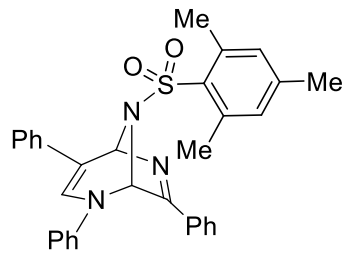

Compound 3c was obtained as a pale yellow solid (100 mg, yield 96\%) according to the general procedure (eluent hexane-EtOAc, 2:1) from pyrazole 1a and triazole $2 \mathbf{c}$; mp $170-172{ }^{\circ} \mathrm{C}$; ${ }^{1} \mathrm{H}$ NMR $\left(\mathrm{CDCl}_{3}\right) \delta 7.64(\mathrm{~d}, J=8.3 \mathrm{~Hz}, 2 \mathrm{H}), 7.44-7.29(\mathrm{~m}, 9 \mathrm{H}), 7.23-7.13(\mathrm{~m}, 2 \mathrm{H}), 7.11(\mathrm{~d}, J=8.3$ $\mathrm{Hz}, 2 \mathrm{H}), 6.96(\mathrm{~s}, 2 \mathrm{H}), 6.62-6.60(\mathrm{~m}, 1 \mathrm{H}), 6.57-6.55(\mathrm{~m}, 1 \mathrm{H}), 6.08-6.06(\mathrm{~m}, 1 \mathrm{H}), 2.61(\mathrm{~s}, 6 \mathrm{H})$, $2.33(\mathrm{~s}, 3 \mathrm{H}) ;{ }^{13} \mathrm{C}\left\{{ }^{1} \mathrm{H}\right\}$ NMR $\left(\mathrm{CDCl}_{3}\right) \delta 158.0,144.2,143.0,140.2,136.8,132.9,132.1,131.0$, 130.6, 129.8, 128.73, 128.65, 128.2, 125.8, 125.0, 124.5, 124.0, 121.1, 113.4, 80.9, 74.5, 22.9, 21.0; HRMS-ESI [M + H] calcd for $\mathrm{C}_{32} \mathrm{H}_{30} \mathrm{~N}_{3} \mathrm{O}_{2} \mathrm{~S}^{+}$520.2053; found 520.2061.

rac-(1R,5R)-8-(4-Methoxyphenylsulfonyl)-2,4,7-triphenyl-2,6,8-triazabicyclo[3.2.1]octa-3,6diene (3d)

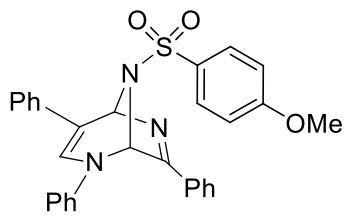

Compound 3d was obtained as a white solid (99 mg, yield 98\%) according to the general procedure (eluent hexane-EtOAc, 2:1) from pyrazole 1a and triazole $2 \mathbf{d}$; mp $153-155{ }^{\circ} \mathrm{C} ;{ }^{1} \mathrm{H}$ NMR $\left(\mathrm{CDCl}_{3}\right)$ $\delta 7.67(\mathrm{~d}, J=8.9 \mathrm{~Hz}, 2 \mathrm{H}), 7.48(\mathrm{~d}, J=7.4 \mathrm{~Hz}, 2 \mathrm{H}), 7.39-7.30(\mathrm{~m}, 5 \mathrm{H}), 7.27-7.12(\mathrm{~m}, 6 \mathrm{H}), 7.05$ $(\mathrm{d}, J=7.7 \mathrm{~Hz}, 2 \mathrm{H}), 6.72(\mathrm{~d}, J=8.9 \mathrm{~Hz}, 2 \mathrm{H}), 6.61(\mathrm{~d}, J=1.3 \mathrm{~Hz}, 1 \mathrm{H}), 6.18-6.15(\mathrm{~m}, 1 \mathrm{H}), 6.14-6.11$ $(\mathrm{m}, 1 \mathrm{H}), 3.78(\mathrm{~s}, 3 \mathrm{H}) ;{ }^{13} \mathrm{C}\left\{{ }^{1} \mathrm{H}\right\} \mathrm{NMR}\left(\mathrm{CDCl}_{3}\right) \delta 163.3,157.8,143.4,136.4,131.0,130.4,130.2$, 129.9, 129.5, 128.6, 128.5, 128.0, 125.9, 124.5, 123.73, 123.7, 120.6, 113.7, 111.7, 80.6, 74.5, 55.5; HRMS-ESI [M+ H] $]^{+}$calcd for $\mathrm{C}_{30} \mathrm{H}_{26} \mathrm{~N}_{3} \mathrm{O}_{3} \mathrm{~S}^{+}$508.1689; found 508.1674.

rac-(1R,5R)-8-(4-Methyl-3-nitrophenylsulfonyl)-2,4,7-triphenyl-2,6,8-triazabicyclo[3.2.1]octa-3,6-diene (3e)

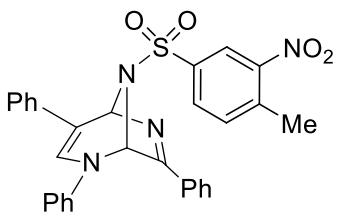

Compound 3e was obtained as a white solid (103 mg, yield 96\%) according to the general procedure (eluent hexane-EtOAc, 2:1) from pyrazole 1a and triazole 2e; mp $155-157{ }^{\circ} \mathrm{C}$; ${ }^{1} \mathrm{H}$ $\operatorname{NMR}\left(\mathrm{CDCl}_{3}\right) \delta 8.35(\mathrm{~d}, J=1.7 \mathrm{~Hz}, 1 \mathrm{H}), 7.82(\mathrm{~d}, J=8.1,1.7 \mathrm{~Hz}, 1 \mathrm{H}), 7.49(\mathrm{~d}, J=7.3 \mathrm{~Hz}, 2 \mathrm{H})$, $7.40-7.30(\mathrm{~m}, 5 \mathrm{H}), 7.28-7.14(\mathrm{~m}, 7 \mathrm{H}), 7.03(\mathrm{~d}, J=7.8 \mathrm{~Hz}, 2 \mathrm{H}), 6.68-6.65(\mathrm{~m}, 1 \mathrm{H}), 6.22-6.19$ $(\mathrm{m}, 1 \mathrm{H}), 6.09-6.06(\mathrm{~m}, 1 \mathrm{H}), 2.59(\mathrm{~s}, 3 \mathrm{H}) ;{ }^{13} \mathrm{C}\left\{{ }^{1} \mathrm{H}\right\} \mathrm{NMR}\left(\mathrm{CDCl}_{3}\right) \delta 157.7,148.4,142.9,138.9$, 137.2 , 135.8, 132.9, 131.7, 131.3, 130.1, 129.9, 128.8, 128.6, 127.9, 126.3, 124.9, 124.7, 123.5, 
123.3, 120.5, 111.4, 80.6, 74.3, 20.5; HRMS-ESI $[\mathrm{M}+\mathrm{H}]^{+}$calcd for $\mathrm{C}_{30} \mathrm{H}_{25} \mathrm{~N}_{4} \mathrm{O}_{4} \mathrm{~S}^{+}$537.1591; found 537.1593 .

rac-(1R,5R)-7-(4-Methoxyphenyl)-8-(4-methylphenylsulfonyl)-2,4-diphenyl-2,6,8-triazabicyclo[3.2.1]octa-3,6-diene (3f)

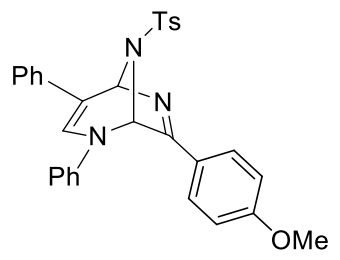

Compound 3f was obtained as a pale yellow solid (100 mg, yield 96\%) according to the general procedure (eluent hexane-EtOAc, 2:1) from pyrazole 1a and triazole 2f; mp $77-79{ }^{\circ} \mathrm{C}$; ${ }^{1} \mathrm{H} \mathrm{NMR}$ $\left(\mathrm{CDCl}_{3}\right) \delta 7.64(\mathrm{~d}, J=8.3 \mathrm{~Hz}, 2 \mathrm{H}), 7.45-7.39(\mathrm{~m}, 2 \mathrm{H}), 7.39-7.29(\mathrm{~m}, 4 \mathrm{H}), 7.24-7.11(\mathrm{~m}, 4 \mathrm{H})$, 7.09-7.03 (m, 4H), 6.76-6.71 (m, 2H), $6.60(\mathrm{~d}, J=1.5 \mathrm{~Hz}, 1 \mathrm{H}), 6.13(\mathrm{~d}, J=1.5 \mathrm{~Hz}, 1 \mathrm{H}), 6.09$ (t, $J=1.5 \mathrm{~Hz}, 1 \mathrm{H}), 3.77(\mathrm{~s}, 3 \mathrm{H}), 2.34(\mathrm{~s}, 3 \mathrm{H}) ;{ }^{13} \mathrm{C}\left\{{ }^{1} \mathrm{H}\right\} \mathrm{NMR}\left(\mathrm{CDCl}_{3}\right) \delta 161.8,157.5,143.9,143.5$, 136.5, 135.0, 129.9, 129.7, 129.1, 128.6, 128.0, 125.8, 124.4, 123.7, 123.6, 122.9, 120.7, 113.9, 112.0, 80.6, 74.5, 55.2, 21.4; HRMS-ESI $[\mathrm{M}+\mathrm{H}]^{+}$calcd for $\mathrm{C}_{31} \mathrm{H}_{28} \mathrm{~N}_{3} \mathrm{O}_{3} \mathrm{~S}^{+}$522.1846; found 522.1831 .

rac-(1R,5R)-7-(4-Chlorophenyl)-8-(4-methylphenylsulfonyl)-2,4-diphenyl-2,6,8-triazabicyclo[3.2.1]octa-3,6-diene (3g)

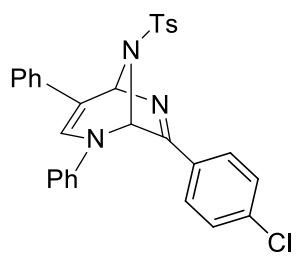

Compound $3 \mathrm{~g}$ was obtained as a yellow solid (103 $\mathrm{mg}$, yield 98\%) according to the general procedure (eluent hexane-EtOAc, 2:1) from pyrazole 1a and triazole $2 \mathrm{~g}$; mp $144-146{ }^{\circ} \mathrm{C}$; ${ }^{1} \mathrm{H}$ NMR $\left(\mathrm{CDCl}_{3}\right) \delta 7.65(\mathrm{~d}, J=8.1 \mathrm{~Hz}, 2 \mathrm{H}), 7.43-7.30(\mathrm{~m}, 6 \mathrm{H}), 7.25-7.13(\mathrm{~m}, 6 \mathrm{H}), 7.10-7.03(\mathrm{~m}$, $4 \mathrm{H}), 6.62(\mathrm{~s}, 1 \mathrm{H}), 6.17(\mathrm{~s}, 1 \mathrm{H}), 6.11(\mathrm{~s}, 1 \mathrm{H}), 2.33(\mathrm{~s}, 3 \mathrm{H}) ;{ }^{13} \mathrm{C}\left\{{ }^{1} \mathrm{H}\right\} \mathrm{NMR}\left(\mathrm{CDCl}_{3}\right) \delta$ 156.2, 144.0, 143.2, 137.1, 136.2, 134.8, 129.9, 129.11, 129.08, 128.8, 128.7, 128.6, 128.0, 125.9, 124.6, 123.7, 123.6, 120.7, 111.5, 80.6, 74.4, 21.4; IR (KBr): 3053, 2958, 2923, 2856, 1593, 1491, 1332, 1253, 1162, 1090, 1010, 811, 757, 698, 673, 589, $543 \mathrm{~cm}^{-1}$; HRMS-ESI $[\mathrm{M}+\mathrm{H}]^{+}$calcd for $\mathrm{C}_{30} \mathrm{H}_{25}{ }^{35} \mathrm{ClN}_{3} \mathrm{O}_{2} \mathrm{~S}^{+}$526.1351; found 526.1363.

rac-(1R,5R)-2-(2-Methylphenyl)-8-(4-methylphenylsulfonyl)-4,7-diphenyl-2-2,6,8triazabicyclo[3.2.1]octa-3,6-diene (3h)

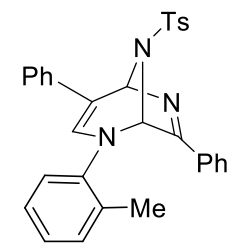


Compound 3h was obtained as a yellow oil (98 mg, yield 97\%) according to the general procedure (eluent hexane-EtOAc, 2:1) from pyrazole $\mathbf{1 b}$ and triazole $\mathbf{2 a} ;{ }^{1} \mathrm{H} \mathrm{NMR}\left(\mathrm{CDCl}_{3}\right) \delta 7.68(\mathrm{~d}, J=8.2$ $\mathrm{Hz}, 2 \mathrm{H}), 7.36-7.29(\mathrm{~m}, 7 \mathrm{H}), 7.25-7.13(\mathrm{~m}, 7 \mathrm{H}), 7.09(\mathrm{~d}, J=8.2 \mathrm{~Hz}, 2 \mathrm{H}), 6.24(\mathrm{~s}, 1 \mathrm{H}), 6.20(\mathrm{~s}$, $1 \mathrm{H}), 6.12(\mathrm{~s}, 1 \mathrm{H}), 2.29(\mathrm{~s}, 3 \mathrm{H}), 2.09(\mathrm{~s}, 3 \mathrm{H}) ;{ }^{13} \mathrm{C}\left\{{ }^{1} \mathrm{H}\right\} \mathrm{NMR}\left(\mathrm{CDCl}_{3}\right) \delta$ 156.6, 144.0, 142.8, 136.6, 135.0, 134.5, 131.8, 130.9, 130.5, 129.6, 128.6, 128.3, 128.0, 127.8, 127.4, 127.1, 127.0, 126.9, 125.6 (2C), 123.6, 81.4, 76.1, 21.4, 18.1; HRMS-ESI $[\mathrm{M}+\mathrm{Na}]^{+}$calcd for $\mathrm{C}_{31} \mathrm{H}_{26} \mathrm{NaN}_{3} \mathrm{O}_{2} \mathrm{~S}^{+}$ 528.1716; found 528.1729.

rac-(1R,5R)-2-(2-Methylphenyl)-8-(4-methylphenylsulfonyl)-4-phenyl-7-(4-(trifluoromethyl)phenyl)-2,6,8-triazabicyclo[3.2.1] octa-3,6-diene (3i)

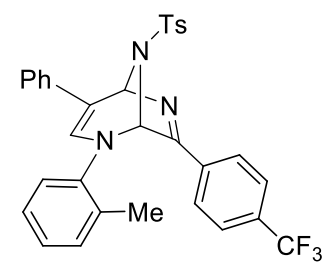

Compound 3i was obtained as a yellow solid (113 mg, yield 98\%) according to the general procedure (eluent hexane-EtOAc, 2:1) from pyrazole $\mathbf{1 b}$ and triazole $\mathbf{2 h}$; $\mathrm{mp} 98-100{ }^{\circ} \mathrm{C}$; ${ }^{1} \mathrm{H}$ NMR $\left(\mathrm{CDCl}_{3}\right) \delta 7.67(\mathrm{~d}, J=8.2 \mathrm{~Hz}, 2 \mathrm{H}), 7.48-7.44(\mathrm{~m}, 2 \mathrm{H}), 7.43-7.39(\mathrm{~m}, 2 \mathrm{H}), 7.36-7.29(\mathrm{~m}, 4 \mathrm{H})$, 7.27-7.16 (m, 4H), 7.13-7.07 (m, 3H), 6.24-6.21 (m, 2H), $6.14(\mathrm{~s}, 1 \mathrm{H}), 2.30(\mathrm{~s}, 3 \mathrm{H}), 2.11(\mathrm{~s}, 3 \mathrm{H})$; ${ }^{13} \mathrm{C}\left\{{ }^{1} \mathrm{H}\right\}$ NMR $\left(\mathrm{CDCl}_{3}\right) \delta 154.8,144.2,142.5,136.3,134.9,134.3,133.9,132.3(\mathrm{q}, J=32.8 \mathrm{~Hz})$, 132.0, 129.6, 128.7, 128.2, 127.8, 127.5, 127.4, 127.0, 126.8, 125.8, 125.2 (q, J = 3.7 Hz), 123.7, $123.6(\mathrm{q}, J=272.8 \mathrm{~Hz}), 110.4,81.5,76.0,21.4,18.1$; IR (KBr): 3053, 2958, 2922, 2856, 1594 , 1491, 1332, 1253, 1164, 1090, 756, 701, 590, $544 \mathrm{~cm}^{-1}$; HRMS-ESI $[\mathrm{M}+\mathrm{Na}]^{+}$calcd for $\mathrm{C}_{32} \mathrm{H}_{26} \mathrm{~F}_{3} \mathrm{NaN}_{3} \mathrm{O}_{2} \mathrm{~S}^{+}$596.1590; found 596.1610.

rac-(1R,5R)-2-(2-Methylphenyl)-8-(4-methylphenylsulfonyl)-7-(4-nitrophenyl)-4-phenyl2,6,8-triazabicyclo[3.2.1]octa-3,6-diene (3j)

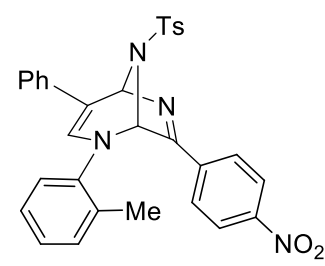

Compound 3j was obtained as an orange solid (101 mg, yield 92\%) according to the general procedure (eluent hexane-EtOAc, $2: 1$ ) from pyrazole $\mathbf{1 b}$ and triazole $2 \mathbf{i}$; mp $150-152{ }^{\circ} \mathrm{C} ;{ }^{1} \mathrm{H} \mathrm{NMR}$ $\left(\mathrm{CDCl}_{3}\right) \delta 8.08-8.03(\mathrm{~m}, 2 \mathrm{H}), 7.69(\mathrm{~d}, J=8.2 \mathrm{~Hz}, 2 \mathrm{H}), 7.50-7.45(\mathrm{~m}, 2 \mathrm{H}), 7.36-7.17(\mathrm{~m}, 8 \mathrm{H})$, $7.13(\mathrm{~d}, J=8.2 \mathrm{~Hz}, 2 \mathrm{H}), 7.11-7.04(\mathrm{~m}, 1 \mathrm{H}), 6.27(\mathrm{~s}, 1 \mathrm{H}), 6.25-6.24(\mathrm{~m}, 1 \mathrm{H}), 6.17-6.14(\mathrm{~m}, 1 \mathrm{H})$, $2.32(\mathrm{~s}, 3 \mathrm{H}), 2.10(\mathrm{~s}, 3 \mathrm{H}) ;{ }^{13} \mathrm{C}\left\{{ }^{1} \mathrm{H}\right\} \mathrm{NMR}\left(\mathrm{CDCl}_{3}\right) \delta 153.5,148.8,144.3,142.3,136.5,136.1$, 134.9, 134.2, 132.1, 129.7, 128.7 (2C), 127.8, 127.62, 127.56, 126.9, 126.7, 126.0, 123.7, 123.5, 110.2, 81.4, 75.9, 21.4, 18.1; IR (KBr): 3050, 2956, 2853, 1595, 1517, 1347, 1243, 1167, 917, $760,697,578 \mathrm{~cm}^{-1}$; HRMS-ESI $[\mathrm{M}+\mathrm{H}]^{+}$calcd for $\mathrm{C}_{31} \mathrm{H}_{27} \mathrm{~N}_{4} \mathrm{O}_{4} \mathrm{~S}^{+}$551.1748; found 551.1729. 
rac-(1R,5R)-8-(4-Methylphenylsulfonyl)-2-(4-nitrophenyl)-4,7-diphenyl-2,6,8-triazabicyclo[3.2.1]octa-3,6-diene (3k)

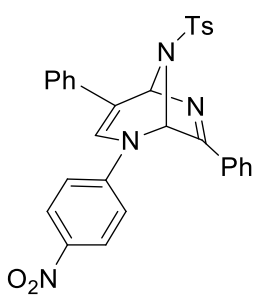

Compound 3k was obtained as a yellow solid (104 mg, yield 97\%) according to the general procedure (eluent hexane-EtOAc, 2:1) from pyrazole 1c and triazole $\mathbf{2 a}$; mp 194-196 ${ }^{\circ} \mathrm{C}$; ${ }^{1} \mathrm{H}$ NMR $\left(\mathrm{CDCl}_{3}\right) \delta 8.27-8.21(\mathrm{~m}, 2 \mathrm{H}), 7.63-7.56(\mathrm{~m}, 4 \mathrm{H}), 7.44-7.25(\mathrm{~m}, 8 \mathrm{H}), 7.15-7.07(\mathrm{~m}, 4 \mathrm{H})$, $6.79(\mathrm{~d}, J=1.6 \mathrm{~Hz}, 1 \mathrm{H}), 6.21-6.17(\mathrm{~m}, 2 \mathrm{H}), 2.36(\mathrm{~s}, 3 \mathrm{H}) ;{ }^{13} \mathrm{C}\left\{{ }^{1} \mathrm{H}\right\} \mathrm{NMR}\left(\mathrm{CDCl}_{3}\right) \delta 159.5,147.9$, 144.5, 142.7, 135.5, 134.8, 131.7, 129.8, 129.4, 128.9, 128.8, 127.94, 127.89, 127.0, 126.0, 124.3, 121.2, 117.8, 116.5, 80.8, 73.3, 21.5; HRMS-ESI [M - H] calcd for $\mathrm{C}_{30} \mathrm{H}_{23} \mathrm{~N}_{4} \mathrm{O}_{4} \mathrm{~S}^{-}$535.1435; found 535.1413 .

rac-(1R,5R)-4-(4-Methylphenyl)-8-(4-methylphenylsulfonyl)-2-(4-nitrophenyl)-7-phenyl2,6,8-triazabicyclo[3.2.1]octa-3,6-diene (3I)

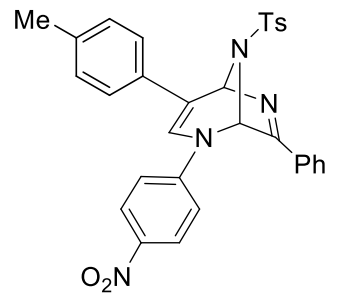

Compound 3l was obtained as a yellow solid (101 mg, yield 92\%) according to the general procedure (eluent hexane-EtOAc, 2:1) from pyrazole $1 \mathbf{d}$ and triazole $2 \mathbf{a}$; mp $137-139{ }^{\circ} \mathrm{C} ;{ }^{1} \mathrm{H}$ $\operatorname{NMR}\left(\mathrm{CDCl}_{3}\right) \delta 8.24(\mathrm{~d}, J=9.2 \mathrm{~Hz}, 2 \mathrm{H}), 7.61-7.56(\mathrm{~m}, 4 \mathrm{H}), 7.41(\mathrm{t}, J=7.4 \mathrm{~Hz}, 1 \mathrm{H}), 7.33(\mathrm{t}, J=$ $7.5 \mathrm{~Hz}, 2 \mathrm{H}), 7.21-7.16(\mathrm{~m}, 4 \mathrm{H}), 7.12-7.07(\mathrm{~m}, 4 \mathrm{H}), 6.77(\mathrm{~d}, J=1.6 \mathrm{~Hz}, 1 \mathrm{H}), 6.17(\mathrm{~d}, J=1.3 \mathrm{~Hz}$, $1 \mathrm{H}), 6.14-6.12(\mathrm{~m}, 1 \mathrm{H}), 2.39(\mathrm{~s}, 3 \mathrm{H}), 2.36(\mathrm{~s}, 3 \mathrm{H}) ;{ }^{13} \mathrm{C}\left\{{ }^{1} \mathrm{H}\right\} \mathrm{NMR}\left(\mathrm{CDCl}_{3}\right) \delta 159.5,147.9,144.4$, 142.6, 136.9, 134.8, 132.6, 131.7, 129.8, 129.5, 129.3, 128.9, 128.0, 127.9, 126.0, 124.3, 120.4, 117.5, 116.6, 80.9, 73.2, 21.5, 21.1; HRMS-ESI $[\mathrm{M}+\mathrm{H}]^{+}$calcd for $\mathrm{C}_{31} \mathrm{H}_{27} \mathrm{~N}_{4} \mathrm{O}_{4} \mathrm{~S}^{+} 551.1748$; found 551.1729 .

rac-(1R,5R)-4-(4-Methoxyphenyl)-8-(4-methylphenylsulfonyl)-2-(4-nitrophenyl)-7-phenyl2,6,8-triazabicyclo[3.2.1] octa-3,6-diene (3m)

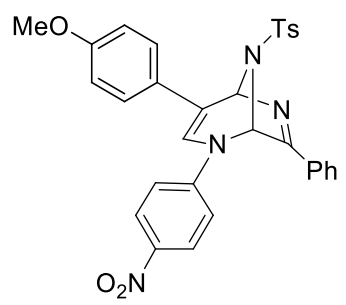

Compound 3m was obtained as a yellow solid (108 $\mathrm{mg}$, yield 96\%) according to the general procedure (eluent hexane-EtOAc, 2:1) from pyrazole 1e and triazole $\mathbf{2 a} ; \mathbf{m p ~} 198-200{ }^{\circ} \mathrm{C} ;{ }^{1} \mathrm{H}$ 
NMR $\left(\mathrm{CDCl}_{3}\right) \delta 8.26-8.20(\mathrm{~m}, 2 \mathrm{H}), 7.61-7.56(\mathrm{~m}, 4 \mathrm{H}), 7.44-7.38(\mathrm{~m}, 1 \mathrm{H}), 7.33(\mathrm{t}, J=7.5 \mathrm{~Hz}$, 2H), 7.24-7.19 (m, 2H), 7.12-7.06 (m, 4H), 6.95-6.90 (m, 2H), $6.78(\mathrm{~d}, J=1.6 \mathrm{~Hz}, 1 \mathrm{H}), 6.15(\mathrm{~d}$, $J=1.3 \mathrm{~Hz}, 1 \mathrm{H}), 6.09-6.07(\mathrm{~m}, 1 \mathrm{H}), 3.86(\mathrm{~s}, 3 \mathrm{H}), 2.36(\mathrm{~s}, 3 \mathrm{H}) ;{ }^{13} \mathrm{C}\left\{{ }^{1} \mathrm{H}\right\} \mathrm{NMR}\left(\mathrm{CDCl}_{3}\right) \delta 159.6$, $158.9,147.9,144.4,142.5,134.8,131.7,129.8,129.3,128.9,128.1,128.0,127.9,126.0,125.7$, 119.7, 117.4, 116.5, 114.3, 80.9, 73.1, 55.4, 21.5; IR (KBr): 3067, 3004, 2965, 2932, 2836, 1592, $1509,1342,1255,1161,1029,919,830,724,708,675,577 \mathrm{~cm}^{-1}$; HRMS-ESI $[\mathrm{M}+\mathrm{H}]^{+}$calcd for $\mathrm{C}_{31} \mathrm{H}_{27} \mathrm{~N}_{4} \mathrm{O}_{5} \mathrm{~S}^{+}$567.1697; found 567.1680.

rac-(1R,5R)-4-(4-Chlorophenyl)-8-(4-methylphenylsulfonyl)-2-(4-nitrophenyl)-7-phenyl2,6,8-triazabicyclo[3.2.1]octa-3,6-diene (3n)

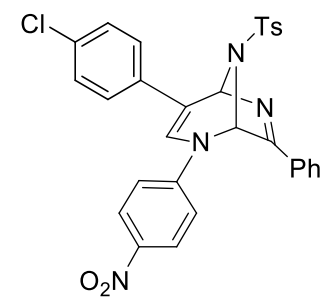

Compound 3n was obtained as a yellow solid (105 mg, yield 92\%) according to the general procedure (eluent hexane-EtOAc, $2: 1$ ) from pyrazole 1 f and triazole $2 \mathbf{2 a}$; mp $186-188{ }^{\circ} \mathrm{C} ;{ }^{1} \mathrm{H} \mathrm{NMR}$ $\left(\mathrm{CDCl}_{3}\right) \delta 8.26(\mathrm{~d}, J=9.1 \mathrm{~Hz}, 2 \mathrm{H}), 7.62-7.54(\mathrm{~m}, 4 \mathrm{H}), 7.42(\mathrm{t}, J=7.4 \mathrm{~Hz}, 1 \mathrm{H}), 7.37-7.30(\mathrm{~m}$, $4 \mathrm{H}), 7.21(\mathrm{~d}, J=8.6 \mathrm{~Hz}, 2 \mathrm{H}), 7.16-7.10(\mathrm{~m}, 4 \mathrm{H}), 6.75(\mathrm{~d}, J=1.6 \mathrm{~Hz}, 1 \mathrm{H}), 6.19-6.17(\mathrm{~m}, 1 \mathrm{H})$, $6.12(\mathrm{~d}, J=1.3 \mathrm{~Hz}, 1 \mathrm{H}), 2.37(\mathrm{~s}, 3 \mathrm{H}) ;{ }^{13} \mathrm{C}\left\{{ }^{1} \mathrm{H}\right\} \mathrm{NMR}\left(\mathrm{CDCl}_{3}\right) \delta$ 159.6, 147.7, 144.6, 142.8, 134.7, 134.1, 132.6, 131.7, 129.6, 129.4, 128.92, 128.86, 127.9, 127.8, 125.9, 125.5, 121.7, 117.9, 115.2, 80.6, 73.2, 21.5; HRMS-ESI $[\mathrm{M}+\mathrm{Na}]^{+}$calcd for $\mathrm{C}_{30} \mathrm{H}_{23}{ }^{35} \mathrm{ClNaN}_{4} \mathrm{O}_{4} \mathrm{~S}^{+}$593.1021; found 593.1034 .

rac-(1R,5R)-4-(3-Bromophenyl)-8-(4-methylphenylsulfonyl)-2-(4-nitrophenyl)-7-phenyl2,6,8-triazabicyclo[3.2.1] octa-3,6-diene (3o)

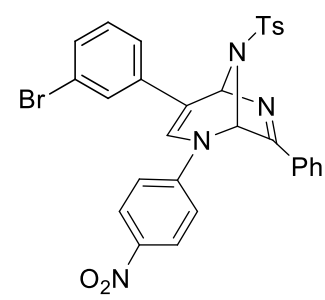

Compound 3o was obtained as a yellow solid (118 mg, yield 96\%) according to the general procedure (eluent hexane-EtOAc, 2:1) from pyrazole $\mathbf{1 g}$ and triazole $\mathbf{2 a} ; \mathrm{mp} 178-180{ }^{\circ} \mathrm{C} ;{ }^{1} \mathrm{H}$ NMR $\left(\mathrm{CDCl}_{3}\right) \delta 8.29-8.23(\mathrm{~m}, 2 \mathrm{H}), 7.61-7.54(\mathrm{~m}, 4 \mathrm{H}), 7.45-7.37(\mathrm{~m}, 2 \mathrm{H}), 7.36-7.23(\mathrm{~m}, 4 \mathrm{H})$, $7.22-7.10(\mathrm{~m}, 5 \mathrm{H}), 6.78(\mathrm{~d}, J=1.6 \mathrm{~Hz}, 1 \mathrm{H}), 6.17-6.15(\mathrm{~m}, 1 \mathrm{H}), 6.08(\mathrm{~d}, J=1.3 \mathrm{~Hz}, 1 \mathrm{H}), 2.40(\mathrm{~s}$, $3 \mathrm{H}) ;{ }^{13} \mathrm{C}\left\{{ }^{1} \mathrm{H}\right\} \mathrm{NMR}\left(\mathrm{CDCl}_{3}\right) \delta 159.5,147.7,144.7,143.1,137.8,134.7,131.8,130.4,129.7,129.6$, 129.5, 129.0, 127.92, 127.89, 127.2, 126.1, 123.1, 122.9, 122.3, 118.3, 114.7, 80.5, 73.4, 21.6; HRMS-ESI [M + H] $]^{+}$calcd for $\mathrm{C}_{30} \mathrm{H}_{24}{ }^{79} \mathrm{BrN}_{4} \mathrm{O}_{4} \mathrm{~S}^{+}$615.0696; found 615.0681. 
rac-(1R,5R)-8-(4-Methylphenylsulfonyl)-2,4-bis(4-nitrophenyl)-7-phenyl-2,6,8triazabicyclo[3.2.1]octa-3,6-diene (3p)

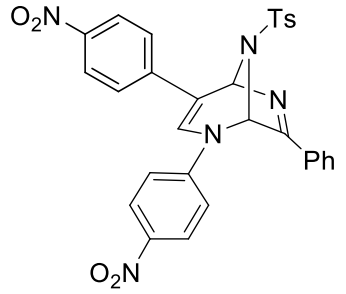

Compound 3p was obtained as an orange solid (103 mg, yield 89\%) according to the general procedure (eluent hexane-EtOAc, 2:1) from pyrazole $\mathbf{1 h}$ and triazole $\mathbf{2 a} ; \mathrm{mp} 191-193{ }^{\circ} \mathrm{C} ;{ }^{1} \mathrm{H}$ NMR $\left(\mathrm{CDCl}_{3}\right) \delta 8.28-8.23(\mathrm{~m}, 2 \mathrm{H}), 8.22-8.17(\mathrm{~m}, 2 \mathrm{H}), 7.63(\mathrm{~d}, J=8.2 \mathrm{~Hz}, 2 \mathrm{H}), 7.57-7.53(\mathrm{~m}$, 2H), 7.45-7.38 (m, 3H), $7.32(\mathrm{t}, J=7.6 \mathrm{~Hz}, 2 \mathrm{H}), 7.26-7.20(\mathrm{~m}, 2 \mathrm{H}), 7.13(\mathrm{~d}, J=8.2 \mathrm{~Hz}, 2 \mathrm{H}), 6.80$ $(\mathrm{d}, J=1.5 \mathrm{~Hz}, 1 \mathrm{H}), 6.47-6.45(\mathrm{~m}, 1 \mathrm{H}), 6.19(\mathrm{~d}, J=1.3 \mathrm{~Hz}, 1 \mathrm{H}), 2.36(\mathrm{~s}, 3 \mathrm{H}) ;{ }^{13} \mathrm{C}\left\{{ }^{1} \mathrm{H}\right\} \mathrm{NMR}$ $\left(\mathrm{CDCl}_{3}\right) \delta 159.8,147.5,146.0,144.9,143.7,142.3,134.7,132.0,129.6,129.3,129.0,127.9,127.8$, 126.0, 125.0, 124.3, 124.1, 119.1, 113.7, 80.2, 73.6, 21.5; HRMS-ESI $[\mathrm{M}+\mathrm{H}]^{+}$calcd for $\mathrm{C}_{30} \mathrm{H}_{24} \mathrm{~N}_{5} \mathrm{O}_{6} \mathrm{~S}^{+}$582.1442; found 582.1454.

rac-(1R,5R)-8-(4-Methylphenylsulfonyl)-4-(naphthalen-2-yl)-2-(4-nitrophenyl)-7-phenyl2,6,8-triazabicyclo[3.2.1] octa-3,6-diene (3q)

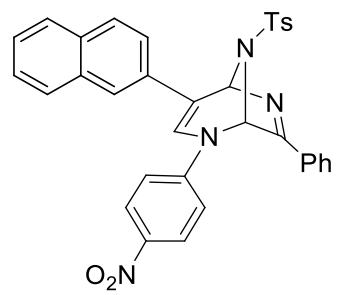

Compound 3q was obtained as a yellow solid (99 $\mathrm{mg}$, yield 85\%) according to the general procedure (eluent hexane-EtOAc, $2: 1$ ) from pyrazole $1 \mathbf{i}$ and triazole $\mathbf{2 a}$; mp $203-205{ }^{\circ} \mathrm{C} ;{ }^{1} \mathrm{H}$ NMR $\left(\mathrm{CDCl}_{3}\right) \delta 8.30-8.24(\mathrm{~m}, 2 \mathrm{H}), 7.89-7.77(\mathrm{~m}, 4 \mathrm{H}), 7.63-7.57(\mathrm{~m}, 4 \mathrm{H}), 7.57-7.46(\mathrm{~m}, 2 \mathrm{H}), 7.43(\mathrm{t}$, $J=7.4 \mathrm{~Hz}, 1 \mathrm{H}), 7.37-7.24(\mathrm{~m}, 3 \mathrm{H}), 7.19-7.14(\mathrm{~m}, 2 \mathrm{H}), 7.08(\mathrm{~d}, J=8.2 \mathrm{~Hz}, 2 \mathrm{H}), 6.82(\mathrm{~d}, J=1.6$ $\mathrm{Hz}, 1 \mathrm{H}), 6.35(\mathrm{~d}, J=1.3 \mathrm{~Hz}, 1 \mathrm{H}), 6.31-6.30(\mathrm{~m}, 1 \mathrm{H}), 2.37(\mathrm{~s}, 3 \mathrm{H}) ;{ }^{13} \mathrm{C}\left\{{ }^{1} \mathrm{H}\right\} \mathrm{NMR}\left(\mathrm{CDCl}_{3}\right) \delta$ $159.8,147.9,144.5,142.9,134.8,133.6,132.8,132.4,131.8,129.8,129.4,129.0,128.5,128.0$, $127.92,127.85,127.7,126.7,126.1,125.9,122.8,122.4,121.6,118.0,116.2,80.7,73.4,21.5$; HRMS-ESI [M + H] $]^{+}$calcd for $\mathrm{C}_{34} \mathrm{H}_{27} \mathrm{~N}_{4} \mathrm{O}_{4} \mathrm{~S}^{+}$587.1748; found 587.1753.

rac-(1R,5R)-4-Bromo-8-(4-methylphenylsulfonyl)-2-(4-nitrophenyl)-7-phenyl-2,6,8triazabicyclo[3.2.1]octa-3,6-diene $(3 \mathrm{r})$

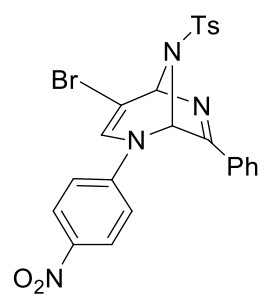

Compound 3r was obtained as a yellow solid (104 mg, yield 96\%) according to the general procedure (eluent hexane-EtOAc, 2:1) from pyrazole $\mathbf{1 j}$ and triazole $\mathbf{2 a}$; mp $92-94{ }^{\circ} \mathrm{C} ;{ }^{1} \mathrm{H}$ NMR 
$\left(\mathrm{CDCl}_{3}\right) \delta 8.23-8.17(\mathrm{~m}, 2 \mathrm{H}), 7.70(\mathrm{~d}, J=8.2 \mathrm{~Hz}, 2 \mathrm{H}), 7.61-7.55(\mathrm{~m}, 2 \mathrm{H}), 7.41(\mathrm{t}, J=7.4 \mathrm{~Hz}$, $1 \mathrm{H}), 7.32(\mathrm{t}, J=7.5 \mathrm{~Hz}, 2 \mathrm{H}), 7.19(\mathrm{~d}, J=8.2 \mathrm{~Hz}, 2 \mathrm{H}), 7.05(\mathrm{~d}, J=9.2 \mathrm{~Hz}, 2 \mathrm{H}), 6.78(\mathrm{~d}, J=1.5$ $\mathrm{Hz}, 1 \mathrm{H}), 6.08-6.06(\mathrm{~m}, 1 \mathrm{H}), 5.68(\mathrm{~d}, J=1.4 \mathrm{~Hz}, 1 \mathrm{H}), 2.37(\mathrm{~s}, 3 \mathrm{H}) ;{ }^{13} \mathrm{C}\left\{{ }^{1} \mathrm{H}\right\} \mathrm{NMR}\left(\mathrm{CDCl}_{3}\right) \delta$ $160.9,147.2$, 144.9, 142.9, 134.3, 132.0, 129.4, 129.3, 128.9, 128.0, 127.9, 125.9, 124.6, 117.6, 93.7, 83.4, 72.5, 21.5; IR (KBr): 3084, 2925, 1591, 1512, 1342, 1303, 1164, 1091, 751, 670, 577, $538 \mathrm{~cm}^{-1}$; HRMS-ESI $[\mathrm{M}+\mathrm{H}]^{+}$calcd for $\mathrm{C}_{24} \mathrm{H}_{20}{ }^{79} \mathrm{BrN}_{4} \mathrm{O}_{4} \mathrm{~S}^{+}$539.0383; found 539.0380.

rac-(1R,5R)-8-(4-Methylphenylsulfonyl)-2-(3-nitrophenyl)-4,7-diphenyl-2,6,8triazabicyclo[3.2.1]octa-3,6-diene (3s)

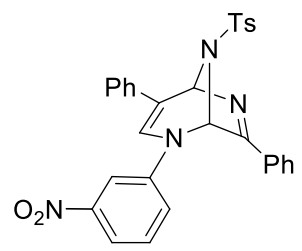

Compound 3s was obtained as an orange solid (105 $\mathrm{mg}$, yield 98\%) according to the general procedure (eluent hexane-EtOAc, 2:1) from pyrazole $\mathbf{1 k}$ and triazole $\mathbf{2 a} ; \mathrm{mp} 164-166{ }^{\circ} \mathrm{C} ;{ }^{1} \mathrm{H}$ $\operatorname{NMR}\left(\mathrm{CDCl}_{3}\right) \delta 7.92(\mathrm{dd}, J=8.1,0.7 \mathrm{~Hz}, 1 \mathrm{H}), 7.68(\mathrm{t}, J=2.2 \mathrm{~Hz}, 1 \mathrm{H}), 7.65-7.59(\mathrm{~m}, 4 \mathrm{H}), 7.53$ $(\mathrm{t}, J=8.1 \mathrm{~Hz}, 1 \mathrm{H}), 7.43-7.30(\mathrm{~m}, 8 \mathrm{H}), 7.29-7.24(\mathrm{~m}, 1 \mathrm{H}), 7.11(\mathrm{~d}, J=8.2 \mathrm{~Hz}, 2 \mathrm{H}), 6.72-6.68$ $(\mathrm{m}, 1 \mathrm{H}), 6.21(\mathrm{~s}, 1 \mathrm{H}), 6.15(\mathrm{~s}, 1 \mathrm{H}), 2.36(\mathrm{~s}, 3 \mathrm{H}) ;{ }^{13} \mathrm{C}\left\{{ }^{1} \mathrm{H}\right\} \mathrm{NMR}\left(\mathrm{CDCl}_{3}\right) \delta 158.7,149.1,144.5$, 144.3, 135.7, 134.8, 131.5, 130.7, 129.9, 129.3, 128.8, 128.7, 128.0, 127.9, 126.6, 124.7, 124.2, 122.1, 118.2, 114.8, 113.8, 80.8, 73.9, 21.4; HRMS-ESI $[\mathrm{M}+\mathrm{Na}]^{+}$calcd for $\mathrm{C}_{30} \mathrm{H}_{25} \mathrm{NaN}_{4} \mathrm{O}_{4} \mathrm{~S}^{+}$ 559.1410; found 559.1407.

rac-(1R,5R)-2-(2,4-Dinitrophenyl)-8-(4-methylphenylsulfonyl)-4,7-diphenyl-2,6,8triazabicyclo[3.2.1]octa-3,6-diene $(3 t)$

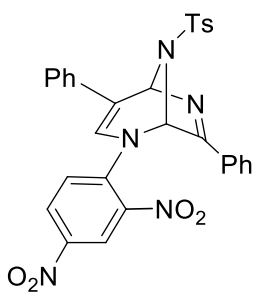

Compound 3t was obtained as an orange solid (110 mg, yield 95\%) according to the general procedure (eluent hexane-EtOAc, $2: 1$ ) from pyrazole 11 and triazole $2 \mathrm{a} ; \mathrm{mp} 148-150{ }^{\circ} \mathrm{C} ;{ }^{1} \mathrm{H}$ NMR $\left(\mathrm{CDCl}_{3}\right) \delta 8.69(\mathrm{~d}, J=2.5 \mathrm{~Hz}, 1 \mathrm{H}), 8.46(\mathrm{dd}, J=9.0,2.5 \mathrm{~Hz}, 1 \mathrm{H}), 7.86(\mathrm{~d}, J=9.0 \mathrm{~Hz}, 1 \mathrm{H}), 7.71$ $(\mathrm{d}, J=8.2 \mathrm{~Hz}, 2 \mathrm{H}), 7.52-7.48(\mathrm{~m}, 2 \mathrm{H}), 7.40-7.32(\mathrm{~m}, 3 \mathrm{H}), 7.30-7.22(\mathrm{~m}, 5 \mathrm{H}), 7.15(\mathrm{~d}, J=8.2$ $\mathrm{Hz}, 2 \mathrm{H}), 6.73-6.70(\mathrm{~m}, 1 \mathrm{H}), 6.20(\mathrm{~d}, J=1.3 \mathrm{~Hz}, 1 \mathrm{H}), 5.65-5.63(\mathrm{~m}, 1 \mathrm{H}), 2.34(\mathrm{~s}, 3 \mathrm{H}) ;{ }^{13} \mathrm{C}\left\{{ }^{1} \mathrm{H}\right\}$ $\mathrm{NMR}\left(\mathrm{CDCl}_{3}\right) \delta 159.7,144.7,143.3,142.2,142.1,135.1,135.0,131.7,129.8,129.7,128.84$, $128.78,128.4,127.8,127.7,127.4,126.6,124.7,122.9,122.6,118.6,81.2,75.4,21.5$; HRMS-ESI $[\mathrm{M}+\mathrm{H}]^{+}$calcd for $\mathrm{C}_{30} \mathrm{H}_{24} \mathrm{~N}_{5} \mathrm{O}_{6} \mathrm{~S}^{+}$582.1442; found 582.1436. 


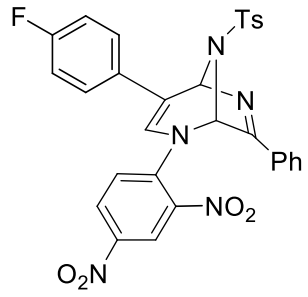

Compound 3u was obtained as an orange solid (115 mg, yield 96\%) according to the general procedure (eluent hexane-EtOAc, 2:1) from pyrazole $1 \mathrm{~m}$ and triazole $\mathbf{2 a}$; mp $164-166{ }^{\circ} \mathrm{C} ;{ }^{1} \mathrm{H}$ NMR $\left(\mathrm{CDCl}_{3}\right) \delta 8.70-8.66(\mathrm{~m}, 1 \mathrm{H}), 8.50-8.45(\mathrm{~m}, 1 \mathrm{H}), 7.89(\mathrm{~d}, J=9.0 \mathrm{~Hz}, 1 \mathrm{H}), 7.72(\mathrm{~d}, J=8.3$ $\mathrm{Hz}, 2 \mathrm{H}), 7.52-7.49(\mathrm{~m}, 2 \mathrm{H}), 7.40-7.34(\mathrm{~m}, 1 \mathrm{H}), 7.28-7.14(\mathrm{~m}, 6 \mathrm{H}), 7.08-7.01(\mathrm{~m}, 2 \mathrm{H}), 6.74-6.68$ $(\mathrm{m}, 1 \mathrm{H}), 6.13-6.11(\mathrm{~m}, 1 \mathrm{H}), 5.60-5.58(\mathrm{~m}, 1 \mathrm{H}), 2.34(\mathrm{~s}, 3 \mathrm{H}) ;{ }^{13} \mathrm{C}\left\{{ }^{1} \mathrm{H}\right\}$ NMR $\left(\mathrm{CDCl}_{3}\right) \delta 162.2(\mathrm{~d}$, $J=247.4 \mathrm{~Hz}), 159.8,144.8,143.3,142.1,142.0,135.0,131.8,131.4$ (d, $J=3.2 \mathrm{~Hz}), 129.8,129.6$, $128.8,128.4,127.8,127.6,126.60,126.56(\mathrm{~d}, J=7.8 \mathrm{~Hz}), 122.9,122.5,117.6,115.8(\mathrm{~d}, J=21.9$ Hz), 81.3, 75.3, 21.5; IR (KBr): 3061, 1600, 1535, 1347, 1163, 918, 834, 726, 680, $601 \mathrm{~cm}^{-1}$; HRMS-ESI $[\mathrm{M}+\mathrm{H}]^{+}$calcd for $\mathrm{C}_{30} \mathrm{H}_{23} \mathrm{FN}_{5} \mathrm{O}_{6} \mathrm{~S}^{+} 600.1348$; found 600.1333.

rac-(1R,5R)-4-(2-Chlorophenyl)-2-(2,4-dinitrophenyl)-8-(4-methylphenylsulfonyl)-7phenyl-2,6,8-triazabicyclo[3.2.1] octa-3,6-diene (3v)

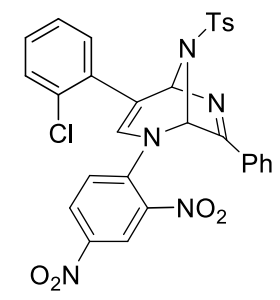

Compound 3v was obtained as an orange solid (120 mg, yield 98\%) according to the general procedure (eluent hexane-EtOAc, 2:1) from pyrazole $\mathbf{1 n}$ and triazole $\mathbf{2 a} ; \mathrm{mp} 202-204{ }^{\circ} \mathrm{C}$; ${ }^{1} \mathrm{H}$ $\operatorname{NMR}\left(\mathrm{CDCl}_{3}\right) \delta 8.72(\mathrm{~d}, J=2.6 \mathrm{~Hz}, 1 \mathrm{H}), 8.50(\mathrm{dd}, J=9.0,2.6 \mathrm{~Hz}, 1 \mathrm{H}), 7.93(\mathrm{~d}, J=9.0 \mathrm{~Hz}, 1 \mathrm{H})$, $7.76(\mathrm{~d}, J=8.2 \mathrm{~Hz}, 2 \mathrm{H}), 7.48-7.44(\mathrm{~m}, 2 \mathrm{H}), 7.43-7.37(\mathrm{~m}, 2 \mathrm{H}), 7.32-7.23(\mathrm{~m}, 5 \mathrm{H}), 7.20(\mathrm{~d}, J=$ $8.2 \mathrm{~Hz}, 2 \mathrm{H}), 6.67(\mathrm{~d}, J=1.4 \mathrm{~Hz}, 1 \mathrm{H}), 6.21-6.19(\mathrm{~m}, 1 \mathrm{H}), 5.85-5.83(\mathrm{~m}, 1 \mathrm{H}), 2.34(\mathrm{~s}, 3 \mathrm{H}) ;{ }^{13} \mathrm{C}\left\{{ }^{1} \mathrm{H}\right\}$ $\mathrm{NMR}\left(\mathrm{CDCl}_{3}\right) \delta 159.4,144.8,143.6,142.5,142.3,135.0,134.1,132.7,131.7,130.9,130.2,130.0$, $129.9,129.0,128.7,128.5,127.9,127.7,127.2,127.0,126.8,122.5,117.7,82.5,76.2,21.5$; HRMS-ESI [M + H] $]^{+}$calcd for $\mathrm{C}_{30} \mathrm{H}_{23}{ }^{35} \mathrm{ClN}_{5} \mathrm{O}_{6} \mathrm{~S}^{+}$616.1052; found 616.1036.

rac-(1R,5R)-4-(4-Bromophenyl)-2-(2,4-dinitrophenyl)-8-(4-methylphenylsulfonyl)-7-phenyl2,6,8-triazabicyclo[3.2.1] octa-3,6-diene (3w)

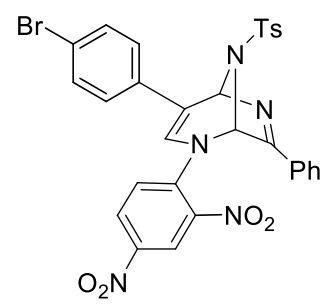


Compound 3w was obtained as an orange solid (128 mg, yield 97\%) according to the general procedure (eluent hexane-EtOAc, 2:1) from pyrazole 10 and triazole $\mathbf{2 a} ; \mathrm{mp} 184-186{ }^{\circ} \mathrm{C} ;{ }^{1} \mathrm{H}$ $\operatorname{NMR}\left(\mathrm{CDCl}_{3}\right) \delta 8.68(\mathrm{~d}, J=2.6 \mathrm{~Hz}, 1 \mathrm{H}), 8.47(\mathrm{dd}, J=9.0,2.6 \mathrm{~Hz}, 1 \mathrm{H}), 7.88(\mathrm{~d}, J=9.0 \mathrm{~Hz}, 1 \mathrm{H})$, $7.71(\mathrm{~d}, J=8.2 \mathrm{~Hz}, 2 \mathrm{H}), 7.52-7.43(\mathrm{~m}, 4 \mathrm{H}), 7.37(\mathrm{t}, J=7.4 \mathrm{~Hz}, 1 \mathrm{H}), 7.29-7.23(\mathrm{~m}, 2 \mathrm{H}), 7.17(\mathrm{~d}$, $J=8.2 \mathrm{~Hz}, 2 \mathrm{H}), 7.11(\mathrm{~d}, J=8.5 \mathrm{~Hz}, 2 \mathrm{H}), 6.70(\mathrm{~d}, J=1.3 \mathrm{~Hz}, 1 \mathrm{H}), 6.11(\mathrm{~d}, J=1.3 \mathrm{~Hz}, 1 \mathrm{H})$, 5.67-5.65 (m, 1H), $2.35(\mathrm{~s}, 3 \mathrm{H}) ;{ }^{13} \mathrm{C}\left\{{ }^{1} \mathrm{H}\right\} \mathrm{NMR}\left(\mathrm{CDCl}_{3}\right) \delta 159.8,144.9,143.4,142.2,141.9$, 135.0, 134.2, 131.9, 131.8, 129.8, 129.6, 128.8, 128.5, 127.8, 127.6, 126.7, 126.3, 123.4, 122.5, 121.2, 117.3, 81.0, 75.3, 21.5; HRMS-ESI $[\mathrm{M}+\mathrm{H}]^{+}$calcd for $\mathrm{C}_{30} \mathrm{H}_{23}{ }^{79} \mathrm{BrN}_{5} \mathrm{O}_{6} \mathrm{~S}^{+} 660.0547$; found 660.0527 .

rac-5-\{(1R,5R)-8-(4-Methylphenylsulfonyl)-4,7-diphenyl-2,6,8-triazabicyclo[3.2.1]octa-3,6dien-2-yl\}-3-phenylisoxazole $(3 x)$

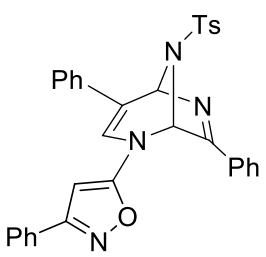

Compound 3x was obtained as a yellow solid (99 $\mathrm{mg}$, yield 89\%) according to the general procedure (eluent hexane-EtOAc, 2:1) from pyrazole 1p and triazole $\mathbf{2 a}$; mp $159-161{ }^{\circ} \mathrm{C} ;{ }^{1} \mathrm{H}$ NMR $\left(\mathrm{CDCl}_{3}\right) \delta 7.93-7.88(\mathrm{~m}, 2 \mathrm{H}), 7.81-7.77(\mathrm{~m}, 2 \mathrm{H}), 7.64(\mathrm{~d}, J=8.2 \mathrm{~Hz}, 2 \mathrm{H}), 7.51-7.39(\mathrm{~m}$, $8 \mathrm{H}), 7.38-7.29(\mathrm{~m}, 3 \mathrm{H}), 7.11(\mathrm{~d}, J=8.2 \mathrm{~Hz}, 2 \mathrm{H}), 6.86(\mathrm{~d}, J=1.4 \mathrm{~Hz}, 1 \mathrm{H}), 6.26(\mathrm{~d}, J=1.2 \mathrm{~Hz}$, $1 \mathrm{H}), 6.09-6.07(\mathrm{~m}, 1 \mathrm{H}), 5.64(\mathrm{~s}, 1 \mathrm{H}), 2.34(\mathrm{~s}, 3 \mathrm{H}) ;{ }^{13} \mathrm{C}\left\{{ }^{1} \mathrm{H}\right\} \mathrm{NMR}\left(\mathrm{CDCl}_{3}\right) \delta 163.7,163.5,161.2$, 144.6, 135.1, 134.4, 131.9, 130.3, 129.4, 129.3, 128.92, 128.88, 128.87, 128.6, 128.04, 127.95, 127.4, 126.6, 124.6, 119.1, 117.0, 80.9, 79.5, 71.0, 21.5; HRMS-ESI $[\mathrm{M}+\mathrm{Na}]^{+}$calcd for $\mathrm{C}_{33} \mathrm{H}_{26} \mathrm{NaN}_{4} \mathrm{O}_{3} \mathrm{~S}^{+}$581.1618; found 581.1632.

rac-(2R,5R)-10-(4-Methylphenylsulfonyl)-1-(4-nitrophenyl)-3-phenyl-10-2,5-dihydro-1H2,5-epiminobenzo[e][1,4]diazepine $(3 y)$

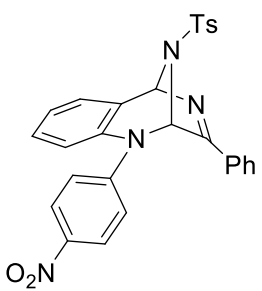

Compound 3y was obtained as a white solid (94 mg, yield 92\%) according to the general procedure (eluent hexane-EtOAc, 2:1) from pyrazole 1q and triazole $2 \mathbf{a}$; mp $176-178{ }^{\circ} \mathrm{C} ;{ }^{1} \mathrm{H} \mathrm{NMR}\left(\mathrm{CDCl}_{3}\right)$ $\delta 8.22(\mathrm{~d}, J=9.0 \mathrm{~Hz}, 2 \mathrm{H}), 7.56(\mathrm{~d}, J=8.2 \mathrm{~Hz}, 2 \mathrm{H}), 7.45-7.35(\mathrm{~m}, 5 \mathrm{H}), 7.30(\mathrm{dd}, J=7.5,1.4 \mathrm{~Hz}$, $1 \mathrm{H}), 7.25(\mathrm{t}, J=7.7 \mathrm{~Hz}, 2 \mathrm{H}), 7.03-6.95(\mathrm{~m}, 3 \mathrm{H}), 6.89(\mathrm{t}, J=7.1 \mathrm{~Hz}, 1 \mathrm{H}), 6.45(\mathrm{~s}, 1 \mathrm{H}), 6.43$ (d, $J$ $=8.2 \mathrm{~Hz}, 1 \mathrm{H}), 6.29(\mathrm{~s}, 1 \mathrm{H}), 2.24(\mathrm{~s}, 3 \mathrm{H}) ;{ }^{13} \mathrm{C}\left\{{ }^{1} \mathrm{H}\right\} \mathrm{NMR}\left(\mathrm{CDCl}_{3}\right) \delta 161.4,149.8,145.0,144.2$, 137.7, 134.2, 131.7, 129.7, 129.3, 129.1, 128.6, 128.2, 127.5, 126.7, 126.1, 125.5, 121.1, 120.8, 116.0, 83.8, 77.7, 21.3; IR (KBr): 3104, 3073, 2925, 2853, 1595, 1516, 1491, 1344, 1217, 1163 , $965,866,756,710,677,582,543 \mathrm{~cm}^{-1}$; HRMS-ESI $[\mathrm{M}+\mathrm{H}]^{+}$calcd for $\mathrm{C}_{28} \mathrm{H}_{23} \mathrm{~N}_{4} \mathrm{O}_{4} \mathrm{~S}^{+} 511.1435$; found 511.1432 . 


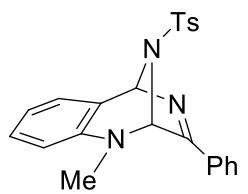

Compound $3 \mathbf{z}$ was obtained as a white solid $(65 \mathrm{mg}$, yield $81 \%$ ) according to the general procedure (eluent hexane-EtOAc, 2:1) from pyrazole 1r and triazole $2 \mathbf{a}(0.8 \mathrm{mmol})$ using $5 \mathrm{~mol}$. \% of $\mathrm{Rh}_{2}(\mathrm{Piv})_{4}$ (reaction time $0.5 \mathrm{~h}$ ); $\mathrm{mp} 217-219{ }^{\circ} \mathrm{C} ;{ }^{1} \mathrm{H} \mathrm{NMR}\left(\mathrm{CDCl}_{3}\right) \delta$ 7.71-7.67 (m, 2H), 7.48-7.37 $(\mathrm{m}, 5 \mathrm{H}), 7.19(\mathrm{dd}, J=7.4,1.4 \mathrm{~Hz}, 2 \mathrm{H}), 7.05-6.97(\mathrm{~m}, 3 \mathrm{H}), 6.73(\mathrm{td}, J=7.4,0.7 \mathrm{~Hz}, 1 \mathrm{H}), 6.16(\mathrm{~d}$, $J=8.2 \mathrm{~Hz}, 1 \mathrm{H}), 6.11(\mathrm{~s}, 1 \mathrm{H}), 5.96(\mathrm{~s}, 1 \mathrm{H}), 2.98(\mathrm{~s}, 3 \mathrm{H}), 2.27(\mathrm{~s}, 3 \mathrm{H}) ;{ }^{13} \mathrm{C}\left\{{ }^{1} \mathrm{H}\right\} \operatorname{NMR}\left(\mathrm{CDCl}_{3}\right) \delta$ 159.8, 143.6, 139.7, 134.5, 131.4, 130.6, 129.3, 128.79, 128.75, 128.3, 127.6, 125.6, 118.6, 118.2, 112.4, 83.7, 77.4, 37.6, 21.4; IR (KBr): 3028, 2976, 2924, 2884, 2883, 1595, 1492, 1374, 1165, $1089,905,813,744,709,674,579,544 \mathrm{~cm}^{-1}$; HRMS-ESI $[\mathrm{M}+\mathrm{H}]^{+}$calcd for $\mathrm{C}_{23} \mathrm{H}_{22} \mathrm{~N}_{3} \mathrm{O}_{2} \mathrm{~S}^{+}$ 404.1427; found 404.1442.

rac-(1R,5R)-Ethyl 3-methyl-2,7-diphenyl-8-(4-methylphenylsulfonyl)-2,6,8-triazabicyclo[3.2.1]octa-3,6-diene-4-carboxylate (3za)

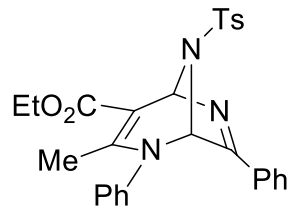

Compound 3za was obtained as a colorless foam (80 mg, yield $80 \%$ ) according to the general procedure (eluent hexane-EtOAc, 2:1) from pyrazole 1s and triazole $\mathbf{2 a} ;{ }^{1} \mathrm{H} \mathrm{NMR}\left(\mathrm{CDCl}_{3},-40{ }^{\circ} \mathrm{C}\right)$ $\delta 7.73(\mathrm{~d}, J=8.2 \mathrm{~Hz}, 2 \mathrm{H}), 7.65-7.60(\mathrm{~m}, 2 \mathrm{H}), 7.39-7.33(\mathrm{~m}, 2 \mathrm{H}), 7.25-7.12(\mathrm{~m}, 7 \mathrm{H}), 6.40-6.35$ $(\mathrm{m}, 2 \mathrm{H}), 6.21(\mathrm{~s}, 1 \mathrm{H}), 4.34-4.28(\mathrm{~m}, 1 \mathrm{H}), 4.18-4.11(\mathrm{~m}, 1 \mathrm{H}), 2.31(\mathrm{~s}, 3 \mathrm{H}), 1.56(\mathrm{~s}, 3 \mathrm{H}), 1.41(\mathrm{~J}=$ $7.1 \mathrm{~Hz}, 3 \mathrm{H}) ;{ }^{13} \mathrm{C}\left\{{ }^{1} \mathrm{H}\right\}$ NMR $\left(\mathrm{CDCl}_{3},-40{ }^{\circ} \mathrm{C}\right) \delta 165.1,158.8,149.6,144.4,140.3,133.1,131.4$, 130.6, 129.3, 129.09, 129.08, 128.7, 128.3, 128.00, 127.96, 127.5, 126.4, 95.5, 79.2, 76.2, 59.9, 21.5, 18.3, 14.5; HRMS-ESI [M + H] calcd for $\mathrm{C}_{28} \mathrm{H}_{28} \mathrm{~N}_{3} \mathrm{O}_{4} \mathrm{~S}^{+}$502.1795; found 502.1798. 


\section{Synthesis of imidazoles 4,15}
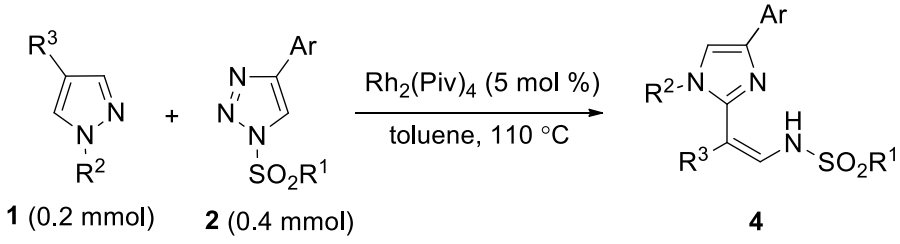

General procedure: Pyrazole $1(0.2 \mathrm{mmol})$, triazole $2(0.4 \mathrm{mmol})$, and toluene $(1 \mathrm{~mL})$ were placed into a screw cap glass tube. After the addition of $\mathrm{Rh}_{2}(\mathrm{Piv})_{4}(6.2 \mathrm{mg}, 0.05$ equiv), cap was rapidly screwed and the tube was placed into an oil bath preheated to $110^{\circ} \mathrm{C}$. The reaction mixture was stirred at this temperature for 1.5-3 h. The solvent was removed in vacuo, and the residue was purified by column chromatography on silica gel (eluent hexane-EtOAc, 2:1) to give the imidazole 4,15.

(Z)-4-Methyl- $N$-(2-(1-methyl-4-phenyl-1H-imidazol-2-yl)-2-phenylvinyl)benzenesulfonamide (4a)<smiles>CN(C)C=C(c1ccccc1)c1nc(-c2ccccc2)cn1C</smiles>

Compound 4a was obtained as a white solid (75 mg, yield $87 \%$ ) according to the general procedure (eluent hexane-EtOAc, 2:1) from pyrazole $1 \mathrm{t}$ and triazole $\mathbf{2 a}$; mp $139-141{ }^{\circ} \mathrm{C}$; ${ }^{1} \mathrm{H} \mathrm{NMR}\left(\mathrm{CDCl}_{3}\right)$ $\delta 10.93$ (br. s, $1 \mathrm{H}), 7.86(\mathrm{~d}, J=8.3 \mathrm{~Hz}, 2 \mathrm{H}), 7.75(\mathrm{~d}, J=7.3 \mathrm{~Hz}, 2 \mathrm{H}), 7.46-7.40(\mathrm{~m}, 2 \mathrm{H}), 7.40-7.29$ (m, 6H), 7.27-7.23 (m, 2H), $7.07(\mathrm{~s}, 1 \mathrm{H}), 6.82(\mathrm{~s}, 1 \mathrm{H}), 3.03(\mathrm{~s}, 3 \mathrm{H}), 2.42(\mathrm{~s}, 3 \mathrm{H}) ;{ }^{13} \mathrm{C}\left\{{ }^{1} \mathrm{H}\right\} \mathrm{NMR}$ $\left(\mathrm{CDCl}_{3}\right) \delta 145.0,143.5,139.4,138.0,137.7,133.1,129.8,129.3,128.8,128.7,128.1,127.5,127.0$, 126.6, 124.5, 117.6, 110.6, 35.2, 21.5; IR (KBr): 3138, 3056, 3028, 2942, 2509 (br), 1646, 1596, 1449, 1342, 1162, 1142, 1088, 969, 855, 752, 698, 663, 550, $537 \mathrm{~cm}^{-1}$; HRMS-ESI [M + H $]^{+}$calcd for $\mathrm{C}_{25} \mathrm{H}_{24} \mathrm{~N}_{3} \mathrm{O}_{2} \mathrm{~S}^{+} 430.1584$; found 430.1572 .

(Z)-4-Methyl- $N$-(2-(1-methyl-4-phenyl-1H-imidazol-2-yl)-2-(4-methylphenyl)vinyl)benzenesulfonamide $(4 \mathrm{~b})$<smiles>Cc1ccc(/C(=C/NS)c2nc(-c3ccccc3)cn2[N+](=O)[O-])cc1</smiles>

Compound $\mathbf{4 b}$ was obtained as a yellow oil (79 $\mathrm{mg}$, yield 89\%) according to the general procedure (eluent hexane-EtOAc, 2:1) from pyrazole 1u and triazole $2 \mathbf{a} ;{ }^{1} \mathrm{H}$ NMR $\left(\mathrm{CDCl}_{3}\right) \delta 11.26$ (br. s, $1 \mathrm{H}), 7.85(\mathrm{~d}, J=8.3 \mathrm{~Hz}, 2 \mathrm{H}), 7.77-7.73(\mathrm{~m}, 2 \mathrm{H}), 7.46-7.40(\mathrm{~m}, 2 \mathrm{H}), 7.34-7.29(\mathrm{~m}, 3 \mathrm{H})$, $7.20-7.12(\mathrm{~m}, 4 \mathrm{H}), 7.06(\mathrm{~s}, 1 \mathrm{H}), 6.77(\mathrm{~s}, 1 \mathrm{H}), 3.06(\mathrm{~s}, 3 \mathrm{H}), 2.42(\mathrm{~s}, 3 \mathrm{H}), 2.38(\mathrm{~s}, 3 \mathrm{H}) ;{ }^{13} \mathrm{C}\left\{{ }^{1} \mathrm{H}\right\}$ NMR $\left(\mathrm{CDCl}_{3}\right) \delta 145.2,143.5,139.5,138.1,137.3,134.7,133.2,129.8,129.6,128.8,128.7,128.0$, 127.1, 126.6, 124.6, 117.5, 110.7, 35.3, 21.5, 21.1; HRMS-ESI $[\mathrm{M}+\mathrm{H}]^{+}$calcd for $\mathrm{C}_{26} \mathrm{H}_{26} \mathrm{~N}_{3} \mathrm{O}_{2} \mathrm{~S}^{+}$ 444.1740; found 444.1733. 
(Z)- $N$-(2-(4-Methoxyphenyl)-2-(1-methyl-4-phenyl-1H-imidazol-2-yl)vinyl)-4methylbenzenesulfonamide $(4 \mathrm{c})$<smiles>COc1ccc(/C(=C/NS(=O)(=O)c2ccccc2)c2nc(-c3ccccc3)cn2C)cc1</smiles>

Compound $\mathbf{4 c}$ was obtained as a white solid (82 $\mathrm{mg}$, yield $89 \%$ ) according to the general procedure (eluent hexane-EtOAc, 2:1) from pyrazole $\mathbf{1 v}$ and triazole $2 \mathbf{a} ; \mathrm{mp} 66-68{ }^{\circ} \mathrm{C} ;{ }^{1} \mathrm{H}$ NMR $\left(\mathrm{CDCl}_{3}\right) \delta$ 11.24 (br. s, 1H), 7.85 (d, $J=8.3 \mathrm{~Hz}, 2 \mathrm{H}), 7.75$ (d, $J=7.3 \mathrm{~Hz}, 2 \mathrm{H}), 7.43$ (t, $J=7.7 \mathrm{~Hz}, 2 \mathrm{H}$ ), 7.34-7.29 (m, 3H), $7.17(\mathrm{~d}, J=8.7 \mathrm{~Hz}, 2 \mathrm{H}), 7.05(\mathrm{~s}, 1 \mathrm{H}), 6.91(\mathrm{~d}, J=8.7 \mathrm{~Hz}, 2 \mathrm{H}), 6.74(\mathrm{~s}, 1 \mathrm{H})$, 3.84 (s, 3H), 3.06 (s, 3H), $2.42(\mathrm{~s}, 3 \mathrm{H}),{ }^{13} \mathrm{C}\left\{{ }^{1} \mathrm{H}\right\} \mathrm{NMR}\left(\mathrm{CDCl}_{3}\right) \delta$ 159.2, 145.2, 143.4, 139.4, 138.1, 133.2, 130.0, 129.8, 129.4, 128.7, 128.5, 127.0, 126.6, 124.6, 117.6, 114.3, 110.4, 55.3, 35.3, 21.5; HRMS-ESI $[\mathrm{M}+\mathrm{H}]^{+}$calcd for $\mathrm{C}_{26} \mathrm{H}_{26} \mathrm{~N}_{3} \mathrm{O}_{3} \mathrm{~S}^{+} 460.1689$; found 460.1682 .

(Z)- $N$-(2-(4-Chlorophenyl)-2-(1-methyl-4-phenyl-1H-imidazol-2-yl)vinyl)-4methylbenzenesulfonamide (4d)<smiles>CNC=C(c1ccc(Cl)cc1)c1nc(-c2ccccc2)cn1[N+](=O)[O-]</smiles>

Compound $\mathbf{4 d}$ was obtained as a white solid ( $81 \mathrm{mg}$, yield $87 \%$ ) according to the general procedure (eluent hexane-EtOAc, 2:1) from pyrazole $1 \mathbf{w}$ and triazole $2 \mathbf{a} ; \mathrm{mp} 153-155{ }^{\circ} \mathrm{C} ;{ }^{1} \mathrm{H} \mathrm{NMR}\left(\mathrm{CDCl}_{3}\right)$ $\delta 10.79$ (br. s, 1H), $7.85(\mathrm{~d}, J=8.3 \mathrm{~Hz}, 2 \mathrm{H}), 7.73(\mathrm{~d}, J=7.3 \mathrm{~Hz}, 2 \mathrm{H}), 7.43(\mathrm{~d}, J=7.7 \mathrm{~Hz}, 2 \mathrm{H})$, $7.37-7.30(\mathrm{~m}, 5 \mathrm{H}), 7.19(\mathrm{~d}, J=8.3 \mathrm{~Hz}, 2 \mathrm{H}), 7.07(\mathrm{~s}, 1 \mathrm{H}), 6.81(\mathrm{~s}, 1 \mathrm{H}), 3.07(\mathrm{~s}, 3 \mathrm{H}), 2.43(\mathrm{~s}, 3 \mathrm{H})$; ${ }^{13} \mathrm{C}\left\{{ }^{1} \mathrm{H}\right\} \mathrm{NMR}\left(\mathrm{CDCl}_{3}\right) \delta 144.6,143.6,139.6,137.9,136.2,133.4,133.0,129.8,129.7,129.2$, $129.1,128.7,127.2,126.6,124.6,117.6,109.3,35.3,21.5$; HRMS-ESI $[\mathrm{M}+\mathrm{H}]^{+}$calcd for $\mathrm{C}_{25} \mathrm{H}_{23}{ }^{35} \mathrm{ClN}_{3} \mathrm{O}_{2} \mathrm{~S}^{+}$464.1194; found 464.1196.

(Z)- $N$-(2-(4-Bromophenyl)-2-(1-methyl-4-phenyl-1H-imidazol-2-yl)vinyl)-4-methylbenzenesulfonamide (4e)<smiles>CNC=C(c1ccc(Br)cc1)c1nc(-c2ccccc2)cn1C</smiles>

Compound $4 \mathbf{e}$ was obtained as a white solid (87 $\mathrm{mg}$, yield $86 \%$ ) according to the general procedure (eluent hexane-EtOAc, 2:1) from pyrazole 1x and triazole $\mathbf{2 a}$; mp $132-134{ }^{\circ} \mathrm{C} ;{ }^{1} \mathrm{H}$ NMR $\left(\mathrm{CDCl}_{3}\right)$ $\delta 10.82$ (br. s, $1 \mathrm{H}), 7.85(\mathrm{~d}, J=8.3 \mathrm{~Hz}, 2 \mathrm{H}), 7.76-7.71(\mathrm{~m}, 2 \mathrm{H}), 7.50(\mathrm{~d}, J=8.4 \mathrm{~Hz}, 2 \mathrm{H}), 7.43(\mathrm{t}$, $J=7.7 \mathrm{~Hz}, 2 \mathrm{H}), 7.35-7.29(\mathrm{~m}, 3 \mathrm{H}), 7.13(\mathrm{~d}, J=8.3 \mathrm{~Hz}, 2 \mathrm{H}), 7.07(\mathrm{~s}, 1 \mathrm{H}), 6.81(\mathrm{~s}, 1 \mathrm{H}), 3.07(\mathrm{~s}$, $3 \mathrm{H}), 2.43(\mathrm{~s}, 3 \mathrm{H}) ;{ }^{13} \mathrm{C}\left\{{ }^{1} \mathrm{H}\right\} \mathrm{NMR}\left(\mathrm{CDCl}_{3}\right) \delta 144.5,143.6,139.6,137.9,136.7,133.0,132.0,129.8$, 129.7, 129.5, 128.7, 127.1, 126.6, 124.6, 121.4, 117.6, 109.3, 35.3, 21.5; IR (KBr): 3065, 2949, 1621, 1496, 1454, 1342, 1164, 1146, 1088, 954, 864, 741, 685, 664, $545 \mathrm{~cm}^{-1}$; HRMS-ESI [M + $\mathrm{H}]^{+}$calcd for $\mathrm{C}_{25} \mathrm{H}_{23}{ }^{79} \mathrm{BrN}_{3} \mathrm{O}_{2} \mathrm{~S}^{+}$508.0689; found 508.0704. 
(Z)-4-Methyl- $N$-(2-(1-methyl-4-phenyl-1H-imidazol-2-yl)-2-(4-nitrophenyl)vinyl)benzenesulfonamide (4f)<smiles>CNC=C(c1ccc([N+](=O)[O-])cc1)c1nc(-c2ccccc2)cn1C</smiles>

Compound $4 \mathbf{f}$ was obtained as an orange solid (80 $\mathrm{mg}$, yield $84 \%$ ) according to the general procedure (eluent hexane-EtOAc, 2:1) from pyrazole 1y and triazole $\mathbf{2 a} ; \mathrm{mp} 112-114{ }^{\circ} \mathrm{C} ;{ }^{1} \mathrm{H}$ NMR $\left(\mathrm{CDCl}_{3}\right) \delta 9.82$ (br. s, $\left.1 \mathrm{H}\right), 8.20(\mathrm{~d}, J=8.8 \mathrm{~Hz}, 2 \mathrm{H}), 7.82(\mathrm{~d}, J=8.3 \mathrm{~Hz}, 2 \mathrm{H}), 7.72-7.68(\mathrm{~m}$, 2H), $7.40(\mathrm{t}, J=7.6 \mathrm{~Hz}, 2 \mathrm{H}), 7.34-7.26(\mathrm{~m}, 5 \mathrm{H}), 7.09(\mathrm{~s}, 1 \mathrm{H}), 7.06(\mathrm{~s}, 1 \mathrm{H}), 3.06(\mathrm{~s}, 3 \mathrm{H}), 2.42(\mathrm{~s}$, $3 \mathrm{H}) ;{ }^{13} \mathrm{C}\left\{{ }^{1} \mathrm{H}\right\} \mathrm{NMR}\left(\mathrm{CDCl}_{3}\right) \delta 146.5,144.9,143.82,143.77,139.7,137.9,133.2,132.5,129.9$, $128.8,127.4,127.3,126.6,124.6,124.3,117.8,107.1,35.0,21.5$; HRMS-ESI $[\mathrm{M}+\mathrm{H}]^{+}$calcd for $\mathrm{C}_{25} \mathrm{H}_{23} \mathrm{~N}_{4} \mathrm{O}_{4} \mathrm{~S}^{+} 475.1435$; found 475.1418 .

(Z)-4-Methyl- $N$-(2-(1-methyl-4-phenyl-1H-imidazol-2-yl)-2-(naphthalen-2-yl)vinyl)benzenesulfonamide $(\mathbf{4 g})$

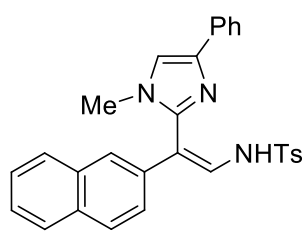

Compound 4g was obtained as a white solid (88 $\mathrm{mg}$, yield $92 \%$ ) according to the general procedure (eluent hexane-EtOAc, 2:1) from pyrazole $\mathbf{~ z}$ and triazole $\mathbf{2 a}$; mp $210-212{ }^{\circ} \mathrm{C} ;{ }^{1} \mathrm{H}$ NMR $\left(\mathrm{CDCl}_{3}\right)$

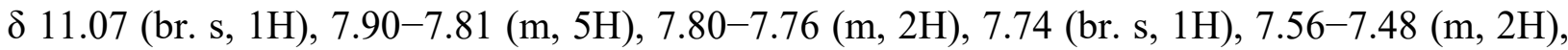
$7.45(\mathrm{t}, J=7.7 \mathrm{~Hz}, 2 \mathrm{H}), 7.37-7.29(\mathrm{~m}, 4 \mathrm{H}), 7.10(\mathrm{~s}, 1 \mathrm{H}), 6.94(\mathrm{~s}, 1 \mathrm{H}), 3.05(\mathrm{~s}, 3 \mathrm{H}), 2.42(\mathrm{~s}, 3 \mathrm{H})$; ${ }^{13} \mathrm{C}\left\{{ }^{1} \mathrm{H}\right\}$ NMR $\left(\mathrm{CDCl}_{3}\right) \delta 145.0,143.6,139.7,138.0,135.2,133.6,133.1,132.5,129.8,129.7$, $128.8,128.5,127.8,127.7,127.1,126.64,126.63,126.3,126.22,126.17,124.6,117.6,110.7,35.3$, 21.5; HRMS-ESI [M + H] $]^{+}$calcd for $\mathrm{C}_{29} \mathrm{H}_{26} \mathrm{~N}_{3} \mathrm{O}_{2} \mathrm{~S}^{+} 480.1740$; found 480.1735 .

(E)-4-Methyl- $N$-(2-(1-methyl-4-phenyl-1H-imidazol-2-yl)-3-oxo-3-phenylprop-1-en-1yl)benzenesulfonamide (4h)

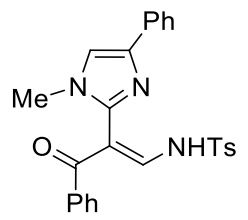

Compound 4h was obtained as a white solid (69 mg, yield 76\%) according to the general procedure (eluent hexane-EtOAc, 2:1) from pyrazole 1za and triazole $\mathbf{2 a}$; mp $284-286{ }^{\circ} \mathrm{C} ;{ }^{1} \mathrm{H} \mathrm{NMR}\left(\mathrm{CDCl}_{3}\right)$ $\delta 13.33$ (br. s, 1H), 8.54 (s, 1H), $7.76(\mathrm{~d}, J=8.0 \mathrm{~Hz}, 2 \mathrm{H}), 7.68(\mathrm{~d}, J=7.4 \mathrm{~Hz}, 2 \mathrm{H}), 7.56(\mathrm{t}, J=7.1$ $\mathrm{Hz}, 1 \mathrm{H}), 7.51-7.42(\mathrm{~m}, 7 \mathrm{H}), 7.29-7.24(\mathrm{~m}, 2 \mathrm{H}), 7.05(\mathrm{~s}, 1 \mathrm{H}), 3.67(\mathrm{~s}, 3 \mathrm{H}), 2.42(\mathrm{~s}, 3 \mathrm{H}),{ }^{13} \mathrm{C}\left\{{ }^{1} \mathrm{H}\right\}$ NMR (DMSO- $\left.d_{6}\right) \delta 190.9,164.2,145.8,141.9,141.1,141.0,131.5,130.5,129.8,129.7,129.5$, 128.6, 128.4, 127.3, 126.3, 125.5, 119.2, 96.8, 35.2, 21.4; IR (KBr): 3144, 3058, 2921, 2853, 2783, $2677,1536,1508,1350,1275,1141,1082,889,824,739,660,555 \mathrm{~cm}^{-1}$; HRMS-ESI [M + Na $]^{+}$ calcd for $\mathrm{C}_{26} \mathrm{H}_{23} \mathrm{NaN}_{3} \mathrm{O}_{3} \mathrm{~S}^{+} 480.1352$; found 480.1343 . 


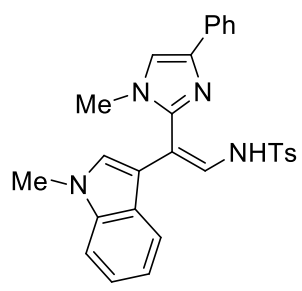

Compound 4i was obtained as a white solid (78 $\mathrm{mg}$, yield $81 \%$ ) according to the general procedure (eluent hexane-EtOAc, 2:1) from pyrazole 1zb and triazole $\mathbf{2 a} ; \mathrm{mp} 229-231{ }^{\circ} \mathrm{C} ;{ }^{1} \mathrm{H} \mathrm{NMR}\left(\mathrm{CDCl}_{3}\right)$ $\delta 11.77$ (br. s, 1H), $7.86(\mathrm{~d}, J=8.1 \mathrm{~Hz}, 2 \mathrm{H}), 7.77(\mathrm{~d}, J=7.5 \mathrm{~Hz}, 2 \mathrm{H}), 7.44(\mathrm{t}, J=7.6 \mathrm{~Hz}, 2 \mathrm{H})$, $7.38-7.23(\mathrm{~m}, 6 \mathrm{H}), 7.12-7.00(\mathrm{~m}, 3 \mathrm{H}), 6.83(\mathrm{~s}, 1 \mathrm{H}), 3.83(\mathrm{~s}, 3 \mathrm{H}), 3.03(\mathrm{~s}, 3 \mathrm{H}), 2.41(\mathrm{~s}, 3 \mathrm{H})$; ${ }^{13} \mathrm{C}\left\{{ }^{1} \mathrm{H}\right\} \mathrm{NMR}\left(\mathrm{CDCl}_{3}\right) \delta 145.7,143.3,139.0,138.2,136.9,133.2,129.7,128.74,128.66,127.9$, 127.2, 127.0, 126.6, 124.6, 122.3, 120.0, 119.4, 117.4, 112.2, 109.5, 102.7, 34.4, 32.9, 21.5; HRMS-ESI [M $+\mathrm{H}]^{+}$calcd for $\mathrm{C}_{28} \mathrm{H}_{27} \mathrm{~N}_{4} \mathrm{O}_{2} \mathrm{~S}^{+} 483.1849$; found 483.1854 .

(Z)- $N$-(2-(1-Ethyl-4-phenyl-1H-imidazol-2-yl)-2-phenylvinyl)-4-methylbenzenesulfonamide $(4 \mathbf{j})$<smiles>CCN(C)c1cn(-c2ccccc2)cc1/C=N\Nc1ccccc1</smiles>

Compound $\mathbf{4} \mathbf{j}$ was obtained as a yellow oil ( $81 \mathrm{mg}$, yield $91 \%)$ according to the general procedure (eluent hexane-EtOAc, 2:1) from pyrazole 1zc and triazole 2a according; ${ }^{1} \mathrm{H}$ NMR $\left(\mathrm{CDCl}_{3}\right) \delta$ 10.60 (br. s, $1 \mathrm{H}), 7.86$ (d, $J=8.3 \mathrm{~Hz}, 2 \mathrm{H}), 7.77(\mathrm{~d}, J=8.2 \mathrm{~Hz}, 2 \mathrm{H}), 7.43$ (t, $J=7.7 \mathrm{~Hz}, 2 \mathrm{H})$, $7.39-7.29(\mathrm{~m}, 6 \mathrm{H}), 7.28-7.25(\mathrm{~m}, 2 \mathrm{H}), 7.15(\mathrm{~s}, 1 \mathrm{H}), 6.83-6.79(\mathrm{~m}, 1 \mathrm{H}), 3.37(\mathrm{q}, J=7.3 \mathrm{~Hz}, 2 \mathrm{H})$, $2.42(\mathrm{~s}, 3 \mathrm{H}), 1.06(\mathrm{t}, J=7.3 \mathrm{~Hz}, 3 \mathrm{H}) ;{ }^{13} \mathrm{C}\left\{{ }^{1} \mathrm{H}\right\} \mathrm{NMR}\left(\mathrm{CDCl}_{3}\right) 144.3,143.5,139.9,138.0,137.7$, 133.2, 129.8, 129.2, 128.8, 128.7, 127.7, 127.5, 127.0, 126.6, 124.5, 115.4, 111.2, 42.1 , 21.5, 15.7; HRMS-ESI $[\mathrm{M}+\mathrm{H}]^{+}$calcd for $\mathrm{C}_{26} \mathrm{H}_{26} \mathrm{~N}_{3} \mathrm{O}_{2} \mathrm{~S}^{+} 444.1740$; found 444.1748 .

\section{(Z)- $N$-(2-(1-Isopropyl-4-phenyl-1H-imidazol-2-yl)-2-phenylvinyl)-4-methylbenzenesulfon-} amide (4k)

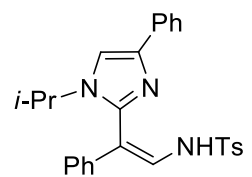

Compound 4k was obtained as a white solid ( $83 \mathrm{mg}$, yield $91 \%$ ) according to the general procedure (eluent hexane-EtOAc, 2:1) from pyrazole 1zd and triazole $2 \mathbf{a} ; \mathrm{mp} 62-64{ }^{\circ} \mathrm{C} ;{ }^{1} \mathrm{H}$ NMR $\left(\mathrm{CDCl}_{3}\right)$ $\delta 9.90$ (br. s, 1H), 7.86 (d, $J=8.3 \mathrm{~Hz}, 2 \mathrm{H}), 7.80-7.86(\mathrm{~m}, 2 \mathrm{H}), 7.43$ (t, $J=7.7 \mathrm{~Hz}, 2 \mathrm{H}), 7.39-7.29$ $(\mathrm{m}, 6 \mathrm{H}), 7.28-7.25(\mathrm{~m}, 3 \mathrm{H}), 6.83(\mathrm{~s}, 1 \mathrm{H}), 3.79(\mathrm{sept}, J=6.6 \mathrm{~Hz}, 1 \mathrm{H}), 2.43(\mathrm{~s}, 3 \mathrm{H}), 1.14(\mathrm{~d}, J=6.6$ $\mathrm{Hz}, 6 \mathrm{H}) ;{ }^{13} \mathrm{C}\left\{{ }^{1} \mathrm{H}\right\} \mathrm{NMR}\left(\mathrm{CDCl}_{3}\right) \delta 143.8,143.6,140.4,138.0,137.9,133.4,129.8,129.1,128.8$,

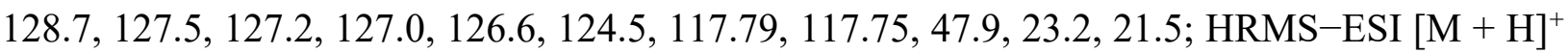
calcd for $\mathrm{C}_{27} \mathrm{H}_{28} \mathrm{~N}_{3} \mathrm{O}_{2} \mathrm{~S}^{+} 458.1897$; found 458.1879 . 
(Z)- $N$-(2-(1-Benzyl-4-phenyl-1H-imidazol-2-yl)-2-phenylvinyl)-4-methylbenzenesulfonamide (4I)

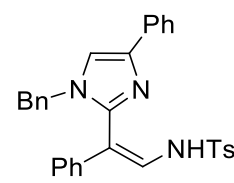

Compound $4 \mathbf{l}$ was obtained as a white solid (92 $\mathrm{mg}$, yield 91\%) according to the general procedure (eluent hexane-EtOAc, 2:1) from pyrazole 1ze and triazole 2a.

Gram-scale synthesis of 4l: Pyrazole 1ze $(702 \mathrm{mg}, 3.0 \mathrm{mmol})$, triazole 2a (1794 mg, $6 \mathrm{mmol})$, $\mathrm{Rh}_{2}$ (Piv) 4 (46 mg, $0.075 \mathrm{mmol}$ ), and toluene $(7 \mathrm{~mL})$ were placed into a round-bottom flask and refluxed in an oil bath for $3 \mathrm{~h}$ under stirring. The solvent was removed in vacuo, and the residue was purified by column chromatography on silica gel (eluent hexane-EtOAc, 3:1). Yield of imidazole 4l: $1.41 \mathrm{~g}(93 \%)$. Mp 95-97 ${ }^{\circ} \mathrm{C} ;{ }^{1} \mathrm{H}$ NMR $\left(\mathrm{CDCl}_{3}\right) \delta 10.91$ (br. s, $\left.1 \mathrm{H}\right), 7.86$ (d, $J=8.3$ $\mathrm{Hz}, 2 \mathrm{H}), 7.77(\mathrm{~d}, J=7.3 \mathrm{~Hz}, 2 \mathrm{H}), 7.43(\mathrm{t}, J=7.7 \mathrm{~Hz}, 2 \mathrm{H}), 7.35-7.28(\mathrm{~m}, 6 \mathrm{H}), 7.27-7.16(\mathrm{~m}, 5 \mathrm{H})$, $7.08(\mathrm{~s}, 1 \mathrm{H}), 6.68(\mathrm{~s}, 1 \mathrm{H}), 6.75(\mathrm{~d}, J=6.7 \mathrm{~Hz}, 2 \mathrm{H}), 4.53(\mathrm{~s}, 2 \mathrm{H}), 2.44(\mathrm{~s}, 3 \mathrm{H}) ;{ }^{13} \mathrm{C}\left\{{ }^{1} \mathrm{H}\right\}$ NMR $\left(\mathrm{CDCl}_{3}\right) \delta 144.9,143.5,139.9,138.0,137.4,136.1,133.1,129.8,129.4,128.8,128.7,128.6,127.9$, 127.8, 127.5, 127.1, 126.7, 126.5, 124.6, 116.8, 110.9, 50.9, 21.5; IR (KBr): 3135, 3059, 3031, 2924, 2585 (br), 1650, 1597, 1445, 1334, 1185, 1156, 1089, 967, 855, 763, 728, 699, 667, $548 \mathrm{~cm}^{-}$ 1; HRMS-ESI [M + H] $]^{+}$calcd for $\mathrm{C}_{31} \mathrm{H}_{28} \mathrm{~N}_{3} \mathrm{O}_{2} \mathrm{~S}^{+}$506.1897; found 506.1911.

\section{(Z)-N-(2-(1-(2-Bromoethyl)-4-phenyl-1H-imidazol-2-yl)-2-phenylvinyl)-4-methylbenzene-} sulfonamide $(\mathbf{4 m})$

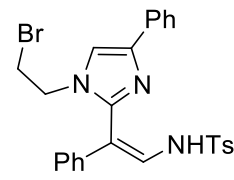

Compound 4m was obtained as a colorless oil (64 mg, yield 61\%) according to the general procedure (eluent hexane-EtOAc, 2:1) from pyrazole 1zf and triazole $\mathbf{2 a} ;{ }^{1} \mathrm{H} \mathrm{NMR}\left(\mathrm{CDCl}_{3}\right) \delta$ 10.92 (br. s, 1H), 7.84 (d, $J=8.3 \mathrm{~Hz}, 2 \mathrm{H}), 7.78$ (d, $J=7.4 \mathrm{~Hz}, 2 \mathrm{H}), 7.47-7.42$ (m, 2H), 7.42-7.30 $(\mathrm{m}, 6 \mathrm{H}), 7.28-7.24(\mathrm{~m}, 2 \mathrm{H}), 7.18(\mathrm{~s}, 1 \mathrm{H}), 6.81(\mathrm{~s}, 1 \mathrm{H}), 3.72(\mathrm{t}, J=6.7 \mathrm{~Hz}, 2 \mathrm{H}), 3.13(\mathrm{t}, J=6.7$ $\mathrm{Hz}, 2 \mathrm{H}), 2.43(\mathrm{~s}, 3 \mathrm{H}) ;{ }^{13} \mathrm{C}\left\{{ }^{1} \mathrm{H}\right\} \mathrm{NMR}\left(\mathrm{CDCl}_{3}\right) \delta 144.4,143.7,140.0,137.9,137.1,132.7,129.8$, 129.7, 129.2, 128.8, 127.9, 127.8, 127.4, 126.6, 124.8, 116.5, 110.5, 48.3, 29.3, 21.5; HRMS-ESI $[\mathrm{M}+\mathrm{H}]^{+}$calcd for $\mathrm{C}_{26} \mathrm{H}_{25}{ }^{79} \mathrm{BrN}_{3} \mathrm{O}_{2} \mathrm{~S}^{+}$522.0845; found 522.0844 .

(Z)-Methyl 2-(2-(2-(4-methylphenylsulfonamido)-1-phenylvinyl)-4-phenyl-1H-imidazol-1yl)acetate $(4 n)$

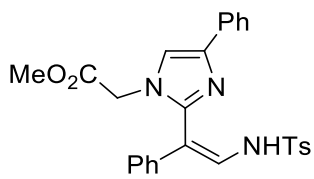

Compound 4n was obtained as a colorless oil (78 $\mathrm{mg}$, yield $80 \%$ ) according to the general procedure (eluent hexane-EtOAc, 2:1) from pyrazole 1zg and triazole $2 \mathbf{2} ;{ }^{1} \mathrm{H} \mathrm{NMR}\left(\mathrm{CDCl}_{3}\right) \delta$ 11.92 (br. s, 1H), 7.85 (d, $J=8.3 \mathrm{~Hz}, 2 \mathrm{H}), 7.79-7.75(\mathrm{~m}, 2 \mathrm{H}), 7.43$ (t, $J=7.7 \mathrm{~Hz}, 2 \mathrm{H}), 7.38-7.30$ $(\mathrm{m}, 6 \mathrm{H}), 7.23-7.19(\mathrm{~m}, 2 \mathrm{H}), 7.11(\mathrm{~s}, 1 \mathrm{H}), 6.79(\mathrm{~s}, 1 \mathrm{H}), 4.13(\mathrm{~s}, 2 \mathrm{H}), 3.58(\mathrm{~s}, 3 \mathrm{H}), 2.43(\mathrm{~s}, 3 \mathrm{H})$; ${ }^{13} \mathrm{C}\left\{{ }^{1} \mathrm{H}\right\}$ NMR $\left(\mathrm{CDCl}_{3}\right) \delta 167.4,145.2,143.6,140.2,137.9,137.2,132.9,129.8,129.6,129.0$, 
$128.7,128.2,127.8,127.2,126.6,124.7,117.2,110.0,52.4,48.2,21.5$; HRMS-ESI [M + H $]^{+}$ calcd for $\mathrm{C}_{27} \mathrm{H}_{26} \mathrm{~N}_{3} \mathrm{O}_{4} \mathrm{~S}^{+} 488.1639$; found 488.1638 .

\section{(Z)-N-(2-(1,4-Diphenyl-1H-imidazol-2-yl)-2-phenylvinyl)-4-methylbenzenesulfonamide (4o)}

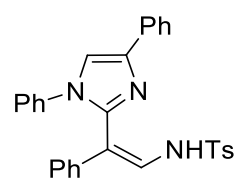

Compound $4 \mathbf{o}$ was obtained as a yellow oil ( $87 \mathrm{mg}$, yield $89 \%)$ according to the general procedure (eluent hexane-EtOAc, 2:1) from pyrazole 1a and triazole 2a (reaction time $16 \mathrm{~h}$ ); ${ }^{1} \mathrm{H}$ NMR $\left(\mathrm{CDCl}_{3}\right) \delta 11.46($ br. s, $1 \mathrm{H}), 7.90(\mathrm{~d}, J=8.3 \mathrm{~Hz}, 2 \mathrm{H}), 7.85(\mathrm{~d}, J=7.3 \mathrm{~Hz}, 2 \mathrm{H}), 7.48(\mathrm{t}, J=7.7 \mathrm{~Hz}$, 2H), 7.39-7.31 (m, 4H), 7.11-7.02 (m, 3H), 6.99-6.88 (m, 7H), $6.84(\mathrm{~s}, 1 \mathrm{H}), 2.44(\mathrm{~s}, 3 \mathrm{H}) ;{ }^{13} \mathrm{C}\left\{{ }^{1} \mathrm{H}\right\}$ $\mathrm{NMR}\left(\mathrm{CDCl}_{3}\right) \delta 144.4,143.6,140.1,137.9,137.4,136.6,132.9,129.8,129.5,128.8,128.5,128.0$, 127.8, 127.6, 127.3, 126.6, 126.5, 125.4, 124.7, 117.4, 111.5, 21.5; IR (KBr): 3249, 3141, 3055, 2923, 1646, 1597, 1497, 1329, 1168, 1138, 1088, 854, 737, 692, 489, $465 \mathrm{~cm}^{-1}$; HRMS-ESI [M + $\mathrm{Na}]^{+}$calcd for $\mathrm{C}_{30} \mathrm{H}_{25} \mathrm{NaN}_{3} \mathrm{O}_{2} \mathrm{~S}^{+}$514.1560; found 514.1561.

(Z)-4-Methyl- $N$-(2-(1-(2-methylphenyl)-4-phenyl-1H-imidazol-2-yl)-2-phenylvinyl)benzenesulfonamide (4p)

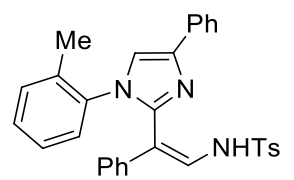

Compound $4 \mathbf{p}$ was obtained as a yellow oil (91 mg, yield 90\%) according to the general procedure (eluent hexane-EtOAc, 2:1) from pyrazole 1b and triazole 2a (reaction time $14 \mathrm{~h}$ ).

Silica gel-catalyzed synthesis from $\mathbf{1 b}$ and $2 a$ : Pyrazole $\mathbf{1 b}(0.2 \mathrm{mmol})$, triazole $2 \mathbf{a}(0.4 \mathrm{mmol})$, and toluene $(1 \mathrm{~mL})$ were placed into a screw cap glass tube. After the addition of $\mathrm{Rh}_{2}(\mathrm{Piv})_{4}(6.2$ $\mathrm{mg}, 0.05$ equiv), cap was rapidly screwed and the tube was placed into an oil bath preheated to $110{ }^{\circ} \mathrm{C}$. The reaction mixture was stirred at this temperature for $5 \mathrm{~min}$. Then silica gel $(500 \mathrm{mg})$ was added and the mixture was stirred in an oil bath at $120^{\circ} \mathrm{C}$ for $1.5 \mathrm{~h}$. Silica gel was filtered off and washed consequently with dichloromethane and EtOAc. The solvent was removed in vacuo, and the residue was purified by column chromatography on silica gel (eluent hexane-EtOAc, 2:1) to give imidazole $4 p$ (97 mg, yield 96\%).

Silica gel-catalyzed synthesis from $3 \mathbf{h}$ : Compound $\mathbf{3 h}$ (101 mg, $0.2 \mathrm{mmol})$, silica gel (500 mg), and toluene $(1 \mathrm{~mL})$ were placed into a screw cap glass tube and stirred in an oil bath at $120{ }^{\circ} \mathrm{C}$ for $2 \mathrm{~h}$. Silica gel was filtered off and washed consequently with dichloromethane and EtOAc. The solvent was removed in vacuo, and the residue was purified by column chromatography on silica gel (eluent hexane-EtOAc, 2:1) to give imidazole $4 \mathbf{p}(81 \mathrm{mg}$, yield $80 \%) ;{ }^{1} \mathrm{H} \mathrm{NMR}\left(\mathrm{CDCl}_{3}\right) \delta$ 11.58 (br. s, 1H), 7.91-7.83 (m, 4H), $7.48(\mathrm{t}, J=7.7 \mathrm{~Hz}, 2 \mathrm{H}), 7.39-7.32(\mathrm{~m}, 3 \mathrm{H}), 7.18(\mathrm{~s}, 1 \mathrm{H})$, $7.02-6.87(\mathrm{~m}, 8 \mathrm{H}), 6.83-6.79(\mathrm{~m}, 1 \mathrm{H}), 6.72(\mathrm{~s}, 1 \mathrm{H}), 2.44(\mathrm{~s}, 3 \mathrm{H}), 1.89(\mathrm{~s}, 3 \mathrm{H}) ;{ }^{13} \mathrm{C}\left\{{ }^{1} \mathrm{H}\right\} \mathrm{NMR}$ $\left(\mathrm{CDCl}_{3}\right) \delta 145.5,143.5,140.0,138.0,136.5,136.3,134.1,132.9,130.3,129.8,129.3,128.8,128.5$, 128.3, 127.53, 127.51, 127.3, 126.7, 126.5, 126.0, 124.7, 117.3, 111.8, 21.5, 17.3; HRMS-ESI [M $+\mathrm{H}]^{+}$calcd for $\mathrm{C}_{31} \mathrm{H}_{28} \mathrm{~N}_{3} \mathrm{O}_{2} \mathrm{~S}^{+}$506.1883; found 506.1897. 


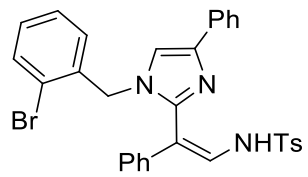

Compound 4q was obtained as a white solid (108 $\mathrm{mg}$, yield 92\%) according to the general procedure (eluent hexane-EtOAc, 2:1) from pyrazole 1zh and triazole $\mathbf{2 a} ; \mathrm{mp} 181-183{ }^{\circ} \mathrm{C} ;{ }^{1} \mathrm{H}$ NMR $\left(\mathrm{CDCl}_{3}\right) \delta 11.24$ (br. s, $\left.1 \mathrm{H}\right), 7.87(\mathrm{~d}, J=8.2 \mathrm{~Hz}, 2 \mathrm{H}), 7.79(\mathrm{~d}, J=7.8 \mathrm{~Hz}, 2 \mathrm{H}), 7.48-7.41$ $(\mathrm{m}, 3 \mathrm{H}), 7.36-7.22(\mathrm{~m}, 6 \mathrm{H}), 7.20-7.08(\mathrm{~m}, 4 \mathrm{H}), 7.05(\mathrm{~s}, 1 \mathrm{H}), 6.77-6.75(\mathrm{~m}, 1 \mathrm{H}), 6.62(\mathrm{dd}, J=$ 7.3, $1.9 \mathrm{~Hz}, 1 \mathrm{H}), 4.57(\mathrm{~s}, 2 \mathrm{H}), 2.45(\mathrm{~s}, 3 \mathrm{H}) ;{ }^{13} \mathrm{C}\left\{{ }^{1} \mathrm{H}\right\} \mathrm{NMR}\left(\mathrm{CDCl}_{3}\right) \delta 145.2,143.5,140.1,137.9$, 137.0, 135.5, 133.0, 132.7, 129.8, 129.3, 129.2, 128.7 (2C), 128.0 (2C), 127.7, 127.6, 127.2, 126.5, 124.6, 122.4, 116.5, 110.9, 51.4, 21.5; HRMS-ESI $[\mathrm{M}+\mathrm{H}]^{+}$calcd for $\mathrm{C}_{31} \mathrm{H}_{27}{ }^{79} \mathrm{BrN}_{3} \mathrm{O}_{2} \mathrm{~S}^{+}$ 584.1002; found 584.0997.

(Z)-N-(2-(1-Methyl-4-phenyl-1H-imidazol-2-yl)-2-phenylvinyl)methanesulfonamide (4r)

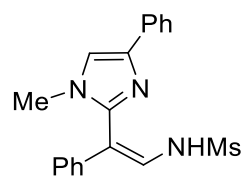

Compound $4 \mathbf{r}$ was obtained as a white solid (68 mg, yield 96\%) according to the general procedure (eluent hexane-EtOAc, 2:1) from pyrazole $1 \mathrm{t}$ and triazole $2 \mathbf{b} ; \mathrm{mp} 152-154{ }^{\circ} \mathrm{C} ;{ }^{1} \mathrm{H} \mathrm{NMR}\left(\mathrm{CDCl}_{3}\right)$ $\delta 10.38$ (br. s, 1H), 7.78-7.74 (m, 2H), 7.46-7.29 (m, 8H), 7.05 (s, 1H), 6.85 (s, 1H), 3.11 (s, 3H), $3.10(\mathrm{~s}, 3 \mathrm{H}) ;{ }^{13} \mathrm{C}\left\{{ }^{1} \mathrm{H}\right\} \mathrm{NMR}\left(\mathrm{CDCl}_{3}\right) \delta 144.9,139.6,137.7,133.0,129.1,128.9,128.7,127.8$, 128.5, 128.1, 124.6, 117.6, 110.4, 41.9, 35.1; IR (KBr): 3132, 3057, 3024, 2944, 2636 (br), 1651, 1599, 1462, 1329, 1308, 1202, 1159, 1128, 976, 849, 762, 697, $510 \mathrm{~cm}^{-1}$; HRMS-ESI [M + H] ${ }^{+}$ calcd for $\mathrm{C}_{19} \mathrm{H}_{20} \mathrm{~N}_{3} \mathrm{O}_{2} \mathrm{~S}^{+} 354.1271$; found 354.2171.

(Z)-4-Methoxy- $N$-(2-(1-methyl-4-phenyl-1H-imidazol-2-yl)-2-phenylvinyl)benzenesulfonamide (4s)

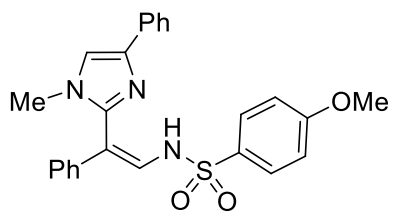

Compound $4 \mathbf{s}$ was obtained as an orange solid (86 mg, yield 97\%) according to the general procedure (eluent hexane-EtOAc, 2:1) from pyrazole $1 \mathrm{t}$ and triazole $2 \mathrm{~d}$; mp $126-128{ }^{\circ} \mathrm{C} ;{ }^{1} \mathrm{H} \mathrm{NMR}$ $\left(\mathrm{CDCl}_{3}\right) \delta 10.96$ (br. s, 1H), 7.93-7.88 (m, 2H), 7.78-7.73 (m, 2H), 7.46-7.29 (m, 6H), 7.27-7.23 $(\mathrm{m}, 2 \mathrm{H}), 7.07(\mathrm{~s}, 1 \mathrm{H}), 7.01-6.96(\mathrm{~m}, 2 \mathrm{H}), 6.80(\mathrm{~s}, 1 \mathrm{H}), 3.86(\mathrm{~s}, 3 \mathrm{H}), 3.04(\mathrm{~s}, 3 \mathrm{H}) ;{ }^{13} \mathrm{C}\left\{{ }^{1} \mathrm{H}\right\} \mathrm{NMR}$ $\left(\mathrm{CDCl}_{3}\right) \delta 162.9,145.0,139.5,137.7,133.2,132.6,129.2,128.9,128.70,128.69,128.0,127.4$, $127.0,124.5,117.6,114.3,110.6,55.6,35.2$; HRMS-ESI $[\mathrm{M}+\mathrm{H}]^{+}$calcd for $\mathrm{C}_{25} \mathrm{H}_{24} \mathrm{~N}_{3} \mathrm{O}_{3} \mathrm{~S}^{+}$ 446.1533 ; found 446.1546 . 

sulfonamide (4t)

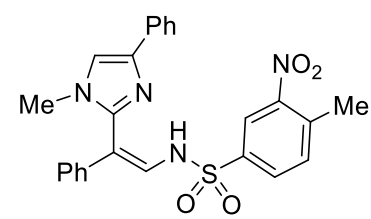

Compound 4t was obtained as an orange solid (88 $\mathrm{mg}$, yield 93\%) according to the general procedure (eluent hexane-EtOAc, 2:1) from pyrazole $\mathbf{1 t}$ and triazole $2 \mathrm{e} ; \mathrm{mp} 116-118^{\circ} \mathrm{C} ;{ }^{1} \mathrm{H}$ NMR $\left(\mathrm{CDCl}_{3}\right) \delta 8.56(\mathrm{~d}, J=1.8 \mathrm{~Hz}, 1 \mathrm{H}), 8.06(\mathrm{dd}, J=8.1,1.8 \mathrm{~Hz}, 1 \mathrm{H}), 7.73-7.68(\mathrm{~m}, 2 \mathrm{H}), 7.51(\mathrm{~d}, J=$ $8.1 \mathrm{~Hz}, 1 \mathrm{H}), 7.45(\mathrm{t}, J=7.7 \mathrm{~Hz}, 2 \mathrm{H}), 7.42-7.30(\mathrm{~m}, 4 \mathrm{H}), 7.29-7.24(\mathrm{~m}, 2 \mathrm{H}), 7.05(\mathrm{~s}, 1 \mathrm{H}), 6.93(\mathrm{~s}$, $1 \mathrm{H}), 3.07$ (s, 3H), 2.67 (s, 3H) (NH signal is not observed); ${ }^{13} \mathrm{C}\left\{{ }^{1} \mathrm{H}\right\} \mathrm{NMR}\left(\mathrm{CDCl}_{3}\right) \delta 149.0,144.9$, 141.0, 137.9, 137.5, 137.0, 133.8, 132.7, 131.6, 130.2, 128.9 (2C), 128.3, 127.6, 127.5, 124.5, 122.9, 117.5, 109.0, 35.5, 20.4; HRMS-ESI [M + H] $]^{+}$calcd for $\mathrm{C}_{25} \mathrm{H}_{23} \mathrm{~N}_{4} \mathrm{O}_{4} \mathrm{~S}^{+}$475.1435; found 475.1443 .

(Z)- $N$-(2-(4-(4-Methoxyphenyl)-1-methyl-1H-imidazol-2-yl)-2-phenylvinyl)-4-methylbenzenesulfonamide (4u)

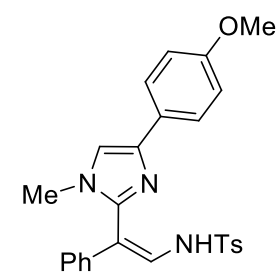

Compound $\mathbf{4 u}$ was obtained as an orange solid (72 $\mathrm{mg}$, yield $78 \%$ ) according to the general procedure (eluent hexane-EtOAc, $2: 1$ ) from pyrazole 1t and triazole $2 \mathrm{f} ; \mathrm{mp} 170-172{ }^{\circ} \mathrm{C} ;{ }^{1} \mathrm{H}$ NMR $\left(\mathrm{CDCl}_{3}\right) \delta 10.70$ (br. s, $\left.1 \mathrm{H}\right), 7.85(\mathrm{~d}, J=8.2 \mathrm{~Hz}, 2 \mathrm{H}), 7.67(\mathrm{~d}, J=8.7 \mathrm{~Hz}, 2 \mathrm{H}), 7.40-7.29(\mathrm{~m}, 5 \mathrm{H})$, $7.28-7.23(\mathrm{~m}, 2 \mathrm{H}), 7.00-6.95(\mathrm{~m}, 3 \mathrm{H}), 6.80(\mathrm{~s}, 1 \mathrm{H}), 3.88(\mathrm{~s}, 3 \mathrm{H}), 3.02(\mathrm{~s}, 3 \mathrm{H}), 2.42(\mathrm{~s}, 3 \mathrm{H})$; ${ }^{13} \mathrm{C}\left\{{ }^{1} \mathrm{H}\right\} \mathrm{NMR}\left(\mathrm{CDCl}_{3}\right) \delta 158.9,144.8,143.4,139.3,138.1,137.7,129.8,129.5,128.8,128.2$, 127.4, 126.6, 125.9, 125.8, 116.4, 114.2, 110.6, 55.3, 35.2, 21.5; HRMS-ESI $[\mathrm{M}+\mathrm{H}]^{+}$calcd for $\mathrm{C}_{26} \mathrm{H}_{26} \mathrm{~N}_{3} \mathrm{O}_{3} \mathrm{~S}^{+} 460.1689$; found 460.1700 .

(Z)- $N$-(2-(4-(4-Chlorophenyl)-1-methyl-1H-imidazol-2-yl)-2-phenylvinyl)-4-methylbenzenesulfonamide $(4 \mathrm{v})$

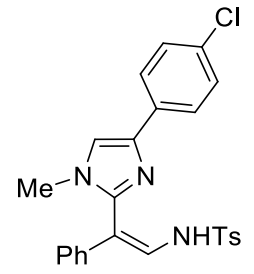

Compound $4 \mathbf{v}$ was obtained as a yellow solid (84 $\mathrm{mg}$, yield 90\%) according to the general procedure (eluent hexane-EtOAc, $2: 1$ ) from pyrazole $1 \mathrm{t}$ and triazole $2 \mathrm{~g}$; $\mathrm{mp} 128-130{ }^{\circ} \mathrm{C} ;{ }^{1} \mathrm{H}$ NMR $\left(\mathrm{CDCl}_{3}\right) \delta 10.81$ (br. s, $\left.1 \mathrm{H}\right), 7.84(\mathrm{~d}, J=8.3 \mathrm{~Hz}, 2 \mathrm{H}), 7.68-7.64(\mathrm{~m}, 2 \mathrm{H}), 7.40-7.30(\mathrm{~m}, 7 \mathrm{H})$, $7.26-7.22(\mathrm{~m}, 2 \mathrm{H}), 7.05(\mathrm{~s}, 1 \mathrm{H}), 6.80(\mathrm{~s}, 1 \mathrm{H}), 3.02(\mathrm{~s}, 3 \mathrm{H}), 2.42(\mathrm{~s}, 3 \mathrm{H}) ;{ }^{13} \mathrm{C}\left\{{ }^{1} \mathrm{H}\right\} \mathrm{NMR}\left(\mathrm{CDCl}_{3}\right)$ $\delta 145.1,143.6,138.5,137.8,137.5,132.4,131.8,129.8,129.0,128.9,128.8,127.9,127.5,126.5$, 125.7, 117.8, 110.7, 35.2, 21.5; IR (KBr): 3081, 2951, 1622, 1597, 1492, 1449, 1336, 1163, 1145 , 
1090, 954, 774, 703, 664, $547 \mathrm{~cm}^{-1}$; HRMS-ESI $[\mathrm{M}+\mathrm{H}]^{+}$calcd for $\mathrm{C}_{25} \mathrm{H}_{23}{ }^{35} \mathrm{ClN}_{3} \mathrm{O}_{2} \mathrm{~S}^{+} 464.1194$; found 464.1200 .

(Z)-4-Methyl- $N$-(2-(1-methyl-4-(4-nitrophenyl)-1H-imidazol-2-yl)-2-phenylvinyl)benzenesulfonamide $(4 \mathrm{w})$

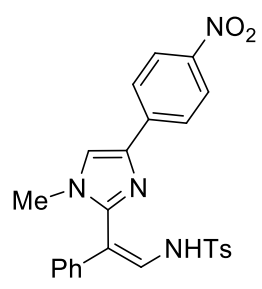

Compound $\mathbf{4 w}$ was obtained as an orange solid (75 $\mathrm{mg}$, yield $79 \%$ ) according to the general procedure (eluent hexane-EtOAc, 2:1) from pyrazole 1 t and triazole $2 \mathbf{i}$; mp $195-197{ }^{\circ} \mathrm{C}$; ${ }^{1} \mathrm{H}$ NMR $\left(\mathrm{CDCl}_{3}\right) \delta 10.79$ (br. s, $\left.1 \mathrm{H}\right), 8.27$ (d, $\left.J=8.9 \mathrm{~Hz}, 2 \mathrm{H}\right), 7.88-7.83(\mathrm{~m}, 4 \mathrm{H}), 7.42-7.33(\mathrm{~m}, 5 \mathrm{H})$, $7.27-7.23(\mathrm{~m}, 3 \mathrm{H}), 6.84(\mathrm{~s}, 1 \mathrm{H}), 3.09(\mathrm{~s}, 3 \mathrm{H}), 2.44(\mathrm{~s}, 3 \mathrm{H}) ;{ }^{13} \mathrm{C}\left\{{ }^{1} \mathrm{H}\right\} \mathrm{NMR}\left(\mathrm{CDCl}_{3}\right) \delta$ 146.4, 146.1, 143.9, 139.6, 137.7, 137.3, 129.9, 129.3, 129.0, 127.9, 127.7, 126.6, 126.4, 124.7, 124.3, 120.2, 110.4, 35.5, 21.5; HRMS-ESI [M + H] $]^{+}$calcd for $\mathrm{C}_{25} \mathrm{H}_{23} \mathrm{~N}_{4} \mathrm{O}_{4} \mathrm{~S}^{+} 475.1435$; found 475.1434.

(Z)-4-Methyl- $N$-(2-phenyl-2-(1-(2-(1-(4-methylphenylsulfonyl)-4-phenyl-1H-imidazol-2yl)benzyl-4-phenyl-)-1H-imidazol-2-yl)vinyl)benzenesulfonamide (4x)

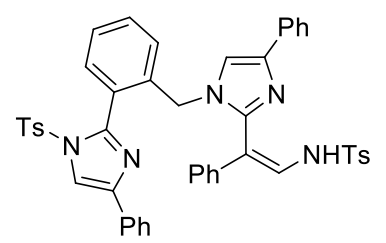

Compound $\mathbf{4 x}$ was obtained as a yellow oil (125 mg, yield $78 \%$ ) according to the general procedure (eluent hexane-EtOAc, 2:1) from pyrazole 1zi and triazole $2 \mathbf{a}(0.6 \mathrm{mmol}) ;{ }^{1} \mathrm{H} \mathrm{NMR}\left(\mathrm{CDCl}_{3}\right) \delta$ 10.47 (br. s, 1H), $7.83(\mathrm{~d}, J=8.2 \mathrm{~Hz}, 2 \mathrm{H}), 7.77-7.75(\mathrm{~m}, 1 \mathrm{H}), 7.59-7.52(\mathrm{~m}, 4 \mathrm{H}), 7.42$ (td, $J=$ 7.6, $1.2 \mathrm{~Hz}, 1 \mathrm{H}), 7.34-7.20(\mathrm{~m}, 13 \mathrm{H}), 7.20-7.14(\mathrm{~m}, 3 \mathrm{H}), 7.09-7.03(\mathrm{~m}, 3 \mathrm{H}), 6.87(\mathrm{t}, J=7.9 \mathrm{~Hz}$, $2 \mathrm{H}), 6.74-6.72(\mathrm{~m}, 1 \mathrm{H}), 4.60(\mathrm{~s}, 2 \mathrm{H}), 2.43(\mathrm{~s}, 3 \mathrm{H}), 2.41(\mathrm{~s}, 3 \mathrm{H}) ;{ }^{13} \mathrm{C}\left\{{ }^{1} \mathrm{H}\right\} \mathrm{NMR}\left(\mathrm{CDCl}_{3}\right) \delta 146.3$, 145.2, 143.8, 143.2, 141.6, 140.2, 138.2, 137.9, 137.4, 134.1, 133.2, 131.9, 131.3, 130.4, 129.9, 129.6, 128.7, 128.5, 128.4, 128.2, 128.1, 127.74, 127.66, 127.14, 127.09, 126.6 (3C), 126.4, 125.3, 124.3, 117.2, 113.8, 111.1, 49.6, 21.7, 21.4; HRMS-ESI $[\mathrm{M}+\mathrm{H}]^{+}$calcd for $\mathrm{C}_{47} \mathrm{H}_{40} \mathrm{~N}_{5} \mathrm{O}_{4} \mathrm{~S}_{2}{ }^{+}$ 802.2516; found 802.2535.

(Z)-2,4-Dinitro- $N$-(2-phenyl-2-(1-methylphenylsulfonyl-4-phenyl-1H-imidazol-2-yl)vinyl)aniline $(4 y)$<smiles></smiles>

Compound $4 \mathbf{y}$ was obtained as an orange solid (93 $\mathrm{mg}$, yield $80 \%$ ) according to the general procedure (eluent hexane-EtOAc, 2:1) from pyrazole $\mathbf{1 l}$ and triazole $\mathbf{2 a}$ (reaction time $6 \mathrm{~h}$ ); $\mathrm{mp}$ 191-193 ${ }^{\circ} \mathrm{C} ;{ }^{1} \mathrm{H}$ NMR (DMSO-d $) \delta 9.89(\mathrm{~d}, J=11.5 \mathrm{~Hz}, 1 \mathrm{H}), 8.77$ (d, $J=2.7 \mathrm{~Hz}, 1 \mathrm{H}$ ), 8.63 (s, 
$1 \mathrm{H}), 8.41(\mathrm{dd}, J=9.5,2.7 \mathrm{~Hz}, 1 \mathrm{H}), 8.06-7.95(\mathrm{~m}, 4 \mathrm{H}), 7.60(\mathrm{~d}, J=8.3 \mathrm{~Hz}, 2 \mathrm{H}), 7.45(\mathrm{t}, J=7.6$ $\mathrm{Hz}, 2 \mathrm{H}), 7.38-7.25(\mathrm{~m}, 6 \mathrm{H}), 7.07(\mathrm{~d}, J=8.3 \mathrm{~Hz}, 2 \mathrm{H}), 2.02(\mathrm{~s}, 3 \mathrm{H}) ;{ }^{13} \mathrm{C}\left\{{ }^{1} \mathrm{H}\right\} \mathrm{NMR}\left(\mathrm{CDCl}_{3}\right) \delta$ 145.6, 143.20, 142.5, 142.3, 138.4, 136.9, 134.2, 131.9, 131.7, 130.1, 129.7, 128.8, 128.7, 128.3, 127.7, 127.4, 126.4, 126.3, 125.6, 123.9, 117.6, 115.9, 114.7, 21.5; IR (KBr): 3261, 3057, 2923, 1612, 1592, 1520, 1369, 1336, 1173, 1144, 1117, 1090, 737, 696, 670, $590 \mathrm{~cm}^{-1}$; HRMS-ESI [M - $\mathrm{H}]^{-}$calcd for $\mathrm{C}_{30} \mathrm{H}_{22} \mathrm{~N}_{5} \mathrm{O}_{6} \mathrm{~S}^{-} 580.1285$; found 580.1268 .

(Z)- $N$-(2-(4-Fluorophenyl)-2-(1-(methylphenylsulfonyl)-4-phenyl-1H-imidazol-2-yl)vinyl)2,4-dinitroaniline $(4 z)$

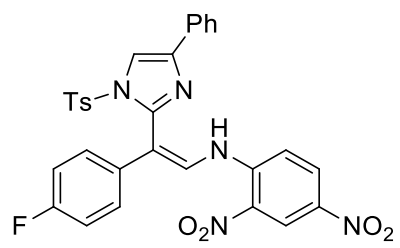

Compound $\mathbf{4 z}$ was obtained as an orange solid (73 mg, yield 61\%) according to the general procedure (eluent hexane-EtOAc, 2:1) from pyrazole $\mathbf{1 m}$ and triazole $\mathbf{2 a}$ (reaction time $7 \mathrm{~h}$ ); $\mathrm{mp}$ $188-190{ }^{\circ} \mathrm{C} ;{ }^{1} \mathrm{H}$ NMR $\left(\mathrm{CDCl}_{3}\right) \delta 10.77(\mathrm{~d}, J=11.1 \mathrm{~Hz}, 1 \mathrm{H}), 9.12(\mathrm{~d}, J=2.7 \mathrm{~Hz}, 1 \mathrm{H}), 8.38(\mathrm{dd}, J$ $=9.5,2.7 \mathrm{~Hz}, 1 \mathrm{H}), 8.00(\mathrm{~s}, 1 \mathrm{H}), 7.94(\mathrm{~d}, J=7.5 \mathrm{~Hz}, 2 \mathrm{H}), 7.47(\mathrm{t}, J=7.6 \mathrm{~Hz}, 2 \mathrm{H}), 7.42-7.35(\mathrm{~m}$, $3 \mathrm{H}), 7.32(\mathrm{~d}, J=9.5 \mathrm{~Hz}, 1 \mathrm{H}), 7.26(\mathrm{~d}, J=11.1 \mathrm{~Hz}, 1 \mathrm{H}), 7.17-7.10(\mathrm{~m}, 2 \mathrm{H}), 7.07(\mathrm{~d}, J=8.0 \mathrm{~Hz}$, $2 \mathrm{H}), 6.90(\mathrm{t}, J=8.4 \mathrm{~Hz}, 2 \mathrm{H}), 2.28(\mathrm{~s}, 3 \mathrm{H}) ;{ }^{13} \mathrm{C}\left\{{ }^{1} \mathrm{H}\right\} \mathrm{NMR}\left(\mathrm{CDCl}_{3}\right) \delta 162.5(\mathrm{~d}, J=248.2 \mathrm{~Hz})$, $145.8,143.1,142.5,142.4,138.5,134.3,133.0(\mathrm{~d}, J=3.1 \mathrm{~Hz}), 132.0,131.6,130.1,129.7,128.9$, 128.4, 128.1 (d, $J=8.1 \mathrm{~Hz}), 127.2,126.3,125.6,123.9,116.4,116.1,115.7(\mathrm{~d}, J=21.8 \mathrm{~Hz})$, 114.7, 21.5; HRMS-ESI [M + H] $]^{+}$calcd for $\mathrm{C}_{30} \mathrm{H}_{21} \mathrm{FN}_{5} \mathrm{O}_{6} \mathrm{~S}^{+}$598.1191; found 598.1164.

(Z)-Ethyl (2-(1-methyl-4-phenyl-1H-imidazol-2-yl)-2-phenylvinyl)(4-methylphenylsulfonyl)carbamate (4za)

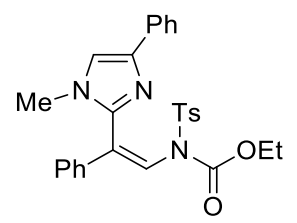

Compound 4za was obtained as a white solid (37 mg, yield 37\%) according to the general procedure (eluent hexane-EtOAc, 2:1) from pyrazole 1zj and triazole $\mathbf{2 a}$; mp $124-126{ }^{\circ} \mathrm{C}$; ${ }^{1} \mathrm{H}$ NMR $\left(\mathrm{CDCl}_{3}\right) \delta 7.89(\mathrm{~d}, J=8.3 \mathrm{~Hz}, 2 \mathrm{H}), 7.44-7.35(\mathrm{~m}, 7 \mathrm{H}), 7.28-7.18(\mathrm{~m}, 5 \mathrm{H}), 7.09(\mathrm{~s}, 1 \mathrm{H})$, 6.99 (s, 1H), $3.95(\mathrm{q}, J=7.1 \mathrm{~Hz}, 2 \mathrm{H}), 3.17(\mathrm{~s}, 3 \mathrm{H}), 2.39(\mathrm{~s}, 3 \mathrm{H}), 1.04(\mathrm{t}, J=7.1 \mathrm{~Hz}, 3 \mathrm{H}) ;{ }^{13} \mathrm{C}\left\{{ }^{1} \mathrm{H}\right\}$ NMR $\left(\mathrm{CDCl}_{3}\right) \delta 151.3,144.3,143.4,141.0,137.3,136.2,134.1,131.4,129.0,128.9,128.8(2 \mathrm{C})$, 128.2, 127.2, 126.4, 124.8, 124.5, 117.9, 63.6, 34.0, 21.6, 13.7; IR (KBr): 3058, 3030, 2987, 2951, 1742, 1610, 1595, 1372, 1356, 1252, 1201, 1168, 1146, 1086, 808, 745, 699, 668, $569 \mathrm{~cm}^{-1}$; HRMS-ESI $[\mathrm{M}+\mathrm{H}]^{+}$calcd for $\mathrm{C}_{28} \mathrm{H}_{28} \mathrm{~N}_{3} \mathrm{O}_{4} \mathrm{~S}^{+}$502.1795; found 502.1777.

(Z)-N-(Dimethylcarbamoyl)-4-methyl- $N$-(2-(1-methyl-4-phenyl-1H-imidazol-2-yl)-2-phenylvinyl)benzenesulfonamide (4zb)

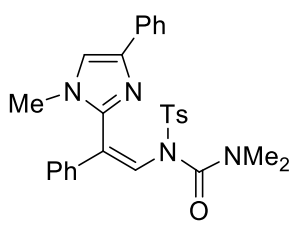


Compound 4zb was obtained as a white solid (30 mg, yield 30\%) according to the general procedure (eluent hexane-EtOAc, 2:1) from pyrazole 1zk and triazole $\mathbf{2 a}$; mp $185-187{ }^{\circ} \mathrm{C} ;{ }^{1} \mathrm{H}$ $\operatorname{NMR}\left(\mathrm{CDCl}_{3}\right) \delta 7.93(\mathrm{~d}, J=8.3 \mathrm{~Hz}, 2 \mathrm{H}), 7.80-7.75(\mathrm{~m}, 2 \mathrm{H}), 7.41-7.21(\mathrm{~m}, 9 \mathrm{H}), 7.16(\mathrm{~s}, 1 \mathrm{H})$, 7.14-7.09 (m, 2H), $3.02(\mathrm{~s}, 3 \mathrm{H}), 3.18-2.40$ (br. m, 6H), $2.48(\mathrm{~s}, 3 \mathrm{H}) ;{ }^{13} \mathrm{C}\left\{{ }^{1} \mathrm{H}\right\} \mathrm{NMR}\left(\mathrm{CDCl}_{3}\right) \delta$ 151.6, 144.8, 142.8, 141.2, 138.0, 135.8, 134.1, 129.6, 128.7, 128.6, 128.5, 127.8, 126.59, 126.56, 126.0, 124.6, 117.8, 117.1, 38.5, 37.1, 32.7, 21.7; HRMS-ESI $[\mathrm{M}+\mathrm{H}]^{+}$calcd for $\mathrm{C}_{28} \mathrm{H}_{29} \mathrm{~N}_{4} \mathrm{O}_{3} \mathrm{~S}^{+}$ 501.1955; found 501.1956.

Methyl rac-(R)-2-phenyl-2-((2S,5S)-1-(4-methylphenylsulfonyl)-4-phenyl-5-(phenylamino)2,5-dihydro-1H-imidazol-2-yl)acetate (15)

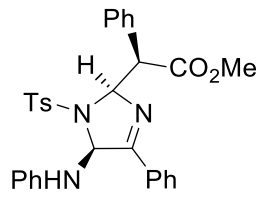

Compound 15 was obtained as a white solid (77 $\mathrm{mg}$, yield 71\%) according to the general procedure (eluent hexane-EtOAc, 2:1) from pyrazole 1zl and triazole $2 \mathbf{a}(0.8 \mathrm{mmol}) ; \mathrm{mp} 172-174{ }^{\circ} \mathrm{C} ;{ }^{1} \mathrm{H}$ $\operatorname{NMR}\left(\mathrm{CDCl}_{3}\right) \delta 7.76(\mathrm{~d}, J=7.6 \mathrm{~Hz}, 2 \mathrm{H}), 7.68(\mathrm{~d}, J=8.0 \mathrm{~Hz}, 2 \mathrm{H}), 7.47-7.23(\mathrm{~m}, 8 \mathrm{H}), 7.18(\mathrm{t}, J$ $=7.6 \mathrm{~Hz}, 2 \mathrm{H}), 7.09(\mathrm{~d}, J=7.4 \mathrm{~Hz}, 2 \mathrm{H}), 6.80(\mathrm{t}, J=7.4 \mathrm{~Hz}, 1 \mathrm{H}), 6.61(\mathrm{~d}, J=4.0 \mathrm{~Hz}, 1 \mathrm{H}), 6.45(\mathrm{~d}$, $J=8.0 \mathrm{~Hz}, 2 \mathrm{H}), 6.39(\mathrm{~d}, J=11.4 \mathrm{~Hz}, 1 \mathrm{H}), 4.33(\mathrm{~d}, J=4.0 \mathrm{~Hz}, 1 \mathrm{H}), 3.88(\mathrm{~s}, 3 \mathrm{H}), 2.42(\mathrm{~s}, 3 \mathrm{H}), 1.79$ $(\mathrm{d}, J=11.4 \mathrm{~Hz}, 1 \mathrm{H}) ;{ }^{13} \mathrm{C}\left\{{ }^{1} \mathrm{H}\right\} \mathrm{NMR}\left(\mathrm{CDCl}_{3}\right) \delta 170.7,167.7,144.1,144.0,136.1,132.4,132.1$, 131.6, 130.1, 129.9, 129.2, 128.6, 128.4, 128.00, 127.97, 127.3, 119.2, 113.7, 87.8, 75.4, 56.5, 52.4, 21.5; HRMS-ESI $[\mathrm{M}+\mathrm{Na}]^{+}$calcd for $\mathrm{C}_{31} \mathrm{H}_{29} \mathrm{NaN}_{3} \mathrm{O}_{4} \mathrm{~S}^{+}$562.1771; found 562.1761.

\section{Synthesis of compounds 16-19}

Synthesis of 7-(4-methylphenylsulfonyl)-2,9-diphenyl-6,7-dihydro-5H-imidazo[1,2- $d][1,4]$ diazepine (16)

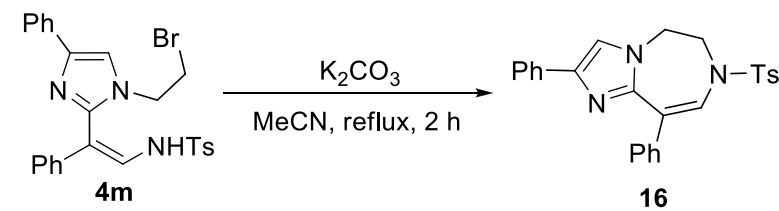

Imidazole 4m (55 mg, $0.1 \mathrm{mmol}), \mathrm{K}_{2} \mathrm{CO}_{3}(69 \mathrm{mg}, 0.5 \mathrm{mmol})$, and $\mathrm{MeCN}$ (4 mL) were placed into a screw cap glass tube and stirred in an oil bath at $82{ }^{\circ} \mathrm{C}$ for $2 \mathrm{~h}$. The solvent was removed in vacuo, and the residue was purified by column chromatography on silica gel (eluent hexane-EtOAc, 3:1) to give compound 16 as a white solid (41 mg, yield 93\%); $\mathrm{mp} 160-162{ }^{\circ} \mathrm{C}$; ${ }^{1} \mathrm{H} \mathrm{NMR}\left(\mathrm{CDCl}_{3}\right) \delta 7.75(\mathrm{~d}, J=8.3 \mathrm{~Hz}, 2 \mathrm{H}), 7.68-7.64(\mathrm{~m}, 2 \mathrm{H}), 7.55-7.50(\mathrm{~m}, 2 \mathrm{H}), 7.44-7.34$ $(\mathrm{m}, 5 \mathrm{H}), 7.33-7.27(\mathrm{~m}, 2 \mathrm{H}), 7.23-7.16(\mathrm{~m}, 2 \mathrm{H}), 7.14(\mathrm{~s}, 1 \mathrm{H}), 4.17-4.12(\mathrm{~m}, 2 \mathrm{H}), 4.01-3.96(\mathrm{~m}$, $2 \mathrm{H}), 2.46(\mathrm{~s}, 3 \mathrm{H}) ;{ }^{13} \mathrm{C}\left\{{ }^{1} \mathrm{H}\right\} \mathrm{NMR}\left(\mathrm{CDCl}_{3}\right) \delta 144.8,144.4,141.5,139.6,135.1,133.8,130.3,130.1$, 128.4, 127.8, 127.3, 126.9, 126.8, 125.7, 124.9, 116.6, 114.8, 47.6, 47.4, 21.6; HRMS-ESI [M + $\mathrm{Na}]^{+}$calcd for $\mathrm{C}_{26} \mathrm{H}_{23} \mathrm{NaN}_{3} \mathrm{O}_{2} \mathrm{~S}^{+} 464.1403$; found 464.1418 . 
Synthesis of $N$-(2-(1-benzyl-4-phenyl-1H-imidazol-2-yl)-2-phenylethyl)-4-methylbenzenesulfonamide (17)

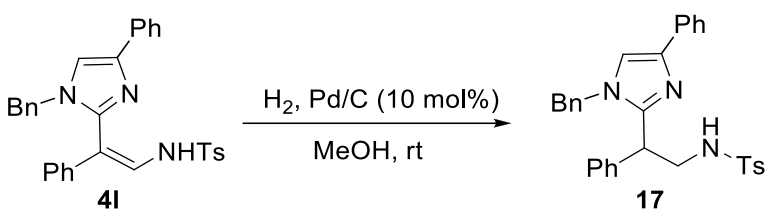

Imidazole $4 \mathrm{l}$ (51 mg, $0.1 \mathrm{mmol}), \mathrm{Pd} / \mathrm{C}(10 \mathrm{wt} \%, 11 \mathrm{mg}, 0.01 \mathrm{mmol})$, and $\mathrm{MeOH}(10 \mathrm{~mL})$ were placed into a round-bottom flask and stirred overnight under a flow of hydrogen at room temperature. The mixture was filtered through Celite, the filtrate was concentrated under reduced pressure. Then the residue was purified by column chromatography on silica gel (eluent hexane-EtOAc, 3:1) to give compound 17 as a white solid (47 mg, yield 93\%); mp 152-154 ${ }^{\circ} \mathrm{C}$; ${ }^{1} \mathrm{H} \mathrm{NMR}\left(\mathrm{CDCl}_{3}\right) \delta$ 7.82-7.72 (m, 4H), 7.44-7.36 (m, 2H), 7.35-7.21 (m, 8H), 7.14-7.05 (m, $3 \mathrm{H}), 6.94-6.85(\mathrm{~m}, 2 \mathrm{H}), 6.34-6.22(\mathrm{~m}, 1 \mathrm{H}), 4.74$ and $4.65(\mathrm{AB}-\mathrm{q}, J=15.6 \mathrm{~Hz}, 2 \mathrm{H}), 4.26-4.16$ (m, 1H), 3.69-3.47 (m, 2H), $2.45(\mathrm{~s}, 3 \mathrm{H}) ;{ }^{13} \mathrm{C}\left\{{ }^{1} \mathrm{H}\right\}$ NMR $\left(\mathrm{CDCl}_{3}\right) \delta 147.4,143.1,140.1,138.5$, 137.3, 135.4, 133.9, 129.7, 129.0, 128.9, 128.6, 128.1, 128.0, 127.7, 127.1, 127.0, 126.8, 124.7, 116.3, 49.5, 47.9, 44.3, 21.5; HRMS-ESI $[\mathrm{M}+\mathrm{H}]^{+}$calcd for $\mathrm{C}_{31} \mathrm{H}_{30} \mathrm{~N}_{3} \mathrm{O}_{2} \mathrm{~S}^{+}$508.2050; found 508.2053 .

\section{Synthesis of zinc complex 18}

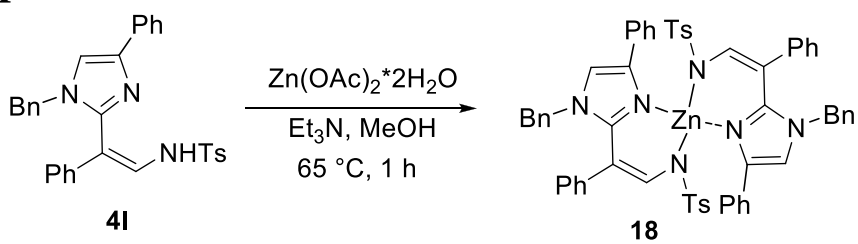

Imidazole $4 \mathrm{l}$ (101 mg, $0.2 \mathrm{mmol}), \mathrm{Zn}(\mathrm{OAc})_{2} \cdot 2 \mathrm{H}_{2} \mathrm{O}(33 \mathrm{mg}, 0.15 \mathrm{mmol}), \mathrm{Et}_{3} \mathrm{~N}(20 \mathrm{mg}, 0.2 \mathrm{mmol})$ and $\mathrm{MeOH}(5 \mathrm{~mL})$ were placed into a round-bottom flask and refluxed in an oil bath for $1 \mathrm{~h}$ under stirring. The solvent was removed in vacuo, and the residue was purified by column chromatography on silica gel (eluent hexane-EtOAc, 1:1).Complex 18 was obtained as a white solid (93 mg, yield 87\%); mp 164-166 ${ }^{\circ} \mathrm{C}$ (dec.); ${ }^{1} \mathrm{H}$ NMR $\left(\mathrm{CDCl}_{3}\right) \delta 7.60(\mathrm{~d}, J=8.0 \mathrm{~Hz}, 4 \mathrm{H})$, 7.33-7.24 (m, 14H), 7.24-7.14 (m, 6H), $7.09(\mathrm{~s}, 2 \mathrm{H}), 6.99-6.93(\mathrm{~m}, 10 \mathrm{H}), 6.91-6.85(\mathrm{~m}, 4 \mathrm{H})$, $6.58(\mathrm{~s}, 2 \mathrm{H}), 4.45(\mathrm{~s}, 4 \mathrm{H}), 2.24(\mathrm{~s}, 6 \mathrm{H}) ;{ }^{13} \mathrm{C}\left\{{ }^{1} \mathrm{H}\right\} \mathrm{NMR}\left(\mathrm{CDCl}_{3}\right) \delta$ 148.3, 144.6, 141.0, 140.4, 140.1, 139.7, 135.4, 131.1, 128.9, 128.7, 128.6, 128.2, 127.9, 127.8, 127.5, 127.0, 126.7, 126.6, 126.3, 116.8, 104.6, 51.7, 21.3; IR (KBr): 3105, 3060, 3029, 2921, 1595, 1499, 1456, 1443, 1295, 1240, 1144, 1089, 989, 882, 730, 695, 667, 554, $538 \mathrm{~cm}^{-1}$; HRMS-ESI $[\mathrm{M}+\mathrm{Na}]^{+}$calcd for $\mathrm{C}_{62} \mathrm{H}_{52} \mathrm{NaN}_{6} \mathrm{O}_{4} \mathrm{~S}_{2} \mathrm{Zn}^{+}$1095.2675; found 1095.2715.

\section{General procedure for the synthesis of boron complexes 19}
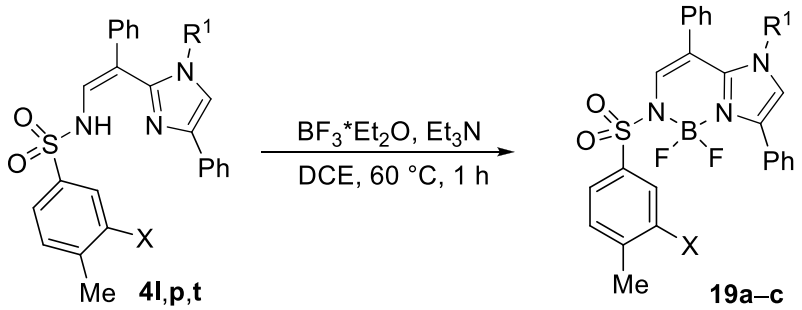
To a solution of imidazole $4(0.2 \mathrm{mmol})$ and $\mathrm{Et}_{3} \mathrm{~N}(60 \mathrm{mg}, 0.6 \mathrm{mmol})$ in DCE $(1 \mathrm{~mL})$ under a stream of argon $\mathrm{BF}_{3} \cdot \mathrm{Et}_{2} \mathrm{O}(142 \mathrm{mg}, 1.0 \mathrm{mmol})$ was added. The resulting mixture was stirred in an oil bath at $60{ }^{\circ} \mathrm{C}$ for $1 \mathrm{~h}$. The solvent was removed in vacuo, and the residue was purified by column chromatography on silica gel (eluent hexane-EtOAc, 1:1).

\section{5-Benzyl-1,1-difluoro-2-((4-methylphenyl)sulfonyl)-4,7-diphenyl-2,5-dihydro- $1 H-1 \lambda^{4}, 8 \lambda^{4}$ - imidazo[2,1-f][1,3,2]diazaborinine (19a)}

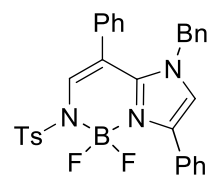

Compound 19a was obtained as a white solid (106 mg, yield 96\%) according to the general procedure (eluent hexane-EtOAc, 1:1) from imidazole 4l; mp $215-217{ }^{\circ} \mathrm{C} ;{ }^{1} \mathrm{H}$ NMR $\left(\mathrm{CDCl}_{3}\right) \delta$ $7.86(\mathrm{~d}, J=8.3 \mathrm{~Hz}, 2 \mathrm{H}), 7.70-7.64(\mathrm{~m}, 2 \mathrm{H}), 7.49$ (br. s, 1H), 7.43-7.22 (m, 13H), 6.85-6.79 (m, $2 \mathrm{H}), 6.68(\mathrm{~s}, 1 \mathrm{H}), 4.63(\mathrm{~s}, 2 \mathrm{H}), 2.40(\mathrm{~s}, 3 \mathrm{H}) ;{ }^{13} \mathrm{C}\left\{{ }^{1} \mathrm{H}\right\} \mathrm{NMR}\left(\mathrm{CDCl}_{3}\right) \delta$ 143.2, 139.8, 138.4, 136.0, 134.3, 133.9, 130.3, 129.4, 129.10, 129.07, 129.04 (2C), 128.98, 128.63, 128.62, 128.5, 128.3, 127.5, 127.0, 119.4, 102.1, 51.8, 21.5; ${ }^{19} \mathrm{~F}\left\{{ }^{1} \mathrm{H}\right\}$ NMR $\left(\mathrm{CDCl}_{3}\right) \delta-126.9--127.2(\mathrm{~m}, 2 \mathrm{~F})$; IR (KBr): 3076, 2919, 1603, 1517, 1448, 1350, 1276, 1167, 1125, 1093, 989, 927, 813, 744, 707, 665, 559 $\mathrm{cm}^{-1}$; HRMS-ESI [M + Na] ${ }^{+}$calcd for $\mathrm{C}_{31} \mathrm{H}_{26} \mathrm{BF}_{2} \mathrm{NaN}_{3} \mathrm{O}_{2} \mathrm{~S}^{+}$576.1704; found 576.1700.

\section{1,1-Difluoro-5-(2-methylphenyl)-2-((4-methylphenyl)sulfonyl)-4,7-diphenyl-2,5-dihydro-} $1 H-1 \lambda^{4}, 8 \lambda^{4}$-imidazo[2,1-f][1,3,2]diazaborinine (19b)

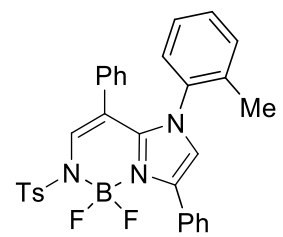

Compound 19b was obtained as a white solid (108 $\mathrm{mg}$, yield 98\%) according to the general procedure (eluent hexane-EtOAc, 1:1) from imidazole 4p; mp $113-115{ }^{\circ} \mathrm{C}$ (dec.); ${ }^{1} \mathrm{H}$ NMR $\left(\mathrm{CDCl}_{3}\right) \delta 7.89(\mathrm{~d}, J=8.1 \mathrm{~Hz}, 2 \mathrm{H}), 7.81-7.75(\mathrm{~m}, 2 \mathrm{H}), 7.54-7.43(\mathrm{~m}, 4 \mathrm{H}), 7.30-7.24(\mathrm{~m}, 2 \mathrm{H})$, $7.89(\mathrm{td}, J=7.5,1.5 \mathrm{~Hz}, 1 \mathrm{H}), 7.02-6.91(\mathrm{~m}, 5 \mathrm{H}), 6.91-6.87(\mathrm{~m}, 3 \mathrm{H}), 6.86(\mathrm{~s}, 1 \mathrm{H}), 2.41(\mathrm{~s}, 3 \mathrm{H})$, $2.02(\mathrm{~s}, 3 \mathrm{H}) ;{ }^{13} \mathrm{C}\left\{{ }^{1} \mathrm{H}\right\} \mathrm{NMR}\left(\mathrm{CDCl}_{3}\right) \delta 143.3,140.2,138.4,136.0,134.8,133.9,132.7,130.8$, $129.8,129.7,129.4,129.3,129.22,129.19,129.17,128.42,128.37,127.62,127.55,127.5,127.3$, 126.6, 120.3, 102.3, 21.5, 17.6; ${ }^{19} \mathrm{~F}\left\{{ }^{1} \mathrm{H}\right\}$ NMR $\left(\mathrm{CDCl}_{3}\right) \delta-124.8--125.4(\mathrm{~m}, 1 \mathrm{~F}),-125.7--126.4$ (m, 1F); HRMS-ESI [M + Na] $]^{+}$calcd for $\mathrm{C}_{31} \mathrm{H}_{26} \mathrm{BF}_{2} \mathrm{NaN}_{3} \mathrm{O}_{2} \mathrm{~S}^{+}$576.1704; found 576.1714.

\section{1,1-Difluoro-5-methyl-2-((4-methyl-3-nitrophenyl)sulfonyl)-4,7-diphenyl-2,5-dihydro-1H-} $1 \lambda^{4}, 8 \lambda^{4}$-imidazo[2,1-f][1,3,2]diazaborinine (19c)

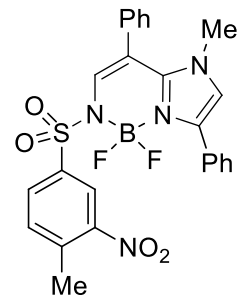

Compound 19c was obtained as a white solid (94 mg, yield 90\%) according to the general procedure (eluent hexane-EtOAc, 1:1) from imidazole 4t; mp 150-152 ${ }^{\circ} \mathrm{C}$ (dec.); ${ }^{1} \mathrm{H}$ NMR 
$\left(\mathrm{CDCl}_{3}\right) \delta 8.46(\mathrm{~s}, 1 \mathrm{H}), 8.07(\mathrm{~d}, J=8.0 \mathrm{~Hz}, 1 \mathrm{H}), 7.69-7.60(\mathrm{~m}, 2 \mathrm{H}), 7.52-7.38(\mathrm{~m}, 8 \mathrm{H}), 7.37-7.31$ $(\mathrm{m}, 2 \mathrm{H}), 6.80(\mathrm{~s}, 1 \mathrm{H}), 3.14(\mathrm{~s}, 3 \mathrm{H}), 2.62(\mathrm{~s}, 3 \mathrm{H}) ;{ }^{13} \mathrm{C}\left\{{ }^{1} \mathrm{H}\right\} \mathrm{NMR}\left(\mathrm{CDCl}_{3}\right) \delta 148.8,140.6,138.1$, 137.8, 135.6, 133.9, 133.5, 131.6, 130.1, 129.2, 129.1, 128.92, 128.90, 128.8, 128.4, 128.3, 123.6, 121.0, 103.8, 36.7, 20.4; ${ }^{19} \mathrm{~F}\left\{{ }^{1} \mathrm{H}\right\}$ NMR $\left(\mathrm{CDCl}_{3}\right) \delta-127.0--127.4(\mathrm{~m}, 1 \mathrm{~F}),-150.1--150.3(\mathrm{~m}$, $1 \mathrm{~F})$; HRMS-ESI [M + Na] ${ }^{+}$calcd for $\mathrm{C}_{25} \mathrm{H}_{21} \mathrm{BF}_{2} \mathrm{NaN}_{4} \mathrm{O}_{4} \mathrm{~S}^{+}$545.1241; found 545.1265.

\section{X-ray data}

\section{Compound 3j (CCDC 2058053)}

Single crystals of $\mathbf{3} \mathbf{j}$ were grown by slow evaporation of its solution in $\mathrm{Et}_{2} \mathrm{O}$-hexane mixture. A suitable crystal was selected, and intensity data were collected on a SuperNova, Single source at offset/far, HyPix3000 diffractometer using monochromated $\mathrm{CuK} \alpha$ radiation. The crystal was kept at 99.97(15) K during data collection. Using Olex $2,{ }^{17}$ the structure was solved with the ShelXT ${ }^{18}$ structure solution program using Intrinsic Phasing and refined with the ShelXL ${ }^{19}$ refinement package using Least Squares minimization.

Figure S1. X-Ray crystal structure of compound 3j with $50 \%$ ellipsoid probability (CCDC 2058053)
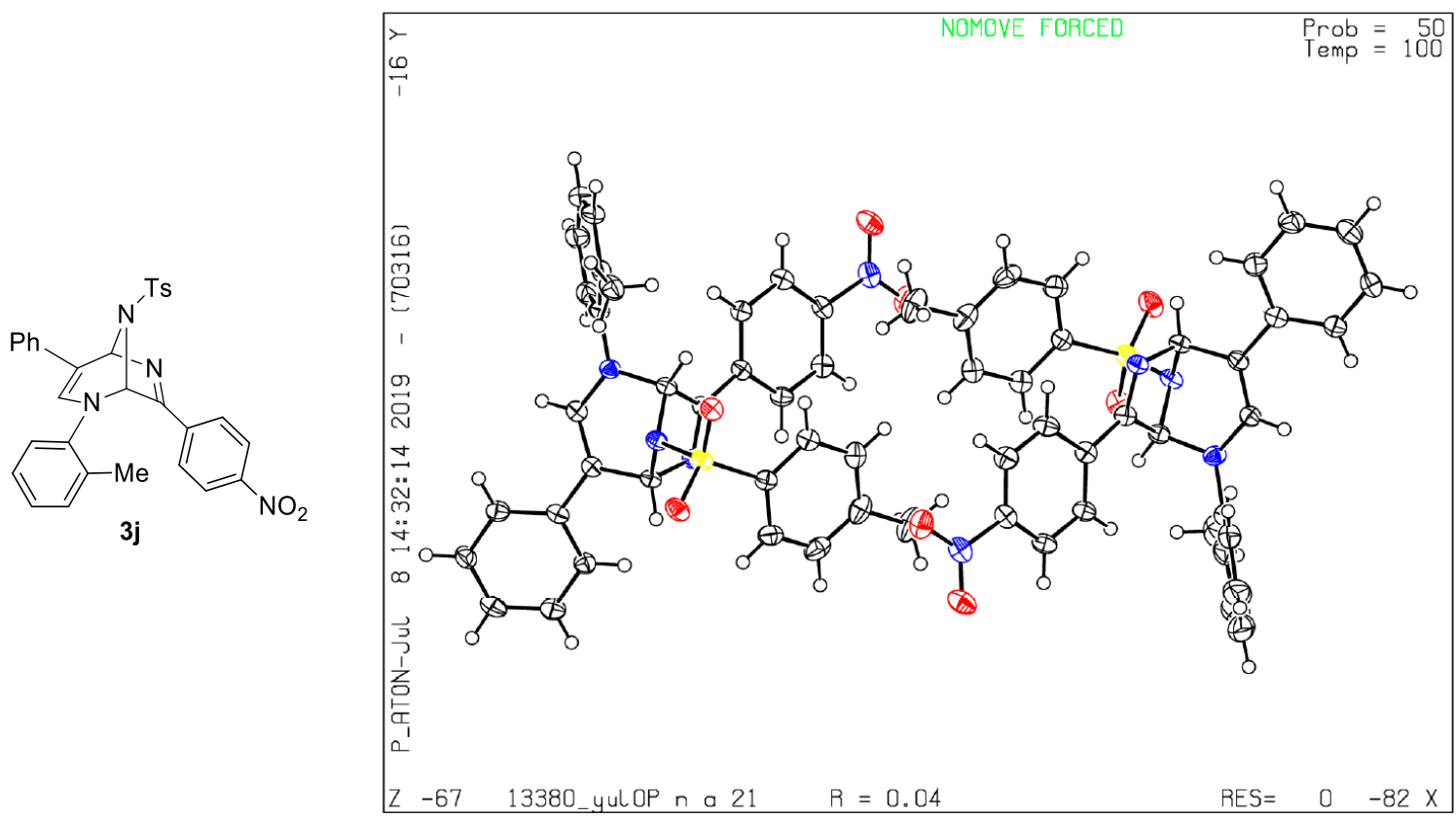

Table S2. Crystal data and structure refinement for $\mathbf{3 j}$.

Empirical formula

Formula weight

Temperature/K

Crystal system

Space group

$\mathrm{a} / \AA$
$\mathrm{C}_{63} \mathrm{H}_{52} \mathrm{~N}_{7} \mathrm{O}_{8} \mathrm{~S}_{2}$

1099.23

99.97(15)

orthorhombic

Pna2 1

$10.90480(10)$ 


\begin{tabular}{|c|c|}
\hline $\mathrm{b} / \AA ̊$ & $12.1918(2)$ \\
\hline $\mathrm{c} / \AA$ & $40.2742(5)$ \\
\hline$\alpha /^{\circ}$ & 90 \\
\hline$\beta /{ }^{\circ}$ & 90 \\
\hline$\gamma /{ }^{\circ}$ & 90 \\
\hline Volume $/ \AA^{3}$ & $5354.42(12)$ \\
\hline $\mathrm{Z}$ & 4 \\
\hline$\rho_{\text {calc }} / \mathrm{cm}^{3}$ & 1.364 \\
\hline$\mu / \mathrm{mm}^{-1}$ & 1.440 \\
\hline $\mathrm{F}(000)$ & 2300.0 \\
\hline Crystal size $/ \mathrm{mm}^{3}$ & $0.2 \times 0.17 \times 0.1$ \\
\hline Radiation & $\mathrm{CuK} \alpha(\lambda=1.54184)$ \\
\hline $2 \Theta$ range for data collection $/^{\circ}$ & 4.388 to 141.22 \\
\hline Index ranges & $-13 \leq \mathrm{h} \leq 12,-14 \leq \mathrm{k} \leq 14,-49 \leq 1 \leq 49$ \\
\hline Reflections collected & 89681 \\
\hline Independent reflections & $10176\left[\mathrm{R}_{\mathrm{int}}=0.0426, \mathrm{R}_{\mathrm{sigma}}=0.0258\right]$ \\
\hline Data/restraints/parameters & $10176 / 1 / 725$ \\
\hline Goodness-of-fit on $\mathrm{F}^{2}$ & 1.050 \\
\hline Final $\mathrm{R}$ indexes $[\mathrm{I}>=2 \sigma(\mathrm{I})]$ & $\mathrm{R}_{1}=0.0388, \mathrm{wR}_{2}=0.1026$ \\
\hline Final $\mathrm{R}$ indexes [all data] & $\mathrm{R}_{1}=0.0399, \mathrm{wR}_{2}=0.1035$ \\
\hline Largest diff. peak/hole / e $\AA^{-3}$ & $0.47 /-0.28$ \\
\hline Flack parameter & $0.050(5)$ \\
\hline
\end{tabular}




\section{Compound 4q (CCDC 1935705)}

Single crystals of $\mathbf{4 q}$ were grown by slow evaporation of its solution in $\mathrm{Et}_{2} \mathrm{O}$-hexane mixture. A suitable crystal was selected, and intensity data were collected on a SuperNova, Single source at offset/far, HyPix3000 diffractometer using monochromated $\mathrm{CuK} \alpha$ radiation. The crystal was kept at $100(2) \mathrm{K}$ during data collection. Using Olex $2,{ }^{17}$ the structure was solved with the ShelXT ${ }^{18}$ structure solution program using Intrinsic Phasing and refined with the ShelXL ${ }^{19}$ refinement package using Least Squares minimization.

Figure S2. X-Ray crystal structure of compound $\mathbf{4 q}$ with $50 \%$ ellipsoid probability (CCDC 1935705)
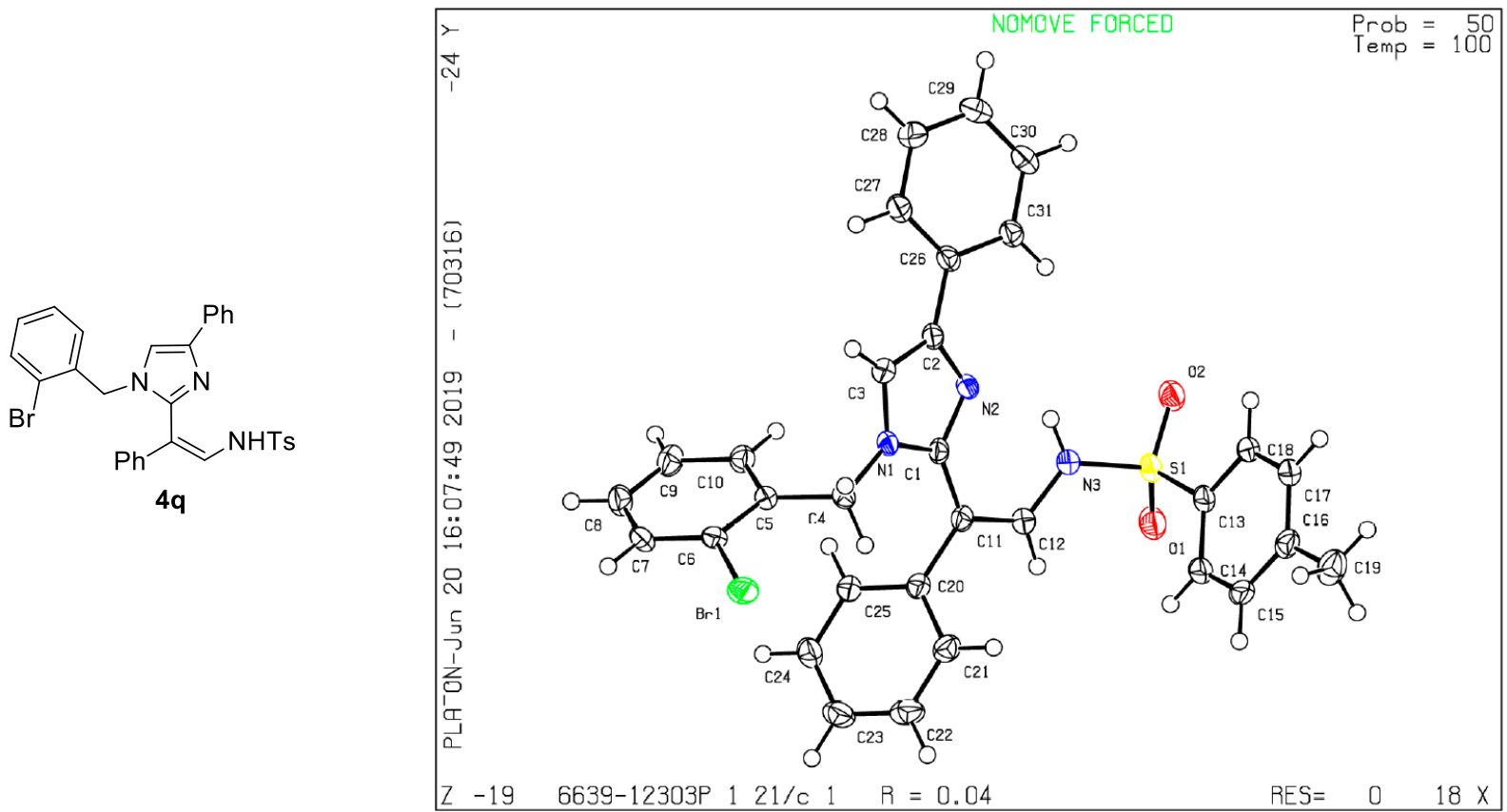

Table S3. Crystal data and structure refinement for $\mathbf{4 q}$.

$\begin{array}{ll}\text { Empirical formula } & \mathrm{C}_{31} \mathrm{H}_{26} \mathrm{BrN}_{3} \mathrm{O}_{2} \mathrm{~S} \\ \text { Formula weight } & 584.52 \\ \text { Temperature/K } & 100(2) \\ \text { Crystal system } & \text { monoclinic } \\ \text { Space group } & \mathrm{P} 2{ }_{1} / \mathrm{c} \\ \mathrm{a} / \AA & 14.2962(3) \\ \mathrm{b} / \AA & 14.0595(2) \\ \mathrm{c} / \AA & 14.4076(3) \\ \alpha /{ }^{\circ} & 90 \\ \beta /{ }^{\circ} & 114.331(2) \\ \gamma /{ }^{\circ} & 90 \\ \mathrm{Volume} / \AA^{3} & 2638.68(9) \\ \mathrm{Z} & 4 \\ \rho_{\text {calcg }} / \mathrm{cm}^{3} & 1.471\end{array}$




$\begin{array}{ll}\mu / \mathrm{mm}^{-1} & 3.138 \\ \mathrm{~F}(000) & 1200.0 \\ \text { Crystal size } / \mathrm{mm}^{3} & 0.24 \times 0.22 \times 0.17 \\ \text { Radiation } & \mathrm{CuK} \alpha(\lambda=1.54184) \\ 2 \Theta \text { range for data collection } /{ }^{\circ} & 6.786 \text { to } 147.602 \\ \text { Index ranges } & -17 \leq \mathrm{h} \leq 17,-16 \leq \mathrm{k} \leq 17,-17 \leq 1 \leq 17 \\ \text { Reflections collected } & 20548 \\ \text { Independent reflections } & 5302\left[\mathrm{R}_{\text {int }}=0.0355, \mathrm{R}_{\text {sigma }}=0.0270\right] \\ \text { Data/restraints/parameters } & 5302 / 0 / 344 \\ \text { Goodness-of-fit on } \mathrm{F}^{2} & 1.038 \\ \text { Final } \mathrm{R} \text { indexes }[\mathrm{I}>=2 \sigma(\mathrm{I})] & \mathrm{R}_{1}=0.0440, \mathrm{wR}_{2}=0.1120 \\ \text { Final } \mathrm{R} \text { indexes [all data] } & \mathrm{R}_{1}=0.0461, \mathrm{wR}_{2}=0.1137 \\ \text { Largest diff. peak/hole } / \mathrm{e} \AA^{-3} & 2.03 /-0.93\end{array}$

\section{Compound 4u (CCDC 2058052)}

Single crystals of $\mathbf{4} \mathbf{u}$ were grown by slow evaporation of its solution in $\mathrm{Et}_{2} \mathrm{O}$-hexane mixture. A suitable crystal was selected, and intensity data were collected on a Xcalibur, Eos diffractometer using monochromated MoK $\alpha$ radiation. The crystal was kept at 293(2) K during data collection. Using Olex $2,{ }^{17}$ the structure was solved with the ShelXT ${ }^{18}$ structure solution program using Intrinsic Phasing and refined with the ShelXL ${ }^{19}$ refinement package using Least Squares minimization.

Figure S3. X-Ray crystal structure of compound $\mathbf{4 u}$ with $50 \%$ ellipsoid probability (CCDC 2058052)
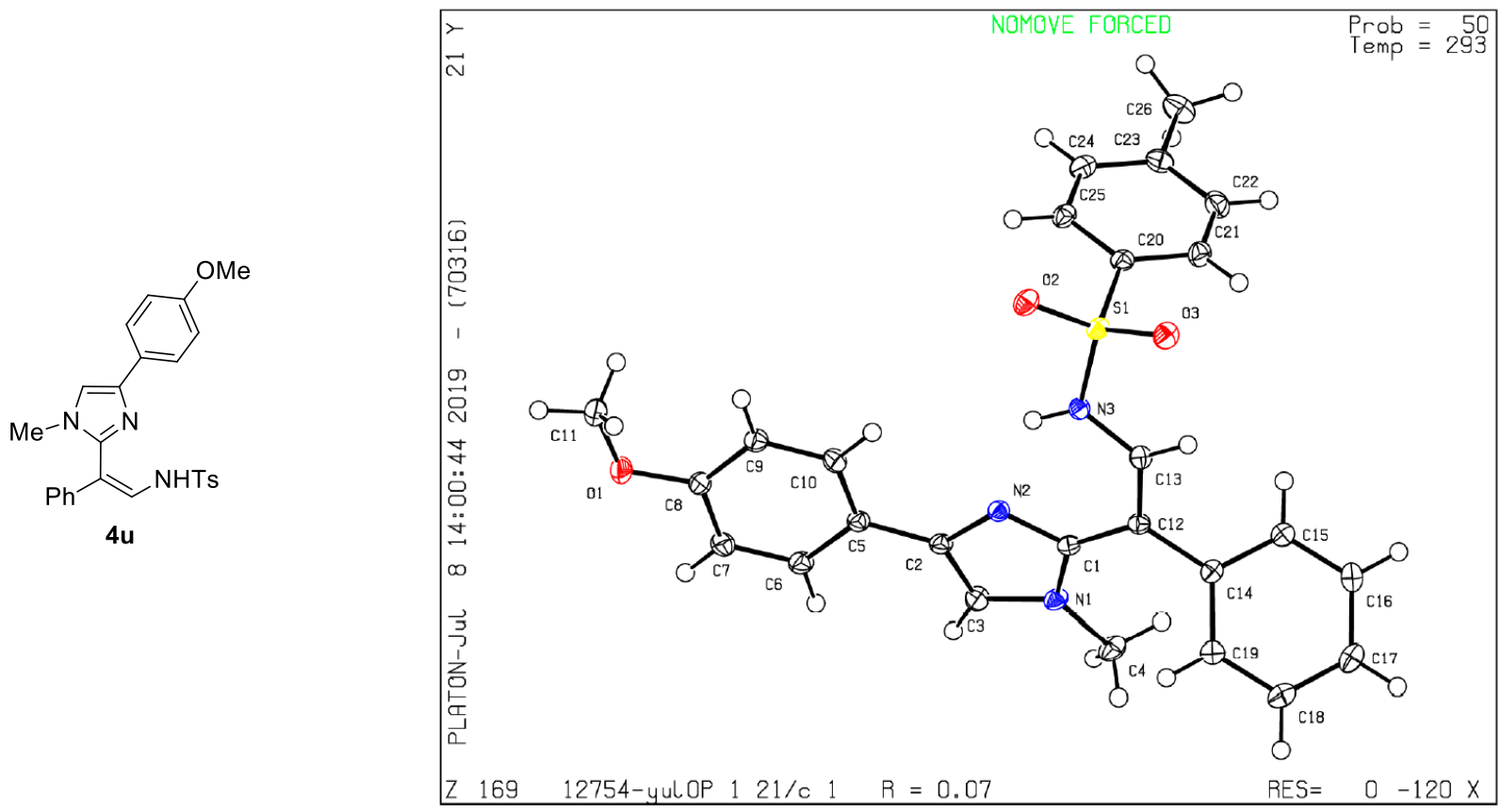
Table S4. Crystal data and structure refinement for $\mathbf{4 u}$.

\begin{tabular}{|c|c|}
\hline Empirical formula & $\mathrm{C}_{26} \mathrm{H}_{25} \mathrm{~N}_{3} \mathrm{O}_{3} \mathrm{~S}$ \\
\hline Formula weight & 459.55 \\
\hline Temperature/K & 293(2) \\
\hline Crystal system & monoclinic \\
\hline Space group & $\mathrm{P} 2{ }_{1} / \mathrm{c}$ \\
\hline $\mathrm{a} / \AA ̊$ & $6.8948(2)$ \\
\hline $\mathrm{b} / \AA ̊$ & $9.9919(2)$ \\
\hline $\mathrm{c} / \AA$ & $32.3713(8)$ \\
\hline$\alpha /^{\circ}$ & 90 \\
\hline$\beta /{ }^{\circ}$ & $93.495(2)$ \\
\hline$\gamma /{ }^{\circ}$ & 90 \\
\hline Volume $/ \AA^{3}$ & $2225.98(10)$ \\
\hline $\mathrm{Z}$ & 4 \\
\hline$\rho_{\text {calc }} g / \mathrm{cm}^{3}$ & 1.371 \\
\hline$\mu / \mathrm{mm}^{-1}$ & 0.180 \\
\hline $\mathrm{F}(000)$ & 968.0 \\
\hline Crystal size $/ \mathrm{mm}^{3}$ & $0.3 \times 0.25 \times 0.2$ \\
\hline Radiation & $\operatorname{MoK} \alpha(\lambda=0.71073)$ \\
\hline $2 \Theta$ range for data collection ${ }^{\circ}$ & 5.562 to 64.738 \\
\hline Index ranges & $-9 \leq \mathrm{h} \leq 9,-14 \leq \mathrm{k} \leq 14,-45 \leq 1 \leq 48$ \\
\hline Reflections collected & 21832 \\
\hline Independent reflections & $7129\left[\mathrm{R}_{\text {int }}=0.0481, \mathrm{R}_{\text {sigma }}=0.0724\right]$ \\
\hline Data/restraints/parameters & $7129 / 0 / 301$ \\
\hline Goodness-of-fit on $\mathrm{F}^{2}$ & 1.202 \\
\hline Final $R$ indexes $[\mathrm{I}>=2 \sigma(\mathrm{I})]$ & $\mathrm{R}_{1}=0.0705, \mathrm{wR}_{2}=0.1421$ \\
\hline Final $\mathrm{R}$ indexes [all data] & $\mathrm{R}_{1}=0.0896, \mathrm{wR}_{2}=0.1480$ \\
\hline Largest diff. peak/hole / e $\AA^{-3}$ & $0.72 /-0.56$ \\
\hline
\end{tabular}




\section{Compound 4za (CCDC 1935704)}

Single crystals of $\mathbf{4 z a}$ were grown by slow evaporation of its solution in $\mathrm{Et}_{2} \mathrm{O}$-hexane mixture. A suitable crystal was selected, and intensity data were collected on a SuperNova, Single source at offset/far, HyPix3000 diffractometer using monochromated $\mathrm{CuK} \alpha$ radiation. The crystal was kept at $100(2) \mathrm{K}$ during data collection. Using Olex $2,{ }^{17}$ the structure was solved with the ShelXT ${ }^{18}$ structure solution program using Intrinsic Phasing and refined with the ShelXL ${ }^{19}$ refinement package using Least Squares minimization.

Figure S4. X-Ray crystal structure of compound 4za with 50\% ellipsoid probability (CCDC 1935704)
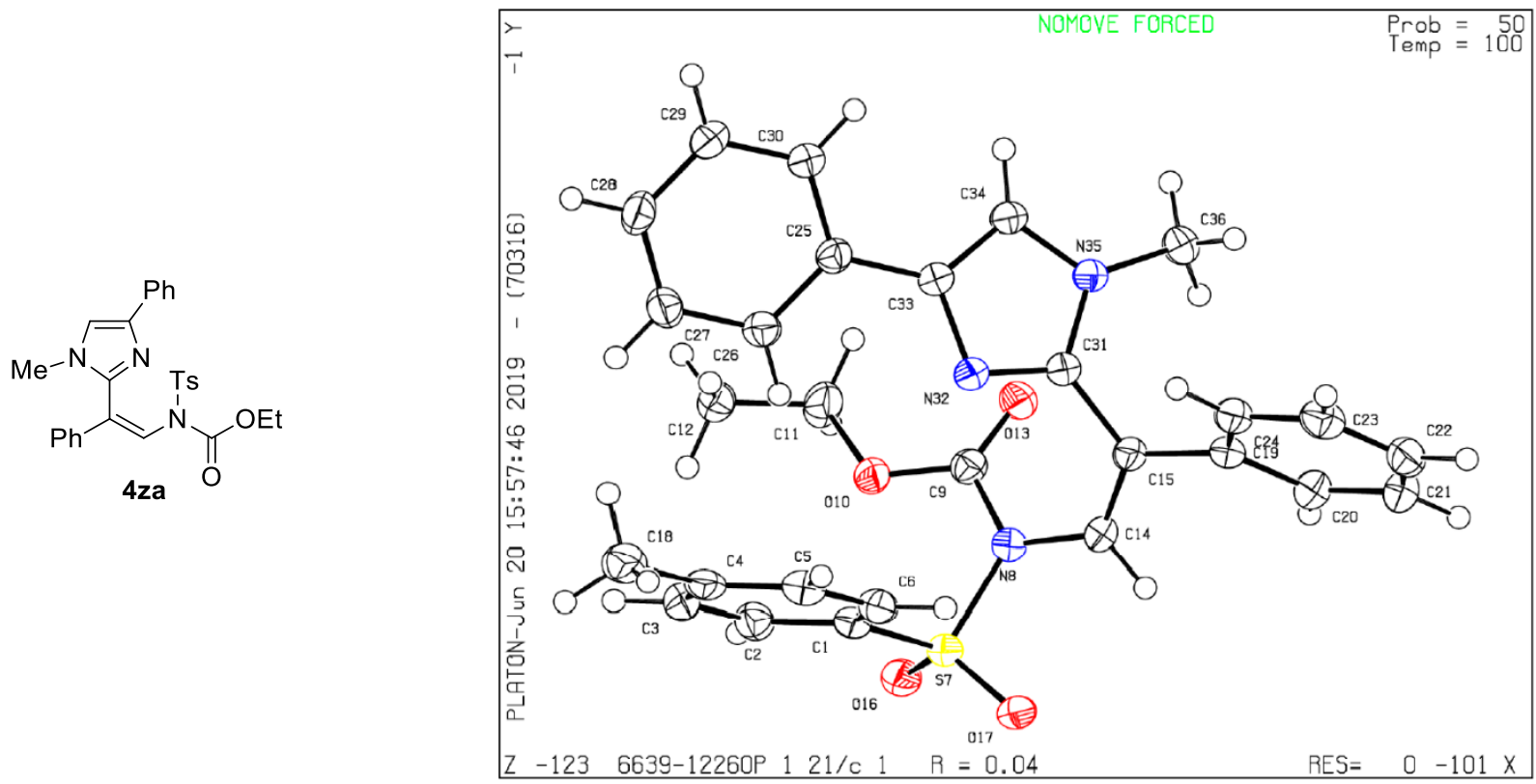

Table S5. Crystal data and structure refinement for 4za.

$\begin{array}{ll}\text { Empirical formula } & \mathrm{C}_{28} \mathrm{H}_{27} \mathrm{~N}_{3} \mathrm{O}_{4} \mathrm{~S} \\ \text { Formula weight } & 501.58 \\ \text { Temperature/K } & 100(2) \\ \text { Crystal system } & \text { monoclinic } \\ \text { Space group } & \mathrm{P} 2{ }_{1} / \mathrm{c} \\ \mathrm{a} / \AA & 14.0894(2) \\ \mathrm{b} / \AA & 7.11626(10) \\ \mathrm{c} / \AA & 25.4818(4) \\ \alpha /{ }^{\circ} & 90 \\ \beta /{ }^{\circ} & 103.8274(16) \\ \gamma /{ }^{\circ} & 90 \\ \text { Volume } / \AA^{3} & 2480.85(7) \\ \mathrm{Z} & 4 \\ \rho_{\text {calc }} / \mathrm{cm}^{3} & 1.343\end{array}$


$\mu / \mathrm{mm}^{-1}$

$\mathrm{F}(000)$

Crystal size $/ \mathrm{mm}^{3}$

Radiation

$2 \Theta$ range for data collection ${ }^{\circ}$

Index ranges

Reflections collected

Independent reflections

Data/restraints/parameters

Goodness-of-fit on $\mathrm{F}^{2}$

Final $R$ indexes $[\mathrm{I}>=2 \sigma(\mathrm{I})]$

Final $\mathrm{R}$ indexes [all data]

Largest diff. peak/hole / e $\AA^{-3}$
1.490

1056.0

$0.2 \times 0.2 \times 0.1$

$\mathrm{CuK} \alpha(\lambda=1.54184)$

6.46 to 148.244

$-17 \leq \mathrm{h} \leq 17,-8 \leq \mathrm{k} \leq 8,-31 \leq 1 \leq 30$

20945

$5003\left[\mathrm{R}_{\text {int }}=0.0468, \mathrm{R}_{\text {sigma }}=0.0343\right]$

$5003 / 0 / 328$

1.080

$\mathrm{R}_{1}=0.0436, \mathrm{wR}_{2}=0.1212$

$\mathrm{R}_{1}=0.0473, \mathrm{wR}_{2}=0.1248$

$0.44 /-0.56$

\section{Compound 15 (CCDC 1935706)}

Single crystals of $\mathbf{1 5}$ were grown by slow evaporation of its solution in $\mathrm{Et}_{2} \mathrm{O}$-hexane mixture. A suitable crystal was selected, and intensity data were collected on a SuperNova, Single source at offset/far, HyPix3000 diffractometer using monochromated $\mathrm{CuK} \alpha$ radiation. The crystal was kept at $100(3) \mathrm{K}$ during data collection. Using Olex $2,{ }^{17}$ the structure was solved with the ShelXT ${ }^{18}$ structure solution program using Intrinsic Phasing and refined with the ShelXL ${ }^{19}$ refinement package using Least Squares minimization.

Figure S5. X-Ray crystal structure of compound $\mathbf{1 5}$ with $50 \%$ ellipsoid probability (CCDC 1935706)
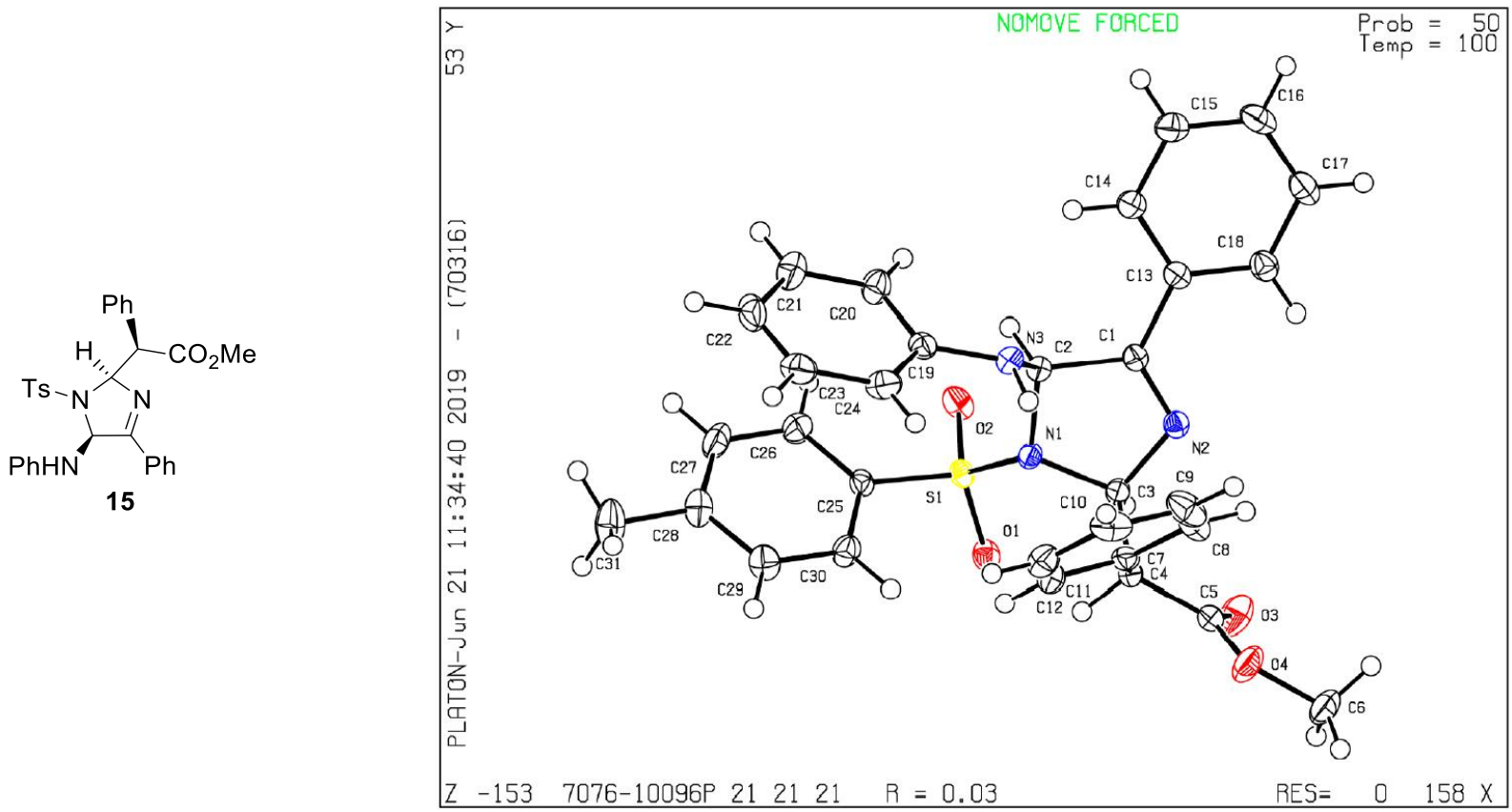
Table S6. Crystal data and structure refinement for $\mathbf{1 5}$.

\begin{tabular}{|c|c|}
\hline Empirical formula & $\mathrm{C}_{31} \mathrm{H}_{29} \mathrm{~N}_{3} \mathrm{O}_{4} \mathrm{~S}$ \\
\hline Formula weight & 539.63 \\
\hline Temperature/K & $100(3)$ \\
\hline Crystal system & orthorhombic \\
\hline Space group & $\mathrm{P} 2{ }_{1} 2_{1} 2_{1}$ \\
\hline $\mathrm{a} / \AA$ & $9.77468(15)$ \\
\hline $\mathrm{b} / \AA$ & $11.1297(2)$ \\
\hline $\mathrm{c} / \AA$ & $24.2596(4)$ \\
\hline$\alpha /^{\circ}$ & 90 \\
\hline$\beta /^{\circ}$ & 90 \\
\hline$\gamma /{ }^{\circ}$ & 90 \\
\hline Volume $/ \AA^{3}$ & $2639.18(8)$ \\
\hline $\mathrm{Z}$ & 4 \\
\hline$\rho_{\text {calc }} \mathrm{g} / \mathrm{cm}^{3}$ & 1.358 \\
\hline$\mu / \mathrm{mm}^{-1}$ & 1.441 \\
\hline $\mathrm{F}(000)$ & 1136.0 \\
\hline Crystal size $/ \mathrm{mm}^{3}$ & $0.5 \times 0.35 \times 0.22$ \\
\hline Radiation & $\operatorname{CuK} \alpha(\lambda=1.54184)$ \\
\hline $2 \Theta$ range for data collection ${ }^{\circ}$ & 7.288 to 143.844 \\
\hline Index ranges & $-12 \leq \mathrm{h} \leq 11,-13 \leq \mathrm{k} \leq 13,-29 \leq 1 \leq 29$ \\
\hline Reflections collected & 33911 \\
\hline Independent reflections & $5156\left[R_{\text {int }}=0.0475, R_{\text {sigma }}=0.0273\right]$ \\
\hline Data/restraints/parameters & $5156 / 0 / 354$ \\
\hline Goodness-of-fit on $\mathrm{F}^{2}$ & 1.026 \\
\hline Final $R$ indexes $[\mathrm{I}>=2 \sigma(\mathrm{I})]$ & $\mathrm{R}_{1}=0.0300, \mathrm{wR}_{2}=0.0774$ \\
\hline Final R indexes [all data] & $\mathrm{R}_{1}=0.0314, \mathrm{wR}_{2}=0.0787$ \\
\hline Largest diff. peak/hole / e $\AA^{-3}$ & $0.20 /-0.37$ \\
\hline Flack parameter & $-0.009(8)$ \\
\hline
\end{tabular}




\section{Calculation details}

All calculations were performed by using the Gaussian 09 suite of quantum chemical programs. ${ }^{20}$ Geometry optimizations of compounds $\mathbf{3 z b}, \mathbf{3 z b}-\mathbf{H}^{+}, \mathbf{4 r}, \mathbf{7 - 1 4}$, and transition states TS2, TS4, TS6-TS12, were performed at the DFT B3LYP/6-31+G(d,p) level using PCM model for toluene. Complex 6, $\mathrm{Rh}_{2}(\mathrm{OAc})_{4}$, and transition states TS1 and TS3 were optimized employing a Stuttgart RSC 1997 ECP basis set for rhodium and a 6-31+G(d,p) basis set for other atoms. Stationary points on the respective potential-energy surfaces were characterized at the same level of theory by evaluating the corresponding Hessian indices. Careful verification of the unique imaginary frequencies for transition states was carried out to check whether the frequency indeed pertains to the desired reaction coordinate.

Table S7. Energies (au) and cartesian coordinates of stationary points for compounds 3zb, 3zb$\mathbf{H}^{+}, \mathbf{4 r}, \mathbf{6}-\mathbf{1 4}, \mathrm{Rh}_{2}(\mathrm{OAc})_{4}$ and transition states TS1-TS12.

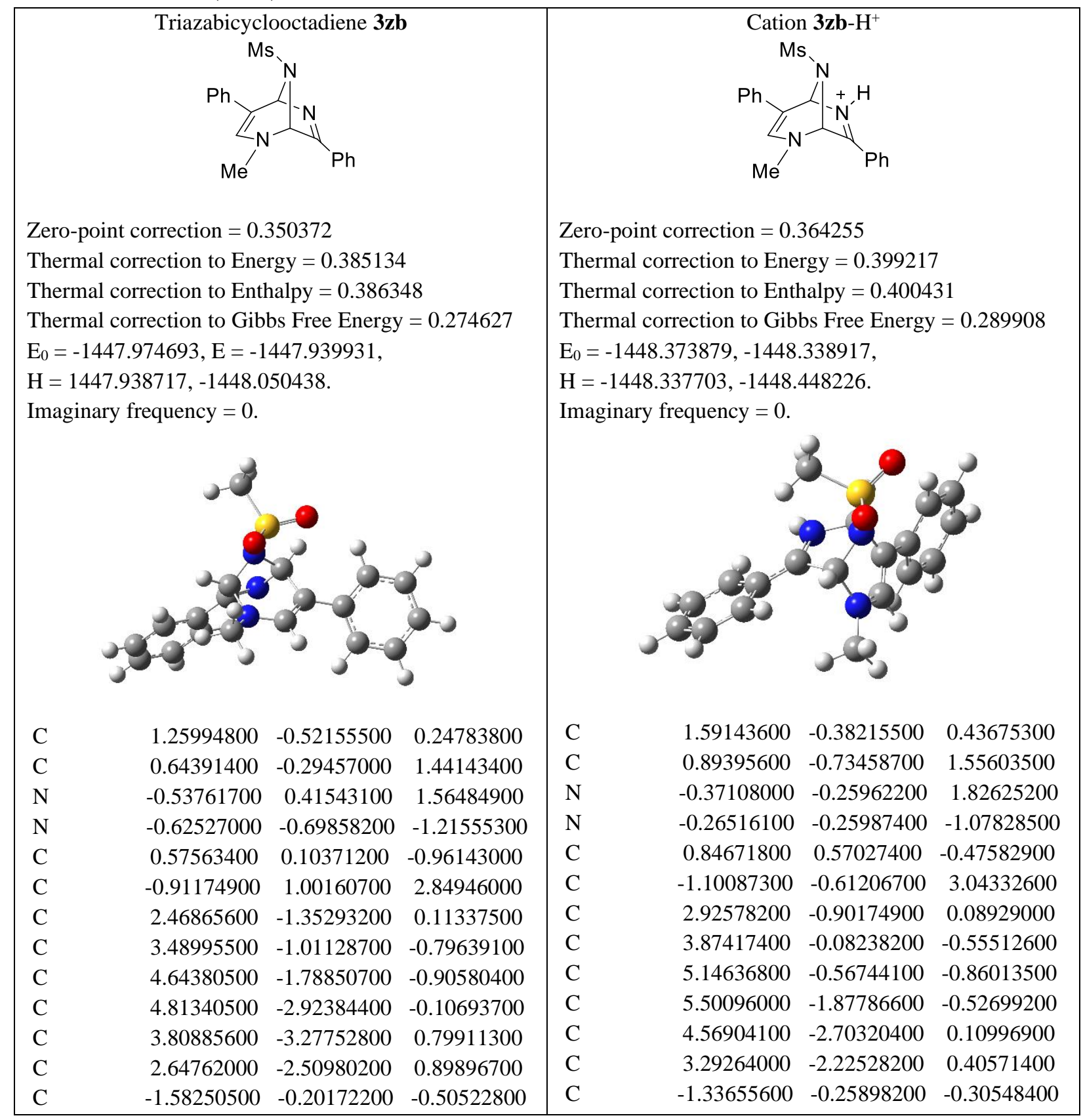




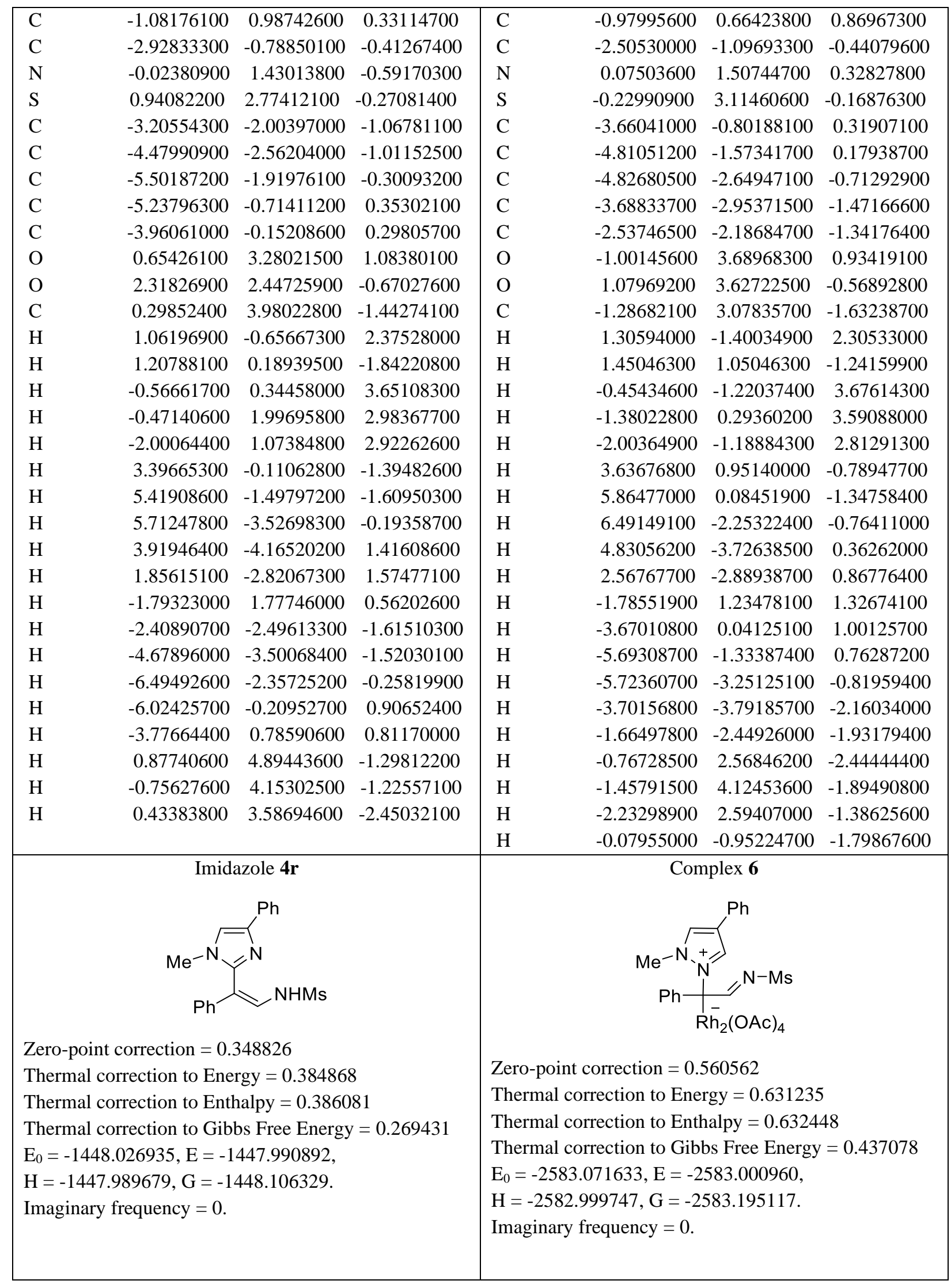




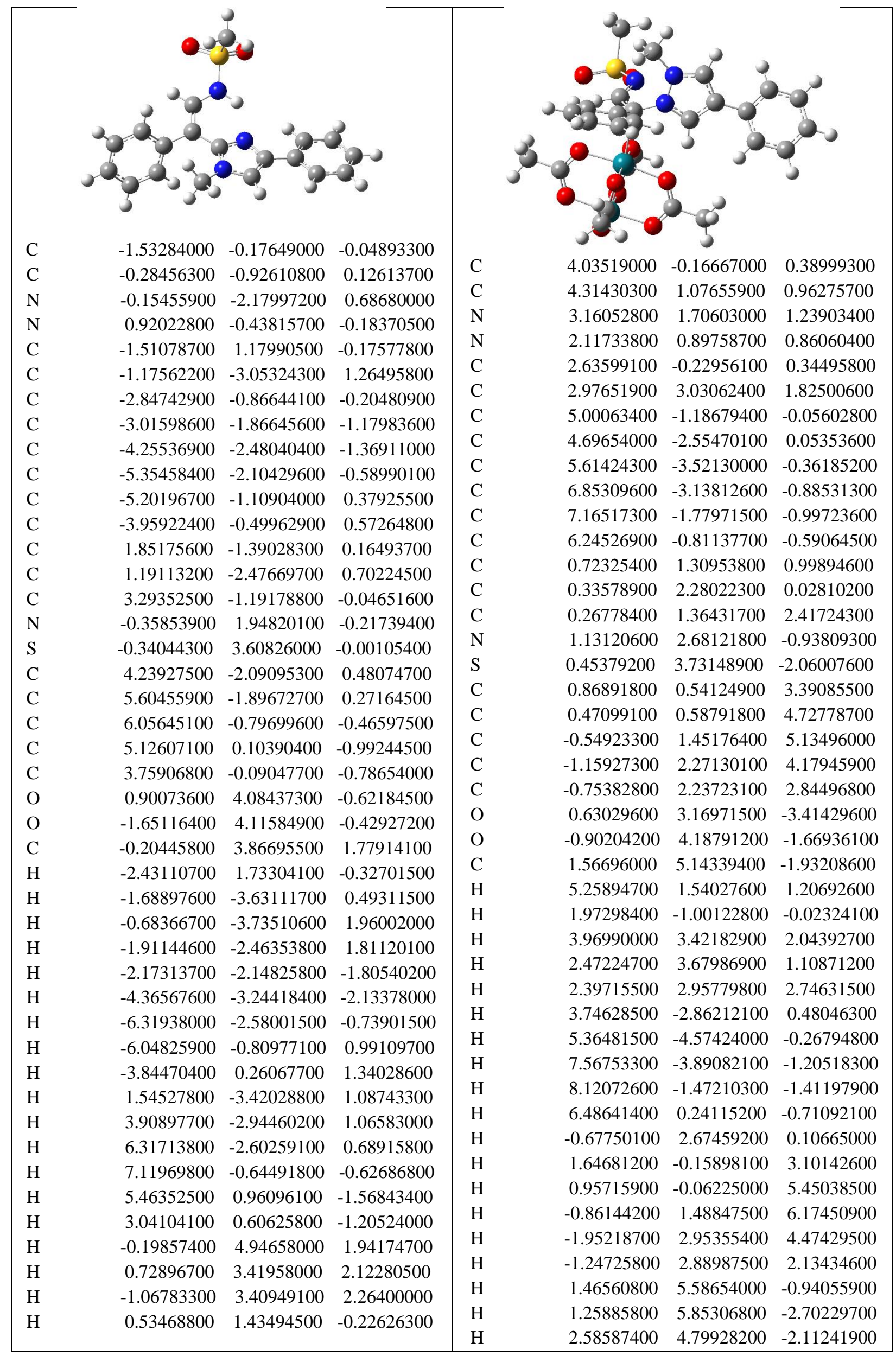




\begin{tabular}{|c|c|c|c|c|}
\hline & $\begin{array}{l}\mathrm{O} \\
\mathrm{O} \\
\mathrm{C} \\
\mathrm{O} \\
\mathrm{O} \\
\mathrm{C} \\
\mathrm{O} \\
\mathrm{O} \\
\mathrm{C} \\
\mathrm{O} \\
\mathrm{C} \\
\mathrm{H} \\
\mathrm{H} \\
\mathrm{H} \\
\mathrm{C} \\
\mathrm{H} \\
\mathrm{H} \\
\mathrm{H} \\
\mathrm{C} \\
\mathrm{H} \\
\mathrm{H} \\
\mathrm{H} \\
\mathrm{C} \\
\mathrm{H} \\
\mathrm{H} \\
\mathrm{H}\end{array}$ & $\begin{array}{c}-2.36940500 \\
-3.11944200 \\
-2.42861500 \\
-3.64242200 \\
-4.09239200 \\
-0.71480800 \\
-1.44964000 \\
-2.39899100 \\
0.25837400 \\
-0.21209800 \\
-1.42944900 \\
-4.61121600 \\
-5.63620700 \\
-4.49855900 \\
-4.37557600 \\
-1.14782100 \\
-0.48842800 \\
-2.06928300 \\
-0.63767600 \\
-2.60875200 \\
-3.67733600 \\
-2.11088300 \\
-2.19695900 \\
0.77013400 \\
0.26685500 \\
1.56741900 \\
1.23047500\end{array}$ & $\begin{array}{c}-1.74336800 \\
-0.34538700 \\
-1.13089500 \\
-1.97168600 \\
-2.40717500 \\
1.11938000 \\
0.90534700 \\
-0.18326000 \\
0.28624500 \\
-0.16838300 \\
-1.00743400 \\
-1.96004700 \\
-3.04572700 \\
-3.25148800 \\
2.04856500 \\
1.70901200 \\
2.47720500 \\
2.83253800 \\
0.30772100 \\
-0.42410100 \\
0.36593900 \\
1.27155200 \\
-2.52129800 \\
-2.67656200 \\
-3.49386200 \\
-1.84886500 \\
-4.16891700 \\
-5.02961800 \\
-3.82448200 \\
-4.45959700\end{array}$ & $\begin{array}{r}-0.82889600 \\
0.01809700 \\
1.88856800 \\
2.02714800 \\
1.09322400 \\
0.30500400 \\
-0.02893200 \\
-0.50894600 \\
-1.91606600 \\
-2.85511300 \\
-2.70607600 \\
-0.34459900 \\
-0.83214600 \\
-1.12410900 \\
0.15335200 \\
0.00089800 \\
1.15266400 \\
-0.57382400 \\
-4.25427900 \\
-4.73567500 \\
-4.83759800 \\
-4.22194600 \\
3.41490700 \\
3.57867000 \\
3.50013700 \\
4.16876800 \\
-1.07125500 \\
-1.51218600 \\
-1.73659600 \\
-0.12165800 \\
\end{array}$ \\
\hline $\begin{array}{l}\text { Zero-point correction }=0.348420 \\
\text { Thermal correction to Energy }=0.384532 \\
\text { Thermal correction to Enthalpy }=0.385745 \\
\text { Thermal correction to Gibbs Free Energy }=0.267867 \\
\mathrm{E}_{0}=-1447.968411, \mathrm{E}=-1447.932299, \\
\mathrm{H}=-1447.931086, \mathrm{G}=-1448.048964 . \\
\text { Imaginary frequency }=0 .\end{array}$ & $\begin{array}{l}\text { Zerc } \\
\text { The } \\
\text { The } \\
\text { The } \\
\mathrm{E}_{0}= \\
\mathrm{H}= \\
\text { Ima }\end{array}$ & $\begin{array}{l}\text { orrection }=0.3 \\
\text { rection to Ener } \\
\text { rection to Enth } \\
\text { rection to Gibb } \\
61709, \mathrm{E}=-14 \\
23070, \mathrm{G}=-14 \\
\text { equency }=0 .\end{array}$ & $\begin{array}{l}\text { tatetraene } 8 \\
\text { N-Ms } \\
\text { ralpy }=0.382240 \\
\text { alpy }=0.38345 \\
447.924283 \text {, } \\
448.042834 \text {. } \\
\end{array}$ & $=0.263690$ \\
\hline
\end{tabular}




\begin{tabular}{|c|c|c|c|c|c|c|c|}
\hline $\mathrm{C}$ & -2.27946100 & 0.19741800 & -0.36574700 & $\mathrm{C}$ & -1.73572600 & -1.38402300 & 0.03224500 \\
\hline $\mathrm{C}$ & -1.71577600 & 0.50612800 & -1.61020100 & $\mathrm{C}$ & -1.49129400 & -2.47953300 & -0.90824200 \\
\hline $\mathrm{N}$ & -0.41672800 & 0.16515700 & -1.60327300 & $\mathrm{~N}$ & -0.39057300 & -2.64040200 & -1.54399300 \\
\hline $\mathrm{N}$ & -0.10054400 & -0.34545900 & -0.37556200 & $\mathrm{~N}$ & 0.60944500 & -1.02014700 & 0.49633200 \\
\hline $\mathrm{C}$ & -1.21612400 & -0.32701600 & 0.37690600 & $\mathrm{C}$ & -0.70655000 & -0.68658300 & 0.61072000 \\
\hline $\mathrm{C}$ & 0.58599400 & 0.32874400 & -2.65044200 & $\mathrm{C}$ & -0.26983100 & -3.81068000 & -2.39622800 \\
\hline $\mathrm{C}$ & -3.67503100 & 0.39289000 & 0.06470100 & $\mathrm{C}$ & -3.13701600 & -1.01047800 & 0.34909900 \\
\hline $\mathrm{C}$ & -4.25778300 & -0.46631300 & 1.01191100 & $\mathrm{C}$ & -3.50117000 & 0.34133000 & 0.51238500 \\
\hline $\mathrm{C}$ & -5.57886700 & -0.27957600 & 1.42114200 & $\mathrm{C}$ & -4.80896900 & 0.70014300 & 0.84237200 \\
\hline $\mathrm{C}$ & -6.34165700 & 0.76214200 & 0.88531900 & $\mathrm{C}$ & -5.79101800 & -0.28113300 & 1.00435000 \\
\hline $\mathrm{C}$ & -5.77173700 & 1.62015000 & -0.05988400 & $\mathrm{C}$ & -5.44878700 & -1.62615700 & 0.83592600 \\
\hline $\mathrm{C}$ & -4.44811200 & 1.44059900 & -0.46392600 & $\mathrm{C}$ & -4.14032700 & -1.98738000 & 0.51117000 \\
\hline $\mathrm{C}$ & 1.22084500 & -0.77829200 & -0.01183100 & $\mathrm{C}$ & 1.61313000 & -0.20937400 & 0.41433200 \\
\hline $\mathrm{C}$ & 2.14237500 & 0.22017800 & 0.24797100 & $\mathrm{C}$ & 1.48901800 & 1.23781800 & 0.10804400 \\
\hline $\mathrm{C}$ & 1.43968000 & -2.22669400 & 0.07428600 & $\mathrm{C}$ & 2.98602000 & -0.77122900 & 0.51408600 \\
\hline $\mathrm{N}$ & 1.87390200 & 1.52716400 & 0.13304800 & $\mathrm{~N}$ & 0.34473200 & 1.80767800 & -0.02944800 \\
\hline S & 3.08439200 & 2.56261500 & 0.57789100 & S & 0.40078900 & 3.49339700 & -0.41870500 \\
\hline $\mathrm{C}$ & 0.53810400 & -3.14263400 & -0.51252700 & $\mathrm{C}$ & 3.19793100 & -2.12679800 & 0.19900400 \\
\hline $\mathrm{C}$ & 0.75232200 & -4.52082800 & -0.44390600 & $\mathrm{C}$ & 4.47045800 & -2.68483400 & 0.29514600 \\
\hline $\mathrm{C}$ & 1.87494300 & -5.03522300 & 0.20825000 & $\mathrm{C}$ & 5.55359000 & -1.90485800 & 0.71792400 \\
\hline $\mathrm{C}$ & 2.77655000 & -4.14223400 & 0.79980400 & $\mathrm{C}$ & 5.35299600 & -0.56108500 & 1.04210500 \\
\hline $\mathrm{C}$ & 2.56295600 & -2.76680100 & 0.74300800 & $\mathrm{C}$ & 4.08071100 & 0.00536200 & 0.93589200 \\
\hline $\mathrm{O}$ & 2.54836400 & 3.53561500 & 1.55546600 & $\mathrm{O}$ & -0.38835900 & 4.17378000 & 0.61846200 \\
\hline $\mathrm{O}$ & 4.34431700 & 1.85900100 & 0.93798600 & $\mathrm{O}$ & 1.78114100 & 3.94328800 & -0.68424400 \\
\hline $\mathrm{C}$ & 3.40639200 & 3.48643200 & -0.93963100 & $\mathrm{C}$ & -0.52194700 & 3.49047500 & -1.96021700 \\
\hline $\mathrm{H}$ & -2.16346400 & 0.93434800 & -2.49476100 & $\mathrm{H}$ & -2.32860300 & -3.17565600 & -1.06417600 \\
\hline $\mathrm{H}$ & -1.16958600 & -0.66889400 & 1.39951300 & $\mathrm{H}$ & -0.95079500 & 0.13079200 & 1.28794600 \\
\hline $\mathrm{H}$ & 0.05977500 & 0.62050100 & -3.55855700 & $\mathrm{H}$ & -1.17167700 & -4.44362200 & -2.41354500 \\
\hline $\mathrm{H}$ & 1.29433800 & 1.10313300 & -2.35031300 & $\mathrm{H}$ & -0.03785400 & -3.49124500 & -3.41882400 \\
\hline $\mathrm{H}$ & 1.10652500 & -0.61641300 & -2.81016300 & $\mathrm{H}$ & 0.57741300 & -4.41732600 & -2.05472100 \\
\hline $\mathrm{H}$ & -3.68296000 & -1.29350500 & 1.41808400 & $\mathrm{H}$ & -2.75740200 & 1.11721200 & 0.35984200 \\
\hline $\mathrm{H}$ & -6.01375000 & -0.95314500 & 2.15348500 & $\mathrm{H}$ & -5.06158600 & 1.74991300 & 0.96156200 \\
\hline $\mathrm{H}$ & -7.36996500 & 0.90544400 & 1.20297600 & $\mathrm{H}$ & -6.80972000 & -0.00195700 & 1.25651000 \\
\hline $\mathrm{H}$ & -6.35343000 & 2.43784600 & -0.47488700 & $\mathrm{H}$ & -6.19989800 & -2.39985600 & 0.96755700 \\
\hline $\mathrm{H}$ & -4.00695700 & 2.13165900 & -1.17630000 & $\mathrm{H}$ & -3.89470500 & -3.04055000 & 0.41811900 \\
\hline $\mathrm{H}$ & 3.14453700 & -0.09441900 & 0.54418700 & $\mathrm{H}$ & 2.41418600 & 1.79682500 & -0.05505300 \\
\hline $\mathrm{H}$ & -0.34183600 & -2.77798800 & -1.03491800 & $\mathrm{H}$ & 2.35153900 & -2.71984000 & -0.12919200 \\
\hline $\mathrm{H}$ & 0.03600700 & -5.19303200 & -0.90881600 & $\mathrm{H}$ & 4.62065300 & -3.72934600 & 0.03792100 \\
\hline $\mathrm{H}$ & 2.04214800 & -6.10656800 & 0.26181700 & $\mathrm{H}$ & 6.54486500 & -2.34190400 & 0.79397100 \\
\hline $\mathrm{H}$ & 3.65016300 & -4.51969400 & 1.32424800 & $\mathrm{H}$ & 6.18454500 & 0.04924000 & 1.38127700 \\
\hline $\mathrm{H}$ & 3.26905900 & -2.10807200 & 1.23775700 & $\mathrm{H}$ & 3.94918700 & 1.04633100 & 1.21416200 \\
\hline $\mathrm{H}$ & 3.79533000 & 2.79990300 & -1.69291700 & $\mathrm{H}$ & -0.61389400 & 4.53508300 & -2.26395500 \\
\hline $\mathrm{H}$ & 4.14779100 & 4.24997300 & -0.69678800 & $\mathrm{H}$ & -1.50155600 & 3.05054200 & -1.77380400 \\
\hline $\mathrm{H}$ & 2.47446000 & 3.94771500 & -1.26821900 & $\mathrm{H}$ & 0.03789200 & 2.91866100 & -2.70098100 \\
\hline \multicolumn{4}{|c|}{ Betaine 9} & \multicolumn{4}{|c|}{ Cation 10} \\
\hline \multicolumn{4}{|c|}{ 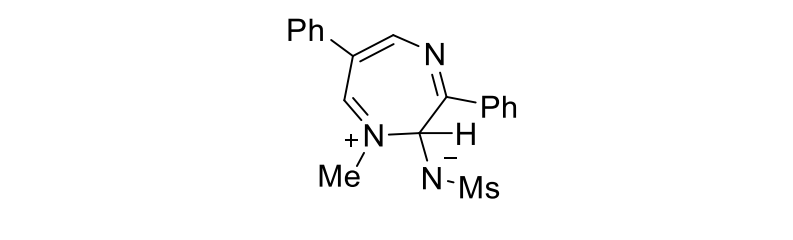 } & \multicolumn{4}{|c|}{ Ms } \\
\hline \multicolumn{4}{|c|}{$\begin{array}{l}\text { Zero-point correction }=0.348576 \\
\text { Thermal correction to Energy }=0.384313 \\
\text { Thermal correction to Enthalpy }=0.385526\end{array}$} & \multicolumn{4}{|c|}{$\begin{array}{l}\text { Zero-point correction }=0.362461 \\
\text { Thermal correction to Energy }=0.398664 \\
\text { Thermal correction to Enthalpy }=0.399878\end{array}$} \\
\hline
\end{tabular}




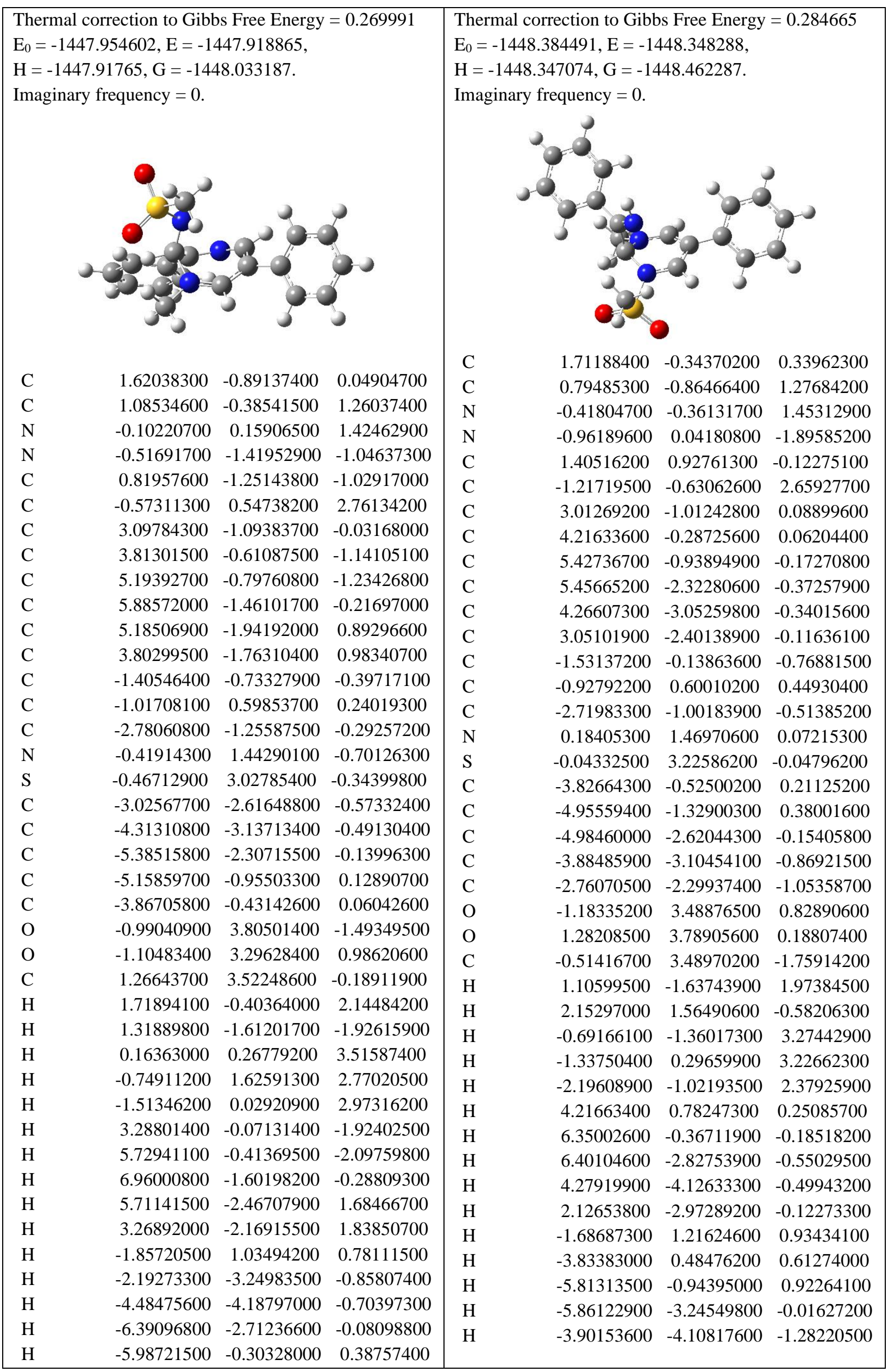




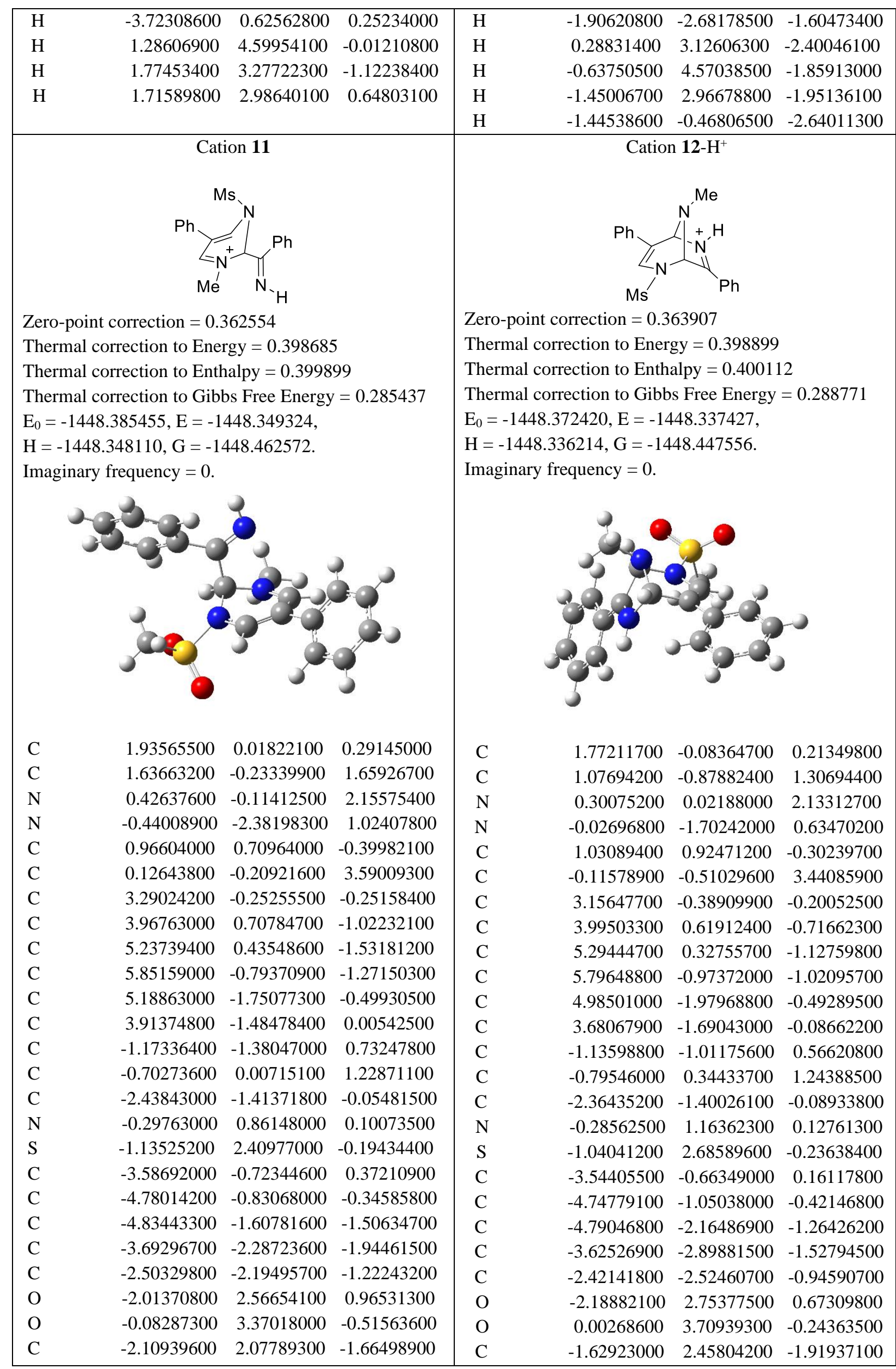




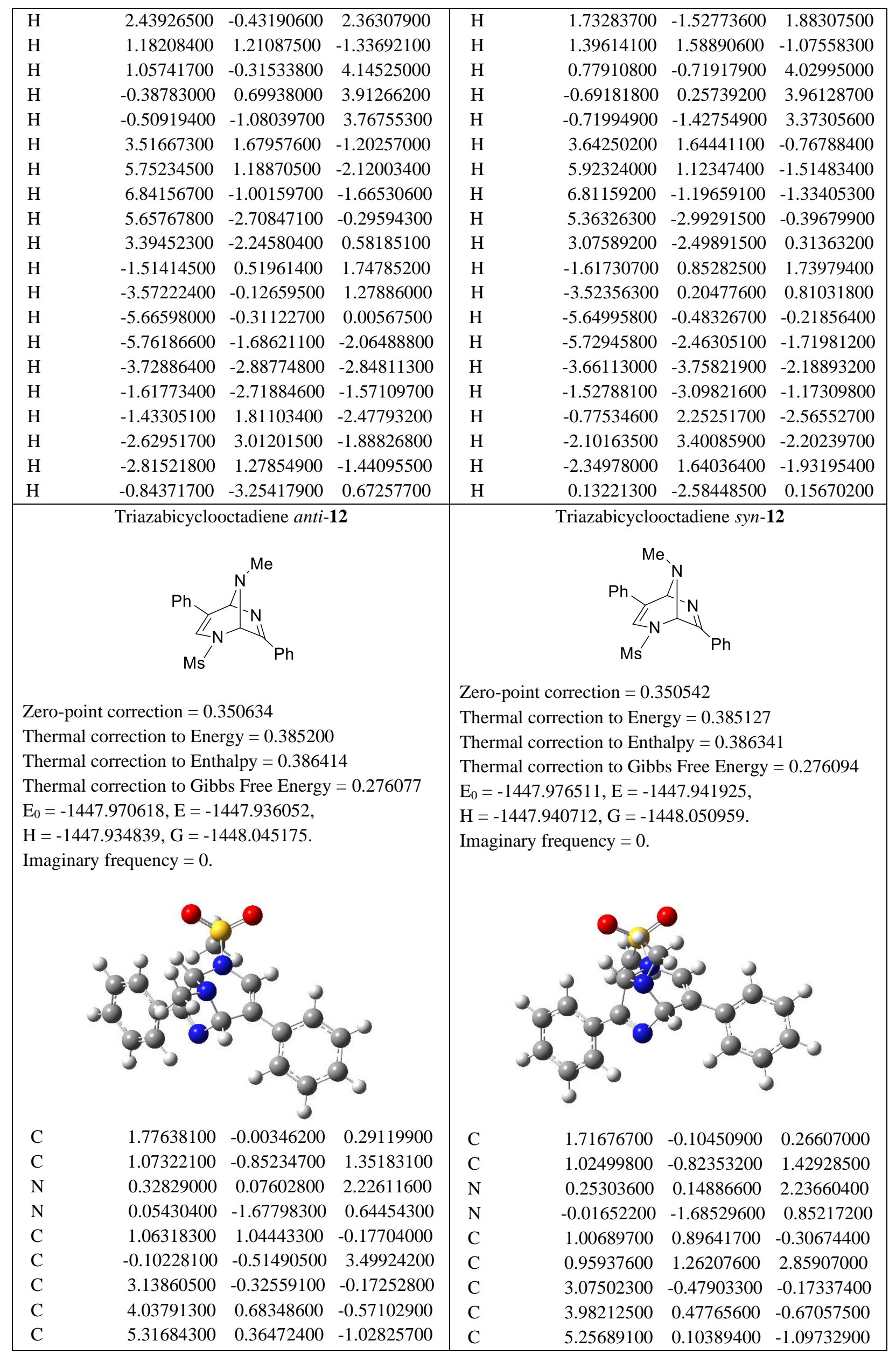




\begin{tabular}{|c|c|c|c|c|c|c|c|}
\hline $\mathrm{C}$ & 5.73746100 & -0.96823400 & -1.08220200 & C & 5.66463900 & -1.23198200 & -1.02217000 \\
\hline $\mathrm{C}$ & 4.86250800 & -1.97889200 & -0.67368700 & $\mathrm{C}$ & 4.78101500 & -2.19020800 & -0.51734700 \\
\hline $\mathrm{C}$ & 3.57899400 & -1.66269600 & -0.22450800 & $\mathrm{C}$ & 3.50169400 & -1.81945900 & -0.09860500 \\
\hline $\mathrm{C}$ & -1.02661900 & -0.97775000 & 0.63495000 & $\mathrm{C}$ & -1.09097300 & -0.97283400 & 0.80279400 \\
\hline $\mathrm{C}$ & -0.77859500 & 0.38664000 & 1.33714900 & $\mathrm{C}$ & -0.81928500 & 0.44120600 & 1.33311600 \\
\hline $\mathrm{C}$ & -2.27251200 & -1.39074400 & -0.03294400 & $\mathrm{C}$ & -2.36254900 & -1.43385300 & 0.22160900 \\
\hline $\mathrm{N}$ & -0.23339900 & 1.31022500 & 0.30279000 & $\mathrm{~N}$ & -0.28156500 & 1.23308100 & 0.14890200 \\
\hline $\mathrm{S}$ & -1.13845800 & 2.58544600 & -0.33602700 & $\mathrm{~S}$ & -1.13240400 & 2.50127200 & -0.56978600 \\
\hline $\mathrm{C}$ & -3.46997400 & -0.67755600 & 0.15667100 & $\mathrm{C}$ & -3.53756200 & -0.67027200 & 0.33900000 \\
\hline $\mathrm{C}$ & -4.65184400 & -1.09930200 & -0.45859200 & $\mathrm{C}$ & -4.74156700 & -1.13542100 & -0.19668400 \\
\hline $\mathrm{C}$ & -4.65284000 & -2.23482400 & -1.27162800 & $\mathrm{C}$ & -4.78722800 & -2.36538200 & -0.85671800 \\
\hline $\mathrm{C}$ & -3.46454000 & -2.95070900 & -1.46794500 & $\mathrm{C}$ & -3.62111700 & -3.13229600 & -0.97909700 \\
\hline $\mathrm{C}$ & -2.28535100 & -2.53423400 & -0.85565400 & $\mathrm{C}$ & -2.41984900 & -2.67277200 & -0.44579400 \\
\hline $\mathrm{O}$ & -2.26583000 & 2.77789800 & 0.58626200 & $\mathrm{O}$ & -2.22472000 & 2.82502800 & 0.35903500 \\
\hline $\mathrm{O}$ & -0.20391300 & 3.67674600 & -0.63388200 & $\mathrm{O}$ & -0.16092600 & 3.52458000 & -0.97829400 \\
\hline $\mathrm{C}$ & -1.78525600 & 1.99297600 & -1.91070200 & $\mathrm{C}$ & -1.84955800 & 1.80942200 & -2.07098800 \\
\hline $\mathrm{H}$ & 1.76594700 & -1.47207000 & 1.92135600 & $\mathrm{H}$ & 1.72396500 & -1.38682900 & 2.05047400 \\
\hline $\mathrm{H}$ & 1.43255000 & 1.73407700 & -0.92578900 & $\mathrm{H}$ & 1.38274500 & 1.49441600 & -1.12836400 \\
\hline $\mathrm{H}$ & 0.78600900 & -0.75693600 & 4.08805800 & $\mathrm{H}$ & 1.43783300 & 1.94332000 & 2.14067800 \\
\hline $\mathrm{H}$ & -0.68362700 & 0.22515700 & 4.05590600 & $\mathrm{H}$ & 0.25150200 & 1.83361700 & 3.46605400 \\
\hline $\mathrm{H}$ & -0.70649400 & -1.42888300 & 3.38521300 & $\mathrm{H}$ & 1.72924100 & 0.85880100 & 3.52307400 \\
\hline $\mathrm{H}$ & 3.74239700 & 1.72584200 & -0.49633500 & $\mathrm{H}$ & 3.69619600 & 1.52512500 & -0.69936400 \\
\hline $\mathrm{H}$ & 5.99291400 & 1.16149600 & -1.32556300 & $\mathrm{H}$ & 5.93926300 & 0.86118800 & -1.47315300 \\
\hline $\mathrm{H}$ & 6.73637800 & -1.21443100 & -1.42994200 & $\mathrm{H}$ & 6.66023100 & -1.52026000 & -1.34630200 \\
\hline $\mathrm{H}$ & 5.17555800 & -3.01857600 & -0.71065600 & $\mathrm{H}$ & 5.08387100 & -3.23168700 & -0.45523800 \\
\hline $\mathrm{H}$ & 2.90326500 & -2.46282300 & 0.06313500 & $\mathrm{H}$ & 2.81825500 & -2.58075500 & 0.26598000 \\
\hline $\mathrm{H}$ & -1.61351400 & 0.86689800 & 1.84058800 & $\mathrm{H}$ & -1.63412500 & 1.00822900 & 1.77603900 \\
\hline $\mathrm{H}$ & -3.48819100 & 0.20863300 & 0.78291500 & $\mathrm{H}$ & -3.52242900 & 0.28746900 & 0.84920900 \\
\hline $\mathrm{H}$ & -5.56866900 & -0.53941600 & -0.30030600 & $\mathrm{H}$ & -5.64118700 & -0.53586400 & -0.09497200 \\
\hline $\mathrm{H}$ & -5.57143200 & -2.56176900 & -1.74993200 & $\mathrm{H}$ & -5.72319600 & -2.72638200 & -1.27280100 \\
\hline $\mathrm{H}$ & -3.46041700 & -3.83324000 & -2.10094100 & $\mathrm{H}$ & -3.65151800 & -4.08903000 & -1.49219600 \\
\hline $\mathrm{H}$ & -1.36123600 & -3.08322500 & -1.00122000 & $\mathrm{H}$ & -1.51313900 & -3.26155400 & -0.53445300 \\
\hline $\mathrm{H}$ & -0.94415200 & 1.71648100 & -2.54774800 & $\mathrm{H}$ & -1.04028100 & 1.44938700 & -2.70752300 \\
\hline $\mathrm{H}$ & -2.34193600 & 2.82389300 & -2.34841100 & $\mathrm{H}$ & -2.39085100 & 2.62180400 & -2.55976000 \\
\hline $\mathrm{H}$ & -2.43821700 & 1.14062800 & -1.72303100 & $\mathrm{H}$ & -2.52582800 & 1.00054000 & -1.79360200 \\
\hline \multicolumn{4}{|c|}{ Betaine 13} & \multicolumn{4}{|c|}{ Betaine 14} \\
\hline \multicolumn{4}{|c|}{$\begin{array}{l}\text { Zero-point correction }=0.347355 \\
\text { Thermal correction to Energy }=0.384868 \\
\text { Thermal correction to Enthalpy }=0.386081 \\
\text { Thermal correction to Gibbs Free Energy }=0.269431 \\
E_{0}=-1447.961120, E=-1447.924909 \\
H=-1447.923696, G=-1448.040841 \\
\text { Imaginary frequency }=0\end{array}$} & \multicolumn{4}{|c|}{$\begin{array}{l}\text { Zero-point correction }=0 ., 347234 \\
\text { Thermal correction to Energy }=0.383349 \\
\text { Thermal correction to Enthalpy }=0.384563 \\
\text { Thermal correction to Gibbs Free Energy }=0.267164 \\
E_{0}=-1447.958332, E=-1447.922216 \\
H=-1447.921003, G=-1448.038401 \\
\text { Imaginary frequency }=0\end{array}$} \\
\hline
\end{tabular}




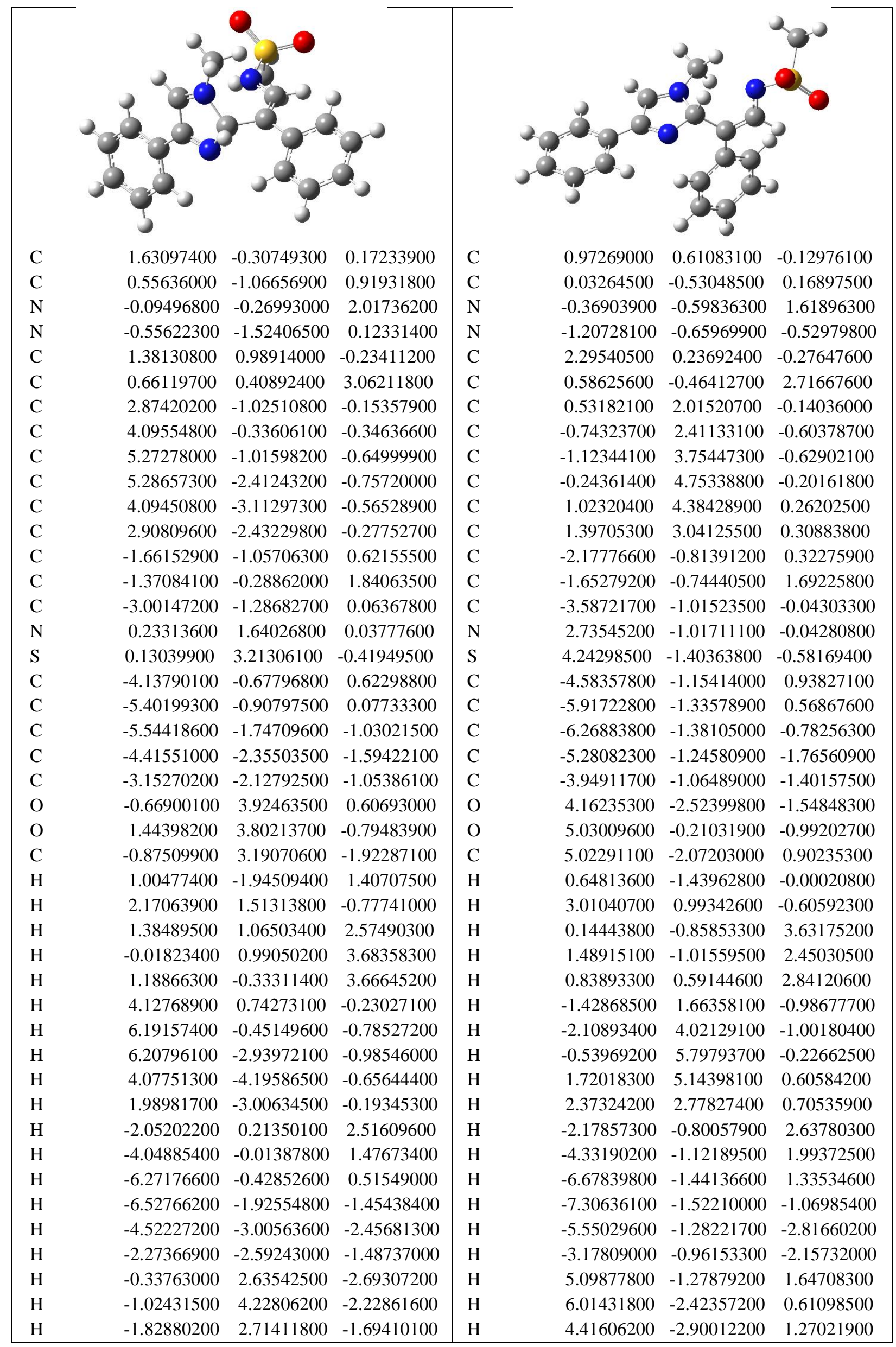




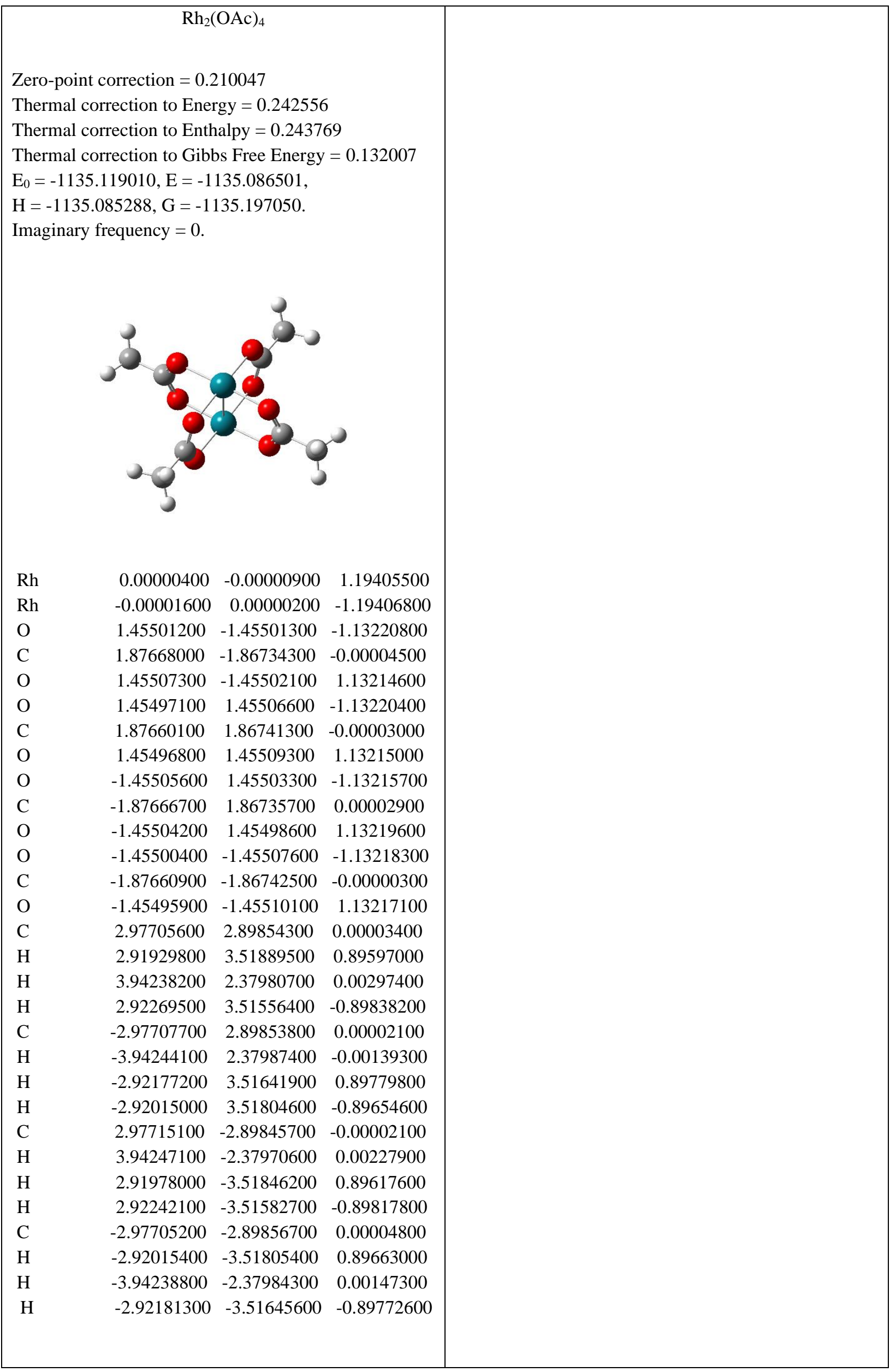




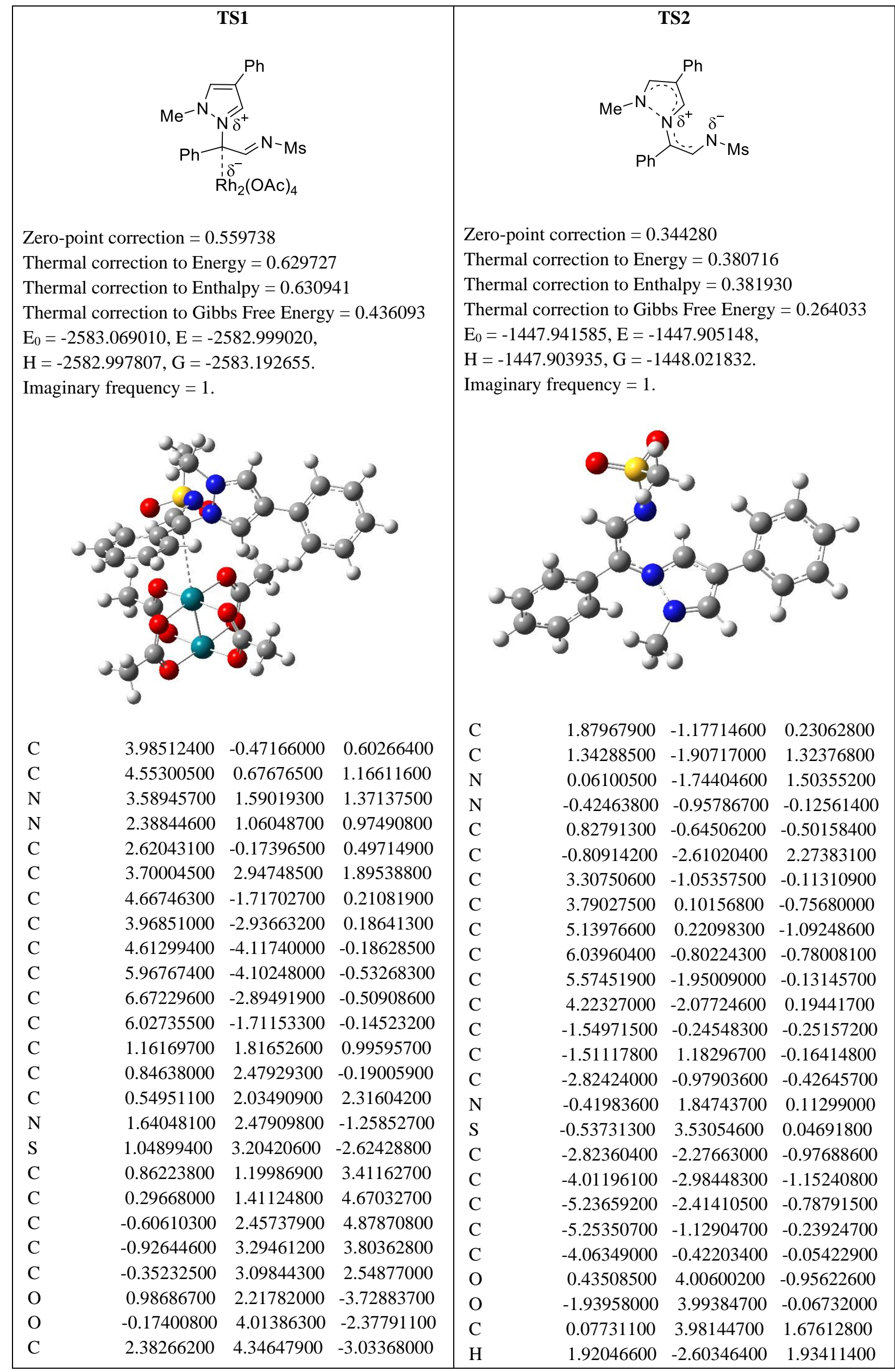




\begin{tabular}{|c|c|c|c|c|c|c|c|}
\hline $\mathrm{H}$ & 5.57440300 & 0.88893400 & 1.44649200 & $\mathrm{H}$ & 0.94395500 & -0.05183800 & -1.40265300 \\
\hline $\mathrm{H}$ & 1.80082700 & -0.74204100 & 0.08387200 & $\mathrm{H}$ & -0.30302300 & -2.90717900 & 3.20069800 \\
\hline $\mathrm{H}$ & 4.76089800 & 3.15595600 & 2.03030600 & $\mathrm{H}$ & -1.72343900 & -2.07133100 & 2.53099400 \\
\hline $\mathrm{H}$ & 3.27276000 & 3.64912900 & 1.17728500 & $\mathrm{H}$ & -1.07577100 & -3.51095900 & 1.71115100 \\
\hline $\mathrm{H}$ & 3.17790700 & 3.02888300 & 2.85059800 & $\mathrm{H}$ & 3.11140100 & 0.92062000 & -0.97481600 \\
\hline $\mathrm{H}$ & 2.92131500 & -2.96395900 & 0.47288700 & $\mathrm{H}$ & 5.48919500 & 1.12226200 & -1.58780100 \\
\hline $\mathrm{H}$ & 4.05762500 & -5.05071600 & -0.19931600 & $\mathrm{H}$ & 7.09014200 & -0.70604700 & -1.03744500 \\
\hline $\mathrm{H}$ & 6.46847300 & -5.02201500 & -0.82064900 & $\mathrm{H}$ & 6.26228000 & -2.75494100 & 0.11194100 \\
\hline $\mathrm{H}$ & 7.72243200 & -2.87104000 & -0.78535900 & $\mathrm{H}$ & 3.87671700 & -2.98957600 & 0.67133600 \\
\hline $\mathrm{H}$ & 6.58004800 & -0.77636200 & -0.15956700 & $\mathrm{H}$ & -2.43906100 & 1.72006900 & -0.37547200 \\
\hline $\mathrm{H}$ & -0.10645300 & 3.00926900 & -0.22200900 & $\mathrm{H}$ & -1.88024100 & -2.72234600 & -1.27701000 \\
\hline $\mathrm{H}$ & 1.53882600 & 0.36089600 & 3.27470300 & $\mathrm{H}$ & -3.98308300 & -3.98099900 & -1.58450900 \\
\hline $\mathrm{H}$ & 0.55760200 & 0.74449300 & 5.48808800 & $\mathrm{H}$ & -6.16251500 & -2.96371900 & -0.92856300 \\
\hline $\mathrm{H}$ & -1.04819500 & 2.62044400 & 5.85705800 & $\mathrm{H}$ & -6.19495400 & -0.67522500 & 0.05701600 \\
\hline $\mathrm{H}$ & -1.62003700 & 4.11941500 & 3.94446400 & $\mathrm{H}$ & -4.10640200 & 0.56327700 & 0.39956300 \\
\hline $\mathrm{H}$ & -0.60395800 & 3.78273900 & 1.74501800 & $\mathrm{H}$ & 0.11363700 & 5.07207000 & 1.70424500 \\
\hline $\mathrm{H}$ & 2.46396700 & 5.09096500 & -2.24065600 & $\mathrm{H}$ & 1.07351000 & 3.55575000 & 1.79691100 \\
\hline $\mathrm{H}$ & 2.11618800 & 4.81831800 & -3.98139400 & $\mathrm{H}$ & -0.61134600 & 3.59690000 & 2.42911900 \\
\hline $\mathrm{H}$ & 3.30665200 & 3.77594700 & -3.13338000 & & & & \\
\hline $\mathrm{Rh}$ & -2.85026600 & -1.63487500 & -0.87137700 & & & & \\
\hline $\mathrm{Rh}$ & -1.21428200 & -0.28544100 & 0.22975300 & & & & \\
\hline $\mathrm{O}$ & -2.51425400 & -0.11091800 & 1.81560900 & & & & \\
\hline $\mathrm{C}$ & -3.64770900 & -0.68977000 & 1.75126500 & & & & \\
\hline $\mathrm{O}$ & -4.05968700 & -1.40226700 & 0.77510700 & & & & \\
\hline $\mathrm{O}$ & -2.08656700 & 1.34778600 & -0.63908700 & & & & \\
\hline C & -3.07351800 & 1.19288000 & -1.43223100 & & & & \\
\hline $\mathrm{O}$ & -3.62167900 & 0.07290400 & -1.70644800 & & & & \\
\hline $\mathrm{O}$ & -0.00482400 & -0.56041000 & -1.40191000 & & & & \\
\hline $\mathrm{C}$ & -0.43754600 & -1.21215300 & -2.41210700 & & & & \\
\hline $\mathrm{O}$ & -1.57401500 & -1.78796800 & -2.47395500 & & & & \\
\hline $\mathrm{O}$ & -0.42314100 & -2.00664100 & 1.05350300 & & & & \\
\hline $\mathrm{C}$ & -0.97965700 & -3.12373900 & 0.78311100 & & & & \\
\hline $\mathrm{O}$ & -1.98046000 & -3.28236900 & 0.01109600 & & & & \\
\hline $\mathrm{C}$ & -3.63110200 & 2.43663500 & -2.07557700 & & & & \\
\hline $\mathrm{H}$ & -4.29916900 & 2.17710200 & -2.89760400 & & & & \\
\hline $\mathrm{H}$ & -4.18978000 & 3.00584400 & -1.32445700 & & & & \\
\hline $\mathrm{H}$ & -2.80621600 & 3.06241300 & -2.42658000 & & & & \\
\hline $\mathrm{C}$ & 0.47206100 & -1.28928000 & -3.61017600 & & & & \\
\hline $\mathrm{H}$ & 1.39323000 & -1.81311100 & -3.33483100 & & & & \\
\hline $\mathrm{H}$ & -0.01741300 & -1.81264100 & -4.43197200 & & & & \\
\hline $\mathrm{H}$ & 0.74458700 & -0.27126000 & -3.90590000 & & & & \\
\hline $\mathrm{C}$ & -4.59187400 & -0.49590000 & 2.91329100 & & & & \\
\hline $\mathrm{H}$ & -5.18380700 & 0.40897000 & 2.73528800 & & & & \\
\hline $\mathrm{H}$ & -5.27531300 & -1.34276700 & 2.99498900 & & & & \\
\hline $\mathrm{H}$ & -4.03107600 & -0.35888200 & 3.83953500 & & & & \\
\hline $\mathrm{C}$ & -0.42470900 & -4.36196600 & 1.44663700 & & & & \\
\hline $\mathrm{H}$ & -1.11559900 & -4.67809100 & 2.23565500 & & & & \\
\hline $\mathrm{H}$ & -0.35603000 & -5.17657700 & 0.72156900 & & & & \\
\hline $\mathrm{H}$ & 0.55069100 & -4.16073600 & 1.89116100 & & & & \\
\hline
\end{tabular}




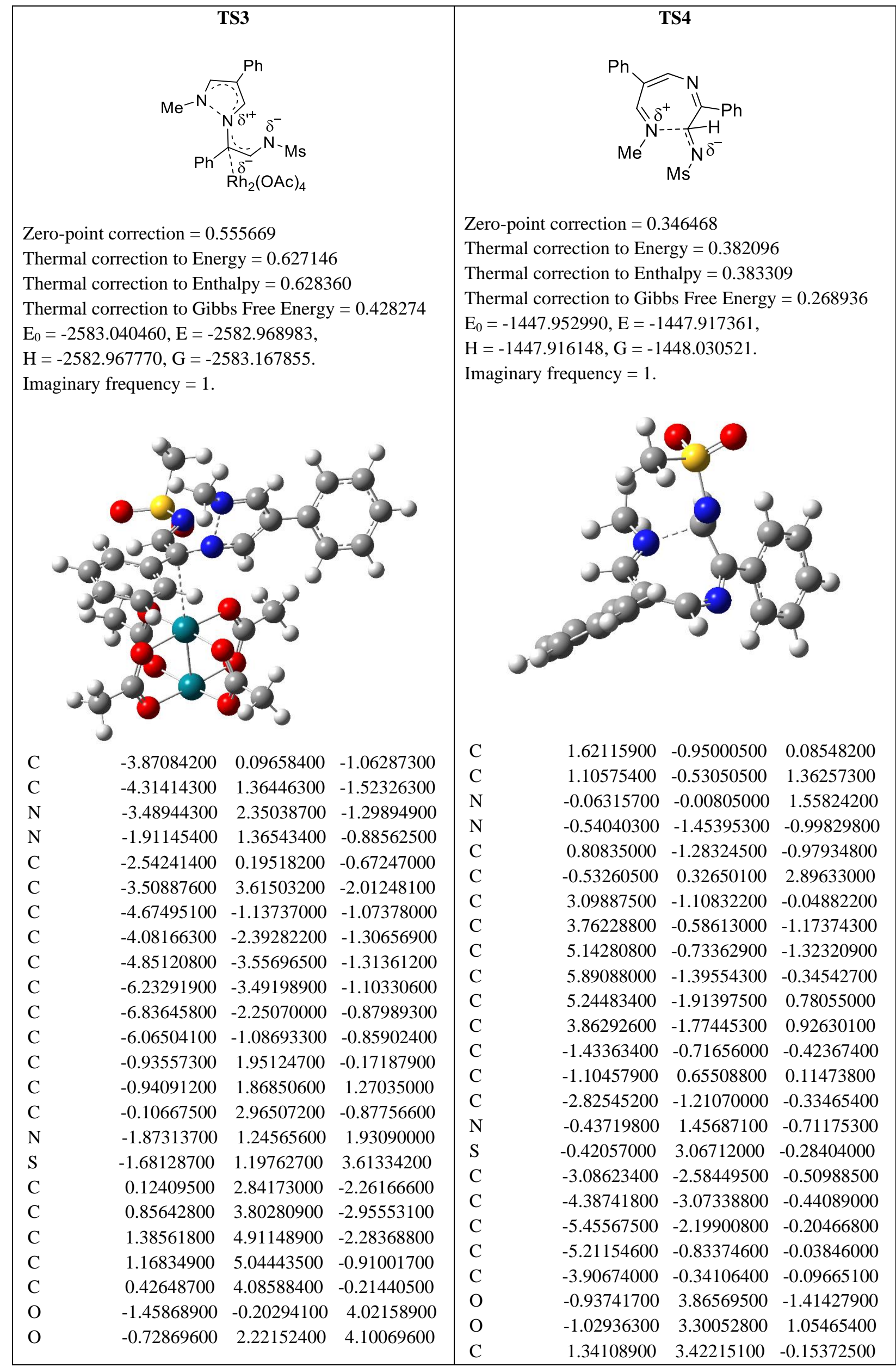




\begin{tabular}{|c|c|c|c|c|c|c|c|}
\hline $\mathrm{C}$ & -3.33750400 & 1.67012500 & 4.12901700 & $\mathrm{H}$ & 1.76743000 & -0.63499900 & 2.22793300 \\
\hline $\mathrm{H}$ & -5.22016800 & 1.50364600 & -2.11700000 & $\mathrm{H}$ & 1.29664800 & -1.64246100 & -1.88315800 \\
\hline $\mathrm{H}$ & -1.95842800 & -0.61599600 & -0.24633200 & $\mathrm{H}$ & 0.19030400 & 0.02528700 & 3.66220600 \\
\hline $\mathrm{H}$ & -4.52770100 & 3.83551400 & -2.35766700 & $\mathrm{H}$ & -0.71004300 & 1.40440400 & 2.95465700 \\
\hline $\mathrm{H}$ & -3.17147700 & 4.41842000 & -1.35399900 & $\mathrm{H}$ & -1.48098200 & -0.18785000 & 3.08533600 \\
\hline $\mathrm{H}$ & -2.84632100 & 3.57633600 & -2.88240100 & $\mathrm{H}$ & 3.19376700 & -0.04756200 & -1.92645700 \\
\hline $\mathrm{H}$ & -3.01485800 & -2.45327800 & -1.50207000 & $\mathrm{H}$ & 5.63435400 & -0.32015000 & -2.19909500 \\
\hline $\mathrm{H}$ & -4.37335200 & -4.51534200 & -1.49680400 & $\mathrm{H}$ & 6.96512600 & -1.50593800 & -0.45916700 \\
\hline $\mathrm{H}$ & -6.83145700 & -4.39815600 & -1.11430700 & $\mathrm{H}$ & 5.81411400 & -2.43877500 & 1.54235800 \\
\hline $\mathrm{H}$ & -7.90756100 & -2.18847100 & -0.70920300 & $\mathrm{H}$ & 3.37552100 & -2.21244000 & 1.79305800 \\
\hline $\mathrm{H}$ & -6.54253800 & -0.13225700 & -0.65503900 & $\mathrm{H}$ & -1.83675900 & 1.07827000 & 0.80011200 \\
\hline $\mathrm{H}$ & -0.11227200 & 2.34442500 & 1.79714700 & $\mathrm{H}$ & -2.25470800 & -3.25370900 & -0.70174800 \\
\hline $\mathrm{H}$ & -0.26187800 & 1.97215800 & -2.78402800 & $\mathrm{H}$ & -4.57200800 & -4.13543900 & -0.57190200 \\
\hline $\mathrm{H}$ & 1.02524100 & 3.68108500 & -4.02218400 & $\mathrm{H}$ & -6.47115800 & -2.58060500 & -0.15373100 \\
\hline $\mathrm{H}$ & 1.95806400 & 5.66004600 & -2.82404500 & $\mathrm{H}$ & -6.03573300 & -0.14818900 & 0.13271400 \\
\hline $\mathrm{H}$ & 1.56645600 & 5.90207900 & -0.37480400 & $\mathrm{H}$ & -3.74641300 & 0.72550900 & 0.01508400 \\
\hline $\mathrm{H}$ & 0.25282700 & 4.23174200 & 0.84703000 & $\mathrm{H}$ & 1.43397900 & 4.48874400 & 0.05915700 \\
\hline $\mathrm{H}$ & -3.53589600 & 2.68602200 & 3.78558800 & $\mathrm{H}$ & 1.80971800 & 3.17588900 & -1.10667800 \\
\hline $\mathrm{H}$ & -3.34950300 & 1.61924400 & 5.21942600 & $\mathrm{H}$ & 1.76502900 & 2.82840600 & 0.65671200 \\
\hline $\mathrm{H}$ & -4.04570000 & 0.96210900 & 3.69812600 & & & & \\
\hline $\mathrm{Rh}$ & 2.87155900 & -1.89132200 & 0.00055700 & & & & \\
\hline $\mathrm{Rh}$ & 1.21319700 & -0.17345400 & -0.16781400 & & & & \\
\hline $\mathrm{O}$ & 0.92804700 & -0.72759600 & -2.13431400 & & & & \\
\hline $\mathrm{C}$ & 1.62489300 & -1.68303200 & -2.61282100 & & & & \\
\hline $\mathrm{O}$ & 2.49508300 & -2.35647900 & -1.96851600 & & & & \\
\hline $\mathrm{O}$ & 2.71404100 & 1.11250800 & -0.73564300 & & & & \\
\hline C & 3.90885000 & 0.67976500 & -0.82288100 & & & & \\
\hline $\mathrm{O}$ & 4.27750700 & -0.52093400 & -0.59396900 & & & & \\
\hline $\mathrm{O}$ & 1.59230000 & 0.27573900 & 1.79645400 & & & & \\
\hline $\mathrm{C}$ & 2.46177700 & -0.39703900 & 2.44454000 & & & & \\
\hline $\mathrm{O}$ & 3.16114100 & -1.34752100 & 1.95922800 & & & & \\
\hline $\mathrm{O}$ & -0.17910600 & -1.57512400 & 0.41284200 & & & & \\
\hline $\mathrm{C}$ & 0.20052600 & -2.75832300 & 0.71109900 & & & & \\
\hline $\mathrm{O}$ & 1.39521300 & -3.18649400 & 0.61079600 & & & & \\
\hline $\mathrm{C}$ & 4.98181100 & 1.67083700 & -1.20295800 & & & & \\
\hline $\mathrm{H}$ & 5.74381100 & 1.18609200 & -1.81681700 & & & & \\
\hline $\mathrm{H}$ & 4.54595800 & 2.52241100 & -1.72703900 & & & & \\
\hline $\mathrm{H}$ & 5.46454700 & 2.03254900 & -0.28818800 & & & & \\
\hline $\mathrm{C}$ & 2.67066200 & -0.02744700 & 3.89076500 & & & & \\
\hline $\mathrm{H}$ & 3.36787100 & -0.71710600 & 4.36724600 & & & & \\
\hline $\mathrm{H}$ & 3.06182300 & 0.99315700 & 3.94922700 & & & & \\
\hline $\mathrm{H}$ & 1.70738400 & -0.03860200 & 4.40789900 & & & & \\
\hline $\mathrm{C}$ & 1.42428800 & -2.02684400 & -4.06936100 & & & & \\
\hline $\mathrm{H}$ & 1.62791700 & -3.08513600 & -4.24209400 & & & & \\
\hline $\mathrm{H}$ & 0.41187900 & -1.77159700 & -4.38704000 & & & & \\
\hline $\mathrm{H}$ & 2.13123500 & -1.44080600 & -4.66748300 & & & & \\
\hline $\mathrm{C}$ & -0.84152100 & -3.70547000 & 1.24930800 & & & & \\
\hline $\mathrm{H}$ & -0.97511500 & -3.49825900 & 2.31684100 & & & & \\
\hline $\mathrm{H}$ & -1.80195100 & -3.54030500 & 0.75704100 & & & & \\
\hline $\mathrm{H}$ & -0.51727600 & -4.73997900 & 1.12826200 & & & & \\
\hline
\end{tabular}




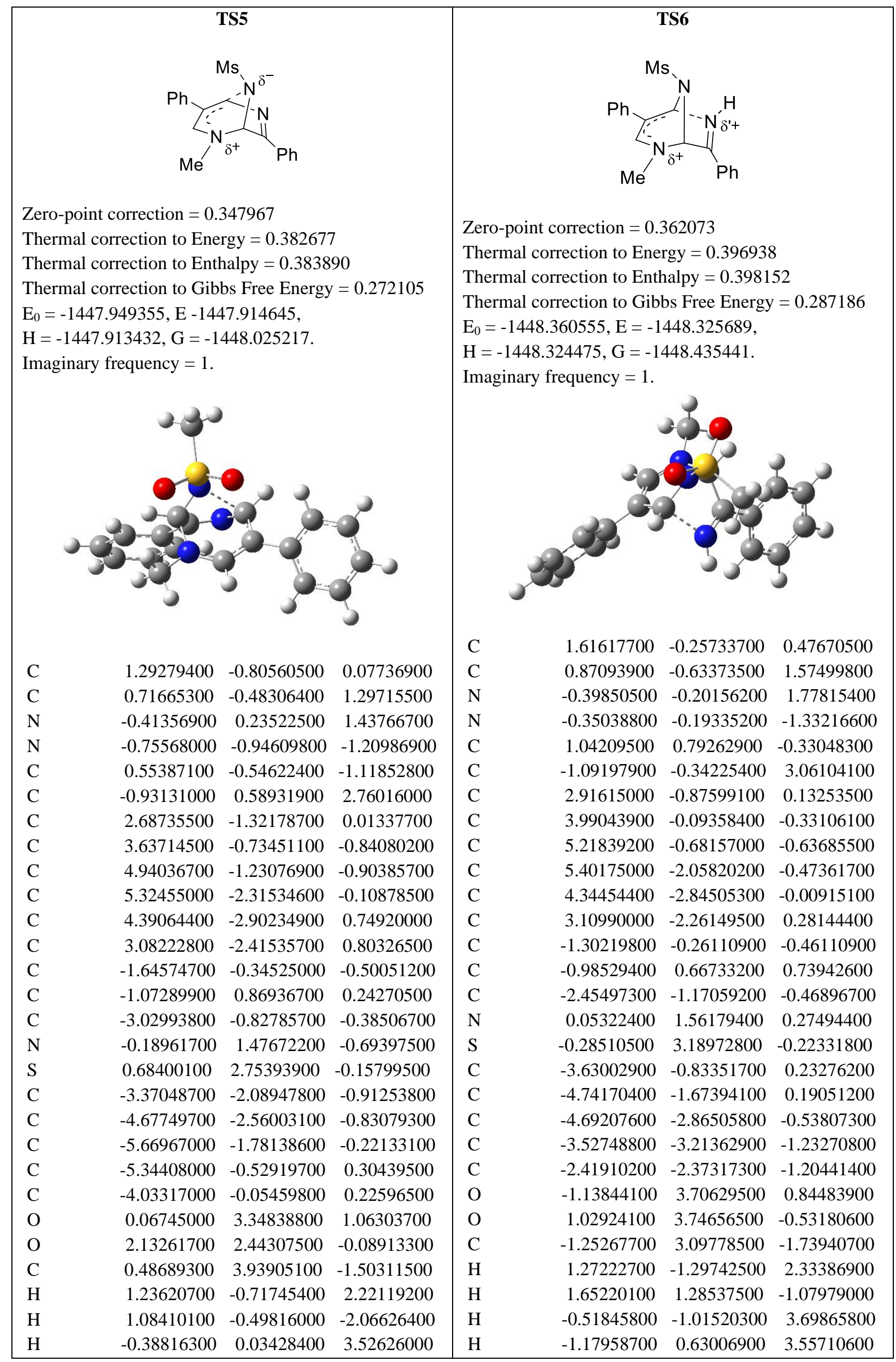




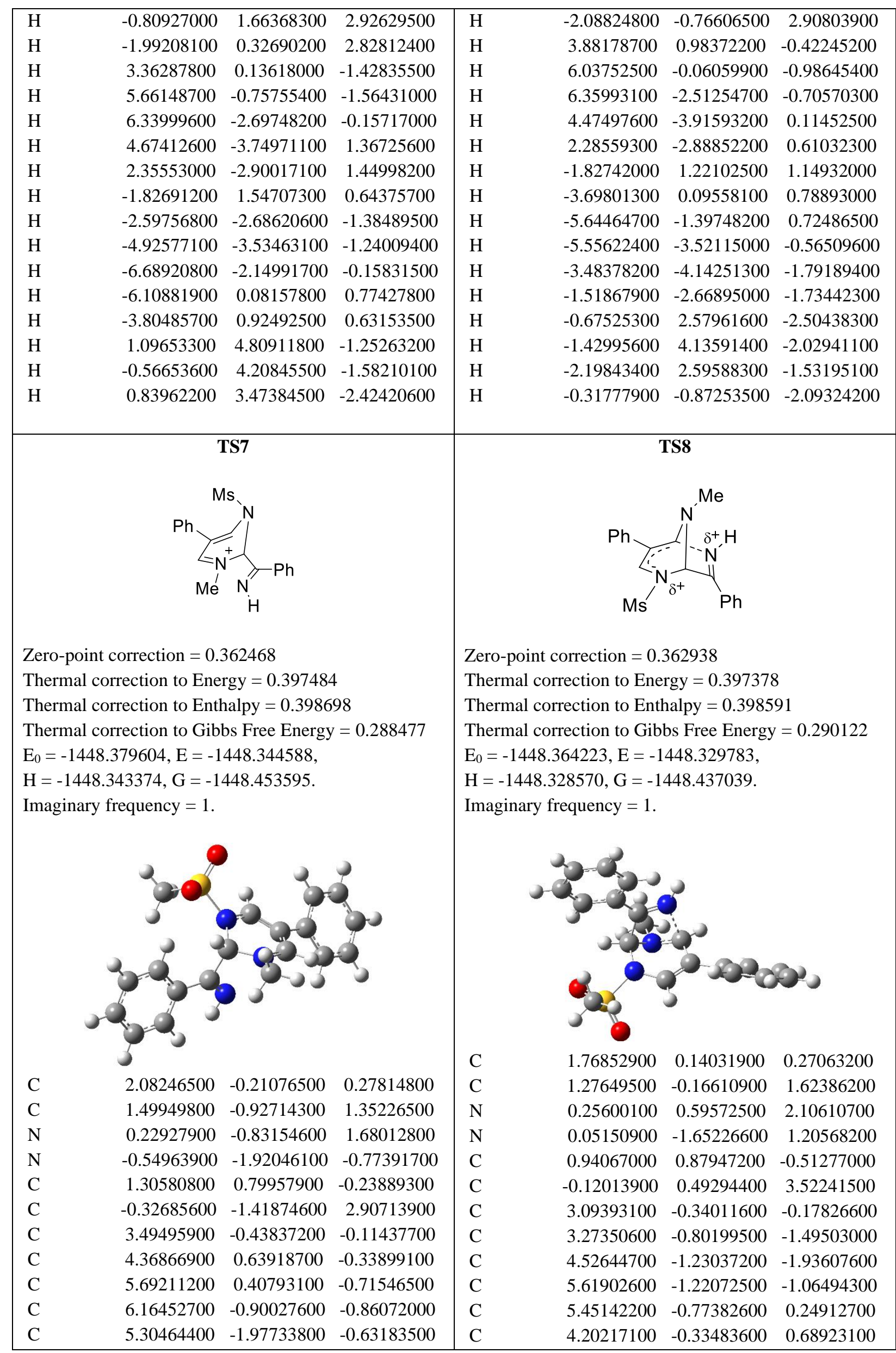




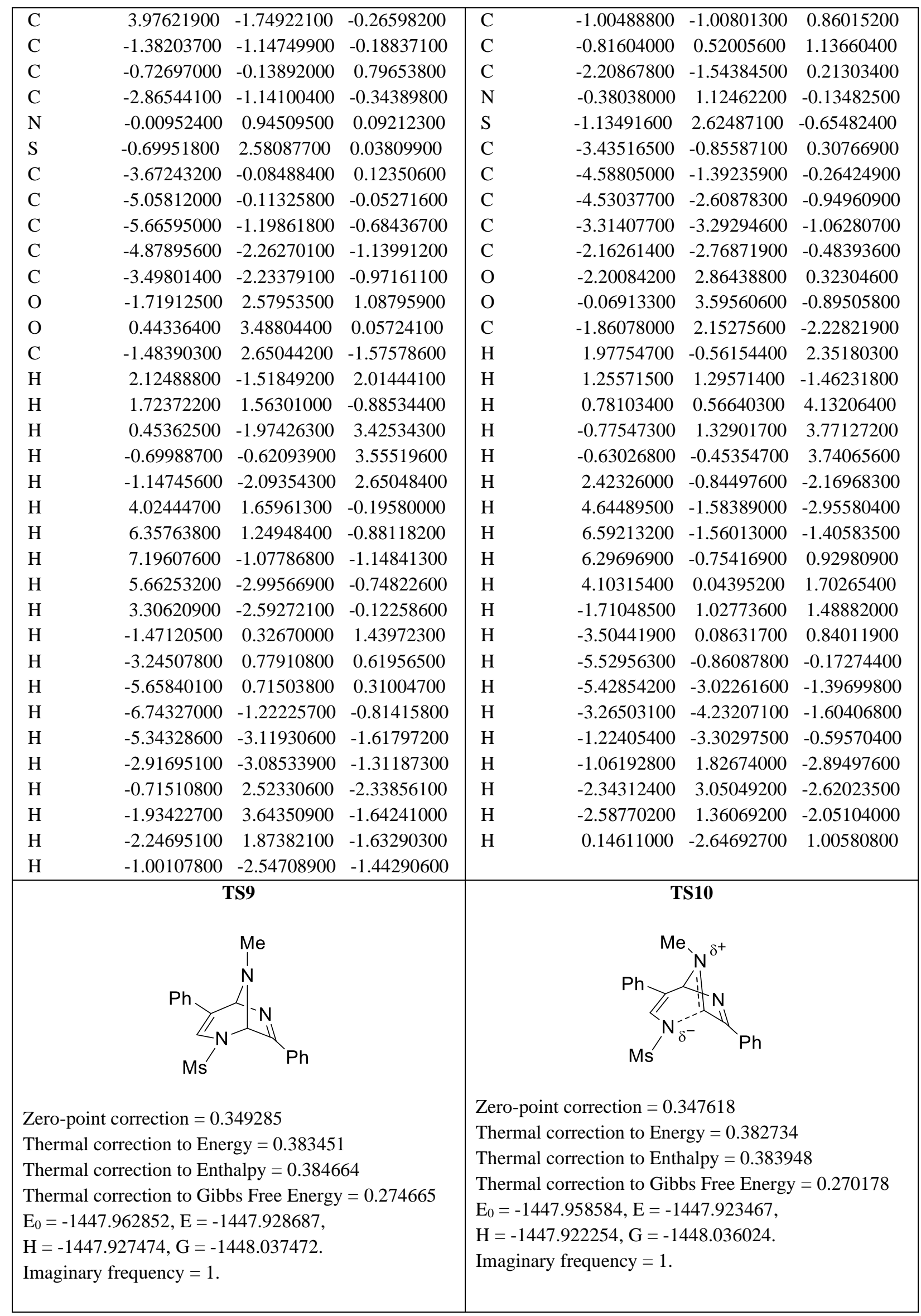




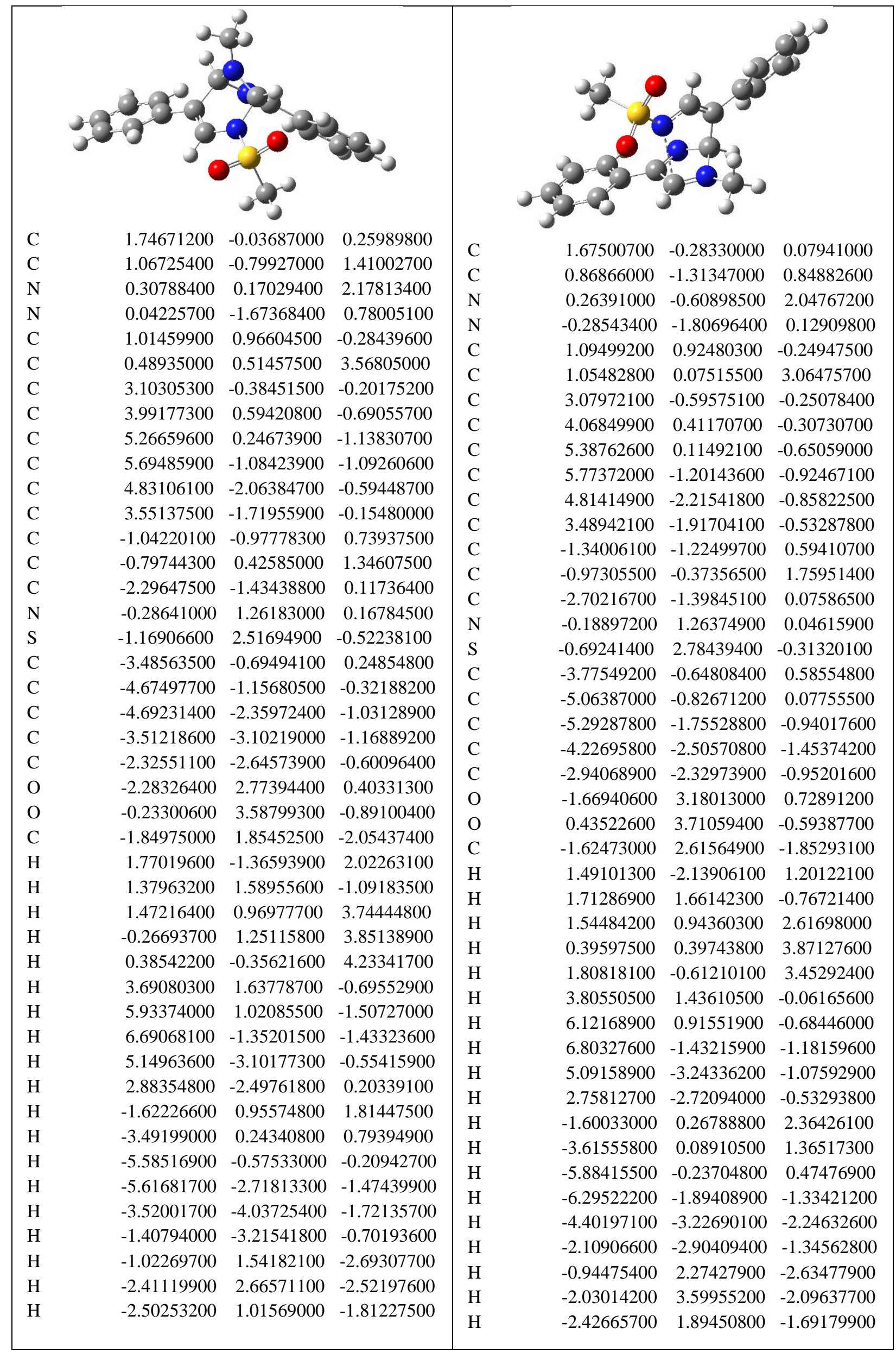




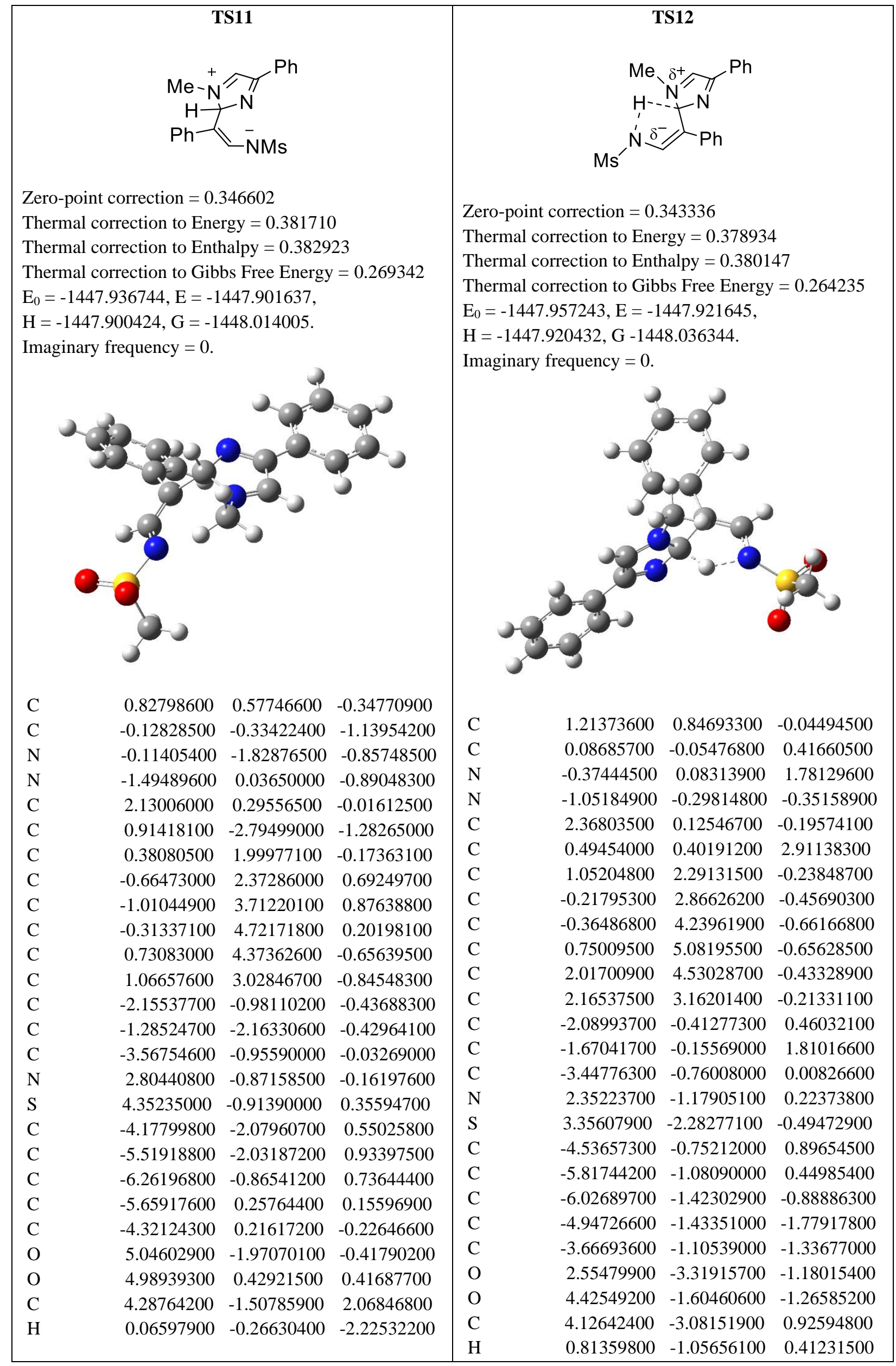




\begin{tabular}{|lrrr|lrrr|}
\hline $\mathrm{H}$ & 2.66888800 & 1.14199600 & 0.41885200 & $\mathrm{H}$ & 3.27404400 & 0.54171200 & -0.62851800 \\
$\mathrm{H}$ & 0.86234300 & -2.87338500 & -2.37261100 & $\mathrm{H}$ & -0.01166900 & 0.14201800 & 3.84103600 \\
$\mathrm{H}$ & 1.88876200 & -2.41063700 & -0.97114100 & $\mathrm{H}$ & 1.41815800 & -0.17102600 & 2.81456400 \\
$\mathrm{H}$ & 0.68716600 & -3.76103300 & -0.83083600 & $\mathrm{H}$ & 0.73135600 & 1.46853800 & 2.90000800 \\
$\mathrm{H}$ & -1.20125600 & 1.60453700 & 1.24006400 & $\mathrm{H}$ & -1.09438500 & 2.22800300 & -0.49827400 \\
$\mathrm{H}$ & -1.81605600 & 3.97033900 & 1.55877000 & $\mathrm{H}$ & -1.35607400 & 4.64957900 & -0.83561300 \\
$\mathrm{H}$ & -0.57954800 & 5.76456500 & 0.34945600 & $\mathrm{H}$ & 0.63515000 & 6.14974100 & -0.81689700 \\
$\mathrm{H}$ & 1.28386300 & 5.14462100 & -1.18595100 & $\mathrm{H}$ & 2.89410700 & 5.17147100 & -0.41492900 \\
$\mathrm{H}$ & 1.87539300 & 2.76718400 & -1.52199600 & $\mathrm{H}$ & 3.15458300 & 2.76308500 & -0.00989400 \\
$\mathrm{H}$ & -1.52936100 & -3.19154300 & -0.19062600 & $\mathrm{H}$ & -2.23150700 & -0.16439500 & 2.73479100 \\
$\mathrm{H}$ & -3.61604400 & -2.99291000 & 0.71972500 & $\mathrm{H}$ & -4.39564200 & -0.48434200 & 1.93950200 \\
$\mathrm{H}$ & -5.98072400 & -2.90362700 & 1.38695400 & $\mathrm{H}$ & -6.64962800 & -1.06927100 & 1.14727700 \\
$\mathrm{H}$ & -7.30556400 & -0.82938900 & 1.03379300 & $\mathrm{H}$ & -7.02303900 & -1.68046000 & -1.23613100 \\
$\mathrm{H}$ & -6.23441600 & 1.16538000 & 0.00256700 & $\mathrm{H}$ & -5.10294500 & -1.70067500 & -2.82008100 \\
$\mathrm{H}$ & -3.84575700 & 1.08167800 & -0.67517900 & $\mathrm{H}$ & -2.82638000 & -1.11571800 & -2.02217800 \\
$\mathrm{H}$ & 3.72774700 & -0.78733200 & 2.66733900 & $\mathrm{H}$ & 4.71499200 & -2.34096800 & 1.46825400 \\
$\mathrm{H}$ & 5.31598700 & -1.58548100 & 2.42670400 & $\mathrm{H}$ & 4.76533200 & -3.87576000 & 0.53564600 \\
$\mathrm{H}$ & 3.80242500 & -2.48472700 & 2.07950600 & $\mathrm{H}$ & 3.34036000 & -3.49802600 & 1.55682600 \\
\hline
\end{tabular}

\section{Figure S6. Energy profile for the transformation of compound 6 to bicycle} 3zb.

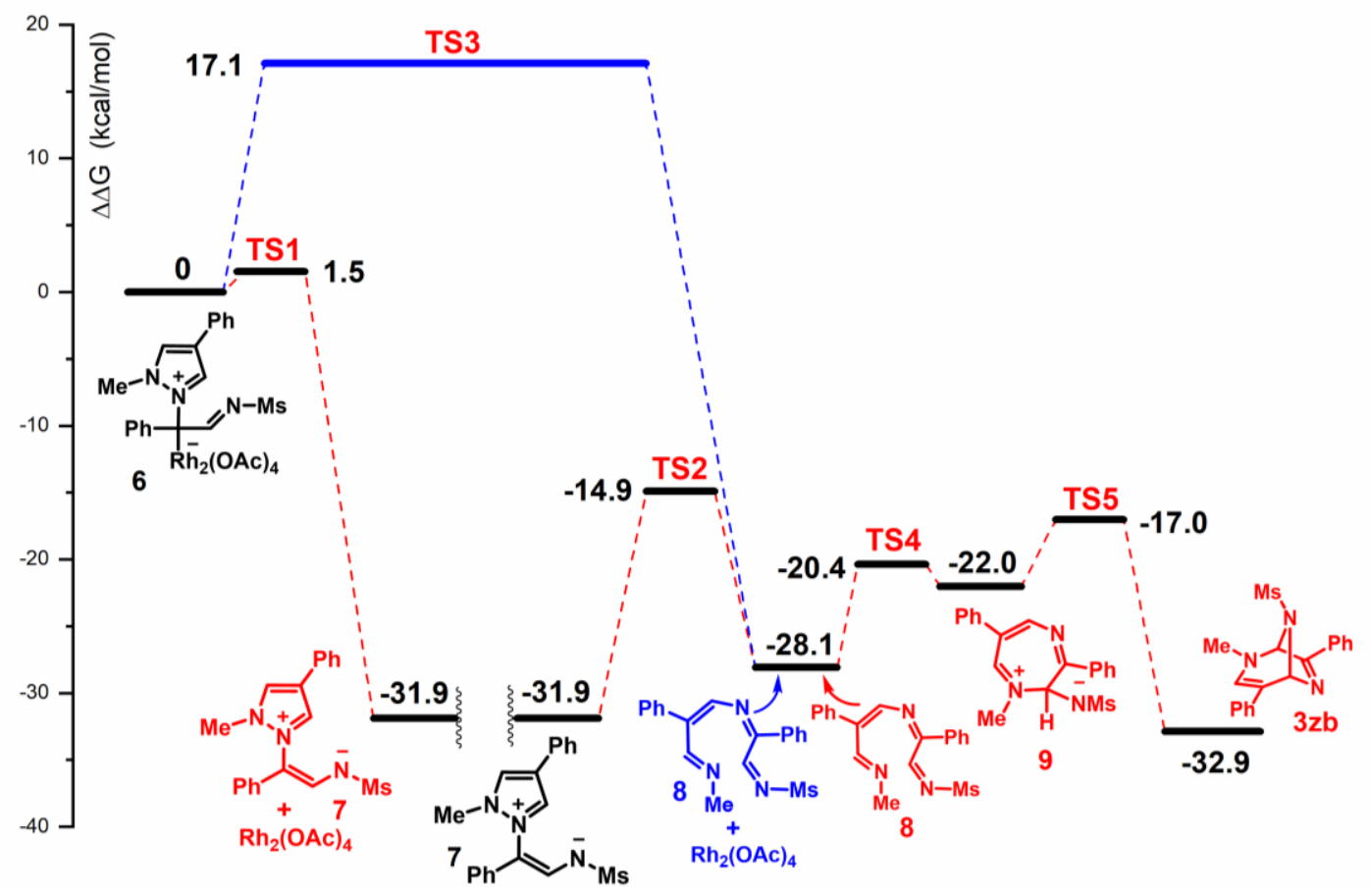


11. Figure S7. Energy profile for the transformation of compound 3zb to imidazole $4 r$.

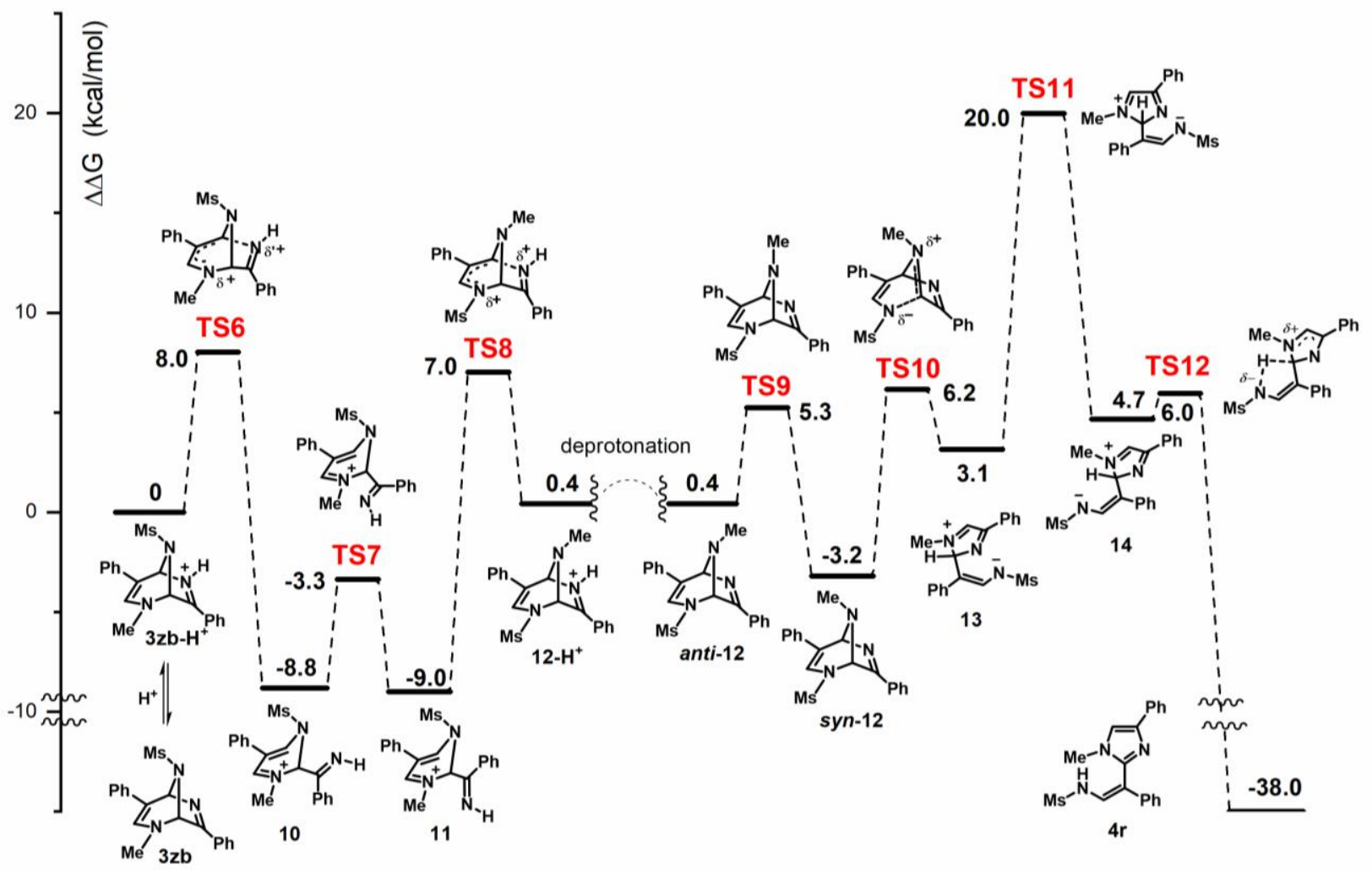




\section{References}

1. Cotton, F. A.; Felthouse, T. R. Inorg. Chem. 1980, 19, 323-328.

2. Thompson, M. E.; Tamayo, A.; Djurovich, P. PCT Int. Appl. WO2004085450; Chem. Abstr. 2004, 141, 340107.

3. Li, S.; Xu, S.; Ding, S.; Zhang, J.; Wang, S.; Li, X. Res. Chem. Intermed. 2013, 40, 1459-1468. 4. Xu, S.; Li, S.; Tang, Y.; Zhang, J.; Wang, S.; Zhou, C.; Li, X. Med. Chem. Res. 2013, 22, 56105616.

5. Koronatov, A. N.; Rostovskii, N. V.; Khlebnikov, A. F.; Novikov, M. S. J. Org. Chem. 2018, 83, 9210-9219.

6. Naas, M.; El Kazzouli, S.; Essassi, E. M.; Bousmina, M.; Guillaumet, G. J. Org. Chem. 2014, 79, 7286-7293.

7. Menozzi, G.; Mosti, L.; Schenone, P. J. Heterocyclic Chem. 1987, 24, 1669-1675.

8. Gant, T. G.; Sarshar, S. PCT Int. Appl. WO2010123999; Chem. Abstr. 2010, 153, 580300.

9. Havez, S. E. PCT Int. Appl. WO2002083622; Chem. Abstr. 2002, 137, 325234.

10. Zheng, K.; Iqbal, S.; Hernandez, P.; Park, H. J.; LoGrasso, P. V.; Feng, Y. J. Med. Chem. 2014, 57, 10013-10030.

11. Burling, S.; Field, L. D.; Messerle, B. A.; Vuong, K. Q.; Turner, P. J. Chem. Soc., Dalton Trans. 2003, 4181-4191.

12. Hernandez-Fernandez, E.; Sanchez-Lara, P. P.; Ordonez, M.; Ramirez-Merroquin, O. A.; Avalos-Alanis, F. G.; Lopez-Cortina, S.; Jimenez-Perez, V. M.; Ibarra-Rivera, T. R. Tetrahedron: Asymmetry 2015, 26, 73-78.

13. Brahim, M.; Ammar, H. B.; Soule, J.-F.; Doucet, H. Tetrahedron 2016, 72, 4312-4320.

14. Berthel, S. J.; Haynes, N.-E.; Keyster, R. F.; Mcdermott, L. A.; Qian, Y.; Sarabu, R.; Scott, N. R.; Tilley, J. W. PCT Int. Appl. WO2009127544; Chem. Abstr. 2009, 151, 470220.

15. Shi, Y.; Yu, X.; Li, C. Y. Eur. J. Org. Chem. 2015, 6429.

16. Gagnon, P. E.; Boivin, J. L.; Tremblay, M. Can. J. Chem. 1953, 31, 673-684.

17. Dolomanov, O. V.; Bourhis, L. J.; Gildea, R. J.; Howard, J.A.K.; Puschmann, H. J. Appl.

Cryst. 2009, 42, 339-341.

18. Sheldrick, G. M. Acta Cryst. A 2015, 71, 3-8.

19. Sheldrick, G. M. Acta Cryst. C 2015, 71, 3-8.

20. Gaussian 09, Revision D.01, Frisch, M. J.; Trucks, G.W.; Schlegel, H. B.; Scuseria, G. E.;

Robb, M. A.; Cheeseman, J. R.; Scalmani, G.; Barone, V.; Mennucci, B.; Petersson, G. A.; Nakatsuji, H.; Caricato, M.; Li, X.; Hratchian, H. P.; Izmaylov, A. F.; Bloino, J.; Zheng, G.; Sonnenberg, J. L.; Hada, M.; Ehara, M.; Toyota, K.; Fukuda, R.; Hasegawa, J.; Ishida, M.; Nakajima, T.; Honda, Y.; Kitao, O.; Nakai, H.; Vreven, T.; Montgomery, J. A.; Peralta, J. E.; Ogliaro, F.; Bearpark, M.; Heyd, J. J.; Brothers, E.; Kudin, K. N.; Staroverov, V. N.; Kobayashi, R.; Normand, J.; Raghavachari, K.; Rendell, A.; Burant, J. C.; Iyengar, S. S.; Tomasi, J.; Cossi, M.; Rega, N.; Millam, N. J.; Klene, M.; Knox, J. E.; Cross, J. B.; Bakken, V.; Adamo, C.; Jaramillo, J.; Gomperts, R.; Stratmann, R. E.; Yazyev, O.; Austin, A. J.; Cammi, R.; Pomelli, C.; Ochterski, J. W.; Martin, R. L.; Morokuma, K.; Zakrzewski, V. G.; Voth, G. A.; Salvador, P.; Dannenberg, J. J.; Dapprich, S.; Daniels, A. D.; Farkas, Ö.; Foresman, J. B.; Ortiz, J. V.; Cioslowski, J.; Fox, D. J. Gaussian 09, Revision C.01; Gaussian: Wallingford CT, 2013. 


\section{3. ${ }^{1} \mathrm{H}$ and ${ }^{13} \mathrm{C}$ NMR spectra of new compounds}

${ }^{1} \mathrm{H}$ NMR spectrum of compound $\mathbf{1 g}\left(400 \mathrm{MHz}, \mathrm{DMSO}-d_{6}\right)$

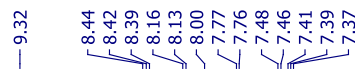

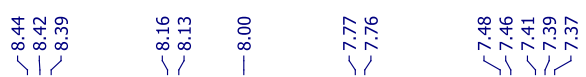
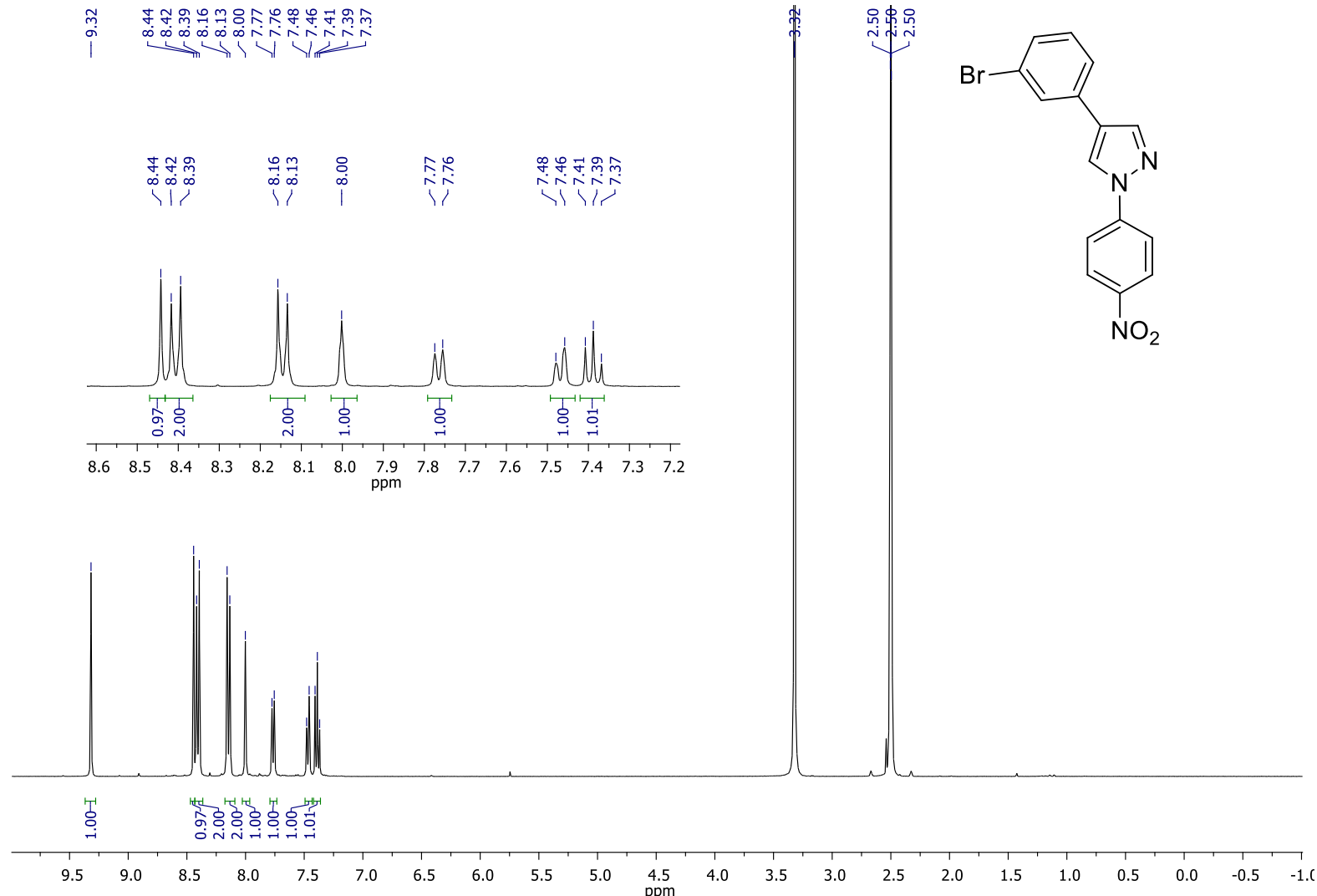

${ }^{13} \mathrm{C}\left\{{ }^{1} \mathrm{H}\right\}$ NMR spectrum of compound $1 \mathrm{~g}\left(100 \mathrm{MHz}\right.$, DMSO- $\left.d_{6}\right)$

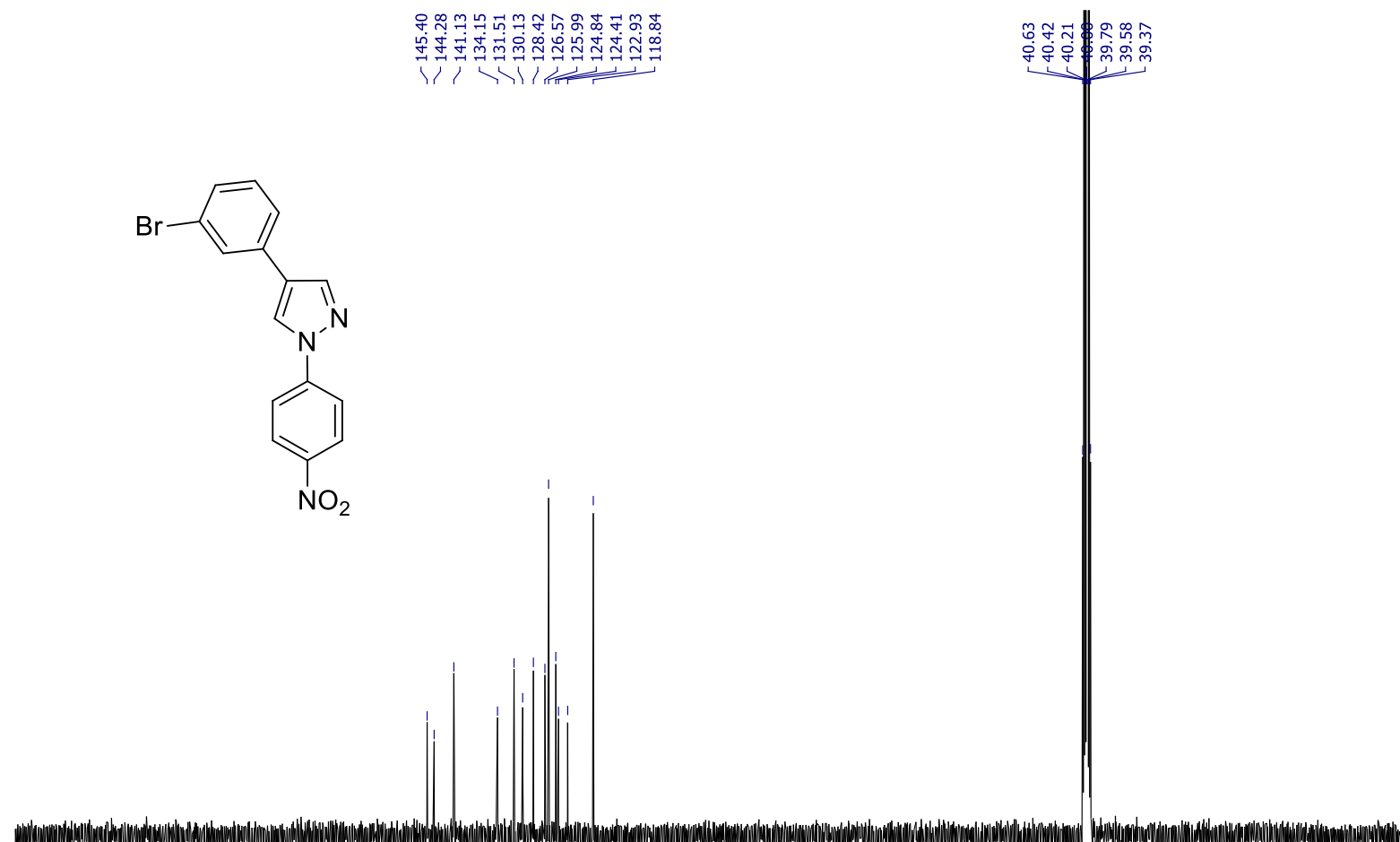

$\begin{array}{llllllllllllllllllllllllll}210 & 200 & 190 & 180 & 170 & 160 & 150 & 140 & 130 & 120 & 110 & 100 & 90 & 80 & 70 & 60 & 50 & 40 & 30 & 20 & 10 & 0 & -10\end{array}$ 
${ }^{1} \mathrm{H}$ NMR spectrum of compound $1 \mathbf{i}\left(400 \mathrm{MHz}, \mathrm{DMSO}-d_{6}\right)$

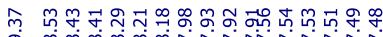

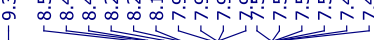

lll
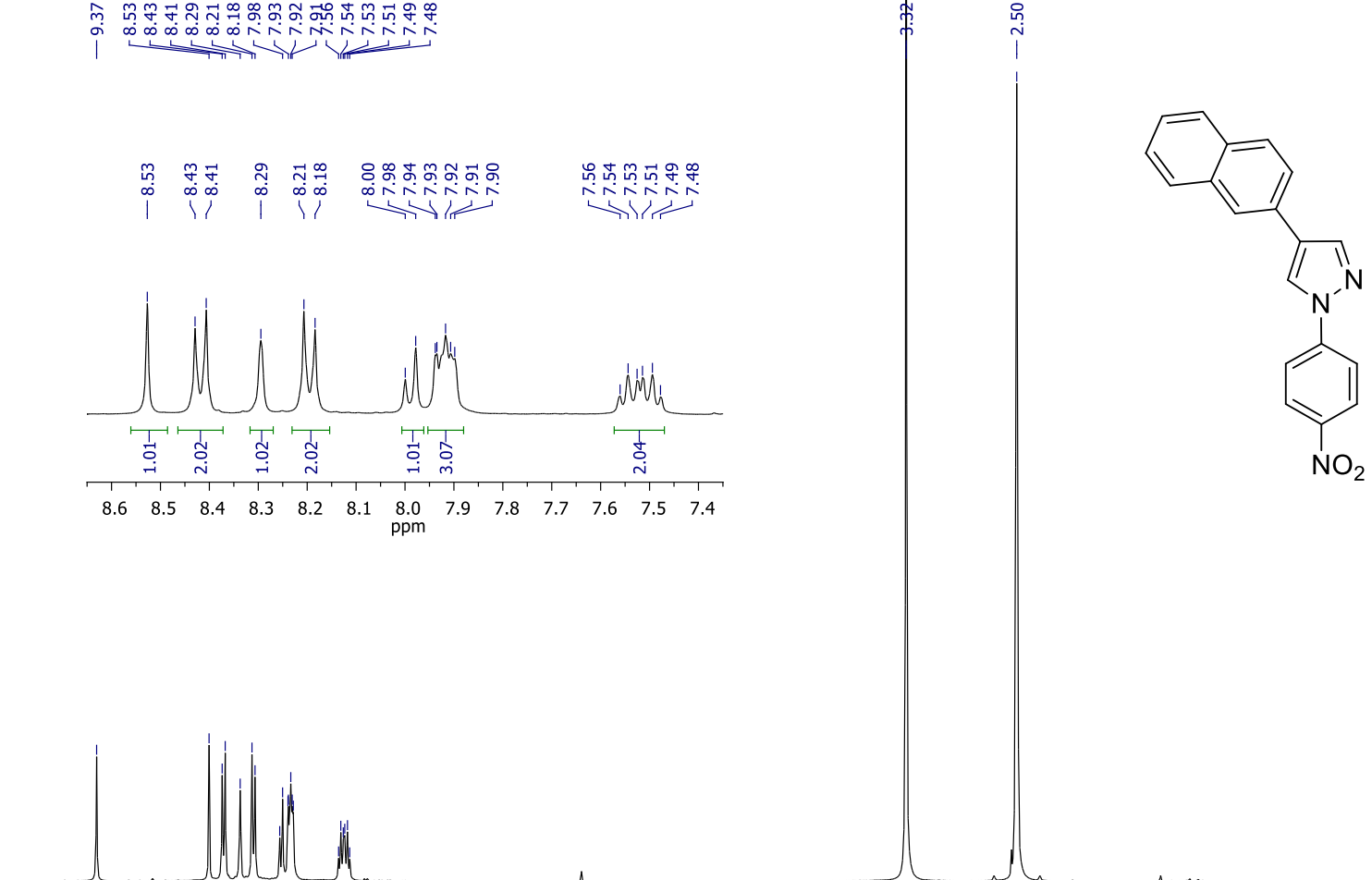

龺

$\begin{array}{lllllllllllllllllllll}9.5 & 9.0 & 8.5 & 8.0 & 7.5 & 7.0 & 6.5 & 6.0 & 5.5 & 5.0 & 4.5 & 4.0 & 3.5 & 3.0 & 2.5 & 2.0 & 1.5 & 1.0 & 0.5 & 0.0 & -0.5\end{array}$

${ }^{13} \mathrm{C}\left\{{ }^{1} \mathrm{H}\right\}$ NMR spectrum of compound $1 \mathbf{i}\left(100 \mathrm{MHz}, \mathrm{DMSO}-d_{6}\right)$

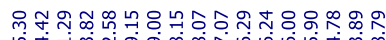

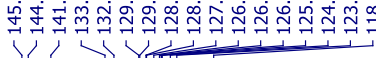

1<smiles>c1cn[nH]c1</smiles><smiles>O=[N+]([O-])c1ccc(I)cc1</smiles>

$\begin{array}{llllllllllll}210 & 200 & 190 & 180 & 170 & 160 & 150 & 140 & 130 & 120 & 110 & 100\end{array}$ 
${ }^{1} \mathrm{H}$ NMR spectrum of compound $\mathbf{1 j}$ (400 MHz, DMSO-d6)

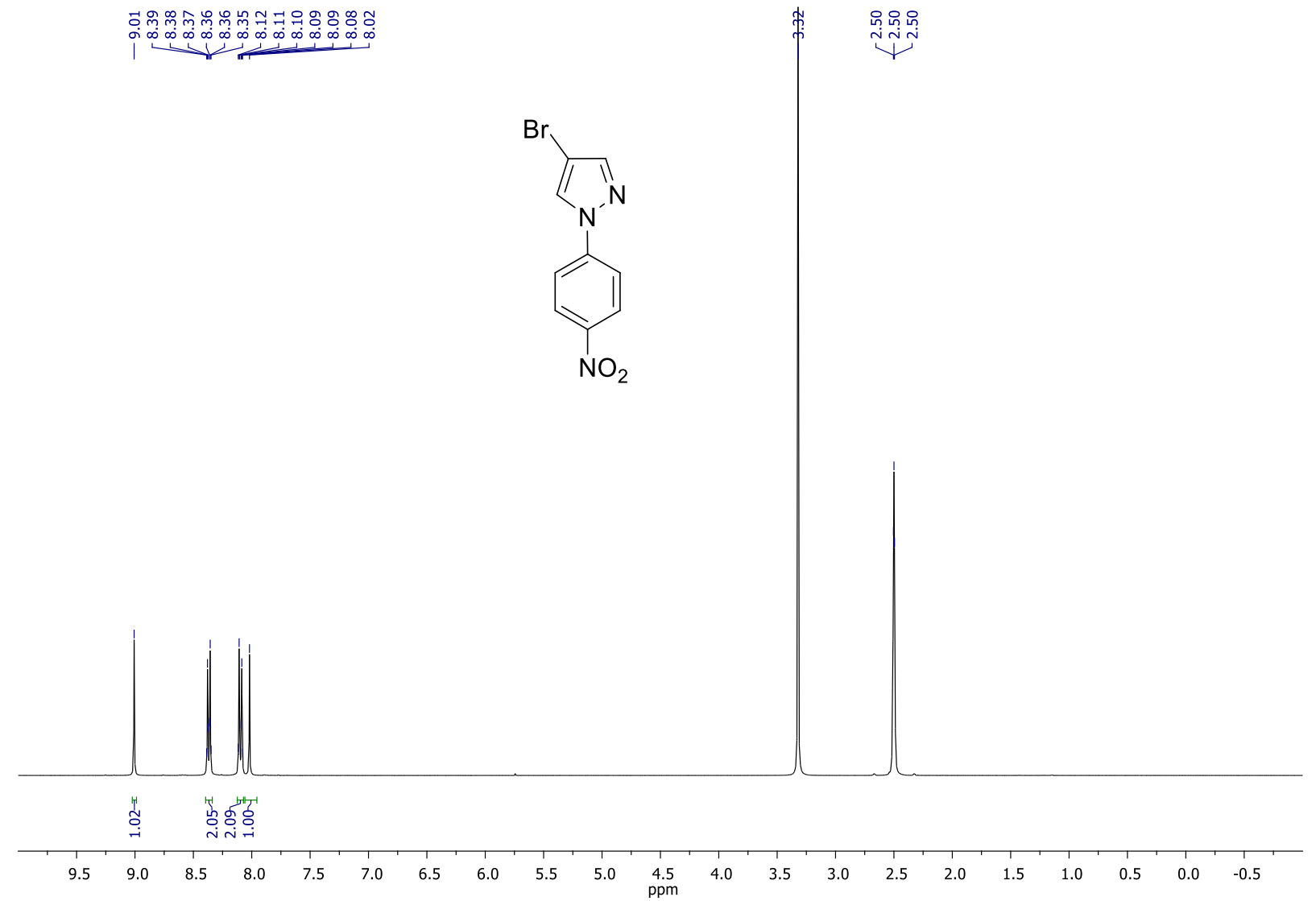

${ }^{13} \mathrm{C}\left\{{ }^{1} \mathrm{H}\right\}$ NMR spectrum of compound $\mathbf{1 j}\left(100 \mathrm{MHz}, \mathrm{DMSO}-d_{6}\right)$

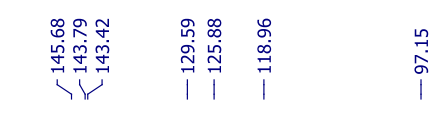<smiles>O=[N+]([O-])c1ccc(-n2cc(Br)cn2)cc1</smiles>

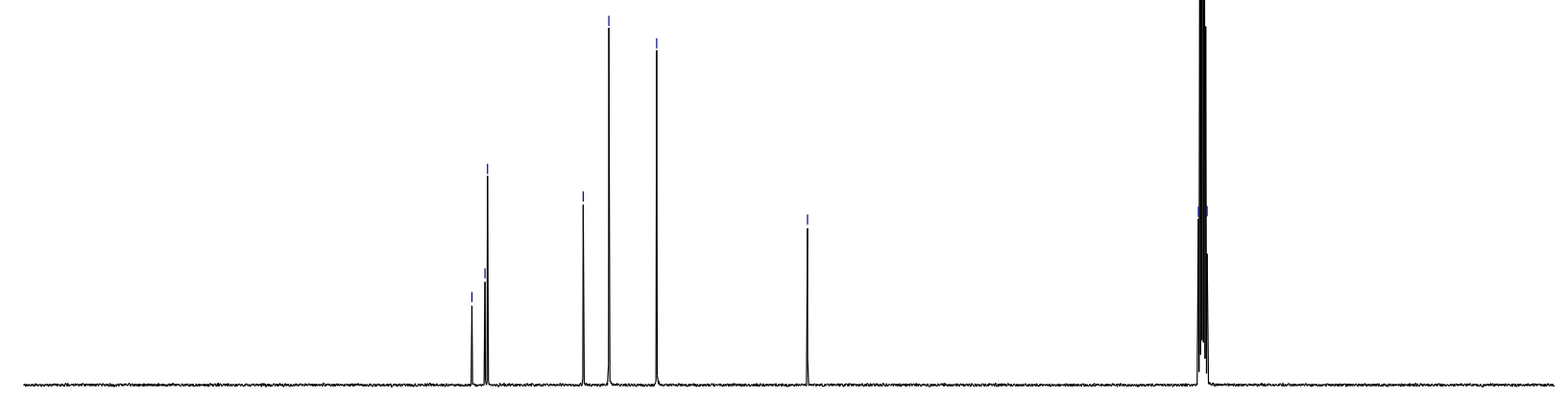

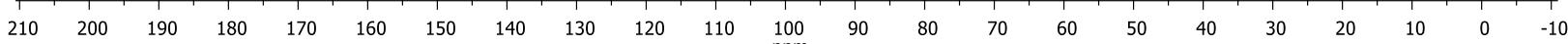


${ }^{1} \mathrm{H}$ NMR spectrum of compound $\mathbf{1 k}\left(400 \mathrm{MHz}, \mathrm{CDCl}_{3}\right)$

후웅

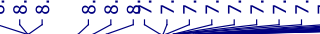

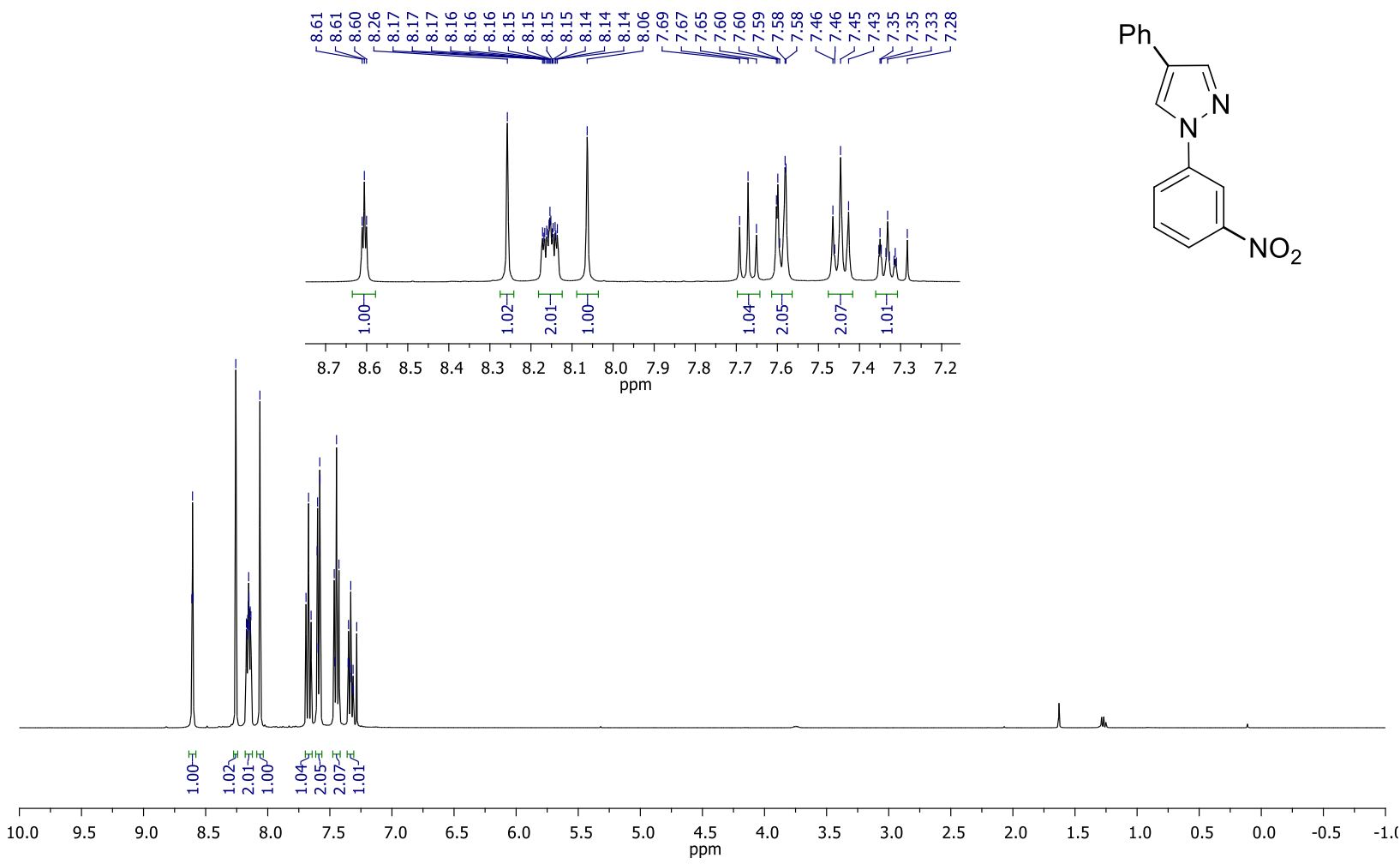

${ }^{13} \mathrm{C}\left\{{ }^{1} \mathrm{H}\right\}$ NMR spectrum of compound $1 \mathbf{k}\left(100 \mathrm{MHz}, \mathrm{CDCl}_{3}\right)$
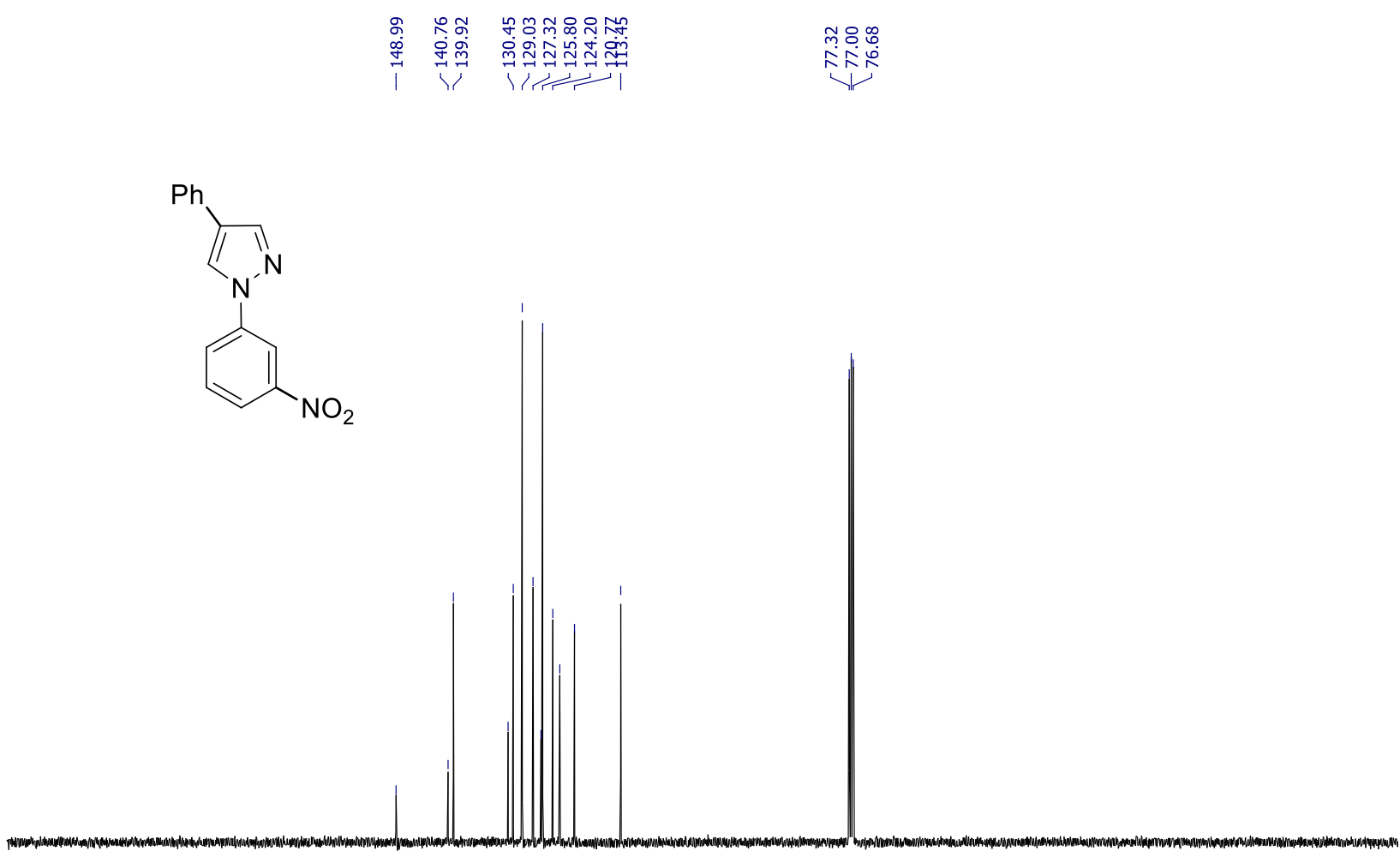

$\begin{array}{llllllllllll}10 & 200 & 190 & 180 & 170 & 160 & 150 & 140 & 130 & 120 & 110 & \begin{array}{r}100 \\ \text { ppm }\end{array}\end{array}$ 
${ }^{1} \mathrm{H}$ NMR spectrum of compound 11 (400 MHz, $\left.\mathrm{CDCl}_{3}\right)$

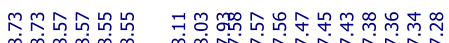

皮我

$\stackrel{\leftrightarrow}{\rightarrow}$

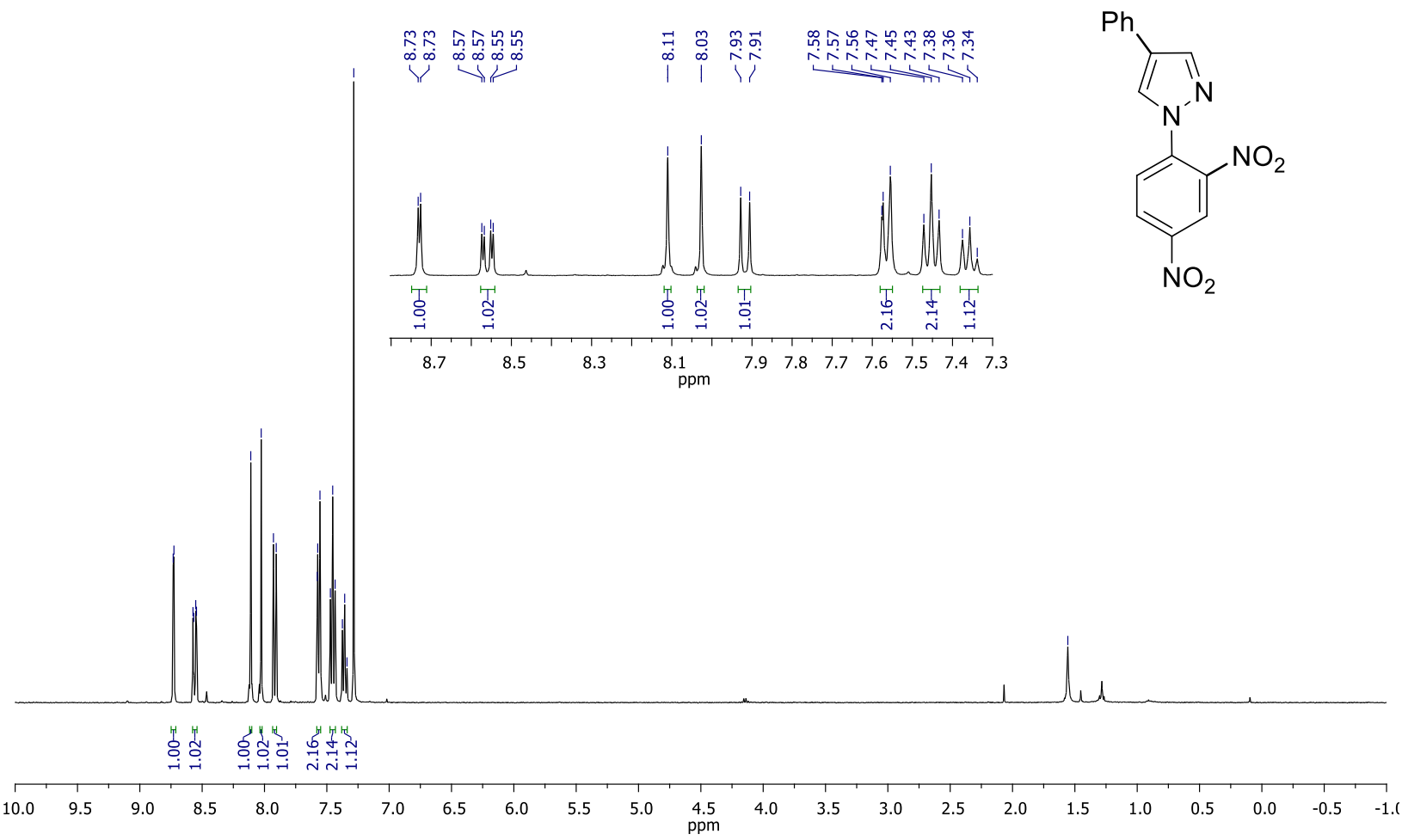

${ }^{13} \mathrm{C}\left\{{ }^{1} \mathrm{H}\right\}$ NMR spectrum of compound $11\left(100 \mathrm{MHz}, \mathrm{CDCl}_{3}\right)$

$\mathrm{Ph}$

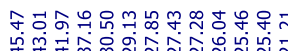

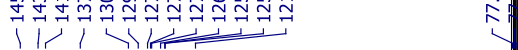<smiles>O=[N+]([O-])c1ccc(-n2cc(I)cn2)c([N+](=O)[O-])c1</smiles>

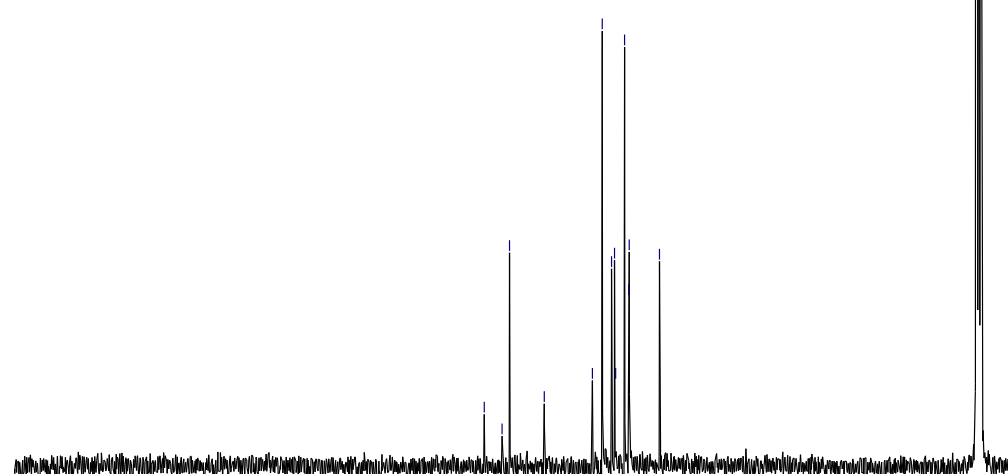

$\begin{array}{llllllllllllllllllllllllll}210 & 200 & 190 & 180 & 170 & 160 & 150 & 140 & 130 & 120 & 110 & 100 & 90 & 80 & 70 & 60 & 50 & 40 & 30 & 20 & 10 & 0 & -10\end{array}$ 
${ }^{1} \mathrm{H}$ NMR spectrum of compound $\mathbf{1 m}\left(400 \mathrm{MHz}, \mathrm{DMSO}-d_{6}\right)$

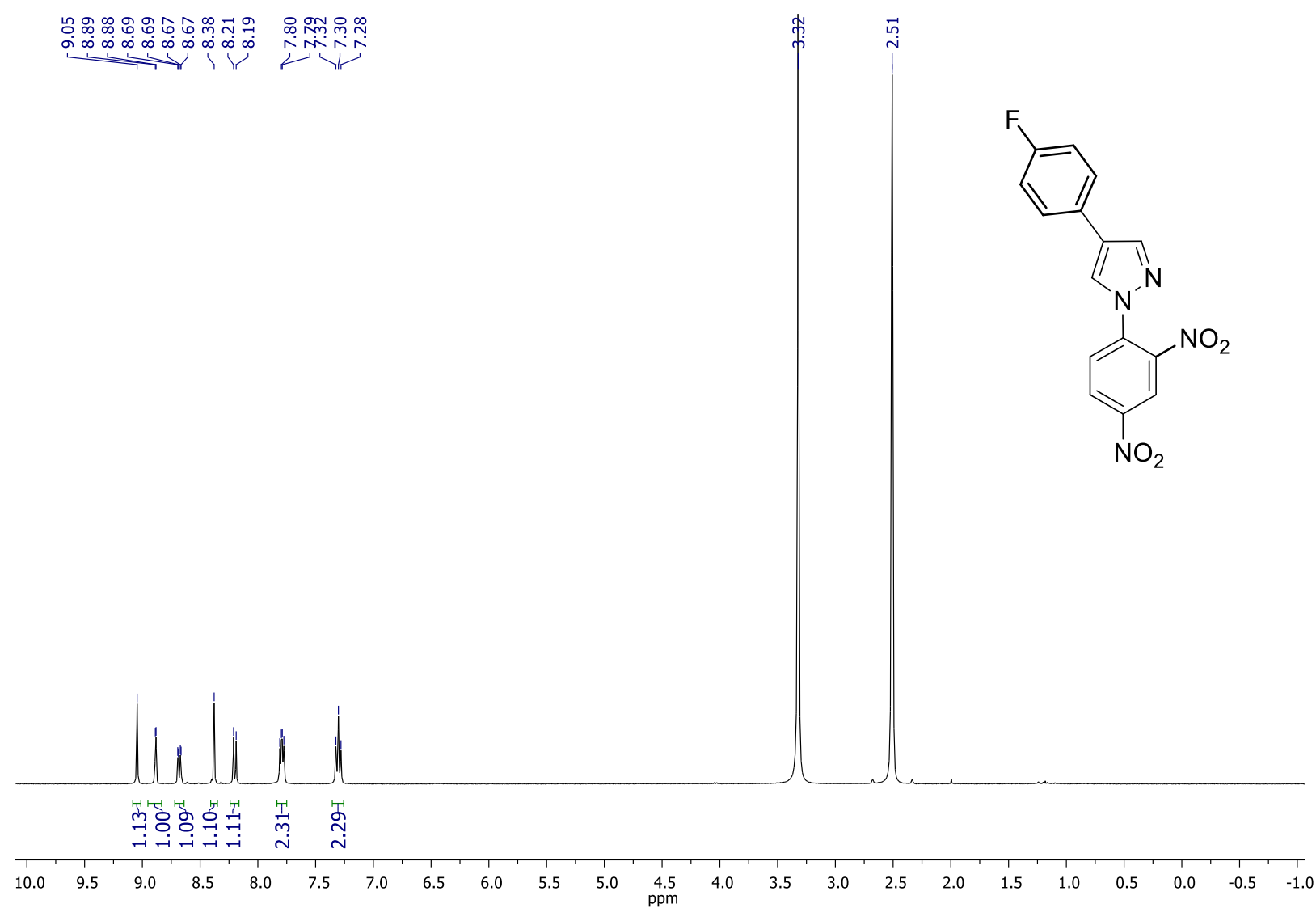

${ }^{13} \mathrm{C}\left\{{ }^{1} \mathrm{H}\right\}$ NMR spectrum of compound $1 \mathbf{m}\left(100 \mathrm{MHz}, \mathrm{DMSO}-d_{6}\right)$

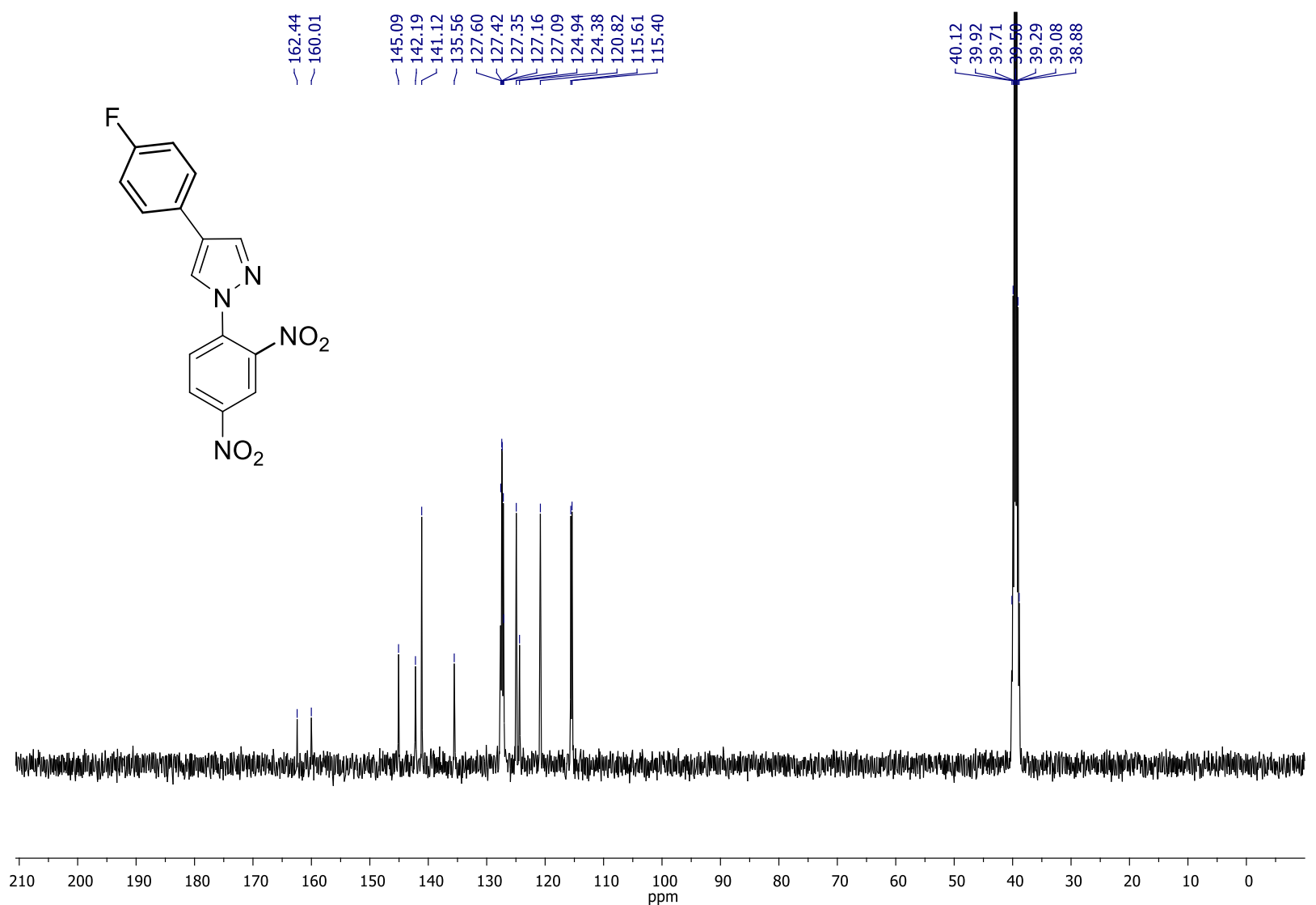


${ }^{1} \mathrm{H}$ NMR spectrum of compound $\mathbf{1 n}\left(400 \mathrm{MHz}, \mathrm{CDCl}_{3}\right)$

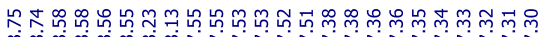

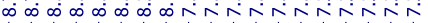

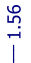

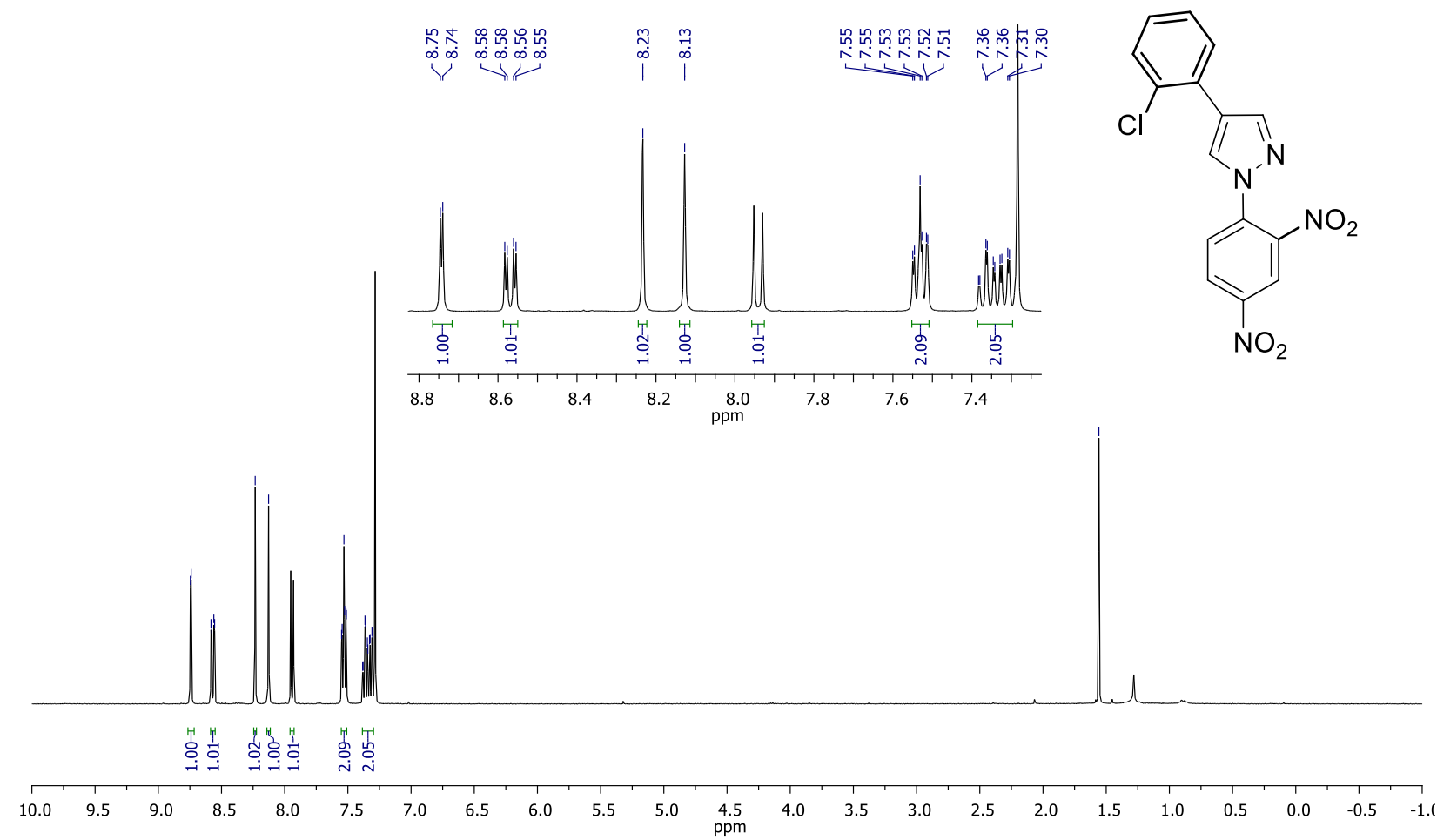

${ }^{13} \mathrm{C}\left\{{ }^{1} \mathrm{H}\right\}$ NMR spectrum of compound 1 n $\left(100 \mathrm{MHz}, \mathrm{CDCl}_{3}\right)$
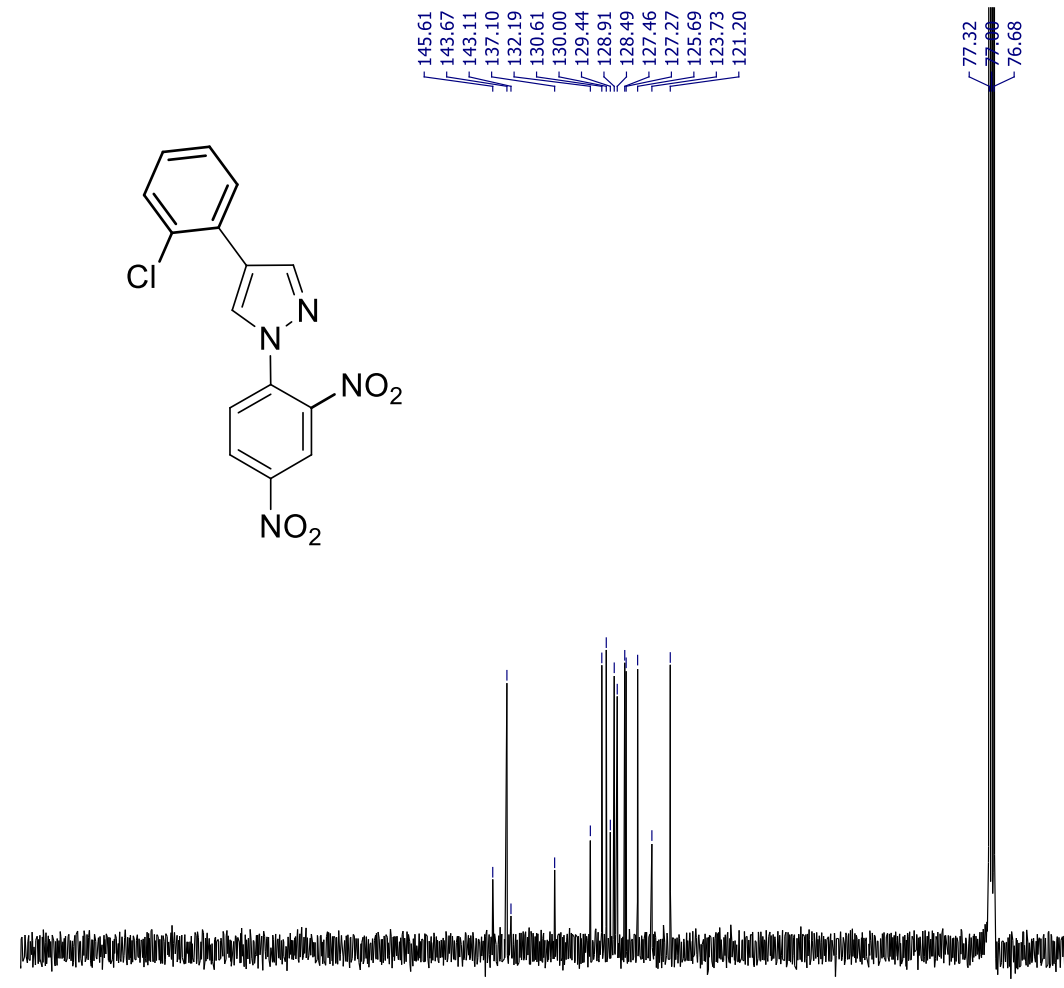

$\begin{array}{llllllllllllllllllllllll}210 & 200 & 190 & 180 & 170 & 160 & 150 & 140 & 130 & 120 & 110 & 100 & 90 & 80 & 70 & 60 & 50 & 40 & 30 & 20 & 10 & 0\end{array}$ 
${ }^{1} \mathrm{H}$ NMR spectrum of compound 10 (400 MHz, DMSO- $d_{6}$ )

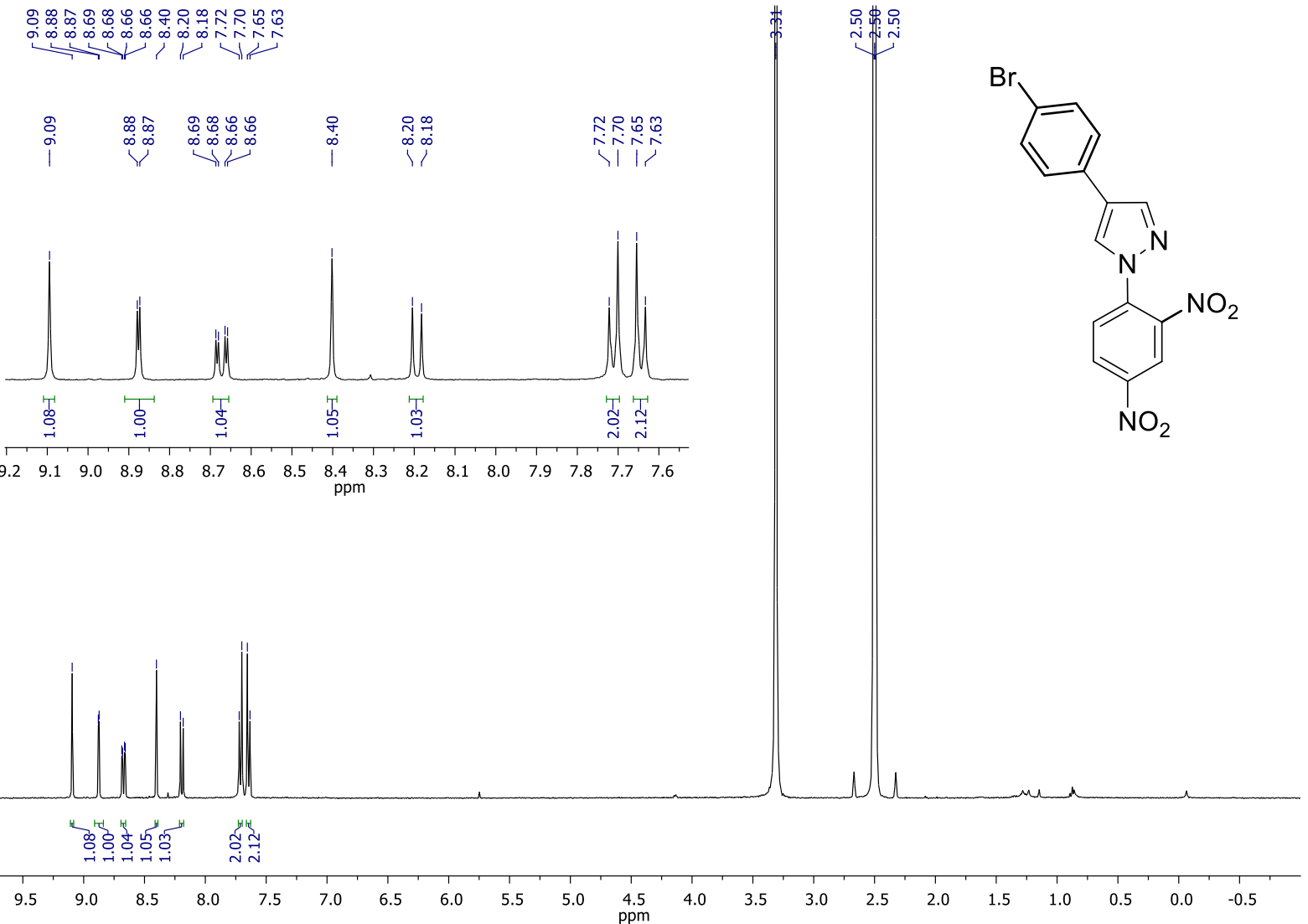

${ }^{13} \mathrm{C}\left\{{ }^{1} \mathrm{H}\right\}$ NMR spectrum of compound $10\left(100 \mathrm{MHz}\right.$, DMSO- $\left.d_{6}\right)$

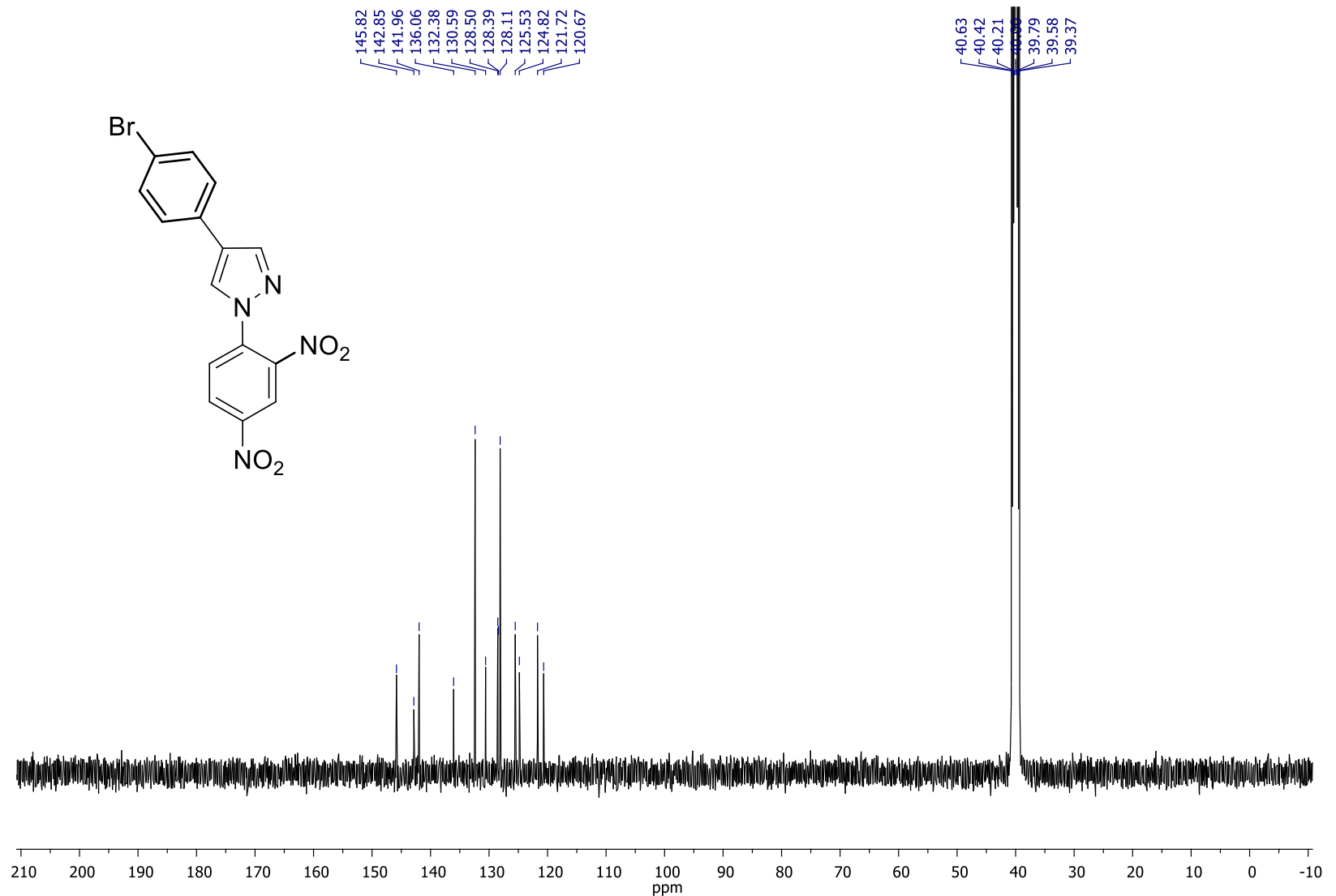


${ }^{1} \mathrm{H}$ NMR spectrum of compound $\mathbf{1 q}\left(400 \mathrm{MHz}, \mathrm{CDCl}_{3}\right)$

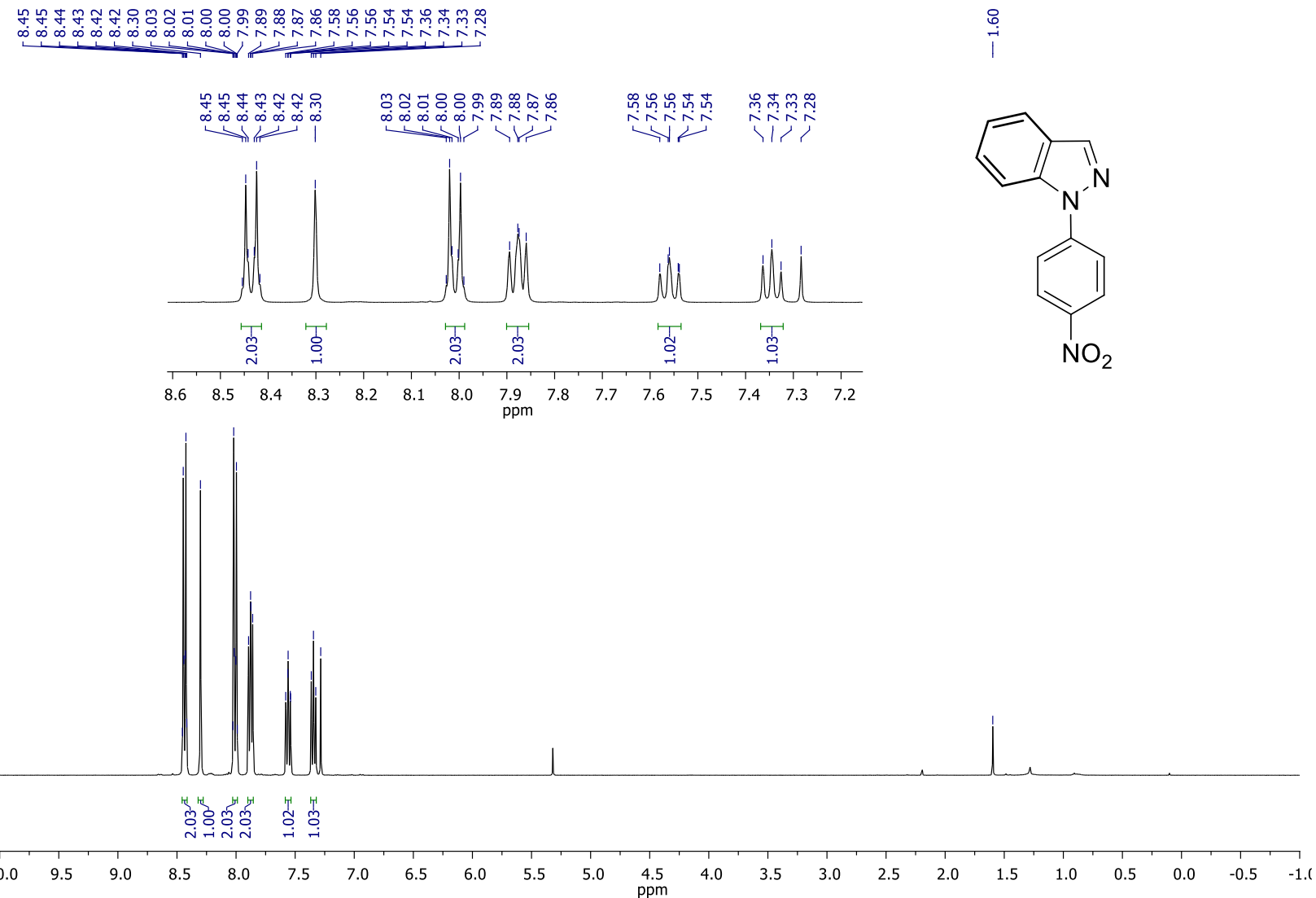

${ }^{13} \mathrm{C}\left\{{ }^{1} \mathrm{H}\right\}$ NMR spectrum of compound $\mathbf{1 q}\left(100 \mathrm{MHz}, \mathrm{CDCl}_{3}\right)$

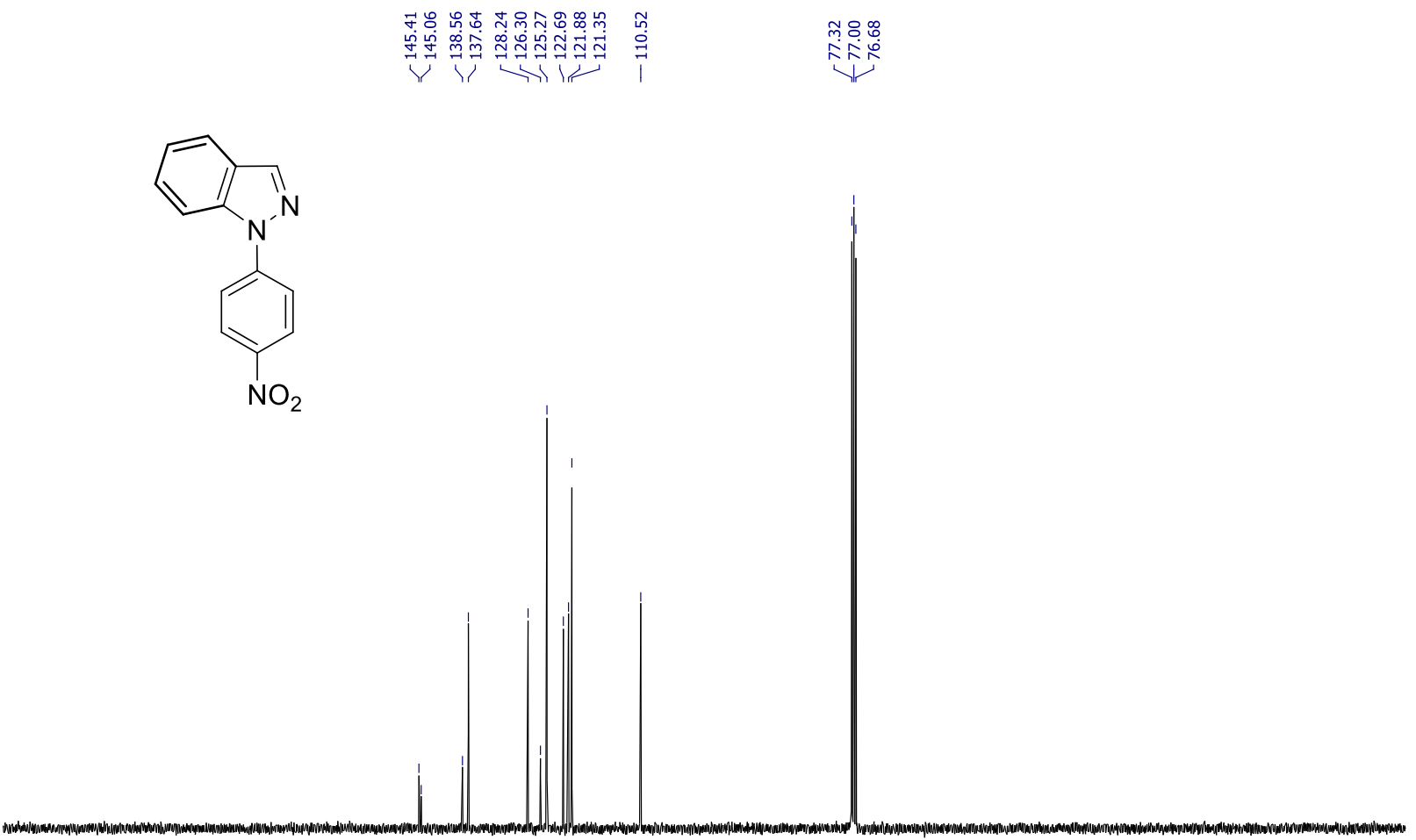

\begin{tabular}{lllllllllllllllllllllllllll}
\hline 210 & 200 & 190 & 180 & 170 & 160 & 150 & 140 & 130 & 120 & 110 & 100 & 90 & 80 & 70 & 60 & 50 & 40 & 30 & 20 & 10 & 0 & 0
\end{tabular} 
${ }^{1} \mathrm{H}$ NMR spectrum of compound 1zi (400 MHz, $\mathrm{CDCl}_{3}$ )

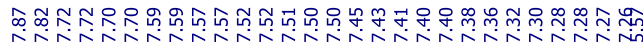

(1)

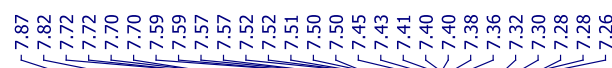

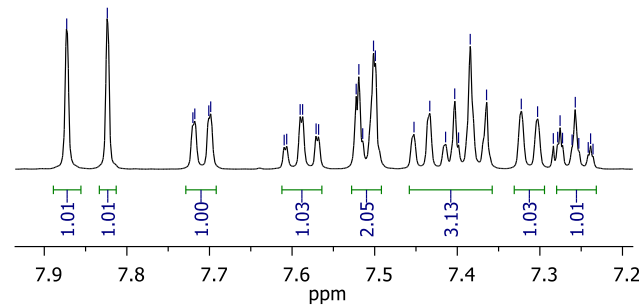

$\mathrm{Ph}$
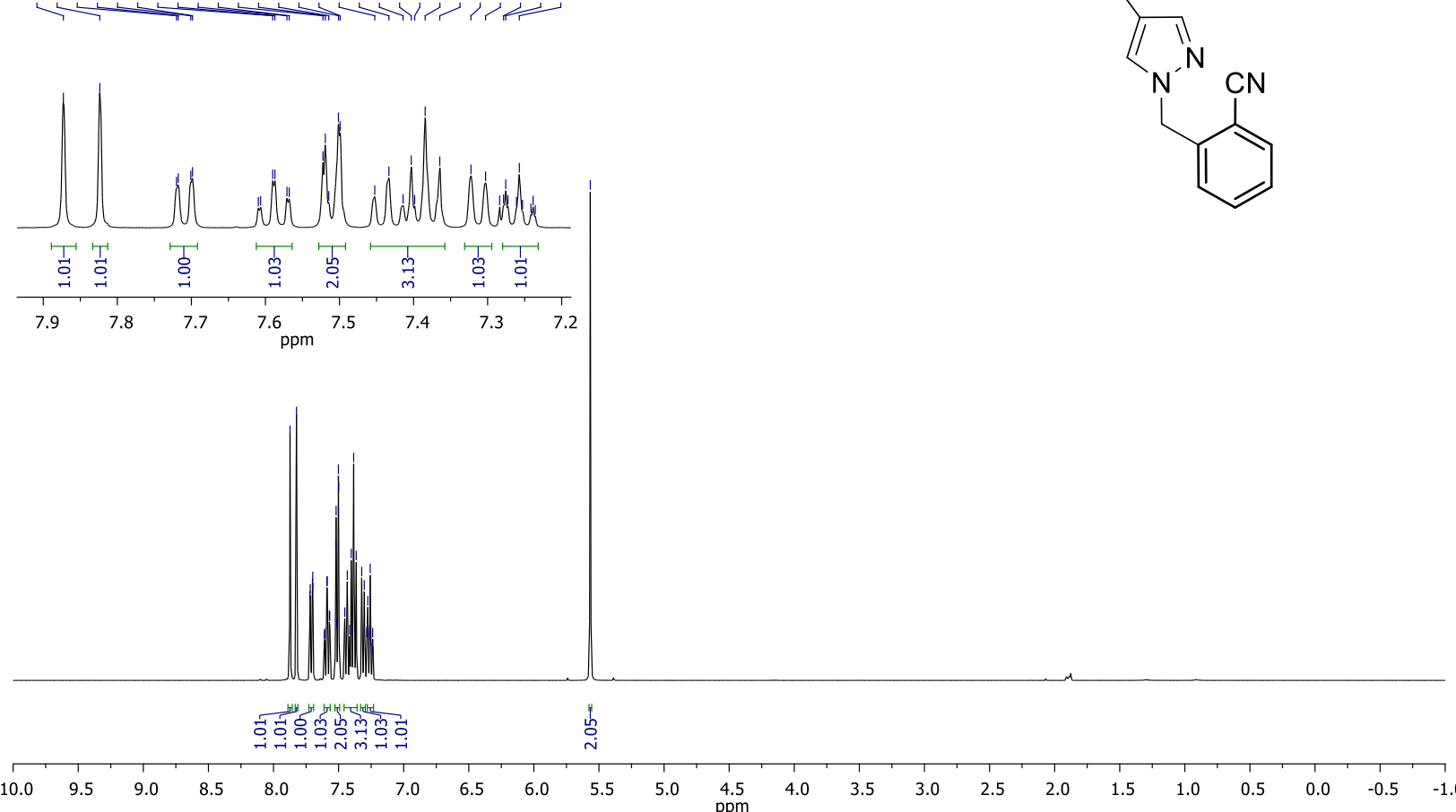

${ }^{13} \mathrm{C}\left\{{ }^{1} \mathrm{H}\right\}$ NMR spectrum of compound 1 zi $\left(100 \mathrm{MHz}, \mathrm{CDCl}_{3}\right)$

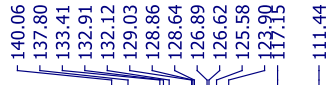

诲
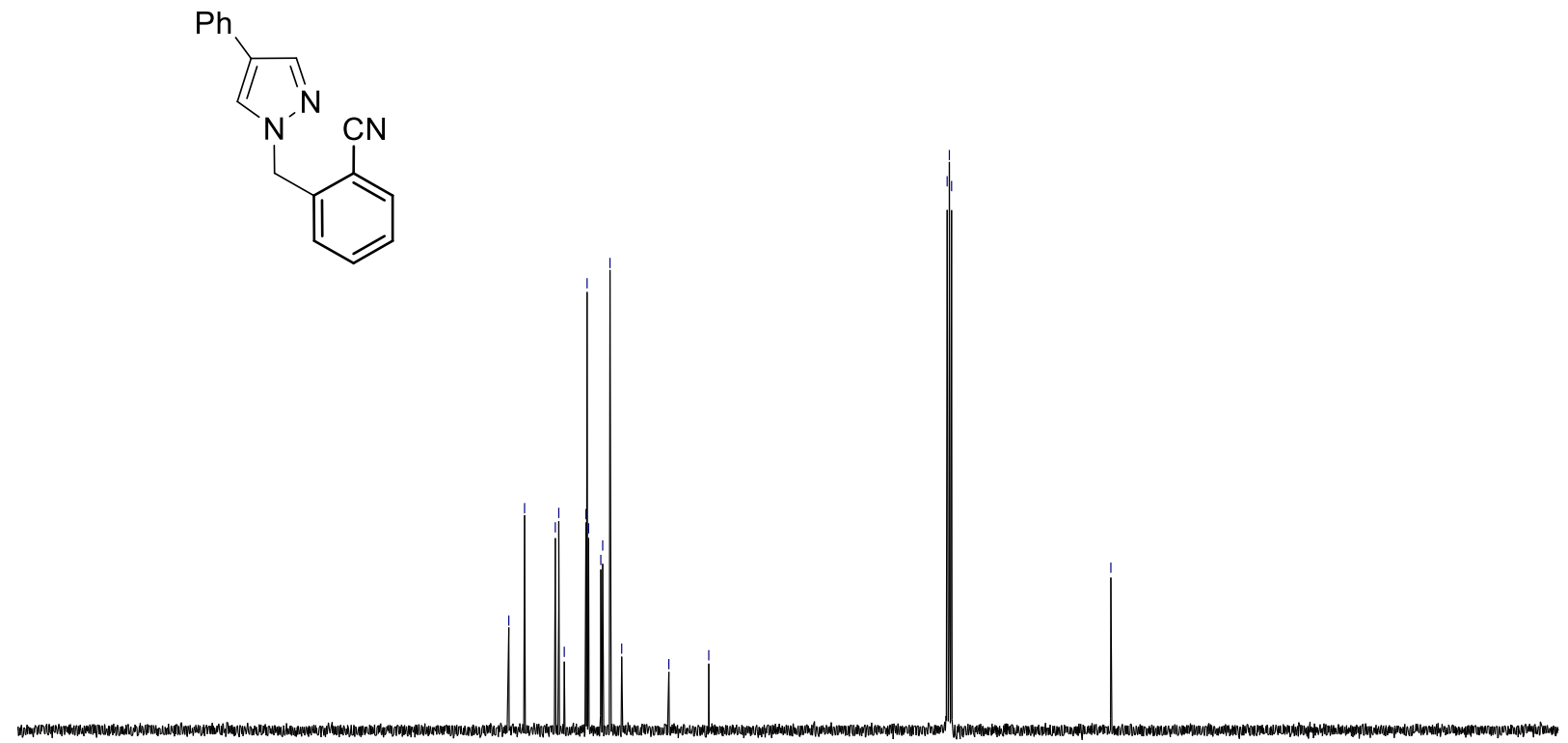

$\begin{array}{lllllllllllllllllllllllll}210 & 200 & 190 & 180 & 170 & 160 & 150 & 140 & 130 & 120 & 110 & 100 & 90 & 80 & 70 & 60 & 50 & 40 & 30 & 20 & 10 & 0 & -10\end{array}$ 
${ }^{1} \mathrm{H}$ NMR spectrum of compound $\mathbf{1 z l}\left(400 \mathrm{MHz}, \mathrm{CDCl}_{3}\right)$

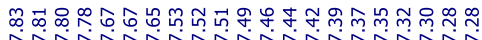
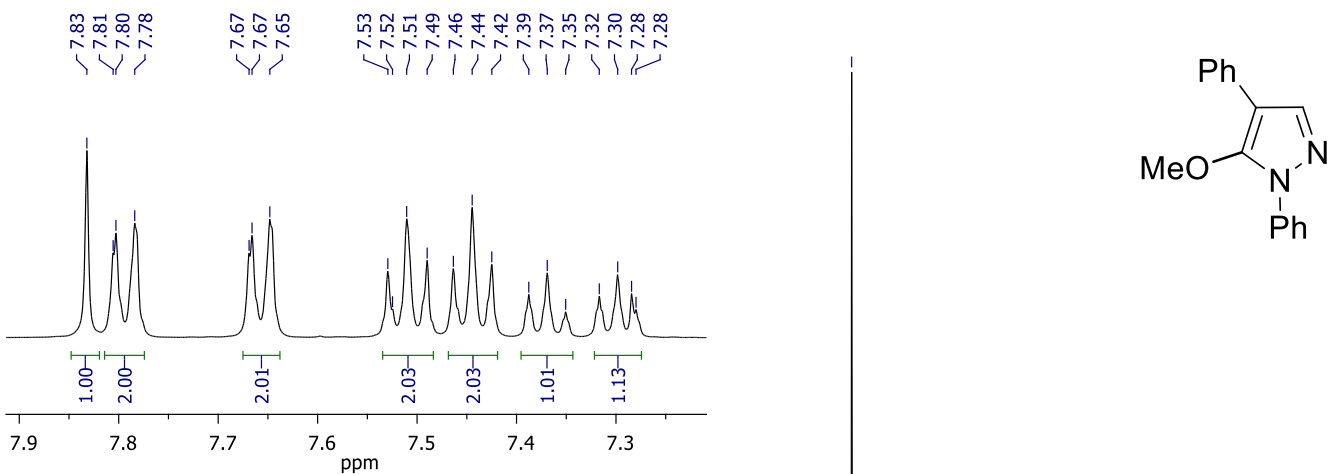

$\mathrm{Ph}$

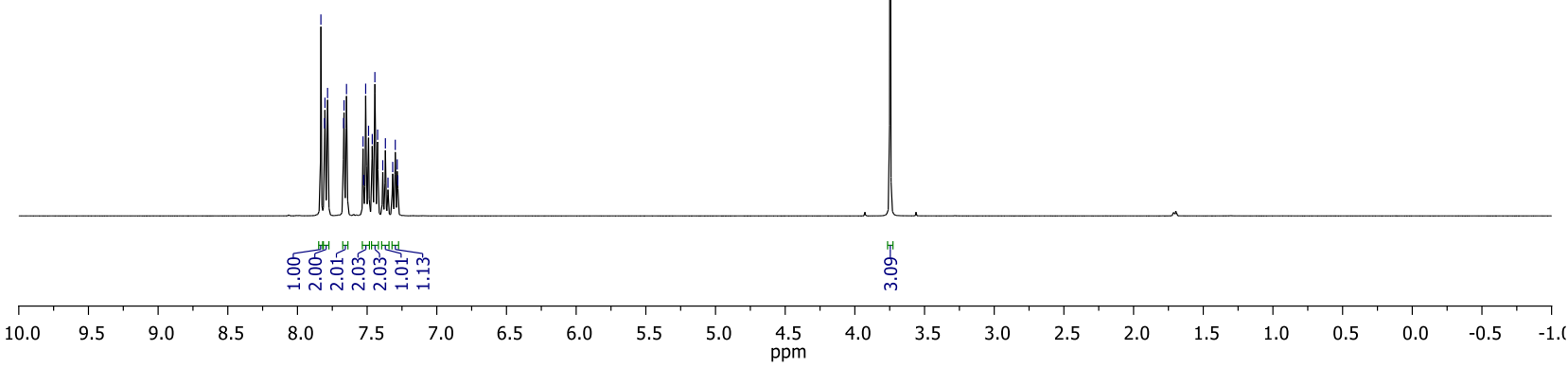

${ }^{13} \mathrm{C}\left\{{ }^{1} \mathrm{H}\right\}$ NMR spectrum of compound $1 z l\left(100 \mathrm{MHz}, \mathrm{CDCl}_{3}\right)$
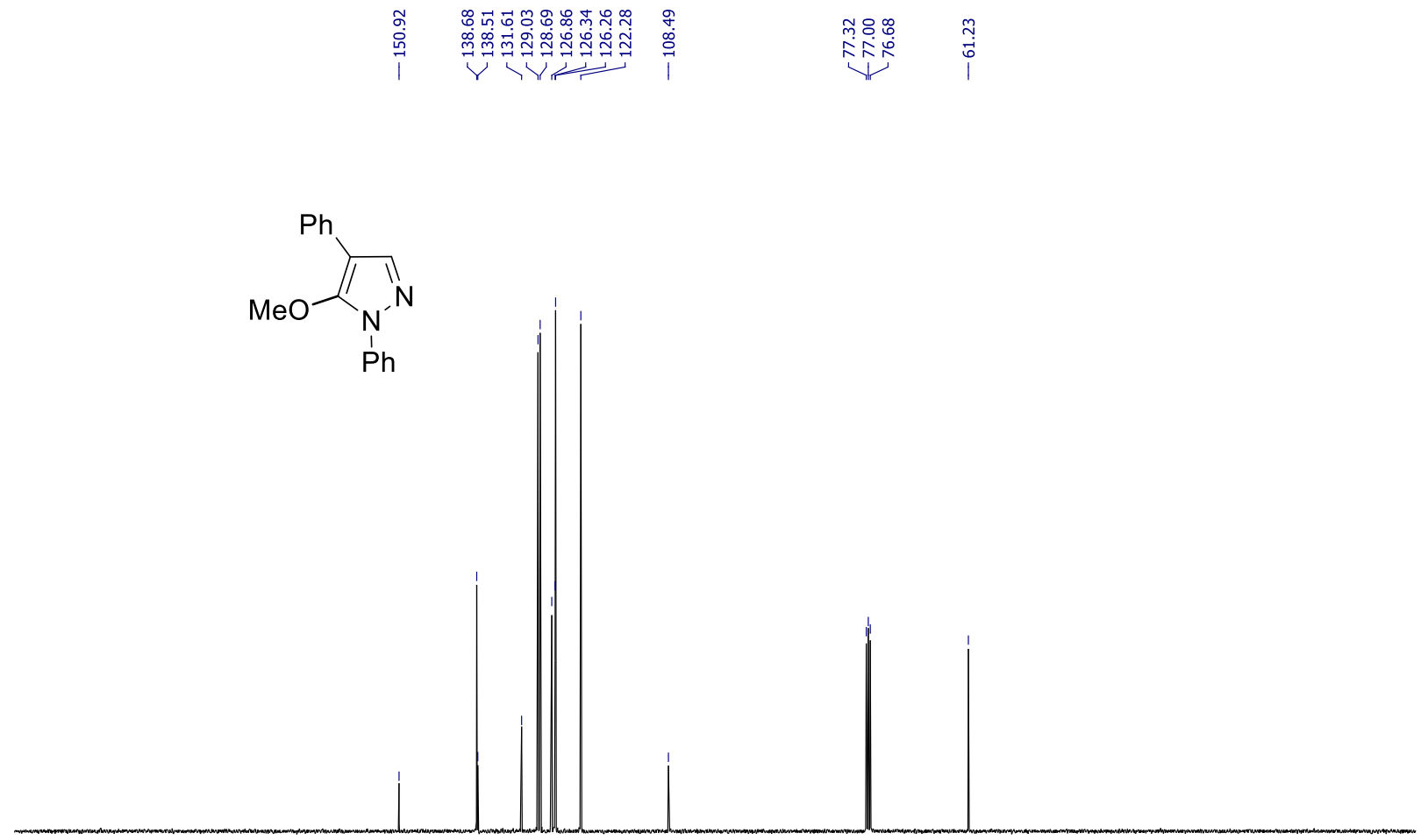

$\begin{array}{llllllllllllllllllllllllll}210 & 200 & 190 & 180 & 170 & 160 & 150 & 140 & 130 & 120 & 110 & 100 & 90 & 80 & 70 & 60 & 50 & 40 & 30 & 20 & 10 & 0\end{array}$ 
${ }^{1} \mathrm{H}$ NMR spectrum of compound $2 \mathbf{e}\left(400 \mathrm{MHz}, \mathrm{CDCl}_{3}\right)$

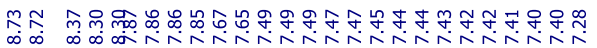

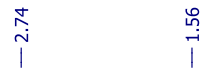
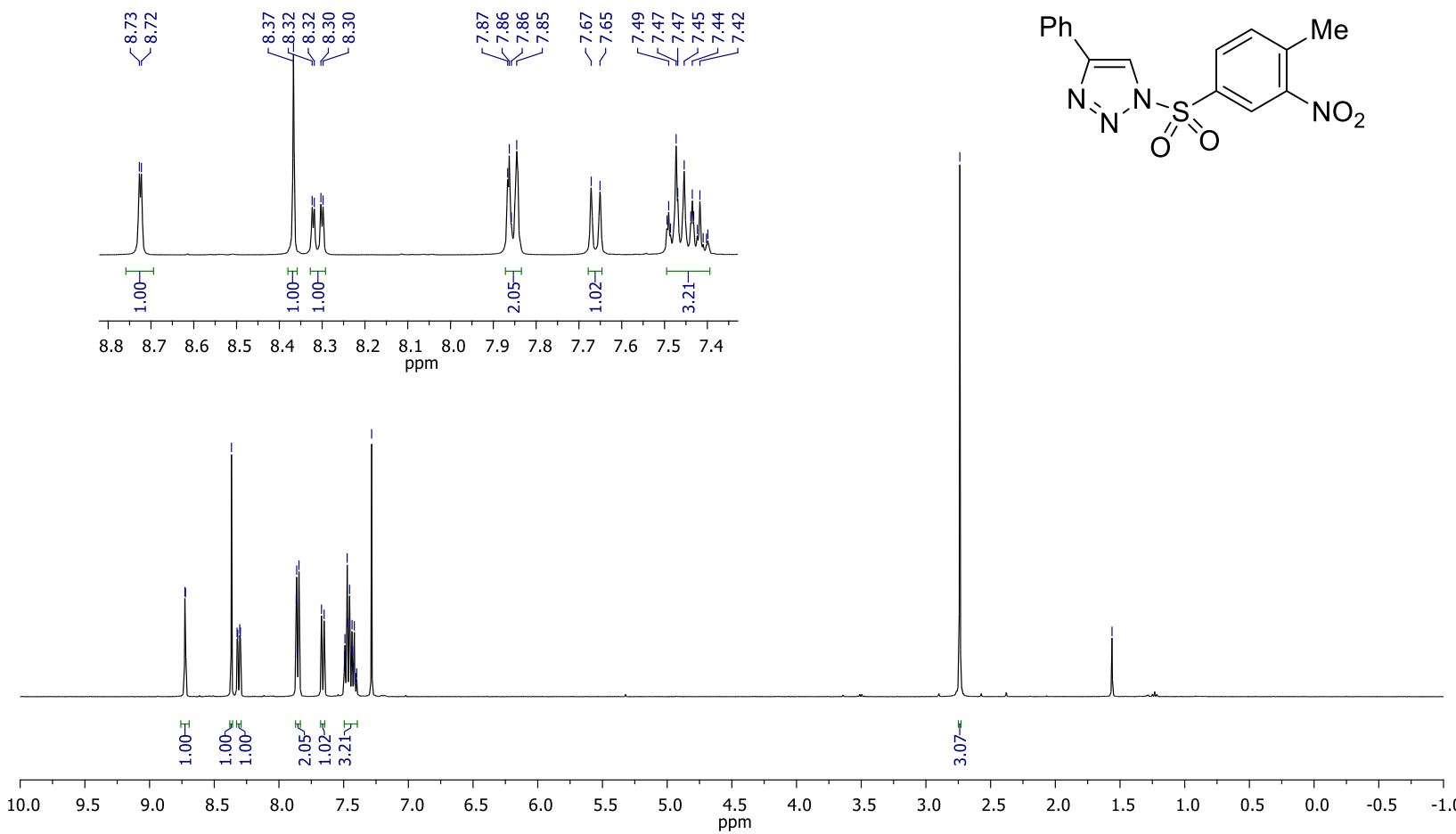

${ }^{13} \mathrm{C}\left\{{ }^{1} \mathrm{H}\right\}$ NMR spectrum of compound $2 \mathbf{e}\left(100 \mathrm{MHz}, \mathrm{CDCl}_{3}\right)$

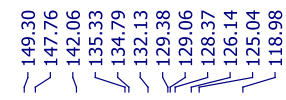

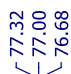

i̊<smiles>Cc1ccc(S(=O)(=O)n2cc(-c3ccccc3)nn2)cc1[N+](=O)[O-]</smiles>

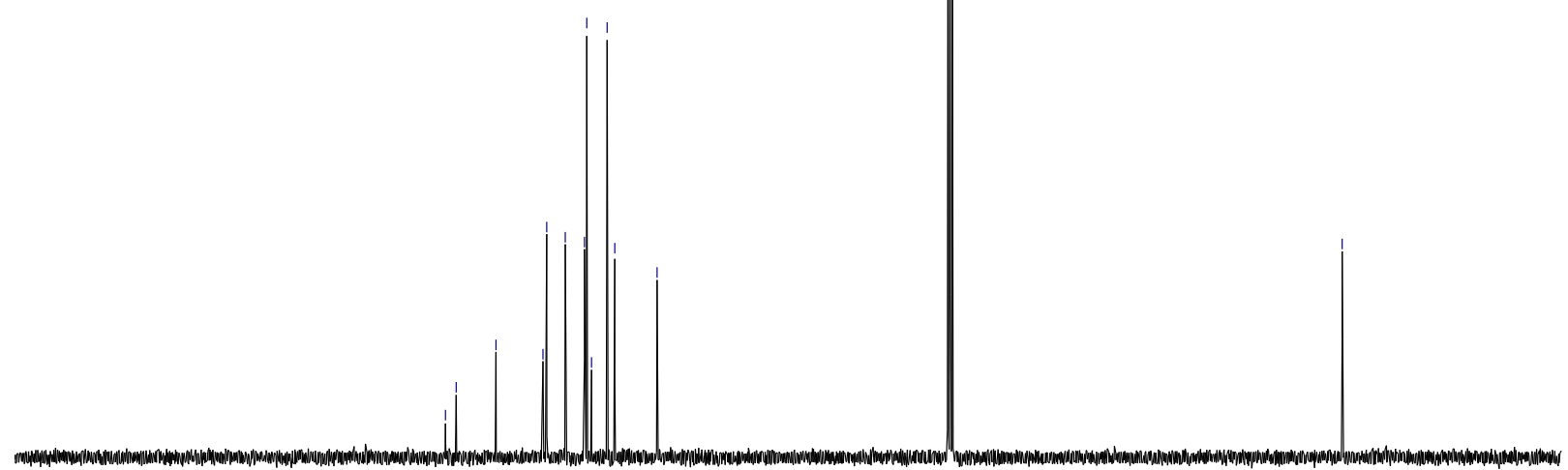

$\begin{array}{lllllllllllllllllllllllllllllllllll} & 210 & 200 & 190 & 180 & 170 & 160 & 150 & 140 & 130 & 120 & 110 & 100 & 90 & 80 & 70 & 60 & 50 & 40 & 30 & 20 & 10 & 0 & -10\end{array}$ 
${ }^{1} \mathrm{H}$ NMR spectrum of compound $\mathbf{3 a}\left(400 \mathrm{MHz}, \mathrm{CDCl}_{3}\right)$

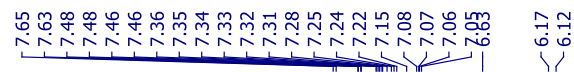
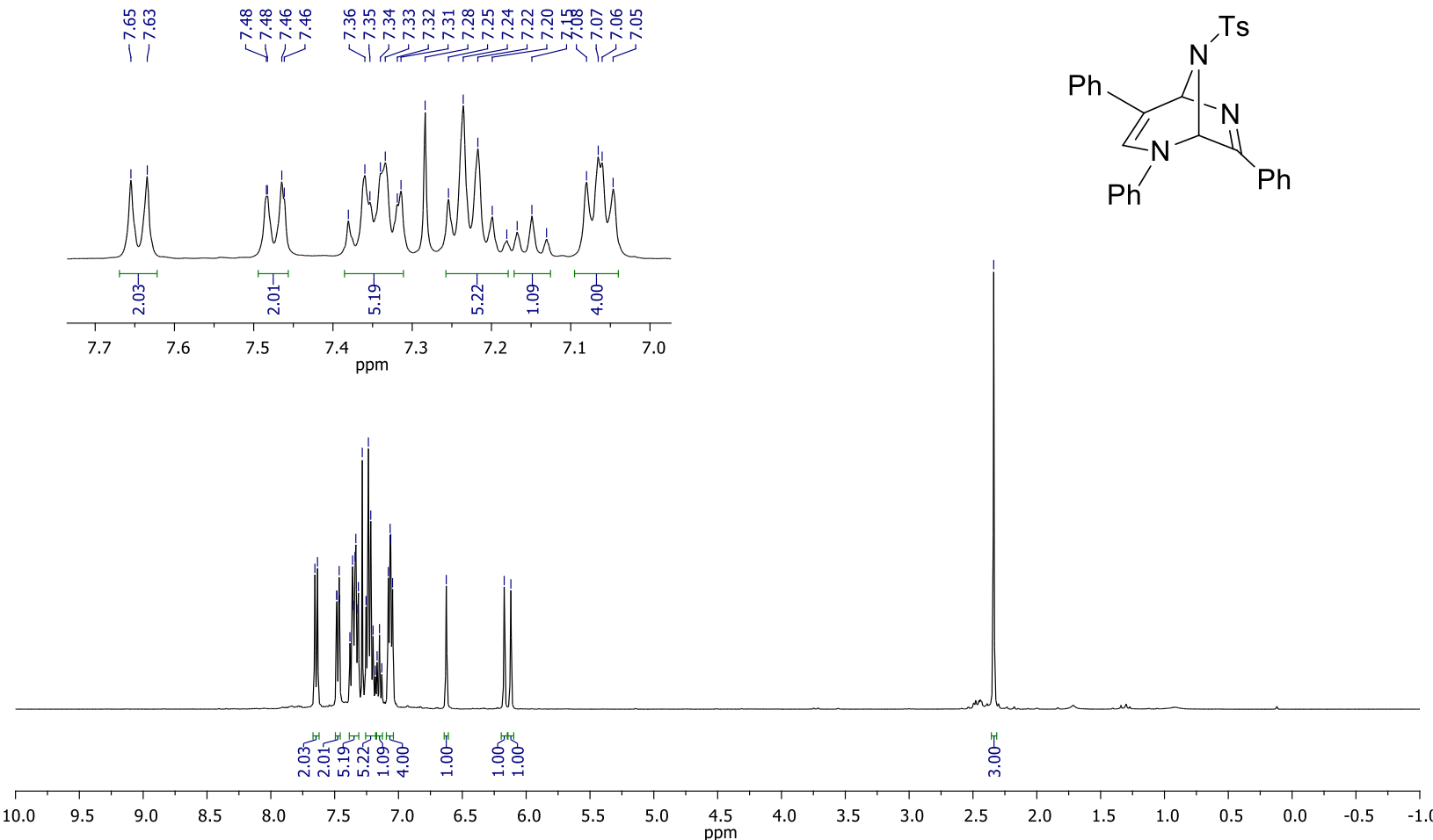

${ }^{13} \mathrm{C}\left\{{ }^{1} \mathrm{H}\right\}$ NMR spectrum of compound 3a $\left(100 \mathrm{MHz}, \mathrm{CDCl}_{3}\right)$

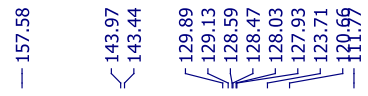

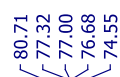<smiles></smiles>

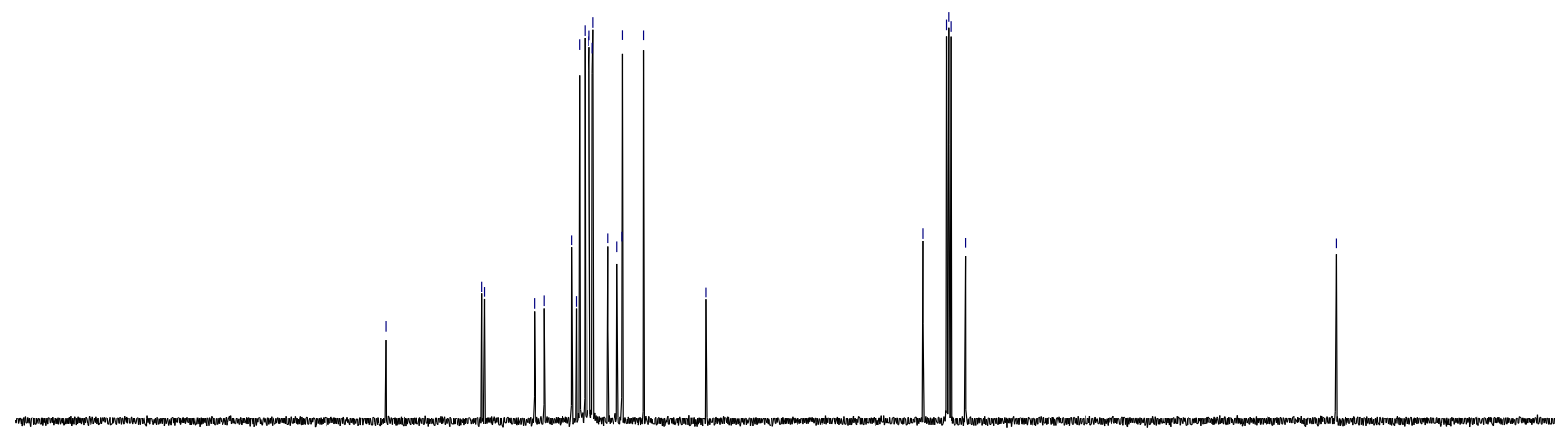

$\begin{array}{llllllllllll}210 & 200 & 190 & 180 & 170 & 160 & 150 & 140 & 130 & 120 & 110 & 100\end{array}$ 
${ }^{1} \mathrm{H}$ NMR spectrum of compound $\mathbf{3 b}\left(400 \mathrm{MHz}, \mathrm{CDCl}_{3}\right)$

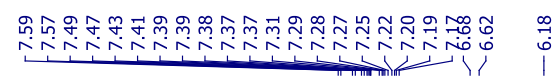
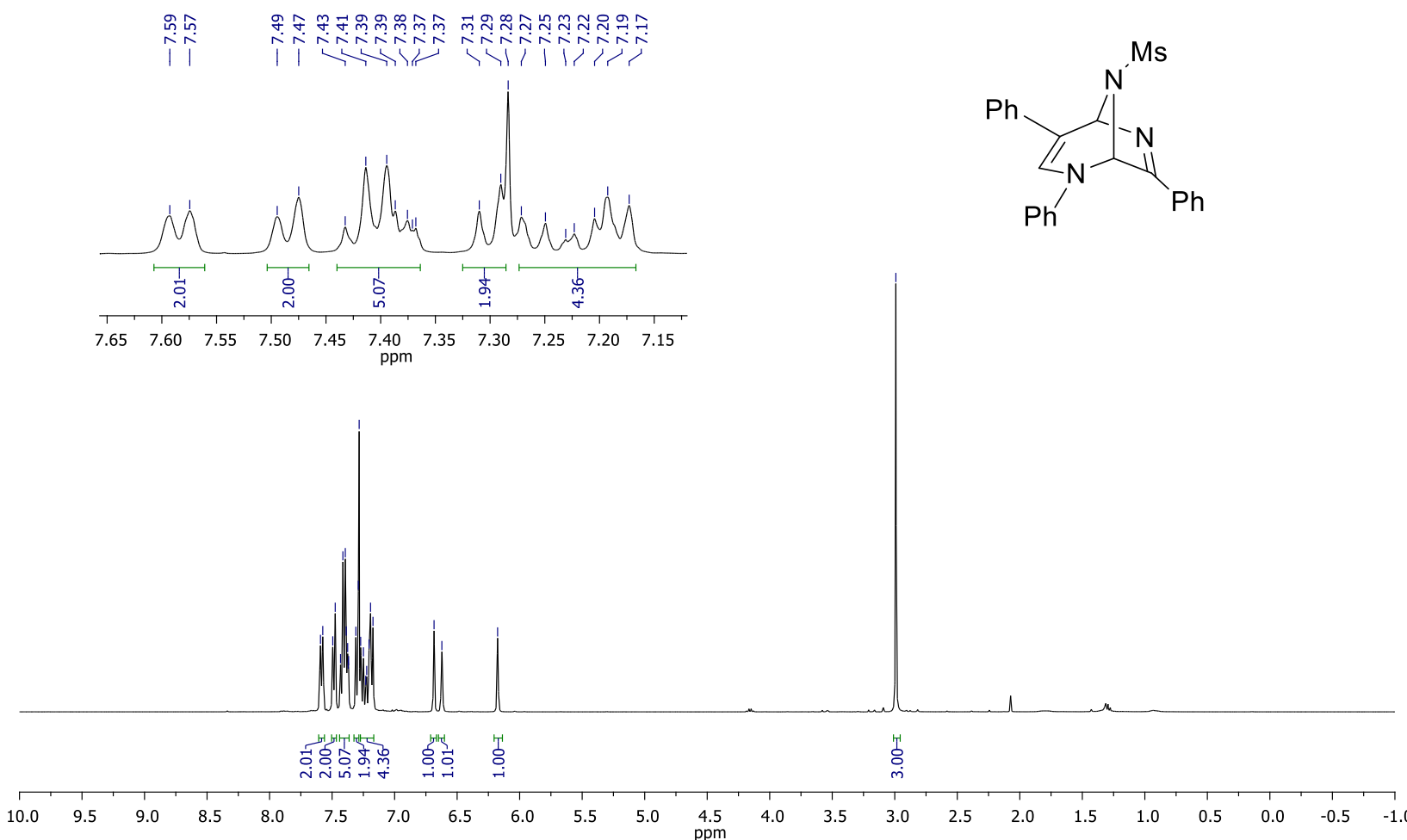

${ }^{13} \mathrm{C}\left\{{ }^{1} \mathrm{H}\right\}$ NMR spectrum of compound $3 \mathbf{b}\left(100 \mathrm{MHz}, \mathrm{CDCl}_{3}\right)$
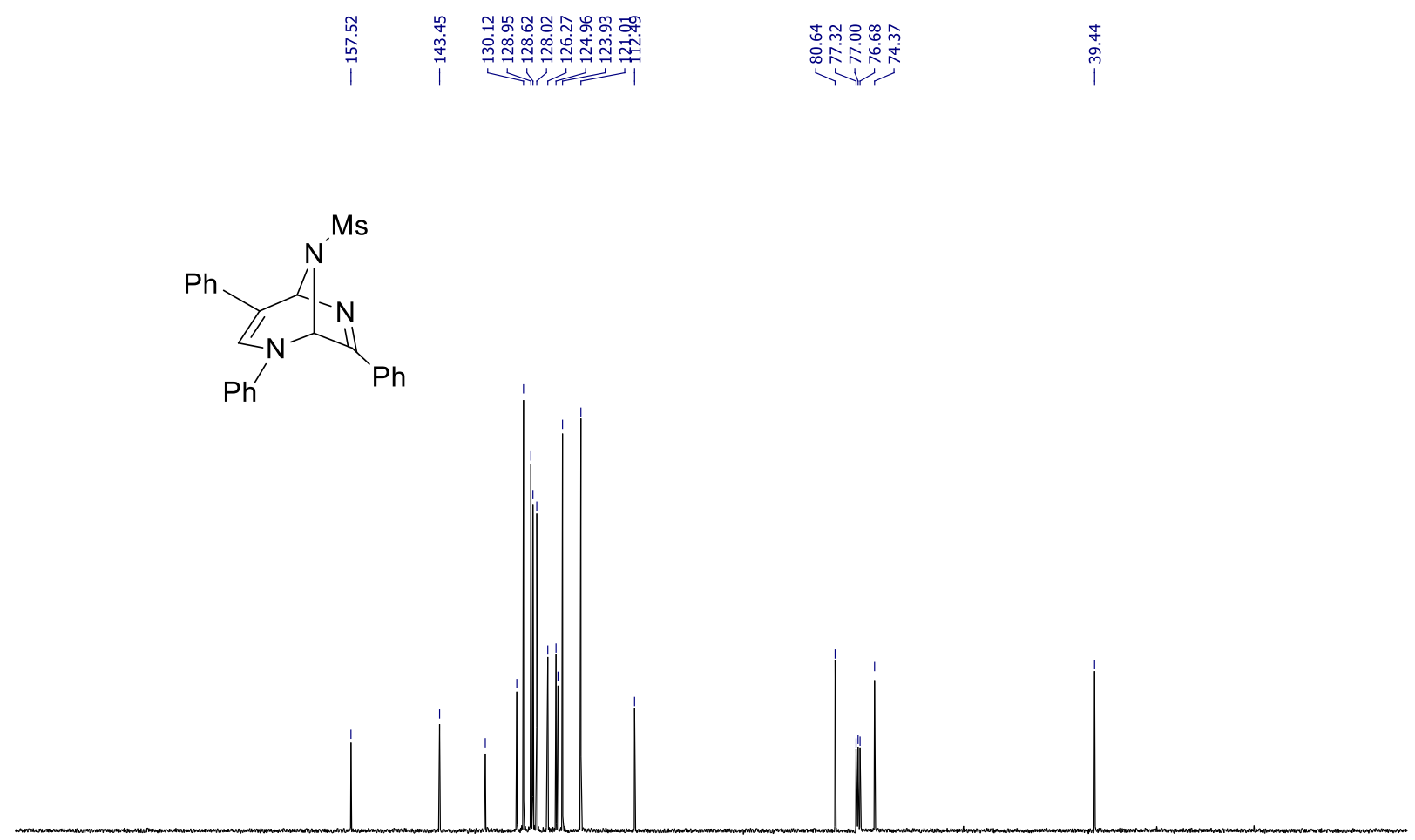

$\begin{array}{llllllllllllllllllllllll}210 & 200 & 190 & 180 & 170 & 160 & 150 & 140 & 130 & 120 & 110 & 100 & 90 & 80 & 70 & 60 & 50 & 40 & 30 & 20 & 10 & 0 & -10\end{array}$ 
${ }^{1} \mathrm{H}$ NMR spectrum of compound $\mathbf{3 c}\left(400 \mathrm{MHz}, \mathrm{CDCl}_{3}\right)$

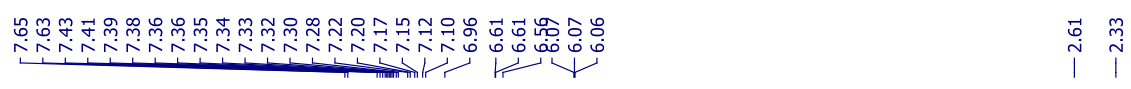

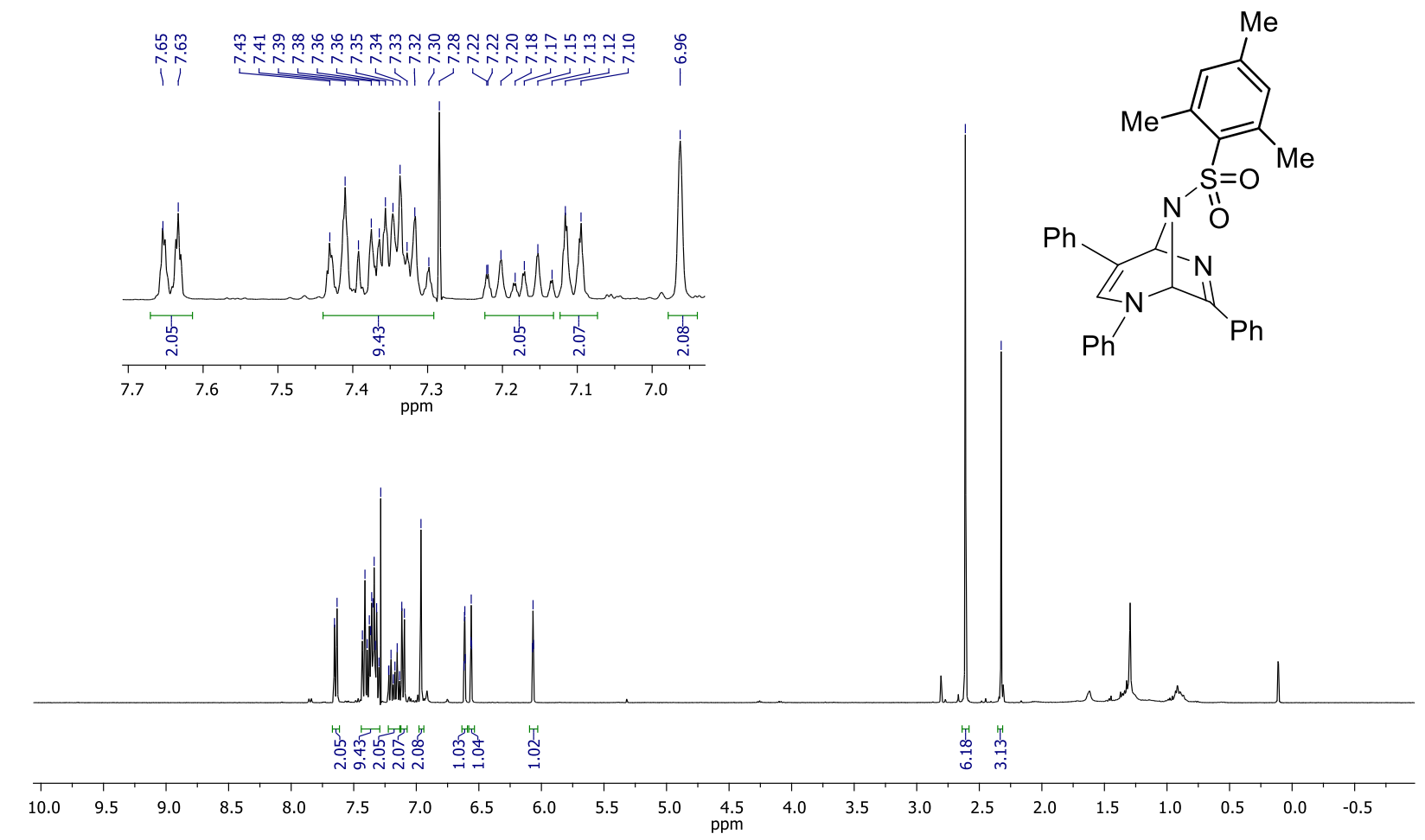

${ }^{13} \mathrm{C}\left\{{ }^{1} \mathrm{H}\right\}$ NMR spectrum of compound $3 \mathrm{c}\left(100 \mathrm{MHz}, \mathrm{CDCl}_{3}\right)$

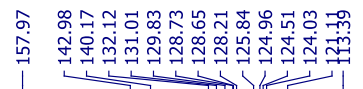

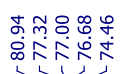
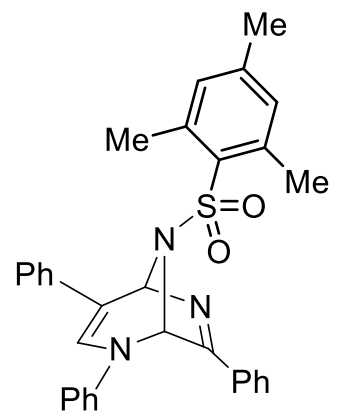

$\begin{array}{llllllllllll}210 & 200 & 190 & 180 & 170 & 160 & 150 & 140 & 130 & 120 & 110 & 100\end{array}$ 
${ }^{1} \mathrm{H}$ NMR spectrum of compound $\mathbf{3 d}\left(400 \mathrm{MHz}, \mathrm{CDCl}_{3}\right)$

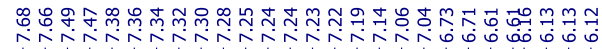

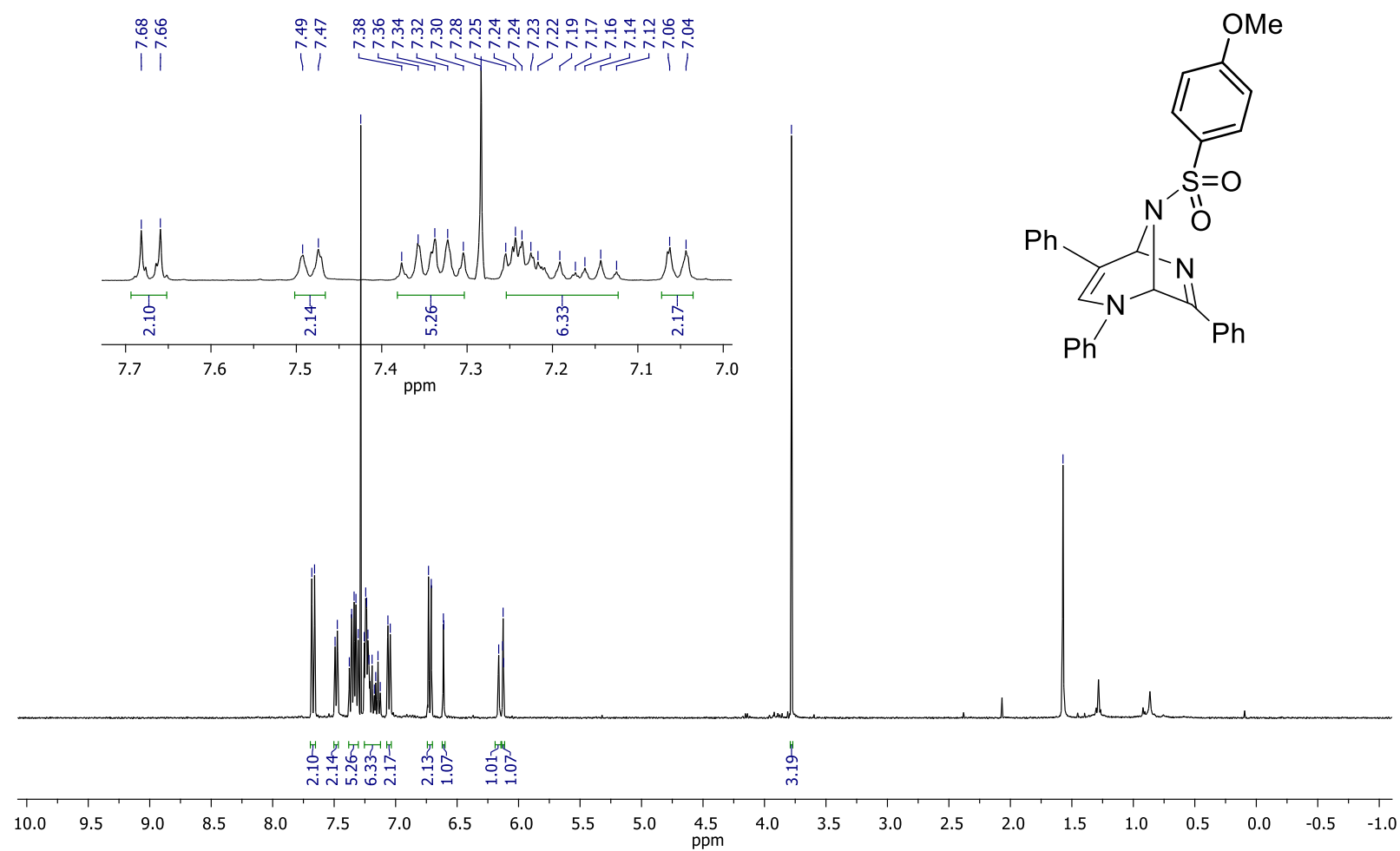

${ }^{13} \mathrm{C}\left\{{ }^{1} \mathrm{H}\right\}$ NMR spectrum of compound 3d $\left(100 \mathrm{MHz}, \mathrm{CDCl}_{3}\right)$

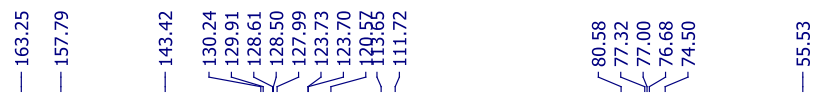

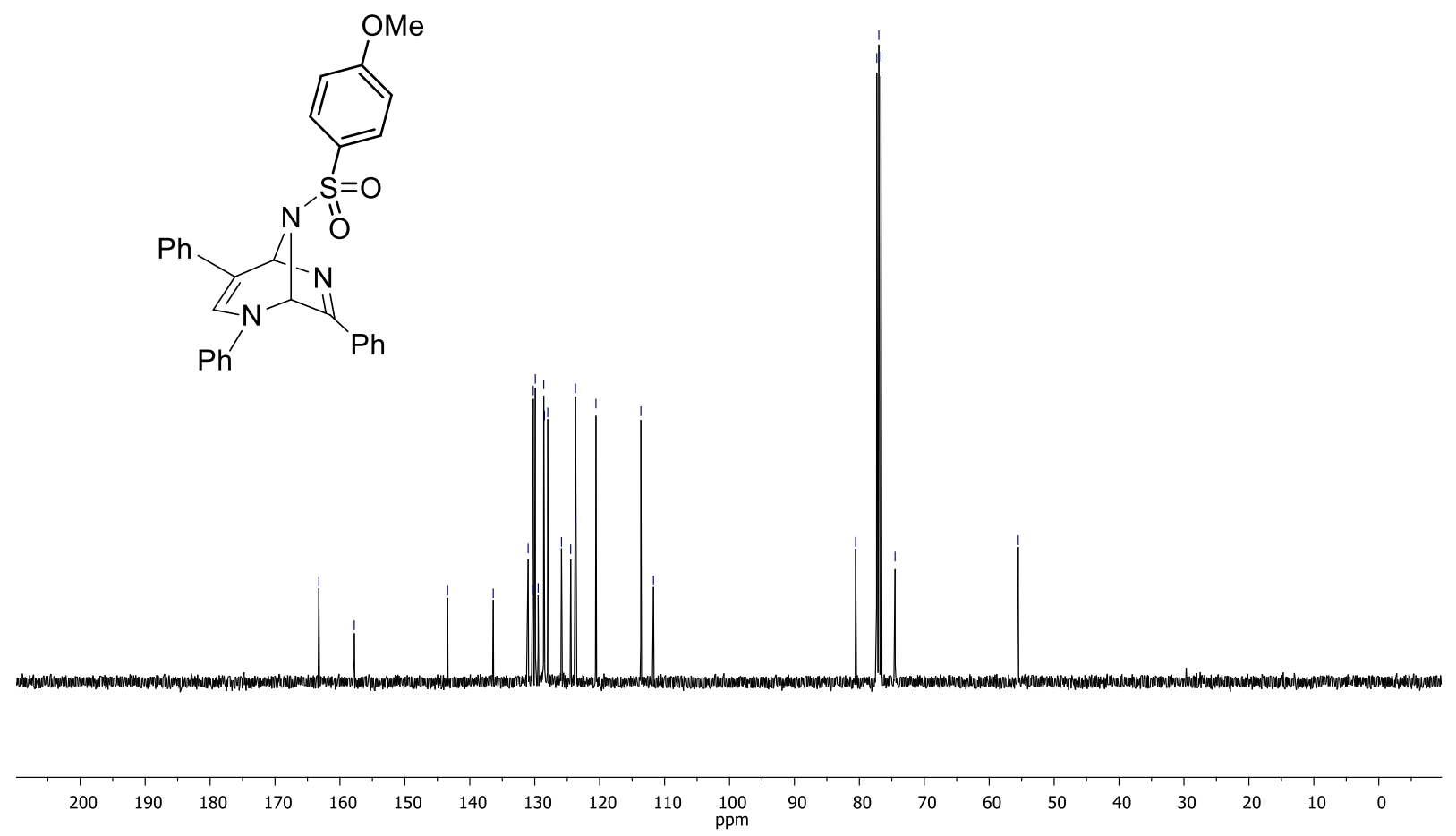


${ }^{1} \mathrm{H}$ NMR spectrum of compound $\mathbf{3 e}\left(400 \mathrm{MHz}, \mathrm{CDCl}_{3}\right)$

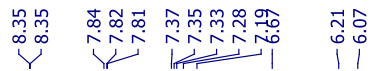

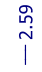
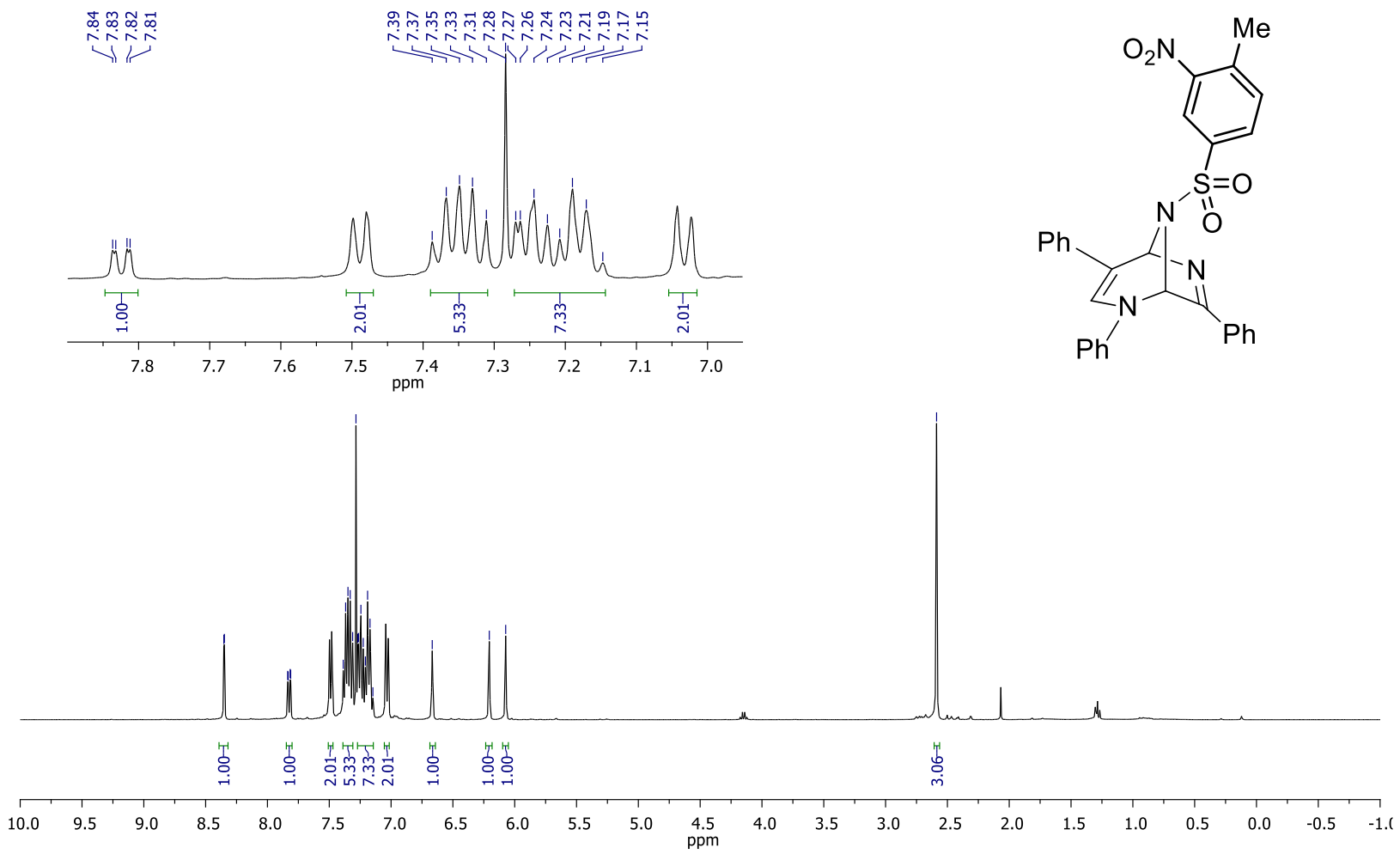

${ }^{13} \mathrm{C}\left\{{ }^{1} \mathrm{H}\right\}$ NMR spectrum of compound $3 \mathbf{e}\left(100 \mathrm{MHz}, \mathrm{CDCl}_{3}\right)$

|

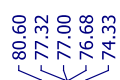

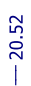
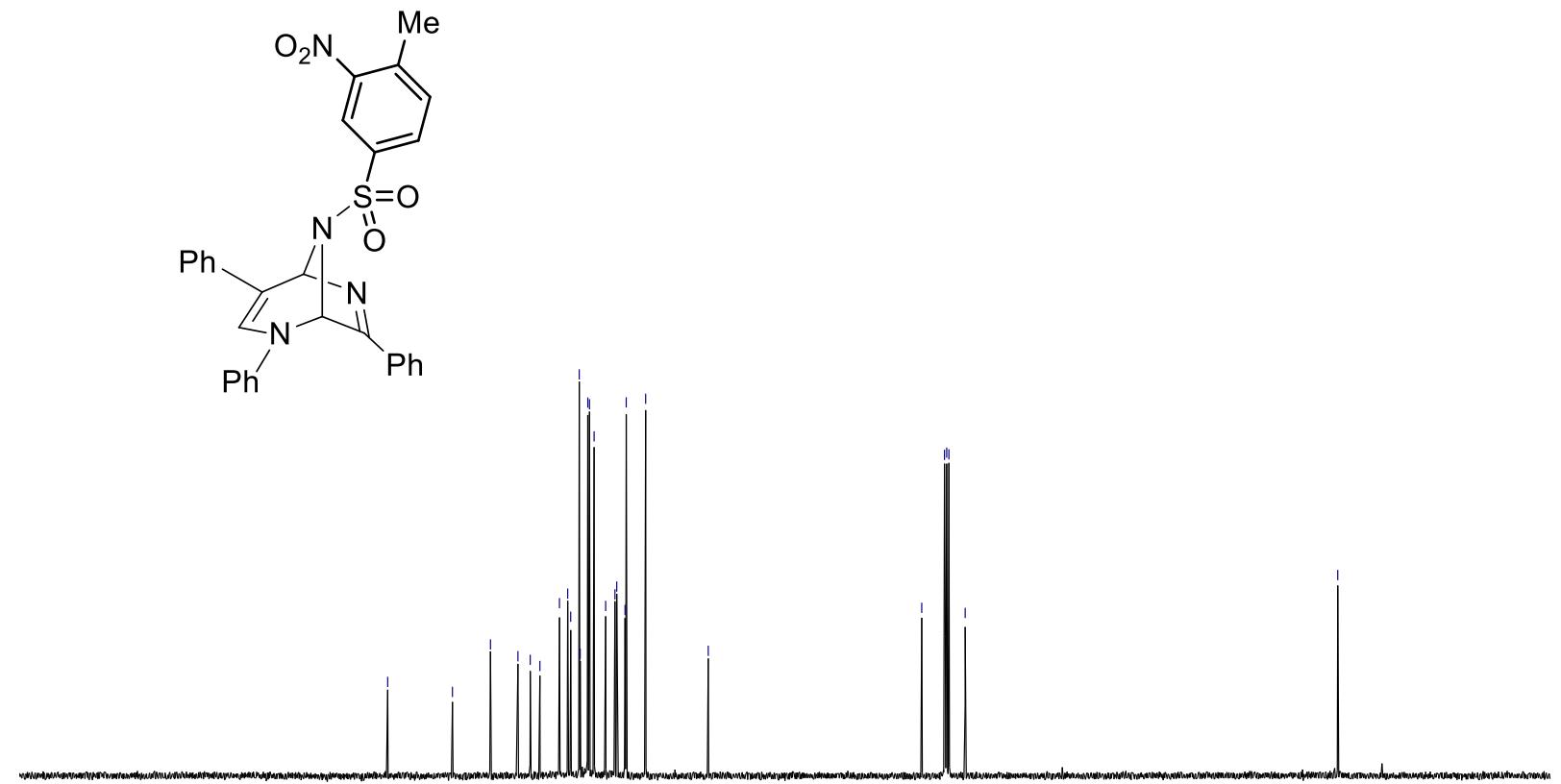

$\begin{array}{llllllllllll}210 & 200 & 190 & 180 & 170 & 160 & 150 & 140 & 130 & 120 & 110 & 100\end{array}$ 
${ }^{1} \mathrm{H}$ NMR spectrum of compound $\mathbf{3 f}\left(400 \mathrm{MHz}, \mathrm{CDCl}_{3}\right)$

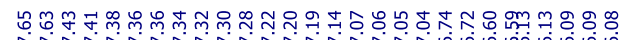

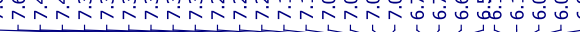

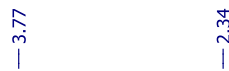
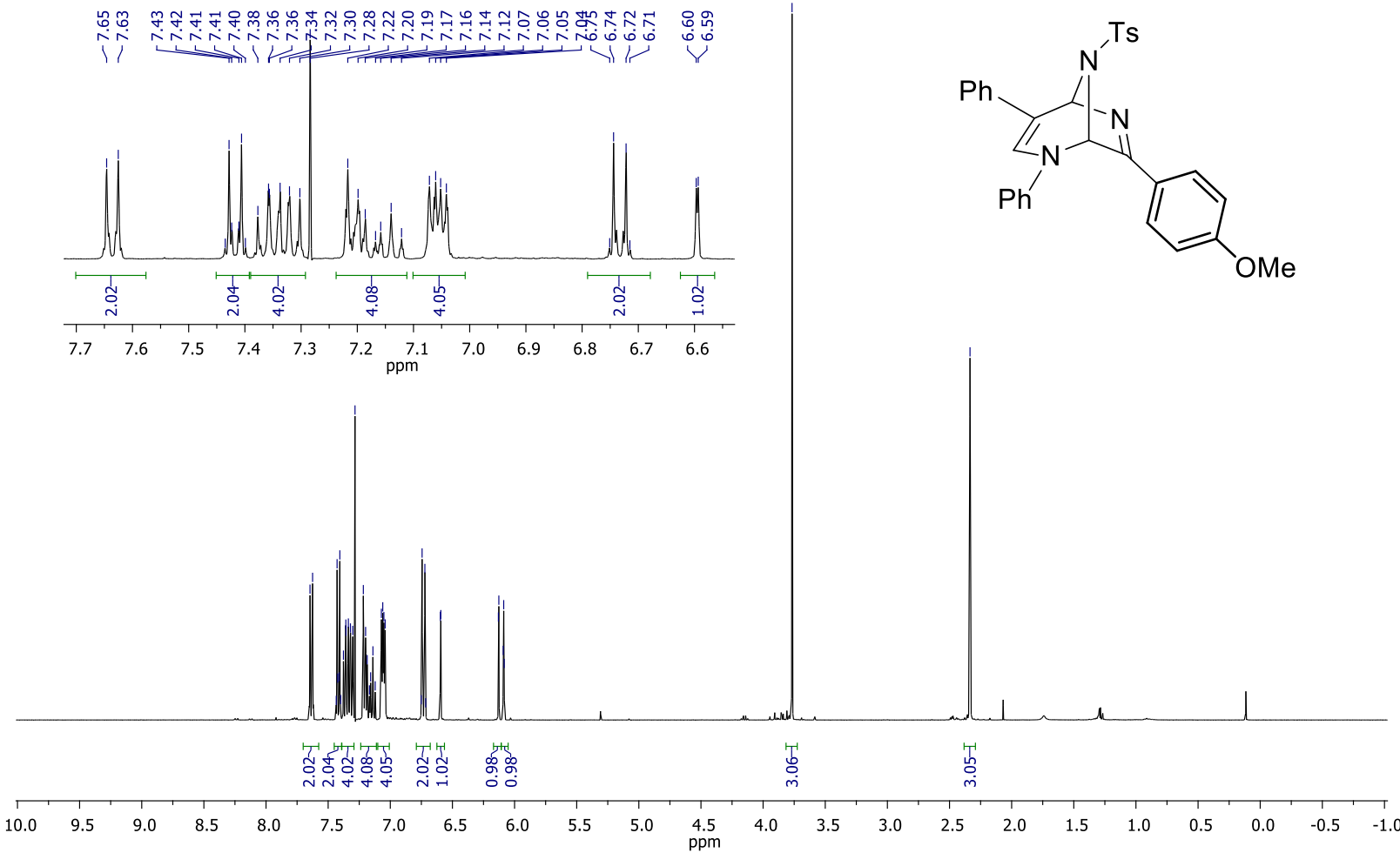

${ }^{13} \mathrm{C}\left\{{ }^{1} \mathrm{H}\right\}$ NMR spectrum of compound $3 f\left(100 \mathrm{MHz}, \mathrm{CDCl}_{3}\right)$

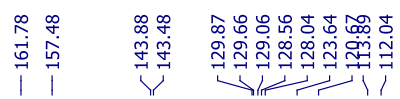

مُ

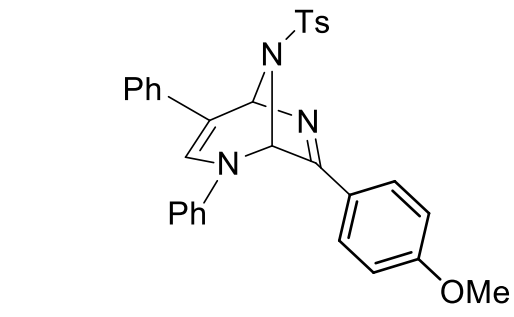

$\begin{array}{lllllllllll}200 & 190 & 180 & 170 & 160 & 150 & 140 & 130 & 120 & 110 & 100\end{array}$ 
${ }^{1} \mathrm{H}$ NMR spectrum of compound $\mathbf{3 g}\left(400 \mathrm{MHz}, \mathrm{CDCl}_{3}\right)$

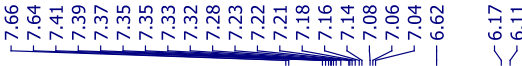

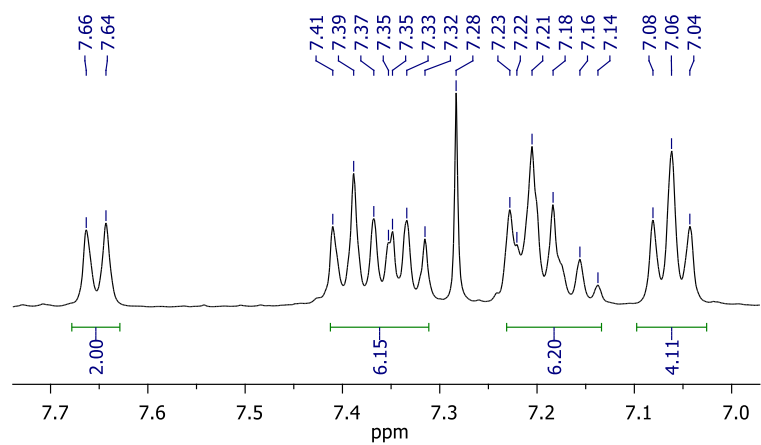

$\stackrel{m}{i}$

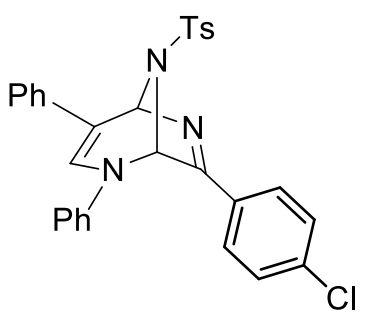

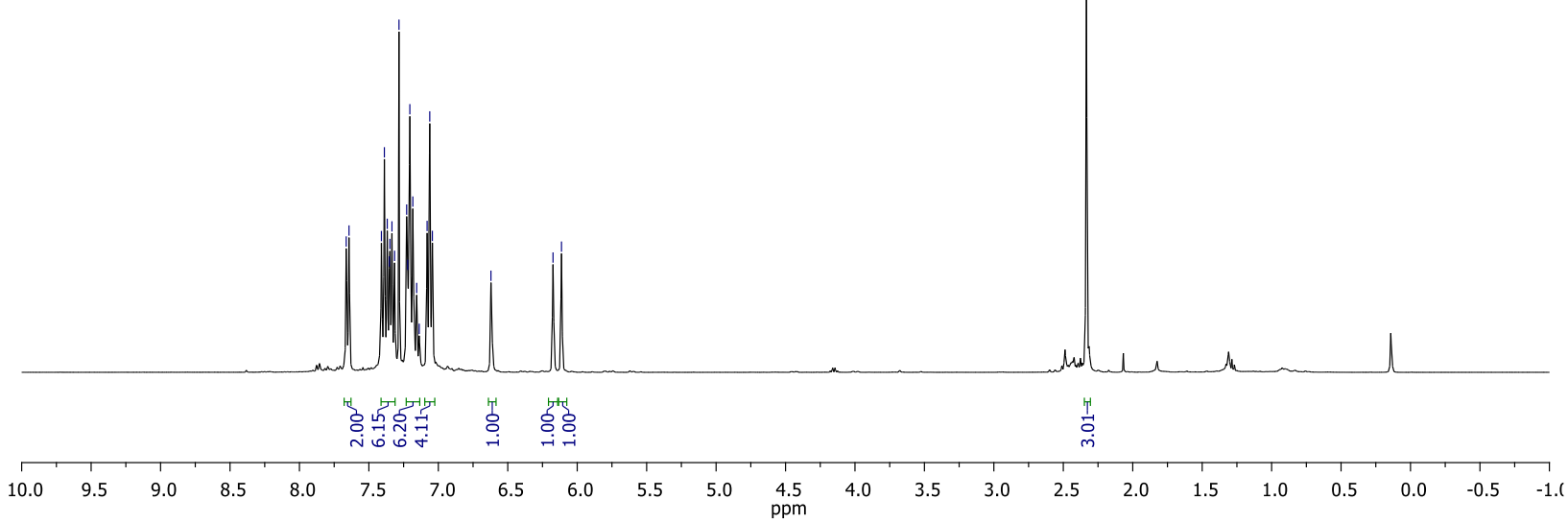

${ }^{13} \mathrm{C}\left\{{ }^{1} \mathrm{H}\right\}$ NMR spectrum of compound $\mathbf{3 g}\left(100 \mathrm{MHz}, \mathrm{CDCl}_{3}\right)$

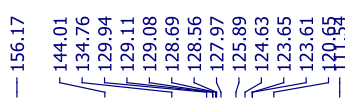

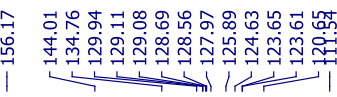

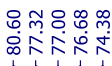

int<smiles>[AlH2]C1C(c2ccccc2)=NC(c2ccc(Cl)cc2)N1c1ccccc1</smiles>

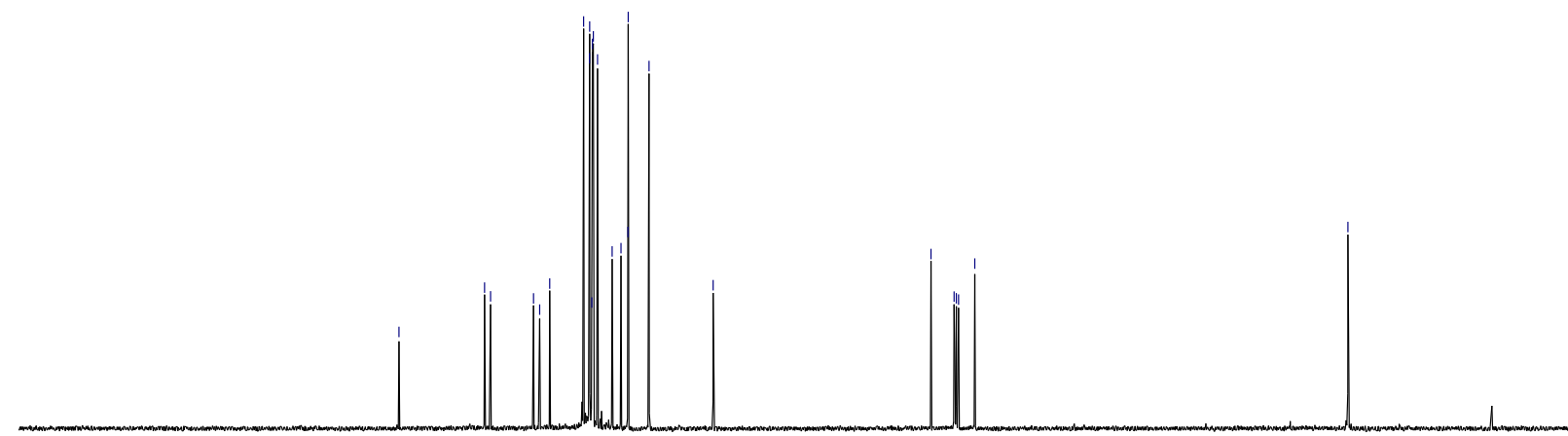

$\begin{array}{llllllllllll}210 & 200 & 190 & 180 & 170 & 160 & 150 & 140 & 130 & 120 & 110 & 100\end{array}$ 
${ }^{1} \mathrm{H}$ NMR spectrum of compound $\mathbf{3 h}\left(400 \mathrm{MHz}, \mathrm{CDCl}_{3}\right)$

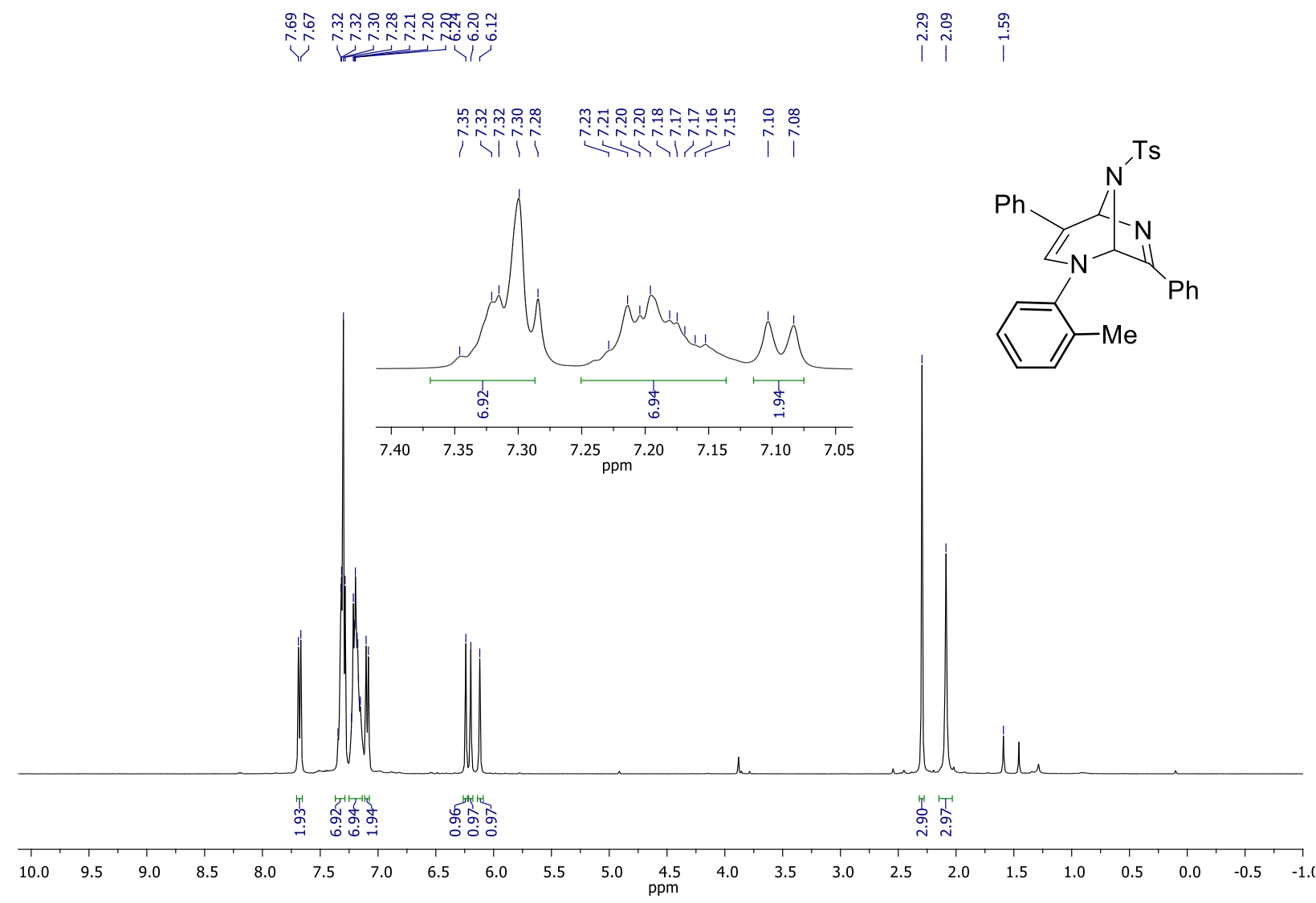

${ }^{13} \mathrm{C}\left\{{ }^{1} \mathrm{H}\right\}$ NMR spectrum of compound $\mathbf{3 h}\left(100 \mathrm{MHz}, \mathrm{CDCl}_{3}\right)$

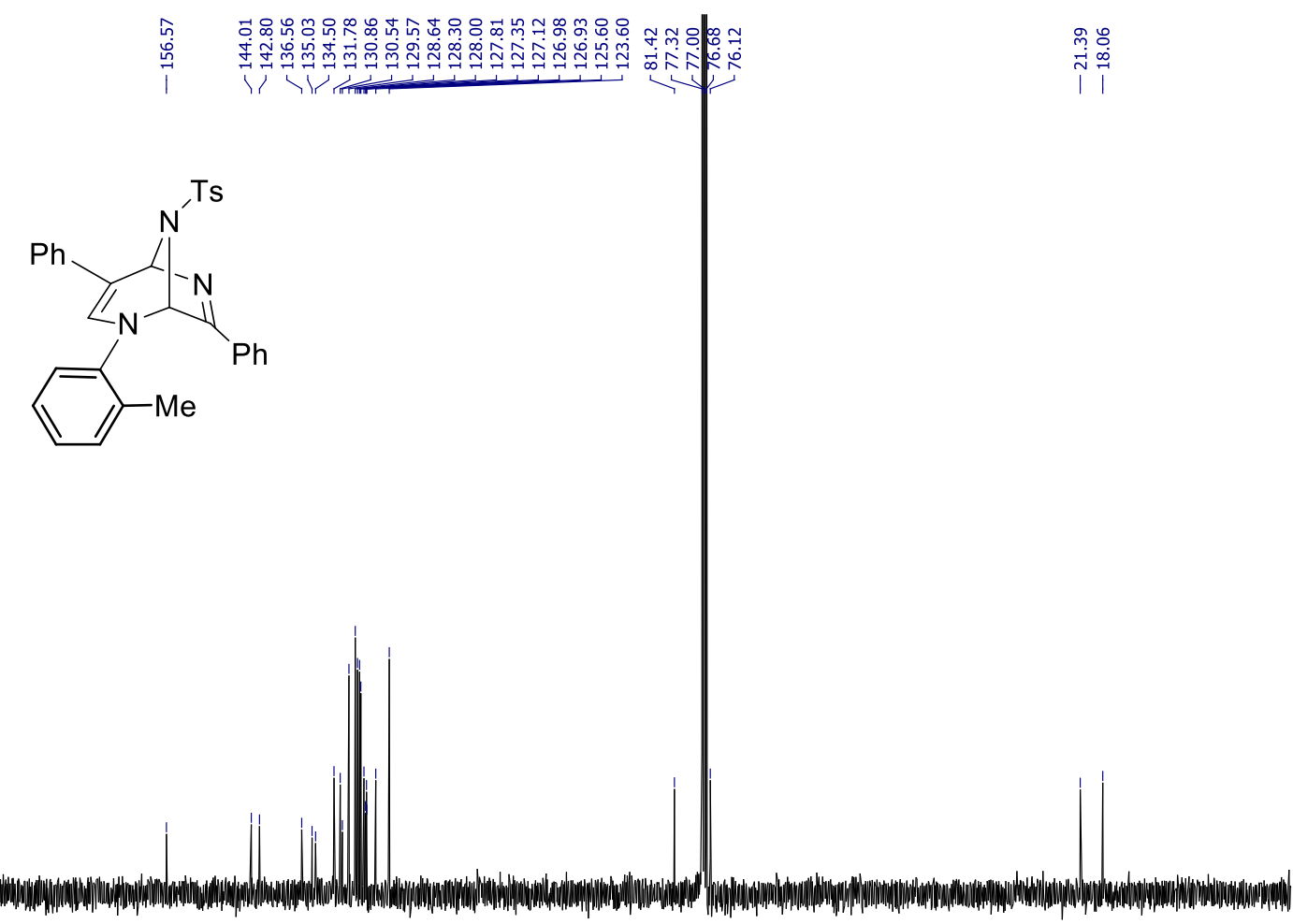

$\begin{array}{lllllllllllllllllllllllll}210 & 200 & 190 & 180 & 170 & 160 & 150 & 140 & 130 & 120 & 110 & 100 & 90 & 80 & 70 & 60 & 50 & 40 & 30 & 20 & 10 & 0 & 10\end{array}$ 
${ }^{1} \mathrm{H}$ NMR spectrum of compound $\mathbf{3 i}\left(400 \mathrm{MHz}, \mathrm{CDCl}_{3}\right)$

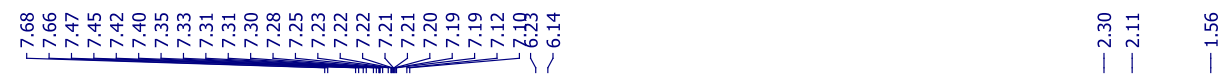

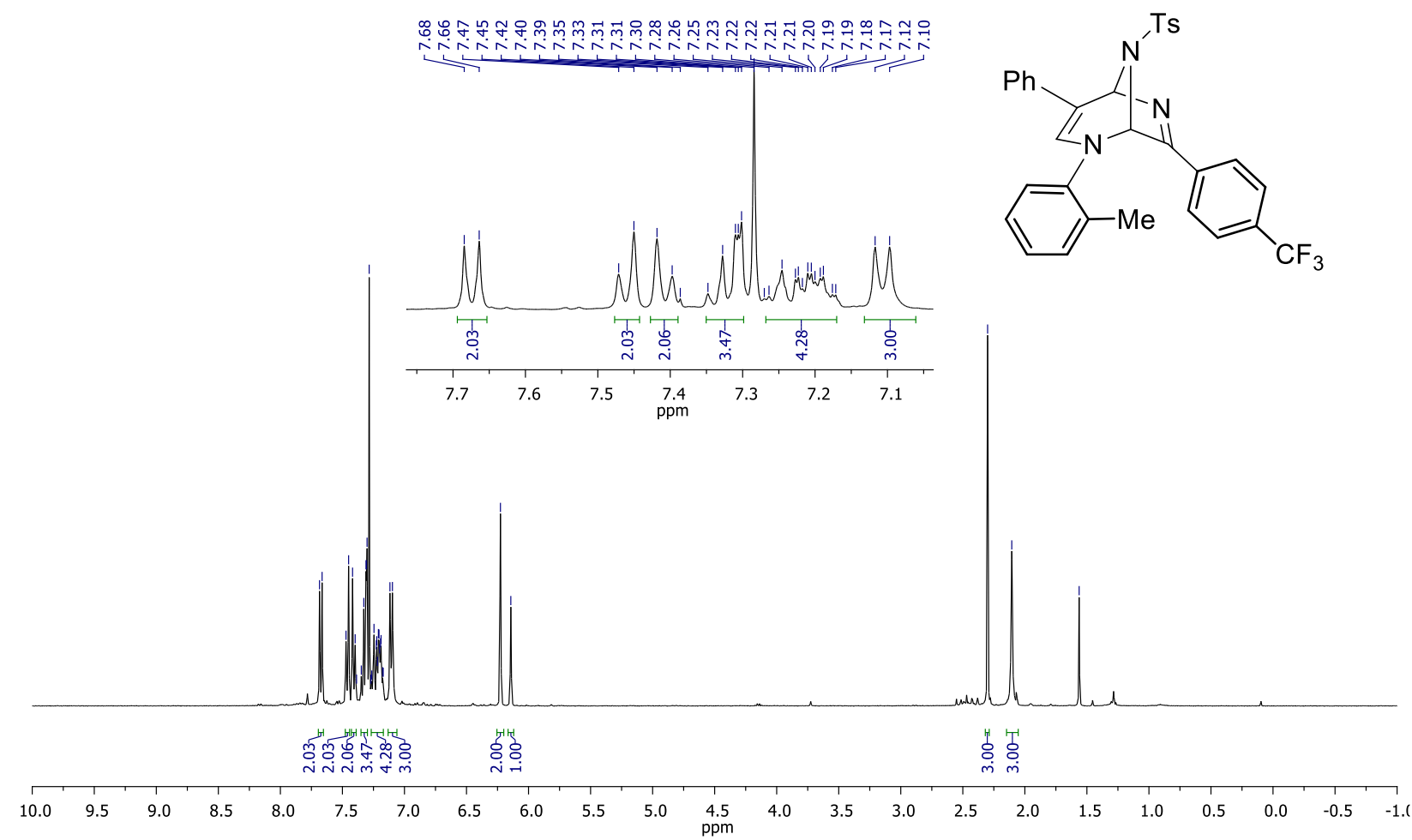

${ }^{13} \mathrm{C}\left\{{ }^{1} \mathrm{H}\right\}$ NMR spectrum of compound $3 \mathbf{i}\left(100 \mathrm{MHz}, \mathrm{CDCl}_{3}\right)$
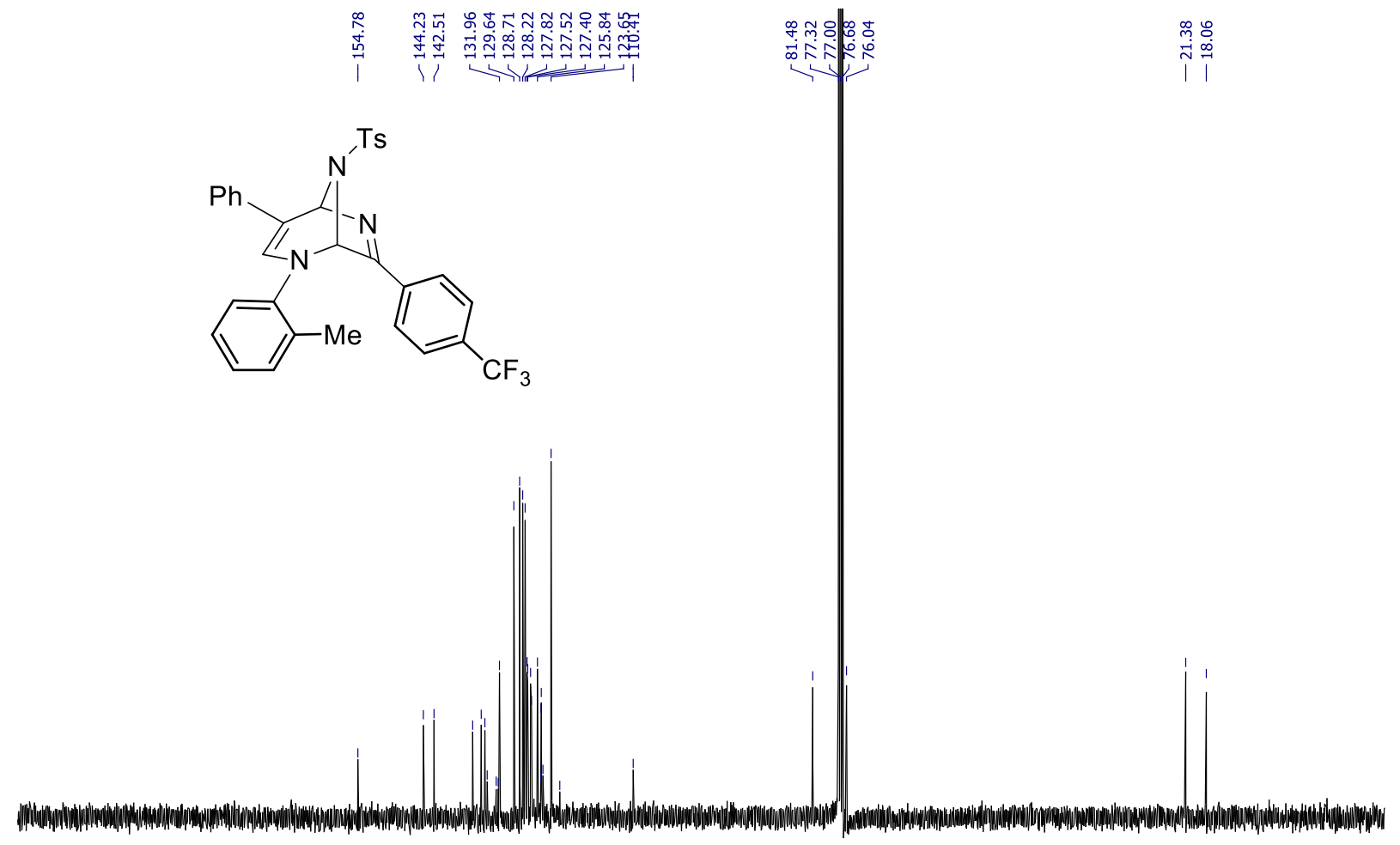

$\begin{array}{llllllllllllllllllllllllllll}200 & 190 & 180 & 170 & 160 & 150 & 140 & 130 & 120 & 110 & 100 & 90 & 80 & 70 & 60 & 50 & 40 & 30 & 20 & 10 & 0 & -10\end{array}$ 
${ }^{1} \mathrm{H}$ NMR spectrum of compound $\mathbf{3 j}$ (400 MHz, $\mathrm{CDCl}_{3}$ )

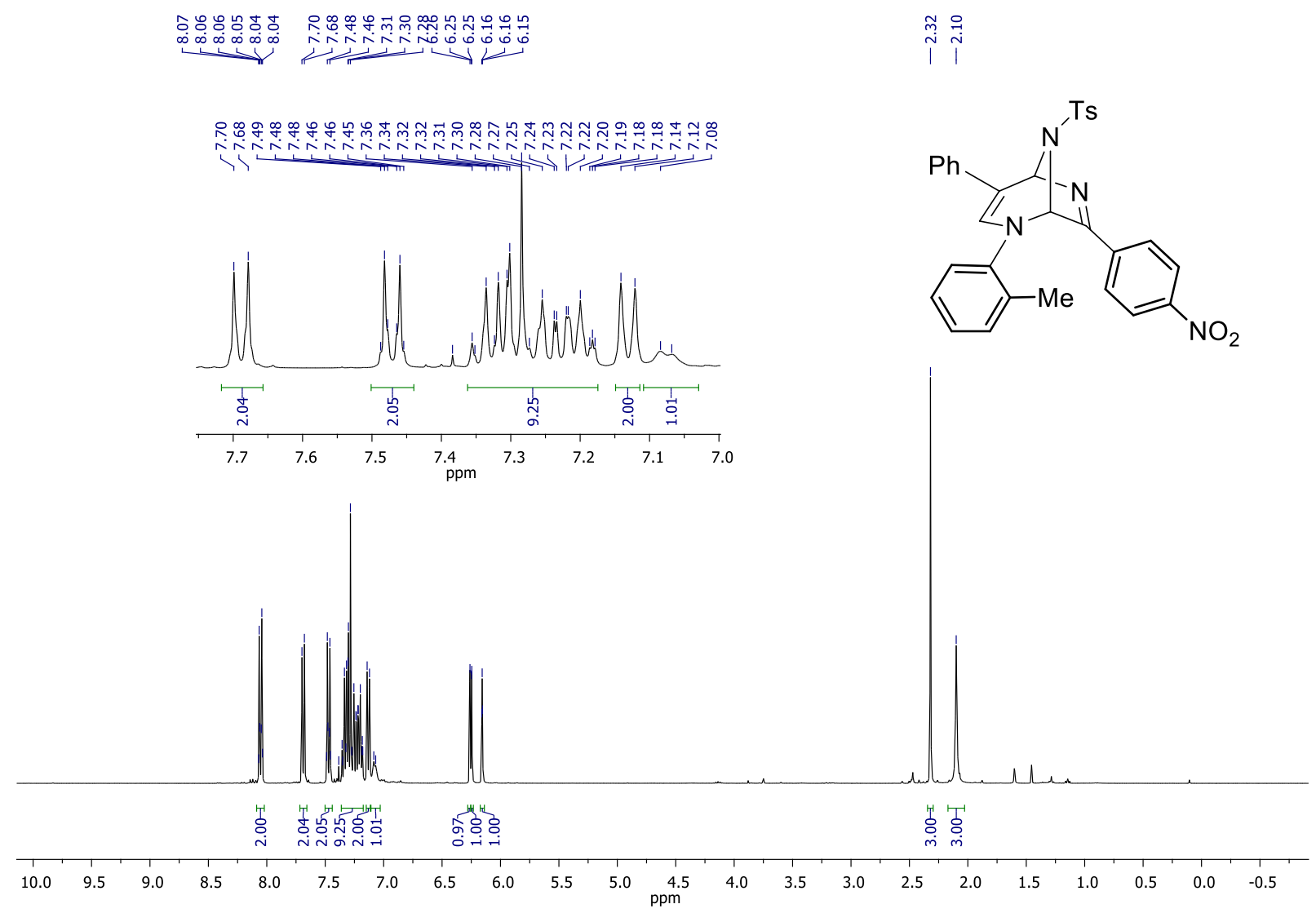

${ }^{13} \mathrm{C}\left\{{ }^{1} \mathrm{H}\right\}$ NMR spectrum of compound $\mathbf{3 j}\left(100 \mathrm{MHz}, \mathrm{CDCl}_{3}\right)$

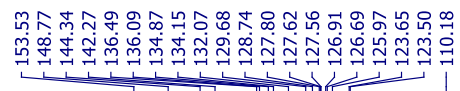

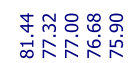

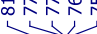<smiles>Cc1ccccc1N1C=C(c2ccccc2)C2C(c3ccc([N+](=O)[O-])cc3)=NC1N2[As]</smiles>

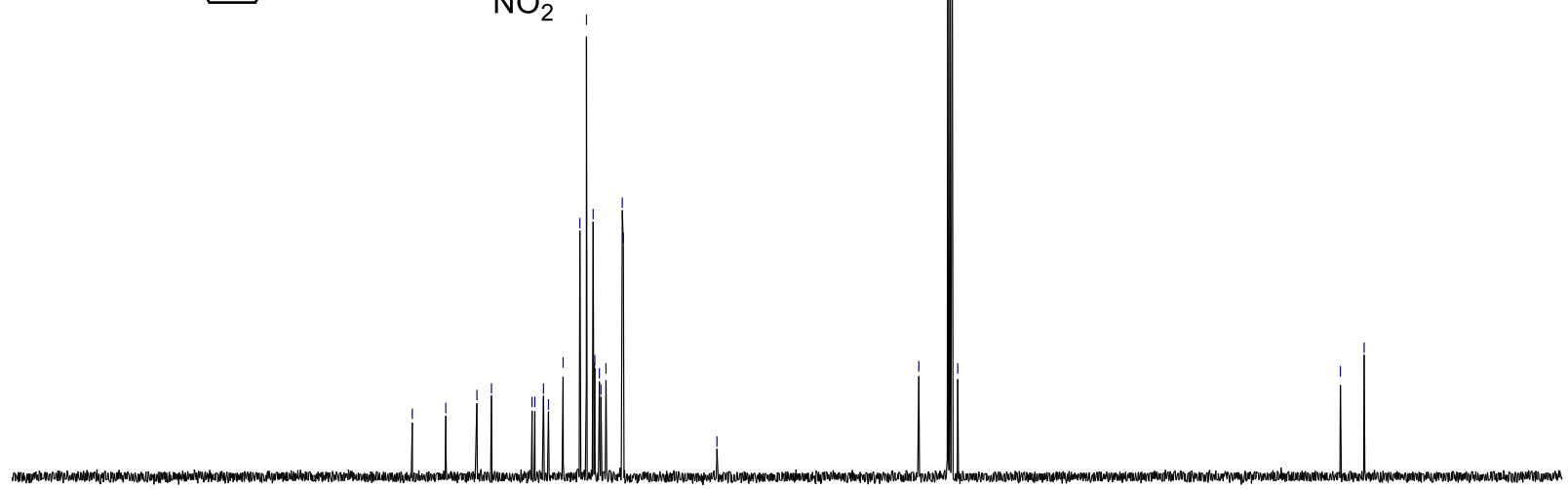


${ }^{1} \mathrm{H}$ NMR spectrum of compound $3 \mathbf{k}\left(400 \mathrm{MHz}, \mathrm{CDCl}_{3}\right)$

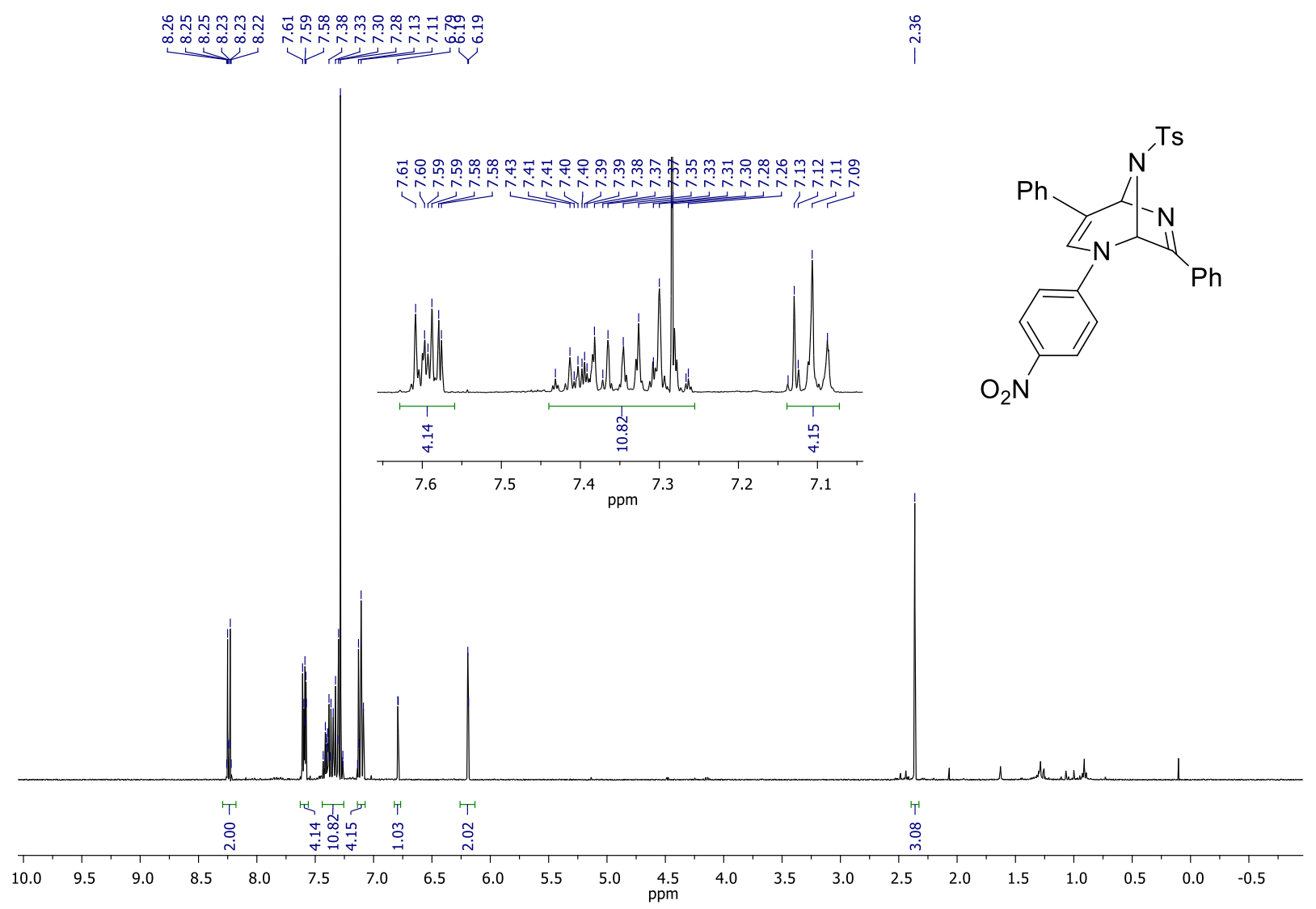

${ }^{13} \mathrm{C}\left\{{ }^{1} \mathrm{H}\right\}$ NMR spectrum of compound 3k (100 MHz, $\left.\mathrm{CDCl}_{3}\right)$

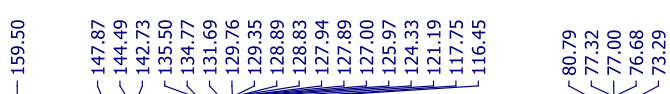<smiles>O=[N+]([O-])c1ccc(N2C=C(c3ccccc3)C3N=C(c4ccccc4)N2C3[AlH2])cc1</smiles>

$\begin{array}{llllllllllll}210 & 200 & 190 & 180 & 170 & 160 & 150 & 140 & 130 & 120 & 110 & \begin{array}{l}100 \\ \mathrm{ppm}\end{array}\end{array}$ 
${ }^{1} \mathrm{H}$ NMR spectrum of compound 31 (400 MHz, $\mathrm{CDCl}_{3}$ )

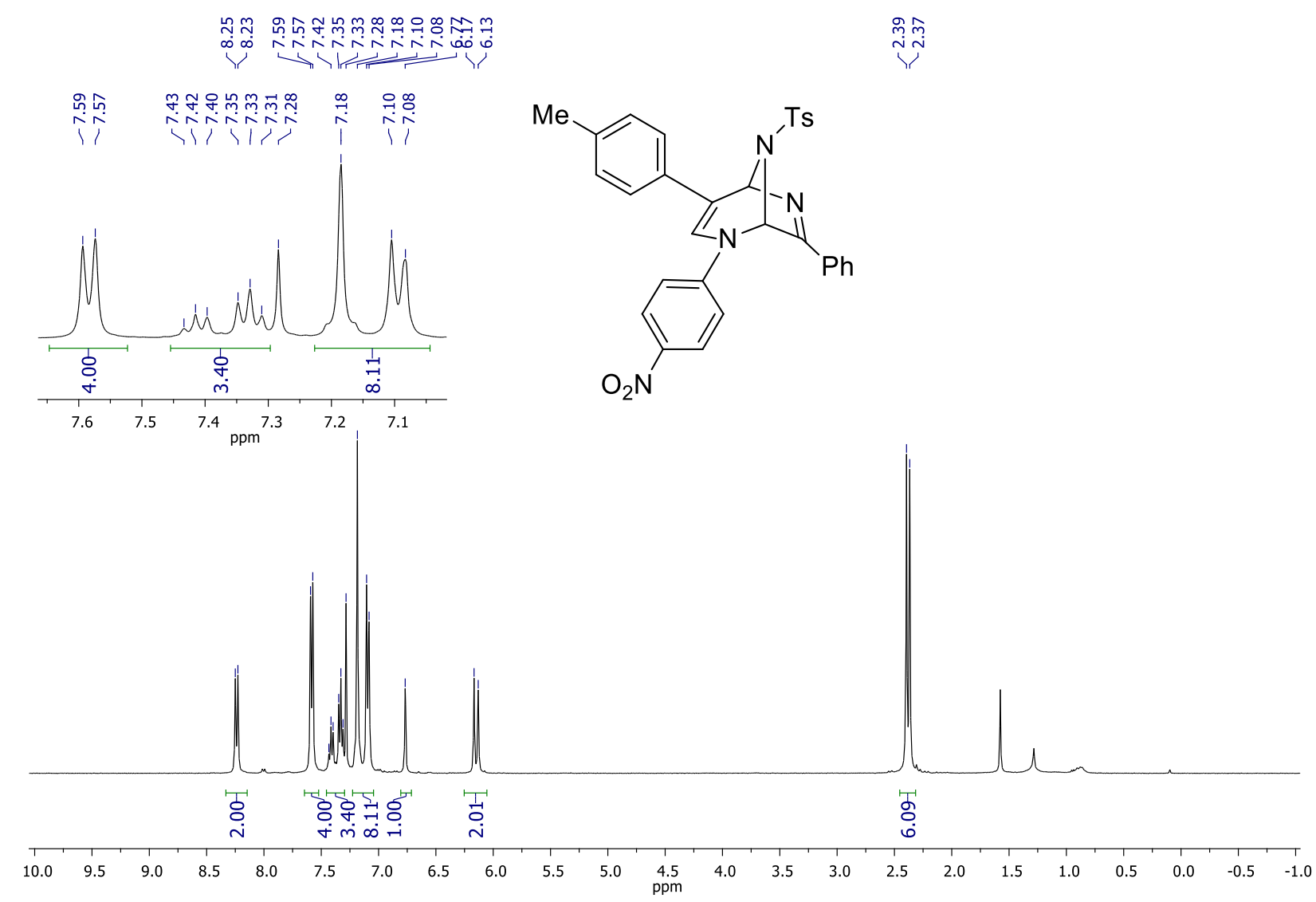

${ }^{13} \mathrm{C}\left\{{ }^{1} \mathrm{H}\right\}$ NMR spectrum of compound $31\left(100 \mathrm{MHz}, \mathrm{CDCl}_{3}\right)$

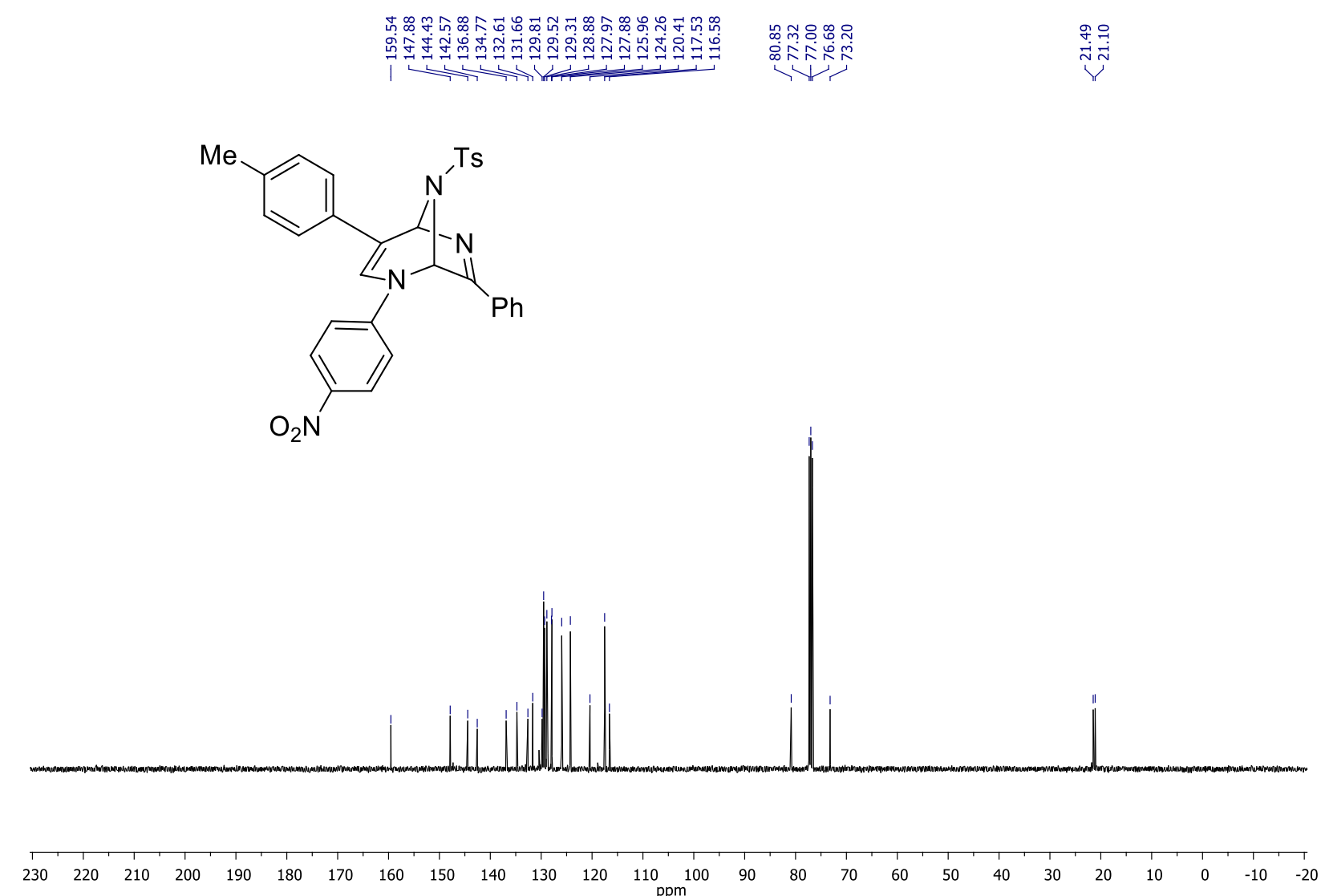


${ }^{1} \mathrm{H}$ NMR spectrum of compound $\mathbf{3 m}\left(400 \mathrm{MHz}, \mathrm{CDCl}_{3}\right)$

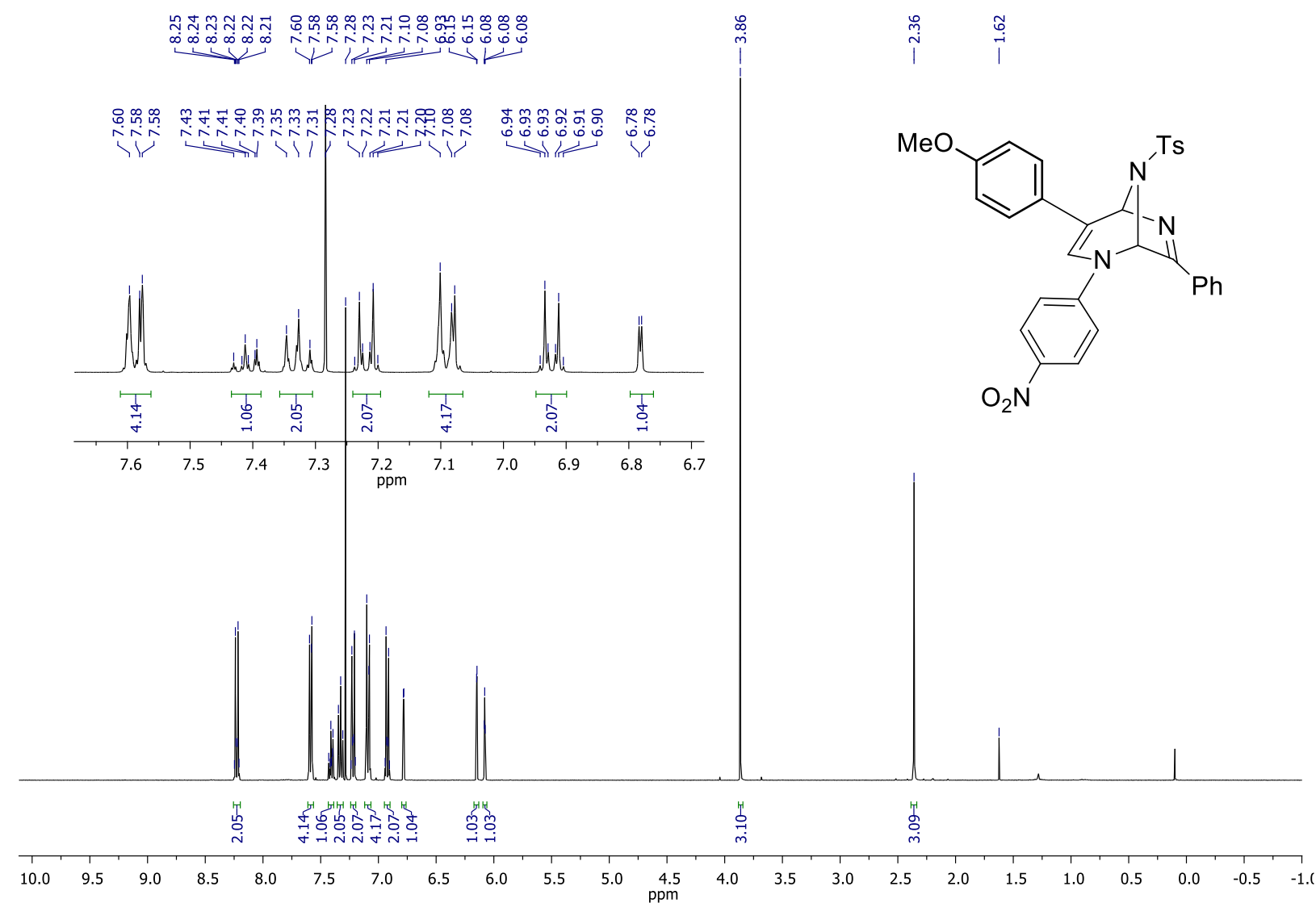

${ }^{13} \mathrm{C}\left\{{ }^{1} \mathrm{H}\right\}$ NMR spectrum of compound $3 \mathbf{m}\left(100 \mathrm{MHz}, \mathrm{CDCl}_{3}\right)$

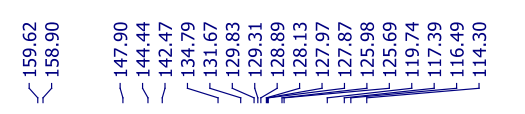<smiles>COc1ccc(C2CN(c3ccc([N+](=O)[O-])cc3)C3C(c4ccccc4)=NC2N3[I-])cc1</smiles>

$\begin{array}{lllllllllll}200 & 190 & 180 & 170 & 160 & 150 & 140 & 130 & 120 & 110 & \begin{array}{l}100 \\ \mathrm{ppm}\end{array}\end{array}$ 
${ }^{1} \mathrm{H}$ NMR spectrum of compound $3 \mathbf{n}\left(400 \mathrm{MHz}, \mathrm{CDCl}_{3}\right)$

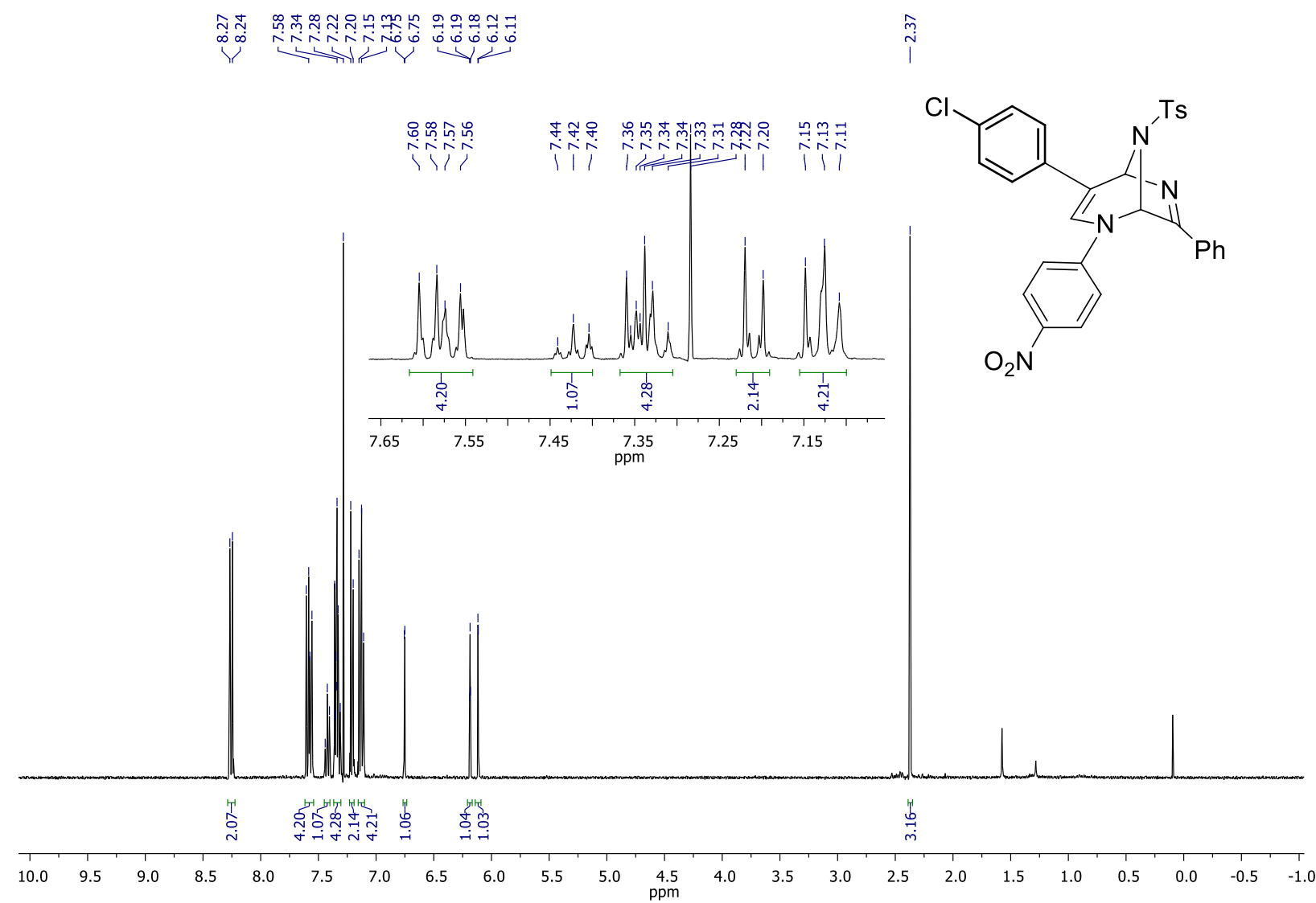

${ }^{13} \mathrm{C}\left\{{ }^{1} \mathrm{H}\right\}$ NMR spectrum of compound 3n $\left(100 \mathrm{MHz}, \mathrm{CDCl}_{3}\right)$

l

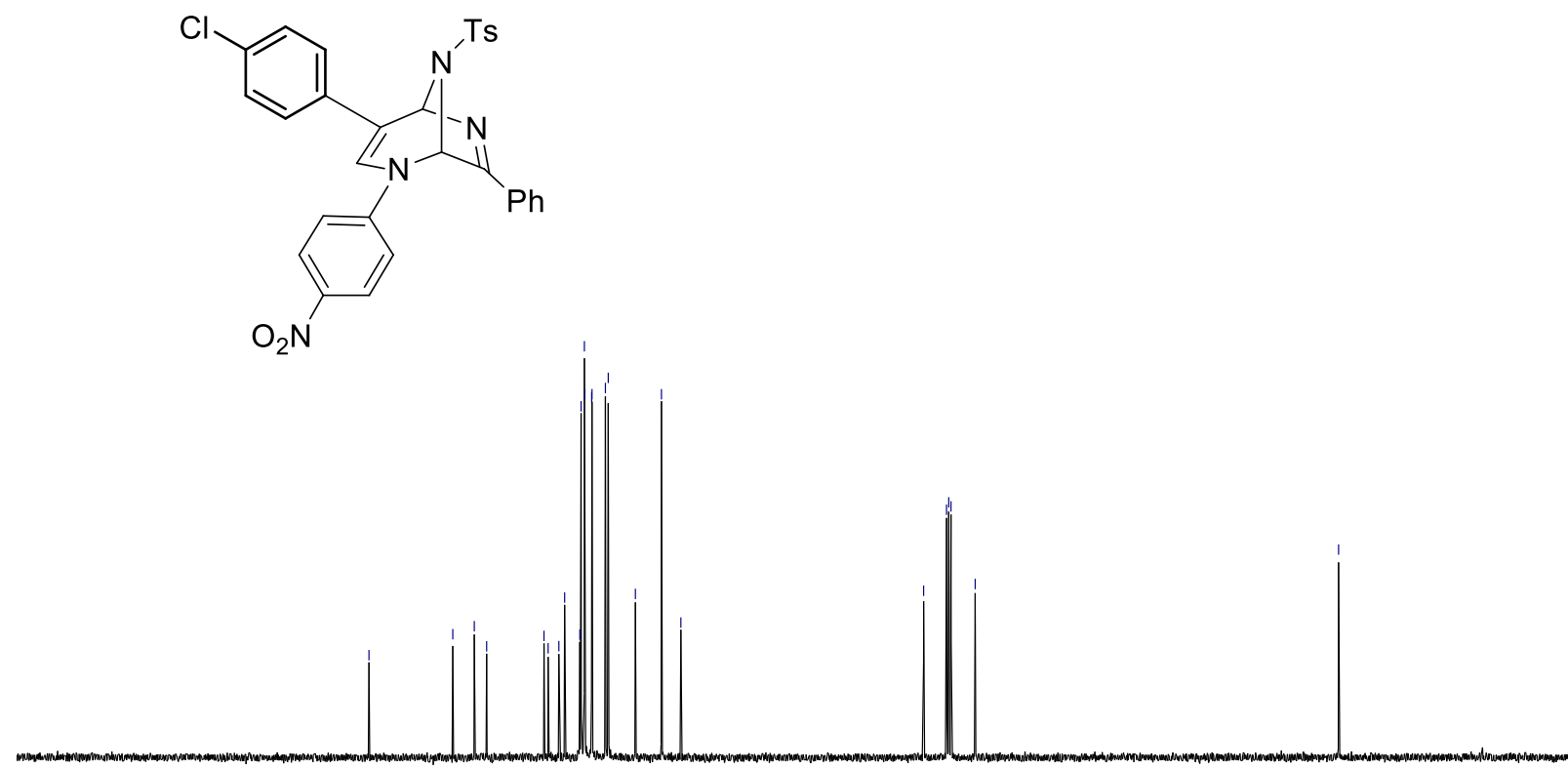

$\begin{array}{lllllllllllllllllllllllll}200 & 190 & 180 & 170 & 160 & 150 & 140 & 130 & 120 & 110 & 100 & 90 & 80 & 70 & 60 & 50 & 40 & 30 & 20 & 10 & 0 & -10\end{array}$ 
${ }^{1} \mathrm{H}$ NMR spectrum of compound $3 \mathbf{0}\left(400 \mathrm{MHz}, \mathrm{CDCl}_{3}\right)$

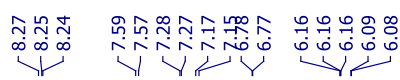

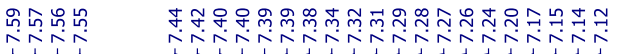

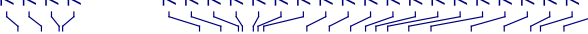

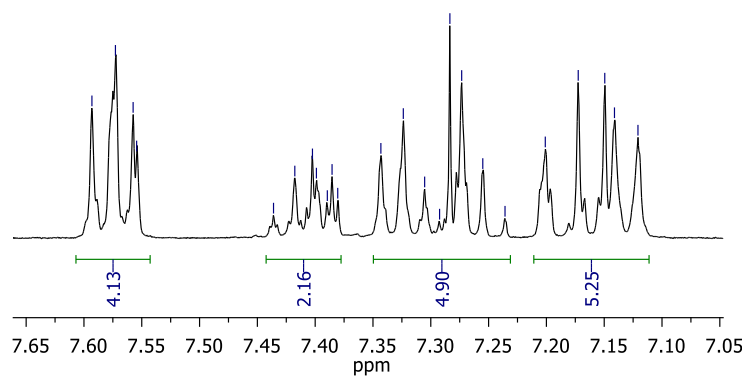

$\begin{array}{lllllllllllll}7.65 & 7.60 & 7.55 & 7.50 & 7.45 & 7.40 & 7.35 & 7.30 & 7.25 & 7.20 & 7.15 & 7.10 & 7.05\end{array}$

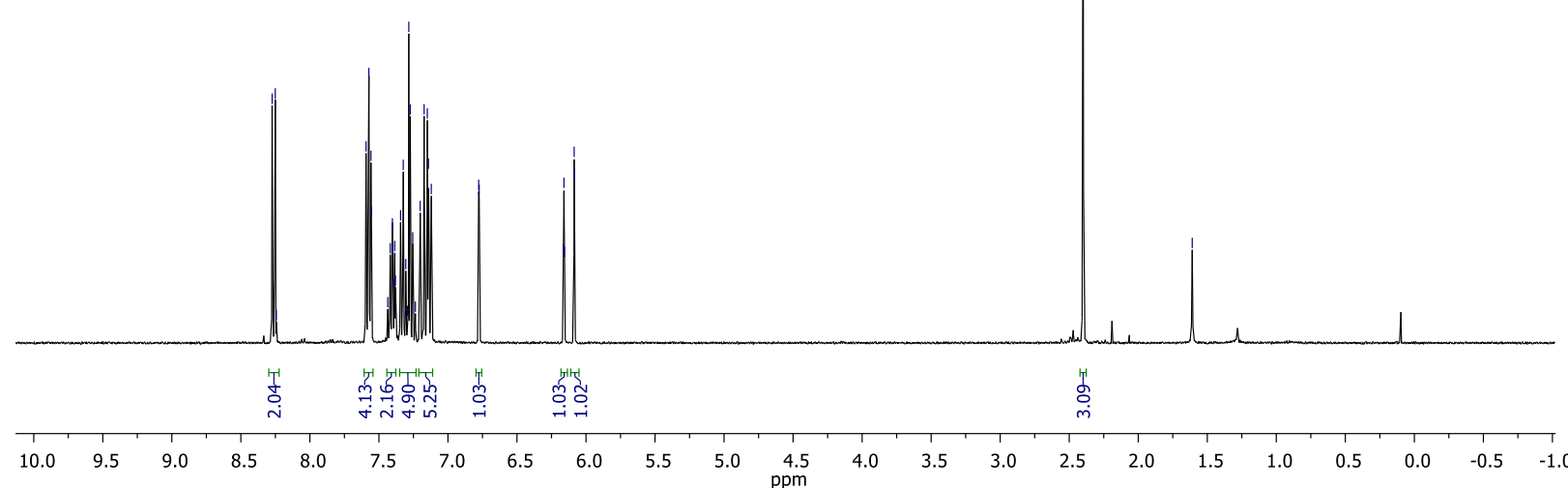

${ }^{13} \mathrm{C}\left\{{ }^{1} \mathrm{H}\right\}$ NMR spectrum of compound $30\left(100 \mathrm{MHz}, \mathrm{CDCl}_{3}\right)$

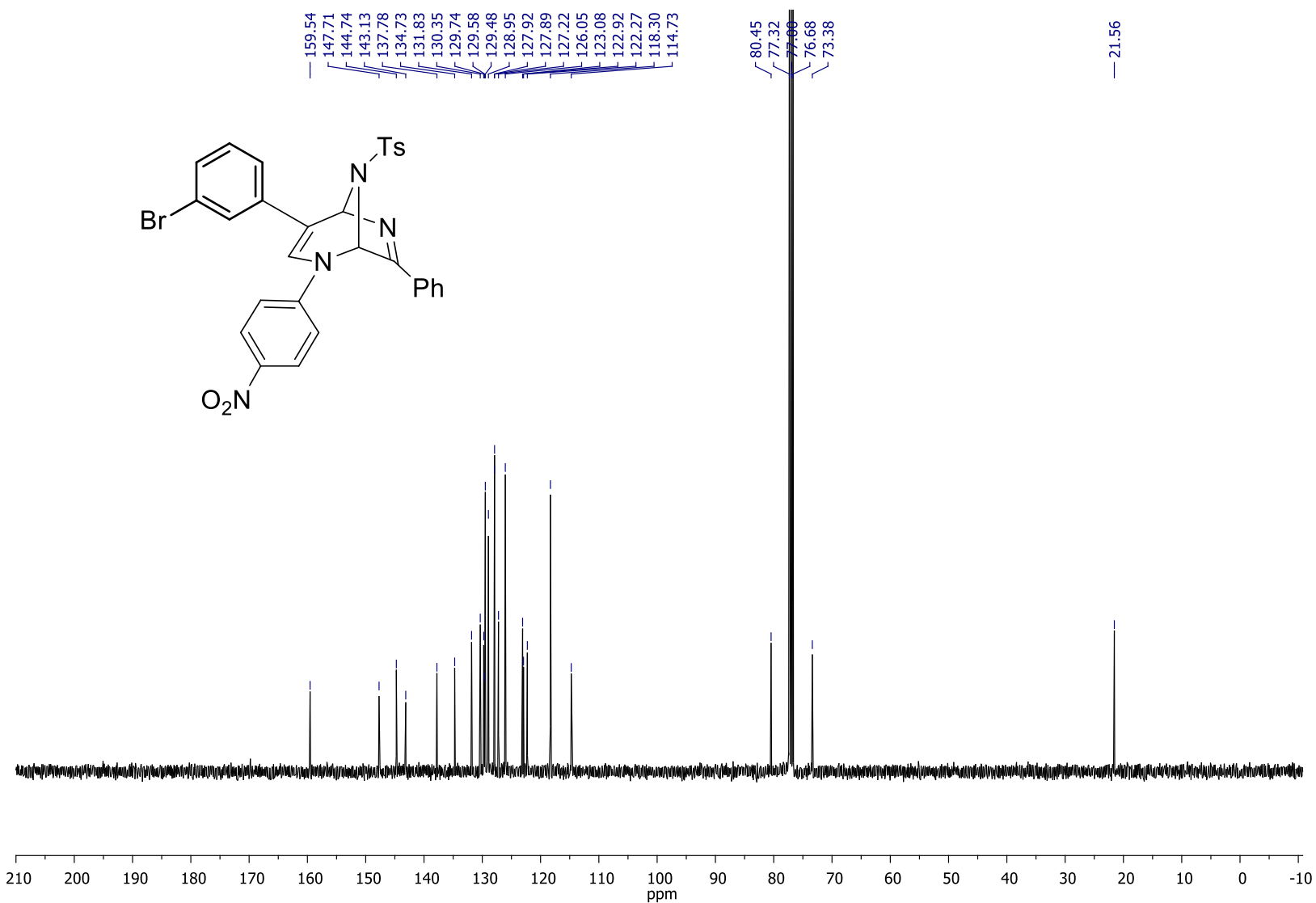

$\stackrel{i}{i} \stackrel{\overrightarrow{0}}{i}$

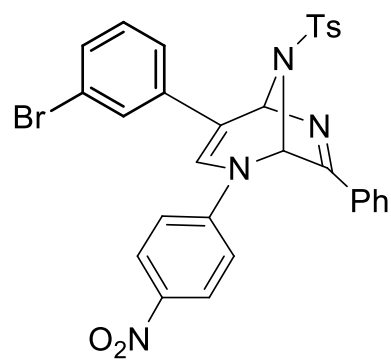


${ }^{1} \mathrm{H}$ NMR spectrum of compound $\mathbf{3 p}\left(400 \mathrm{MHz}, \mathrm{CDCl}_{3}\right)$

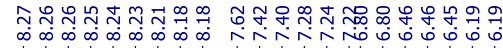

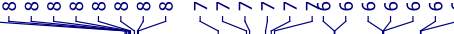

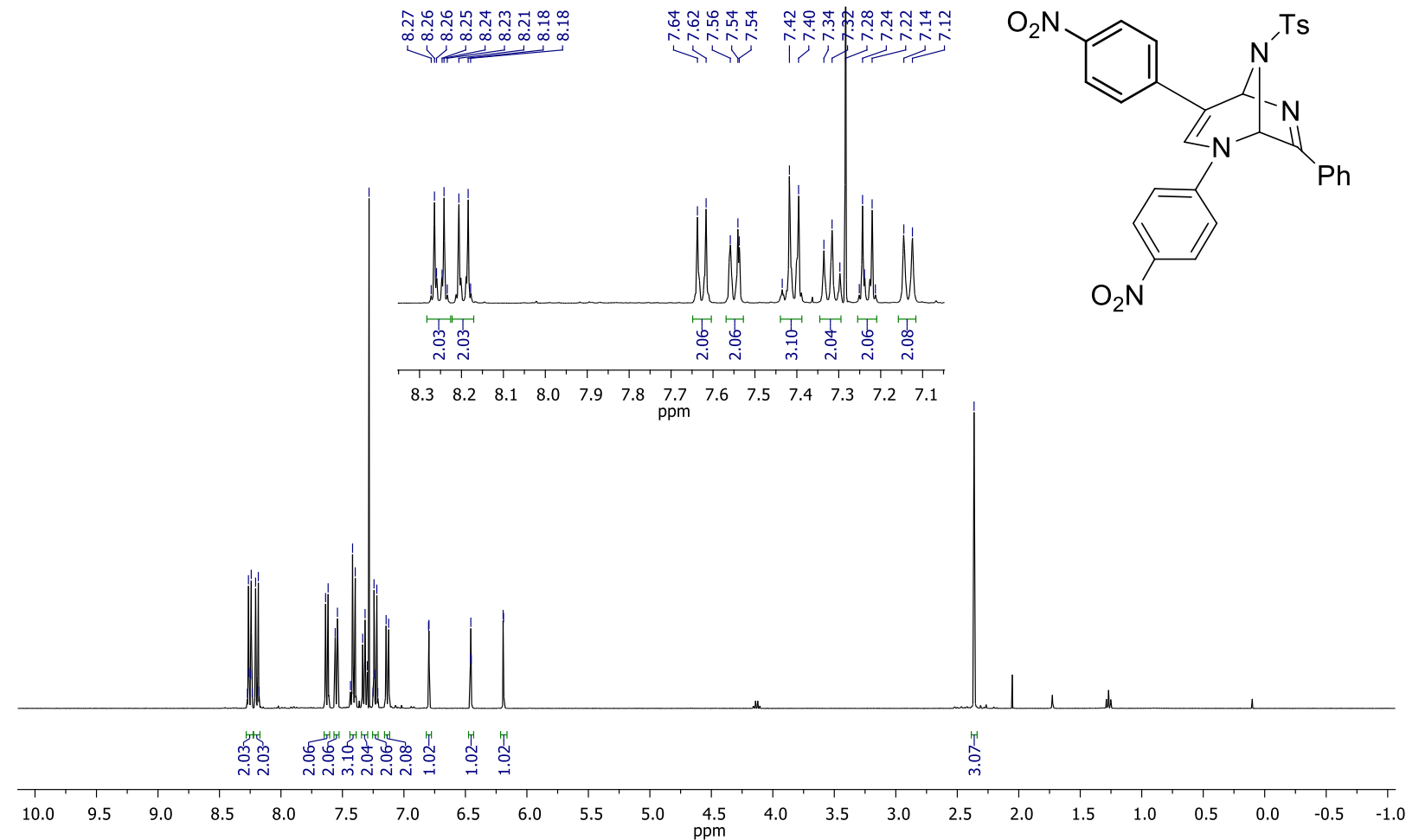

${ }^{13} \mathrm{C}\left\{{ }^{1} \mathrm{H}\right\}$ NMR spectrum of compound $3 \mathbf{p}\left(100 \mathrm{MHz}, \mathrm{CDCl}_{3}\right)$

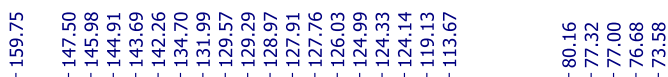

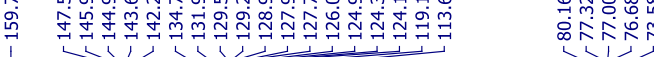
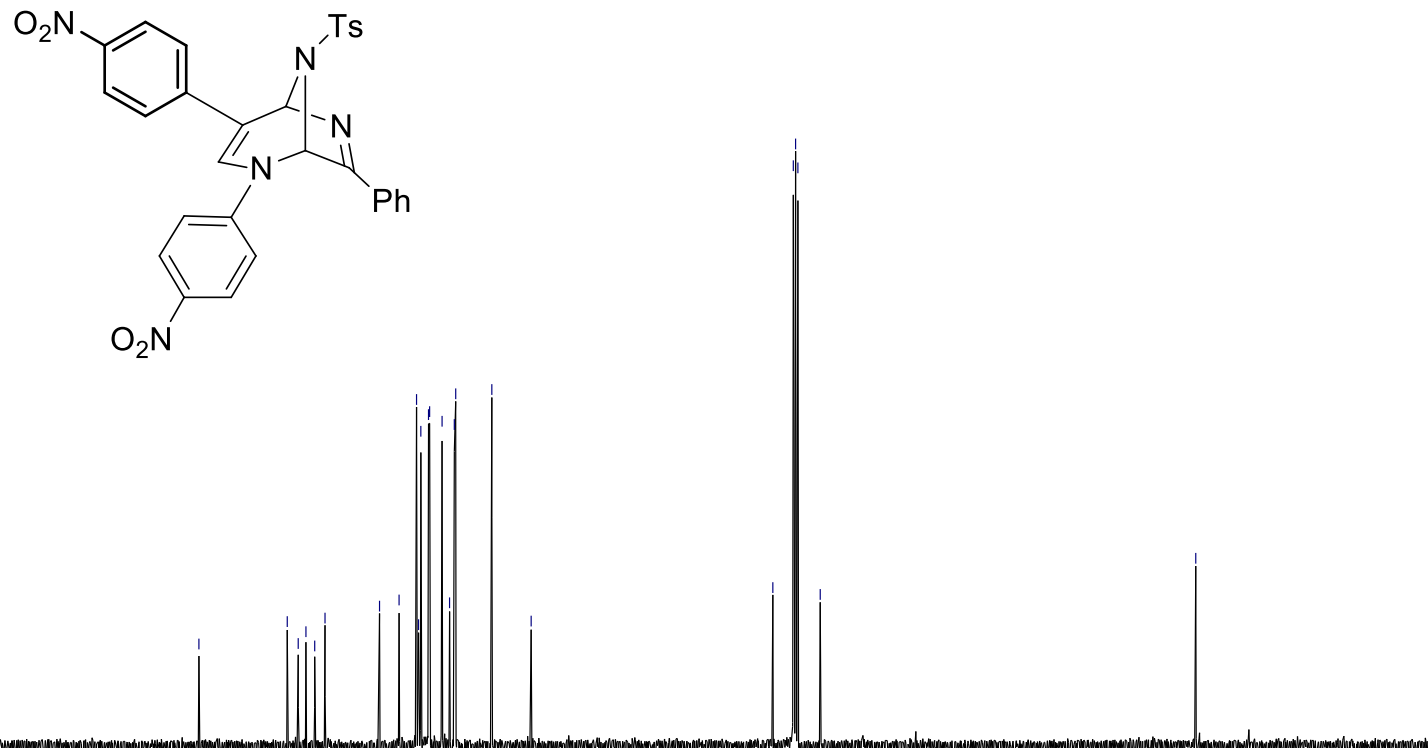

$\begin{array}{llllllllllllllllllllllll}200 & 190 & 180 & 170 & 160 & 150 & 140 & 130 & 120 & 110 & 100 & 90 & 80 & 70 & 60 & 50 & 40 & 30 & 20 & 10 & 0 & -10\end{array}$ 
${ }^{1} \mathrm{H}$ NMR spectrum of compound $\mathbf{3 q}\left(400 \mathrm{MHz}, \mathrm{CDCl}_{3}\right)$
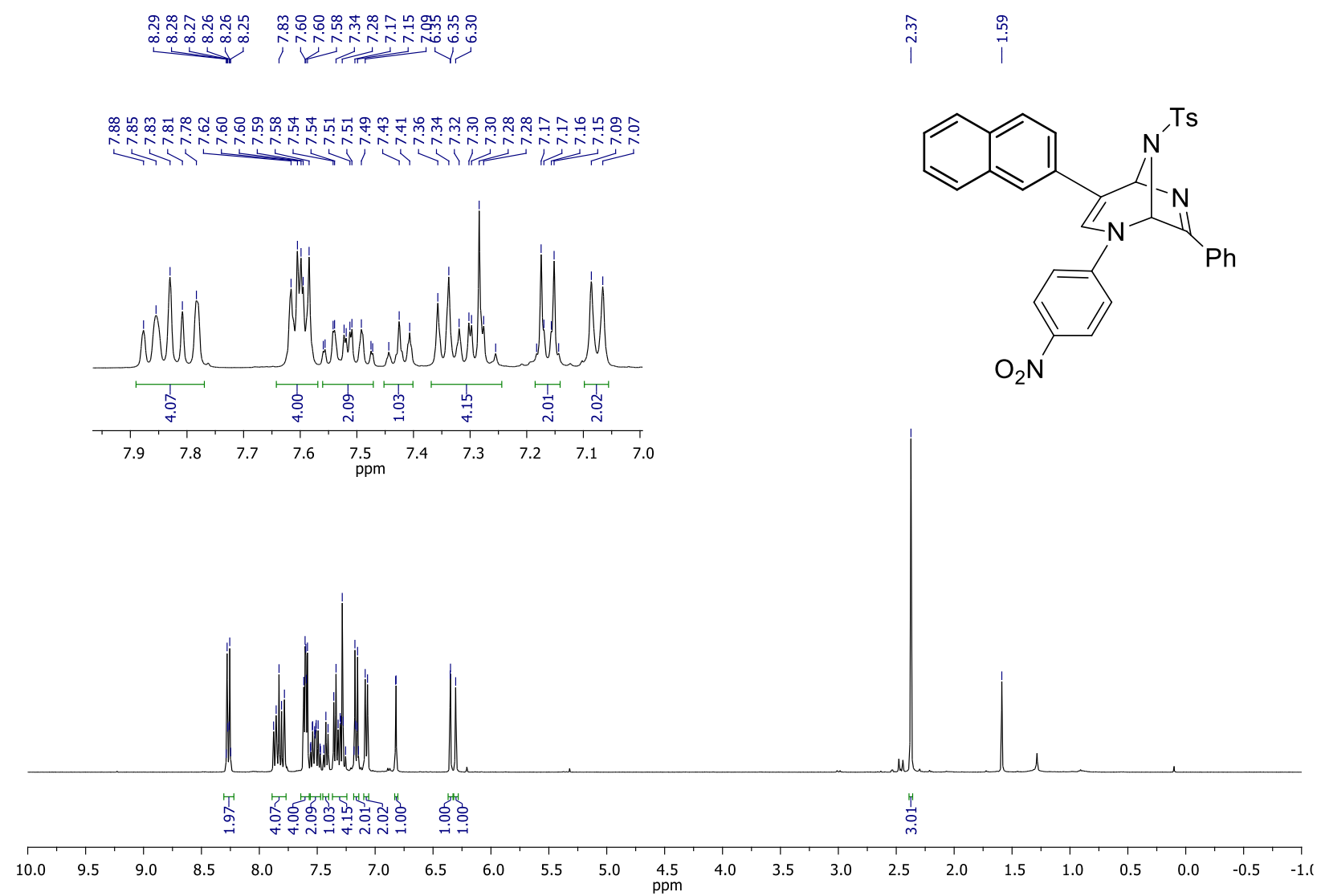

${ }^{13} \mathrm{C}\left\{{ }^{1} \mathrm{H}\right\}$ NMR spectrum of compound $\mathbf{3 q}\left(100 \mathrm{MHz}, \mathrm{CDCl}_{3}\right)$

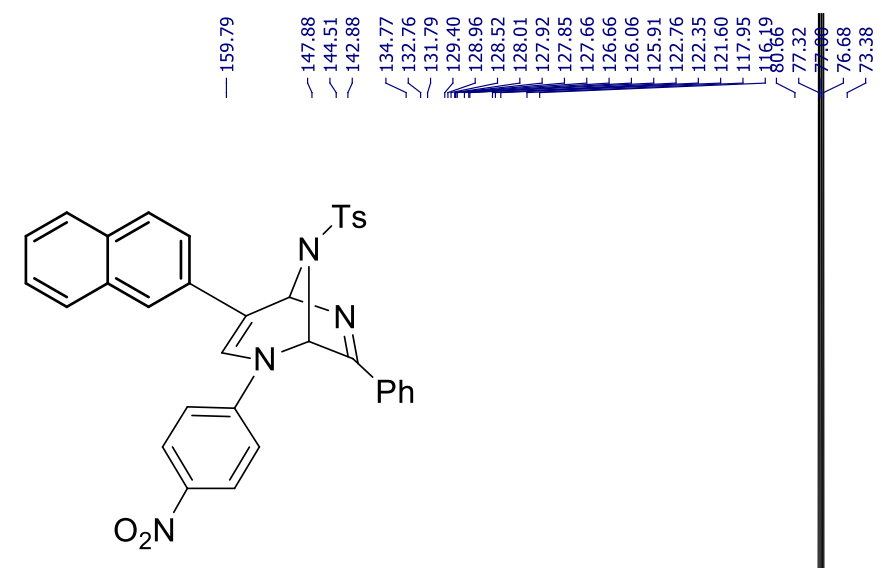

$\stackrel{\text { ñ }}{\text { I }}$

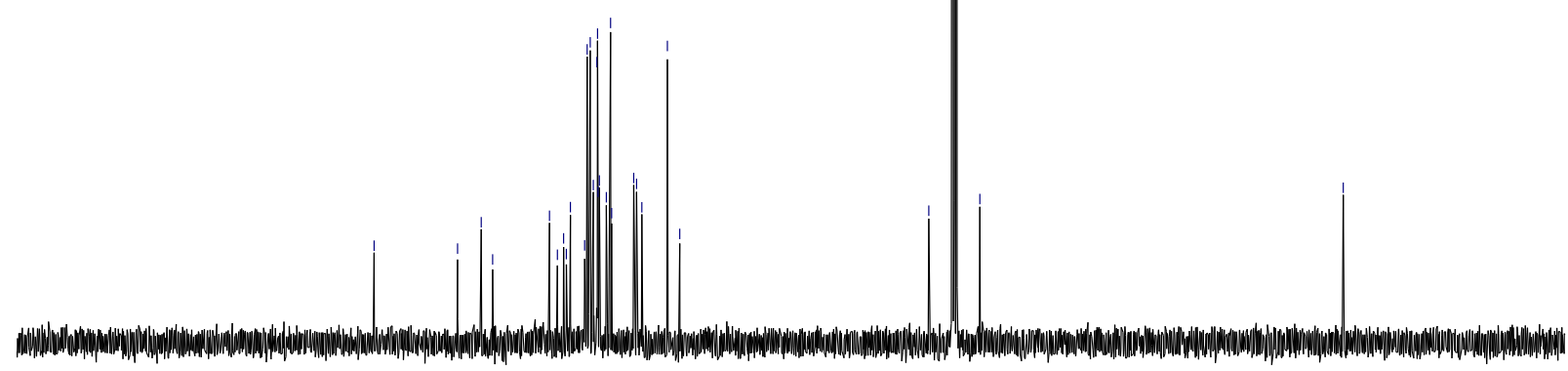

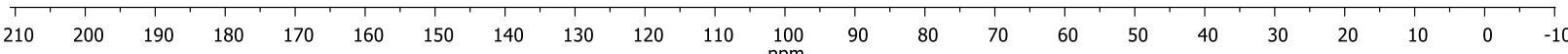


${ }^{1} \mathrm{H}$ NMR spectrum of compound $\mathbf{3 r}\left(400 \mathrm{MHz}, \mathrm{CDCl}_{3}\right)$

لـ
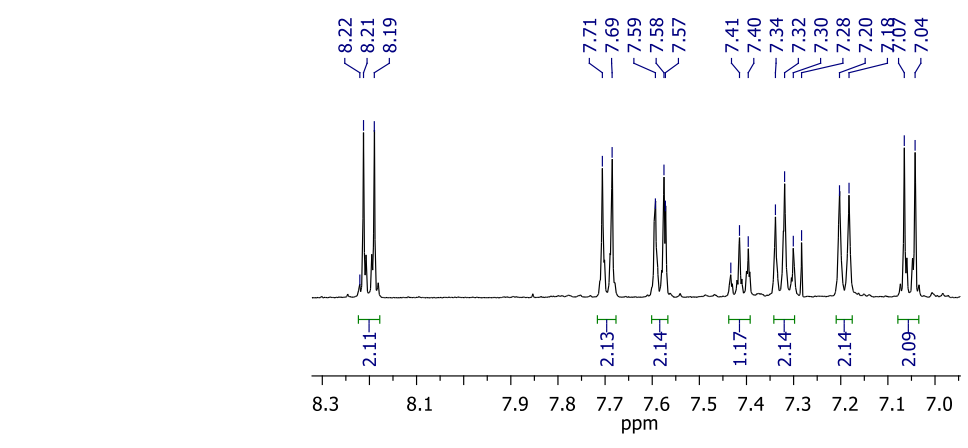

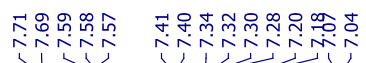

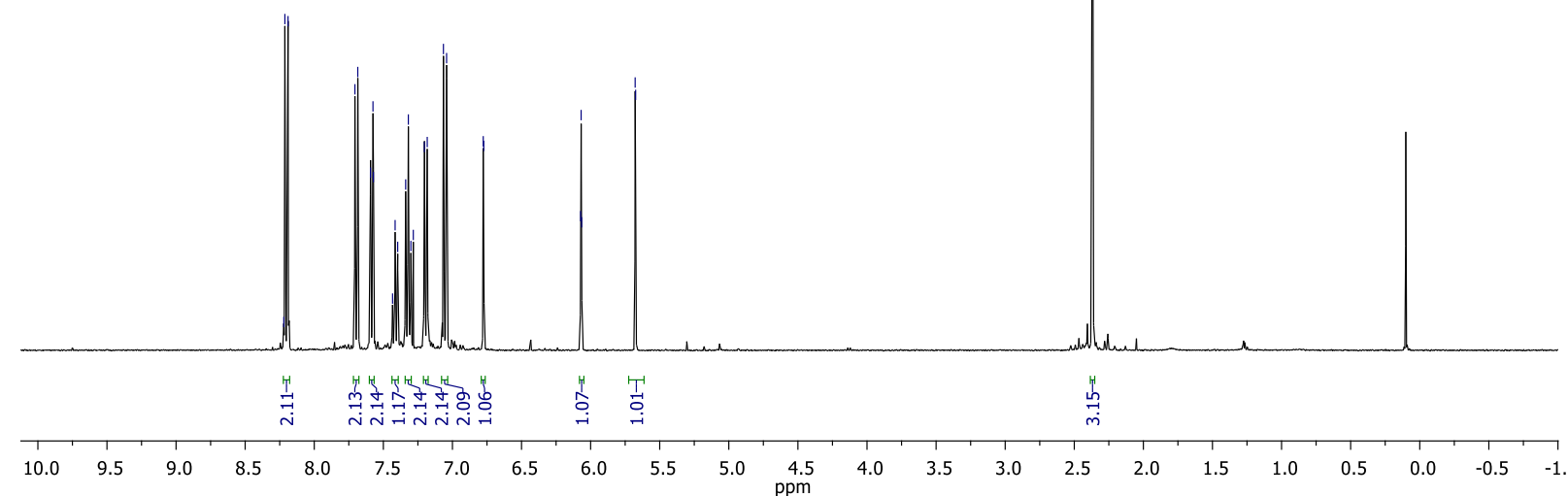

${ }^{13} \mathrm{C}\left\{{ }^{1} \mathrm{H}\right\}$ NMR spectrum of compound $3 \mathbf{r}\left(100 \mathrm{MHz}, \mathrm{CDCl}_{3}\right)$

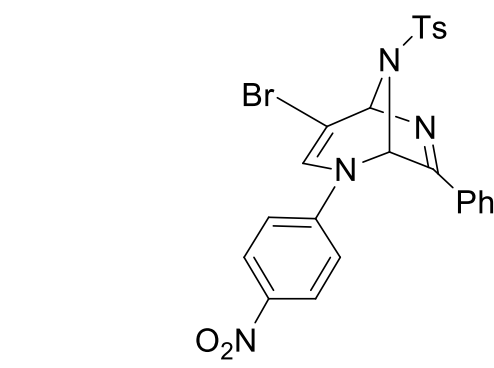

$210 \quad 200 \quad 190$

$180 \quad 170 \quad 160$

$150 \quad 140$

130

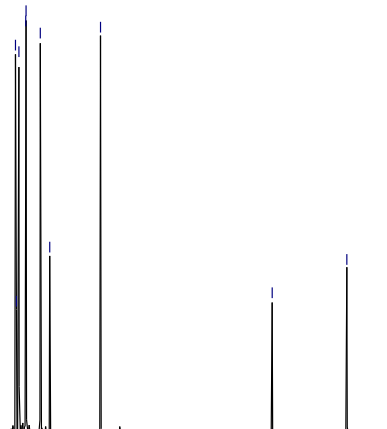


${ }^{1} \mathrm{H}$ NMR spectrum of compound $\mathbf{3 s}\left(400 \mathrm{MHz}, \mathrm{CDCl}_{3}\right)$

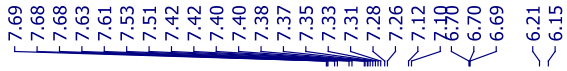

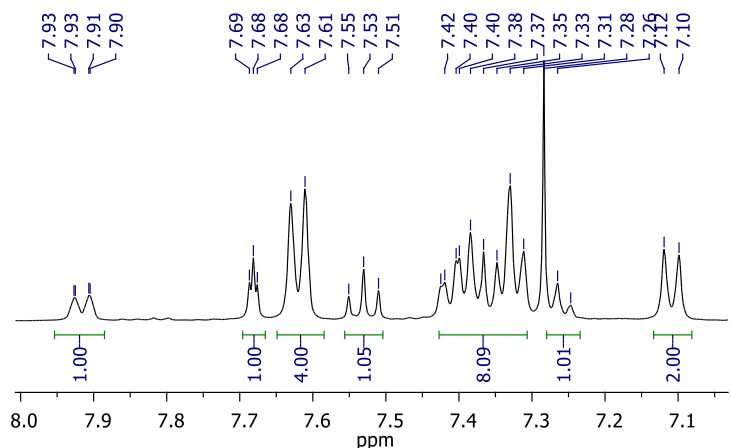

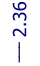

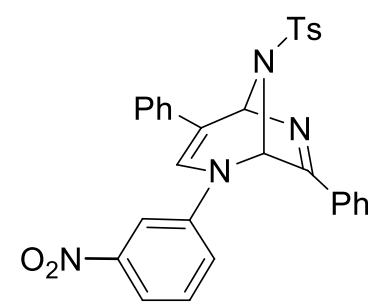

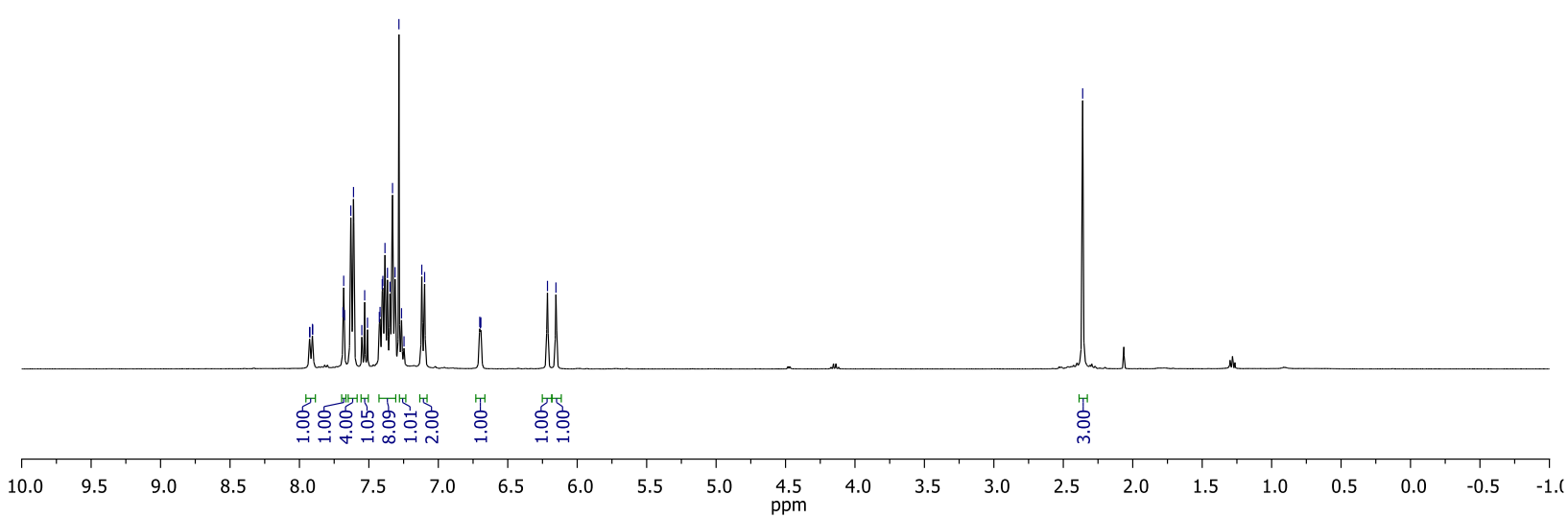

${ }^{13} \mathrm{C}\left\{{ }^{1} \mathrm{H}\right\}$ NMR spectrum of compound 3s $\left(100 \mathrm{MHz}, \mathrm{CDCl}_{3}\right)$

\section{荡

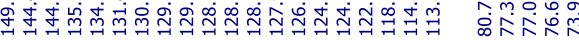

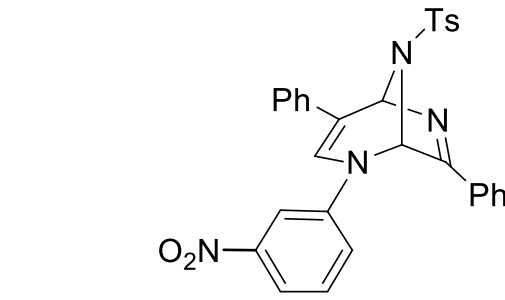


${ }^{1} \mathrm{H}$ NMR spectrum of compound $\mathbf{3 t}\left(400 \mathrm{MHz}, \mathrm{CDCl}_{3}\right)$

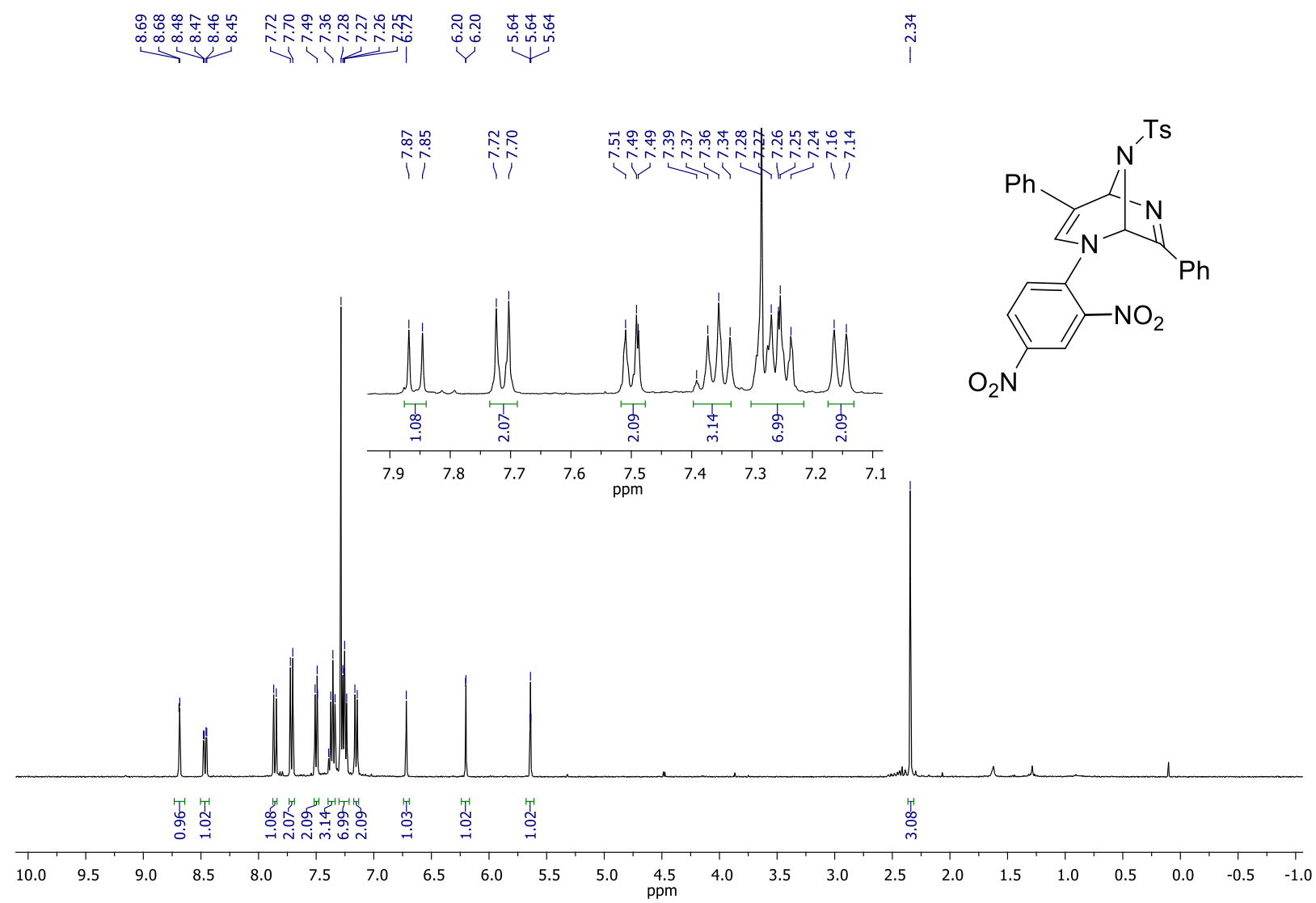

${ }^{13} \mathrm{C}\left\{{ }^{1} \mathrm{H}\right\}$ NMR spectrum of compound $3 \mathrm{t}\left(100 \mathrm{MHz}, \mathrm{CDCl}_{3}\right)$

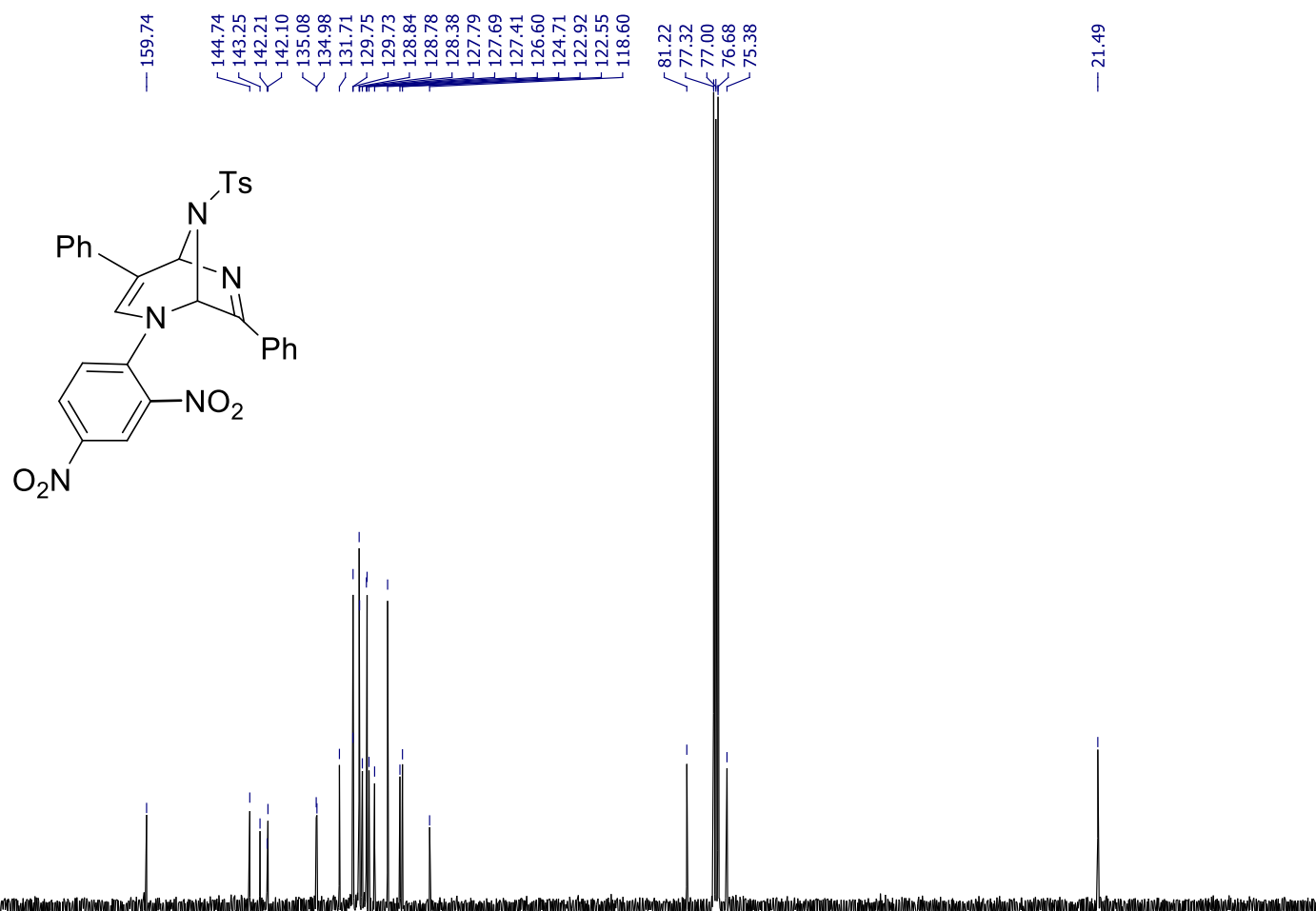

$\begin{array}{lllllllllllllllllllllllll}210 & 200 & 190 & 180 & 170 & 160 & 150 & 140 & 130 & 120 & 110 & 100 & 90 & 80 & 70 & 60 & 50 & 40 & 30 & 20 & 10 & 0 & -10\end{array}$ 
${ }^{1} \mathrm{H}$ NMR spectrum of compound $3 \mathbf{u}\left(400 \mathrm{MHz}, \mathrm{CDCl}_{3}\right)$

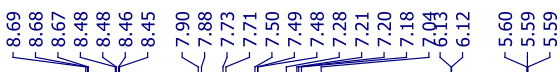

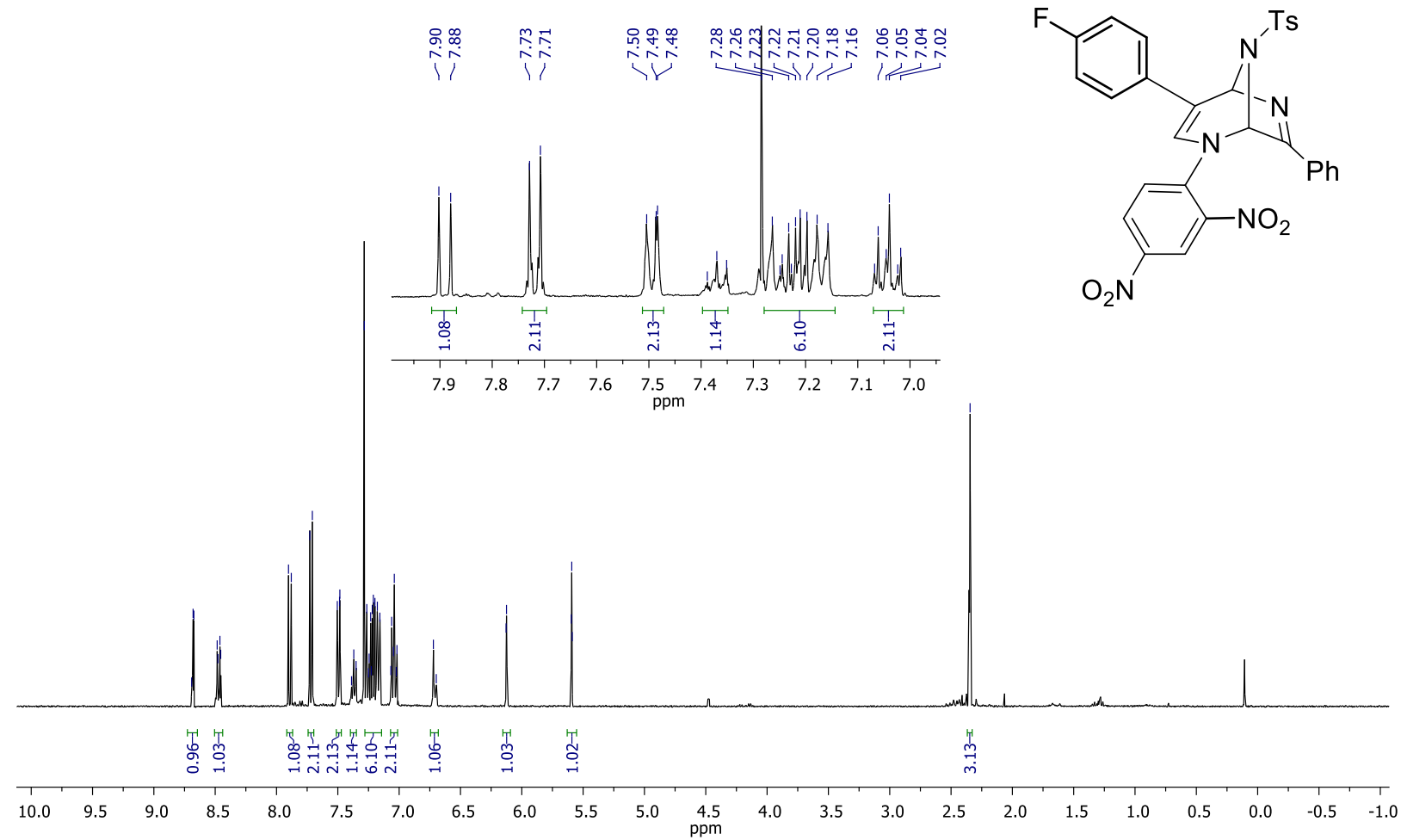

${ }^{13} \mathrm{C}\left\{{ }^{1} \mathrm{H}\right\}$ NMR spectrum of compound $3 \mathbf{u}\left(100 \mathrm{MHz}, \mathrm{CDCl}_{3}\right)$

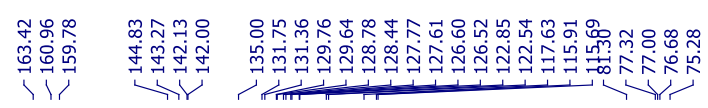<smiles>O=[N+]([O-])c1ccc(N2CC(c3ccc(F)cc3)C3C(c4ccccc4)=NC2N3[As])c([N+](=O)[O-])c1</smiles>
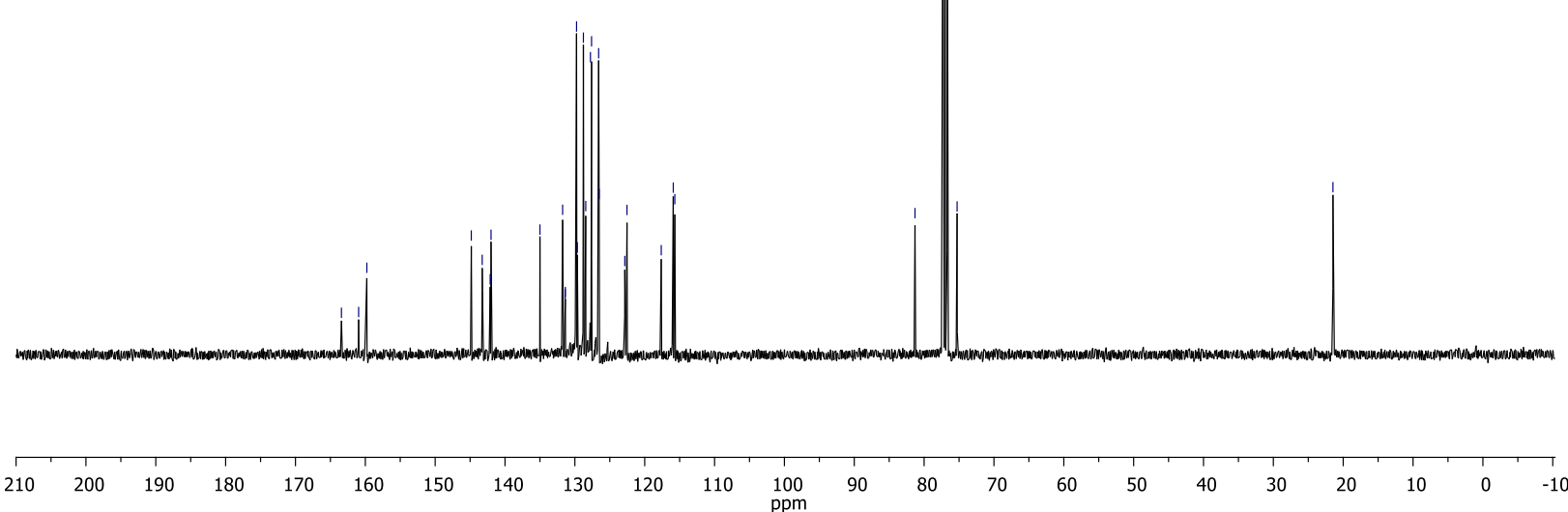
${ }^{1} \mathrm{H}$ NMR spectrum of compound $\mathbf{3 v}\left(400 \mathrm{MHz}, \mathrm{CDCl}_{3}\right)$

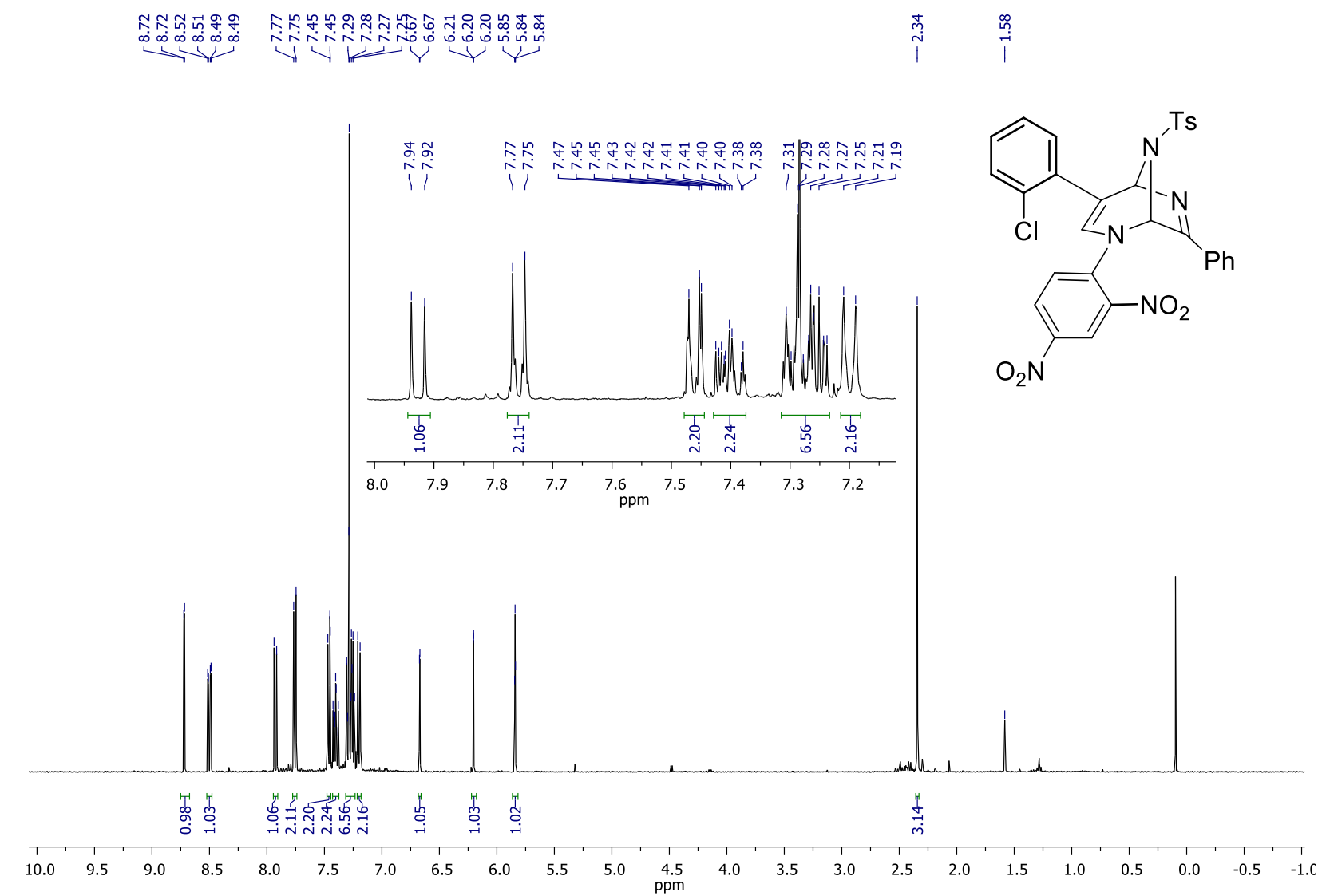

${ }^{13} \mathrm{C}\left\{{ }^{1} \mathrm{H}\right\}$ NMR spectrum of compound $\mathbf{3 v}\left(100 \mathrm{MHz}, \mathrm{CDCl}_{3}\right)$

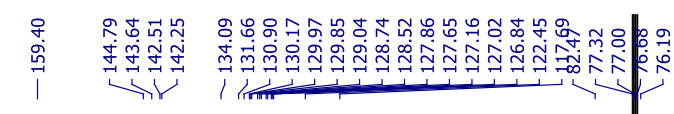<smiles>O=[N+]([O-])c1ccc(N2C=C(c3ccccc3Cl)C3C(c4ccccc4)=NC2N3[AlH2])c([N+](=O)[O-])c1</smiles>

$\begin{array}{lllllllllll}200 & 190 & 180 & 170 & 160 & 150 & 140 & 130 & 120 & 110 & \begin{array}{l}100 \\ \text { ppm }\end{array}\end{array}$ 
${ }^{1} \mathrm{H}$ NMR spectrum of compound $\mathbf{3 w}\left(400 \mathrm{MHz}, \mathrm{CDCl}_{3}\right)$

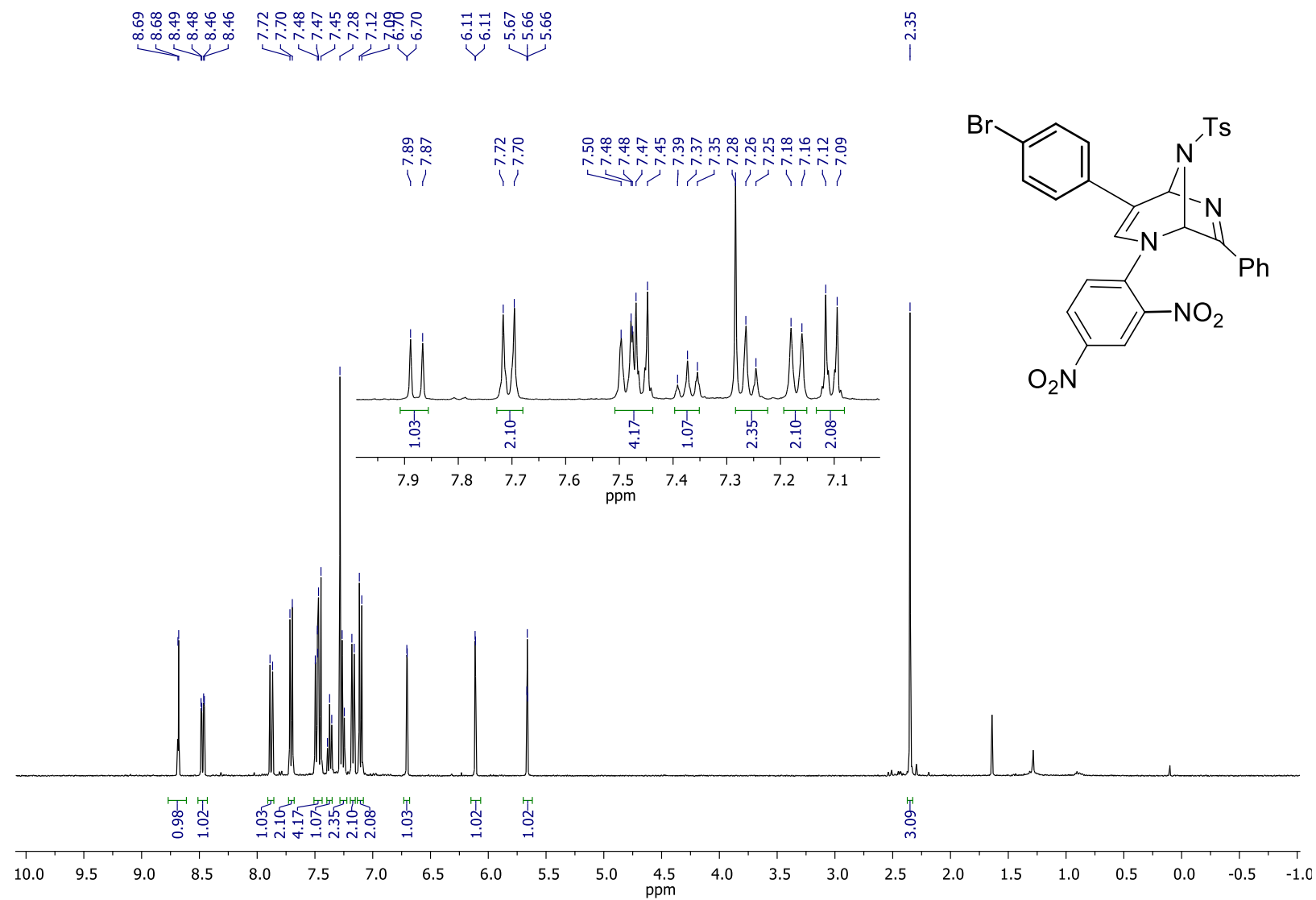

${ }^{13} \mathrm{C}\left\{{ }^{1} \mathrm{H}\right\}$ NMR spectrum of compound 3w $\left(100 \mathrm{MHz}, \mathrm{CDCl}_{3}\right)$

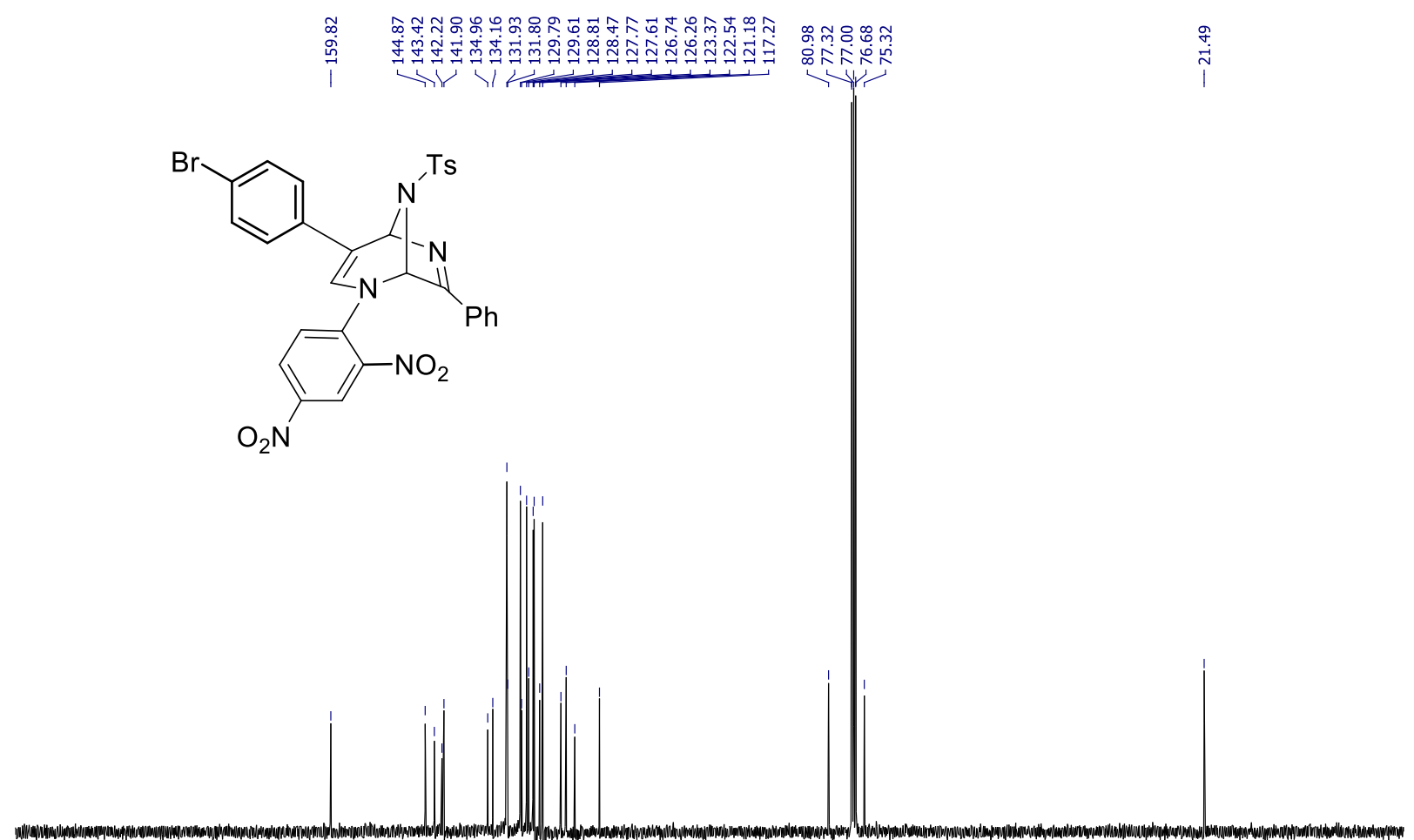

$\begin{array}{llllllllllllllllllllllllllll}200 & 190 & 180 & 170 & 160 & 150 & 140 & 130 & 120 & 110 & 100 & 90 & 80 & 70 & 60 & 50 & 40 & 30 & 20 & 10 & 0 & -10\end{array}$ 
${ }^{1} \mathrm{H}$ NMR spectrum of compound $\mathbf{3 x}\left(400 \mathrm{MHz}, \mathrm{CDCl}_{3}\right)$

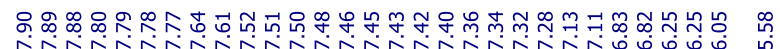

$\stackrel{\stackrel{n}{i}}{i} \stackrel{\stackrel{0}{\leftrightarrow}}{i}$

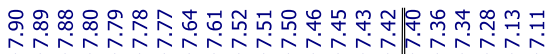

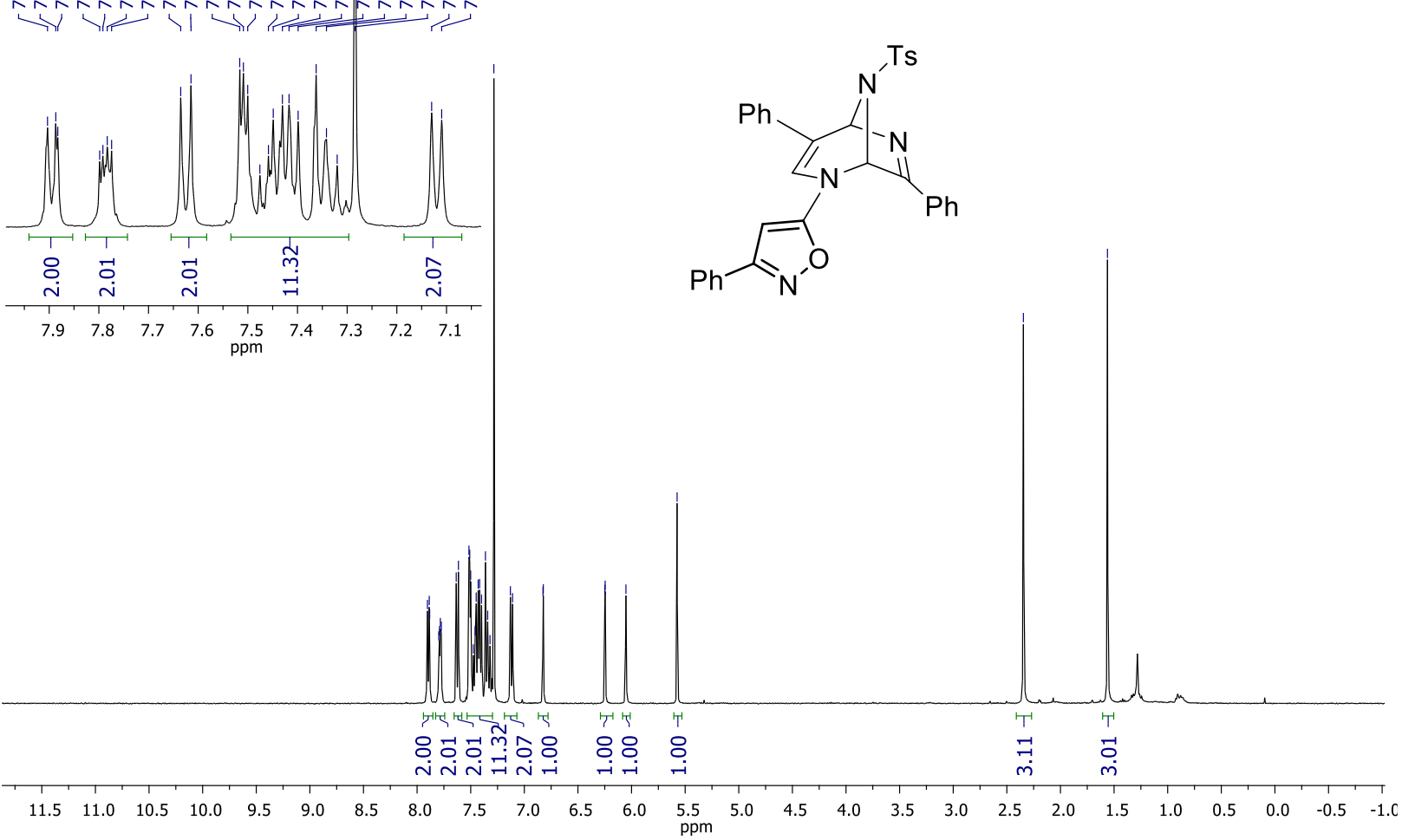

${ }^{13} \mathrm{C}\left\{{ }^{1} \mathrm{H}\right\}$ NMR spectrum of compound $\mathbf{3 x}\left(100 \mathrm{MHz}, \mathrm{CDCl}_{3}\right)$
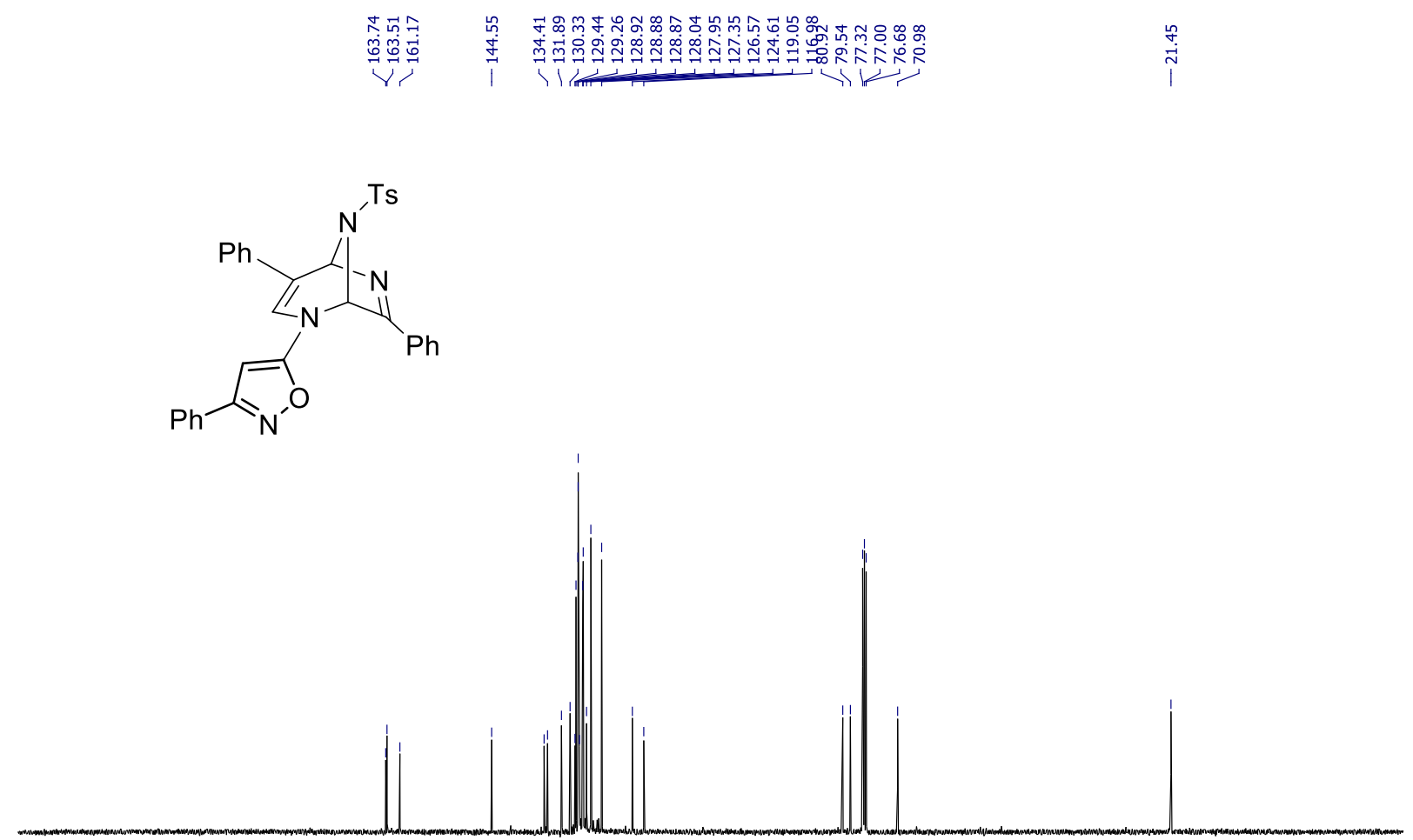

$\begin{array}{llllllllllllllllllllllllllllllll}230 & 220 & 210 & 200 & 190 & 180 & 170 & 160 & 150 & 140 & 130 & 120 & 110 & 100 & 90 & 80 & 70 & 60 & 50 & 40 & 30 & 20 & 10 & 0 & -10 & -20\end{array}$ 
${ }^{1} \mathrm{H}$ NMR spectrum of compound $\mathbf{3 y}\left(400 \mathrm{MHz}, \mathrm{CDCl}_{3}\right)$

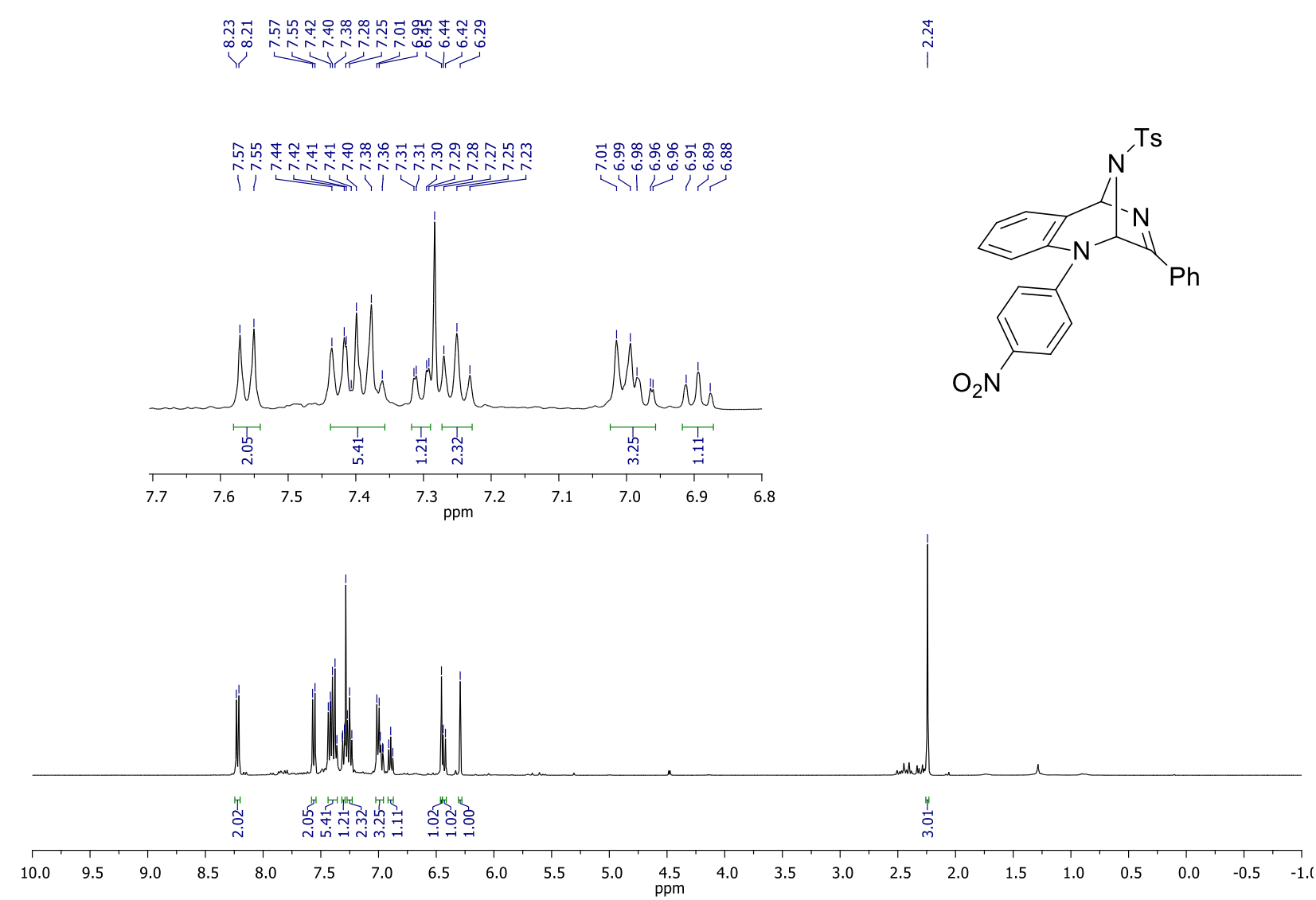

${ }^{13} \mathrm{C}\left\{{ }^{1} \mathrm{H}\right\} \mathrm{NMR}$ spectrum of compound $\mathbf{3 y}\left(100 \mathrm{MHz}, \mathrm{CDCl}_{3}\right)$
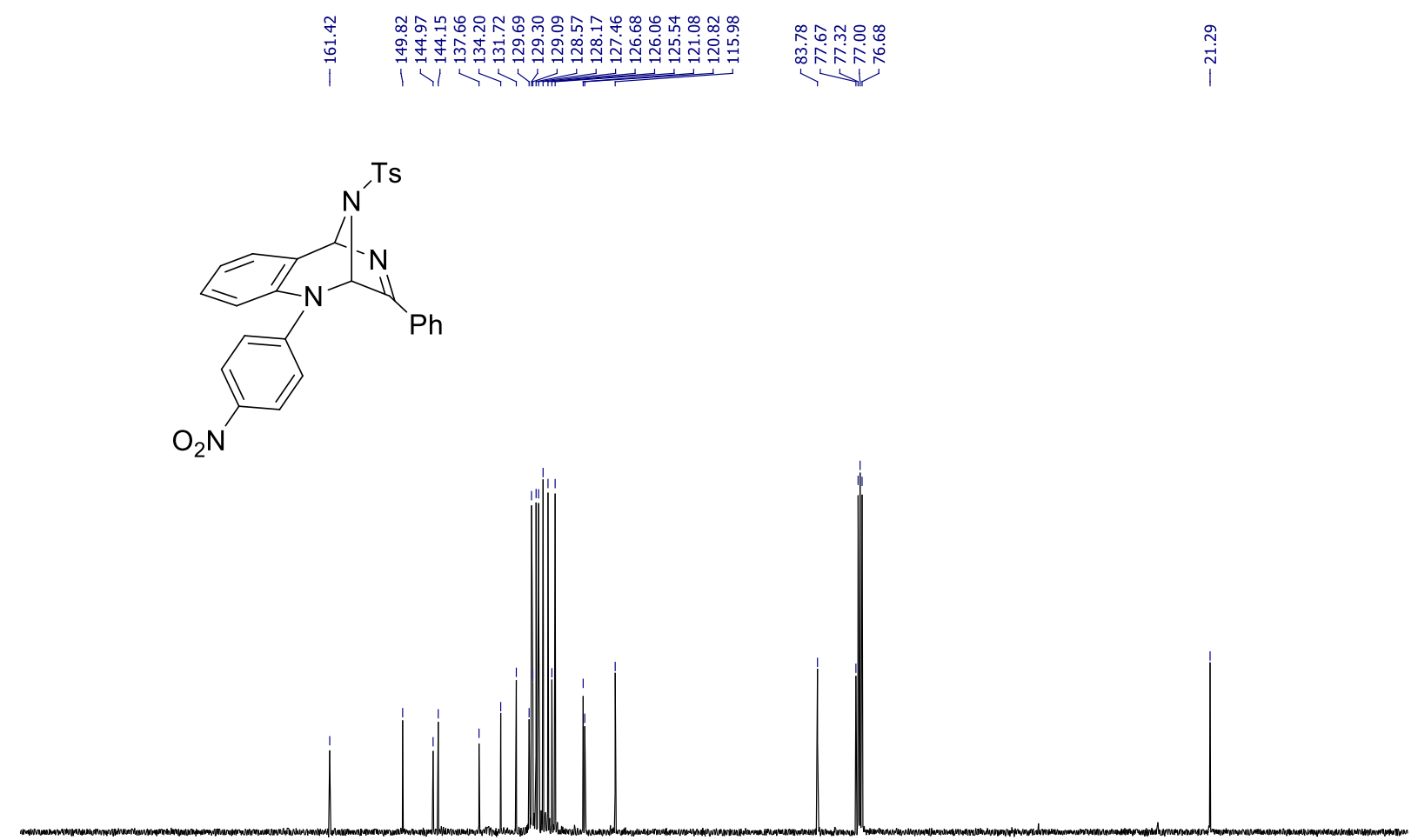

$\begin{array}{lllllllllllllllllllllllllllll}1 & 210 & 200 & 190 & 180 & 170 & 160 & 150 & 140 & 130 & 120 & 110 & 100 & 90 & 80 & 70 & 60 & 50 & 40 & 30 & 20 & 10 & 0 & -10\end{array}$ 
${ }^{1} \mathrm{H}$ NMR spectrum of compound $\mathbf{3 z}\left(400 \mathrm{MHz}, \mathrm{CDCl}_{3}\right)$

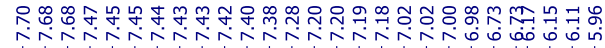

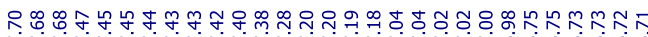
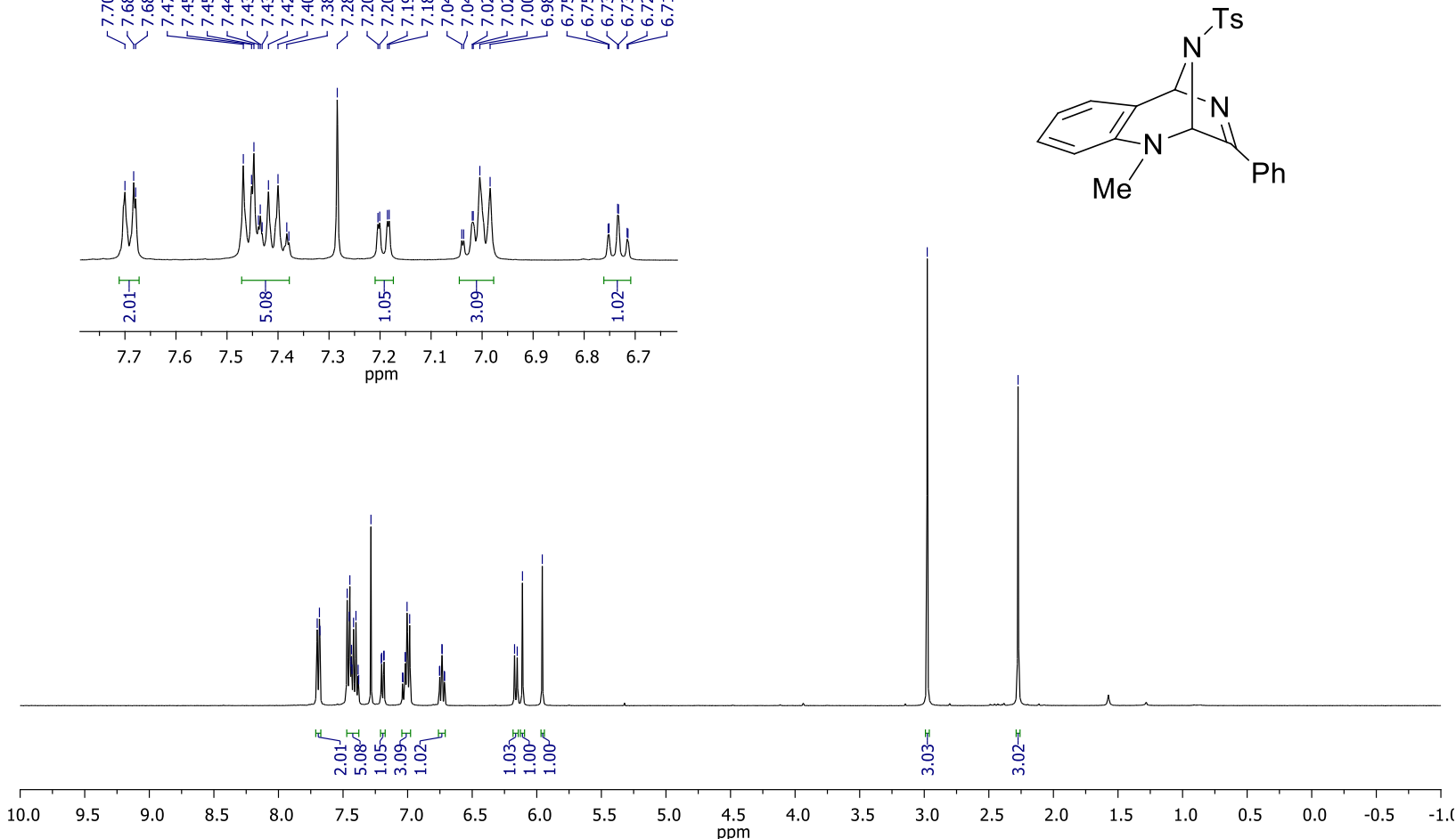

${ }^{13} \mathrm{C}\left\{{ }^{1} \mathrm{H}\right\}$ NMR spectrum of compound $\mathbf{3 z}\left(100 \mathrm{MHz}, \mathrm{CDCl}_{3}\right)$

চ

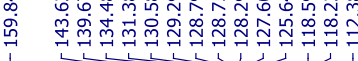

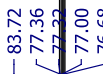

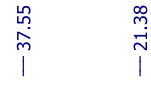
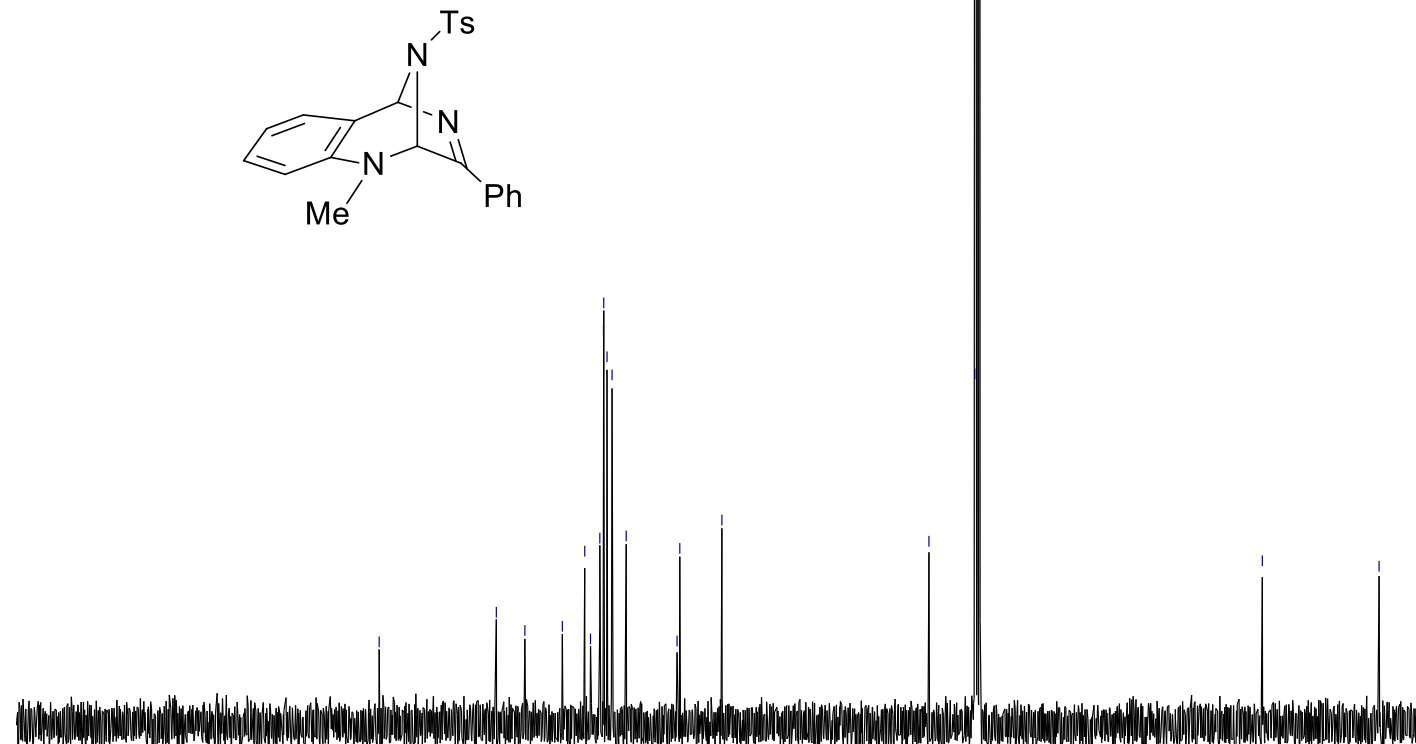

$\begin{array}{lllllllllllllllllllllllllll}210 & 200 & 190 & 180 & 170 & 160 & 150 & 140 & 130 & 120 & 110 & 100 & 90 & 80 & 70 & 60 & 50 & 40 & 30 & 20 & 10 & 0 & -10\end{array}$ 
${ }^{1} \mathrm{H}$ NMR spectrum of compound $3 z a\left(400 \mathrm{MHz}, \mathrm{CDCl}_{3},-40{ }^{\circ} \mathrm{C}\right.$ )

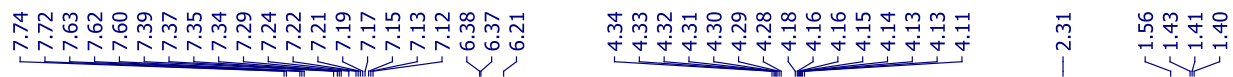
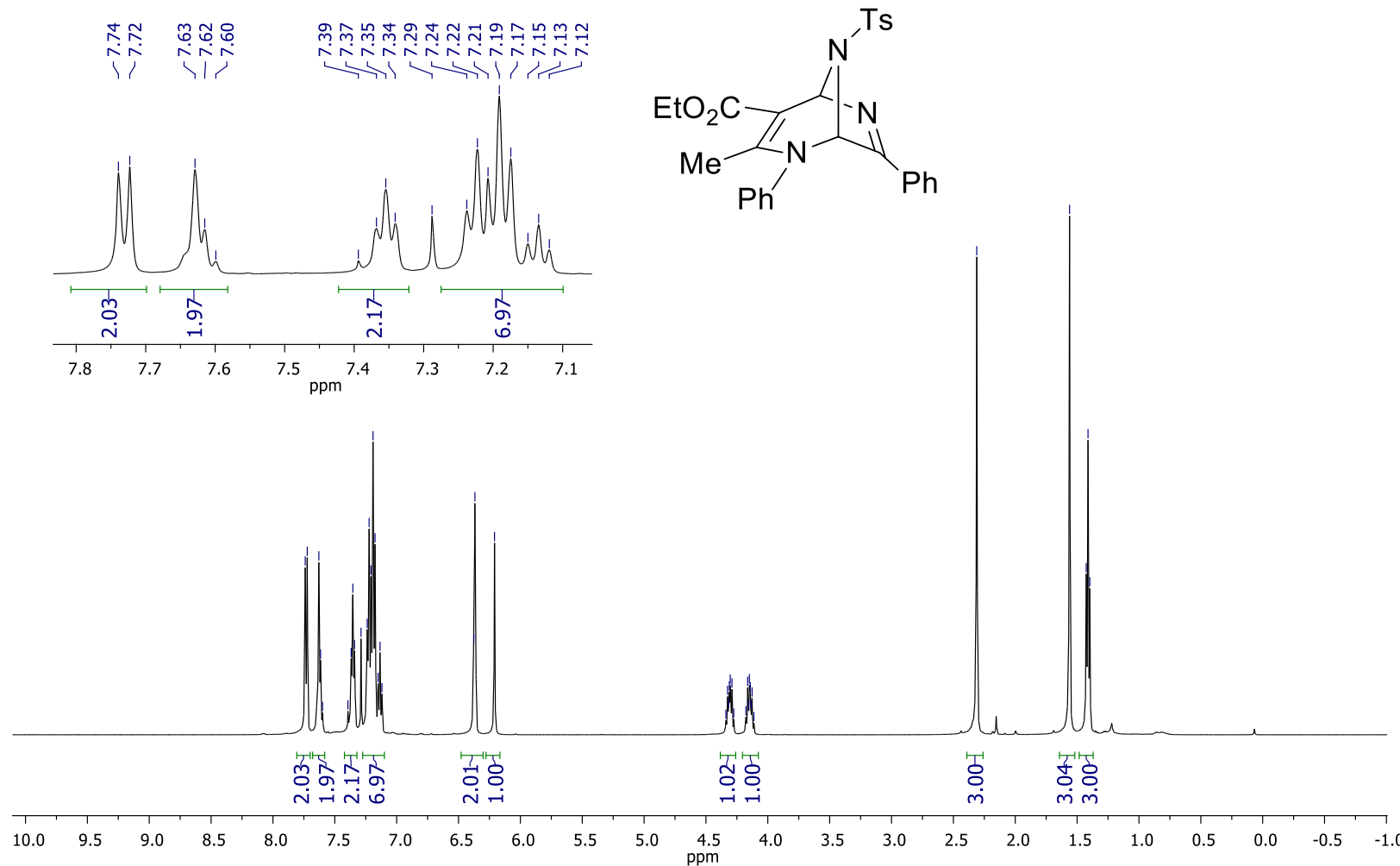

${ }^{1} \mathrm{H}$ NMR spectrum of compound 3za (400 $\mathrm{MHz}, \mathrm{CDCl}_{3}, 20{ }^{\circ} \mathrm{C}$ )
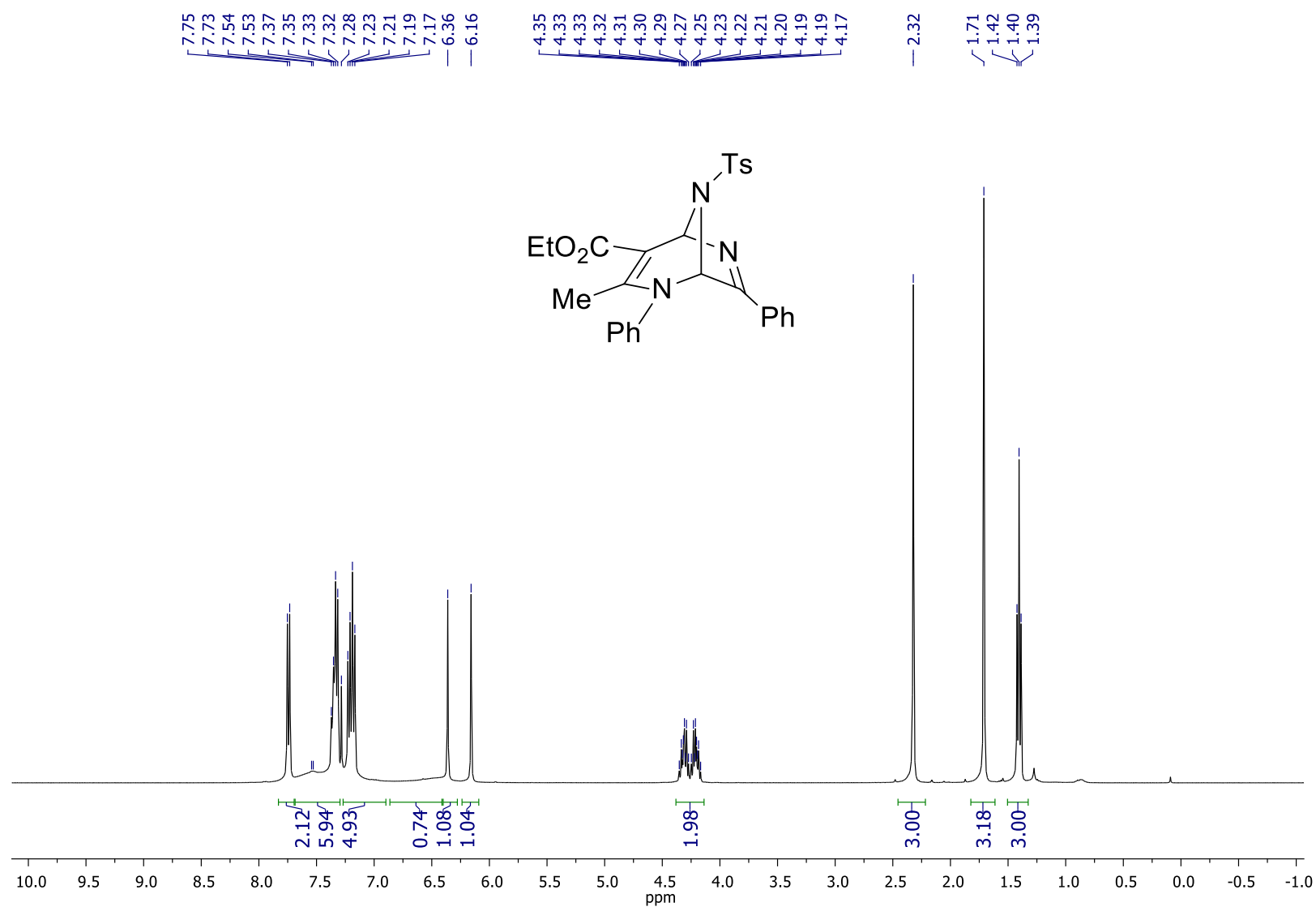
${ }^{13} \mathrm{C}\left\{{ }^{1} \mathrm{H}\right\}$ NMR spectrum of compound $3 z a\left(100 \mathrm{MHz}, \mathrm{CDCl}_{3},-40{ }^{\circ} \mathrm{C}\right)$

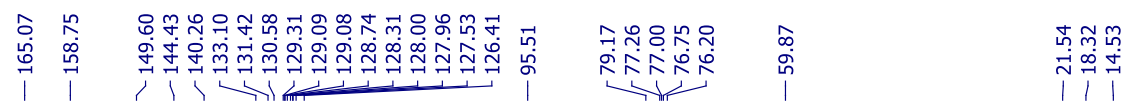
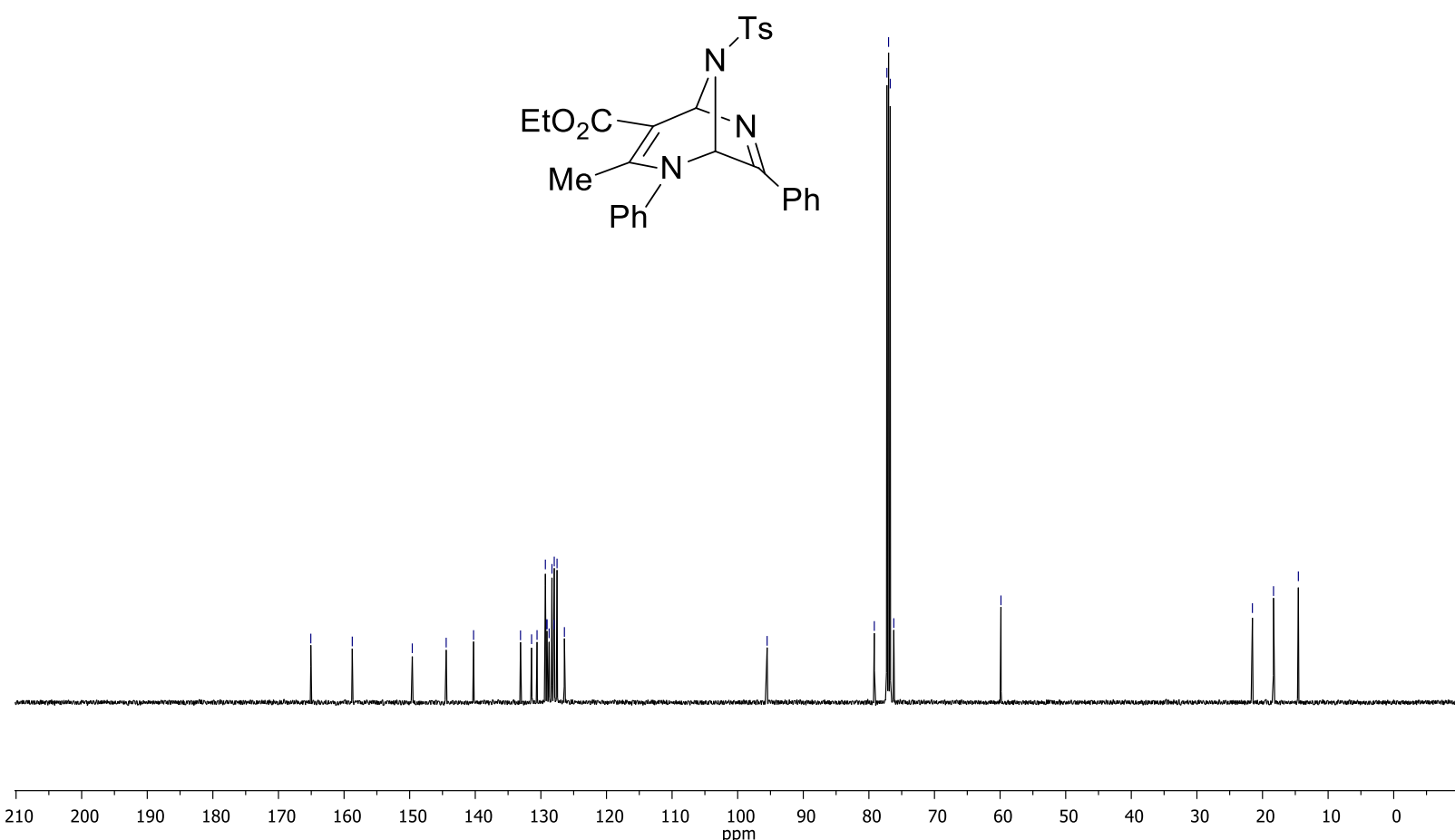

${ }^{1} \mathrm{H}-{ }^{13} \mathrm{C}$ HSQC spectrum of compound 3za $\left(\mathrm{CDCl}_{3},-40{ }^{\circ} \mathrm{C}\right)$

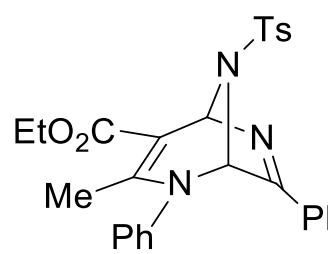

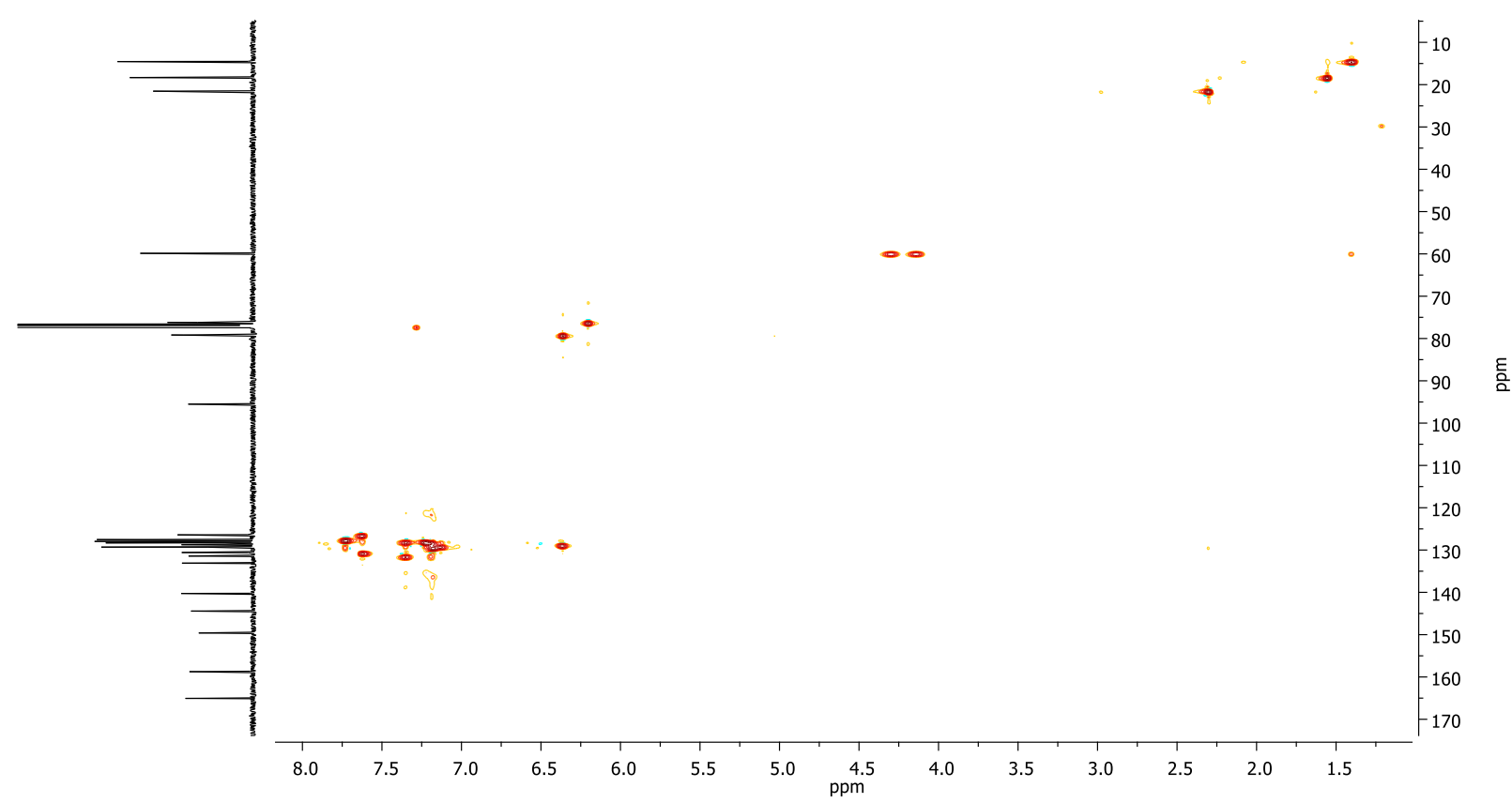


${ }^{1} \mathrm{H}$ NMR spectrum of compound $\mathbf{4 a}\left(400 \mathrm{MHz}, \mathrm{CDCl}_{3}\right)$

$\stackrel{m}{\stackrel{m}{i}}$

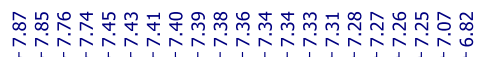

$\stackrel{m}{\stackrel{m}{i}} \stackrel{\sim}{\sim}$

ii
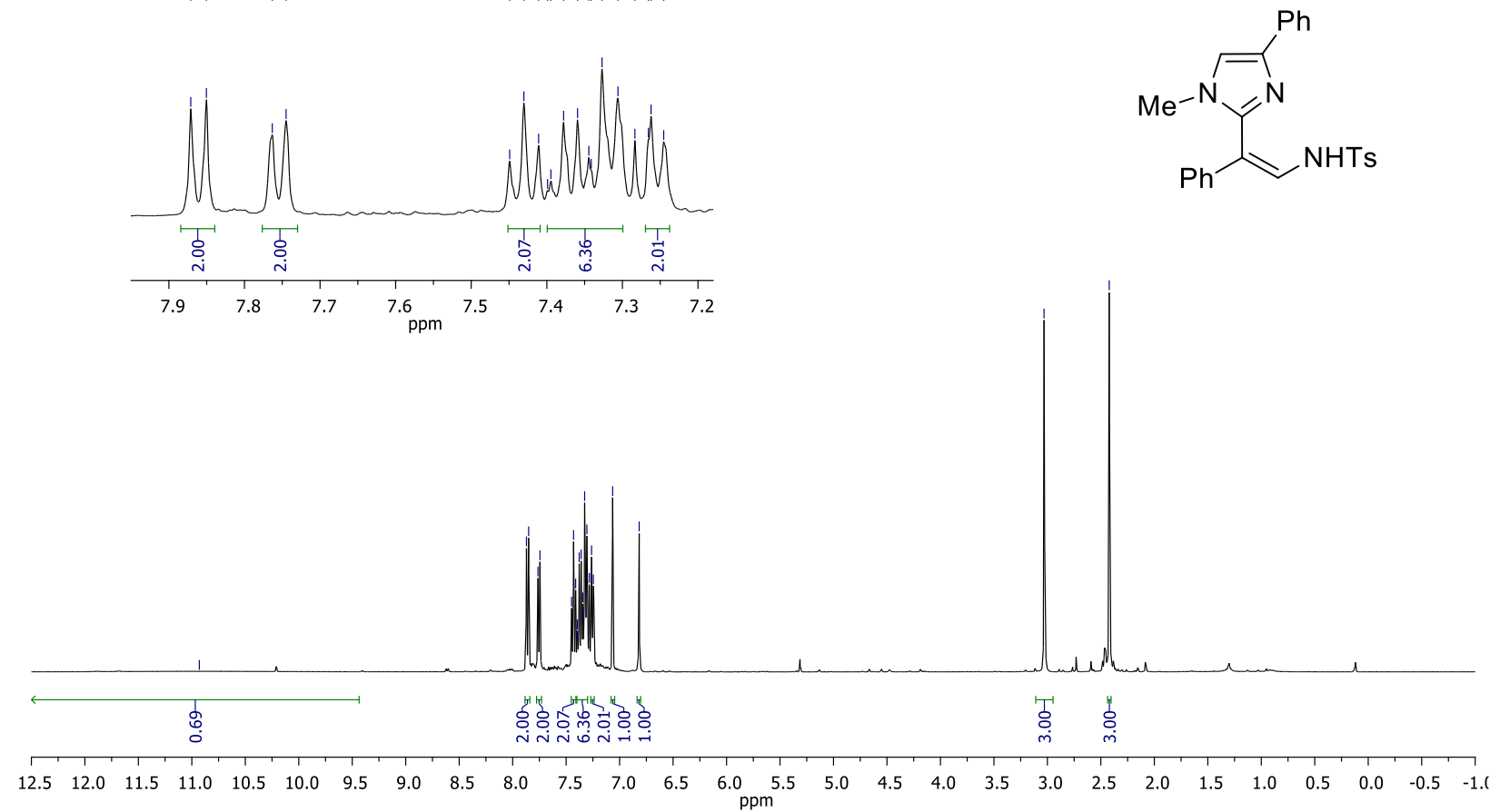

${ }^{13} \mathrm{C}\left\{{ }^{1} \mathrm{H}\right\}$ NMR spectrum of compound $4 \mathbf{a}\left(100 \mathrm{MHz}, \mathrm{CDCl}_{3}\right)$

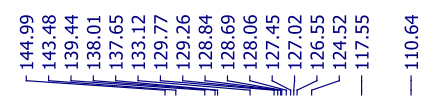

일

송

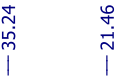<smiles>Cn1cc(-c2ccccc2)nc1/C(=C\N[15F])c1ccccc1</smiles>

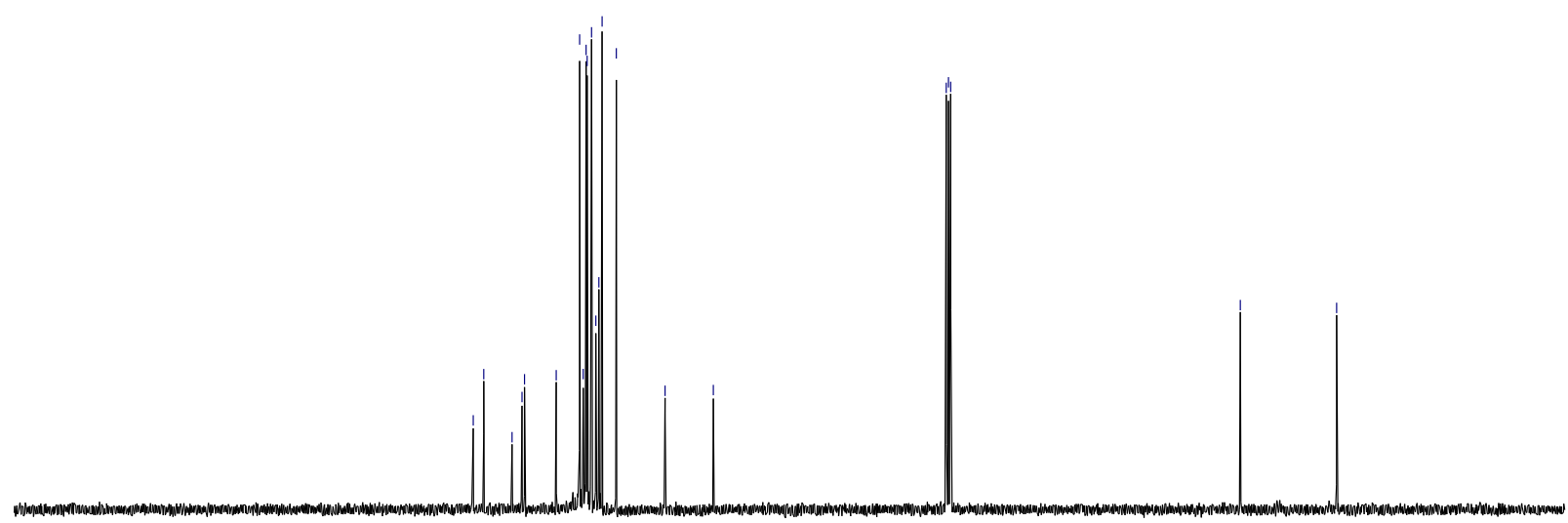

$\begin{array}{llllllllllllllllllllllllllllll}210 & 200 & 190 & 180 & 170 & 160 & 150 & 140 & 130 & 120 & 110 & 100 & 90 & 80 & 70 & 60 & 50 & 40 & 30 & 20 & 10 & 0 & -10\end{array}$ 
${ }^{1} \mathrm{H}$ NMR spectrum of compound $\mathbf{4 b}\left(400 \mathrm{MHz}, \mathrm{CDCl}_{3}\right)$

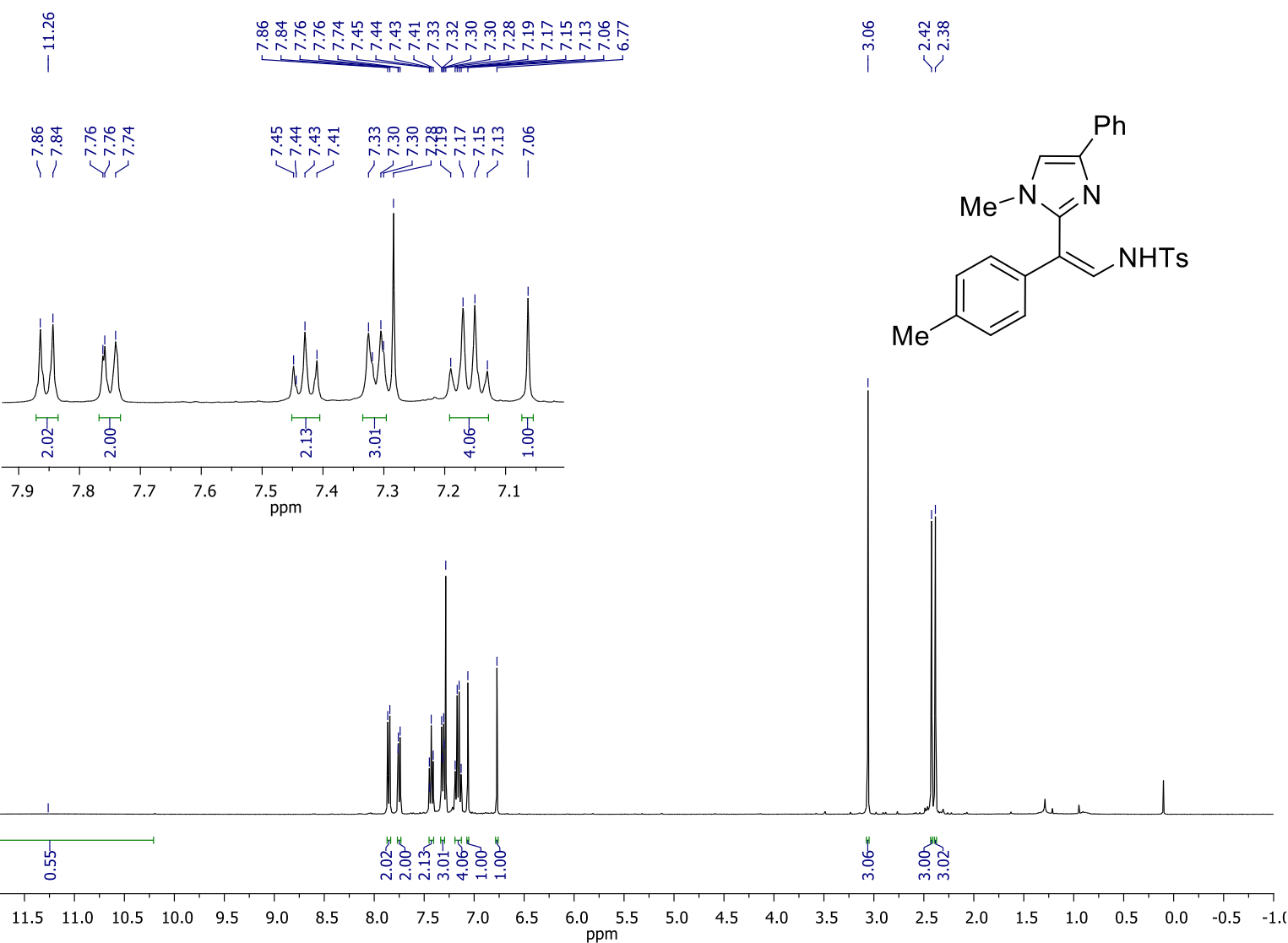

${ }^{13} \mathrm{C}\left\{{ }^{1} \mathrm{H}\right\}$ NMR spectrum of compound $\mathbf{4 b}\left(100 \mathrm{MHz}, \mathrm{CDCl}_{3}\right)$<smiles>COc1cn(C)c(/C(=C\N[15F])c2ccc(C)cc2)n1</smiles>

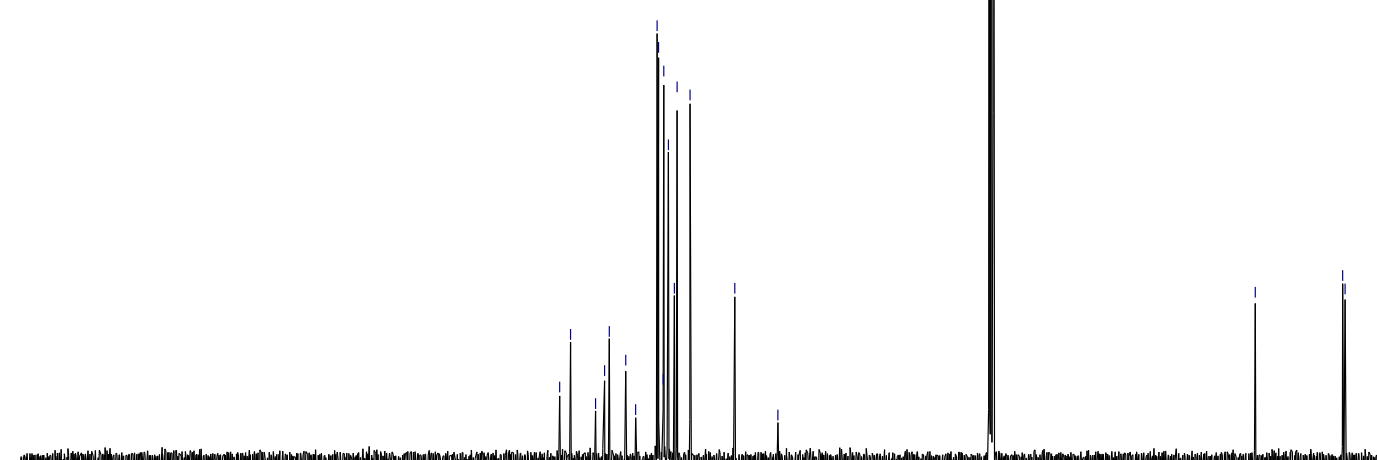

$\begin{array}{llllllllllllllllllllllllllllllllllllll}230 & 220 & 210 & 200 & 190 & 180 & 170 & 160 & 150 & 140 & 130 & 120 & 110 & 100 & 90 & 80 & 70 & 60 & 50 & 40 & 30 & 20 & 10 & 0 & -10 & -20\end{array}$ 
${ }^{1} \mathrm{H}$ NMR spectrum of compound $\mathbf{4 c}\left(400 \mathrm{MHz}, \mathrm{CDCl}_{3}\right)$

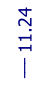

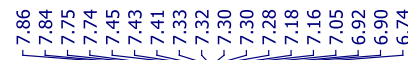

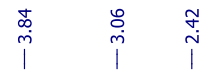

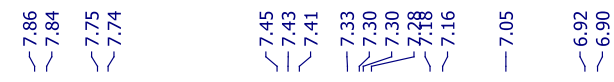
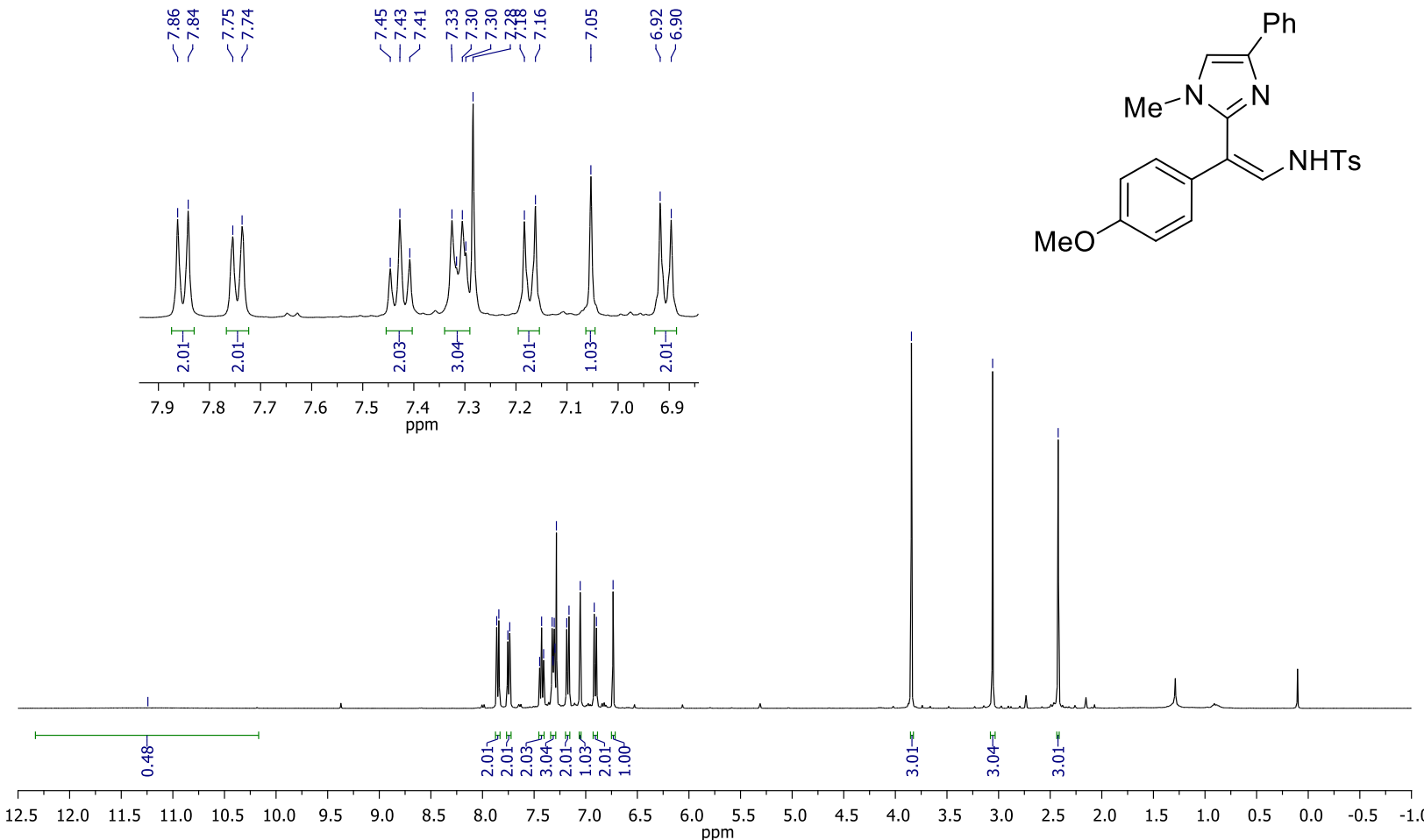

${ }^{13} \mathrm{C}\left\{{ }^{1} \mathrm{H}\right\}$ NMR spectrum of compound $4 \mathbf{c}\left(100 \mathrm{MHz}, \mathrm{CDCl}_{3}\right)$

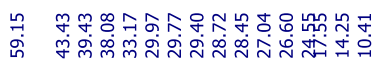

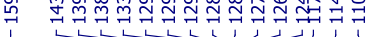<smiles>COc1ccc(/C(=C/N[Sn])c2nc(-c3ccccc3)cn2C)cc1</smiles>

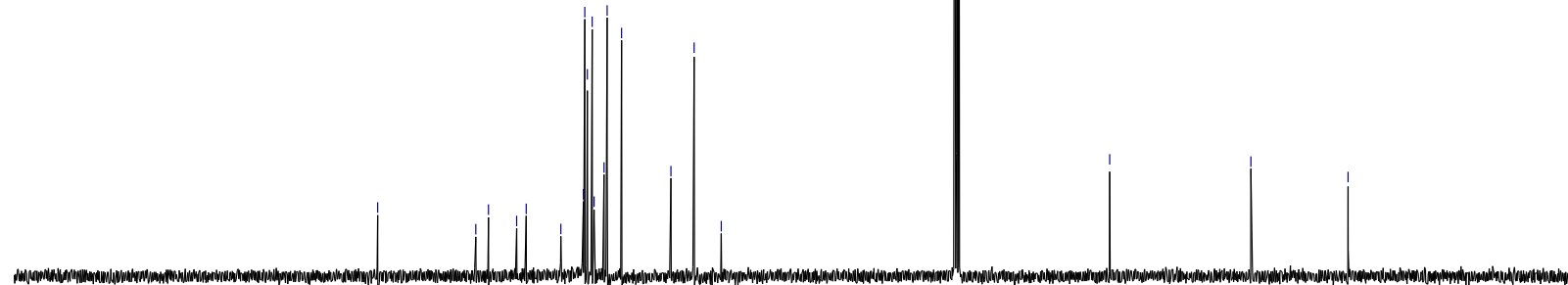

$\begin{array}{llllllllllllllllllllllllllllll}210 & 200 & 190 & 180 & 170 & 160 & 150 & 140 & 130 & 120 & 110 & 100 & 90 & 80 & 70 & 60 & 50 & 40 & 30 & 20 & 10 & 0 & -10\end{array}$ 
${ }^{1} \mathrm{H}$ NMR spectrum of compound $\mathbf{4 d}\left(400 \mathrm{MHz}, \mathrm{CDCl}_{3}\right)$
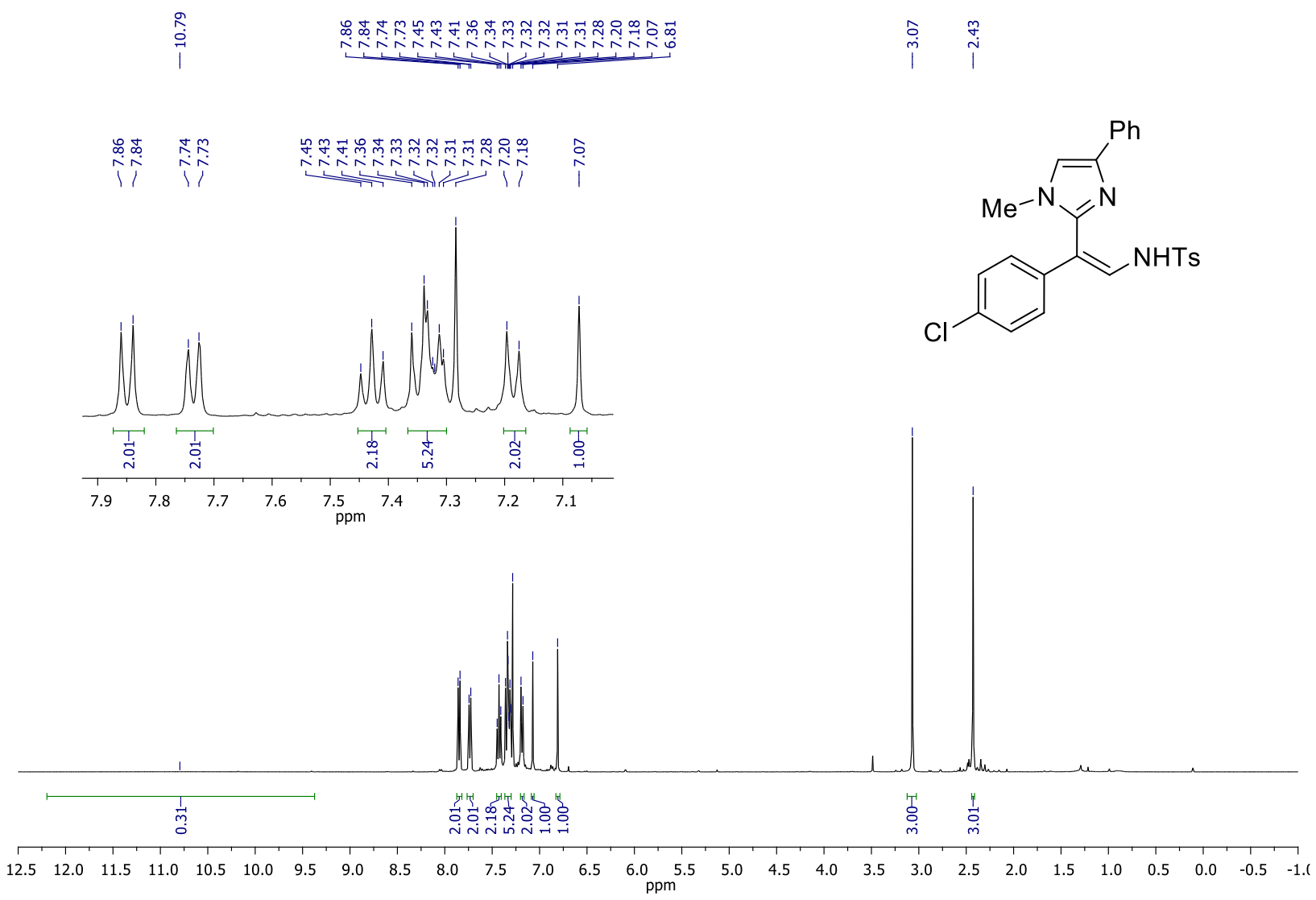

${ }^{13} \mathrm{C}\left\{{ }^{1} \mathrm{H}\right\}$ NMR spectrum of compound $4 \mathbf{d}\left(100 \mathrm{MHz}, \mathrm{CDCl}_{3}\right)$

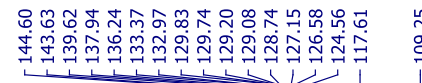<smiles></smiles>

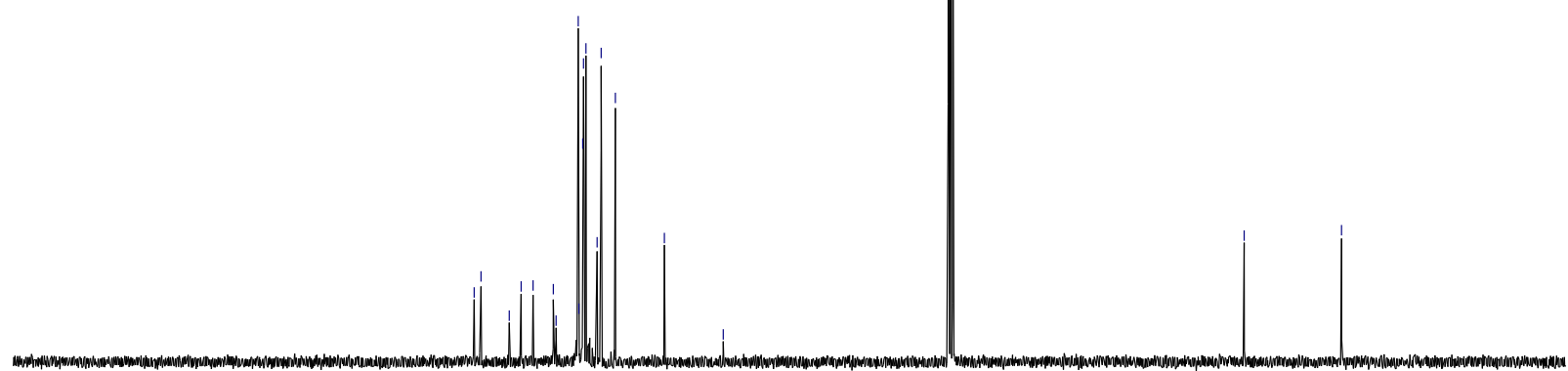

$\begin{array}{llllllllllll}210 & 200 & 190 & 180 & 170 & 160 & 150 & 140 & 130 & 120 & 110 & 100\end{array}$ 
${ }^{1} \mathrm{H}$ NMR spectrum of compound $\mathbf{4 e}\left(400 \mathrm{MHz}, \mathrm{CDCl}_{3}\right)$

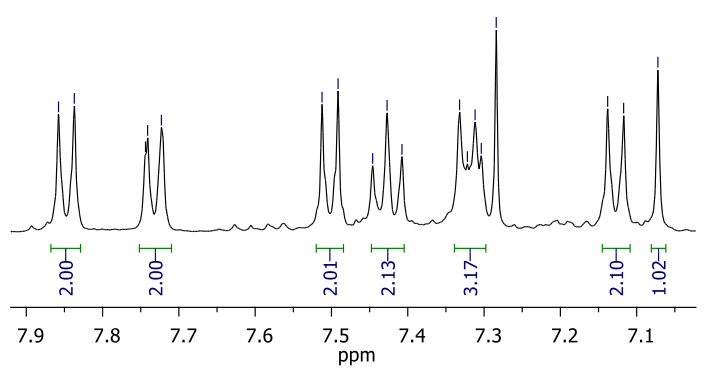<smiles>Cn1cc(-c2ccccc2)nc1/C(=C\N[15F])c1ccc(Br)cc1</smiles>

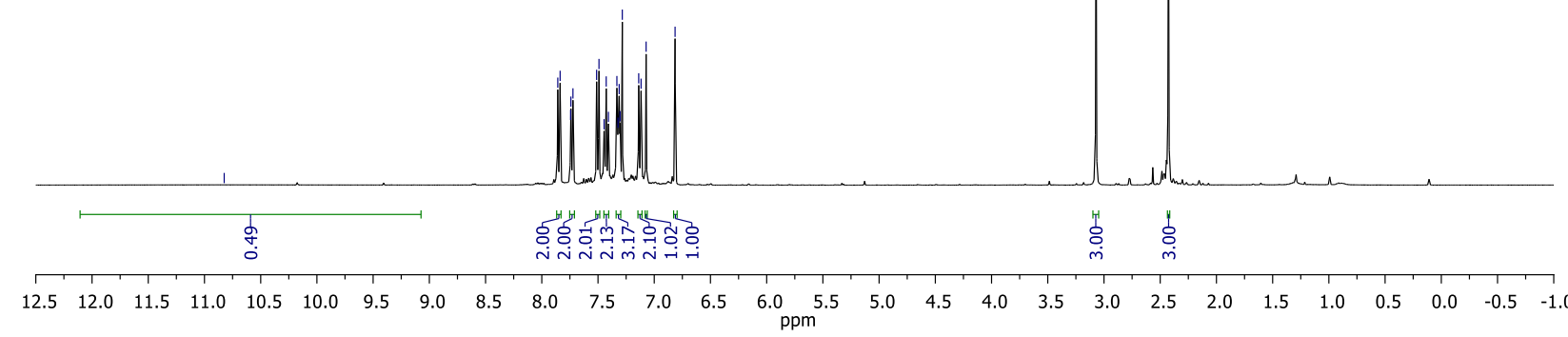

${ }^{13} \mathrm{C}\left\{{ }^{1} \mathrm{H}\right\}$ NMR spectrum of compound $4 \mathbf{e}\left(100 \mathrm{MHz}, \mathrm{CDCl}_{3}\right)$

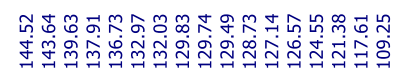<smiles>Cn1cc(-c2ccccc2)nc1/C(=C\N[18F])c1ccc(Br)cc1</smiles>

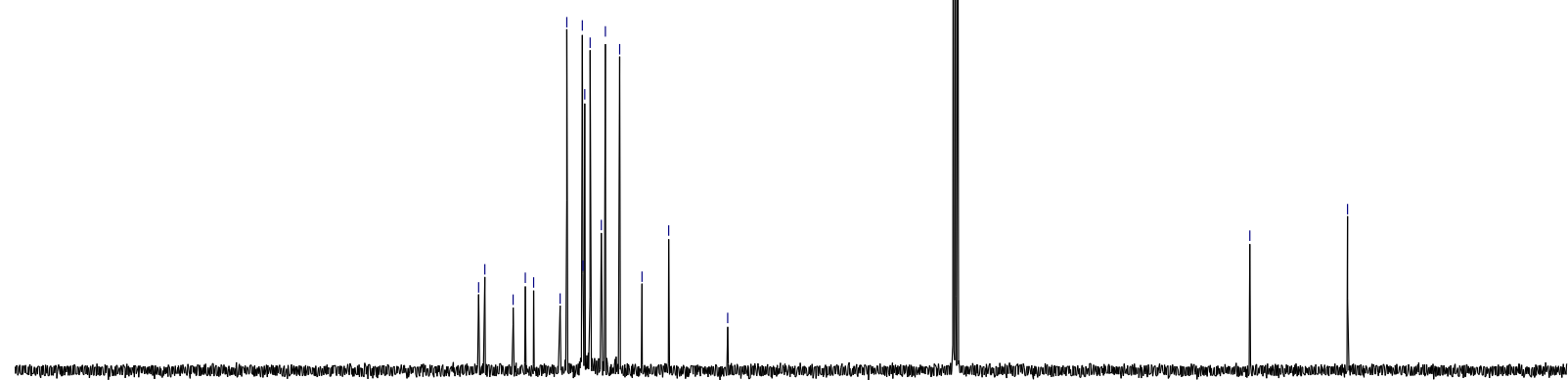


${ }^{1} \mathrm{H}$ NMR spectrum of compound $\mathbf{4 f}\left(400 \mathrm{MHz}, \mathrm{CDCl}_{3}\right)$

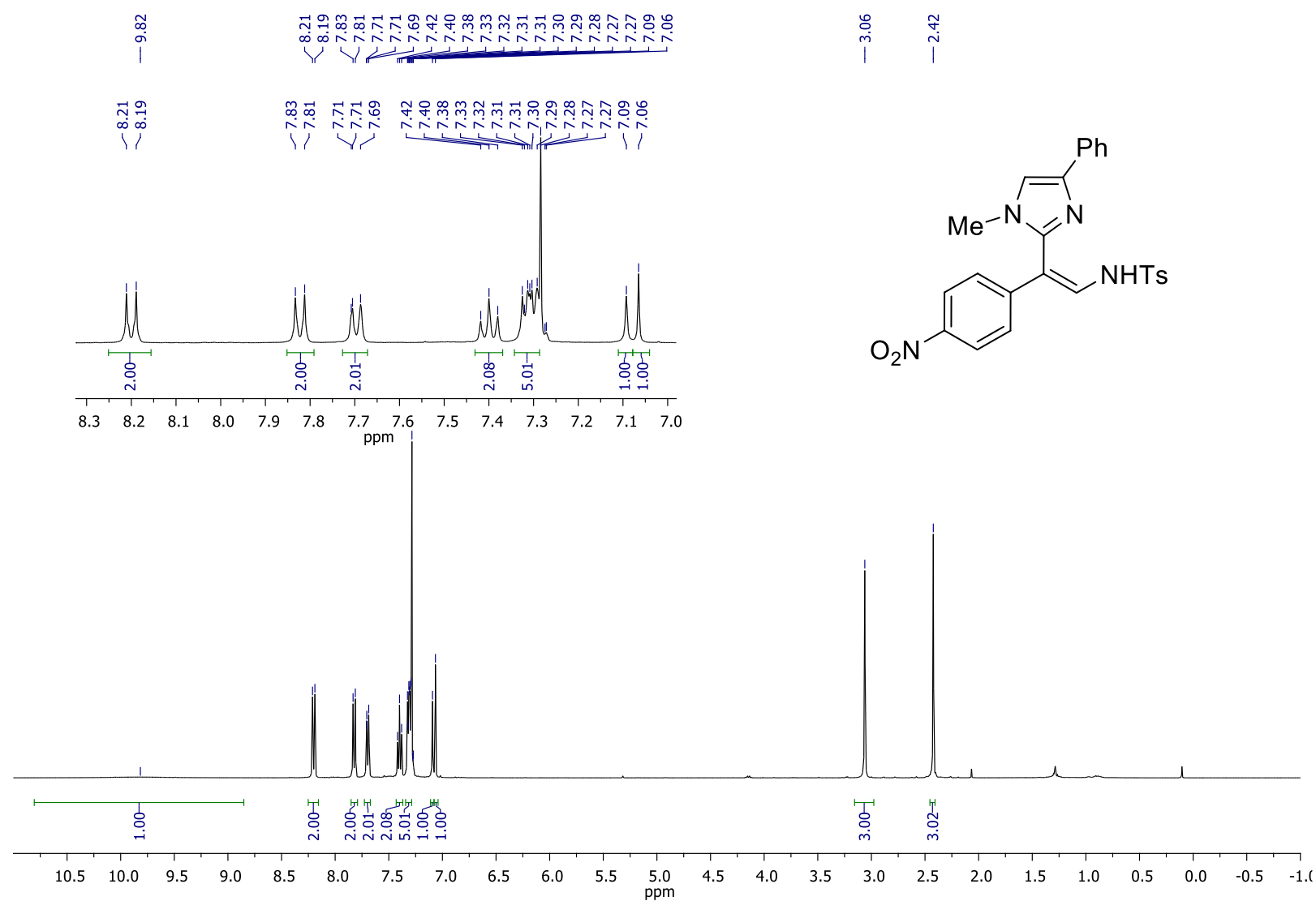

${ }^{13} \mathrm{C}\left\{{ }^{1} \mathrm{H}\right\}$ NMR spectrum of compound $\mathbf{4 f}\left(100 \mathrm{MHz}, \mathrm{CDCl}_{3}\right)$

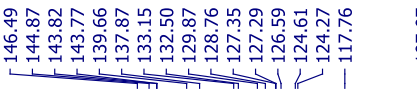

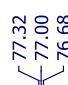

ஓू<smiles>CCN/C=C(/c1ccc([N+](=O)[O-])cc1)c1nc(-c2ccccc2)cn1C</smiles>

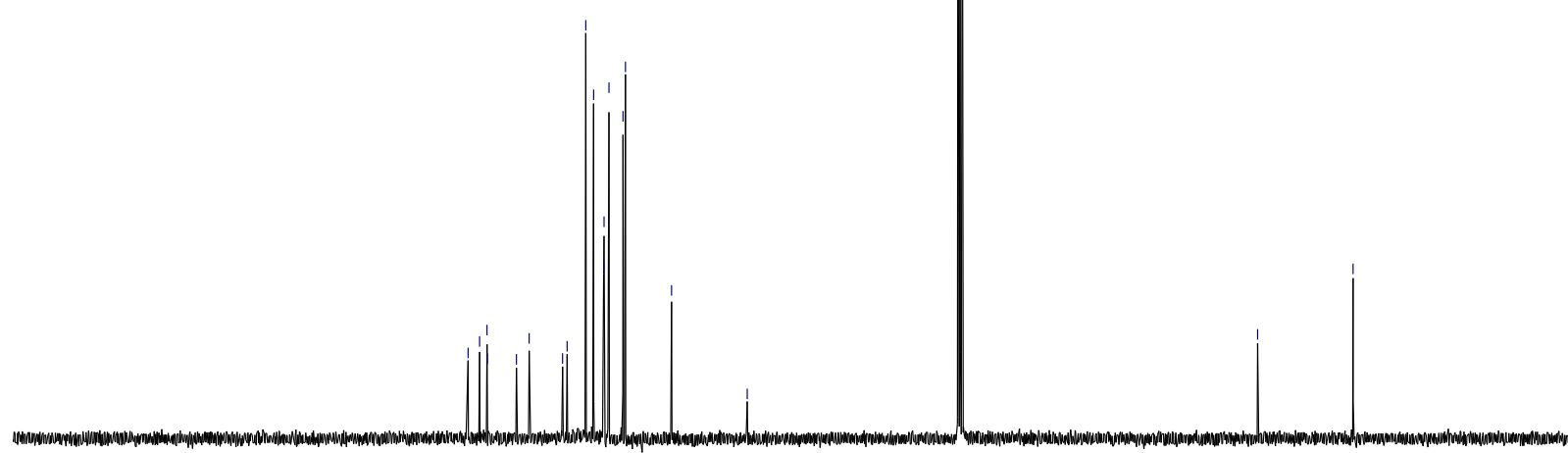

$\begin{array}{llllllllllll}210 & 200 & 190 & 180 & 170 & 160 & 150 & 140 & 130 & 120 & 110 & 100\end{array}$ 
${ }^{1} \mathrm{H}$ NMR spectrum of compound $\mathbf{4 g}\left(400 \mathrm{MHz}, \mathrm{CDCl}_{3}\right)$

l
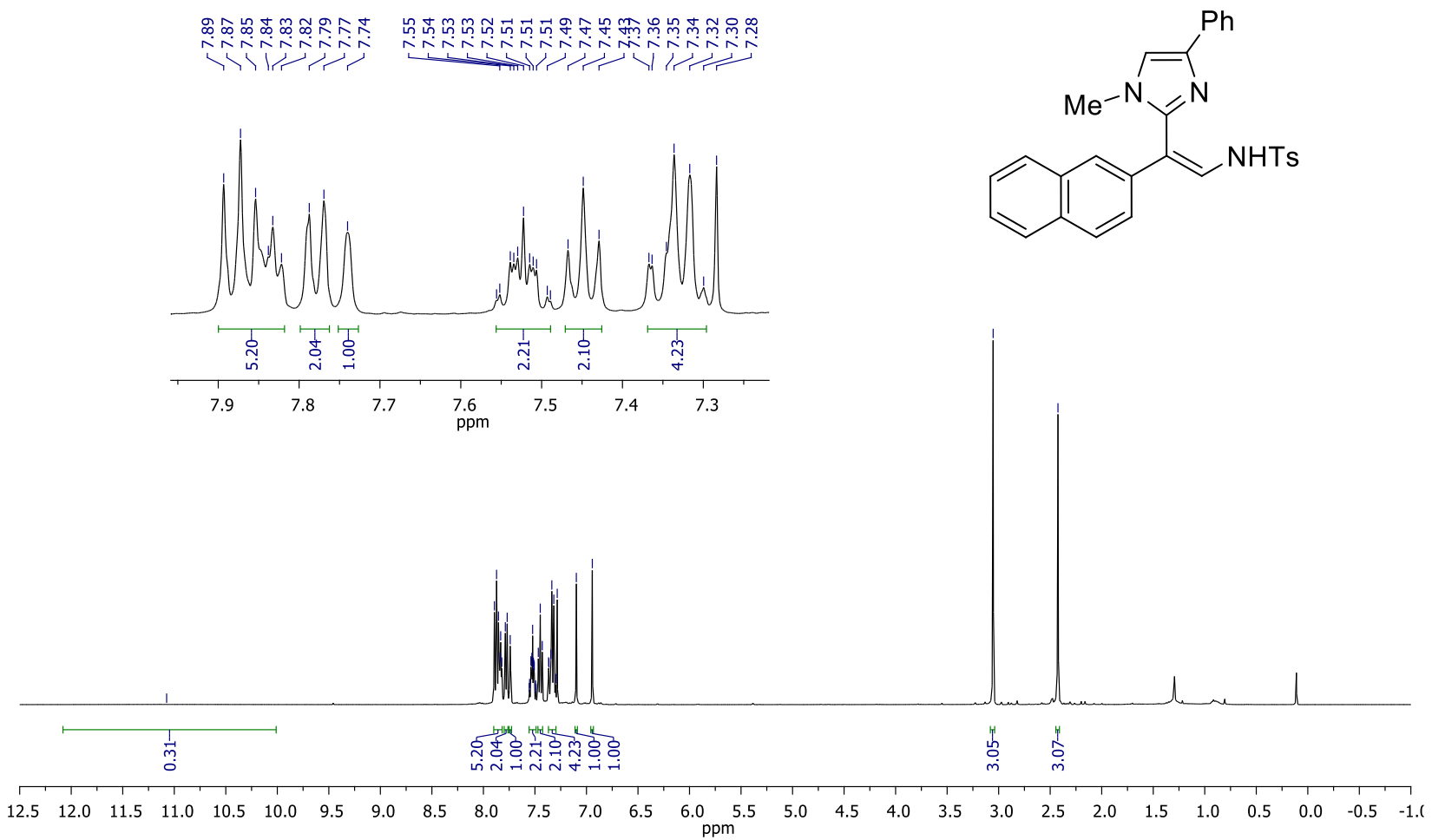

${ }^{13} \mathrm{C}\left\{{ }^{1} \mathrm{H}\right\}$ NMR spectrum of compound $\mathbf{4 g}\left(100 \mathrm{MHz}, \mathrm{CDCl}_{3}\right)$

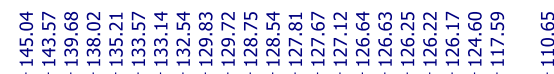<smiles>CN/C=C(/c1ccc2ccccc2c1)c1nc(-c2ccccc2)cn1C</smiles>

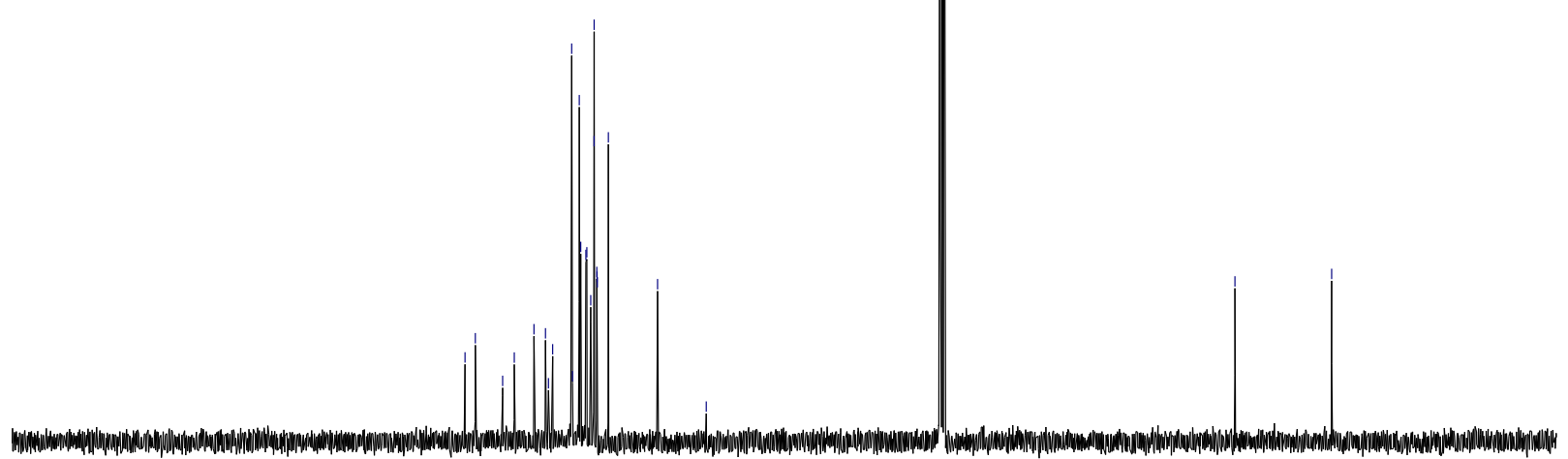

$\begin{array}{lllllllllll}200 & 190 & 180 & 170 & 160 & 150 & 140 & 130 & 120 & 110 & \begin{array}{l}100 \\ \mathrm{ppm}\end{array}\end{array}$

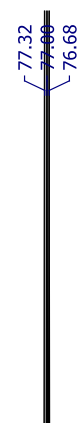

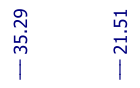


${ }^{1} \mathrm{H}$ NMR spectrum of compound $\mathbf{4 h}\left(400 \mathrm{MHz}, \mathrm{CDCl}_{3}\right)$

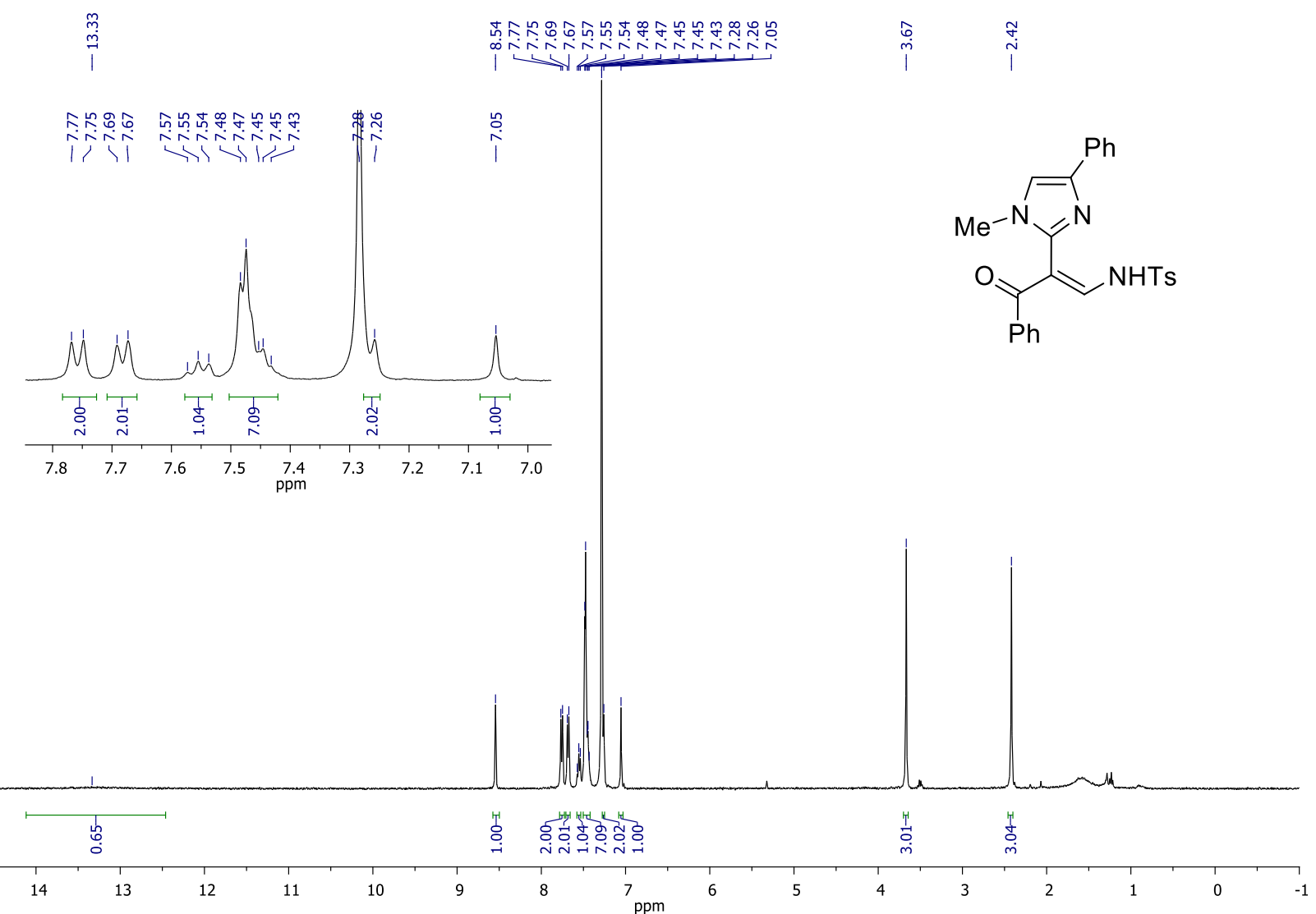

${ }^{13} \mathrm{C}$ NMR spectrum of compound $4 \mathbf{h}\left(100 \mathrm{MHz}, \mathrm{DMSO}-d_{6}\right)$

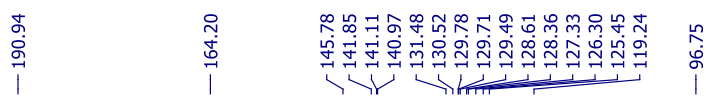

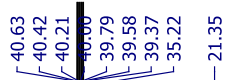<smiles>Cn1cc(-c2ccccc2)nc1/C(=C\N[Sn])C(=O)c1ccccc1</smiles>

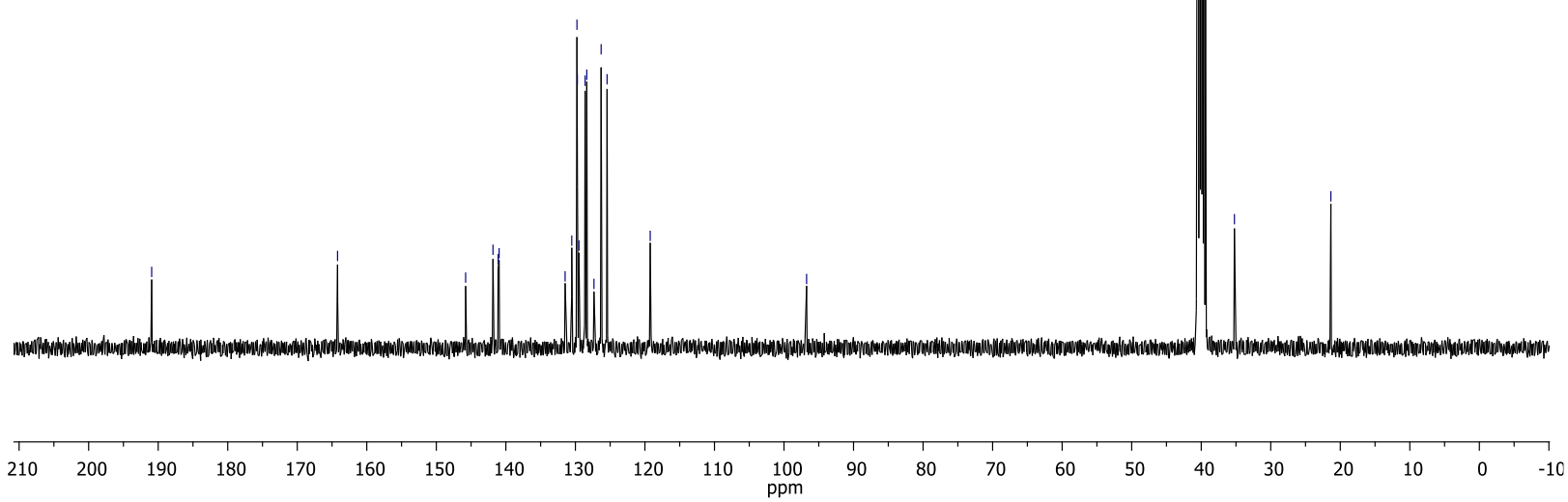


${ }^{1} \mathrm{H}$ NMR spectrum of compound $\mathbf{4 i}\left(400 \mathrm{MHz}, \mathrm{CDCl}_{3}\right)$

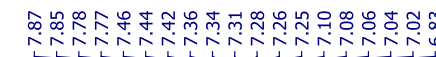

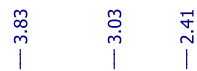
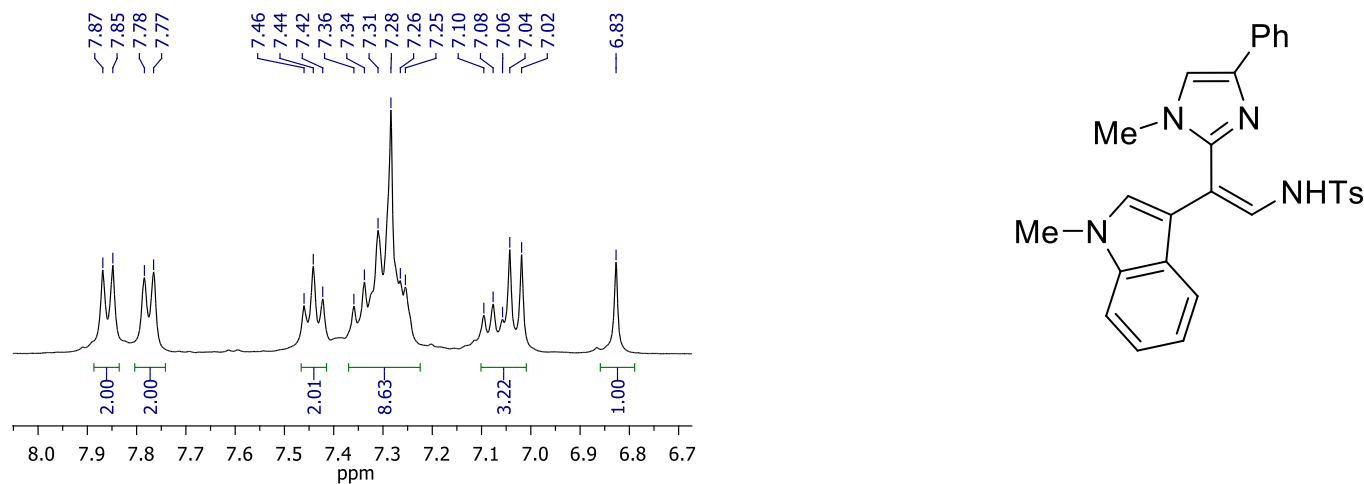

$\begin{array}{lllllllllllllll}8.0 & 7.9 & 7.8 & 7.7 & 7.6 & 7.5 & 7.4 & 7.3 & 7.2 & 7.1 & 7.0 & 6.9 & 6.8 & 6.7\end{array}$

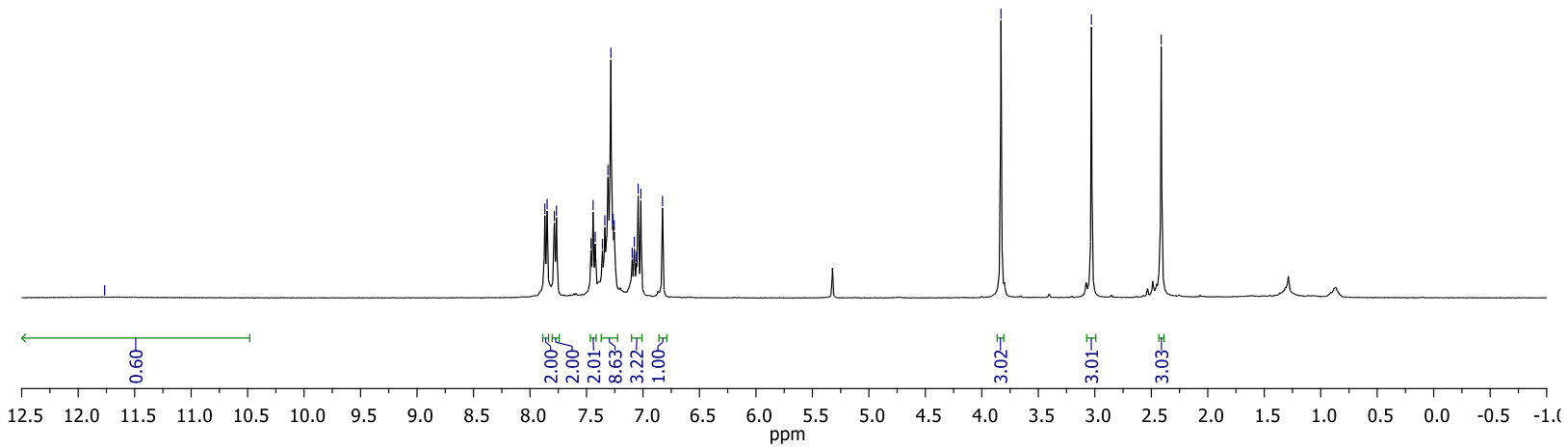

${ }^{13} \mathrm{C}\left\{{ }^{1} \mathrm{H}\right\}$ NMR spectrum of compound $4 \mathbf{i}\left(100 \mathrm{MHz}, \mathrm{CDCl}_{3}\right)$
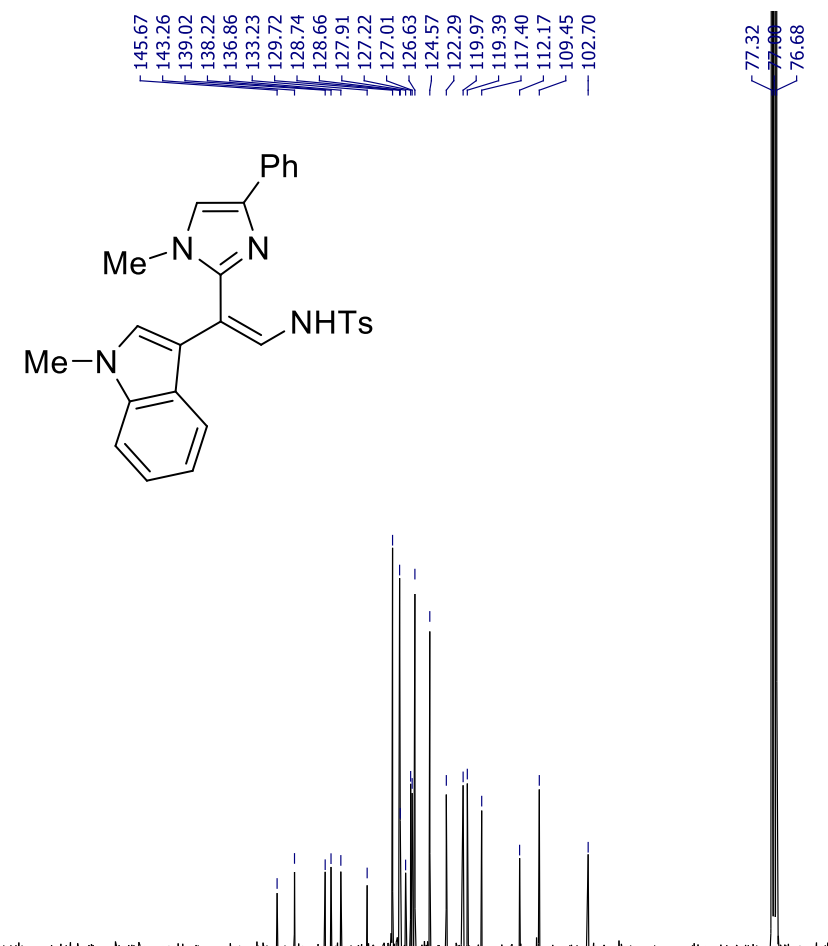

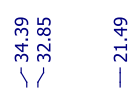

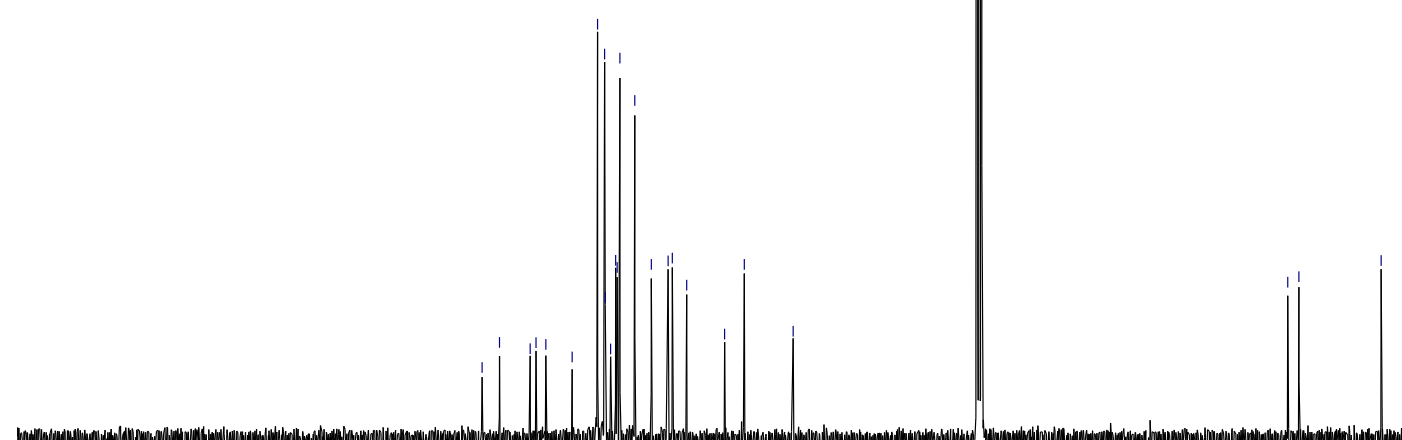

$\begin{array}{lllllllllll}200 & 190 & 180 & 170 & 160 & 150 & 140 & 130 & 120 & 110 & 100\end{array}$ 
${ }^{1} \mathrm{H}$ NMR spectrum of compound $\mathbf{4 j}\left(400 \mathrm{MHz}, \mathrm{CDCl}_{3}\right)$

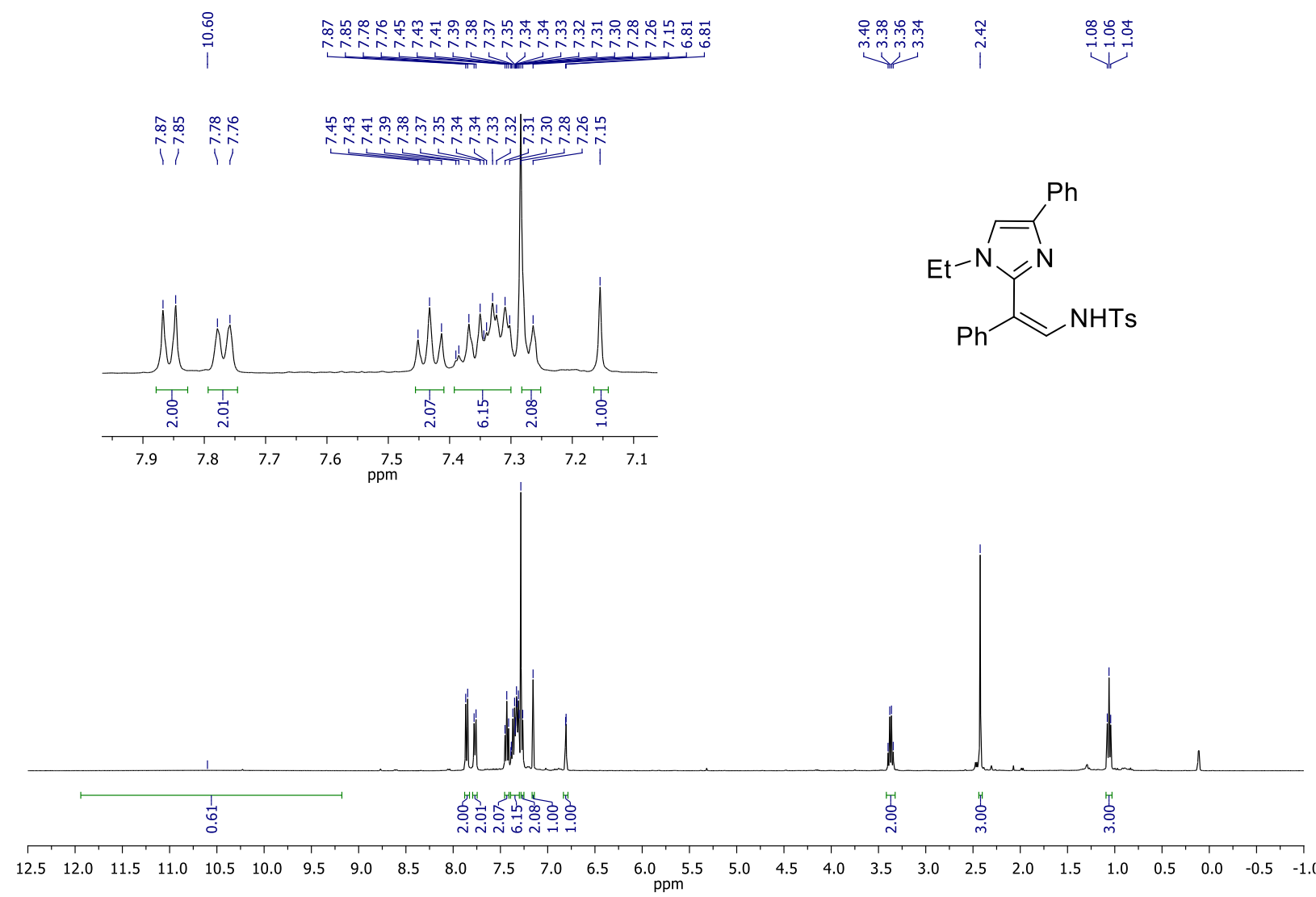

${ }^{13} \mathrm{C}\left\{{ }^{1} \mathrm{H}\right\}$ NMR spectrum of compound $4 \mathbf{j}\left(100 \mathrm{MHz}, \mathrm{CDCl}_{3}\right)$
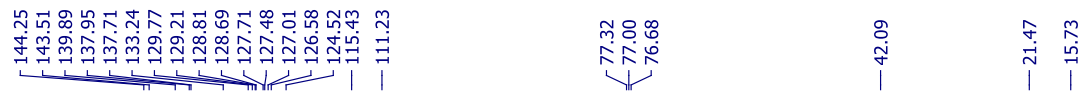<smiles>CCn1cc(-c2ccccc2)nc1/C(=C\N[Sn])c1ccccc1</smiles>

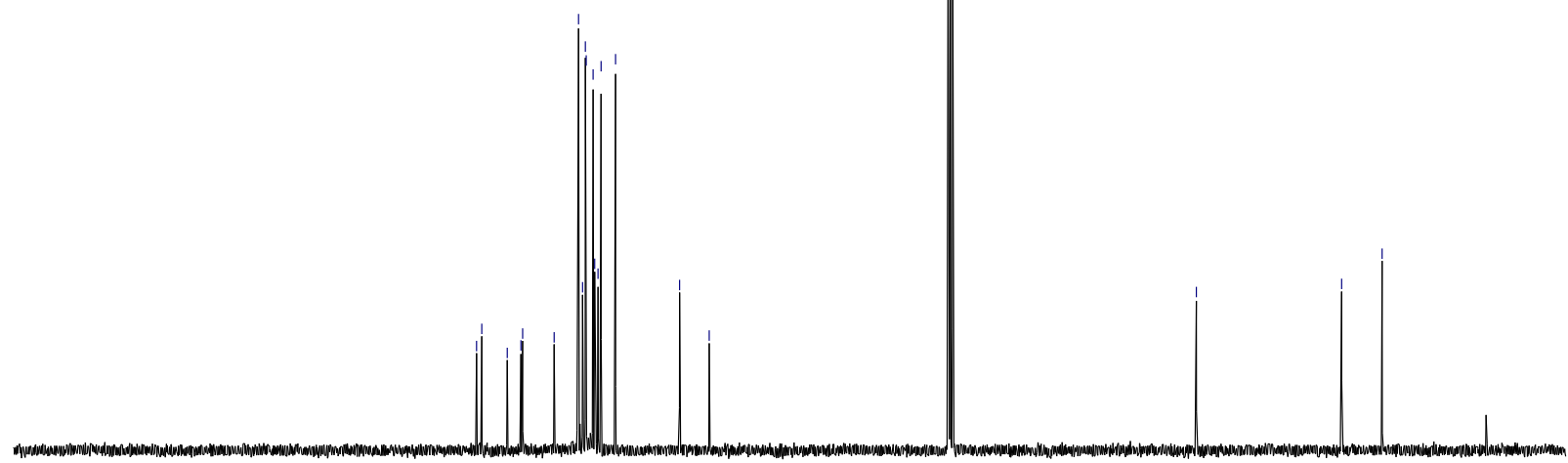

\begin{tabular}{llllllllllllllllllllllllllllllllll}
\hline 210 & 200 & 190 & 180 & 170 & 160 & 150 & 140 & 130 & 120 & 110 & 100 & 90 & 80 & 70 & 60 & 50 & 40 & 30 & 20 & 10 & 0 & -10
\end{tabular} 
${ }^{1} \mathrm{H}$ NMR spectrum of compound $\mathbf{4 k}\left(400 \mathrm{MHz}, \mathrm{CDCl}_{3}\right)$
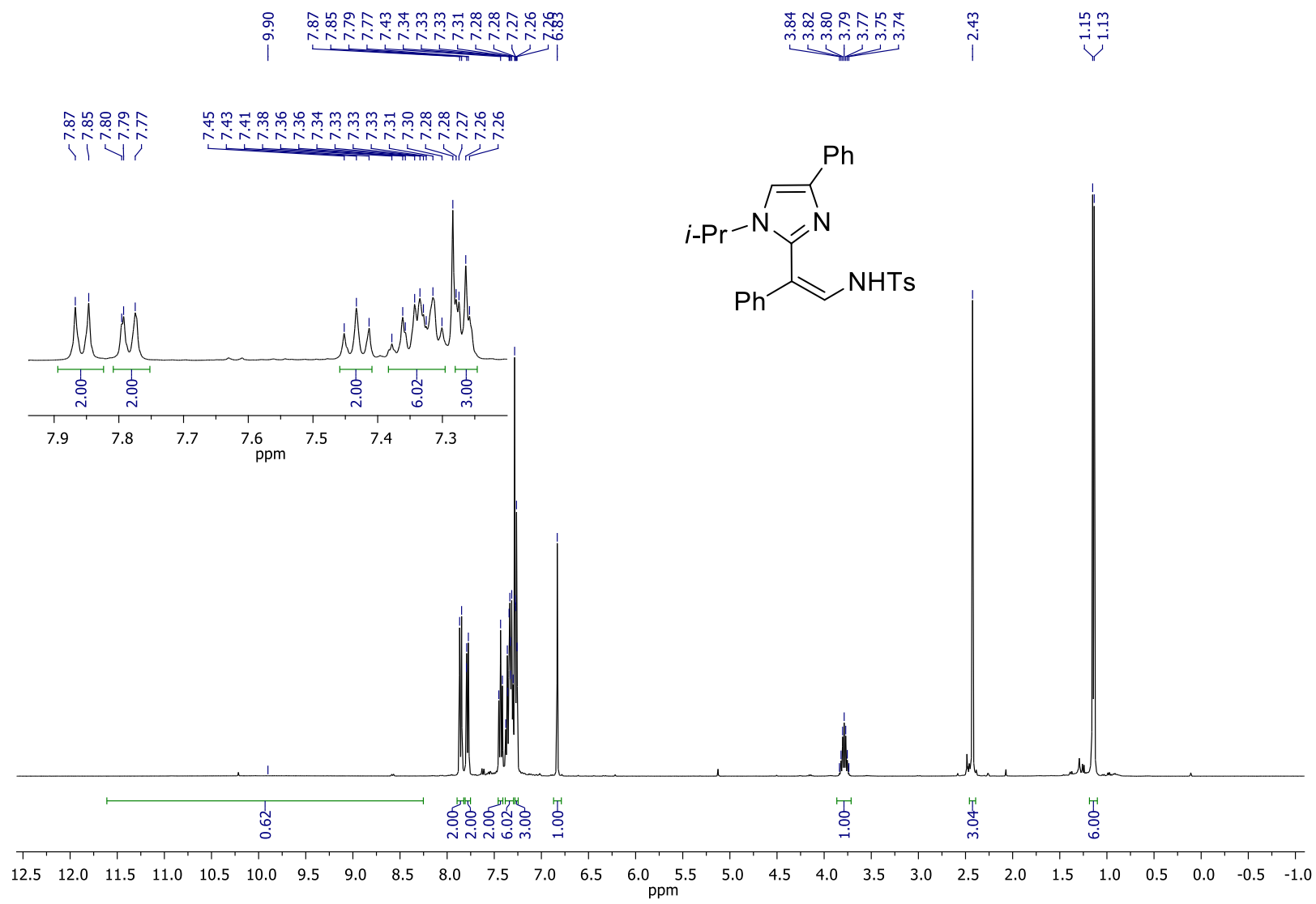

${ }^{13} \mathrm{C}\left\{{ }^{1} \mathrm{H}\right\}$ NMR spectrum of compound $4 \mathbf{k}\left(100 \mathrm{MHz}, \mathrm{CDCl}_{3}\right)$

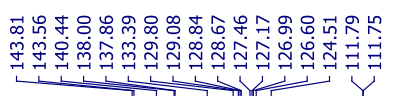<smiles>CC([In])n1cc(-c2ccccc2)nc1/C(=C\N[In])c1ccccc1</smiles>

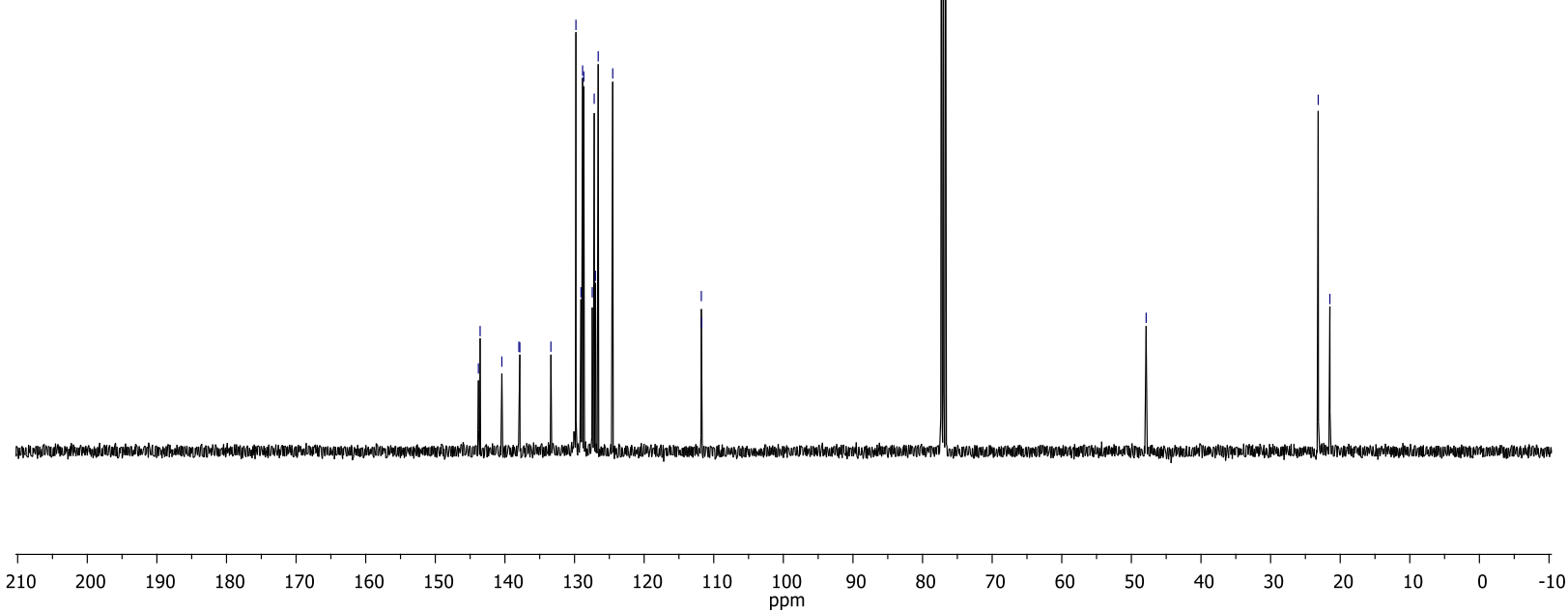


${ }^{1} \mathrm{H}$ NMR spectrum of compound $\mathbf{4 l}\left(400 \mathrm{MHz}, \mathrm{CDCl}_{3}\right)$
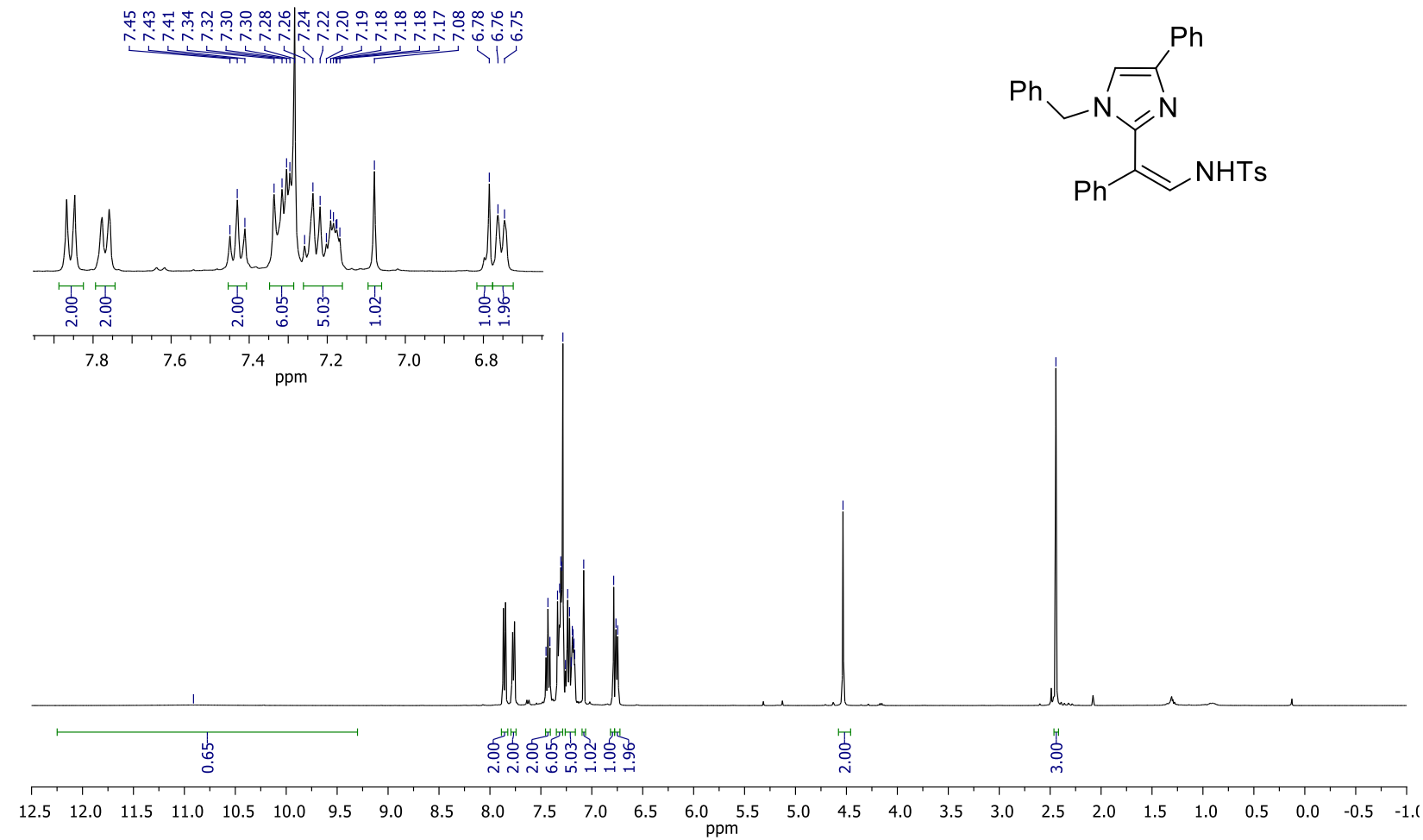

${ }^{13} \mathrm{C}\left\{{ }^{1} \mathrm{H}\right\}$ NMR spectrum of compound $4 \mathrm{l}\left(100 \mathrm{MHz}, \mathrm{CDCl}_{3}\right)$
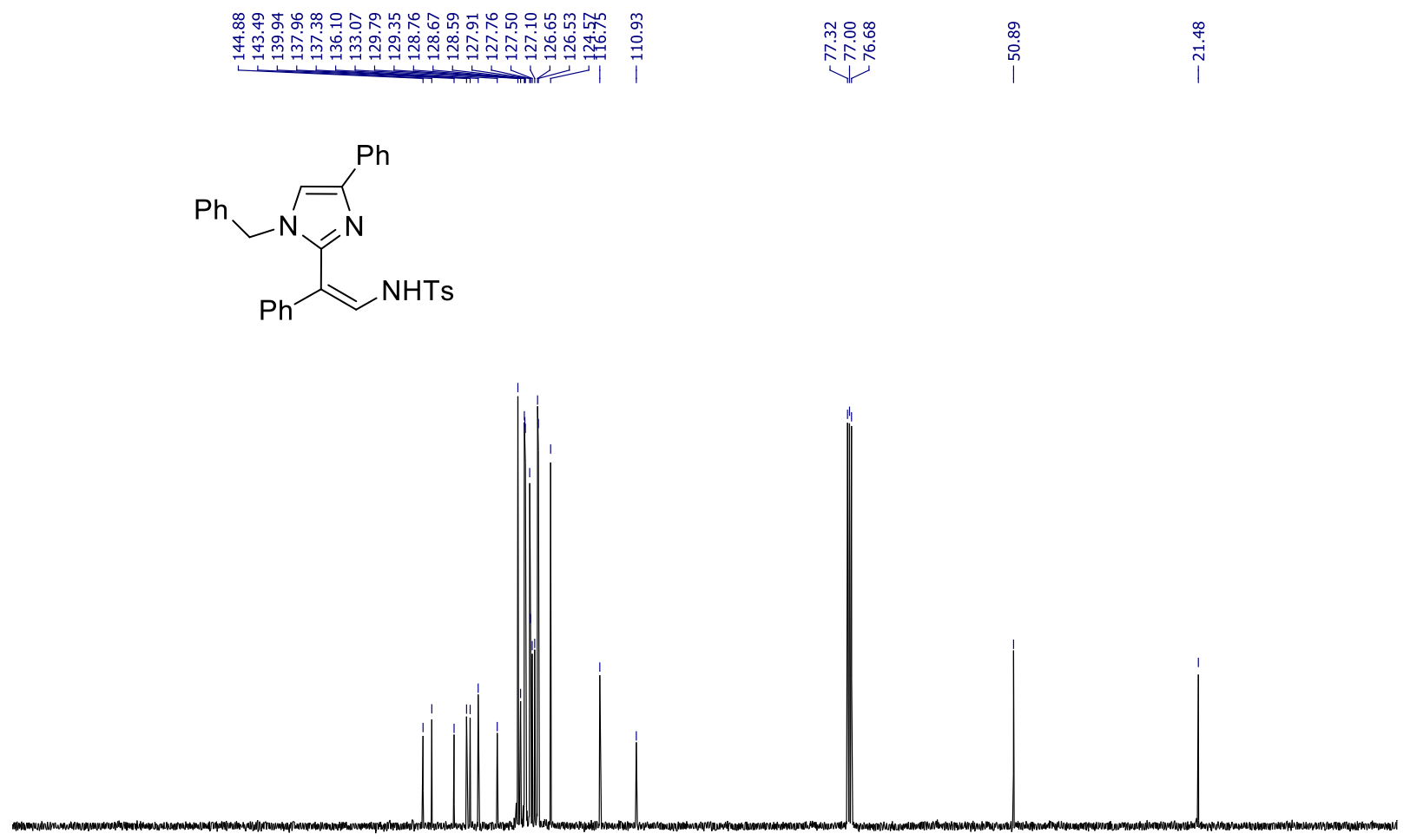

$\begin{array}{lllllllllllllllllllllllll}210 & 200 & 190 & 180 & 170 & 160 & 150 & 140 & 130 & 120 & 110 & 100 & 90 & 80 & 70 & 60 & 50 & 40 & 30 & 20 & 10 & 0 & -10\end{array}$ 
${ }^{1} \mathrm{H}$ NMR spectrum of compound $\mathbf{4 m}\left(400 \mathrm{MHz}, \mathrm{CDCl}_{3}\right)$

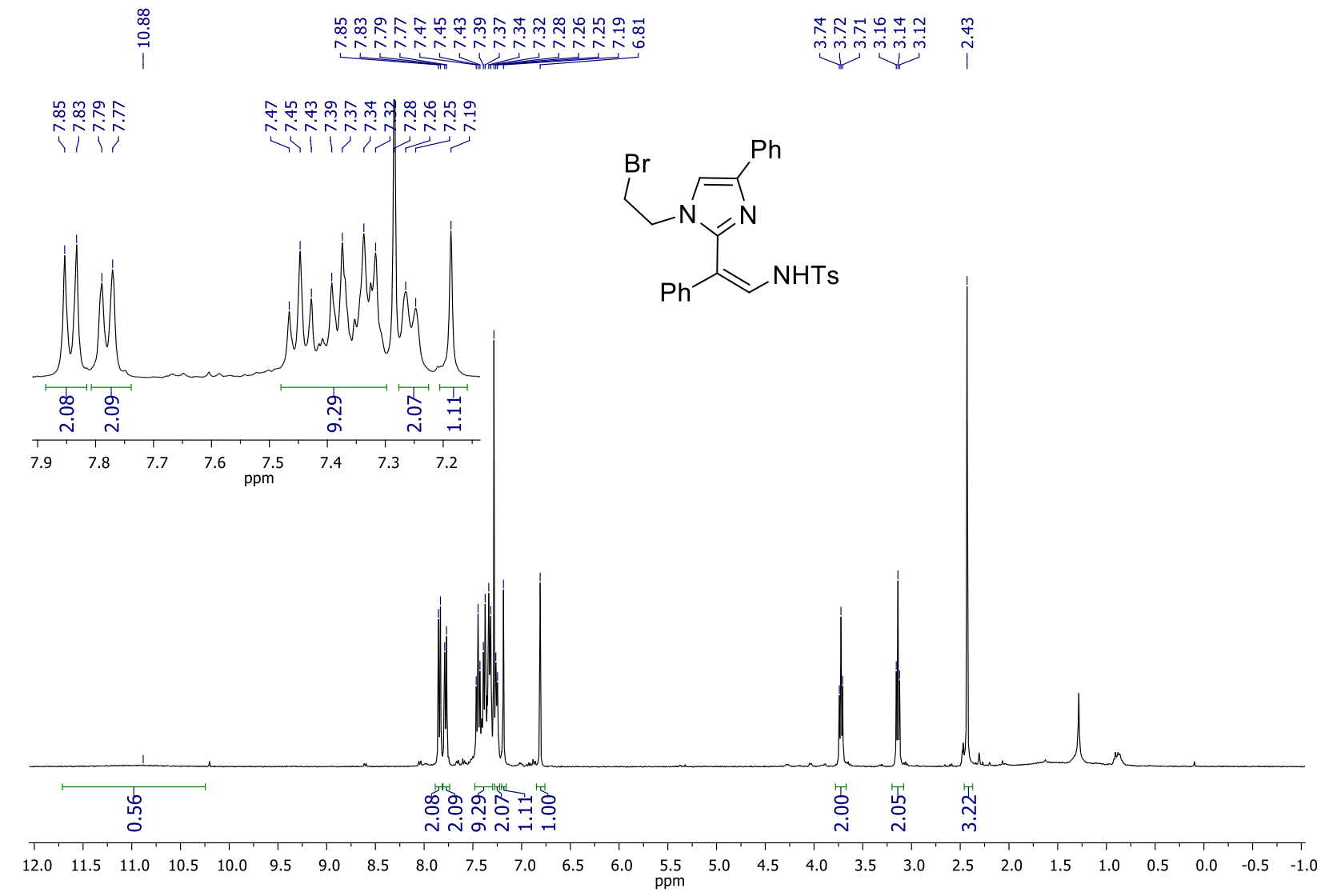

${ }^{13} \mathrm{C}\left\{{ }^{1} \mathrm{H}\right\}$ NMR spectrum of compound $\mathbf{4 m}\left(100 \mathrm{MHz}, \mathrm{CDCl}_{3}\right)$

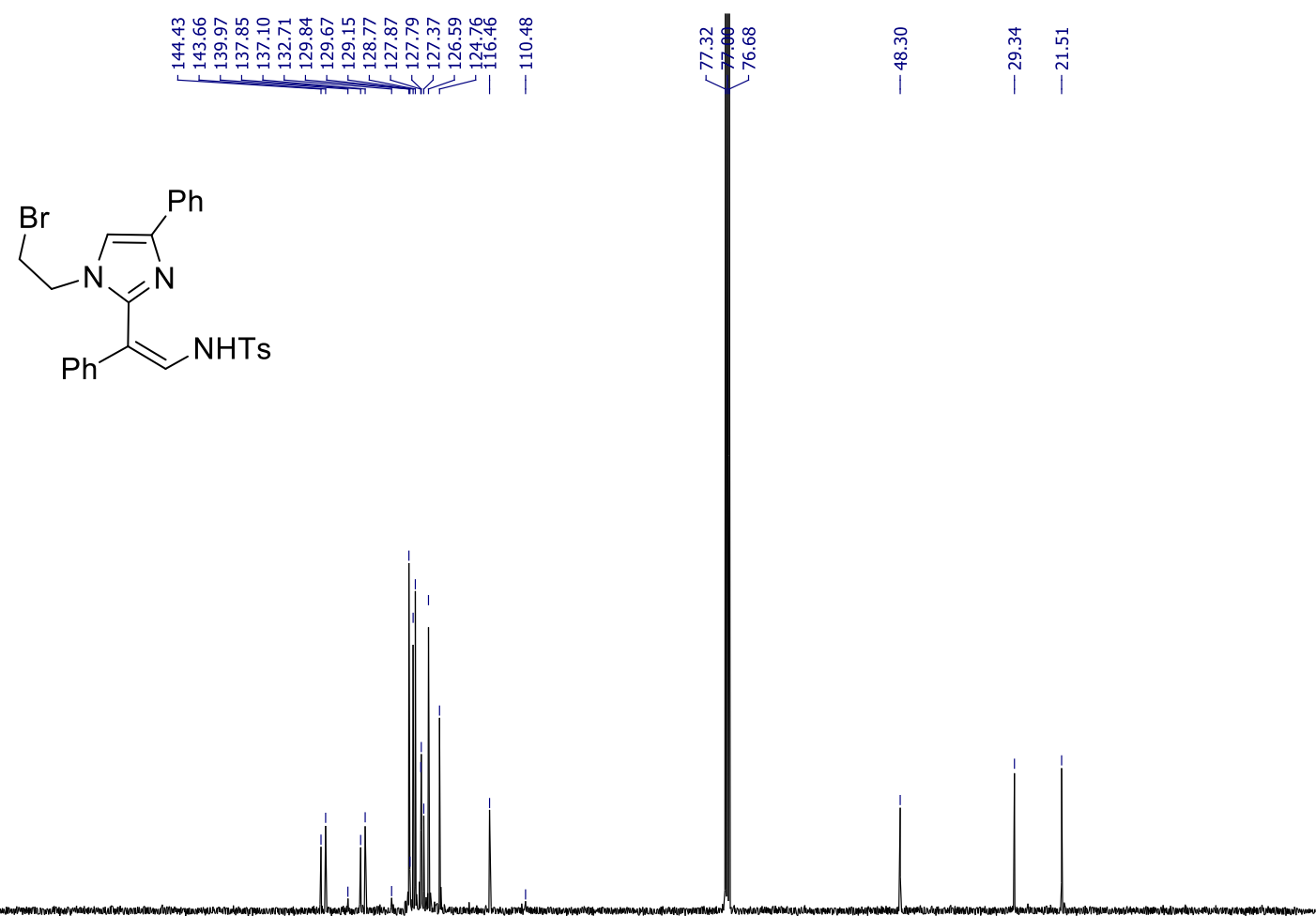

\begin{tabular}{rlllllllllllllllllllllllllllllllllll}
\hline 230 & 220 & 210 & 200 & 190 & 180 & 170 & 160 & 150 & 140 & 130 & 120 & 110 & 100 & 90 & 80 & 70 & 60 & 50 & 40 & 30 & 20 & 10 & 0 & -10 & -20
\end{tabular} 
${ }^{1} \mathrm{H}$ NMR spectrum of compound $4 \mathbf{n}\left(400 \mathrm{MHz}, \mathrm{CDCl}_{3}\right)$

:
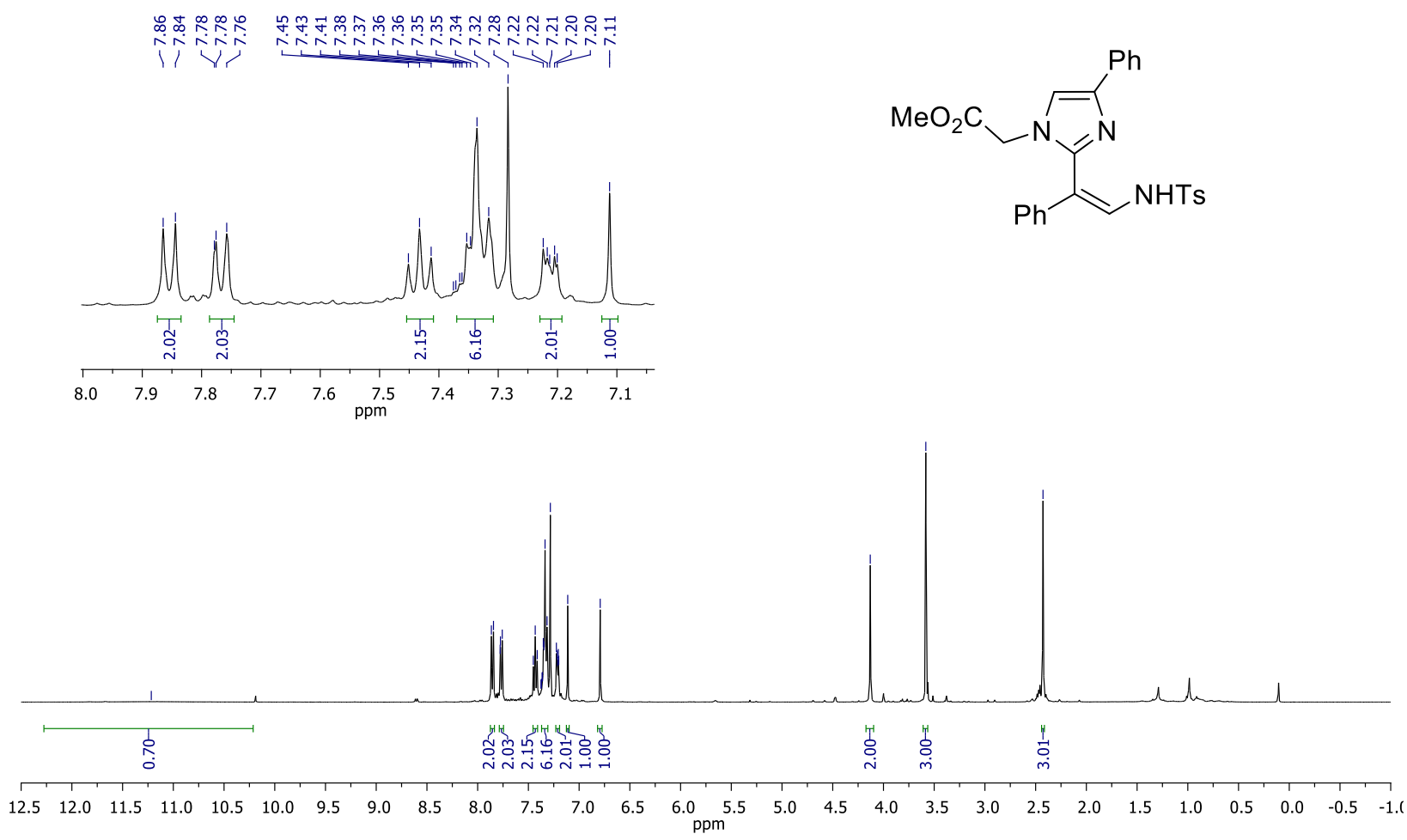

${ }^{13} \mathrm{C}\left\{{ }^{1} \mathrm{H}\right\}$ NMR spectrum of compound $4 \mathbf{n}\left(100 \mathrm{MHz}, \mathrm{CDCl}_{3}\right)$

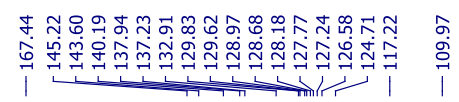<smiles>CC(=O)Cn1cc(-c2ccccc2)nc1/C(=C\N[Sn])c1ccccc1</smiles>
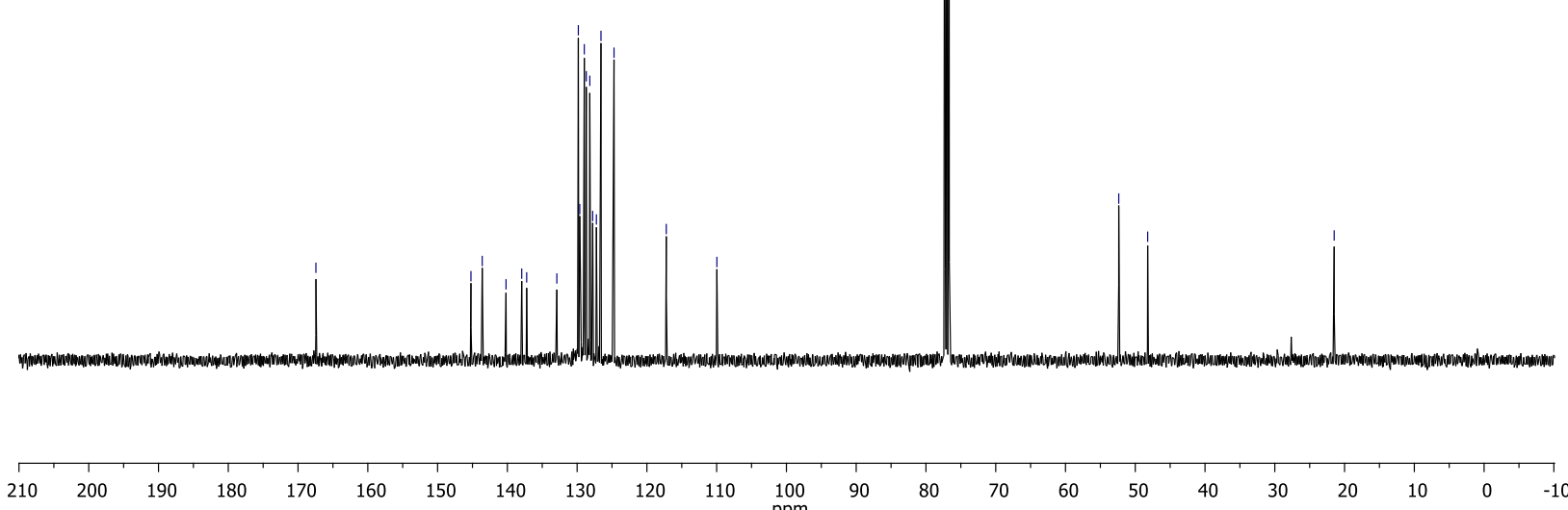
${ }^{1} \mathrm{H}$ NMR spectrum of compound $4 \mathbf{0}\left(400 \mathrm{MHz}, \mathrm{CDCl}_{3}\right)$

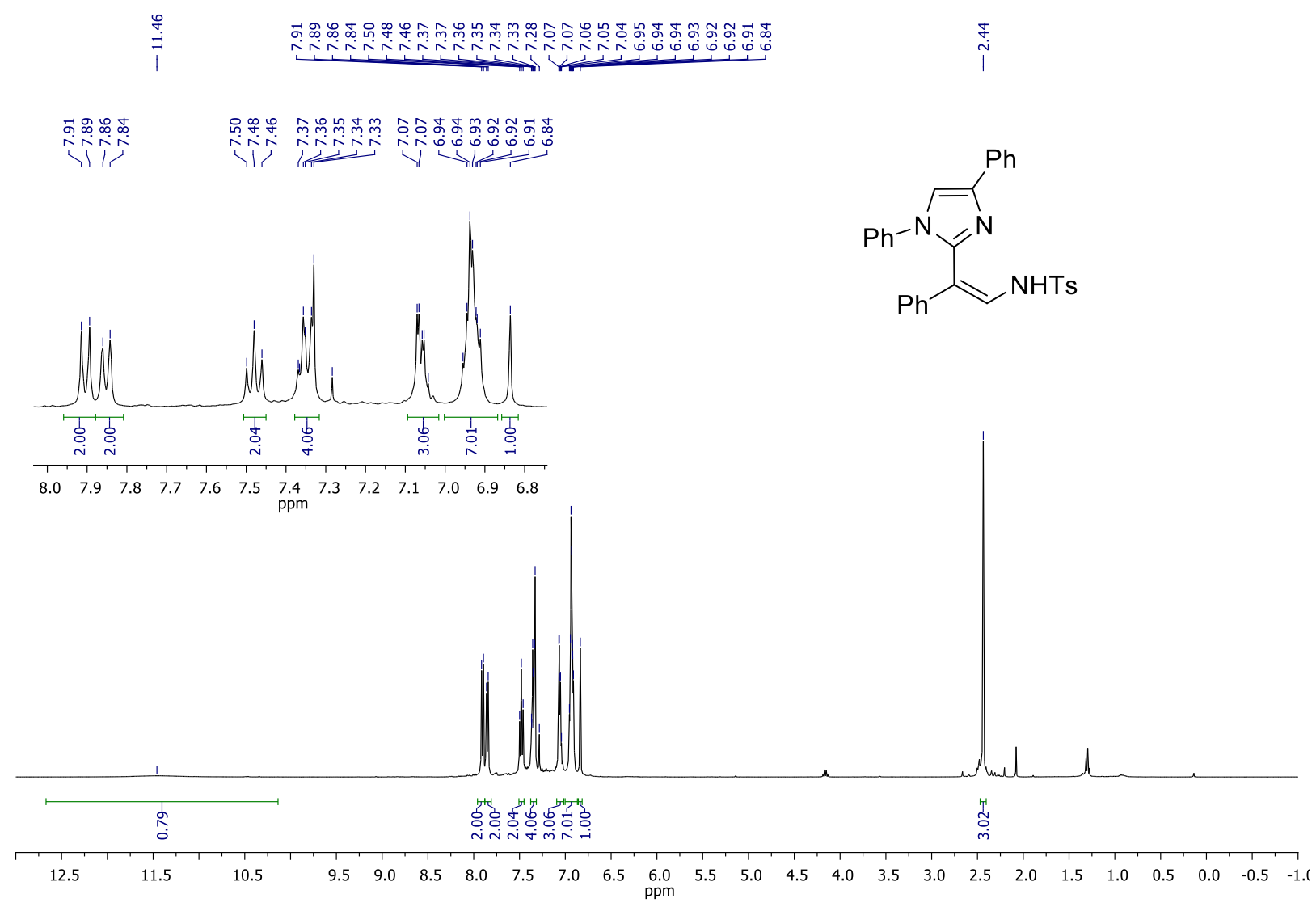

${ }^{13} \mathrm{C}\left\{{ }^{1} \mathrm{H}\right\}$ NMR spectrum of compound $40\left(100 \mathrm{MHz}, \mathrm{CDCl}_{3}\right)$

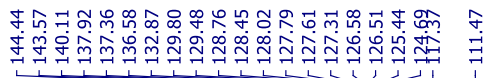

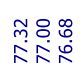

in

$\stackrel{\text { ำ }}{\stackrel{i}{1}}$<smiles>[AsH3-]N/C=C(/c1ccccc1)c1nc(-c2ccccc2)cn1-c1ccccc1</smiles>

PhTs

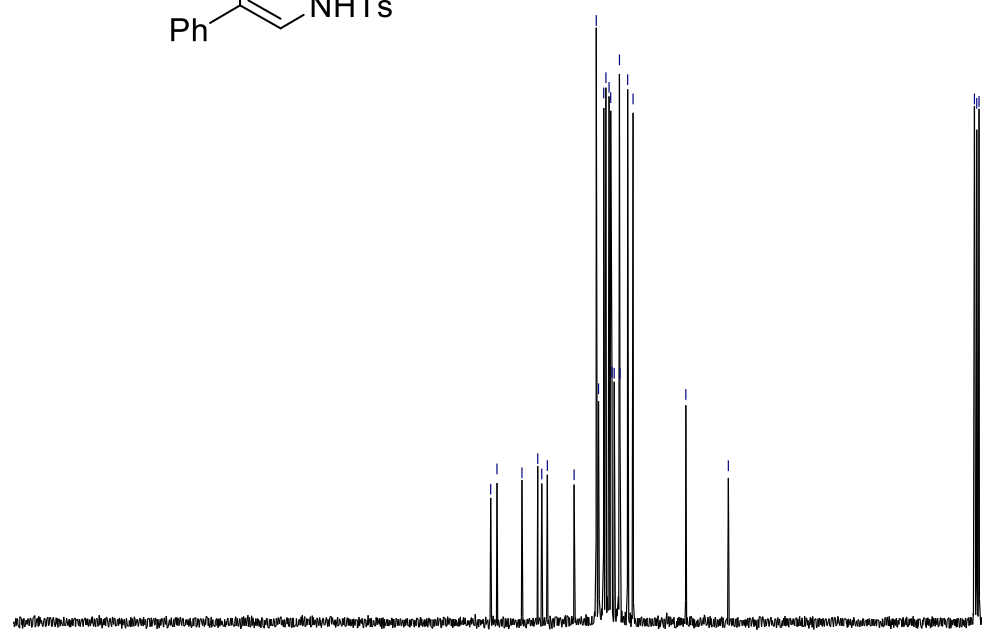

$\begin{array}{lllllllllllllllllllllllllllll}210 & 200 & 190 & 180 & 170 & 160 & 150 & 140 & 130 & 120 & 110 & 100 & 90 & 80 & 70 & 60 & 50 & 40 & 30 & 20 & 10 & 0 & -10\end{array}$ 
${ }^{1} \mathrm{H}$ NMR spectrum of compound $\mathbf{4 p}\left(400 \mathrm{MHz}, \mathrm{CDCl}_{3}\right)$

|

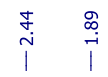

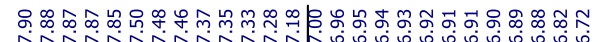
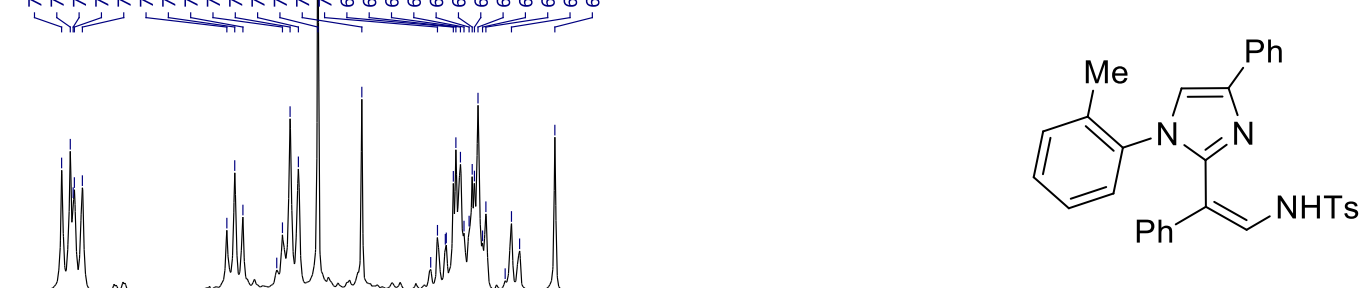

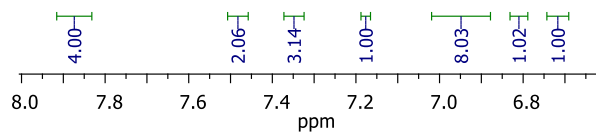
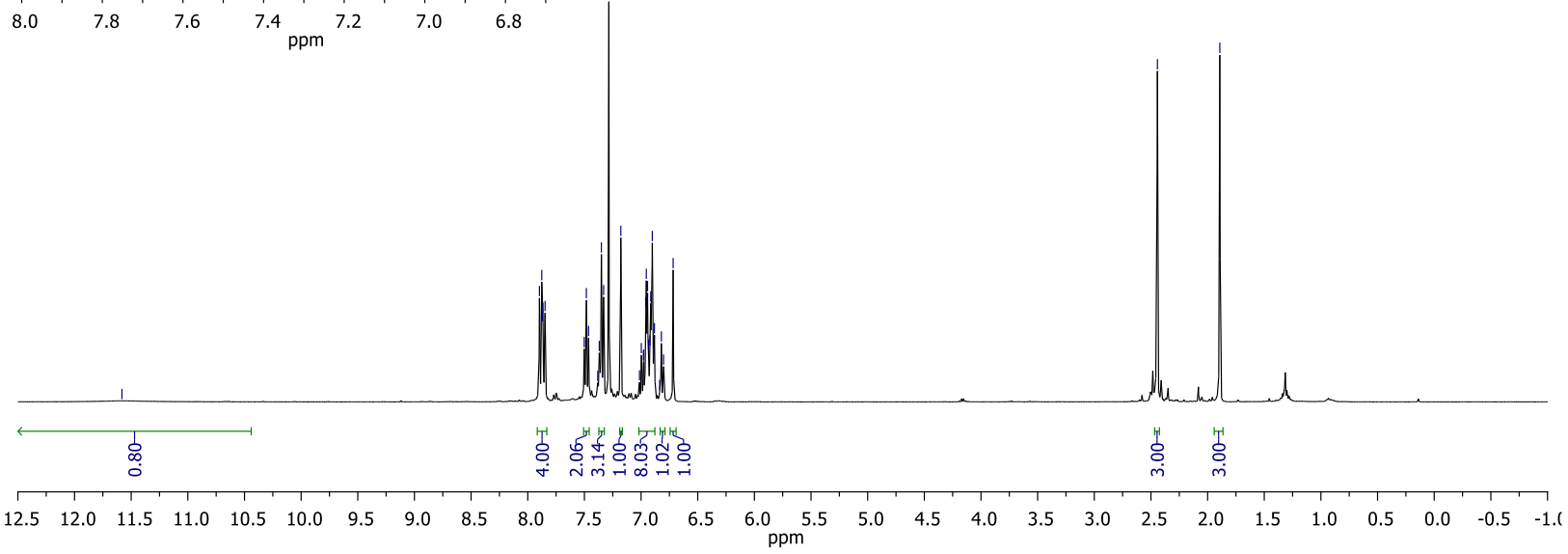

${ }^{13} \mathrm{C}\left\{{ }^{1} \mathrm{H}\right\}$ NMR spectrum of compound $4 \mathbf{p}\left(100 \mathrm{MHz}, \mathrm{CDCl}_{3}\right)$

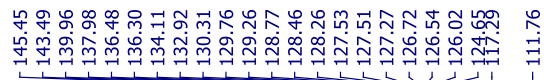

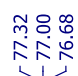

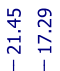<smiles>Cc1ccccc1-n1cc(-c2ccccc2)nc1/C(=C\N[15F])c1ccccc1</smiles>

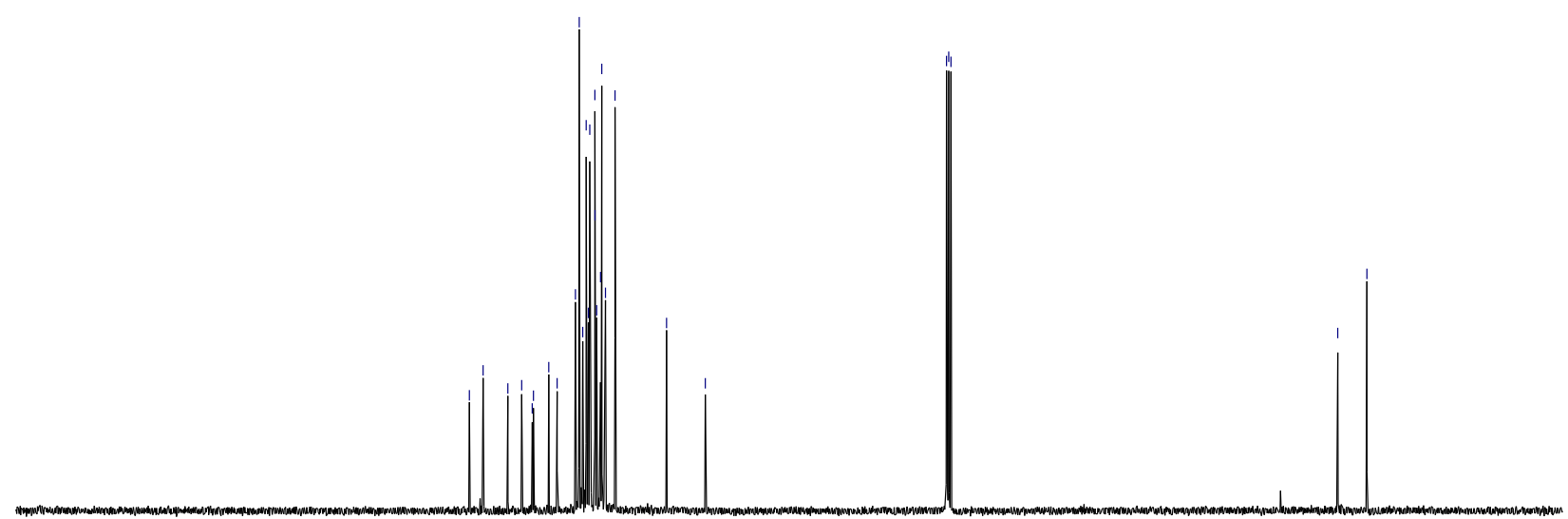

$\begin{array}{llllllllllllllllllllllllllllll}210 & 200 & 190 & 180 & 170 & 160 & 150 & 140 & 130 & 120 & 110 & 100 & 90 & 80 & 70 & 60 & 50 & 40 & 30 & 20 & 10 & 0 & -10\end{array}$ 
${ }^{1} \mathrm{H}$ NMR spectrum of compound $\mathbf{4 q}\left(400 \mathrm{MHz}, \mathrm{CDCl}_{3}\right)$

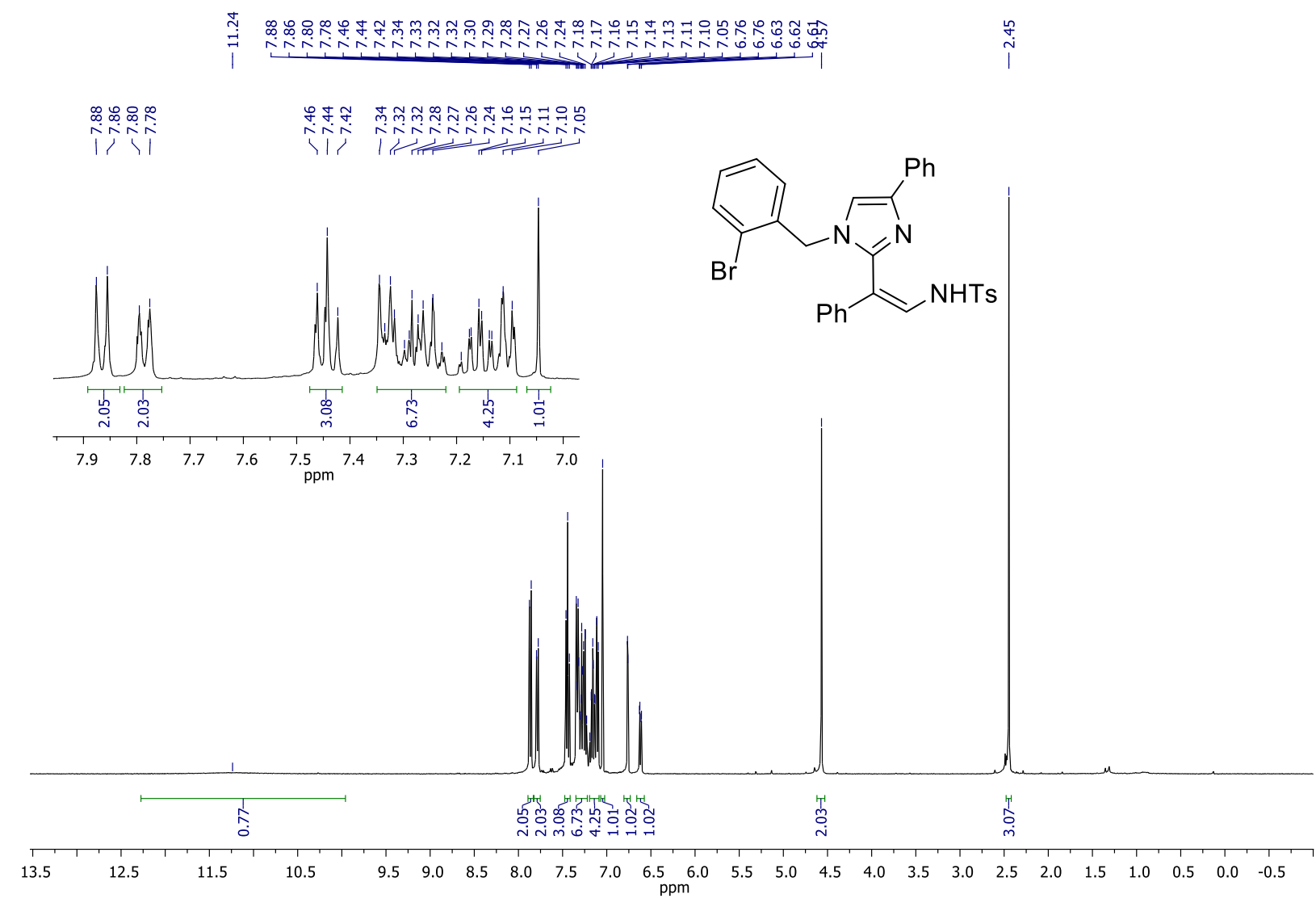

${ }^{13} \mathrm{C}\left\{{ }^{1} \mathrm{H}\right\} \mathrm{NMR}$ spectrum of compound $\mathbf{4 q}\left(100 \mathrm{MHz}, \mathrm{CDCl}_{3}\right)$
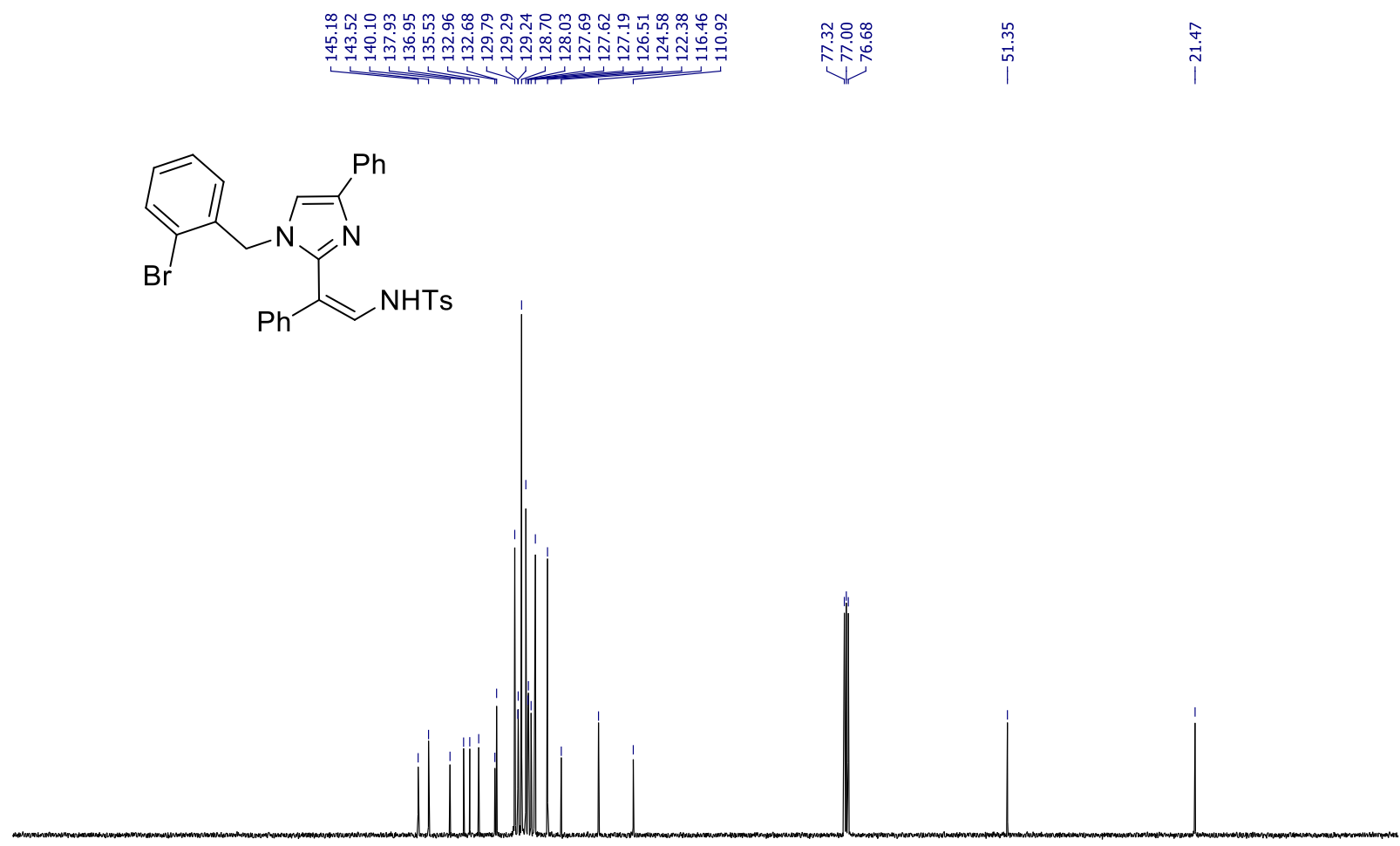

$\begin{array}{lllllllllllllllllllllllllll}200 & 190 & 180 & 170 & 160 & 150 & 140 & 130 & 120 & 110 & 100 & 90 & 80 & 70 & 60 & 50 & 40 & 30 & 20 & 10 & 0 & -10\end{array}$ 
${ }^{1} \mathrm{H}$ NMR spectrum of compound $\mathbf{4 r}\left(400 \mathrm{MHz}, \mathrm{CDCl}_{3}\right)$
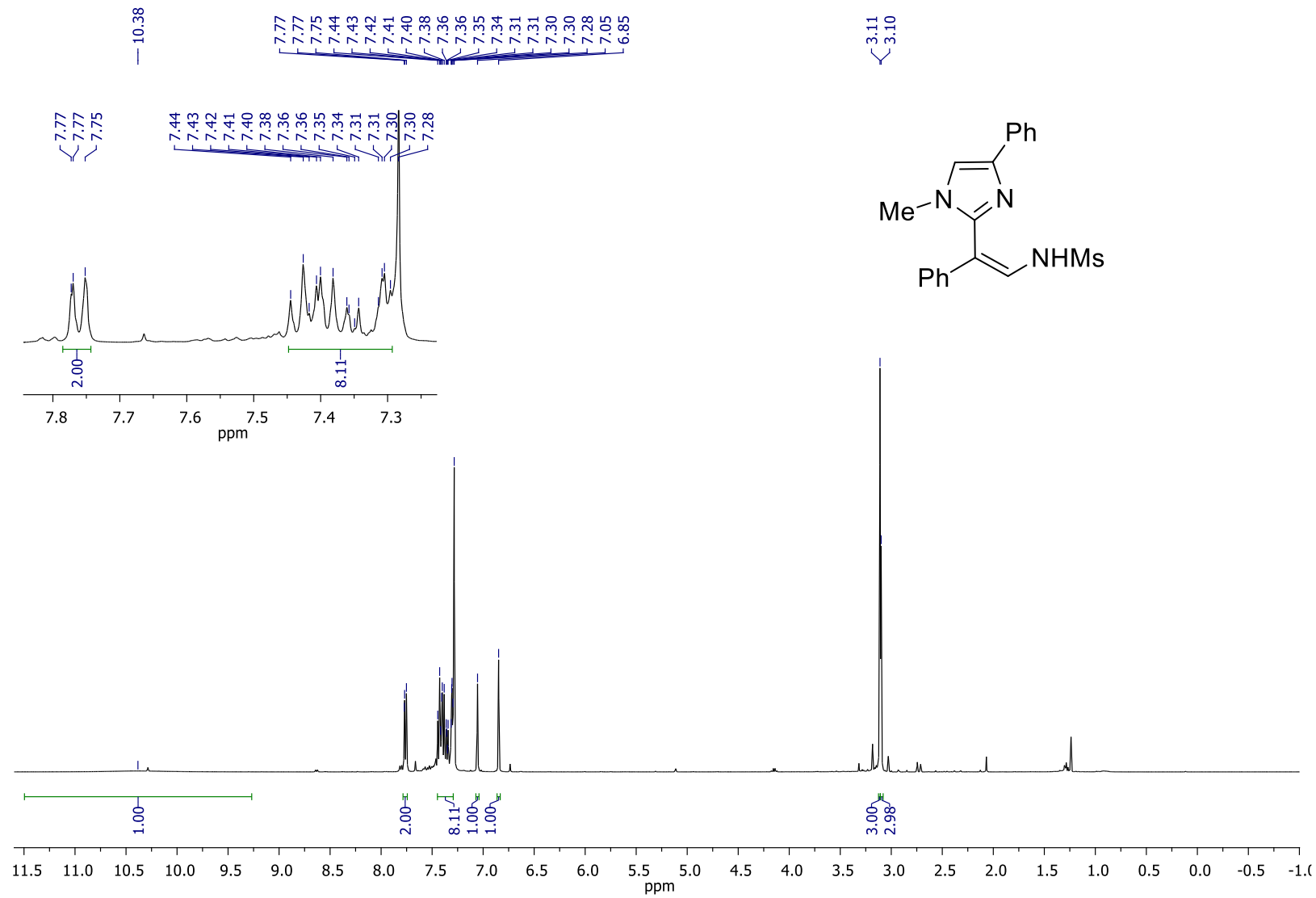

${ }^{13} \mathrm{C}\left\{{ }^{1} \mathrm{H}\right\}$ NMR spectrum of compound $4 \mathbf{r}\left(100 \mathrm{MHz}, \mathrm{CDCl}_{3}\right)$

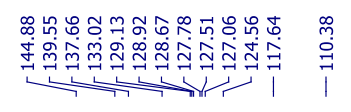

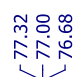

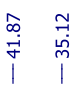<smiles>Cn1cc(-c2ccccc2)nc1/C(=C\NS(C)(=O)=O)c1ccccc1</smiles>

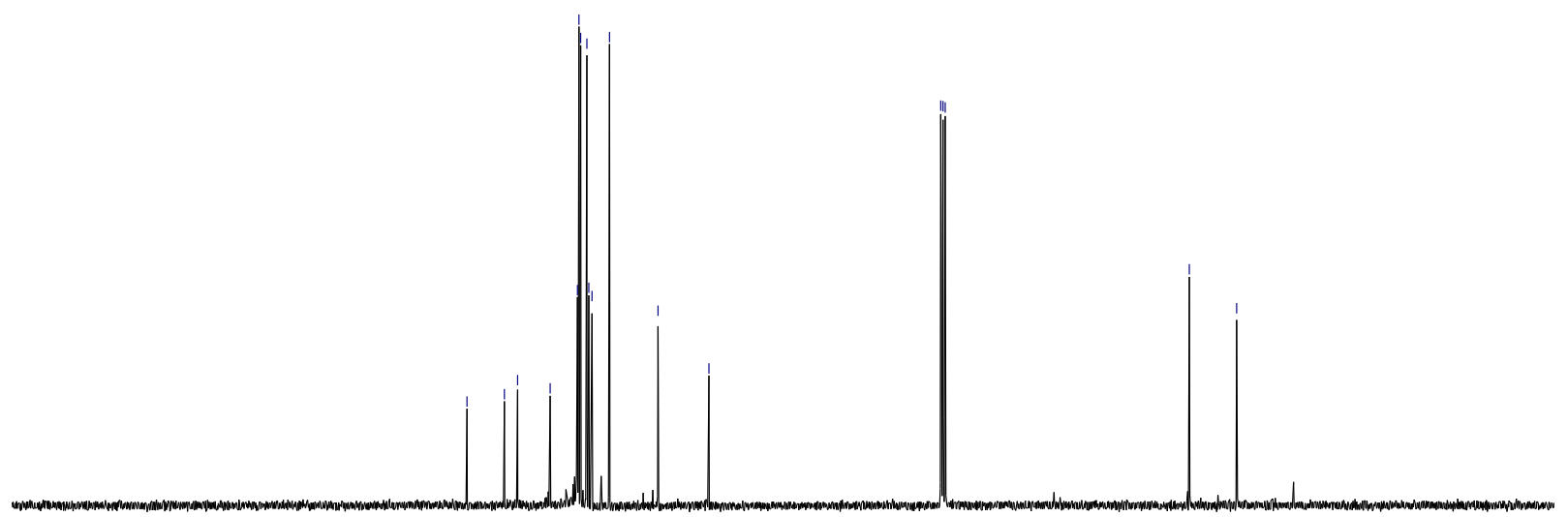

$\begin{array}{lllllllllllllllllllllll}200 & 190 & 180 & 170 & 160 & 150 & 140 & 130 & 120 & 110 & \begin{array}{l}100 \\ \mathrm{ppm}\end{array} & 90 & 80 & 70 & 60 & 50 & 40 & 30 & 20 & 10 & 0 & -10\end{array}$ 
${ }^{1} \mathrm{H}$ NMR spectrum of compound $\mathbf{4 s}\left(400 \mathrm{MHz}, \mathrm{CDCl}_{3}\right)$

|

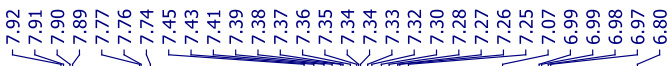
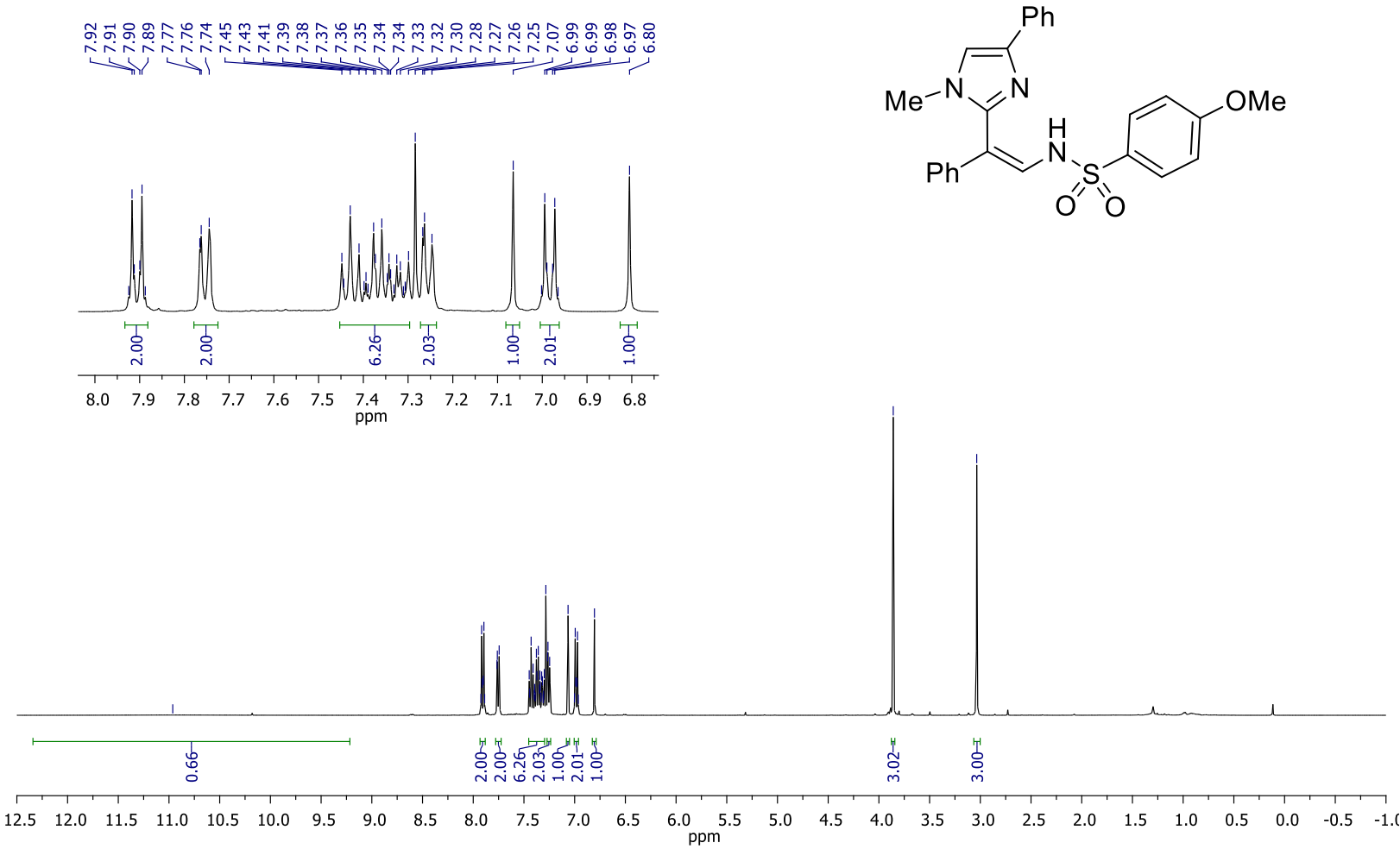

${ }^{13} \mathrm{C}\left\{{ }^{1} \mathrm{H}\right\}$ NMR spectrum of compound $4 \mathbf{s}\left(100 \mathrm{MHz}, \mathrm{CDCl}_{3}\right)$

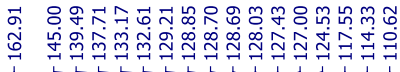

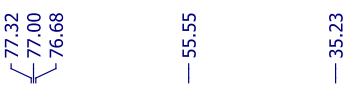<smiles>COc1ccc(S(=O)(=O)N/C=C(/c2ccccc2)c2nc(-c3ccccc3)cn2C)cc1</smiles>

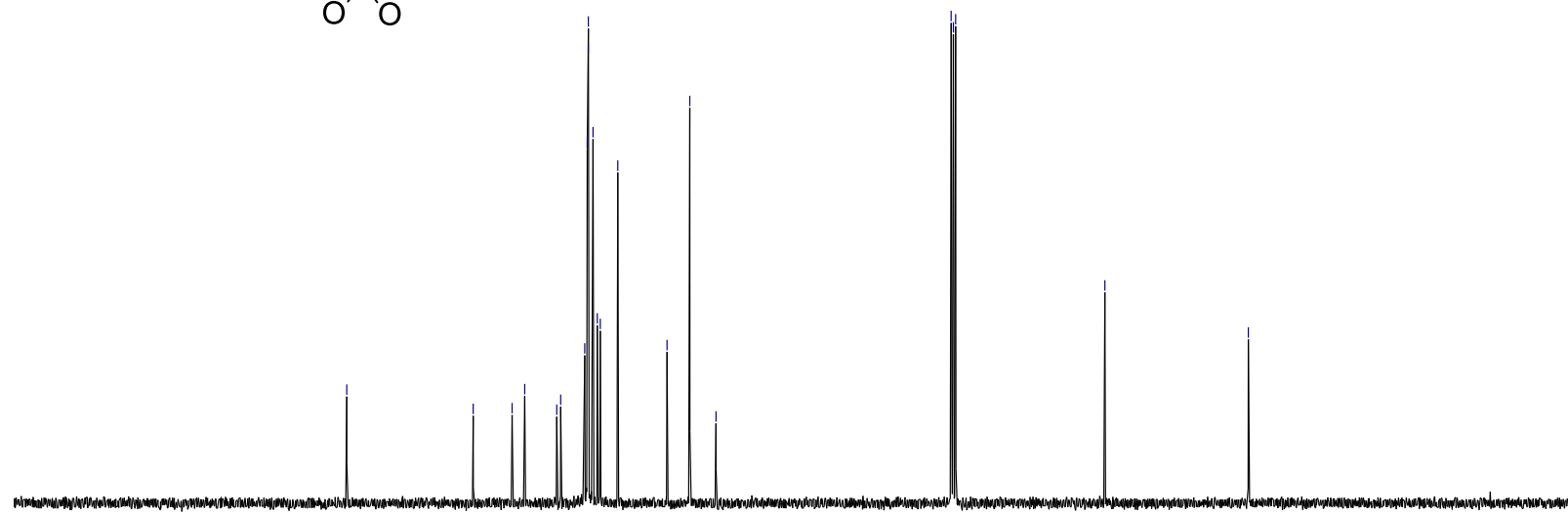

$\begin{array}{llllllllllllllllllllllllllllllllll}200 & 190 & 180 & 170 & 160 & 150 & 140 & 130 & 120 & 110 & 100 & 90 & 80 & 70 & 60 & 50 & 40 & 30 & 20 & 10 & 0 & -10\end{array}$ 
${ }^{1} \mathrm{H}$ NMR spectrum of compound $\mathbf{4 t}\left(400 \mathrm{MHz}, \mathrm{CDCl}_{3}\right)$

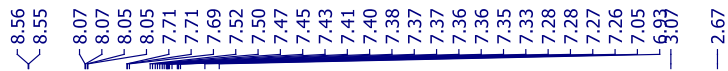

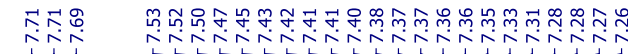

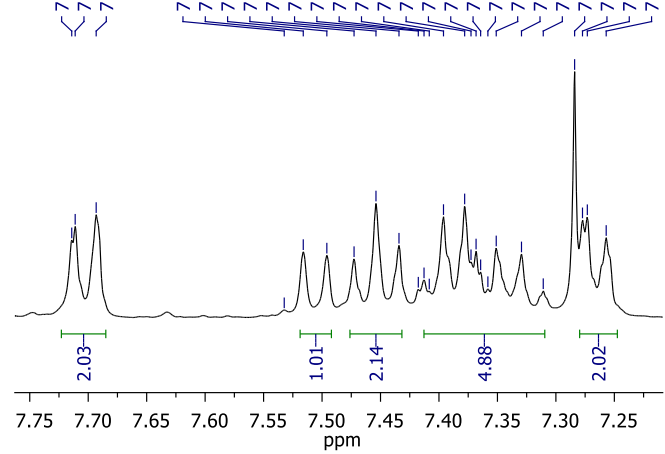<smiles>Cn1cc(-c2ccccc2)nc1/C(=C\NS(=O)(=O)c1ccc([N+](=O)[O-])c([N+](=O)[O-])c1)c1ccccc1</smiles>

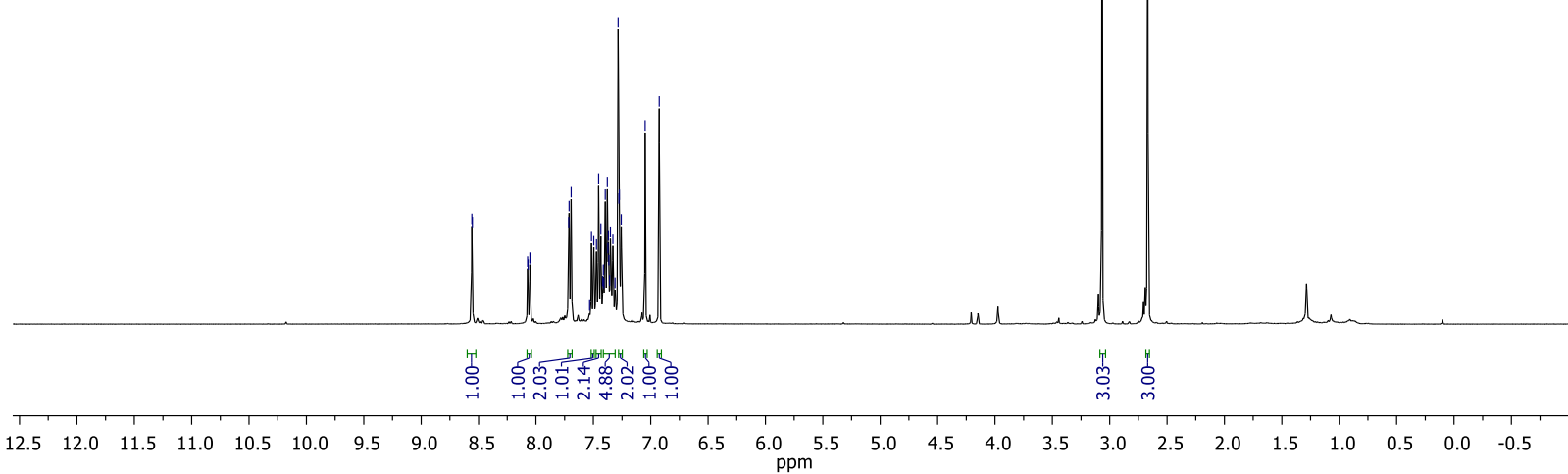

${ }^{13} \mathrm{C}\left\{{ }^{1} \mathrm{H}\right\}$ NMR spectrum of compound $4 \mathbf{t}\left(100 \mathrm{MHz}, \mathrm{CDCl}_{3}\right)$

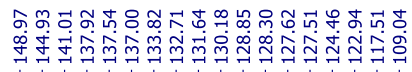

mon

is

点<smiles>Cc1ccc(S(=O)(=O)N/C=C(/c2ccccc2)c2nc(-c3ccccc3)cn2C)cc1[N+](=O)[O-]</smiles> 
${ }^{1} \mathrm{H}$ NMR spectrum of compound $\mathbf{4 u}\left(400 \mathrm{MHz}, \mathrm{CDCl}_{3}\right)$
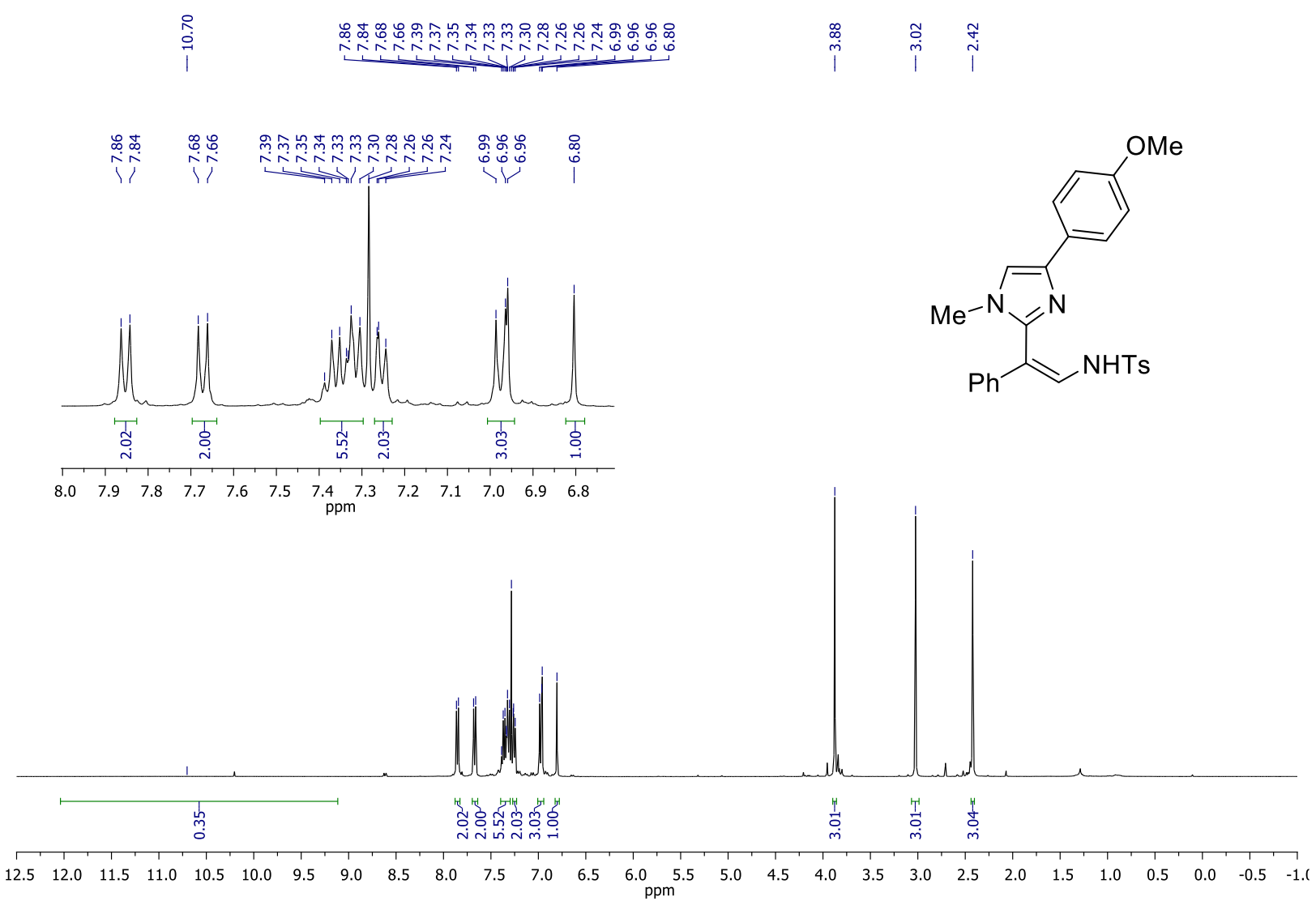

${ }^{13} \mathrm{C}\left\{{ }^{1} \mathrm{H}\right\}$ NMR spectrum of compound $\mathbf{4 u}\left(100 \mathrm{MHz}, \mathrm{CDCl}_{3}\right)$

|
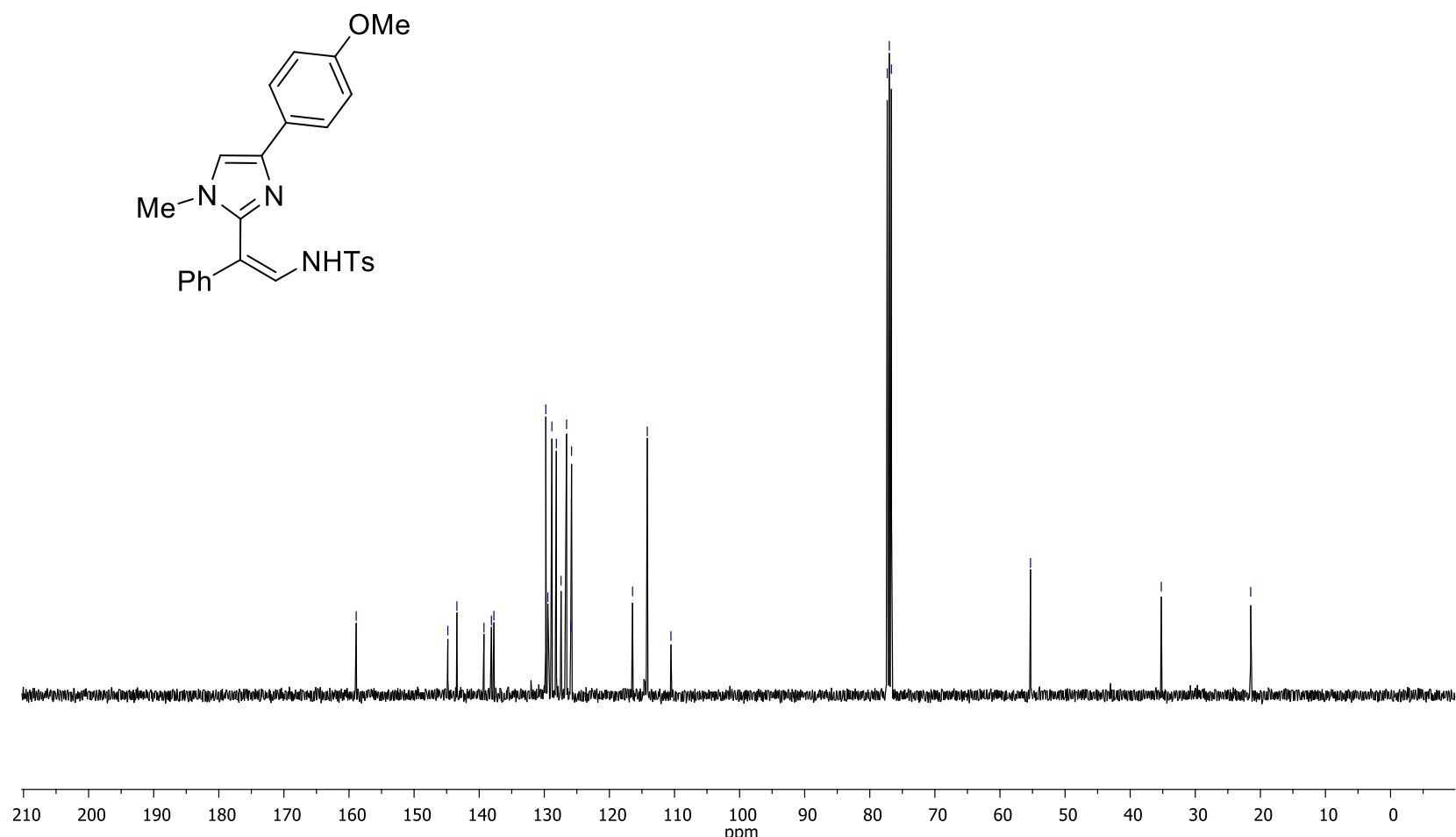
${ }^{1} \mathrm{H}$ NMR spectrum of compound $\mathbf{4 v}\left(400 \mathrm{MHz}, \mathrm{CDCl}_{3}\right)$

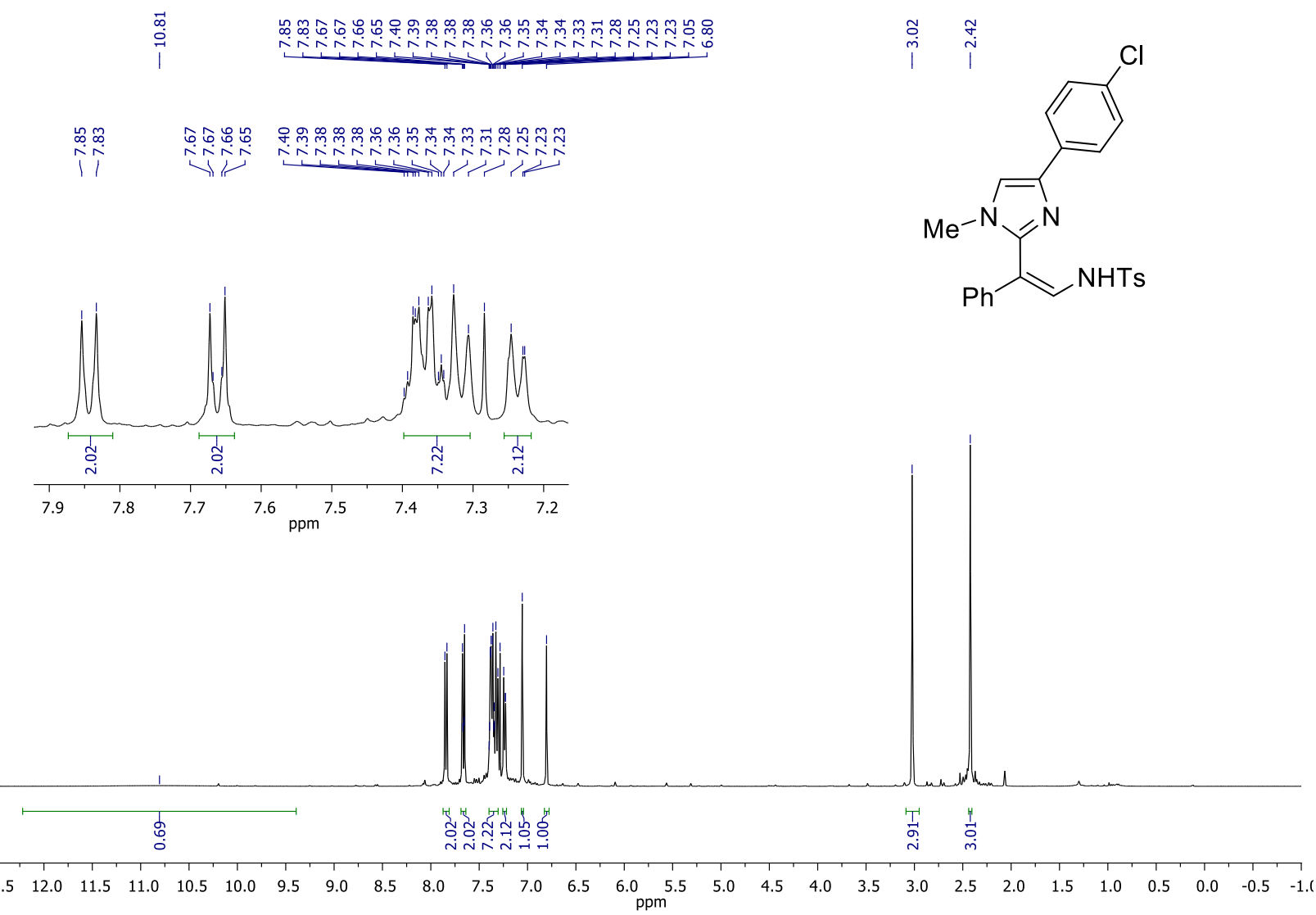

${ }^{13} \mathrm{C}\left\{{ }^{1} \mathrm{H}\right\}$ NMR spectrum of compound $4 \mathbf{v}\left(100 \mathrm{MHz}, \mathrm{CDCl}_{3}\right)$

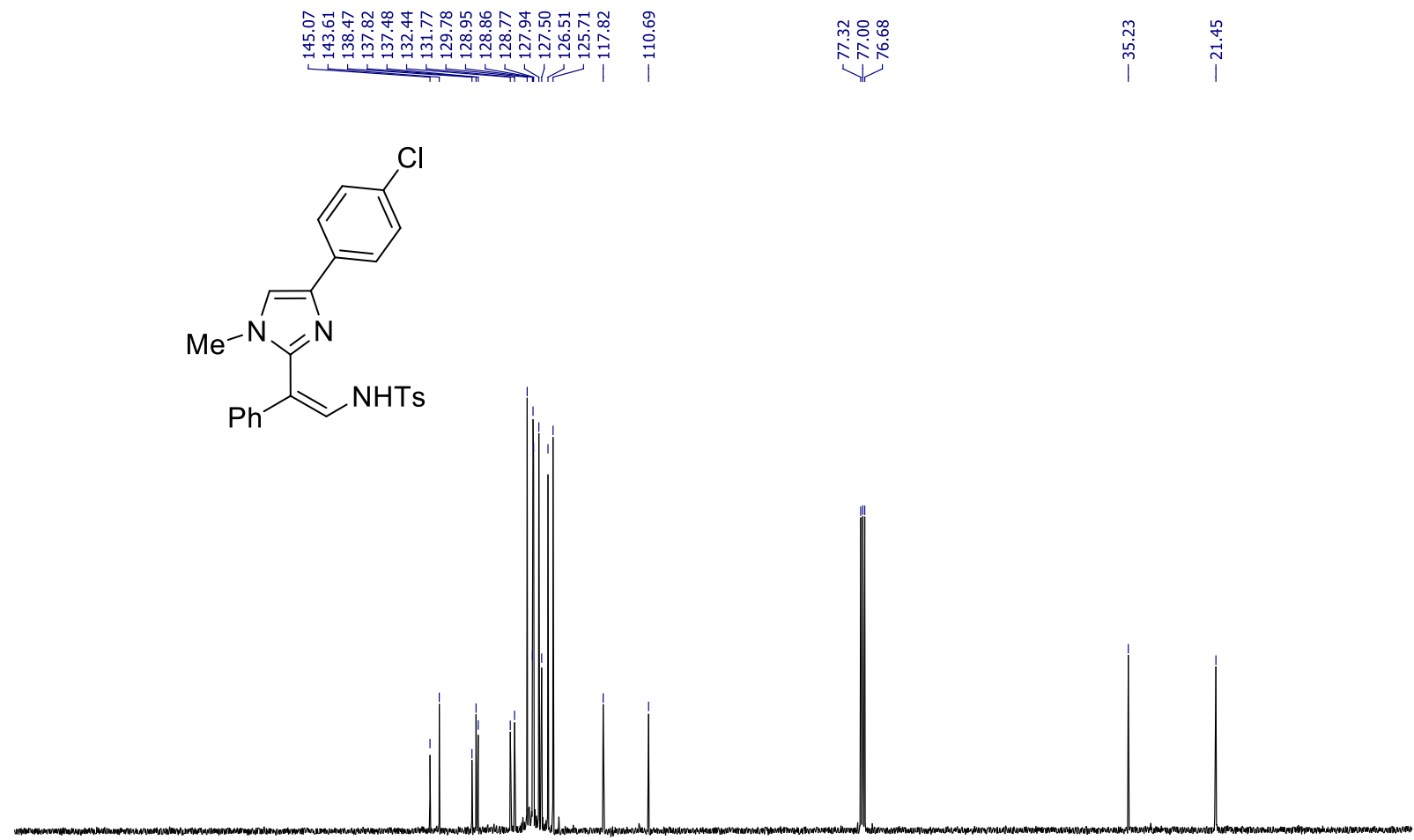

\begin{tabular}{llllllllllllllllllllllllll}
\hline 210 & 200 & 190 & 180 & 170 & 160 & 150 & 140 & 130 & 120 & 110 & 100 & 90 & 80 & 70 & 60 & 50 & 40 & 30 & 20 & 10 & 0
\end{tabular} 
${ }^{1} \mathrm{H}$ NMR spectrum of compound $\mathbf{4 w}\left(400 \mathrm{MHz}, \mathrm{CDCl}_{3}\right)$

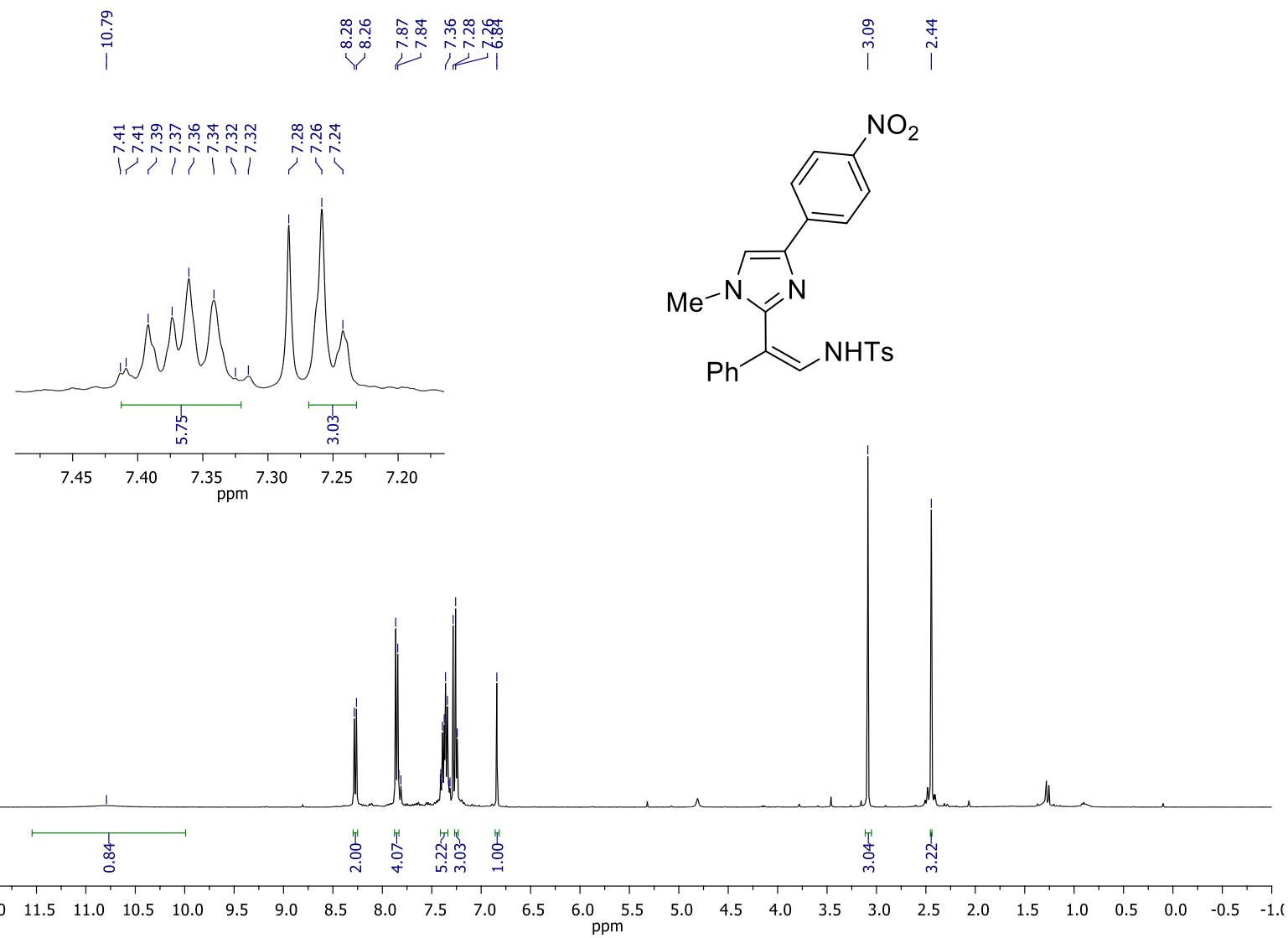

${ }^{13} \mathrm{C}\left\{{ }^{1} \mathrm{H}\right\}$ NMR spectrum of compound $\mathbf{4 w}\left(100 \mathrm{MHz}, \mathrm{CDCl}_{3}\right)$

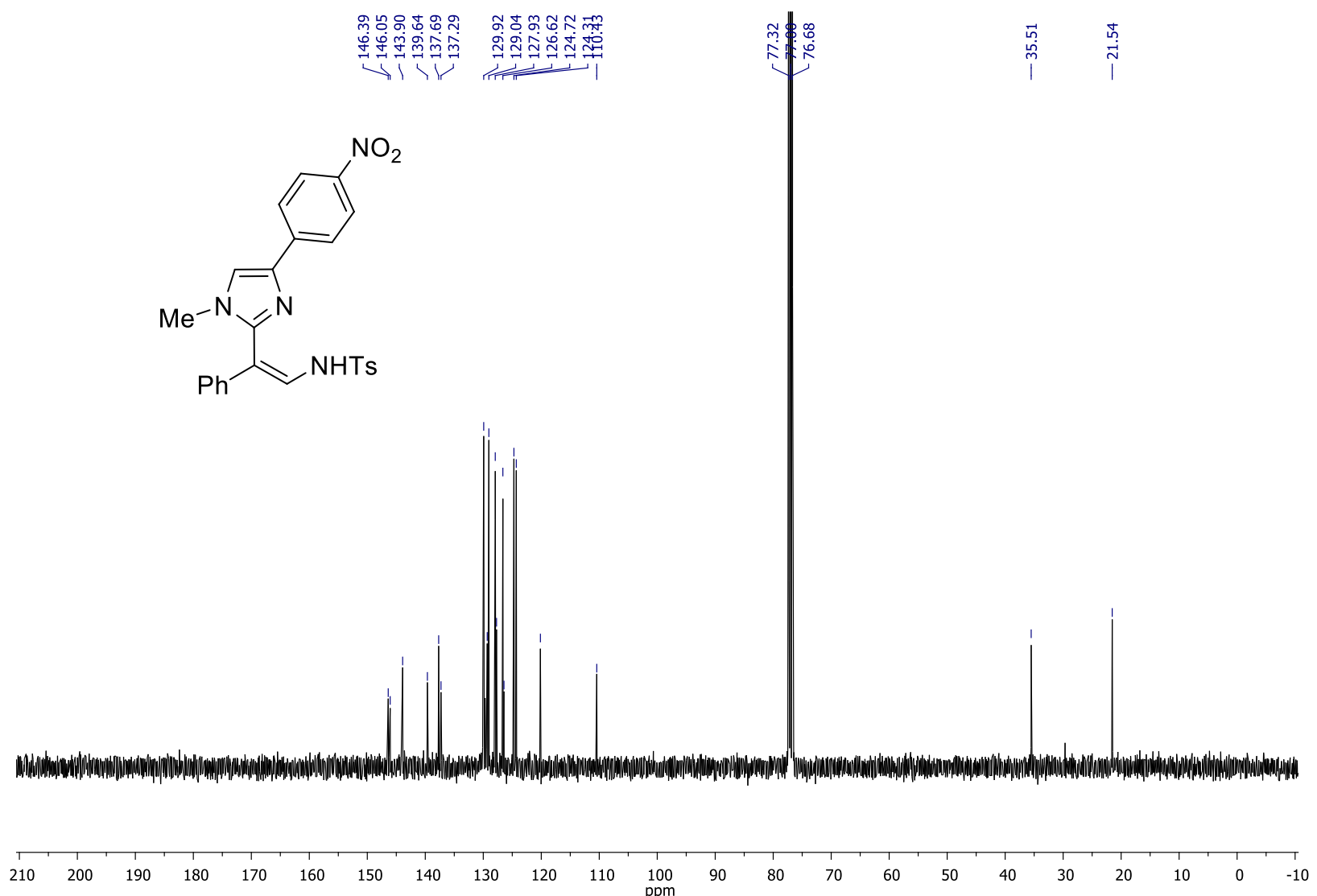


${ }^{1} \mathrm{H}$ NMR spectrum of compound $\mathbf{4 x}\left(400 \mathrm{MHz}, \mathrm{CDCl}_{3}\right)$

等

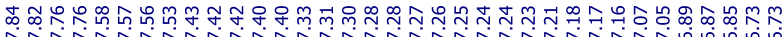

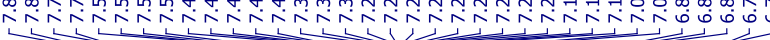
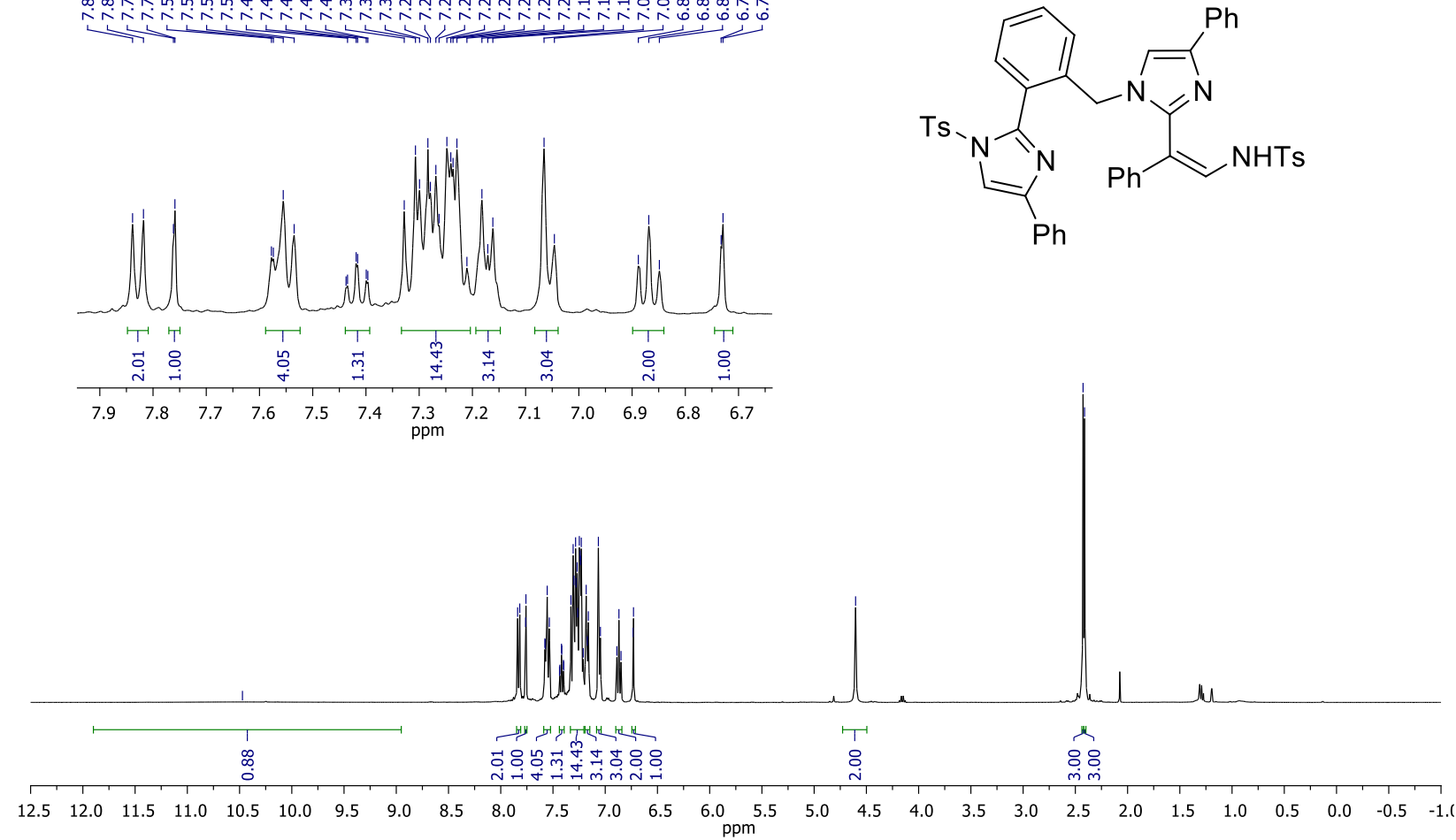

${ }^{13} \mathrm{C}\left\{{ }^{1} \mathrm{H}\right\}$ NMR spectrum of compound $4 \mathbf{x}\left(100 \mathrm{MHz}, \mathrm{CDCl}_{3}\right)$

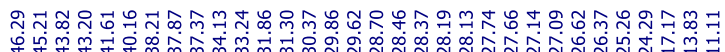

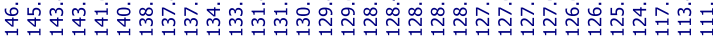

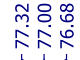

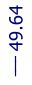

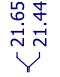

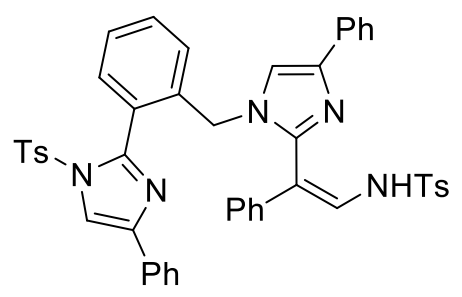

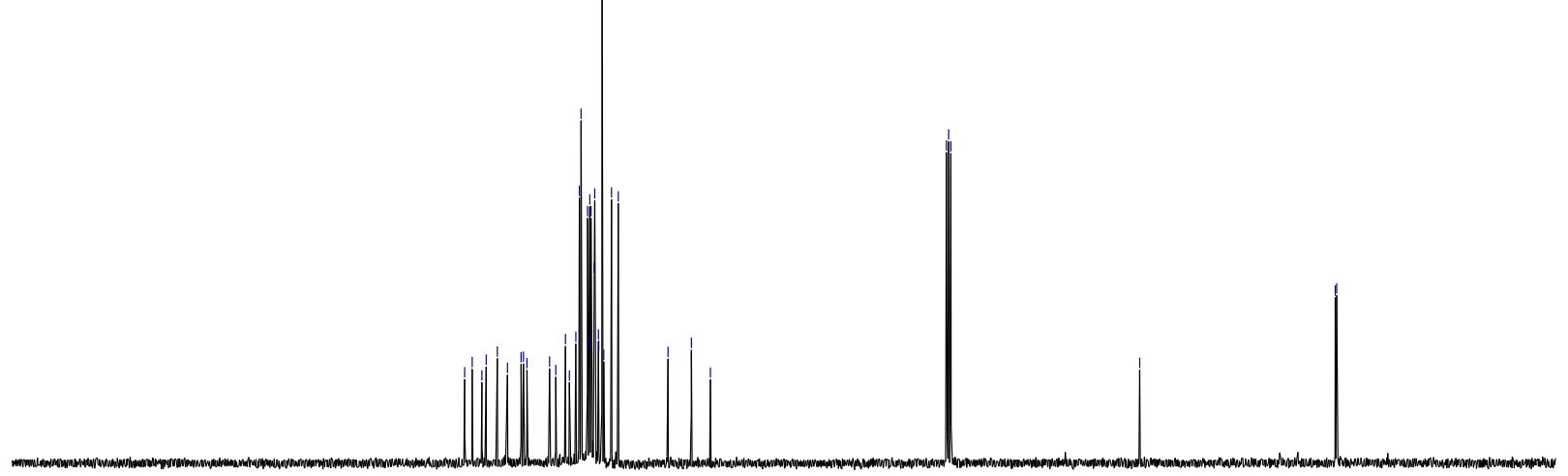

$\begin{array}{llllllllllll}210 & 200 & 190 & 180 & 170 & 160 & 150 & 140 & 130 & 120 & 110 & 100\end{array}$ 
${ }^{1} \mathrm{H}$ NMR spectrum of compound $\mathbf{4 y}\left(400 \mathrm{MHz}, \mathrm{CDCl}_{3}\right)$

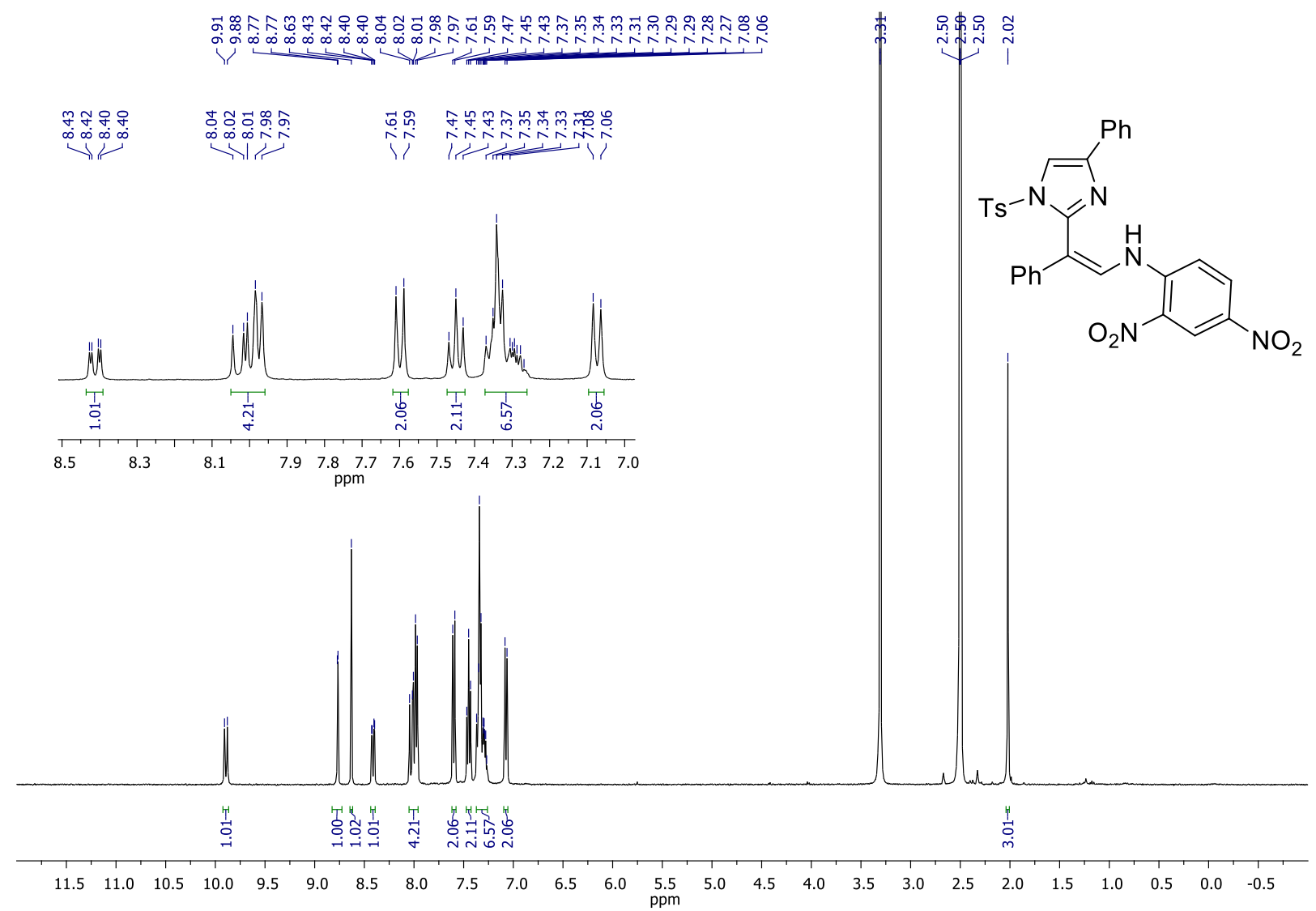

${ }^{13} \mathrm{C}\left\{{ }^{1} \mathrm{H}\right\}$ NMR spectrum of compound $\mathbf{4 y}\left(100 \mathrm{MHz}, \mathrm{CDCl}_{3}\right)$
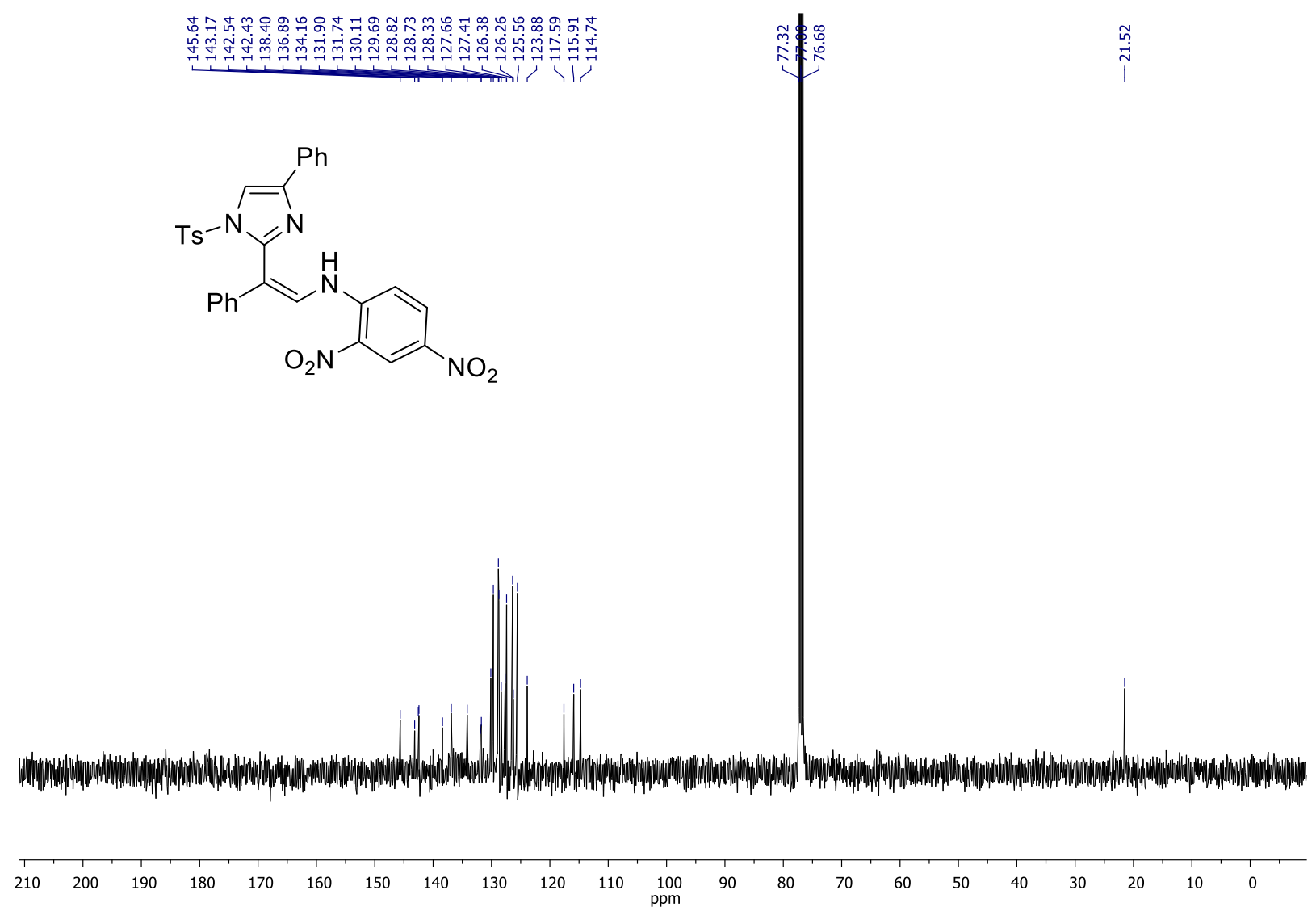
${ }^{1} \mathrm{H}$ NMR spectrum of compound $\mathbf{4 z}\left(400 \mathrm{MHz}, \mathrm{CDCl}_{3}\right)$

V)<smiles>[3H]n1cc(-c2ccccc2)nc1/C(=C\Nc1ccc([N+](=O)[O-])cc1[N+](=O)[O-])c1ccc(F)cc1</smiles>

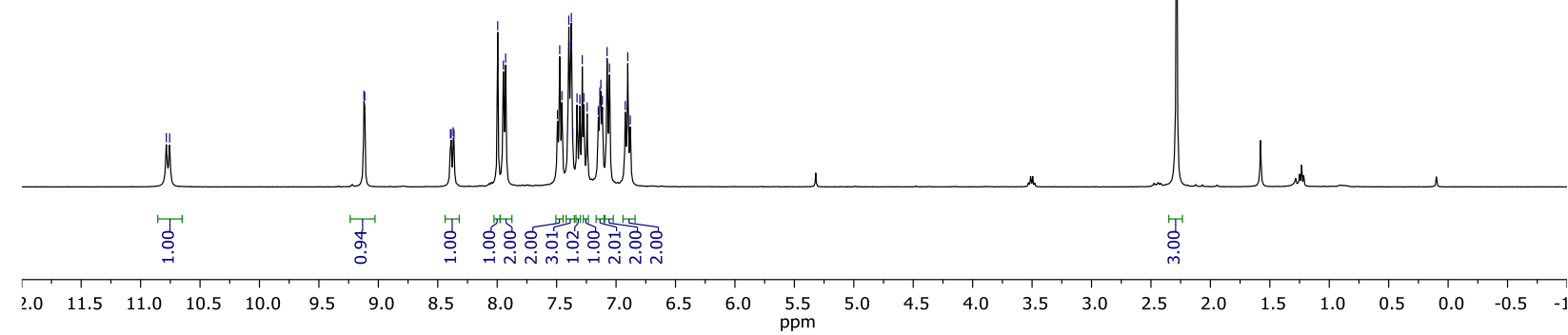

${ }^{13} \mathrm{C}\left\{{ }^{1} \mathrm{H}\right\}$ NMR spectrum of compound $\mathbf{4 z}\left(100 \mathrm{MHz}, \mathrm{CDCl}_{3}\right)$

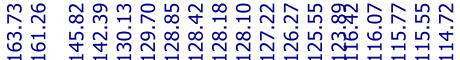

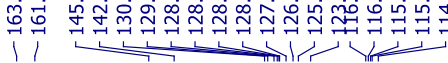<smiles>[3H]n1cc(-c2ccccc2)nc1/C(=C\Nc1ccc([N+](=O)[O-])cc1[N+](=O)[O-])c1ccc(F)cc1</smiles> 
${ }^{1} \mathrm{H}$ NMR spectrum of compound $\mathbf{4 z a}\left(400 \mathrm{MHz}, \mathrm{CDCl}_{3}\right)$

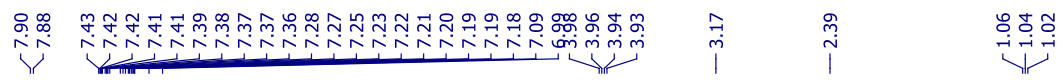

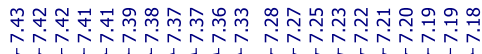
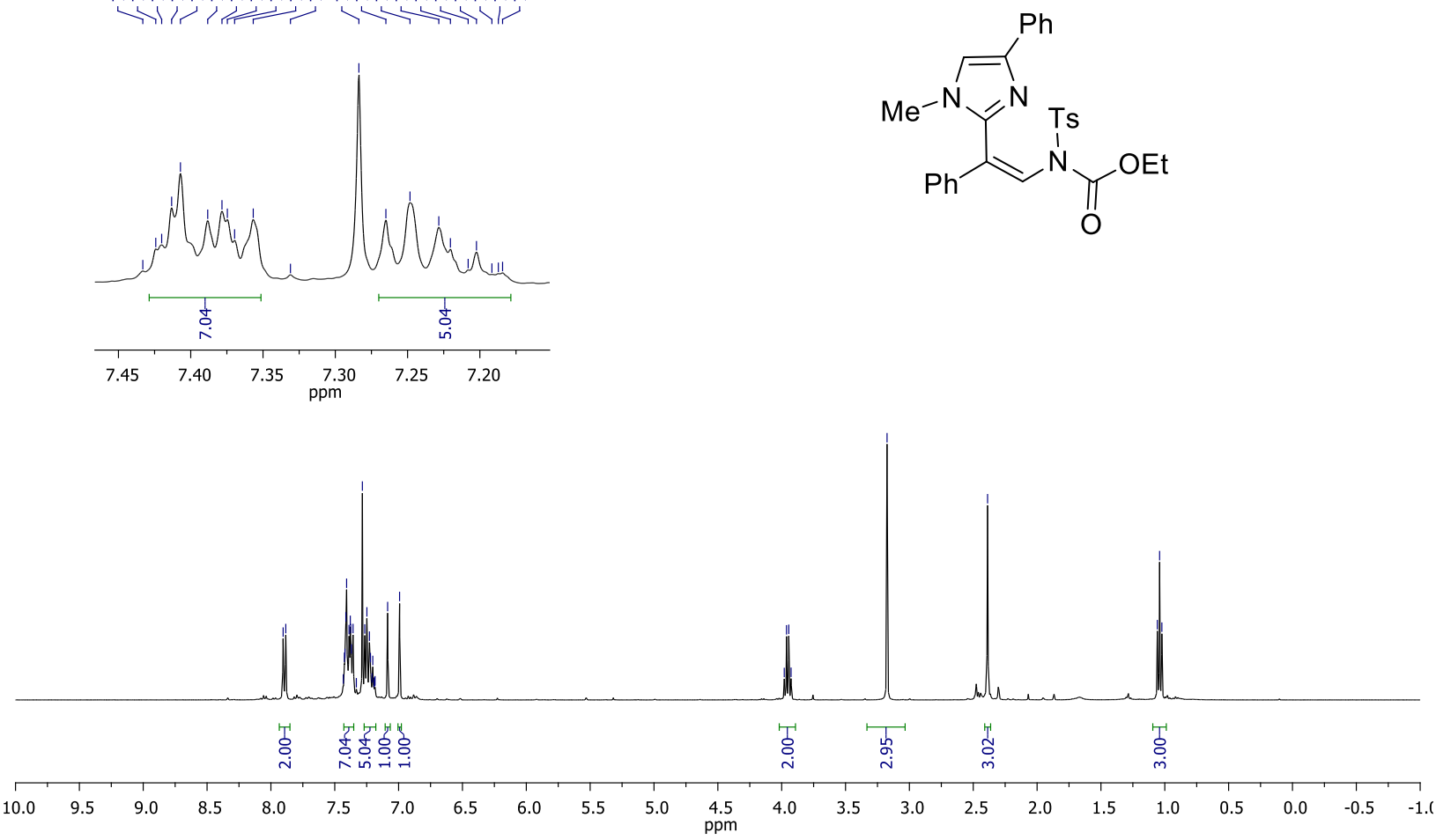

${ }^{13} \mathrm{C}\left\{{ }^{1} \mathrm{H}\right\}$ NMR spectrum of compound $4 \mathbf{z a}\left(100 \mathrm{MHz}, \mathrm{CDCl}_{3}\right)$

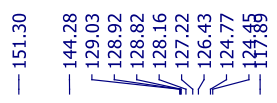<smiles>CCOC(=O)N([13CH3])/C=C(/c1ccccc1)c1nc(-c2ccccc2)cn1C</smiles>

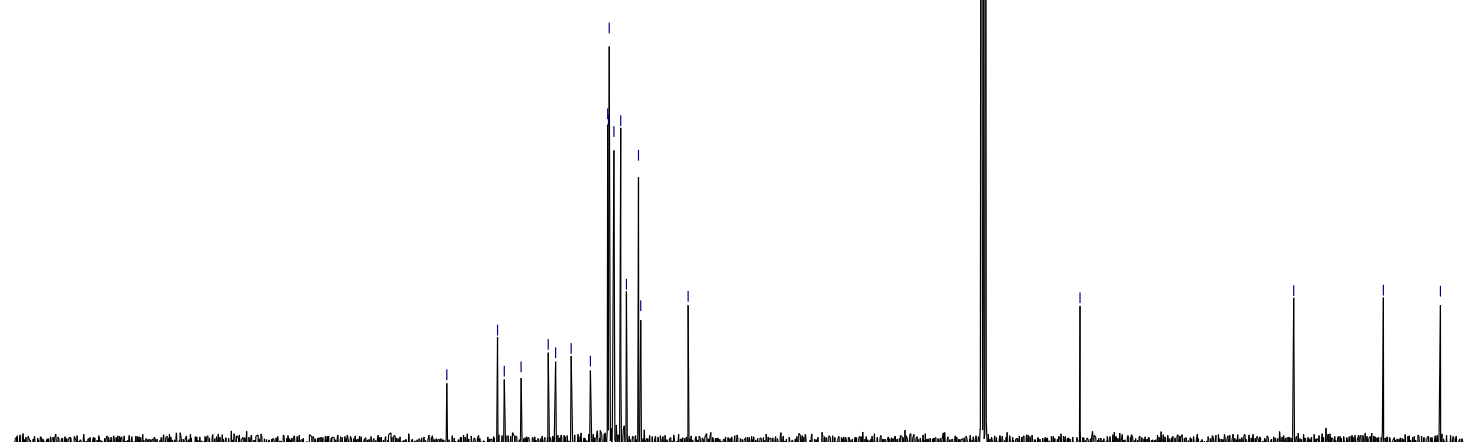


${ }^{1} \mathrm{H}$ NMR spectrum of compound $\mathbf{4 z b}\left(400 \mathrm{MHz}, \mathrm{CDCl}_{3}\right)$

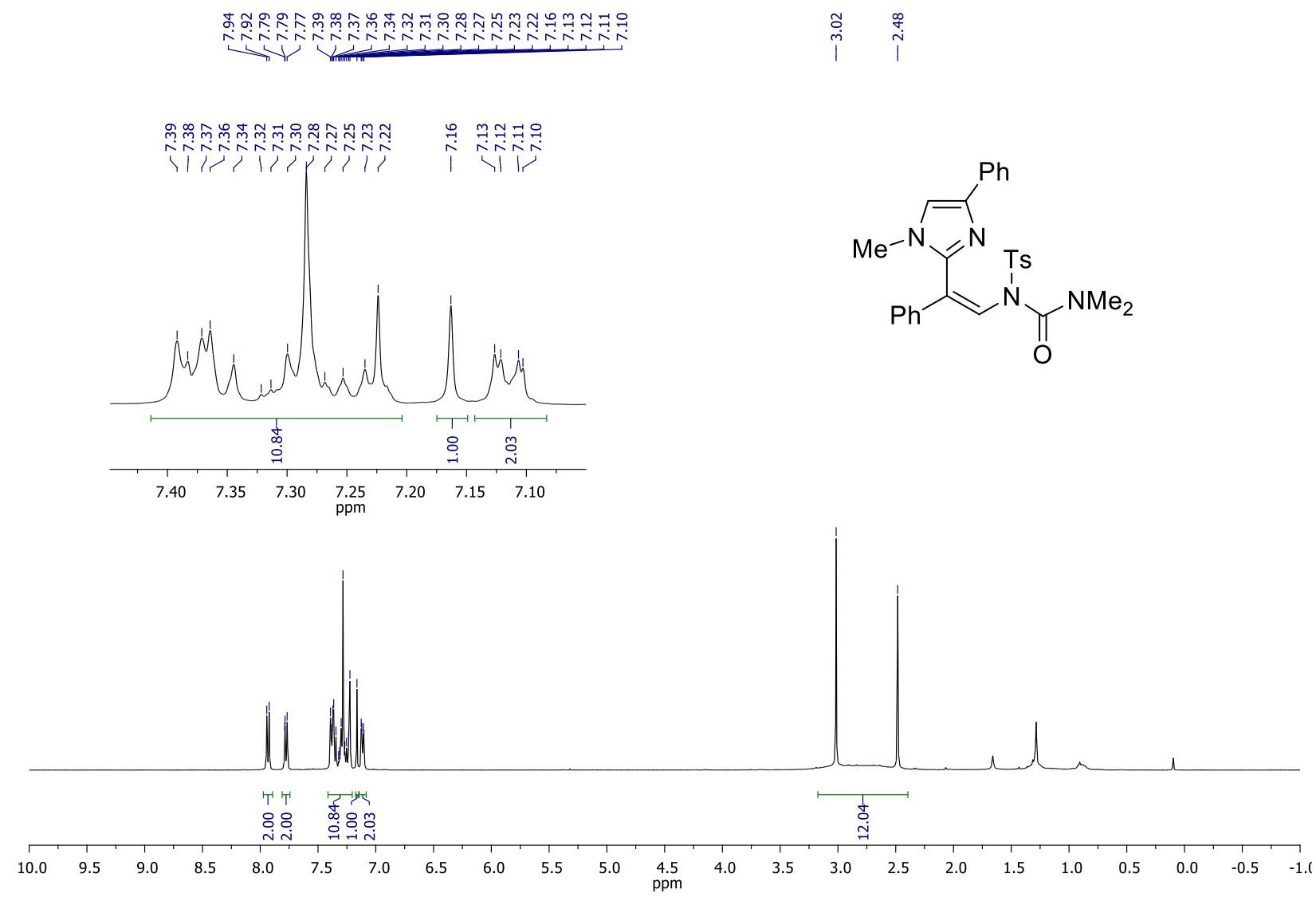

${ }^{13} \mathrm{C}\left\{{ }^{1} \mathrm{H}\right\}$ NMR spectrum of compound $\mathbf{4 z b}\left(100 \mathrm{MHz}, \mathrm{CDCl}_{3}\right)$
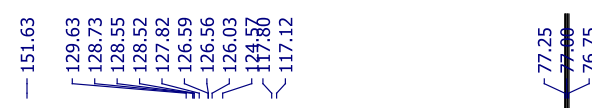

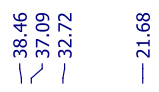

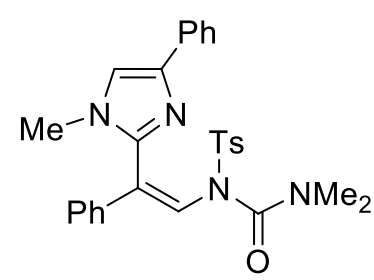

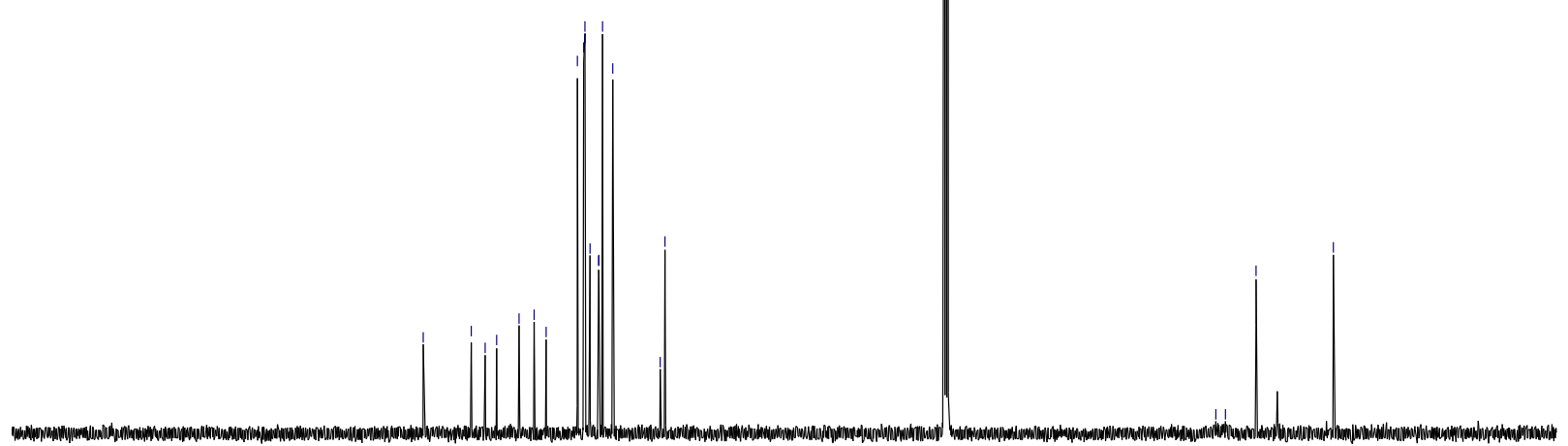

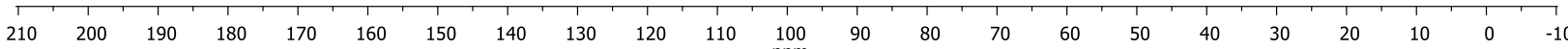


${ }^{1} \mathrm{H}$ NMR spectrum of compound $15\left(400 \mathrm{MHz}, \mathrm{CDCl}_{3}\right)$

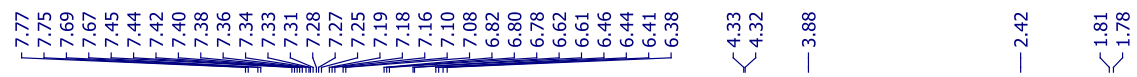

$\underbrace{-11}$
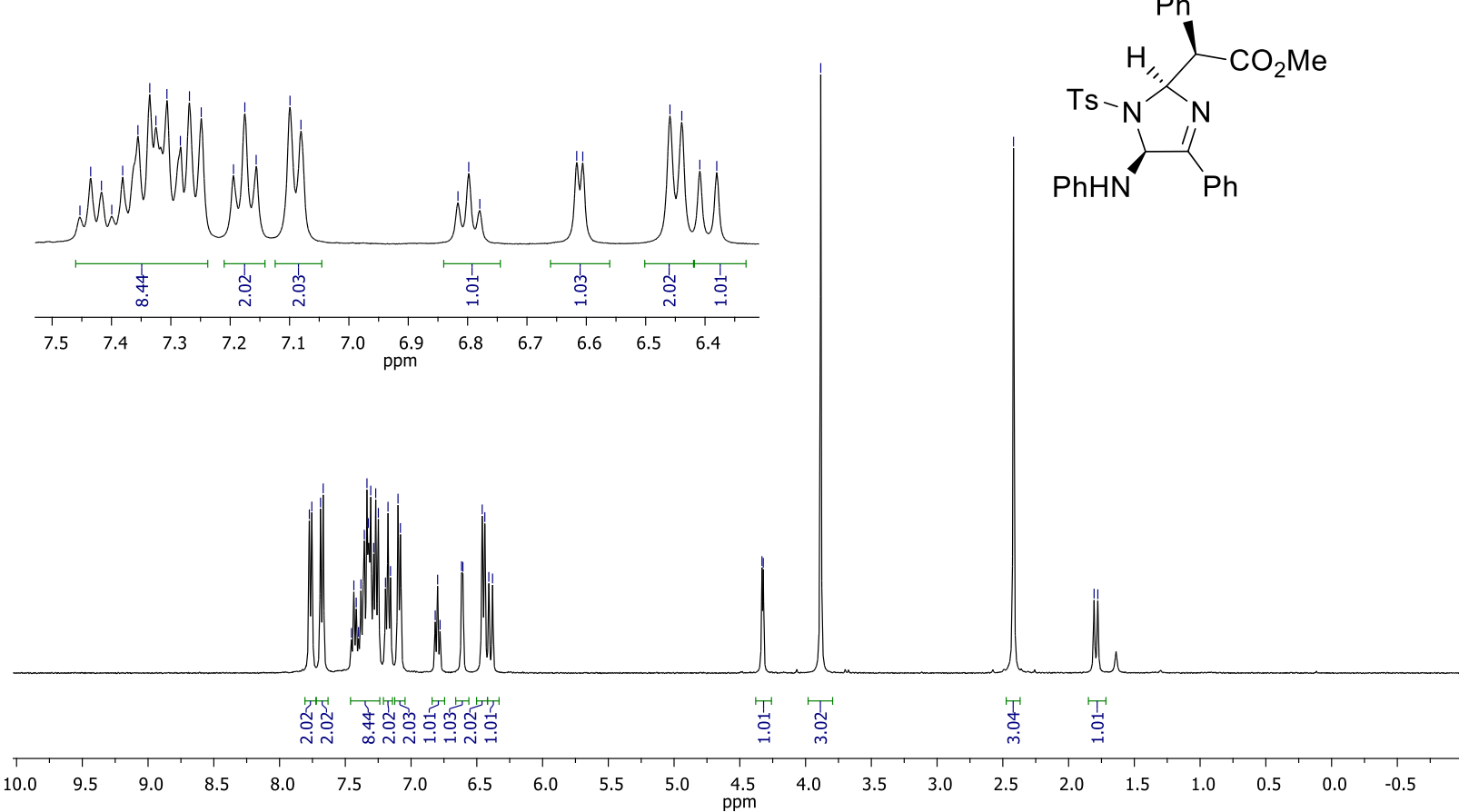

${ }^{13} \mathrm{C}\left\{{ }^{1} \mathrm{H}\right\}$ NMR spectrum of compound $15\left(100 \mathrm{MHz}, \mathrm{CDCl}_{3}\right)$
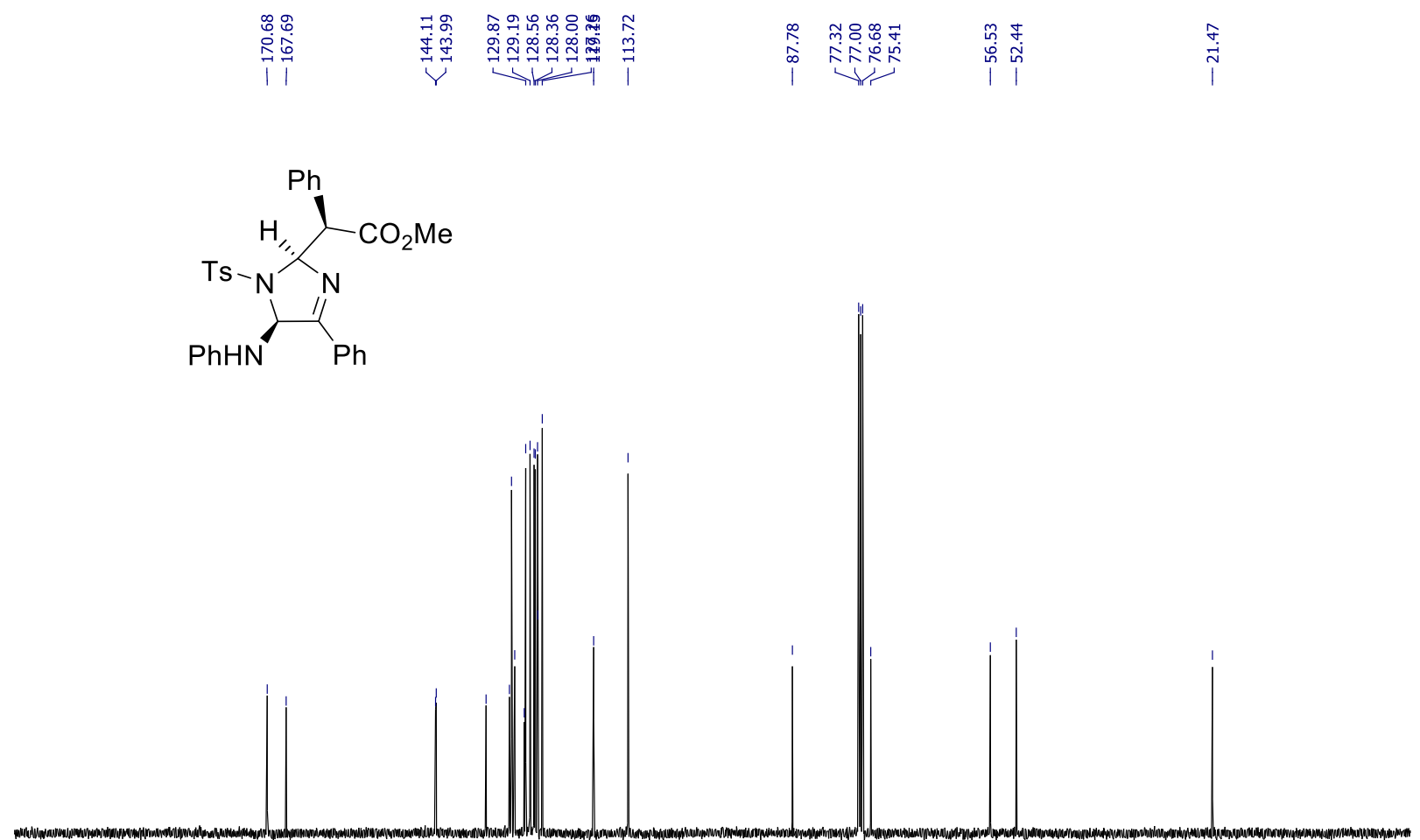

$\begin{array}{lllllllllllllllllllllllll}210 & 200 & 190 & 180 & 170 & 160 & 150 & 140 & 130 & 120 & 110 & 100 & 90 & 80 & 70 & 60 & 50 & 40 & 30 & 20 & 10 & 0 & 0\end{array}$ 
${ }^{1} \mathrm{H}$ NMR spectrum of compound $16\left(400 \mathrm{MHz}, \mathrm{CDCl}_{3}\right)$

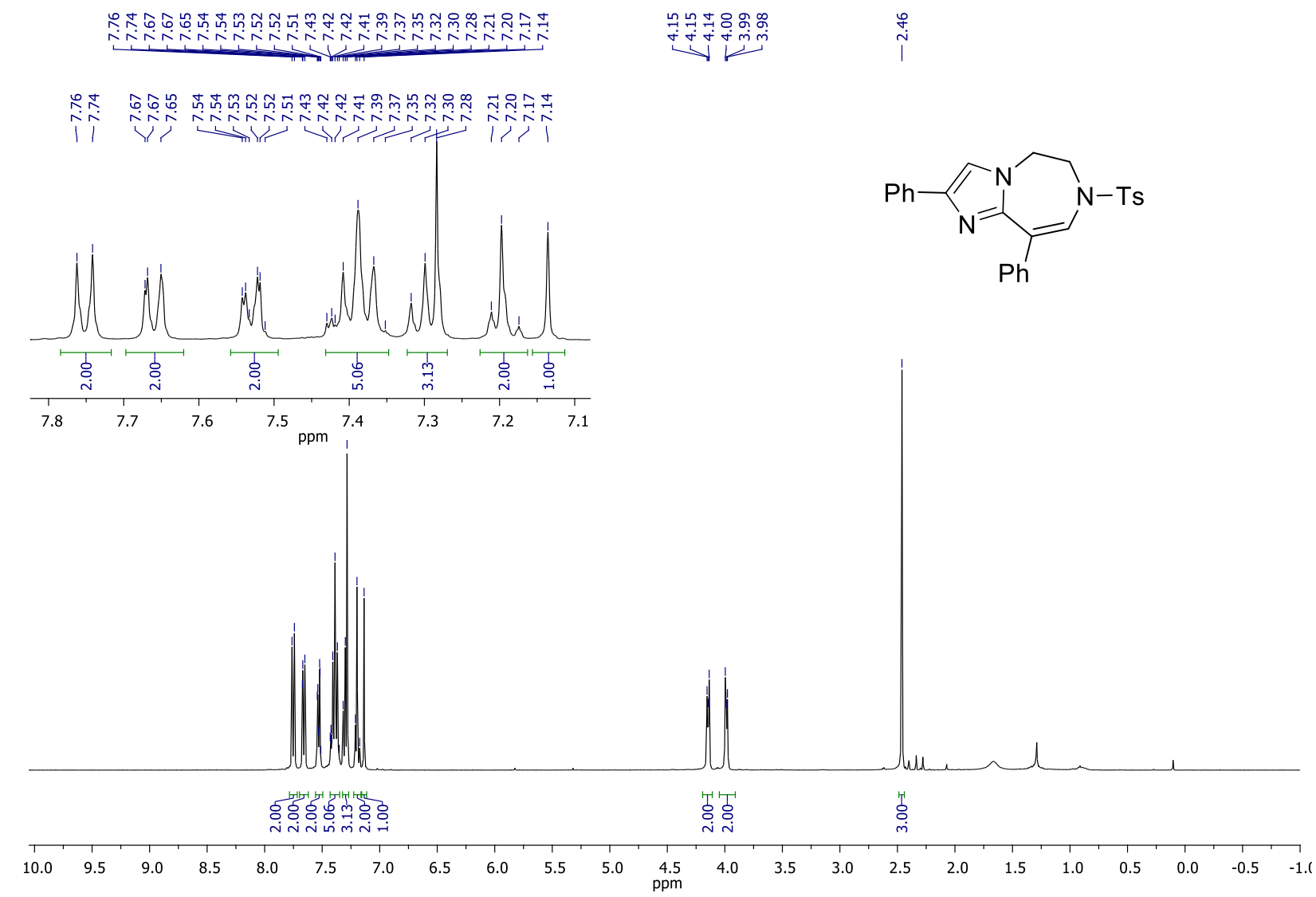

${ }^{13} \mathrm{C}\left\{{ }^{1} \mathrm{H}\right\}$ NMR spectrum of compound $16\left(100 \mathrm{MHz}, \mathrm{CDCl}_{3}\right)$
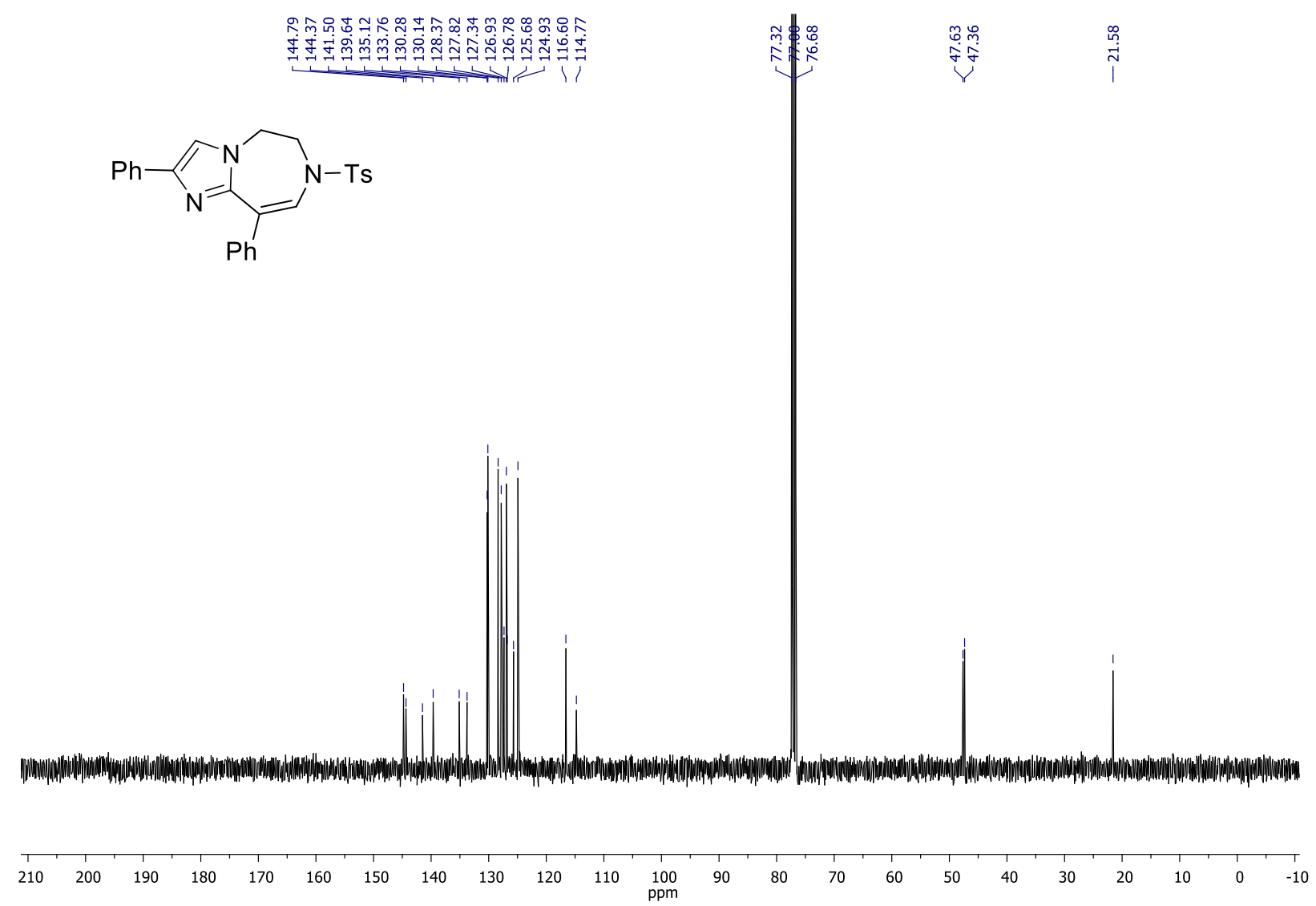
${ }^{1} \mathrm{H}$ NMR spectrum of compound $17\left(400 \mathrm{MHz}, \mathrm{CDCl}_{3}\right)$

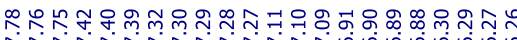

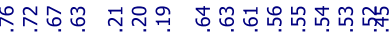

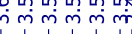<smiles>CNCC(c1ccccc1)c1nc(S)cn1Cc1ccccc1</smiles>

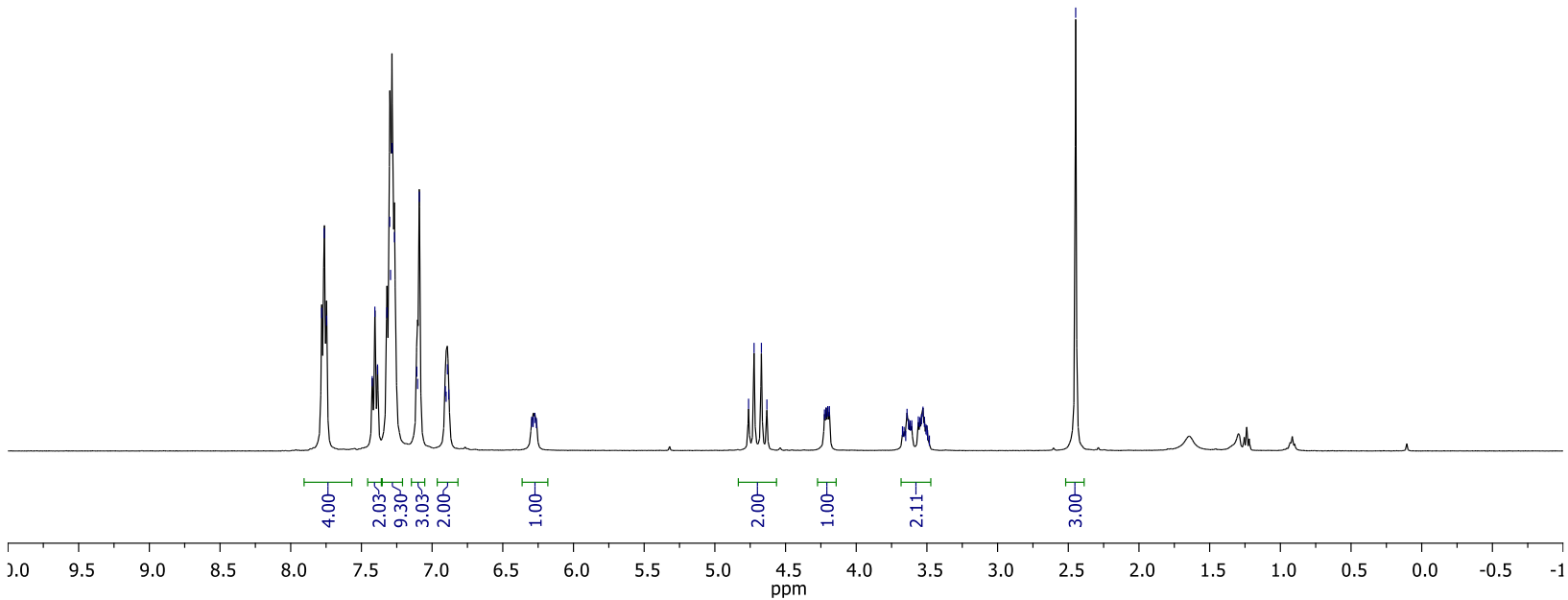

${ }^{13} \mathrm{C}\left\{{ }^{1} \mathrm{H}\right\}$ NMR spectrum of compound $17\left(100 \mathrm{MHz}, \mathrm{CDCl}_{3}\right)$

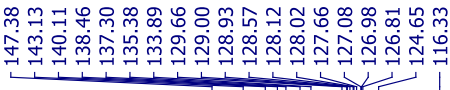<smiles>[As]NCC(c1ccccc1)c1nc(-c2ccccc2)cn1Cc1ccccc1</smiles>

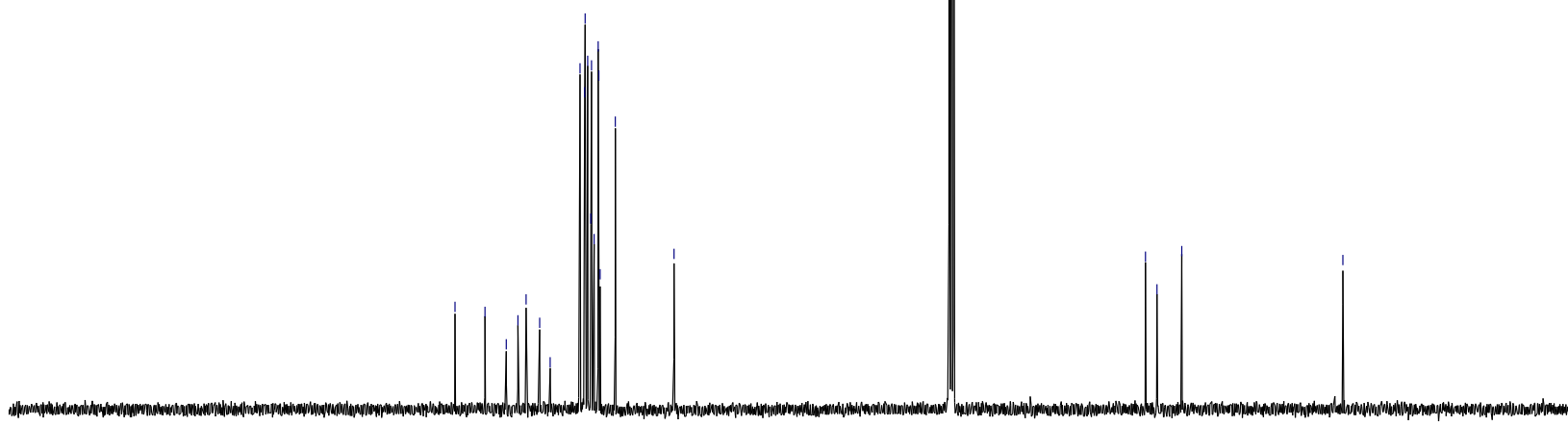

$\begin{array}{llllllllllllllllllllllll}10 & 200 & 190 & 180 & 170 & 160 & 150 & 140 & 130 & 120 & 110 & 100 & 90 & 80 & 70 & 60 & 50 & 40 & 30 & 20 & 10 & 0 & -1\end{array}$ 
${ }^{1} \mathrm{H}$ NMR spectrum of compound $18\left(400 \mathrm{MHz}, \mathrm{CDCl}_{3}\right)$

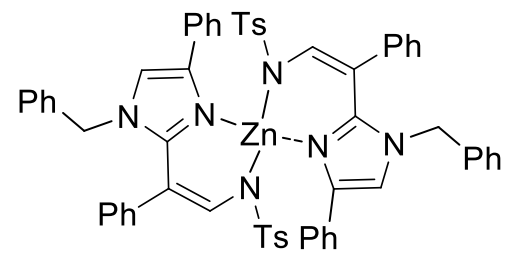

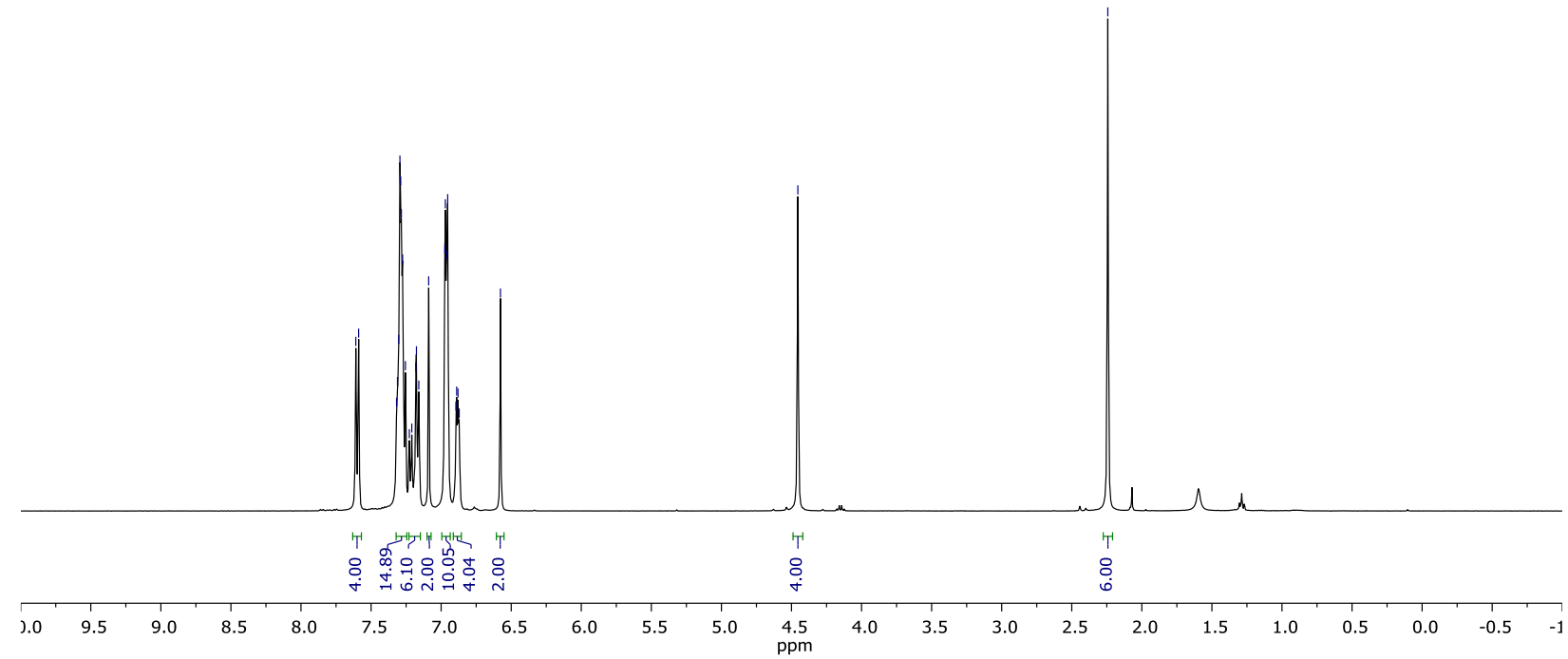

${ }^{13} \mathrm{C}\left\{{ }^{1} \mathrm{H}\right\}$ NMR spectrum of compound $18\left(100 \mathrm{MHz}, \mathrm{CDCl}_{3}\right)$

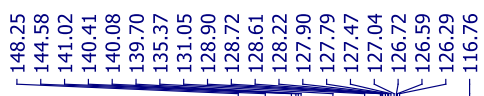<smiles></smiles>
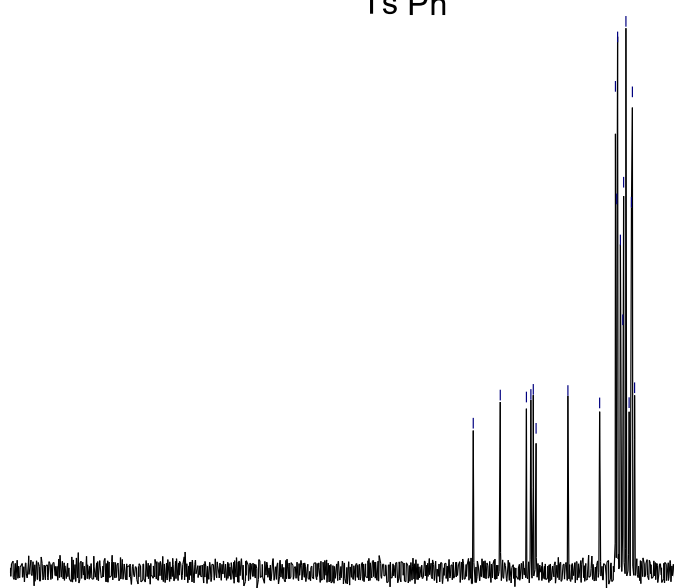

$\begin{array}{lllllllllll}210 & 200 & 190 & 180 & 170 & 160 & 150 & 140 & 130 & 120 & 110\end{array}$ 100
$\mathrm{ppm}$ 
${ }^{1} \mathrm{H}$ NMR spectrum of compound 19a $\left(400 \mathrm{MHz}, \mathrm{CDCl}_{3}\right)$

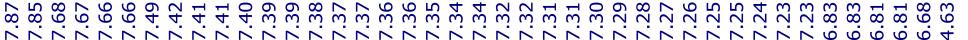

U

$\stackrel{\substack{i \\ i}}{i}$<smiles>FB1Cn2cc(-c3ccccc3)nc2C(c2ccccc2)=CN1[12F]</smiles>

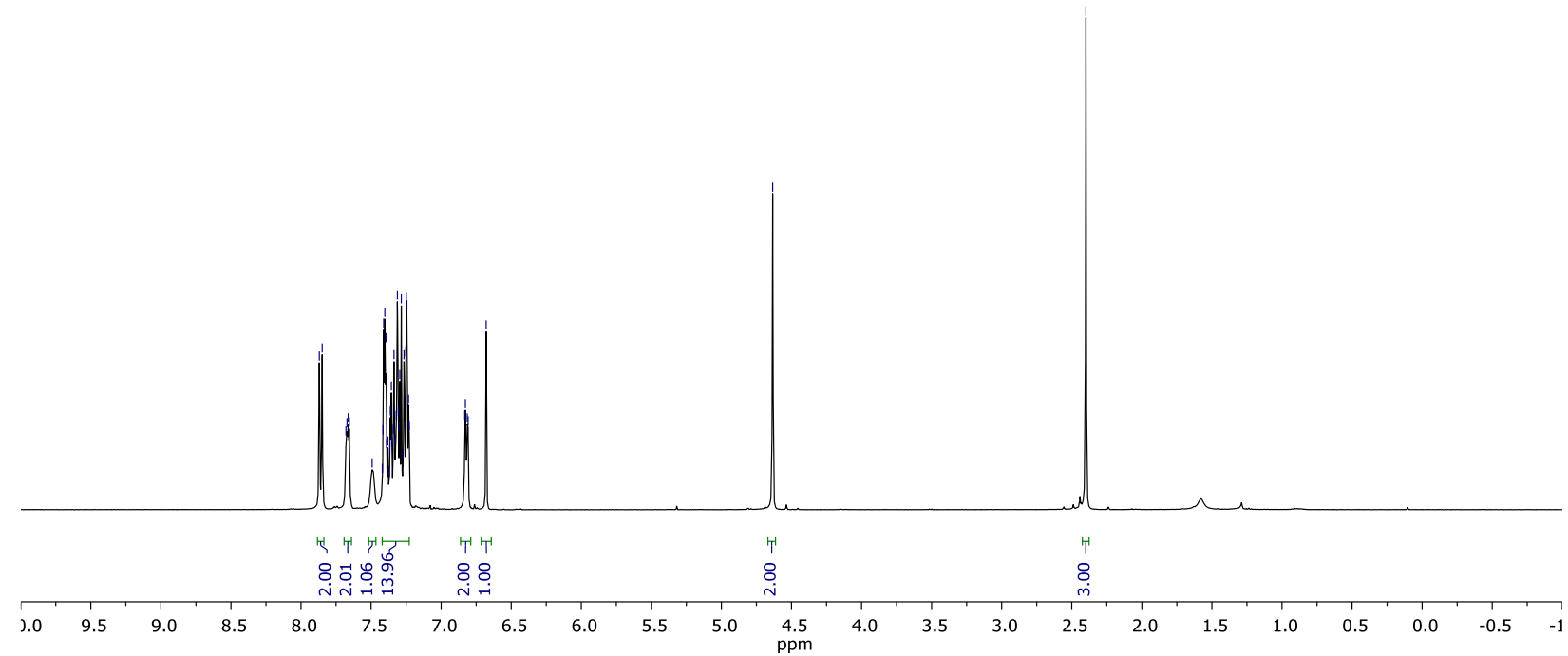

${ }^{13} \mathrm{C}\left\{{ }^{1} \mathrm{H}\right\}$ NMR spectrum of compound 19a (100 $\left.\mathrm{MHz}, \mathrm{CDCl}_{3}\right)$

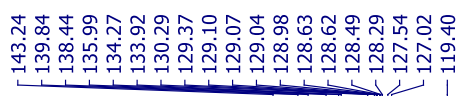

옹

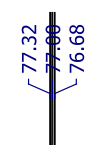

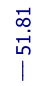

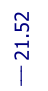<smiles></smiles>

|||||||||||| $\mid$

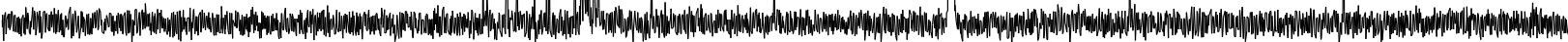

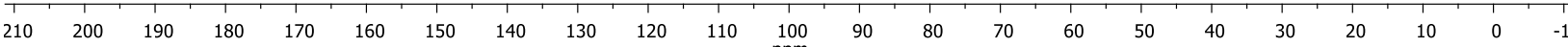


${ }^{19} \mathrm{~F}\left\{{ }^{1} \mathrm{H}\right\}$ NMR spectrum of compound 19a $\left(376.5 \mathrm{MHz}, \mathrm{CDCl}_{3}\right)$<smiles></smiles> 
${ }^{1} \mathrm{H}$ NMR spectrum of compound $19 b\left(400 \mathrm{MHz}, \mathrm{CDCl}_{3}\right)$

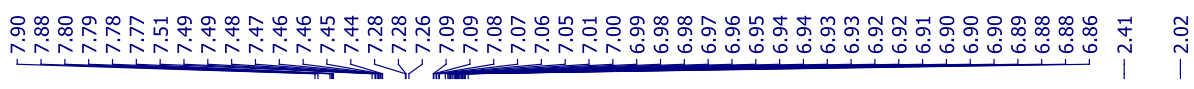<smiles>Cc1ccccc1-n1cc(-c2ccccc2)nc1-c1ccccc1</smiles>

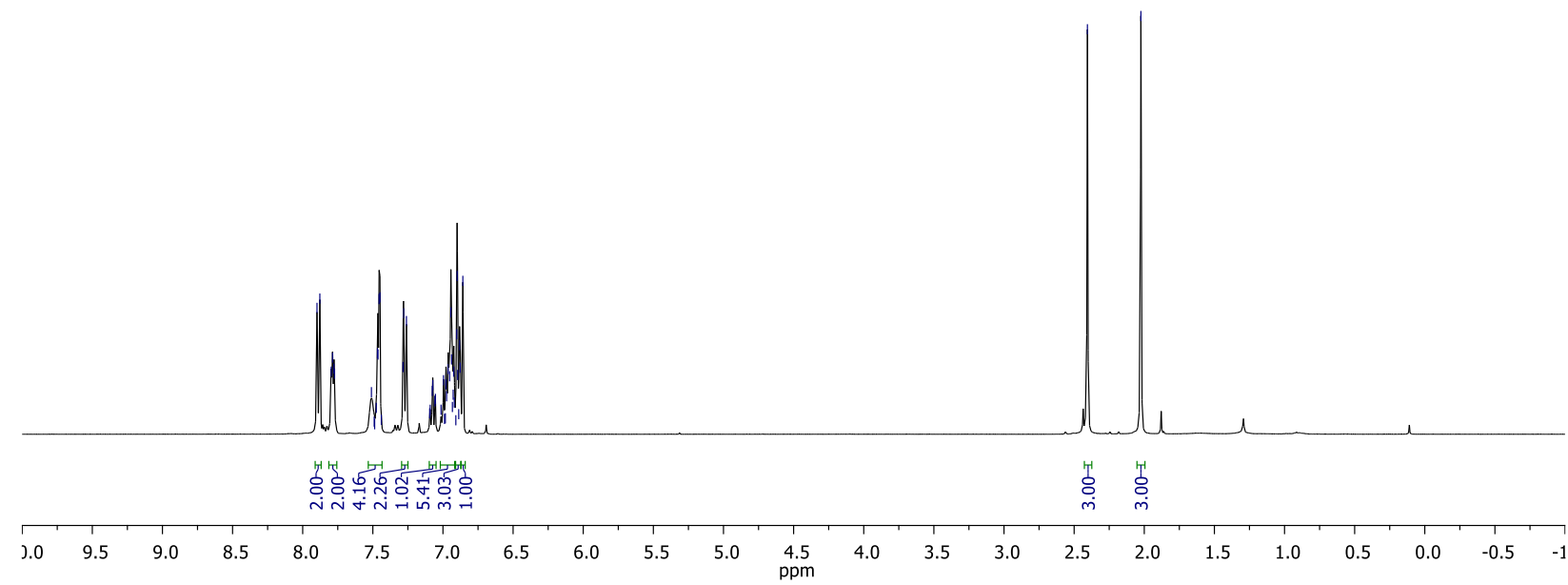

${ }^{13} \mathrm{C}\left\{{ }^{1} \mathrm{H}\right\}$ NMR spectrum of compound $19 b\left(100 \mathrm{MHz}, \mathrm{CDCl}_{3}\right)$

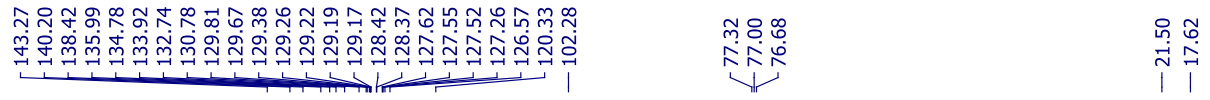<smiles>Cc1ccccc1N1C=C(c2ccccc2)N2NB1C(c1ccccc1)=CN([12F])B2F</smiles>

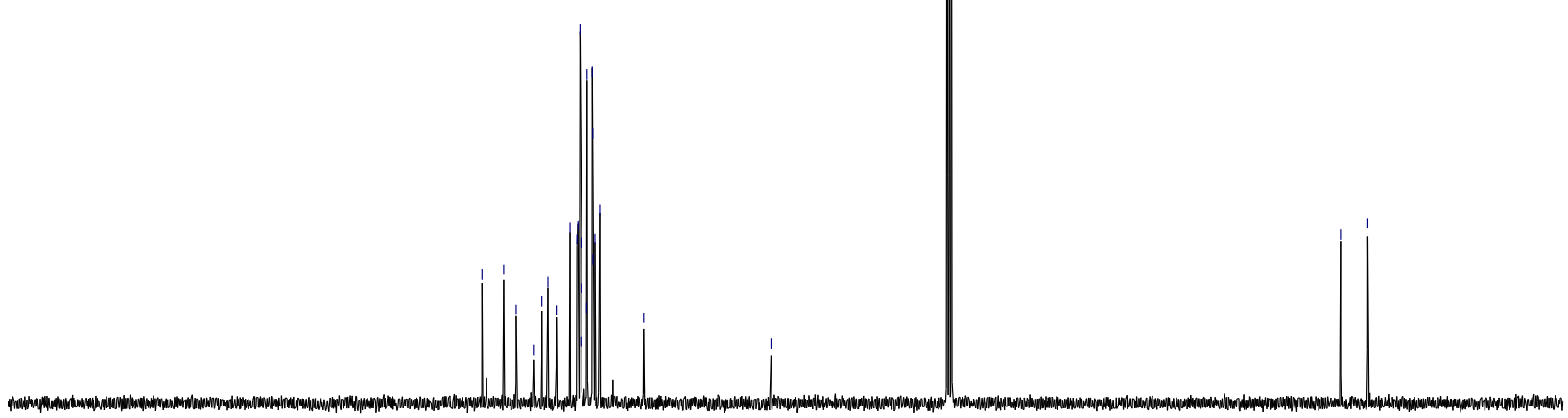

$\begin{array}{llllllllllllllllllllllll}10 & 200 & 190 & 180 & 170 & 160 & 150 & 140 & 130 & 120 & 110 & 100 & 90 & 80 & 70 & 60 & 50 & 40 & 30 & 20 & 10 & 0 & -1\end{array}$ 
${ }^{19} \mathrm{~F}\left\{{ }^{1} \mathrm{H}\right\}$ NMR spectrum of compound $\mathbf{1 9 b}\left(376.5 \mathrm{MHz}, \mathrm{CDCl}_{3}\right)$

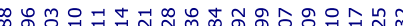

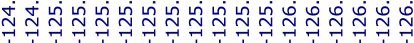<smiles></smiles>

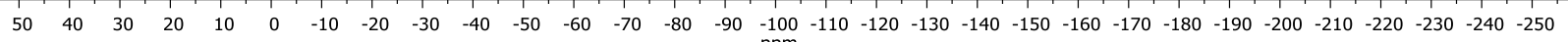


${ }^{1} \mathrm{H}$ NMR spectrum of compound $19 \mathrm{c}\left(400 \mathrm{MHz}, \mathrm{CDCl}_{3}\right)$

$$
\text { 至 }
$$
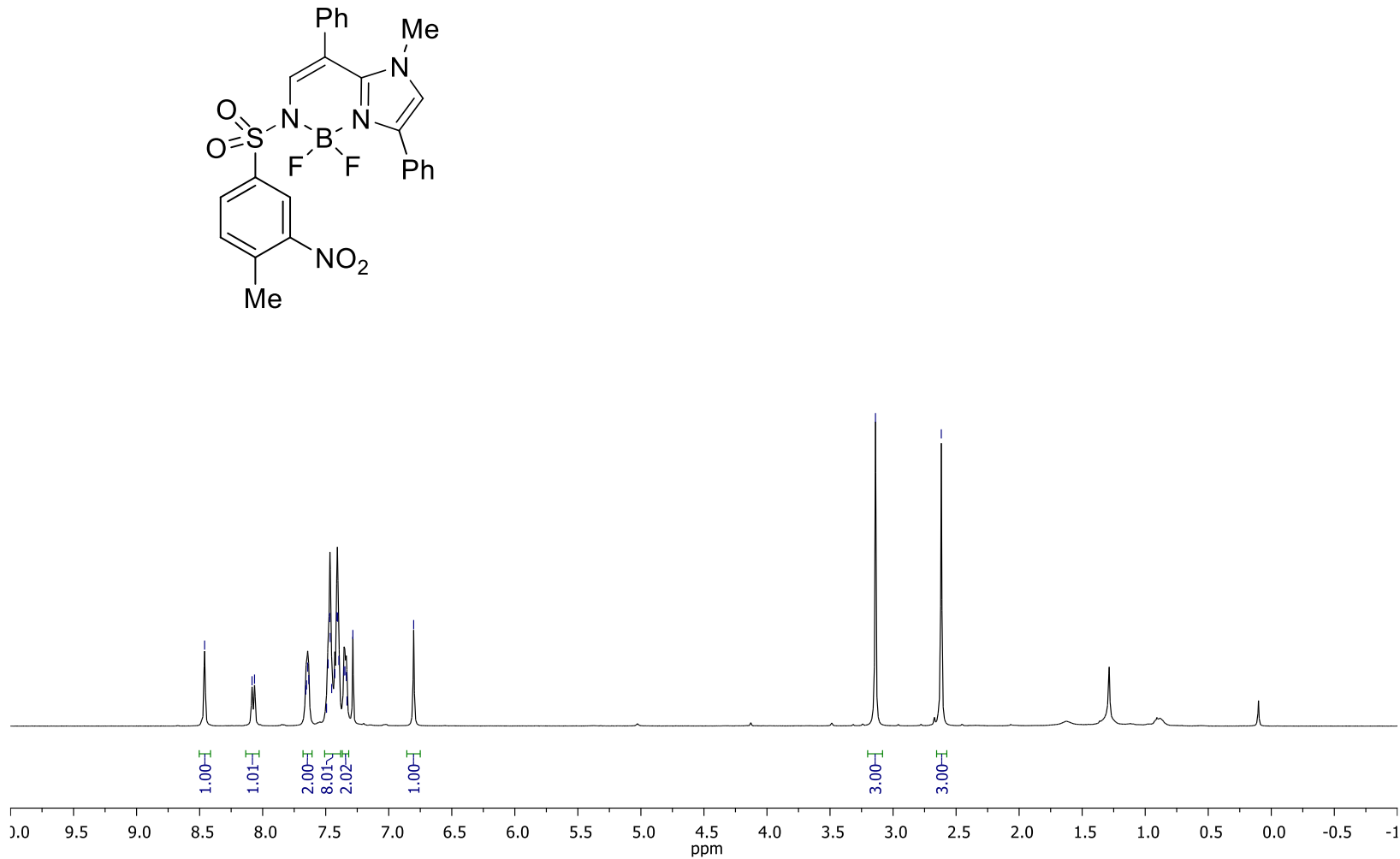

${ }^{13} \mathrm{C}\left\{{ }^{1} \mathrm{H}\right\}$ NMR spectrum of compound $19 \mathrm{c}\left(100 \mathrm{MHz}, \mathrm{CDCl}_{3}\right)$
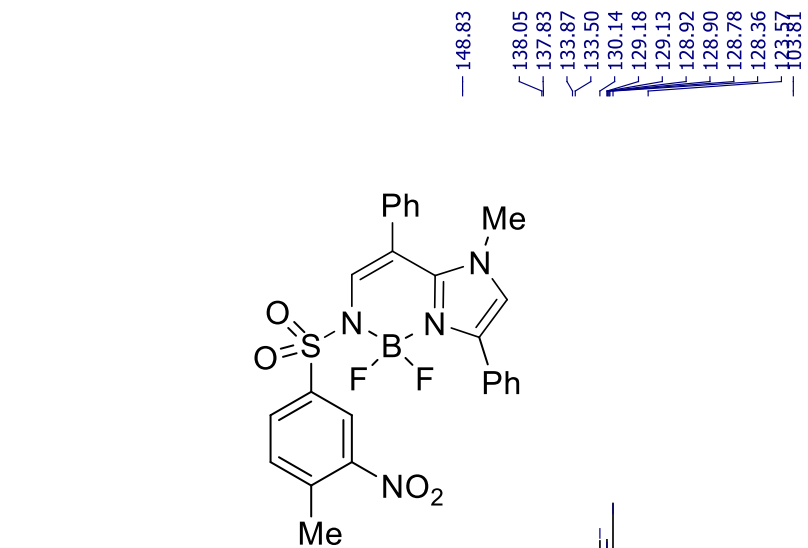

$\begin{array}{lllllllllllllllllllllll}10 & 200 & 190 & 180 & 170 & 160 & 150 & 140 & 130 & 120 & 110 & 100 & 90 & 80 & 70 & 60 & 50 & 40 & 30 & 20 & 10 & 0 & -1\end{array}$ 
${ }^{19} \mathrm{~F}\left\{{ }^{1} \mathrm{H}\right\}$ NMR spectrum of compound 19c $\left(376.5 \mathrm{MHz}, \mathrm{CDCl}_{3}\right)$

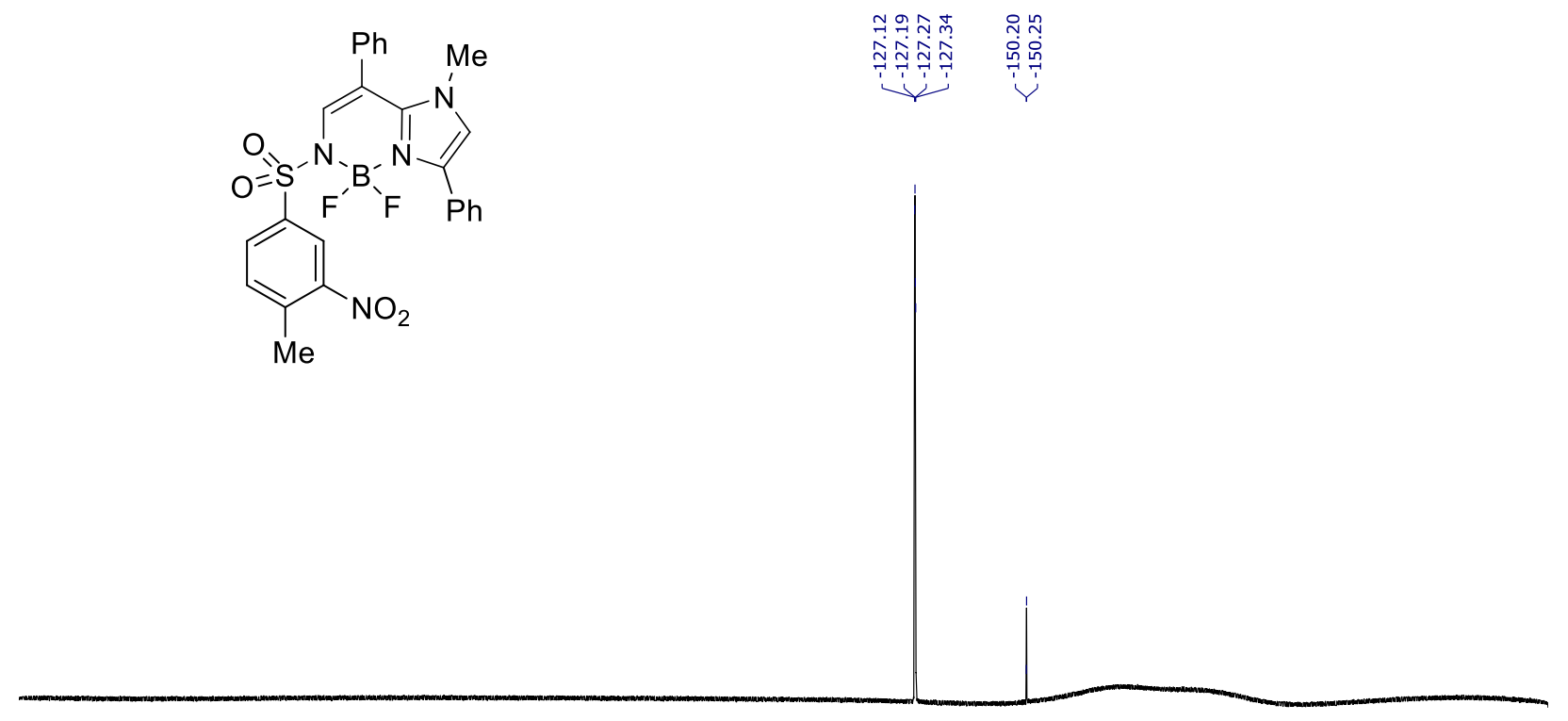

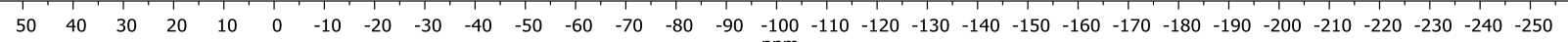

